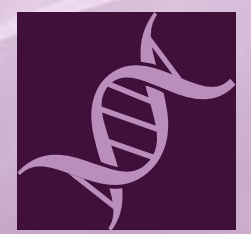

International Journal of Molecular Sciences

Special Protein
Molecules

Computational Identification

Edited by Quan Zou Printed Edition of the Special Issue Published in IJMS 


\section{Special Protein Molecules \\ Computational Identification}





\section{Special Protein Molecules Computational Identification}

Special Issue Editor Quan Zou 
Special Issue Editor

Quan Zou

Tianjin University

China

\section{Editorial Office}

MDPI

St. Alban-Anlage 66

Basel, Switzerland

This is a reprint of articles from the Special Issue published online in the open access journal International Journal of Molecular Sciences (ISSN 1422-0067) from 2017 to 2018 (available at: http:// www.mdpi.com/journal/ijms/special_issues/special_protein_computation)

For citation purposes, cite each article independently as indicated on the article page online and as indicated below:

LastName, A.A.; LastName, B.B.; LastName, C.C. Article Title. Journal Name Year, Article Number, Page Range.

ISBN 978-3-03897-043-9 (Pbk)

ISBN 978-3-03897-044-6 (PDF)

Articles in this volume are Open Access and distributed under the Creative Commons Attribution (CC BY) license, which allows users to download, copy and build upon published articles even for commercial purposes, as long as the author and publisher are properly credited, which ensures maximum dissemination and a wider impact of our publications. The book taken as a whole is (C) 2018 MDPI, Basel, Switzerland, distributed under the terms and conditions of the Creative Commons license CC BY-NC-ND (http:/ / creativecommons.org/licenses/by-nc-nd/4.0/). 


\section{Contents}

About the Special Issue Editor $\ldots \ldots \ldots \ldots \ldots \ldots \ldots \ldots$ vii

Quan Zou and Wenying He

Special Protein Molecules Computational Identification

Reprinted from: Int. J. Mol. Sci. 2018, 19, 536, doi: 10.3390/ijms19020536 . . . . . . . . . . . . .

Yanbin Wang, Zhuhong You, Xiao Li, Xing Chen, Tonghai Jiang and Jingting Zhang

PCVMZM: Using the Probabilistic Classification Vector Machines Model Combined with a

Zernike Moments Descriptor to Predict Protein-Protein Interactions from Protein Sequences

Reprinted from: Int. J. Mol. Sci. 2017, 18, 1029, doi: 10.3390/ijms18051029 . . . . . . . . . . . 10

Shiheng Lu, Yan Yan, Zhen Li, Lei Chen, Jing Yang, Yuhang Zhang, Shaopeng Wang and Lin Liu

Determination of Genes Related to Uveitis by Utilization of the Random Walk with Restart Algorithm on a Protein-Protein Interaction Network

Reprinted from: Int. J. Mol. Sci. 2017, 18, 1045, doi: 10.3390/ijms18051045 . . . . . . . . . . . . .

Haitao Ding, Fen Gao, Yong Yu and Bo Chen

Biochemical and Computational Insights on a Novel Acid-Resistant and Thermal-Stable Glucose 1-Dehydrogenase

Reprinted from: Int. J. Mol. Sci. 2017, 18, 1198, doi: 10.3390/ijms18061198 . . . . . . . . . . . . .

Qiu-Li Hou, Jin-Xiang Luo, Bing-Chuan Zhang, Gao-Fei Jiang, Wei Ding and Yong-Qiang Zhang

3D-QSAR and Molecular Docking Studies on the TcPMCA1-Mediated Detoxification of Scopoletin and Coumarin Derivatives

Reprinted from: Int. J. Mol. Sci. 2017, 18, 1380, doi: 10.3390/ijms18071380 . . . . . . . . . . . . . 58

Tonghui Huang, Jie Sun, Shanshan Zhou, Jian Gao and Yi Liu

Identification of Direct Activator of Adenosine Monophosphate-Activated Protein Kinase

(AMPK) by Structure-Based Virtual Screening and Molecular Docking Approach

Reprinted from: Int. J. Mol. Sci. 2017, 18, 1408, doi: 10.3390/ijms18071408 . . . . . . . . . . . . . 82

Bożena Futoma-Kołoch, Bartłomiej Dudek, Katarzyna Kapczyńska, Eva Krzyżewska, Kamila Korzekwa, Jacek Rybka, Elżbieta Klausa, Martyna Wańczyk and Gabriela Bugla-Płoskońska

Relationship of Triamine-Biocide Tolerance of Salmonella enterica Serovar Senftenberg to Antimicrobial Susceptibility, Serum Resistance and Outer Membrane Proteins

Reprinted from: Int. J. Mol. Sci. 2017, 18, 1459, doi: 10.3390/ijms18071459 . . . . . . . . . . . . .

Jinjian Jiang, Nian Wang, Peng Chen, Chunhou Zheng and Bing Wang

Prediction of Protein Hotspots from Whole Protein Sequences by a Random Projection

Ensemble System

Reprinted from: Int. J. Mol. Sci. 2017, 18, 1543, doi: 10.3390/ijms18071543 . . . . . . . . . . . . . 109

Cong Shen, Yijie Ding, Jijun Tang, Xinying Xu and Fei Guo

An Ameliorated Prediction of Drug-Target Interactions Based on Multi-Scale Discrete Wavelet

Transform and Network Features

Reprinted from: Int. J. Mol. Sci. 2017, 18, 1781, doi: 10.3390/ijms18081781 . . . . . . . . . . . . 122 
Jennifer C. Chandler, Neha S. Gandhi, Ricardo L. Mancera, Greg Smith, Abigail Elizur and Tomer Ventura

Understanding Insulin Endocrinology in Decapod Crustacea: Molecular Modelling Characterization of an Insulin-Binding Protein and Insulin-Like Peptides in the Eastern Spiny Lobster, Sagmariasus verreauxi

Reprinted from: Int. J. Mol. Sci. 2017, 18, 1832, doi: 10.3390/ijms18091832 . . . . . . . . . . . . 137

Ya-Wei Zhao, Zhen-Dong Su, Wuritu Yang, Hao Lin, Wei Chen and Hua Tang

IonchanPred 2.0: A Tool to Predict Ion Channels and Their Types

Reprinted from: Int. J. Mol. Sci. 2017, 18, 1838, doi: 10.3390/ijms18091838 . . . . . . . . . . . . . 156

Jun Zhang and Bin Liu

PSFM-DBT: Identifying DNA-Binding Proteins by Combing Position Specific Frequency Matrix and Distance-Bigram Transformation

Reprinted from: Int. J. Mol. Sci. 2017, 18, 1856, doi: 10.3390/ijms18091856 . . . . . . . . . . . . . 166

Min Li, Dongyan Li, Yu Tang, Fangxiang Wu and Jianxin Wang

CytoCluster: A Cytoscape Plugin for Cluster Analysis and Visualization of Biological Networks Reprinted from: Int. J. Mol. Sci. 2017, 18, 1880, doi: 10.3390/ijms18091880 . . . . . . . . . . . . . . 182

Bo Li, and Bo Liao

Protein Complexes Prediction Method Based on Core-Attachment Structure and Functional Annotations

Reprinted from: Int. J. Mol. Sci. 2017, 18, 1910, doi: 10.3390/ijms18091910 . . . . . . . . . . . . 195

Kirill S. Antonets and Anton A. Nizhnikov

Predicting Amyloidogenic Proteins in the Proteomes of Plants

Reprinted from: Int. J. Mol. Sci. 2017, 18, 2155, doi: 10.3390/ijms18102155 . . . . . . . . . . . . 211

Jun Wang, Long Zhang, Lianyin Jia, Yazhou Ren and Guoxian Yu

Protein-Protein Interactions Prediction Using a Novel Local Conjoint Triad Descriptor of Amino

Acid Sequences

Reprinted from: Int. J. Mol. Sci. 2017, 18, 2373, doi: 10.3390/ijms18112373 . . . . . . . . . . . . 232

Pu-Feng Du, Wei Zhao, Yang-Yang Miao, Le-Yi Wei and Likun Wang

UltraPse: A Universal and Extensible Software Platform for Representing Biological Sequences

Reprinted from: Int. J. Mol. Sci. 2017, 18, 2400, doi: 10.3390/ijms18112400 . . . . . . . . . . . . 249

Shunfang Wang, Bing Nie, Kun Yue, Yu Fei, Wenjia Li and Dongshu Xu

Protein Subcellular Localization with Gaussian Kernel Discriminant Analysis and Its Kernel

Parameter Selection

Reprinted from: Int. J. Mol. Sci. 2017, 18, 2718, doi: 10.3390/ijms18122718 . . . . . . . . . . . . 261

Chun Yan Yu, Xiao Xu Li, Hong Yang, Ying Hong Li, Wei Wei Xue, Yu Zong Chen, Lin Tao and Feng Zhu

Assessing the Performances of Protein Function Prediction Algorithms from the Perspectives of Identification Accuracy and False Discovery Rate

Reprinted from: Int. J. Mol. Sci. 2018, 19, 183, doi: 10.3390/ijms19010183 . . . . . . . . . . . . 277 


\section{About the Special Issue Editor}

Quan Zou, professor, received his Ph.D. from the Harbin Institute of Technology, P.R. China, in 2009. From 2009 to 2015, he was an assistant and associate professor at Xiamen University, P.R. China. He now works in the school of computer science and technology at Tianjin University. His research is focused on the areas of bioinformatics, machine learning and parallel computing. Several related works have been published in Science, Briefings in Bioinformatics, Bioinformatics, etc. Google scholar shows that his more than 100 papers have been cited over 4000 times (as of June, 2018). 



\title{
Editorial
}

\section{Special Protein Molecules Computational Identification}

\author{
Quan Zou *and Wenying He \\ School of Computer Science and Technology, Tianjin University, Tianjin 300354, China; hwying1234@tju.edu.cn \\ * Correspondence: zouquan@tju.edu.cn
}

Received: 16 January 2018; Accepted: 10 February 2018; Published: 10 February 2018

\begin{abstract}
Computational identification of special protein molecules is a key issue in understanding protein function. It can guide molecular experiments and help to save costs. I assessed 18 papers published in the special issue of Int. J. Mol. Sci., and also discussed the related works. The computational methods employed in this special issue focused on machine learning, network analysis, and molecular docking. New methods and new topics were also proposed. There were in addition several wet experiments, with proven results showing promise. I hope our special issue will help in protein molecules identification researches.
\end{abstract}

Keywords: bioinformatics; machine learning; feature selection; protein classification; network analysis; molecular docking

\section{Introduction}

With the development of next generation sequencing technologies, the size of biological databases have increased dramatically in terms of the number of samples. It is fast and cheap to obtain biological sequences but relatively slow and expensive to extract function information because of limitations of traditional biological experimental technologies. Protein, as the product of gene expression and the important material basis of life activity, participates in almost all life activities and biological processes. For some special protein molecules, the detection of new ones is time-consuming and costly. Some special proteins are present, such as cytokines, enzymes, cell-penetrating peptides, anticancer peptides, cancerlectins, and $G$ protein-coupled receptors. In order to save the wet experimental costs, researches first select some candidates through computer programs. The "computer program" is the key step in selecting candidates. High false positive software would lead to high spending on the validation process.

In this special issue, these "computer program" approaches and algorithms are discussed. Numerous sequence-based "golden features" have been proposed for these problems, such as Chou's PseAAC. Ever since the concept of PseAAC was proposed, it has penetrated into nearly all fields of protein identification. However, it is suggested that special features and classification methods should be proposed for special protein molecular. "Golden features" could hardly apply to all kinds of proteins. In this special issue, submissions focused on a kind of special protein molecules, collected related data sets, got better prediction performance (especially low false positive), and developed friendly software tools or web servers.

We received 36 submissions. After rigorous reviewing process, 18 papers were published. They come from different countries, including China, Russia, Canada, Australia, USA, Poland, etc. These papers could be categorized into three subtopics. As shown in Figure 1. 


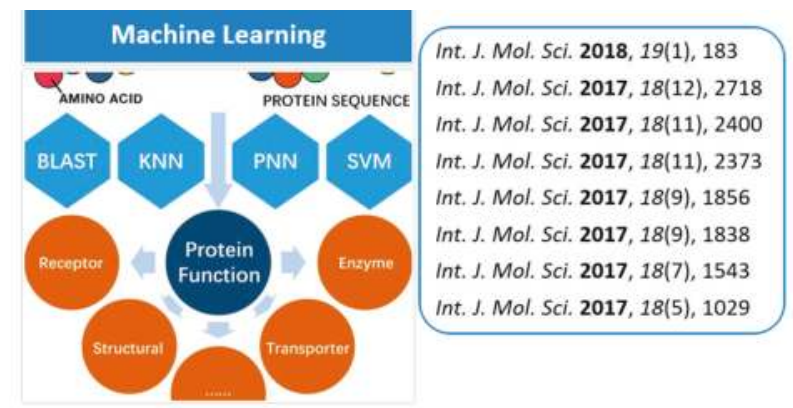

Int. J. Mol. Sci. 2017, 18(10), 2155 Int. J. Mol. Sci. 2017, 18(9), 1832 Int. J. Mol. Sci. 2017, 18(7), 1459 Int. J. Mol. Sci. 2017, 18(7), 1408 int. J. Mol, Sci. 2017, 18(7), 1380 int. J. Mol. Sci. 2017, 18(6), 1198
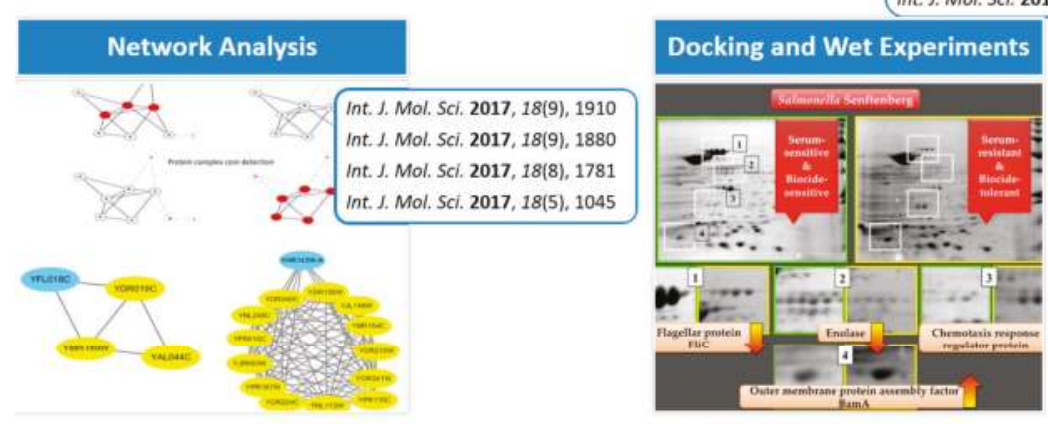

Figure 1. Subtopics of our special issue $[38,52,63]$.

\section{Machine Learning Related Researches}

\subsection{Protein-Protein Interaction Prediction}

The first subtopic is to identify or predict protein function with machine learning methods. Two papers focused on protein-protein interaction prediction. Protein-protein interactions (PPIs) play crucial roles in almost all cellular processes. Correctly predicting protein-protein interactions contributes to precise protein function prediction [1,2]. Most of them focus on the PPIs predictions from various data types, including 3D structural information, gene ontology and annotations, and gene fusion. Wang et al. [3] proposed a sequence-based approach (DNN-LCTD) combining deep neural networks (DNN) and Local Conjoint Triad Description (LCTD) feature representation. Experimental results showed that DNN-LCTD is very promising for predicting PPIs. Wang et al. [4] using the Zernike moments (ZM) descriptor on the PSSM combined with Probabilistic Classification Vector Machines (PCVM) classifier developed the PCVMZM predictor for predicting the PPIs from protein amino acids sequences. It was proved to be a robust, powerful and feasible PPI prediction method. Ding et al. [5] developed a random forest algorithm based predictor using a multivariate mutual information feature representation scheme and normalized Moreau-Broto Autocorrelation information from protein sequence. Another work [6] is a novel matrix-based protein sequence representation approach to identify PPIs, using an ensemble learning method for classification. The matrix of Amino Acid Contact (AAC) was constructed based on the statistical analysis of residue-pairing frequencies in a data-set of 6323 protein-protein complexes. The feature vector was extracted by applying algorithms of Histogram of Oriented Gradient (HOG) and Singular Value Decomposition (SVD) on the Substitution Matrix Representation (SMR) matrix of protein sequence.

Drug-target interaction is a special PPI. Because the experimental prediction of drug-target interaction (DTIs) is time-consuming and expensive, computational technology with high accuracy plays a crucial rule in the large-scale rapid prediction of DTIs. Shen et al. [7] proposed DAWN a kind 
of Drug-target interactions predictor combining discrete Wavelet transform and Network features. Most importantly, DAWN as a kind of machine learning approach of feature vector-based method, has the desired effect under the condition of without network information. In the same year, they also developed the second tool [8] using molecular substructure fingerprints, Multivariate Mutual Information (MMI) of proteins, and network topology.

Hotspot has important significance in the determination of protein-protein interactions [9]. Many methods have been developed for the hotspot predictions $[10,11]$ and even protein binding site predictions [12]. Most of the works focused on the hotspot predictions from a curated small partial dataset of the whole protein sequences [13]. In Jiang's work [14], the issue of hotspot determination was approached from whole natural protein sequences, and a random projection ensemble system based on $\mathrm{k}$ nearest neighbor algorithm to identify hotspot residues by sequence information alone was developed. Experimental results showed that although this method did not perform well enough in the real applications of hotspots, it was very promising in the determination of hotspot residues from whole sequences.

\subsection{Special Proteins Identification}

Besides protein-protein interaction, DNA binding proteins, ion channel proteins, and amyloids have also attracted researchers' attentions. DNA binding protein is a kind of special protein molecule, whose identification is one of the most important tasks in studying the function of proteins. In this regard, many computational predictors have been proposed [15-21]. In a special issue, Zhang et al. [22] proposed a new approach to extract evolutionary information from the Position Specific Frequency Matrix (PSFM) and incorporate the evolutionary information, and a computational predictor was proposed for DNA binding protein identification. Experimental results showed that this predictor outperformed some existing state-of-the-art approaches in this field. DNA-protein interactions play a key role in a variety of biological processes, especially in cellular metabolism. Endowed with a ditto multi-scale idea in essence, Shen et al. [23] addressed a kind of competitive method called Multi-scale Local Average Blocks (MLAB) algorithm. Different from the structure-based route, MLAB exploited a strategy that not only extracted local evolutionary information from primary sequence, but also used predicted solvent accessibility. Moreover, the construction on the predictor of DNA-protein binding sites wields an ensemble weighted sparse representation model with random under-sampling.

Ion channels are membrane proteins which are widely distributed in all cells. They have been shown to be extensively involved in various physiological and pathological processes, including regulating neuronal and cardiac excitability, muscle contraction, hormone secretion, fluid movement, and immune cell activation. Different ion channels play their unique roles in different biological processes. With the rapid development of next-generation sequencing technologies, the accumulation of proteomic data provides uswith a platform to systematically investigate and predict ion channels and their types. Several studies have focused on the prediction of ion channels and their types [24-26]. The paper published in the special issue [27] proposed a new prediction model to quickly predict ion channels and their types. An improved feature extraction method combining dipeptide composition with the physicochemical property correlation between two residues was developed to formulate protein samples. Subsequently, the analysis of variance (ANOVA) combined with the incremental feature selection (IFS) was employed to find out the optimal features which can produce the maximum accuracy. As a result, authors achieved the overall accuracies of $87.8 \%$ for discriminating ion channels from non-ion channels, $94.0 \%$ for distinguishing between voltage-gated ion channel and ligand-gated ion channels and $92.6 \%$ for four types of voltage-gated ion channels, respectively. Based on the proposed models, a web server called IonchanPred 2.0 (http://lin.uestc.edu.cn/server/IonchanPredv2.0) was established. The free predictor will be most useful to most wet-experimental scholars. A few groups have focused on the outer membrane protein recently, Wang et al. introduced the predicted topology structure as a mainly structure-specific 
feature to this classical type of ion channel protein, improved the precision of outer membrane identification [28], inter-barrel contact prediction [29] and fold recognition [30].

In this special issue, Antonets et al. [31] detected amyloidogenic proteins in the proteomes of plants. Amyloids are protein fibrils with characteristic spatial structure. The main computational method for them was phylogenetic analysis together with machine learning techniques. This kind of protein also includes DNA and RNA binding ones, which showed that different kinds of proteins have comment characters. To summarize, effective protein features and machine learning techniques are still essential and challenging in the future.

\subsection{Protein Subcellular Localization and Function Analysis}

Besides PPI, special proteins, protein subcellular localization, and function prediction are traditional challenges and attract researchers. In general, only when the protein is located in the correct subcellular location, can the protein function normally. Therefore, prediction of protein subcellular localization is an important component of proteomics, and it can aid the identification of drug targets. Due to the technical limitation and high cost of time and money in traditional experimental methods, research on protein subcellular location annotation with the machine learning technique has become a focused research problem in bioinformatics. When we use machine learning technologies to predict protein subcellular location, we need to extract the features of protein sequences, and then use the classifier to realize the protein classification. Thus feature extraction and dimension reduction are important techniques for analyzing the complex and high dimensional biological data in protein subcellular location. In order to improve the prediction accuracy of protein subcellular location, an appropriate algorithm for reducing data dimension should be used before classification. Wang et al. [32] proposed two feature fusion expressions and then used the linear discriminant analysis (LDA) method for dimension reduction. Considering the general nonlinear property in protein sequence data, they [33] introduced the nonlinear kernel discriminant analysis (KDA) method to reduce the high dimensionality in some feature data in this special issue. In this paper, an improved Gauss kernel parameter selection algorithm was proposed to predict subcellular location. It was proposed by maximizing the differences of reconstruction errors between edge normal samples and internal normal samples. The proposed method did not only show the same effect as traditional methods, but also reduced the computational time and improved the efficiency. It should be noted that LDA and KDA methods cannot only reduce the data dimensionality, but also take use of some classification information in the data, resulting in an ideal classification effect. Besides, there have been some new dimensional reduction algorithms which have been tried in other pattern recognition fields, such as face recognition [34].

Knowledge of protein function is the key to the understanding of the biological process and disease development and to the discovery of new therapeutic targets [35]. Various in-silico methods have been developed for protein function prediction [36], which complement one another due to their distinct underlying theory [37]. A comprehensive comparison of the performances between those popular prediction algorithms was conducted based on the information from 93 functional protein families [38], which observed a substantially higher sensitivity of BLAST and a significantly reduced false discovery rate of machine learning.

Since machine learning is a key issue in protein research, it is essential to extract numerical features from the protein primary sequence. Some recent studies showed that evolutionary information and the sequence-order effects are very important for extracting the features of proteins [39,40]. In their special issue, Du et al. [41] developed the UltraPse program to convert biological sequences into digital features. Unlike the PseAAC-Builder [42] or PseAAC-General [43], the UltraPse program can be used on DNA/RNA sequences as well as protein sequences. The program is a good starting point in predicting special protein functional characters, especially the exact subcellular localization of proteins [44]. 


\section{Network Techniques Related Researches}

Network analysis is also an important technique for protein identification and function research. Identification of disease genes is very important in medicine. For a disease, extracting its disease genes as completely as possible is helpful in understanding its pathogenesis, thereby designing effective treatments. To date, several network methods have been proposed to identify genes related to different diseases, such as the guilt by association (GBA) based method [45], the shortest path algorithm based method [46-48], the flow propagation algorithm [49], and the random walk with restart (RWR) algorithm based method [50]. In view of the fact that the RWR algorithm can make full use of the whole network, a RWR algorithm based method was proposed by Lu et al. [51] to identify disease genes of uveitis, a serious eye disease that may cause blindness in both young and middle-aged people. The method first applied the RWR algorithm on a protein-protein interaction (PPI) network using validated uveitis-related genes as seed nodes. Second, the obtained genes were filtered by a permutation test that can exclude false positive genes produced by the PPI network. Finally, they extracted important genes from the remaining genes by evaluating their associations to validate genes. Several putative genes were accessed and some have been determined to be important for the pathogenesis of uveitis.

Li et al. [52] employed the advanced network clustering algorithm for protein complex identification. Their method could detect the overlapping complex from the PPI network. Cluster analysis of biological networks is an important topic in systems biology. Up to now, a number of computational methods and tools have been proposed for analyzing biological networks and identifying protein complexes [53]. Various plugins based on cytoscape, such as CytoNCA [54], ClusterViz [55], DyNetViewer [56], CytoCtrlAnalyser [57], were developed to analyze biological networks from different perspectives. CytoCluster [58] in our special issue is a popular clustering tool which integrates six clustering algorithms and BinGO function. Since it was established in July 2013, CytoCluster has been downloaded more than 11,200 times from the Cytoscape App Store and has been applied to different biological networks analyses.

\section{Docking and Wet Experiments Researches}

Docking is still an interesting and hot topic in protein structure and function analysis, especially in the drug design process. Adenosine monophosphate-activated protein kinase (AMPK) plays a critical role in the regulation of energy metabolism. Huang et al. [59] employed molecular docking to get potential $\beta 1$-selective AMPK activators. Finally, 12 novel compounds were selected as potential starting points for the design of direct $\beta 1$-selective AMPK activators. Hou et al. [60] investigated the relationship between scopoletin structure and TCPMCA1(a gene name)-inhibiting activity of scopoletin and other 30 coumarin derivatives by employing docking and three-dimensional quantitative structure-activity relationships (3D-QSAR). This work offers additional insights into the mechanism underlying the interaction of scopoletin with TCPMCA1 gene. Together with this work, the other three works in this special issue also carried out wet experiments. Besides wet experiments, Ding et al. [61] completed bioinformatics analysis and molecular dynamics simulation on glucose 1-dehydrogenase (GDH). Chandler et al. [62] extracted insulin-binding protein and insulin-like peptides in the Eastern spiny lobster, Sagmariasus verreauxi. Molecular modelling, including docking, showed various interaction and regulation. Futoma-Koloch et al. [63] laid special stress on analyzing the relationship between triamine-biocide tolerance of Salmonella enterica serovar Senftenberg with antimicrobial susceptibility, serum resistance, and outer membrane proteins.

To conclude, papers in this special issue cover several emerging topics of computational identification and bioinformatics analysis of special protein molecules. We fervently hope that this particular issue will attract considerable interest in the relevant fields. We are grateful to Int. J. Mol. Sci. for providing the chance to organize this special issue. We also thank the reviewers for their efforts in guaranteeing the high quality of this special issue. Finally, we thank all those who contributed to this special issue. Int. J. Mol. Sci. has promised to continue with the same topic as a new special issue in 
2018. Besides special protein molecules, nucleic acids with special modifications identification (such as RNA m6A [64], protein phosphorylation [65] and methylation, etc.) will also be welcomed in the 2018 special issue. I hope more authors and readers will contribute, especially to the follow-up works from this special issue.

Acknowledgments: The work was supported by the Natural Science Foundation of China (No. 61771331).

Conflicts of Interest: The authors declare no conflict of interest.

\section{References}

1. Zeng, J; Zou, Q.; Wu, Y.; Li, D.; Liu, X. An Empirical Study of Features Fusion Techniques for Protein-protein Interaction Prediction. Curr. Bioinform. 2016, 11, 4-12. [CrossRef]

2. Garzón, J.I.; Deng, L.; Murray, D.; Shapira, S.; Petrey, D.; Honig, B. A computational interactome and functional annotation for the human proteome. eLife 2016, 5, e18715. [CrossRef] [PubMed]

3. Wang, J.; Zhang, L.; Jia, L.; Ren, Y.; Yu, G. Protein-protein Interactions Prediction Using a Novel Local Conjoint Triad Descriptor of Amino Acid Sequences. Int. J. Mol. Sci. 2017, 18, 2373. [CrossRef] [PubMed]

4. Wang, Y.; You, Z.; Li, X.; Chen, X.; Jiang, T.; Zhang, J. PCVMZM: Using the Probabilistic Classification Vector Machines Model Combined with a Zernike Moments Descriptor to Predict Protein-Protein Interactions from Protein Sequences. Int. J. Mol. Sci. 2017, 18, 1029. [CrossRef] [PubMed]

5. Ding, Y.; Tang, J.; Guo, F. Predicting protein-protein interactions via multivariate mutual information of protein sequences. BMC Bioinform. 2016, 17, 398. [CrossRef] [PubMed]

6. Ding, Y.; Tang, J.; Guo, F. Identification of Protein-Protein Interactions via a Novel Matrix-Based Sequence Representation Model with Amino Acid Contact Information. Int. J. Mol. Sci. 2016, 17, 1623. [CrossRef] [PubMed]

7. Shen, C.; Ding, Y.; Tang, J.; Xu, X.; Guo, F. An Ameliorated Prediction of Drug-Target Interactions Based on Multi-Scale Discrete Wavelet Transform and Network Features. Int. J. Mol. Sci. 2017, 18, 1781. [CrossRef] [PubMed]

8. Ding, Y.; Tang, J.; Guo, F. Identification of Drug-Target Interactions via Multiple Information Integration. Inf. Sci. 2017, 418-419, 546-560. [CrossRef]

9. Chen, P.; Li, J. Sequence-based identification of interface residues by an integrative profile combining hydrophobic and evolutionary information. BMC Bioinform. 2010, 11, 402. [CrossRef] [PubMed]

10. Chen, P.; Li, J.; Wong, L.; Kuwahara, H.; Huang, J.Z.; Gao, X. Accurate prediction of hot spot residues through physicochemical characteristics of amino acid sequences. Proteins-Struct. Funct. Bioinform. 2013, 81, 1351-1362. [CrossRef] [PubMed]

11. Pan, Y.; Wang, Z.; Zhan, W.; Deng, L. Computational identification of binding energy hot spots in protein-RNA complexes using an ensemble approach. Bioinformatics 2017. [CrossRef] [PubMed]

12. Chen, P.; Hu, S.; Zhang, J.; Gao, X.; Li, J.; Xia, J.; Wang, B. A sequence-based dynamic ensemble learning system for protein ligand-binding site prediction. IEEE/ACM Trans. Comput. Biol. Bioinform. 2016, 13, 901-912. [CrossRef] [PubMed]

13. Hu, S.S.; Peng, C.; Bing, W.; Li, J. Protein binding hot spots prediction from sequence only by a new ensemble learning method. Amino Acids 2017, 49, 1773-1785. [CrossRef] [PubMed]

14. Jiang, J.; Wang, N.; Chen, P.; Zheng, C.; Wang, B. Prediction of Protein Hotspots from Whole Protein Sequences by a Random Projection Ensemble System. Int. J. Mol. Sci. 2017, 18, 1543. [CrossRef] [PubMed]

15. Liu, B.; Wang, S.; Dong, Q.; Li, S.; Liu, X. Identification of DNA-binding proteins by combining auto-cross covariance transformation and ensemble learning. IEEE Trans. NanoBiosci. 2016, 15, 328-334. [CrossRef] [PubMed]

16. Liu, B.; Xu, J.; Fan, S.; Xu, R.; Zhou, J.; Wang, X. PseDNA-Pro: DNA-Binding Protein Identification by Combining Chou's PseAAC and Physicochemical Distance Transformation. Mol. Inform. 2015, 34, 8-17. [CrossRef] [PubMed]

17. Liu, B.; Wang, S.; Wang, X. DNA binding protein identification by combining pseudo amino acid composition and profile-based protein representation. Sci. Rep. 2015, 5, 15479. [CrossRef] [PubMed]

18. Liu, B.; Xu, J.; Lan, X.; Xu, R.; Zhou, J.; Wang, X.; Chou, K.-C. iDNA-Prot I dis: Identifying DNA-Binding Proteins by Incorporating Amino Acid Distance-Pairs and Reduced Alphabet Profile into the General Pseudo Amino Acid Composition. PLoS ONE 2014, 9, e106691. [CrossRef] [PubMed] 
19. Song, L.; Li, D.; Zeng, X.; Wu, Y.; Guo, L.; Zou, Q. nDNA-Prot: Identification of DNA-binding Proteins Based on Unbalanced Classification. BMC Bioinform. 2014, 15, 298. [CrossRef] [PubMed]

20. Wei, L.; Tang, J.; Zou, Q. Local-DPP: An Improved DNA-binding Protein Prediction Method by Exploring Local Evolutionary Information. Inf. Sci. 2017, 384, 135-144. [CrossRef]

21. Qu, K.; Han, K.; Wu, S.; Wang, G.; Wei, L. Identification of DNA-Binding Proteins Using Mixed Feature Representation Methods. Molecules 2017, 22, 1602. [CrossRef] [PubMed]

22. Zhang, J.; Liu, B. PSFM-DBT: Identifying DNA-Binding Proteins by Combing Position Specific Frequency Matrix and Distance-Bigram Transformation. Int. J. Mol. Sci. 2017, 18, 1856. [CrossRef] [PubMed]

23. Shen, C.; Ding, Y.; Tang, J.; Song, J.; Guo, F. Identification of DNA-protein Binding Sites through Multi-Scale Local Average Blocks on Sequence Information. Molecules 2017, 22, 2079. [CrossRef] [PubMed]

24. Liu, W.X.; Deng, E.Z.; Chen, W.; Lin, H. Identifying the subfamilies of voltage-gated potassium channels using feature selection technique. Int. J. Mol. Sci. 2014, 15, 12940-12951. [CrossRef] [PubMed]

25. Lin, H.; Ding, H. Predicting ion channels and their types by the dipeptide mode of pseudo amino acid composition. J. Theor. Biol. 2011, 269, 64-69. [CrossRef] [PubMed]

26. Chen, W.; Lin, H. Identification of voltage-gated potassium channel subfamilies from sequence information using support vector machine. Comput. Biol. Med. 2012, 42, 504-507. [CrossRef] [PubMed]

27. Zhao, Y.W.; Su, Z.D.; Yang, W.; Lin, H.; Chen, W.; Tang, H. IonchanPred 2.0: A Tool to Predict Ion Channels and Their Types. Int. J. Mol. Sci. 2017, 18, 1838. [CrossRef] [PubMed]

28. Wang, H.; Liu, B.; Sun, P.P.; Ma, Z.Q. A Topology Structure Based Outer Membrane Proteins Segment Alignment Method. Math. Probl. Eng. 2013, 2013, 541359. [CrossRef]

29. Zhang, L.; Wang, H.; Yan, L.; Su, L.; Xu, D. OMPcontact: An Outer Membrane Protein Inter-Barrel Residue Contact Prediction Method. J. Comput. Biol. 2017, 24, 217-228. [CrossRef] [PubMed]

30. Wang, H.; He, Z.; Zhang, C.; Zhang, L.; Xu, D. Transmembrane protein alignment and fold recognition based on predicted topology. PLoS ONE 2013, 8, e69744. [CrossRef] [PubMed]

31. Antonets, K.S.; Nizhnikov, A.A. Predicting Amyloidogenic Proteins in the Proteomes of Plants. Int. J. Mol. Sci. 2017, 18, 2155. [CrossRef] [PubMed]

32. Wang, S.; Liu, S. Protein Sub-Nuclear Localization Based on Effective Fusion Representations and Dimension Reduction Algorithm LDA. Int. J. Mol. Sci. 2015, 16, 30343-30361. [CrossRef] [PubMed]

33. Wang, S.; Nie, B.; Yue, K.; Fei, Y.; Li, W.; Xu, D. Protein Subcellular Localization with Gaussian Kernel Discriminant Analysis and Its Kernel Parameter Selection. Int. J. Mol. Sci. 2017, 18, 2718. [CrossRef] [PubMed]

34. Wang, S.; Liu, P. A New Feature Extraction Method Based on the Information Fusion of Entropy Matrix and Covariance Matrix and Its Application in Face Recognition. Entropy 2015, 17, 4664-4683. [CrossRef]

35. Li, Y.H.; Yu, C.Y.; Li, X.X.; Zhang, P.; Tang, J.; Yang, Q.; Fu, T.; Zhang, X.; Cui, X.; Tu, G. Therapeutic target database update 2018: Enriched resource for facilitating bench-to-clinic research of targeted therapeutics. Nucleic Acids Res. 2017, 46, D1121-D1127.

36. Li, B.; Tang, J.; Yang, Q.; Li, S.; Cui, X.; Li, Y.; Chen, Y.; Xue, W.; Li, X.; Zhu, F. NOREVA: Normalization and evaluation of MS-based metabolomics data. Nucleic Acids Res. 2017, 45, W162-W170. [CrossRef] [PubMed]

37. Li, Y.H.; Xu, J.Y.; Tao, L.; Li, X.F.; Li, S.; Zeng, X.; Chen, S.Y.; Zhang, P.; Qin, C.; Zhang, C. SVM-Prot 2016: A Web-Server for Machine Learning Prediction of Protein Functional Families from Sequence Irrespective of Similarity. PLoS ONE 2016, 11, e0155290. [CrossRef] [PubMed]

38. Yu, C.; Li, X.; Yang, H.; Li, Y.H.; Xue, W.; Chen, Y.; Tao, L.; Zhu, F. Assessing the Performances of Protein Function Prediction Algorithms from the Perspectives of Identification Accuracy and False Discovery Rate. Int. J. Mol. Sci. 2018, 19, 183. [CrossRef] [PubMed]

39. Liu, B. BioSeq-Analysis: A platform for DNA, RNA, and protein sequence analysis based on machine learning approaches. Brief. Bioinform. 2018. [CrossRef] [PubMed]

40. Liu, B.; Liu, F.; Wang, X.; Chen, J.; Fang, L.; Chou, K.-C. Pse-in-One: A web server for generating various modes of pseudo components of DNA, RNA, and protein sequences. Nucleic Acids Res. 2015, 43, W65-W71. [CrossRef] [PubMed]

41. Du, P.F.; Zhao, W.; Miao, Y.Y.; Wei, L.Y.; Wang, L. UltraPse: A Universal and Extensible Software Platform for Representing Biological Sequences. Int. J. Mol. Sci. 2017, 18, 2400. [CrossRef] [PubMed]

42. Du, P.; Wang, X.; Xu, C.; Gao, Y. PseAAC-Builder: A cross-platform stand-alone program for generating various special Chou's pseudo-amino acid compositions. Analyt. Biochem. 2012, 425, 117-119. [CrossRef] [PubMed] 
43. Du, P.; Gu, S.; Jiao, Y. PseAAC-General: Fast Building Various Modes of General Form of Chou's Pseudo-Amino Acid Composition for Large-Scale Protein Datasets. Int. J. Mol. Sci. 2014, 15, 3495-3506. [CrossRef] [PubMed]

44. Jiao, Y.S.; Du, P.F. Predicting Golgi-resident protein types using pseudo amino acid compositions: Approaches with positional specific physicochemical properties. J. Theor. Biol. 2015, 391, 35-42. [CrossRef] [PubMed]

45. Oti, M.; Snel, B.; Huynen, M.A.; Brunner, H.G. Predicting disease genes using protein-protein interactions. J. Med. Genet. 2006, 43, 691. [CrossRef] [PubMed]

46. Chen, L.; Hao Xing, Z.; Huang, T.; Shu, Y.; Huang, G.; Li, H.-P. Application of the Shortest Path Algorithm for the Discovery of Breast Cancer-Related Genes. Curr. Bioinform. 2016, 11, 51-58. [CrossRef]

47. Zhang, J.; Yang, J.; Huang, T.; Shu, Y.; Chen, L. Identification of novel proliferative diabetic retinopathy related genes on protein-protein interaction network. Neurocomputing 2016, 217, 63-72. [CrossRef]

48. Chen, L.; Yang, J.; Xing, Z.; Yuan, F.; Shu, Y.; Zhang, Y.; Kong, X.; Huang, T.; Li, H.; Cai, Y.D. An integrated method for the identification of novel genes related to oral cancer. PLoS ONE 2017, 12, e0175185. [CrossRef] [PubMed]

49. Zhang, J.; Zhang, Z.; Chen, Z.; Lei, D. Integrating Multiple Heterogeneous Networks for Novel LncRNA-disease Association Inference. IEEE/ACM Trans. Comput. Biol. Bioinform. 2018. [CrossRef] [PubMed]

50. Zhu, L.; Su, F.; Xu, Y.; Zou, Q. Network-based method for mining novel HPV infection related genes using random walk with restart algorithm. BBA-Mol. Basis Dis. 2018. [CrossRef] [PubMed]

51. Lu, S.; Yan, Y.; Li, Z.; Chen, L.; Yang, J.; Zhang, Y.; Wang, S.; Liu, L. Determination of Genes Related to Uveitis by Utilization of the Random Walk with Restart Algorithm on a Protein-Protein Interaction Network. Int. J. Mol. Sci. 2017, 18, 1045. [CrossRef] [PubMed]

52. Li, B.; Liao, B. Protein Complexes Prediction Method Based on Core-Attachment Structure and Functional Annotations. Int. J. Mol. Sci. 2017, 18, 1910. [CrossRef] [PubMed]

53. Li, M.; Meng, X.; Zheng, R.; Wu, F.X.; Li, Y.; Pan, Y.; Wang, J. Identification of protein complexes by using a spatial and temporal active protein interaction network. IEEE/ACM Trans. Comput. Biol. Bioinform. 2018. [CrossRef] [PubMed]

54. Tang, Y.; Li, M.; Wang, J.; Pan, Y.; Wu, F.X. CytoNCA: A cytoscape plugin for centrality analysis and evaluation of protein interaction networks. BioSystems 2015, 127, 67-72. [CrossRef] [PubMed]

55. Wang, J.; Chen, G.; Chen, G.; Li, M.; Wu, F.X.; Pan, Y. ClusterViz: A cytoscape APP for cluster analysis of biological network. IEEE/ACM Trans. Comput. Biol. Bioinform. 2015, 12, 815-822. [CrossRef] [PubMed]

56. Li, M.; Yang, J.; Wu, F.X.; Pan, Y.; Wang, J. DyNetViewer: A Cytoscape app for dynamic network construction, analysis and visualization. Bioinformatics 2018. [CrossRef] [PubMed]

57. Wu, L.; Min, L.; Wang, J.; Wu, F.X. CytoCtrlAnalyser: A Cytoscape app for biomolecular network controllability analysis. Bioinformatics 2017. [CrossRef] [PubMed]

58. Li, M.; Li, D.; Tang, Y.; Wu, F.; Wang, J. CytoCluster: A Cytoscape Plugin for Cluster Analysis and Visualization of Biological Networks. Int. J. Mol. Sci. 2017, 18, 1880. [CrossRef] [PubMed]

59. Huang, T.; Sun, J.; Zhou, S.; Gao, J.; Liu, Y. Identification of Direct Activator of Adenosine Monophosphate-Activated Protein Kinase (AMPK) by Structure-Based Virtual Screening and Molecular Docking Approach. Int. J. Mol. Sci. 2017, 18, 1408. [CrossRef] [PubMed]

60. Hou, Q.L.; Luo, J.X.; Zhang, B.C.; Jiang, G.F.; Ding, W.; Zhang, Y.Q. 3D-QSAR and Molecular Docking Studies on the TcPMCA1-Mediated Detoxification of Scopoletin and Coumarin Derivatives. Int. J. Mol. Sci. 2017, 18, 1380. [CrossRef] [PubMed]

61. Ding, H.; Gao, F.; Yu, Y.; Chen, B. Biochemical and Computational Insights on a Novel Acid-Resistant and Thermal-Stable Glucose 1-Dehydrogenase. Int. J. Mol. Sci. 2017, 18, 1198. [CrossRef] [PubMed]

62. Chandler, J.C.; Gandhi, N.S.; Mancera, R.L.; Smith, G.; Elizur, A.; Ventura, T. Understanding Insulin Endocrinology in Decapod Crustacea: Molecular Modelling Characterization of an Insulin-Binding Protein and Insulin-Like Peptides in the Eastern Spiny Lobster, Sagmariasus verreauxi. Int. J. Mol. Sci. 2017, 18, 1832. [CrossRef] [PubMed]

63. FutomaKołoch, B.; Dudek, B.; Kapczyńska, K.; Krzyżewska, E.; Wańczyk, M.; Korzekwa, K.; Rybka, J.; Klausa, E. Relationship of Triamine-Biocide Tolerance of Salmonella enterica Serovar Senftenberg to Antimicrobial Susceptibility, Serum Resistance and Outer Membrane Proteins. Int. J. Mol. Sci. 2017, 18, 1459. [CrossRef] [PubMed] 
64. Chen, W.; Xing, P.; Zou, Q. Detecting N6-methyladenosine sites from RNA transcriptomes using ensemble Support Vector Machines. Sci. Rep. 2017, 7, 40242. [CrossRef] [PubMed]

65. Wei, L.; Xing, P.; Tang, J.; Zou, Q. PhosPred-RF: A novel sequence-based predictor for phosphorylation sites using sequential information only. IEEE Trans. NanoBiosci. 2017, 16, 240-247. [CrossRef] [PubMed]

(c) (1)

(C) 2018 by the authors. Licensee MDPI, Basel, Switzerland. This article is an open access article distributed under the terms and conditions of the Creative Commons Attribution (CC BY) license (http:/ / creativecommons.org/licenses/by/4.0/). 


\title{
PCVMZM: Using the Probabilistic Classification Vector Machines Model Combined with a Zernike Moments Descriptor to Predict Protein-Protein Interactions from Protein Sequences
}

\author{
Yanbin Wang ${ }^{1,+}$, Zhuhong You ${ }^{1, *,+}$, Xiao Li ${ }^{1, *}$, Xing Chen ${ }^{2}$, Tonghai Jiang ${ }^{1}$ and Jingting Zhang ${ }^{3}$ \\ 1 Xinjiang Technical Institutes of Physics and Chemistry, Chinese Academy of Science, Urumqi 830011, China; \\ wangyanbin15@mails.ucas.ac.cn (Y.W.); jth@ms.xjb.ac.cn (T.J.) \\ 2 School of Information and Control Engineering, China University of Mining and Technology, \\ Xuzhou 221116, China; xingchen@amss.ac.cn \\ 3 Department of Mathematics and Statistics, Henan University, Kaifeng 100190, China; \\ zhangjingting15@mails.ucas.ac.cn \\ * Correspondence: zhuhongyou@ms.xjb.ac.cn (Z.Y.); xiaoli@ms.xjb.ac.cn (X.L.); \\ Tel.: +86-991-3835-823 (Z.Y.); +86-991-3848-575 (X.L.) \\ + These authors contributed equally to this work.
}

Academic Editor: Christo Z. Christov

Received: 24 March 2017; Accepted: 29 April 2017; Published: 11 May 2017

\begin{abstract}
Protein-protein interactions (PPIs) are essential for most living organisms' process. Thus, detecting PPIs is extremely important to understand the molecular mechanisms of biological systems. Although many PPIs data have been generated by high-throughput technologies for a variety of organisms, the whole interatom is still far from complete. In addition, the high-throughput technologies for detecting PPIs has some unavoidable defects, including time consumption, high cost, and high error rate. In recent years, with the development of machine learning, computational methods have been broadly used to predict PPIs, and can achieve good prediction rate. In this paper, we present here PCVMZM, a computational method based on a Probabilistic Classification Vector Machines (PCVM) model and Zernike moments (ZM) descriptor for predicting the PPIs from protein amino acids sequences. Specifically, a Zernike moments (ZM) descriptor is used to extract protein evolutionary information from Position-Specific Scoring Matrix (PSSM) generated by Position-Specific Iterated Basic Local Alignment Search Tool (PSI-BLAST). Then, PCVM classifier is used to infer the interactions among protein. When performed on PPIs datasets of Yeast and H. Pylori, the proposed method can achieve the average prediction accuracy of $94.48 \%$ and $91.25 \%$, respectively. In order to further evaluate the performance of the proposed method, the state-of-the-art support vector machines (SVM) classifier is used and compares with the PCVM model. Experimental results on the Yeast dataset show that the performance of PCVM classifier is better than that of SVM classifier. The experimental results indicate that our proposed method is robust, powerful and feasible, which can be used as a helpful tool for proteomics research.
\end{abstract}

Keywords: proteins; position-specific scoring matrix; probabilistic classification vector machines

\section{Introduction}

Recognition of protein-protein interactions (PPIs) is essential for elucidating the function of proteins and further understanding the various biological processes in cells. In the last decade, a variety of biological methods have been used for large-scale PPIs detection, such as tandem affinity purification [1], yeast two-hybrid systems [2,3], and protein chip [4]. For the limit of the experimental 
technique, these methods have some disadvantages, including high cost and time-intensive, as well as high rates of both false-positive and false-negative. Hence, computational methods for the detection of protein interactions have become hot research topics of proteomics research. So far, a number of computational methods have been presented for the detection of PPIs based on different data types, such as protein domains, protein structure information, genomic information and phylogenetic profiles [5-13]. However, these approaches cannot be achieved unless prior information of the protein is available. Hence, the mentioned methods are not widespread. Compared to the rapid growth of a large number of protein sequences, other data that can be used to predict the PPIs are scarce. Therefore, computational methods using only protein amino acid sequence information for PPIs prediction is especially interesting [14]. Bock and Gough used a support vector machine (SVM) with protein sequence descriptors to predict PPIs [15]. Martin et al. proposed an approach to predict PPIs by using signature product, which is a descriptor that extends from signature descriptors [16]. Najafabadi et al. attempted to solve this problem with Bayesian network [17]. Shen et al. adopted a SVM model to predict PPI network by combining Skernel function of protein pairs with a conjoint triad feature [18]. Yu-An Huang et al. developed a method by combining discrete cosine transform and using weighted sparse representation-based classifier to predict PPIs, and it has achieved very exciting prediction accuracy when applying this method to detecting yeast PPIs [19]. Yan-Zhi Guo et al. also obtained promising prediction results by adopting support vector machine and auto covariance [20]. Loris Nanni et al. developed several matrix-based protein representation methods, including [21-25]. Other feature extraction approaches based on protein sequence have been proposed in [26-34]. In this study, a novel computational approach for predicting PPIs from amino acid sequences based on a probabilistic classification vector machines model (PCVM) and a Zernike moments descriptor (PCVMZM) was proposed. The major improvement is the development of a more accurate protein sequence representation. Specifically, we employed the Zernike moments feature representation on a Position-Specific Scoring Matrix (PSSM) to extract the evolutionary information from protein sequence, and then a probabilistic classification vector machines classifier is used to infer the PPIs. In more detail, a PSSM representation is used to represent each protein. Afterward, for the sake of obtaining more representative information, we apply a Zernike moments descriptor to extract features in each protein PSSM and use Zernike moments of 12-order information and generate a 42-dimensional feature vector. Finally, we adopt the machine learning method called PCVM to accomplish classification. The proposed method was applied to Yeast and H. Pylori PPIs datasets. The experiments have shown that a PCVM prediction model with a Zernike moments descriptor yields fantastic performance. By further contrast experiment, we found that our proposed method was superior to the state-of-the-art SVM, which clearly shows that the proposed approach is trustworthy in predicting PPIs [35-39].

\section{Results and Discussion}

\subsection{Evaluation Measure}

The proposed method is evaluated against the following criteria: The Accuracy (Acc), Sensitivity (Sen), Precision (Pre), and Matthew's correlation coefficient (MCC). All the computational formula is defined as follows:

$$
\begin{gathered}
\text { Accuracy }=\frac{T P+T N}{T P+F P+T N+F N} \\
\text { Sensitivity }=\frac{T P}{T P+F N} \\
\text { Precision }=\frac{T P}{T P+F P} \\
\text { MCC }=\frac{(T P \times T N)-(F P \times F N)}{\sqrt{(T P+F N) \times(T N+F P) \times(T P+F P) \times(T N+F N)}}
\end{gathered}
$$


where TP represents the number of true positive, that true samples are predicted correctly, TN represents the number of true negative that true noninteracting pairs are predicted correctly. FP represents the number of false positive that non-interacting pairs are predicted to be interaction. FN represents the number of false negative that interacting pairs are predicted to be non-interacting. In addition, the receiver operating characteristic (ROC) curve [40] is applied to evaluate the performance of our method. The area under an ROC curve (AUC) [41] also is computed.

\subsection{Assessment of Prediction}

In order to make our method more reliable, five-fold cross-validation was adopted to divide a whole dataset into five parts. Hence, we obtained five models through separate experiments for each data set. The prediction result of PCVM prediction models with a Zernike moments description of protein sequence on Yeast and H. Pylori datasets are shown in Tables 1 and 2. From Table 1, we can see that our proposed method achieved a good performance on the Yeast dataset. Its average accuracy, sensitivity, precision, and MCC are $94.48 \%, 95.13 \%, 93.92 \%$ and $89.58 \%$, respectively. When using our proposed method on the H. Pylori dataset, as shown in Table 2, we also achieved some satisfactory results of average accuracy, sensitivity, precision, and MCC of $91.25 \%, 92.05 \%, 90.60 \%$ and $84.04 \%$, respectively.

Table 1. Fivefold cross validation results using the proposed method on Yeast dataset.

\begin{tabular}{ccccc}
\hline Testing Set & Acc (\%) & Sen (\%) & Pre (\%) & MCC (\%) \\
\hline 1 & 96.38 & 97.21 & 95.57 & 93.02 \\
2 & 94.05 & 95.23 & 92.77 & 88.81 \\
3 & 93.07 & 96.73 & 90.27 & 87.06 \\
4 & 94.46 & 94.20 & 94.71 & 89.53 \\
5 & 94.42 & 92.26 & 96.26 & 89.46 \\
Average & $94.48 \pm 1.2$ & $95.13 \pm 2.0$ & $93.92 \pm 2.4$ & $89.58 \pm 2.2$ \\
\hline
\end{tabular}

Table 2. Fivefold cross validation results using the proposed method on H. Pylori dataset.

\begin{tabular}{ccccc}
\hline Testing Set & Acc (\%) & Sen (\%) & Pre (\%) & MCC (\%) \\
\hline 1 & 89.54 & 92.11 & 86.82 & 81.24 \\
2 & 92.11 & 92.68 & 91.41 & 85.46 \\
3 & 91.08 & 91.16 & 91.16 & 83.75 \\
4 & 91.42 & 92.25 & 90.34 & 84.31 \\
5 & 92.12 & 92.04 & 93.23 & 85.42 \\
Average & $91.25 \pm 1.1$ & $92.05 \pm 0.6$ & $90.06 \pm 2.4$ & $84.04 \pm 1.7$ \\
\hline
\end{tabular}

From the experimental results, it can be seen that our proposed approach is robust, accurate and practical for predicting PPIs. The outstanding performance for detecting PPIs can be put down to the feature extraction and the classification model of our proposed method. It is effective that Zernike moments are used for feature extraction, and the PCVM model is accurate and robust in dealing with classification problems.

\subsection{Comparison with the Support Vector Machine (SVM)-Based Method}

In order to further evaluate the prediction performance of the proposed entire model, the SVM model is adopted based on the Yeast dataset to predict PPIs using the same Zernike moments to extract feature, and then, we compared the classification result between PCVM and SVM. We employed the SVM through the library for Support Vector Machines (LIBSVM) tool [42]. SVM have two parameters, $c$ and $g$, respectively. A grid search method is used to optimize parameters $c$ and $g$. In our experiment, a radial basis function is used as the kernel function and the initial value $c$ and $g$ was set to 0.4 and 0.5. 
Table 3 gives the prediction results of five-fold cross-validation over two different classification methods on the Yeast dataset. From Table 3, we can see that the classification method of SVM achieved $89.31 \%$ average accuracy, $87.54 \%$ average sensitivity, $90.81 \%$ average precision, $80.91 \%$ average MCC. While the classification results of the PCVM method achieved $94.48 \%$ average accuracy, $95.13 \%$ average sensitivity, 93.92\% average precision, $89.58 \%$ average MCC. Experimental results show that PCVM classification method is significantly better than the SVM classification method. Comparison of ROC curves performed between RVM and SVM on the Yeast dataset from Figures 1 and 2, we have experimental data obtained that the PCVM classifier is more accurate and robust than the SVM classifier for detecting PPIs.

Table 3. Five-fold cross-validation results by using two models on the Yeast dataset.

\begin{tabular}{|c|c|c|c|c|c|}
\hline Model & Testing Set & $\operatorname{Acc}(\%)$ & Sen $(\%)$ & Pre $(\%)$ & $\operatorname{MCC}(\%)$ \\
\hline \multirow{6}{*}{$\begin{array}{c}\text { Probabilistic Classification Vector } \\
\text { Machines (PCVM) }\end{array}$} & 1 & 96.38 & 97.21 & 95.57 & 93.02 \\
\hline & 2 & 94.05 & 95.23 & 92.77 & 88.81 \\
\hline & 3 & 93.07 & 96.73 & 90.27 & 87.06 \\
\hline & 4 & 94.46 & 94.20 & 94.71 & 89.53 \\
\hline & 5 & 94.42 & 92.26 & 96.26 & 89.46 \\
\hline & Average & $94.48 \pm 1.2$ & $95.13 \pm 2.0$ & $93.92 \pm 2.4$ & $89.58 \pm 2.2$ \\
\hline \multirow{6}{*}{ Support Vector Machin (SVM) } & 1 & 89.23 & 87.75 & 90.27 & 80.76 \\
\hline & 2 & 90.48 & 88.73 & 91.49 & 82.74 \\
\hline & 3 & 87.62 & 87.37 & 88.07 & 78.30 \\
\hline & 4 & 89.63 & 88.05 & 90.97 & 81.40 \\
\hline & 5 & 89.60 & 85.79 & 93.23 & 81.32 \\
\hline & Average & $89.31 \pm 1.7$ & $87.54 \pm 1.1$ & $90.81 \pm 1.9$ & $80.91 \pm 1.62$ \\
\hline
\end{tabular}

The main improvement is attributed to three points: (1) the main advantage of PCVM is that the truncated Gaussian priors are adopted to generate robust and sparse results-in other words, the number of weight vectors is less than SVM. Hence, the complexity of the model is reduced, besides, the model is more general; (2) The parameter optimization procedure of the PCVM based on EM algorithm and probabilistic inference not only can improve the performance, but also save the effort to do cross-validation; (3) The PCVM model is simpler and easier to be understood, because the number of basic functions does not grow linearly with the number of training points. In general, the PCVM is a sparse model that makes up the shortcoming of SVM without deskilling the generalization performance and provides probabilistic outputs. Here it is, our proposed approach can produce satisfactory results.

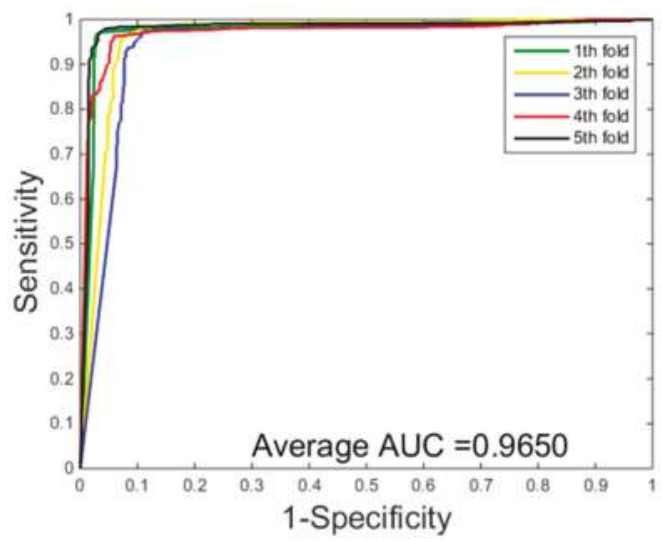

Figure 1. Receiver operating characteristic (ROC) curves performed of a probabilistic classification vector machines model (PCVM) on the Yeast dataset. 


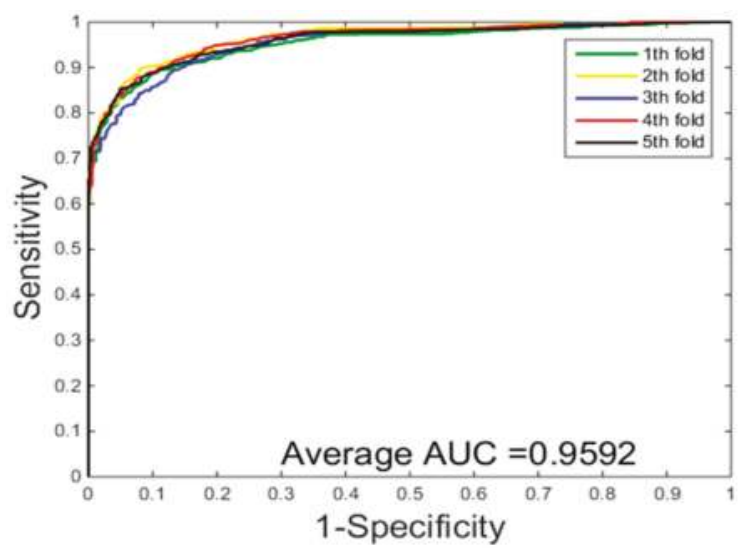

Figure 2. ROC curves performed of support vector machine (SVM) on the Yeast dataset.

\subsection{Comparison with Other Methods}

In recent years, many classification methods have been developed to predict PPIs. To further validate the performance of our proposed method, we compared the predictive performance of our method with other existing several well-known methods. The achieved results of five-fold cross-validation of different methods on the Yeast dataset and H. pylori dataset are shown in Tables 4 and 5. From Table 4, the prediction accuracy of other previous methods on the Yeast dataset varies from $75.08 \%$ to $93.92 \%$, while the proposed method achieved higher value of $94.48 \%$. Similarly, the sensitivity and MCC of our method are also higher than those of other methods. We can find similar results on the H. pylori dataset in Table 5. Our proposed method achieves $91.25 \%$ accuracy, which is higher than the other five methods with the highest prediction accuracy of $87.50 \%$. The same is true for precision, sensitivity and MCC. All prediction results in Tables 4 and 5 indicate that the PCVM classifier is stable and robust and can improve the prediction performance compared with the state-of-the-art methods. The improvement of prediction performance of our method may derive from the novel feature extraction method which extracts the highly discriminative information, and the use of PCVM classifier which ensures accurate and stable prediction.

Table 4. Practical predicting results of different methods on the Yeast dataset.

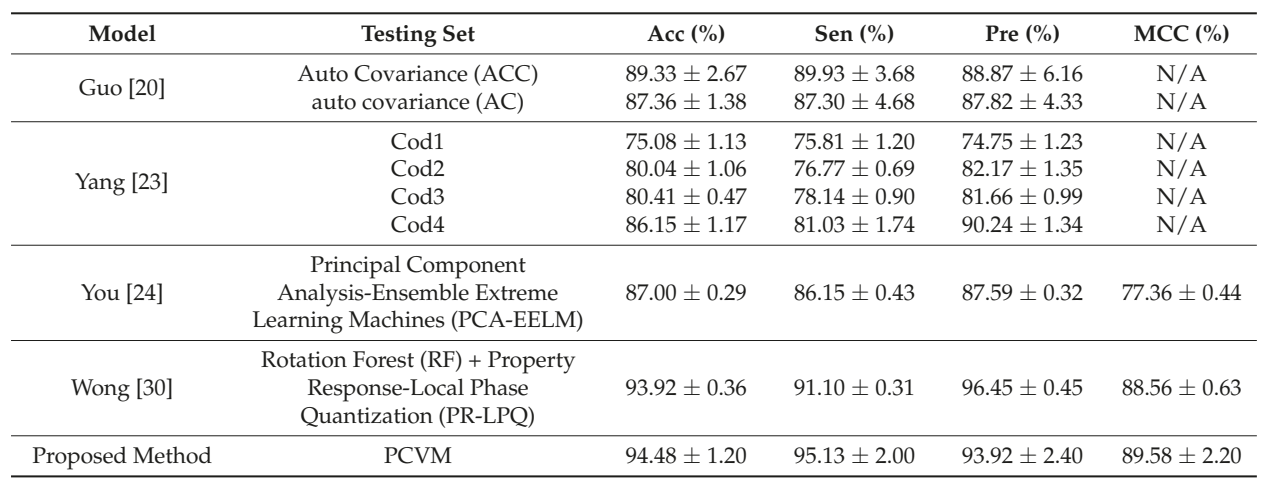


Table 5. Practical predicting results of different methods on the H. Pylori dataset.

\begin{tabular}{ccccc}
\hline Model & Acc (\%) & Sen (\%) & Pre (\%) & MCC (\%) \\
\hline Nanni [23] & 83.00 & 86.00 & 85.10 & N/A \\
Nanni [32] & 84.00 & 86.00 & 84.00 & N/A \\
Nanni and Lumini [25] & 86.60 & 86.70 & 85.00 & N/A \\
Z-H You [29] & 87.50 & 88.95 & 86.15 & 78.13 \\
L Nanni [24] & 84.00 & 84.00 & 84.00 & N/A \\
Proposed Method & 91.25 & 92.05 & 90.06 & 84.04 \\
\hline
\end{tabular}

\section{Materials and Methodology}

\subsection{Dataset}

Up to now, many databases of PPIs data have been generated, such as Database of Interaction Proteins (DIP) [43], Molecular Interaction Database (MINT) [44], and Biomolecular Interaction Network Database (BIND) [45]. To evaluate our approach, we used two publicly available datasets: Yeast and H. Pylori, which were extracted from Database of Interaction Proteins (DIP). In order to ensure the reliability of the tests, we extract 5594 positive protein pairs to constitute the positive dataset and 5594 negative protein pairs to constitute the negative protein dataset from the Yeast dataset. Analogously, we extract 1458 positive protein pairs to constitute the positive dataset and 1458 negative protein pairs to constitute the negative protein dataset from the $H$. Pylori dataset. Therefore, the Yeast dataset consists of 11,188 protein pairs and the H. Pylori dataset consists of 2916 protein pairs.

\subsection{Position-Specific Scoring Matrix}

A Position-Specific Scoring Matrix (PSSM) was usually adopted to find distantly related proteins, protein disulfide, protein quaternary structural attributes and protein folding patterns [46-49]. In this paper, we also adopt PSSM to predict PPIs. Here, each protein was transformed into a PSSM matrix by employing the Position-Specific Iterated Basic Local Alignment Search Tool (PSI-BLAST) [50,51]. A PSSM is represented as

$$
\operatorname{PSSM}=\left(N_{1}, N_{2}, \ldots, N_{i}, \ldots, N_{20}\right)
$$

where $N_{i}=\left(N_{1 i}, N_{2 i}, \ldots, N_{L i}\right)^{T},(i=1,2, \ldots, 20)$. A PSSM contains $L \times 20$ elements, where $L$ denotes the length of an amino acid sequence and 20 columns are owing to 20 amino acids. The $N_{i j}$ of the PSSM element is indicated as a score of $j$ th amino acid in the $i$ th position of the given protein sequence and it can be expressed as $N_{i j}=\sum_{k=1}^{20} p(i, k) \times q(j, k)$ where $p(i, k)$ is the appearing frequency value of the $k_{t h}$ amino acid at position $i$ of the probe, and $q(j, k)$ represents the value of Dayhoff's mutation matrix [52] between the $j_{t h}$ and the $k_{t h}$ amino acids. Consequently, the higher the score, the better the conserved position [53-55].

In our study, the experiment datasets were built by using PSI-BLAST to transform each protein into a PSSM for detecting PPIs. To obtain more extensive homologous sequences, the e-value parameter of PSI-BLAST was set to 0.001 and chose three iterations. As a result, the PSSM of a protein sequence can be represented as a $M \times 20$ matrix, where $M$ is the number of residues and each column represents an amino acid [56-59].

\subsection{Zernike Moments}

Zernike moments have an exciting performance in the field of image recognition for extract image feature, because it is robust against rotation and it can represent information from different angles. In this paper, we first introduced Zernike moments to extract significant information from protein sequences. In this section, Zernike moments and their principal properties are described, and we illustrate how to achieve the rotation invariance. Finally, we describe the process of feature selection. 


\subsubsection{Invariance of Normalized Zernike Moment}

The principle of Zernike moments [60-63] is Zernike polynomials [64-66], that is a set of complete orthogonal polynomials within the unit circle. In two-dimensional space, these polynomials can be expressed as $\left\{V_{n m}(x, y)\right\}$ and expression is as follows:

$$
V_{n m}(x, y)=V_{n m}(\rho, \theta)=R_{n m}(\rho) e^{j m \theta} \quad \text { for } \rho \leq 1
$$

where $n$ is a nonnegative integer and $m$ is an integer subject to constraints $n-|m|$ even, $|m| \leq n$. Here, $\left\{R_{n m}(\rho)\right\}$ is a radial polynomial in the form of

$$
R_{n m}(\rho)=\sum_{s=0}^{(n-|m| / 2)}(-1)^{s} \frac{(n-s) !}{s !\left(\frac{n+|m|}{2}-s\right) !\left(\frac{n+|m|}{2}-s\right) !} \rho^{n-2 s}
$$

Note that $R_{n,-m}(\rho)=R_{n m}(\rho)$.The set of polynomials are orthogonal, i.e.,

$$
\int_{0}^{2 \pi} \int_{0}^{1} V_{n m}^{*}(\rho, \theta) V_{p q}(\rho, \theta) \rho d \rho d \theta=\frac{\pi}{n+1} \delta_{n p} \delta_{m q}
$$

With

$$
\delta_{a b}=\left\{\begin{array}{cc}
1 & a=b \\
0 & \text { otherwise }
\end{array}\right.
$$

The two-dimensional Zernike moments for continuous function $f(\rho, \theta)$ are the projection of $f(\rho, \theta)$ onto these orthogonal basis function and denoted by

$$
A_{n m}=\frac{n+1}{\pi} \int_{0}^{2 \pi} \int_{0}^{1} f(\rho, \theta) V_{n m}^{*}(\rho, \theta) \rho d \rho d \theta
$$

Correspondingly, for a digital function, the two-dimensional Zernike moments are represented by

$$
A_{n m}=\frac{n+1}{\pi} \sum_{(\rho, \theta) \in \text { unit circle }} \sum f(\rho, \theta) V_{n m}^{*}(\rho, \theta)
$$

To compute the Zernike moments of a PSSM matrix [67-70], the center of the matrix is taken as the origin and coordinates are mapped into a unit circle, i.e., $x^{2}+y^{2} \leq 1$. Those values of matrix falling outside the unit disk are not used in the computation. Note that $A_{n m}^{*}=A_{n,-m}$.

\subsubsection{Introduction of a Zernike Moments Descriptor}

When we define $f^{\prime}(\rho, \theta)$ as the rotated function, the equivalence between original and rotated function is

$$
f^{\prime}(\rho, \theta)=f(\rho, \theta-\alpha)
$$

The Zernike moments $A_{n m}^{\prime}$ of the rotated function $f^{\prime}(\rho, \theta)$ become

$$
A_{n m}^{\prime}=A_{n m} e^{-j m \alpha}
$$

Equation (13) indicates that Zernike moments only need phase shift on rotation. Therefore, the magnitude of the Zernike moment, $\left|A_{n m}^{\prime}\right|$, can be adopted as rotation-invariant feature.

Therefore, after moving the origin of PSSM matrix into the centroid, we can compute the Zernike moments and the magnitudes of the moments are rotation-invariant [71,72].

\subsubsection{Feature Selection}

According to the foregoing, we have known that the magnitudes of Zernike moments can be used as rotation-invariant features. One problem that must be considered is how big should $N$ be? 
The lower-order moments extract gross information and high details information are captured by higher-order moments. In our experiments, $N$ is set to 12 . We can obtain 42 features from each protein sequence. The feature vector $\vec{F}$ be represented as:

$$
\vec{F}=\left[\left|A_{11}\right|,\left|A_{22}\right|, \ldots \ldots,\left|A_{N M}\right|\right]^{T}
$$

where $\left|A_{n m}\right|$ represent the Zernike moments magnitude. Here, we do not consider the case of $m=0$, because they do not include useful information regarding the PPIs and Zernike moments with $m<0$ have not been considered, because they are inferred through $A_{n,-m}=A_{n m}^{*}$. Hence, the dimension of the feature vector $\vec{F}$ is 42 [73]. The obtained Zernike moments is shown in Table 6.

Table 6. List of Zernike Moments (ZMs) sorted by $n$ and $m$ in sequence for the case where $(n, m)=(12,12)$.

\begin{tabular}{cccccc}
\hline $\boldsymbol{N}$ & Moments & No. & $\boldsymbol{N}$ & Moments & No. \\
\hline 1 & $A_{11}$ & 1 & 7 & $A_{71}, A_{73}, A_{75}, A_{77}$ & 4 \\
2 & $A_{22}$ & 1 & 8 & $A_{82}, A_{84}, A_{86}, A_{88}$ & 4 \\
3 & $A_{31}, A_{33}$ & 2 & 9 & $A_{91}, A_{93}, A_{95}, A_{97}, A_{99}$ & 5 \\
4 & $A_{42}, A_{44}$ & 2 & 10 & $A_{10,2}, A_{10,4}, A_{10,6}, A_{10,8}, A_{10,10}$ & 5 \\
5 & $A_{51}, A_{53}, A_{55}$ & 3 & 11 & $A_{11,1}, A_{11,3}, A_{11,5}, A_{11,7}, A_{11,9}, A_{11,11}$ & 6 \\
6 & $A_{62}, A_{64}, A_{66}$ & 3 & 12 & $A_{12,2}, A_{12,4}, A_{12,6}, A_{12,8}, A_{12,10}, A_{12,12}$ & 6 \\
\hline
\end{tabular}

\subsection{Related Machine Learning Models}

In the field of machine learning, the Support Vector Machines (SVM) [74] are acknowledged as an excellent supervision model in pattern recognition, classification, and regression analysis. However, there are certain apparent disadvantages when using this method: (1) the count of support vectors grows linearly with the scale of the training set; (2) Outputs of the SVMs are not probabilistic; (3) The parameters of kernel function need to be optimized by cross-validation, the procedure wastes a lot of computing resources. Compared with SVM, the Relevance Vector Machines (RVM) [75] based on Bayesian technique can avoid these problems. The RVM method takes advantage of the Bayesian automatic relevance determination (ARD) [76] framework and gives a zero-mean Gaussian prior over every weight $w_{i}$ to produce a sparse solution. However, for a classification problem, the zero-mean Gaussian prior are given over weights for negative and positive classes, which leads to a problem that some training points belonging to negative classes may be given positive weights and vice-versa. Under this circumstance, it may give rise to produce some unreliable vectors for the decision of RVMs. For the sake of addressing this problem and proposing an appropriate probabilistic model for predicting PPIs, we first adopt the Probabilistic Classification Vector Machine (PCVM) classifier which gives different priors over weights for training points that belong to different classes, i.e., the non-negative, left-truncated Gaussian is used for the positive class and the non-positive, right-truncated Gaussian is used for the negative class. PCVM provides many advantages: (1) PCVM produces the probabilistic outputs for each test point; (2) It is effective that PCVM used expectation maximization (EM) algorithm to optimizing kernel parameters; (3) PCVM introduced a sparser model leading to faster performance in the test stage.

\subsection{PCVM Algorithm}

PCVM is a classification model that supervised learning. Hence, we need a set of input-target training pairs $\left\{x_{i}, y_{i}\right\}_{i=1}^{N}$, where $y_{i}=\{-1,+1\}$ to train a learning model $f(\mathrm{x} ; \mathrm{w})$, which is defined by parameters $W$. The model is a linear combination of $N$ basis functions and is represented as

$$
f(\mathrm{x} ; \mathrm{w})=\sum_{i=1}^{N} w_{i} \varnothing_{i, \theta}(x)+b
$$


where the $\left\{\varnothing_{1, \theta}(x), \ldots \ldots \varnothing_{N, \theta}(x)\right\}$ is basis function, (wherein $\theta$ represent the parameter vector of the basis function), the $W=\left(w_{1}, \ldots \ldots, w_{N}\right)^{T}$ is the parameter of the PCVM model, the $b$ is the bias.

In this paper, we adopt the radial basis function (RBF) [77] as the basis and adopt the probit link function $\psi(x)=\int_{-\infty}^{x} N(t \mid 0,1) d t$ to obtain the binary outputs. Finally, mapping the $f(x ; w)$ into $\psi(x)$, the expression of the PCVM model becomes:

$$
\mathrm{L}(\mathrm{X} ; \mathrm{w}, \mathrm{b})=\psi\left(\sum_{i=1}^{N} w_{i} \varnothing_{i, \theta}(x)+b\right)=\psi\left(\Phi_{\theta}(X) W+b\right)
$$

A truncated Gaussian distribution as a prior is employed over each weight $w_{i}$ as follow

$$
p(\mathrm{~W} \mid \boldsymbol{\alpha})=\prod_{i=1}^{N} p\left(w_{i} \mid \alpha_{i}\right)=\prod_{i=1}^{N} N_{t}\left(w_{i} \mid 0, \alpha_{i}^{-1}\right)
$$

A zero-mean Gaussian distribution as a prior is employed over the bias $b$ :

$$
p(b \mid \beta)=\mathrm{N}\left(b \mid 0, \beta^{-1}\right)
$$

The $N_{t}\left(w_{i} \mid 0, \alpha_{i}^{-1}\right)$ is a truncated Gaussian function, $\alpha_{i}$ is the precision of the corresponding parameter $w_{i}, \beta$ represents the precision of the normal distribution of $b$. When $y_{i}=+1$, the truncated prior is a non-negative, left-truncated Gaussian, and when $y_{i}=-1$, the prior is a non-positive, right-truncated Gaussian. This can be represented as

$$
p\left(w_{i} \mid \alpha_{i}\right)=\left\{\begin{array}{cl}
2 N\left(w_{i} \mid 0, \alpha_{i}^{-1}\right) & y_{i} w_{i} \geq 0 \\
0 & \text { others }
\end{array}\right.
$$

The gamma distribution is adopted as the hyper prior of $\alpha$ and $\beta$. Using the EM algorithm, assign the parameters of a PCVM model, such as parameters $b, W$ and $\theta$. The EM algorithm is an iterative algorithm, which is used to estimate the maximum likelihood or maximum posterior probability involving latent variables. For more details about the PCVM theory, please refer to [78,79].

\subsection{Initial Parameter Selection and Training}

The PCVM algorithm has only one parameter, $\theta$, which can be optimized automatically in the training process. However, the EM algorithm is susceptible to initial point and trap in local maxima. Choosing the best initialization point is an effective method to avoid the local maxima. We train a PCVM model with eight initialization points over the five training folds of each data. Hence, we obtain a $5 \times 8$ matrix of parameters, where the rows represent the folds and the columns represent the initializations. For each row, we select the results of the lowest test error. Hence, we find only five points, and then, we select the medium over those parameters. We have experimental obtained the optimal initial value $\theta$ which is seted as 3.6 on the Yeast dataset and 1.18 on the $H$. pylori dataset.

\section{Conclusions}

Considering time, efficiency and economy, the use of computational methods based on protein amino acid sequences to predict PPIs has attracted the attention of researchers. The computational method is playing an important role in proteomics research, because it saves manpower and material resources and is more accurate and efficient. In this paper, we introduce an accurate computational method based on protein sequence. It is established by using a PCVM classifier combined with a Zernike moments descriptor on the PSSM. The experiments showed that the performance of our proposed method achieves a high classification accuracy and is superior to the SVM. The main improvements of the developed approach come from adopting a Zernike moments descriptor as feature extraction approach that can capture multi-angle useful and representative information. More than this, the use of a PCVM classifier ensures more reliable and accurate recognition, because the 
use of the truncated Gaussian priors can lead to obtaining robust and sparse results—the number of support vectors is less than SVM, and the probabilistic outputs produced by PCVM can assess the uncertainty of prediction on the skewed dataset. In addition, the parameter optimization procedure of the PCVM not only can improve the performance, but also save effort to do cross-validation. Due to the outstanding performance of the Zernike moments descriptor and PCVM, our method can improve the PPIs accuracy rate. All in all, our proposed method is highly efficient and stable and can be a useful tool for predicting PPIs.

Acknowledgments: This work is supported in part by the National Science Foundation of China, under Grants 61373086, 11301517 and 61572506. The authors would like to thank all the editors and anonymous reviewers for their constructive advices.

Author Contributions: Yanbin Wang and Zhuhong You conceived the algorithm, carried out analyses, prepared the data sets, carried out experiments, and wrote the manuscript. Xiao Li, Xing Chen, Tonghai Jiang and Jingting Zhang designed, performed and analyzed experiments. All authors read and approved the final manuscript.

Conflicts of Interest: The authors declare no conflicts of interest.

\section{References}

1. Puig, O.; Caspary, F.; Rigaut, G.; Rutz, B.; Bouveret, E.; Bragado-Nilsson, E.; Wilm, M.; Seraphin, B. The tandem affinity purification (TAP) method: A general procedure of protein complex purification. Methods 2001, 24, 218-229. [CrossRef] [PubMed]

2. Staudinger, J.; Zhou, J.; Burgess, R.; Elledge, S.J.; Olson, E.N. PICK1: A perinuclear binding protein and substrate for protein kinase C isolated by the yeast two-hybrid system. J. Cell Biol. 1995, 128, 263-271. [CrossRef] [PubMed]

3. Koegl, M.; Uetz, P. Improving yeast two-hybrid screening systems. Brief. Funct. Genom. 2007, 6, 302-312. [CrossRef] [PubMed]

4. Zhu, H.; Snyder, M. Protein chip technology. Curr. Opin. Chem. Biol. 2003, 7, 55-63. [CrossRef]

5. Pazos, F.; Valencia, A. Similarity of phylogenetic trees as indicator of protein-protein interaction. Protein Eng. Des. Sel. 2001, 14, 609-614. [CrossRef]

6. Wang, B.; Chen, P.; Huang, D.S.; Li, J.J.; Lok, T.M.; Lyu, M.R. Predicting protein interaction sites from residue spatial sequence profile and evolution rate. FEBS Lett. 2006, 580, 380-384. [CrossRef] [PubMed]

7. Maleki, M.; Hall, M.; Rueda, L. Using structural domains to predict obligate and non-obligate protein-protein interactions. СІВСВ 2012, 252-261. [CrossRef]

8. Huang, C.; Morcos, F.; Kanaan, S.P.; Wuchty, S.; Chen, D.Z.; Izaguirre, J.A. Predicting protein-protein interactions from protein domains using a set cover approach. IEEE/ACM Trans. Comput. Biol. Bioinform. 2007, 4, 78-87. [CrossRef] [PubMed]

9. Jansen, R.; Yu, H.; Greenbaum, D.; Kluger, Y.; Krogan, N.J.; Chung, S.; Emili, A.; Snyder, M.; Greenblatt, J.F.; Gerstein, M. A Bayesian networks approach for predicting protein-protein interactions from genomic data. Science 2003, 302, 449-453. [CrossRef] [PubMed]

10. Qin, S.; Cai, L. Predicting protein-protein interaction based on protein secondary structure information using Bayesian classifier. J. Inn. Mongolia Univ. Sci. Technol. 2010, 1, 021. (In Chinese).

11. Cai, L.; Pei, Z.; Qin, S.; Zhao, X. Prediction of protein-protein interactions in Saccharomyces cerevisiae Based on Protein Secondary Structure. iCBEB 2012, 413-416. [CrossRef]

12. You, Z.H.; Yu, J.Z.; Zhu, L.; Li, S.; Wen, Z.K. A MapReduce based parallel SVM for large-scale predicting protein-protein interactions. Neurocomputing 2014, 145, 37-43. [CrossRef]

13. You, Z.H.; Zheng, Y.; Han, K.; Huang, D.S.; Zhou, X. A semi-supervised learning approach to predict synthetic genetic interactions by combining functional and topological properties of functional gene network. BMC Bioinform. 2010, 11, 1-13. [CrossRef] [PubMed]

14. Zou, Q.; Hu, Q.; Guo, M.; Wang, G. HAlign: Fast multiple similar DNA/RNA sequence alignment based on the centre star strategy. Bioinformatics 2015, 31, 2475. [CrossRef] [PubMed]

15. Bock, J.R.; Gough, D.A. Whole-proteome interaction mining. Bioinformatics 2003, 19, 125-134. [CrossRef] [PubMed] 
16. Martin, S.; Roe, D.; Faulon, J.L. Predicting protein-protein interactions using signature products. Bioinformatics 2005, 21, 218-226. [CrossRef] [PubMed]

17. Najafabadi, H.S. Sequence-based prediction of protein-protein interactions by means of codon usage. Genome Biol. 2008, 9, 1-9. [CrossRef] [PubMed]

18. Shen, J.; Zhang, J.; Luo, X.; Zhu, W.; Yu, K.; Chen, K.; Li, Y.; Jiang, H. Predicting protein-protein interactions based only on sequences information. Proc. Natl. Acad. Sci. USA 2007, 104, 4337-4341. [CrossRef] [PubMed]

19. Huang, Y.A.; You, Z.H.; Xin, G.; Leon, W.; Wang, L. Using Weighted Sparse Representation Model Combined with Discrete Cosine Transformation to Predict Protein-Protein Interactions from Protein Sequence. BioMed Res. Int. 2015, 2015, 1-10. [CrossRef] [PubMed]

20. Guo, Y.; Yu, L.; Wen, Z.; Li, M. Using support vector machine combined with auto covariance to predict protein-protein interactions from protein sequences. Nucleic Acids Res. 2008, 36, 3025-3030. [CrossRef] [PubMed]

21. Nanni, L.; Lumini, A. An ensemble of support vector machines for predicting the membrane protein type directly from the amino acid sequence. Amino Acids 2008, 35, 573-580. [CrossRef] [PubMed]

22. Nanni, L.; Lumini, A. An ensemble of K-local hyperplanes for predicting protein-protein interactions. Bioinformatics 2006, 22, 1207-1210. [CrossRef] [PubMed]

23. Nanni, L. Fusion of classifiers for predicting protein-protein interactions. Neurocomputing 2005, 68, 289-296. [CrossRef]

24. Nanni, L.; Brahnam, S.; Lumini, A. High performance set of PseAAC and sequence based descriptors for protein classification. J. Theor. Biol. 2010, 266, 1-10. [CrossRef] [PubMed]

25. Nanni, L.; Lumini, A. A genetic approach for building different alphabets for peptide and protein classification. BMC Bioinform. 2008, 9, 45. [CrossRef] [PubMed]

26. You, Z.H.; Li, J.; Gao, X.; He, Z.; Zhu, L.; Lei, Y.K.; Ji, Z. Detecting protein-protein interactions with a novel matrix-based protein sequence representation and support vector machines. BioMed Res. Int. 2015, 2015, 1-9. [CrossRef] [PubMed]

27. You, Z.H.; Chan, K.C.C.; Hu, P. Predicting protein-protein interactions from primary protein sequences using a novel multi-scale local feature representation scheme and the random forest. PLoS ONE 2015, 10, e0125811. [CrossRef] [PubMed]

28. Wang, L.; You, Z.H.; Chen, X.; Li, J.Q.; Yan, X.; Zhang, W.; Huang, Y.A. An ensemble approach for large-scale identification of protein- protein interactions using the alignments of multiple sequences. Oncotarget 2016, 8 , 5149-5159. [CrossRef] [PubMed]

29. You, Z.; Le, Y.; Zh, L.; Xi, J.; Wang, B. Prediction of protein-protein interactions from amino acid sequences with ensemble extreme learning machines and principal component analysis. BMC Bioinform. 2013, 14, S10. [CrossRef] [PubMed]

30. Wong, L.; You, Z.H.; Ming, Z.; Li, J.; Chen, X.; Huang, Y.A. Detection of Interactions between Proteins through Rotation Forest and Local Phase Quantization Descriptors. Int. J. Mol. Sci. 2016, 17, 21. [CrossRef] [PubMed]

31. Lei, Y.K.; You, Z.H.; Ji, Z.; Zhu, L.; Huang, D.S. Assessing and predicting protein interactions by combining manifold embedding with multiple information integration. BMC Bioinform. 2012, 13, S3. [CrossRef] [PubMed]

32. Nanni, L. Letters: Hyperplanes for predicting protein-protein interactions. Neurocomputing 2005, 69, 257-263. [CrossRef]

33. You, Z.H.; Li, S.; Gao, X.; Luo, X.; Ji, Z. Large-scale protein-protein interactions detection by integrating big biosensing data with computational model. BioMed Res. Int. 2014, 2014, 598129. [CrossRef] [PubMed]

34. Huang, Y.A.; You, Z.H.; Li, X.; Chen, X.; Hu, P.; Li, S.; Luo, X. Construction of Reliable Protein-Protein Interaction Networks Using Weighted Sparse Representation Based Classifier with Pseudo Substitution Matrix Representation Features. Neurocomputing 2016, 218, 131-138. [CrossRef]

35. An, J.Y.; You, Z.H.; Chen, X.; Huang, D.S.; Yan, G.Y. Robust and accurate prediction of protein self-interactions from amino acids sequence using evolutionary information. Mol. BioSyst. 2016, 12, 3702-3710. [CrossRef] [PubMed]

36. Pan, J.B.; Hu, S.C.; Wang, H.; Zou, Q.; Ji, Z.L. PaGeFinder: Quantitative identification of spatiotemporal pattern genes. Bioinformatics 2012, 28, 1544-1545. [CrossRef] [PubMed] 
37. Zou, Q.; Li, X.B.; Jiang, W.R.; Lin, Z.Y.; Li, G.L.; Chen, K. Survey of MapReduce frame operation in bioinformatics. Brief. Bioinform. 2014, 15, 637. [CrossRef] [PubMed]

38. Zeng, X.; Zhang, X.; Zou, Q. Integrative approaches for predicting microRNA function and prioritizing disease-related microRNA using biological interaction networks. Brief. Bioinform. 2016, 17, 193-203. [CrossRef] [PubMed]

39. Li, P.; Guo, M.; Wang, C.; Liu, X.; Zou, Q. An overview of SNP interactions in genome-wide association studies. Brief. Funct. Genom. 2015, 14, 143-155. [CrossRef] [PubMed]

40. Fawcett, T. An introduction to ROC analysis. Pattern Recognit. Lett. 2006, 27, 861-874. [CrossRef]

41. Huang, J.; Ling, C.X. Using AUC and accuracy in evaluating learning algorithms. Knowl. Data Eng. Trans. 2005, 17, 299-310.

42. Chang, C.C.; Lin, C.J. LIBSVM: A library for support vector machines. ACM Trans. Intell. Syst. Technol. 2007, 2, 389-396. [CrossRef]

43. Quan, Z.; Li, J.; Li, S.; Zeng, X.; Wang, G. Similarity computation strategies in the microRNA-disease network: A survey. Brief. Funct. Genom. 2016, 15, 55.

44. Licata, L.; Briganti, L.; Peluso, D.; Perfetto, L.; Iannuccelli, M.; Galeota, E.; Sacco, F.; Palma, A.; Nardozza, A.P.; Santonico, E. MINT, the molecular interaction database: 2012 update. Nucleic Acids Res. 2012, 40, D857-D861. [CrossRef] [PubMed]

45. Bader, G.D.; Donaldson, I.; Wolting, C.; Ouellette, B.F.F.; Pawson, T.; Hogue, C.W.V. BIND—The Biomolecular Interaction Network Database. Nucleic Acids Res. 2001, 29, 242-245. [CrossRef] [PubMed]

46. Jones, D.T. Protein secondary structure prediction based on position-specific scoring matrices. J. Mol. Biol. 1999, 292, 195-202. [CrossRef] [PubMed]

47. Maurer-Stroh, S.; Debulpaep, M.; Kuemmerer, N.; de la Paz, M.L.; Martins, I.C.; Reumers, J.; Morris, K.L.; Copland, A.; Serpell, L.; Serrano, L. Exploring the sequence determinants of amyloid structure using position-specific scoring matrices. Nat. Methods 2010, 7, 237-242. [CrossRef] [PubMed]

48. Henikoff, J.G.; Henikoff, S. Using substitution probabilities to improve position-specific scoring matrices. Bioinformatics 1996, 12, 135-143. [CrossRef]

49. Paliwal, K.K.; Sharma, A.; Lyons, J.; Dehzangi, A. A Tri-Gram Based Feature Extraction Technique Using Linear Probabilities of Position Specific Scoring Matrix for Protein Fold Recognition. J. Theor. Biol. 2014, 13, 44-50. [CrossRef] [PubMed]

50. Altschul, S.F.; Madden, T.L.; Schäffer, A.A.; Zhang, J.; Zhang, Z.; Miller, W.; Lipman, D.J. Gapped BLAST and PSI-BLAST: A new generation of protein database search programs. Nucleic Acids Res. 1997, 25, 3389-3402. [CrossRef] [PubMed]

51. Huang, Q.Y.; You, Z.H.; Zhang, X.F.; Yong, Z. Prediction of Protein-Protein Interactions with Clustered Amino Acids and Weighted Sparse Representation. Int. J. Mol. Sci. 2015, 16, 10855-10869. [CrossRef] [PubMed]

52. Dayhoff, M. A model of evolutionary change in proteins. Atlas Protein Seq. Struct. 1977, 5, 345-352.

53. Bhagwat, M.; Aravind, L. PSI-BLAST tutorial. Methods Mol. Biol. 2007, 395, 177-186. [PubMed]

54. Xiao, R.Q.; Guo, Y.Z.; Zeng, Y.H.; Tan, H.F.; Tan, H.F.; Pu, X.M.; Li, M.L. Using position specific scoring matrix and auto covariance to predict protein subnuclear localization. J. Biomed. Sci. Eng. 2009, 2, 51-56. [CrossRef]

55. An, J.Y.; Meng, F.R.; You, Z.H.; Fang, Y.H.; Zhao, Y.J.; Ming, Z. Using the Relevance Vector Machine Model Combined with Local Phase Quantization to Predict Protein-Protein Interactions from Protein Sequences. BioMed Res. Int. 2016, 2016, 1-9. [CrossRef] [PubMed]

56. Kim, W.Y.; Kim, Y.S. A region-based shape descriptor using Zernike moments. Signal Process. Image Commun. 2000, 16, 95-102. [CrossRef]

57. Liao, S.X.; Pawlak, M. On the accuracy of Zernike moments for image analysis. IEEE Trans. Pattern Anal. Mach. Intell. 1998, 20, 1358-1364. [CrossRef]

58. Li, S.; Lee, M.C.; Pun, C.M. Complex Zernike moments features for shape-based image retrieval. IEEE Trans. Syst. Man Cybern. Part A Syst. Hum. 2009, 39, 227-237. [CrossRef]

59. Georgiou, D.N.; Karakasidis, T.E.; Megaritis, A.C. A short survey on genetic sequences, chou's pseudo amino acid composition and its combination with fuzzy set theory. Open Bioinform. J. 2013, 7, 41-48. [CrossRef]

60. Liu, T.; Qin, Y.; Wang, Y.; Wang, C. Prediction of Protein Structural Class Based on Gapped-Dipeptides and a Recursive Feature Selection Approach. Int. J. Mol. Sci. 2015, 17, 15. [CrossRef] [PubMed] 
61. Wang, S.; Liu, S. Protein Sub-Nuclear Localization Based on Effective Fusion Representations and Dimension Reduction Algorithm LDA. Int. J. Mol. Sci. 2015, 16, 30343-30361. [CrossRef] [PubMed]

62. Georgiou, D.N.; Karakasidis, T.E.; Nieto, J.J.; Torres, A. A study of entropy/clarity of genetic sequences using metric spaces and fuzzy sets. J. Theor. Biol. 2010, 267, 95. [CrossRef] [PubMed]

63. Hse, H.; Newton, A.R. Sketched symbol recognition using Zernike moments. In Proceedings of the 17th International Conference on Pattern Recognition, Cambridge, UK, 23-26 August 2004; Volume 1, pp. 367-370.

64. Noll, R.J. Zernike polynomials and atmospheric turbulence. JOsA 1976, 66, 207-211. [CrossRef]

65. Wang, J.Y.; Silva, D.E. Wave-front interpretation with Zernike polynomials. Appl. Opt. 1980, 19, 1510-1518. [CrossRef] [PubMed]

66. Schwiegerling, J.; Greivenkamp, J.E.; Miller, J.M. Representation of videokeratoscopic height data with Zernike polynomials. JOsA 1995, 12, 2105-2113. [CrossRef]

67. Chong, C.W.; Raveendran, P.; Mukundan, R. A comparative analysis of algorithms for fast computation of Zernike moments. Pattern Recognit. 2003, 36, 731-742. [CrossRef]

68. Singh, C.; Walia, E.; Upneja, R. Accurate calculation of Zernike moments. Inf. Sci. 2013, 233, 255-275. [CrossRef]

69. Hwang, S.K.; Billinghurst, M.; Kim, W.Y. Local Descriptor by Zernike Moments for Real-Time Keypoint Matching. Image Signal Process. 2008, 2, 781-785.

70. Liao, S.X.; Pawlak, M. A study of Zernike moment computing. Asian Conf. Comput. Vis. 2006, 98, $394-401$.

71. Khotanzad, A.; Hong, Y.H. Invariant Image Recognition by Zernike Moments. IEEE Trans. Pattern Anal. Mach. Intell. 1990, 12, 489-497. [CrossRef]

72. Kim, H.S.; Lee, H.K. Invariant image watermark using Zernike moments. IEEE Trans.Circuits Syst. Video Technol. 2003, 13, 766-775.

73. Zou, Q.; Zeng, J.C.; Cao, L.J.; Ji, R.R. A Novel Features Ranking Metric with Application to Scalable Visual and Bioinformatics Data Classification. Neurocomputing 2016, 173, 346-354. [CrossRef]

74. Burges, C.J.C. A Tutorial on Support Vector Machines for Pattern Recognition. Data Min. Knowl. Discov. 1998, 2, 121-167. [CrossRef]

75. Bishop, C.M.; Tipping, M.E.; Nh, C.C. Variational Relevance Vector Machines. Adv. Neural Inf. Process. Syst. 2000, 12, 299-334.

76. Li, Y.; Campbell, C.; Tipping, M. Bayesian automatic relevance determination algorithms for classifying gene expression data. Bioinformatics 2002, 18, 1332-1339.

77. Wei, L.Y.; Tang, J.J.; Zou, Q. Local-DPP: An Improved DNA-binding Protein Prediction Method by Exploring Local Evolutionary Information. Inf. Sci. 2017, 384, 135-144. [CrossRef]

78. Chen, H.; Tino, P.; Yao, X. Probabilistic classification vector machines. IEEE Trans. Neural Netw. 2009, 20, 901-914. [CrossRef] [PubMed]

79. Chen, H.; Tino, P.; Xin, Y. Efficient Probabilistic Classification Vector Machine With Incremental Basis Function Selection. IEEE Trans. Neural Netw. Learn. Syst. 2014, 25, 356-369. [CrossRef] [PubMed]

(C) 2017 by the authors. Licensee MDPI, Basel, Switzerland. This article is an open access article distributed under the terms and conditions of the Creative Commons Attribution (CC BY) license (http:/ / creativecommons.org/licenses/by/4.0/). 
Article

\title{
Determination of Genes Related to Uveitis by Utilization of the Random Walk with Restart Algorithm on a Protein-Protein Interaction Network
}

\author{
Shiheng Lu ${ }^{1}$, Yan Yan ${ }^{1}$, Zhen Li ${ }^{1}$, Lei Chen ${ }^{2}$, Jing Yang ${ }^{3}$, Yuhang Zhang ${ }^{4}$, Shaopeng Wang ${ }^{3}$ \\ and Lin Liu ${ }^{1, *}$ \\ 1 Department of Ophthalmology, Ren Ji Hospital, School of Medicine, Shanghai Jiao Tong University, \\ Shanghai 200127, China; ludice@163.com (S.L.); hz2004yan@163.com (Y.Y.); lizhen1981_1@126.com (Z.L.) \\ 2 College of Information Engineering, Shanghai Maritime University, Shanghai 201306, China; \\ chen_lei1@163.com \\ 3 School of Life Sciences, Shanghai University, Shanghai 200444, China; mercuryyangjing@sina.com (J.Y.); \\ wsptfb@163.com (S.W.) \\ 4 Institute of Health Sciences, Shanghai Institutes for Biological Sciences, Chinese Academy of Sciences, \\ Shanghai 200031, China; zhangyh825@163.com \\ * Correspondence: liulin20160929@hotmail.com; Tel.: +86-189-1835-8758
}

Academic Editor: Christo Z. Christov

Received: 9 April 2017; Accepted: 9 May 2017; Published: 13 May 2017

\begin{abstract}
Uveitis, defined as inflammation of the uveal tract, may cause blindness in both young and middle-aged people. Approximately $10-15 \%$ of blindness in the West is caused by uveitis. Therefore, a comprehensive investigation to determine the disease pathogenesis is urgent, as it will thus be possible to design effective treatments. Identification of the disease genes that cause uveitis is an important requirement to achieve this goal. To begin to answer this question, in this study, a computational method was proposed to identify novel uveitis-related genes. This method was executed on a large protein-protein interaction network and employed a popular ranking algorithm, the Random Walk with Restart (RWR) algorithm. To improve the utility of the method, a permutation test and a procedure for selecting core genes were added, which helped to exclude false discoveries and select the most important candidate genes. The five-fold cross-validation was adopted to evaluate the method, yielding the average F1-measure of 0.189. In addition, we compared our method with a classic GBA-based method to further indicate its utility. Based on our method, 56 putative genes were chosen for further assessment. We have determined that several of these genes (e.g., CCL4, Jun, and MMP9) are likely to be important for the pathogenesis of uveitis.
\end{abstract}

Keywords: uveitis; protein-protein interaction; random walk with restart algorithm

\section{Introduction}

Uveitis is defined as an inflammation of the uveal tract, which is composed of the ciliary body, iris and choroid [1,2]. Uveitis is one of the leading causes of permanent and irreversible blindness in young and middle-aged people and accounts for $10-15 \%$ of blindness in the Western world [1-3]. Uveitis can be caused by infectious and non-infectious factors; the latter include Vogt-Koyanagi-Harada (VKH) syndrome, Behcet's disease (BD), acute anterior uveitis (AAU), birdshot chorioretinopathy (BCR) and some types of cancers. VKH is an autoimmune disease characterized by systemic disorders including poliosis, vitiligo, alopecia, auditory signs and disorders of the central nervous system [4,5]. $\mathrm{BD}$ is a chronic multi-systemic inflammatory disease characterized by nongranulomatous uveitis, oral ulcers and skin lesions [2,6]. AAU is the most common non-infectious cause of uveitis and is characterized by self-limiting and recurrent inflammation involving the ciliary and iris body [7]. BCR is 
a chronic, bilateral, and posterior uveitis that has an almost 100\% genetic association with HLA-A29 [8]. Uveitis or uveitis masquerade syndrome could also be induced by some intraocular tumors, such as retinoblastoma and intraocular lymphoma, or their therapeutic approaches [9-15].

It has been reported that complex genetic mechanisms coupled with an aberrant immune response may be involved in the development of uveitis. In some cases, the pathogenesis of uveitis seemly has a different cause than those described above, such as sarcoidosis [16]. Mutations in different genes and gene families have been discovered in patients. In this study, we focused on the most important causes of uveitis and research for the putative genes involved in these processes. Human leukocyte antigens (HLAs) are the major molecules that are important for the development of uveitis, including uveitis associated with VKH (HLA-DR4, DRB1/DQA1), BD (HLA-B51), AAU (HLA-B27) and BCR (HLA-A29). In addition, genome-wide association studies revealed that abnormities of many non-HLA genes such as the interleukin (IL) family and the Signal transducer and activator of transcription 4 (STAT4) also participate in the progression of uveitis [17-19]. IL23R is associated with both VKH and AAU [20]. Furthermore, copy number variations (CNVs) of Toll-like receptors (TLRs), a family of cellular receptors that function in innate immune response, are associated with BD, VKH and AAU. These genes include TLRs 1-3, TLRs 5-7, and TLRs 9-10 [21]. SNPs of TLR4 were also shown to be involved in the development of BD [22]. In addition, it has been demonstrated that there is increased expression of T-bet and IFN- $\gamma$, two genes involved in the Th1 cell pathway, in uveitis patients [23]. The activator of STAT4 affects $I L-17$ production and is a shared risk factor for BD in different cohorts $[17,24]$. Finally, interleukins (notably IL-2, IL12B, IL18 and IL23R) are important cytokines that play a pathogenic role in the process of uveitis $[2,17,25]$. In this study, we mainly focused on the genes that play an important role in the immune system, transcription, or cell adhesion.

Using traditional methods, it is quite difficult to collect these large-scale data and analyze genes synthetically. The microarray is a widely used tool for the identification of novel genes. Microarray analysis has been used to determine a number of genes that are associated with uveitis, including the IL10 family and several other transcripts [16,26-29]. In recent years, computational analysis has been applied to identify virulence genes, but many of these genes were identified based on guilt by association (GBA) [30-32]. This approach assumes that the candidate genes, which are neighbors of disease genes, are more likely to be new virulence genes. Thus, the GBA-based methods only consider the neighbors of known disease genes to discover novel candidates. Therefore, these methods only examine part of the gene network. Random Walk with Restart (RWR) is another algorithm that identifies disease-related genes [33-35]. This algorithm utilizes a set of seed nodes that represent disease genes and performs random walking on the gene network. When the probabilities of all nodes are stable, the probability of a node gene correlating with disease is updated. The genes that correspond to nodes that have high probabilities may be potential novel candidate virulence genes. This method is useful for mining disease genes and to better explore the mechanism of disease. In addition, other studies have adopted the shortest path (SP) algorithm to identify novel disease genes [36-41]. By searching the shortest paths that connect any two validated disease genes, genes that are present in these paths could be extracted and considered as novel disease genes. An obvious advantage of the RWR or SP algorithms is that these algorithms utilize the entire gene network and consider more factors, therefore performing a more extensive and reliable analysis.

As discussed above, many genetic factors contribute to the pathogenesis of uveitis. In this study, we utilized computational analyses to build a genetic network based on previously known factors. A computational method was built to identify novel genes related to uveitis. First, a large network was constructed using human protein-protein interactions (PPIs). Next, the RWR algorithm was performed on the network using the validated uveitis-related genes as seed nodes, yielding several possible candidate genes. These candidate genes were filtered based on a set of criteria that were built by $p$-values and their associations with validated uveitis-related genes. To indicate the utility of the method, it was evaluated by five-fold cross-validation, resulting in the average F1-measure of 0.189. Furthermore, the proposed method was compared with a classic GBA-based method [30-32] to 
further prove its effectiveness for identification of uveitis-related genes. Through our method, 56 novel candidate genes were identified and extensively analyzed.

\section{Results and Discussion}

\subsection{Results of Testing Random Walk with Restart (RWR)-Based Method}

Before the RWR-based method was used to identify novel uveitis-related genes, five-fold cross-validation was adopted to evaluate its utility. For each part, the results yielded by the method on the rest four parts were counted as recall, precision and F1-measure, which are listed in Table 1. It can be observed that the average of recall, precision and F1-measure was $0.287,0.141$ and 0.189 , respectively. Although these measurements are not very high, the RWR-based method is still acceptable due to the difficulties for identification of novel genes with given functions. Besides, the utility of the RWR-based method would be further proved by comparing it with other methods, which is described in Section 2.5.

Table 1. The performance of the Random Walk with Restrart (RWR)-based method yielded by five-fold cross-validation.

\begin{tabular}{cccc}
\hline Index of Part & Recall & Precision & F1-Measure \\
\hline 1 & 0.172 & 0.089 & 0.118 \\
2 & 0.172 & 0.088 & 0.116 \\
3 & 0.379 & 0.177 & 0.242 \\
4 & 0.310 & 0.141 & 0.194 \\
5 & 0.400 & 0.211 & 0.276 \\
Mean & 0.287 & 0.141 & 0.189 \\
\hline
\end{tabular}

\subsection{RWR Genes}

Based on the uveitis-related genes, the RWR algorithm yielded a probability for each gene in the PPI network, which indicated the likelihood of the gene being important for uveitis. Then, genes were selected that had probabilities larger than $10^{-5}$. From our analysis, we obtained 3641 RWR genes, which are provided with their RWR probabilities in Supplementary Table S1.

\subsection{Candidate Genes}

According to the RWR-based method detailed in Section 3.3, RWR genes were filtered using a permutation test. For each RWR gene, a $p$-value was assigned to indicate whether the RWR gene is specific for uveitis. The $p$-value for each of the 3641 RWR genes is also provided in Supplementary Table S1. We found 1231 candidate genes that had a $p$-value $<0.05$ (see the first 1231 genes in Supplementary Table S1).

The 1231 candidate genes were then further analyzed using the criteria outlined in Section 3.3. For each candidate gene, MIS (cf. Equation (3)) and MFS (cf. Equation (5)) were calculated, and the values for each gene are available in Supplementary Table S1. The threshold for MIS was set at 900, while 0.8 was used as the threshold for MFS. Finally, we obtained 56 Ensembl IDs (listed in Table 2) corresponding to core candidate genes. These genes were deemed to be highly related to uveitis and could be considered novel candidate genes. As intuitionistic evidence, a sub-network was plotted in Figure 1, which contains the putative and validated genes. Each putative gene had strong associations with validated genes, implying that they had functions similar to those of the validated genes and may be novel uveitis-related genes with high probabilities. 


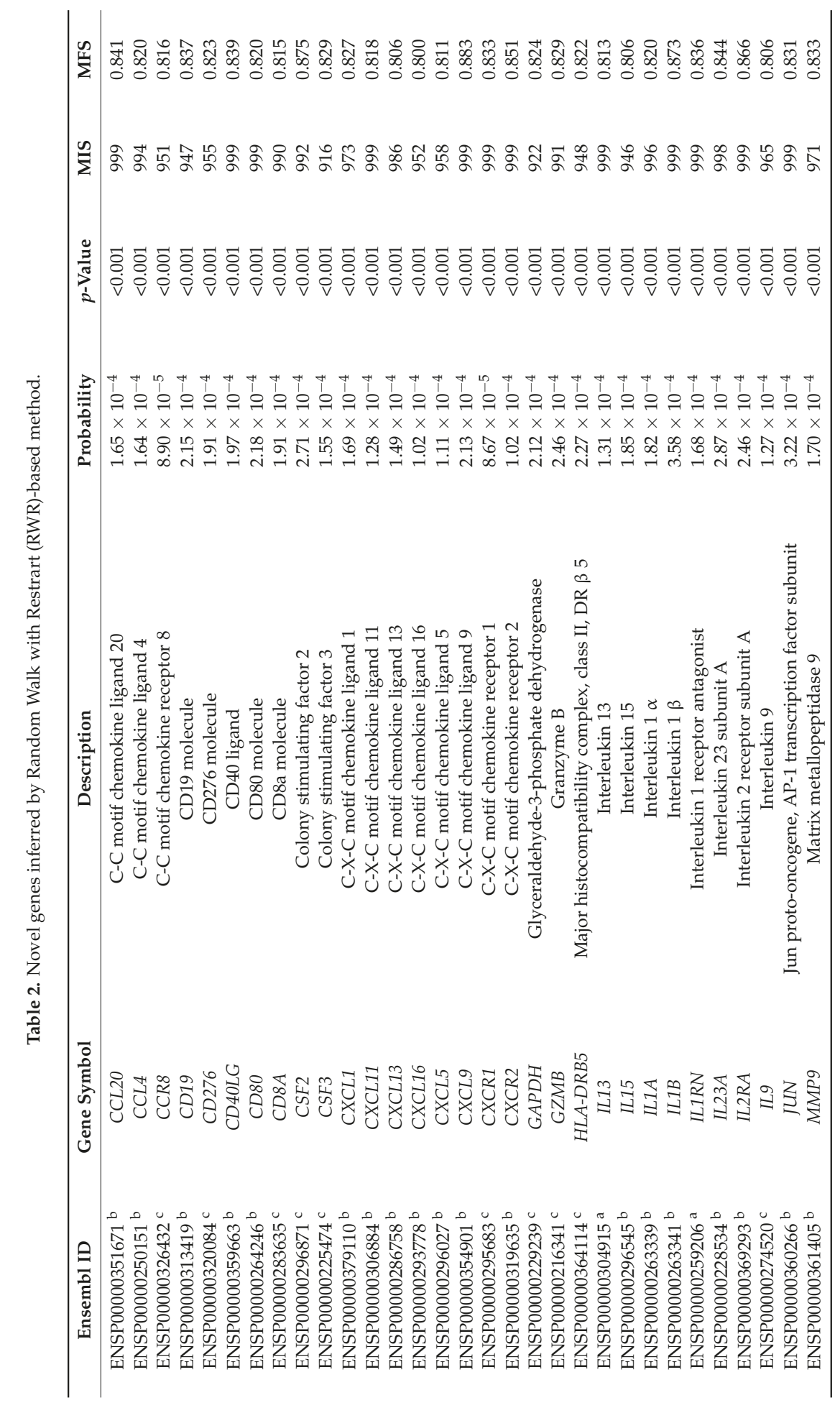




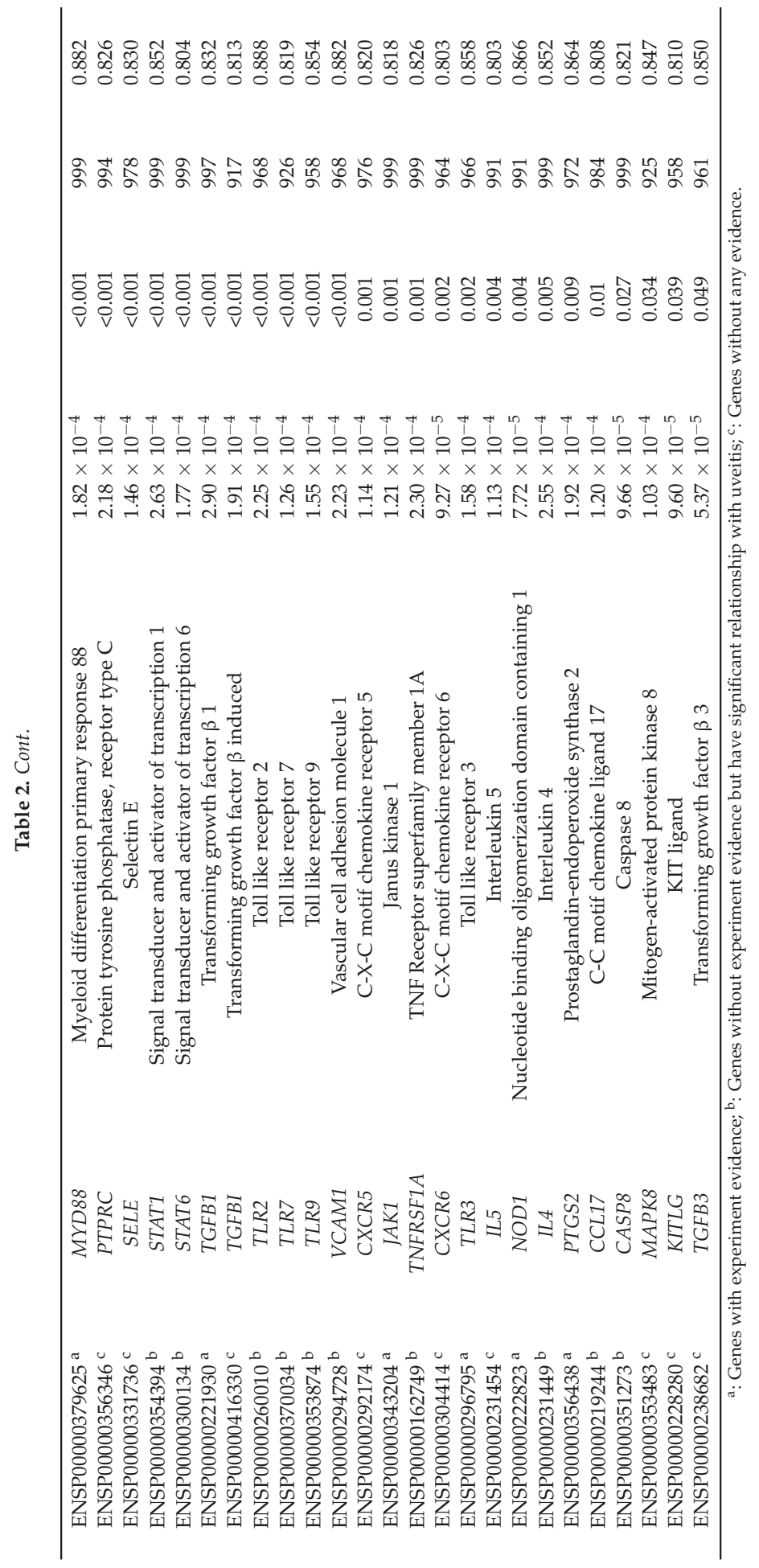




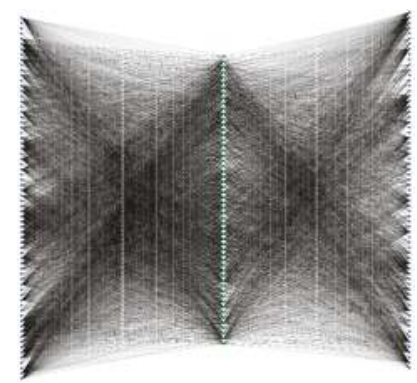

Figure 1. The sub-network of the large network containing Ensembl Identifications (IDs) of validated and putative uveitis-related genes. Blue nodes represent Ensembl IDs of validated uveitis-related genes. Green nodes represent Ensembl IDs of putative uveitis-related genes.

\subsection{Analysis of Novel Genes}

In this study, the RWR-based method yielded fifty-six genes that were deemed to have a significant correlation with uveitis. Detailed information for these genes is provided in Table 2.

\subsubsection{Immune System Regulation Genes}

CCL4 (C-C motif chemokine ligand 4) belongs to the cytokine family and is involved in immunoregulation and inflammation. It has been reported that CCL4 is associated with BD immunopathogenesis [42]. In the majority of VKH cases, the expression of another family member CCL17 was lower in cerebrospinal fluid than in serum, which indicated its potential function in VKH [43]. CCL17 also could be inhibited by overexpression of SOCS1 in the retina to regulate the recruitment of inflammatory cells [44]. The cytokine CCL20 was considered to be a specific biomarker of HLA-B27-associated uveitis [45]. Our study revealed that CCL4, CCL17 and CCL20 likely play essential roles in uveitis.

CD40 ligand (also known as CD154) is a type II transmembrane glycoprotein that has structural homology to the proteins of the TNF (tumor necrosis factor) family [46-49]. The interaction between the CD40 and CD40 ligand is important for both cellular and humoral immune responses [50]. The CD40 and CD40 ligand interaction provides signals in T-cell priming and effecter functions [46,48,49,51-53], whereas monocyte and B-cell apoptosis could be inhibited by their interaction [54]. It has been demonstrated that the $C D 40$ ligand is associated with the immune-pathogenesis of several autoimmune diseases including AU (anterior uveitis) [54,55]. The CD40 ligand is significantly expressed on T-cells in the peripheral blood of patients with AU [56]. The results of the RWR-based method revealed a MIS of 999 had a $p$-value $<0.001$. Expression of CD80 on dendritic cells (DCs) could be induced by activation of NOD1 and NOD2 and is involved in the pathogenesis of VKH syndrome [57]. In another report, it was found that BBR downregulated the expression of costimulatory molecules $C D 40, C D 80$ and CD86 on DCs [58]. The MIS and $p$-value of CD80 were 999 and $<0.001$, respectively. We speculate that these molecules play key roles in uveitis, but their mechanism in uveitis must still be clarified.

CSF2 (colony stimulating factor 2) is a cytokine that functions as a hematological cell growth factor by stimulating stem cells to produce granulocytes and monocytes [59]. Three signaling pathways can be activated by CSF2: the JAK2/STAT pathway, the MAP pathway and the PI3K pathway [60-64]. CSF2 is a valuable prognostic indicator and a therapeutic target in tumors [59]. CSF2 expression in uveitis is reported as rare. However, in this study, the MIS of CSF2 was 992 with a $p$-value $<0.001$. We speculate that CSF2 might be a key factor in the pathogenesis of uveitis.

Interleukins and their receptors are inflammatory cytokines that play an important role in immune system response. Many interleukins and their receptors are involved in uveitis, as discussed above. Our data showed that IL13, IL15, IL1A, IL1B, IL1RN, IL4, IL5, IL9, IL23A and IL2RA had MISs larger 
than 900 with $p$-values $<0.05$. It has been observed that the expression of IL1A is decreased in patients with clinically active BD, while the expression of $I L 1 B$ is increased in patients with active, inactive or ocular BD [65]. IL1B has been associated with ocular Behcet's disease [66]. IL-13 is a strong immunomodulatory cytokine which is a promising mode of treatment for uveitis [67-70]. $I L-15$ and its receptor system is involved in the inflammatory process and pathogenesis of BD and the IL-15/FC fusion protein has been shown to inhibit IRBP1-20 specific CD80+ T cell to decrease the severity of EAU [71,72]. An aberrantly high CNV of IL23A is a common risk factor for VKH and BD [73]. In mice, $I L-1 R N$ suppresses immune-mediated ocular inflammation and is considered a potential biomarker in the management of patients with uveitis [74]. Interleukin 2 receptor $\alpha(I L 2 R A)$ is a risk locus in various autoimmune diseases and a variant of this gene, $r s 2104286$, was demonstrated to be strongly associated with intermediated uveitis [75]. An antibody against IL2RA, daclizumab is used to reduce intermediated uveitis [76]. However, rs2104286 was not related to endogenous non-anterior uveitis [77]. EAU (experimental autoimmune uveoretinitis) disease severity was reduced in mice in which $I L-1 B$ expression was reduced in the retina through deletion of $\mathrm{S} 100 \mathrm{~B}, \mathrm{a} \mathrm{Ca}^{2+}$ binding protein [78]. In a Lewis rat model of EAU, $I L-2$ and $I L-4$ were produced in destructive foci in the retina and uveal tract. $I L-2$ is thought to act as a cytotoxic effector, while $I L-4$ is associated with a helper cell function [79]. In patients with BD, IL-2 is more highly expressed, while $I L-4$ is more lowly expressed [80]. Genetic findings suggest that more work should be done to evaluate both the molecular target and the inhibitor for personalized therapy.

TLR2, TLR3, TLR7 and TLR9 belong to the Toll-like receptor (TLR) family, which are key factors in pathogen recognition and activation of innate immunity. TLRs are thought to be associated with infection and auto-inflammatory or autoimmune diseases, including uveitis [81,82]. Several autoimmune diseases, including BD, are associated with certain TLR gene polymorphisms [83,84]. A significant association has been found between polymorphism of TLR2 and ocular BD patients [85]. The expression of TLR4 was significantly up-regulated in monocyte-derived macrophages from VKH patients [86]. The chitosan-mediated TLR3-siRNA transfection had a potential therapeutic effect on remitting uveitis [87]. In a Chinese Han population, a high copy number of TLR7 conferred risk for BD patients [88]. In the Japanese population, the homozygous genotypes and homozygous deplotype configuration of TLR9 SNPs was associated with the susceptibility to BD [89]. It has been reported that glucocorticoid could improve uveitis by downregulating TLR7 and TLR9 in peripheral blood of patients with uveitis [90]. In our analysis, TLR2, TLR3, TLR7 and TLR9 have MIS scores of 968, 966, 926 and 958, respectively. We argue that TLR2, TLR3, TLR7 and TLR8 play essential roles in uveitis and thus require more attention.

\subsubsection{Transcription Associated Genes}

Jun (also known as jun proto-oncogene) is a critical subunit of the transcription factor AP1, which is an important modulator of diverse biological processes such as cell proliferation, apoptosis and malignant transformation [91]. Jun is activated through phosphorylation at Ser 63 and Ser 73 by JNK [92,93]. A high level of Jun has been observed in various types of cancer including non-small cell lung cancer, oral squamous cell carcinoma, breast cancer and colorectal cancer [94-98]. Overexpression of Jun has led to aberrant tumor growth and progression and inhibited cell apoptosis [94]. The underlying mechanism of Jun as it relates to uveitis is still unclear. In a gene screen assay, it was found that expression of Jun showed a significantly higher index in experimental lens-induced uveitis rabbits [99]. In our analysis, Jun showed a significant index $p$-value and an MIS of 999; therefore, we propose that Jun may be an essential factor in uveitis.

STAT1 and STAT6 encode transcription factors that belong to the STAT family, where phosphorylation is activated by receptor associated kinases. Atopic dermatitis associated uveitis may be driven by TH2-mediated inflammation [100]. IL-4 is a TH2 cytokine, and binding with its receptor can activate STAT6 via the (Jak) Janus kinase/STAT signaling pathway to promote many immunomodulatory genes [101]. Furthermore, the Stat6 VT (STAT6 V547A/T548A) mouse model of 
atopic dermatitis exhibited uveitis symptoms [100]. In the STAT family, TH17 cells can be induced by IL-2 and suppressed by IL-27/STAT1 to contribute to uveitis [102]. In this study, STAT1 and STAT6 both had significant $p$-values and MIS of 999, and therefore we hypothesize that STAT1/6 function has a putative role in uveitis.

\subsubsection{Cell Adhesion and Signal Transduction Related Genes}

Matrix metalloproteinase $(M M P)$ are key factors for the degradation of extracellular matrix components and modification of cytokines, protease inhibitors, and cell surface signaling systems [103-106]. Polymorphisms on the MMP-9 promoter can affect the development of visceral involvement in Korean people with BD [107]. In our RWR analysis, MMP9 had an MIS of 971 and a $p$-value $<0.001$, which suggests that MMP-9 may be a novel susceptibility gene for uveitis.

VCAM1 (vascular cell adhesion molecule 1) belongs to the Ig superfamily and is a cell surface sialoglycoprotein expressed by cytokine-activated endothelium. This protein is mediated by leukocyte-endothelial cell adhesion and signal transduction $[108,109]$. VCAM1 can be regulated by inflammatory cytokines such as IL1B [108]. Uveitis is closely associated with the immune system and immune-related proteins including the interleukin family. In our study, the MIS of VCAM1 was 968 and the $p$-value was less than 0.05 , which makes VCAM1 a candidate gene for uveitis.

We detected 56 novel uveitis-related genes using the RWR-based method. These genes can be clustered into three categories, shown in Figure 2. Among these 56 potential genes, eight $(8 / 56,14.3 \%)$ genes, IL13, IL1RN, JAK1, MYD88, NOD1, PTGS2, TGFB1 and TLR3, were considered as uveitis genes by experimental evidence [110-117], and 29 (29/56, 51.8\%) genes (CASP8, CCL17, CCL20, CCL4, CD19, CD40 LG, CD80, CXCL1, CXCL11, CXCL13, CXCL16, CXCL5, CXCL9, CXCR2, IL15, IL1A, IL1B, IL23A, IL2RA, IL4, JUN, MMP9, STAT1, STAT6, TLR2, TLR7, TLR9, TNFRSF1A and VCAM1) had a correlation with uveitis. However, the pathogenesis is not clear. In our results, we found that these genes have a significant relationship with uveitis genes and therefore need more validation. There are few reports of the rest of the genes (19/56, 33.9\%) (CCR8, CD276, CD8A, CSF2, CSF3, CXCR1, CXCR5, CXCR6, GAPDH, GZMB, HLA-DB5, IL5, IL9, KITLG, MAPK8, PTPRC, SELE, TGFB3 and TGFBI) which participate in the process of uveitis. We considered that they might be novel uveitis genes and merit attention. We argue that some of these may be critical putative virulence genes for uveitis and could be interesting agents for the treatment of human uveitis.

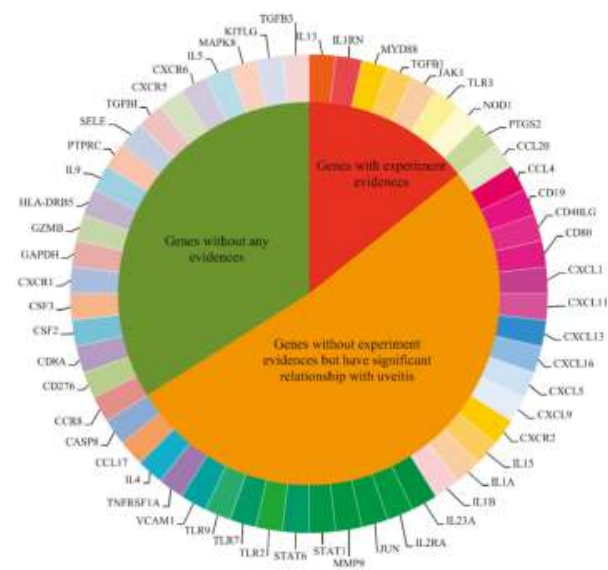

Figure 2. Clustering results of the 56 novel genes according to their evidences for being novel uveitis-related genes. Among 56 novel genes, eight have experiment evidence, 29 have significant relationship with uveitis but without experiment evidence, while no evidence can be found for the rest genes. 


\subsection{Comparison of Other Methods}

The results listed in Sections 2.1-2.4 can partly prove the effectiveness of the RWR-based method. In this section, we compared our method with a classic GBA-based method [30-32], i.e., a method like the nearest neighbor algorithm (NNA). This method identified novel genes from neighbors of the uveitis-related genes in a network. For convenience, we directly used the PPI network that was adopted in the RWR-based method. In addition, we called a neighbor of a node is a nearer neighbor if the edge between them was assigned a higher weight due to the definition of the interaction score reported in STRING. The GBA-based method selected the $k$ nearest neighbors of each uveitis-related genes and collected them together as the predicted genes of the method, where $k$ is a predefined parameter.

The five-fold cross-validation method was also adopted to test the GBA-based method, which used the same partition in testing the RWR-based method. Because we do not know the best value of $k$, we tried the following values: $1,2,3,4,5,6,7,8,9,10,20,30,40,50,60,70,80,90$, and 100 . The testing results are provided in Supplementary Table S2. The best performance of the GBA-based method with different parameter $k$ on each part is shown in Table 3. Compared with the testing results of RWR-based method, also listed in Table 3 for convenience, we can see that GBA-based method provides higher recalls sometimes, however, it always provides lower precisions, indicating the GBA-based method can yield more false positive genes. If only considering the F1-measure, we can conclude that F1-measures of the RWR-based method are always higher than those of the GBA-based method. It is indicated that the RWR-based method is superior to GBA-based method for identification of uveitis-related genes.

Table 3. Comparison of the RWR-based method and GBA-based method.

\begin{tabular}{cccccccc}
\hline \multirow{2}{*}{ Index of Part } & \multicolumn{3}{c}{ RWR-Based Method } & \multicolumn{4}{c}{ GBA-Based Method } \\
\cline { 2 - 8 } & Recall & Precision & F1-Measure & Best Value of $\boldsymbol{k}$ & Recall & Precision & F1-Measure \\
\hline 1 & 0.172 & 0.089 & 0.118 & 1 & 0.207 & 0.061 & 0.094 \\
2 & 0.172 & 0.088 & 0.116 & 1 & 0.207 & 0.059 & 0.092 \\
3 & 0.379 & 0.177 & 0.242 & 3 & 0.345 & 0.039 & 0.069 \\
4 & 0.310 & 0.141 & 0.194 & 1 & 0.172 & 0.052 & 0.079 \\
5 & 0.400 & 0.211 & 0.276 & 3 & 0.500 & 0.061 & 0.109 \\
\hline
\end{tabular}

\section{Materials and Methods}

\subsection{Materials}

Uveitis-related genes were collected from literatures indexed by PubMed (http://www.ncbi. nlm.nih.gov/pubmed/). The keywords "uveitis" and "genes" were used to search the literature in PubMed, which resulted in the collection of 744 papers. Among them, 98 review papers that generally summarized uveitis-related genes were manually reviewed. From those 98 papers, 121 genes were chosen from 96 reviews reporting the functional genes that may be important for uveitis or for specific uveitis symptoms. These genes are provided in Supplementary Table S3. In total, 146 Ensembl IDs for these genes were also determined and are provided in Supplementary Table S3.

\subsection{Protein-protein Interaction (PPI) Network}

PPIs are useful for the investigation of genetic disorders because they play an essential role in intracellular and intercellular biochemical processes. Many computational methods have been developed using this information, such as the prediction engines for the identification of protein functions [118-120] and methods for identification of novel disease genes [36-38]. Several methods were built based on the hypothesis that two proteins in a PPI are more likely to share similar functions. Thus, we can infer novel genes related to uveitis using PPI information and the uveitis-related genes mentioned in Section 3.1. 
In this study, we used the PPI information retrieved from STRING (Search Tool for the Retrieval of Interacting Genes/Proteins, Version 9.1, http://string-db.org/) [121] to construct the PPI network that the RWR algorithm can be applied. To access the PPI information in STRING, we downloaded the file "protein.links.v9.1 txt.gz". Because "9606" is the organism code for the human interactome in STRING, lines in this file that started with "9606" were extracted, obtaining 2,425,314 human PPIs involving 20,770 proteins. According to STRING, these PPIs were derived from the following four sources: (1) genomic context; (2) high-throughput experiments; (3) (conserved) co-expression; and (4) previous knowledge. Thus, the information in STRING contained both the direct (physical) and the indirect (functional) association between proteins, therefore STRING could widely measure the associations between proteins. Each PPI contained two Ensembl IDs and one score that ranged between 150 and 999, which indicated the strength of the interaction. An interaction with a high score meant this interaction has a high probability of occurring. For each interaction containing proteins $p_{a}$ and $p_{b}$, the score was denoted by $S\left(p_{a}, p_{b}\right)$. The PPI network defined the 20,770 proteins as the nodes, and two nodes were adjacent if and only if their corresponding proteins can form a PPI. Additionally, each edge in the network represented a PPI; thus, we assigned a weight to each edge, which was defined as the score of its corresponding PPI. From our analysis, a PPI network containing 20,770 nodes and 2,425,314 edges was obtained.

\subsection{RWR-Based Method}

The RWR algorithm was executed on the PPI network using validated genes as seed nodes to search possible genes. Then, a permutation test was executed to exclude false discoveries found by RWR. The remaining candidate genes with strong associations to validated genes were selected for further analysis. The pseudo-codes of the RWR-based method are listed in Table 4.

Table 4. The pseudo-code of the RWR-based method.

\section{RWR-Based Method}

Input: Ensembl IDs of uveitis-related genes, a PPI network

Output: A number of putative uveitis-related genes

1. Execute the RWR algorithm on the PPI network using the Ensembl IDs of uveitis-related genes as seed nodes, yielding a probability for each gene in the network; genes with probabilities higher than $10^{-5}$ were selected and called RWR genes;

2. Execute a permutation test, producing the $p$-value for each RWR gene; select RWR genes with $p$-values less than 0.05 ; the remaining genes were called candidate genes;

3. For each candidate gene, calculate its MIS (cf. Equation (3)) and MFS (cf. Equation (5)); select candidate genes with MISs no less than 900 and MFSs larger than 0.8;

4. Output the remaining candidate genes as the putative uveitis-related genes.

\subsubsection{Searching Possible Genes Using the RWR Algorithm}

RWR is a type of ranking algorithm [33]. Based on a seed node or a set of seed nodes, it simulates a walker that starts from the nodes and randomly walks in a network. Here, 146 Ensembl IDs listed in Supplementary Table S3 were deemed as seed nodes. Starting from these nodes, we attempted to discover novel nodes (genes) related to uveitis. In the beginning of the RWR algorithm, a 20,770-D vector $P_{0}$ was constructed, in which each composition represented the probability that a node in the network was a uveitis-related gene. Because the 146 Ensembl IDs represented validated uveitis-related genes, their compositions in $P_{0}$ were set to $1 / 146$, while others were set to zero. Then, the RWR algorithm repeatedly updated this probability vector until it became stable. We designated $P_{i}$ to represent the probability vector after the $i$-th step was executed. The probability vector was updated according to the following equation:

$$
P_{i+1}=(1-r) A^{T} P_{i}+r P_{0}
$$


where $A$ represented the column-wise normalized adjacency matrix of the PPI network and $r$ was set to 0.8 . When $\left\|P_{t+1}-P_{t}\right\|_{L_{1}}<10^{-6}$, the update procedure was stopped, and $P_{t+1}$ was the output of the RWR algorithm.

According to the probability vector yielded by the RWR algorithm, some nodes received high probabilities. It was apparent that their corresponding genes are more likely to be uveitis-related genes. To avoid missing possible uveitis-related genes, we set a probability threshold of $10^{-5}$. The corresponding genes of these nodes were designated as RWR genes.

In this study, we used the RWR program on the heterogeneous network that was implemented in Matlab and proposed by Li and Patra [122]. The code can be downloaded at http:/ /www3.ntu.edu. sg/home/aspatra/research/Yongjin_BI2010.zip. By setting the special values of some parameters, this program could be used to execute the RWR algorithm on a single network.

\subsubsection{Excluding False Discoveries Using the Permutation Test}

Based on the validated uveitis-related genes and RWR algorithm, new RWR genes were accessed. However, this result was influenced by the structure of the constructed PPI network, i.e., some RWR genes were selected due to the structure of the network and they were not necessarily unique to uveitis. Furthermore, if we randomly selected some nodes in the network as seed nodes of the RWR algorithm, these genes were still selected for and were therefore deemed as likely to be false positive. To control for these genes, a permutation test was executed. We randomly constructed 1000 Ensembl ID sets, denoted by $E_{1}, E_{2}, \ldots, E_{1000}$, consisting of 146 Ensembl IDs. For each set, the Ensembl IDs were deemed seed nodes of the RWR algorithm. Each RWR gene was given a probability. Thus, there were 1000 probabilities for 1000 sets and one probability for 146 Ensembl IDs of the uveitis-related genes for each RWR gene. Then, a measurement, called the $p$-value, was counted for each RWR gene $g$, which was defined as:

$$
p-\operatorname{value}(g)=\frac{\Theta}{1000}
$$

where $\Theta$ represented the number of randomly constructed sets where the probability assigned to $g$ was larger than that for the 146 Ensembl IDs of uveitis-related genes. Clearly, an RWR gene with a high $p$-value indicated that the gene was not specific for uveitis and should be discarded. RWR genes with $p$-values less than 0.05 were selected for further analysis as potential candidate genes for uveitis.

\subsubsection{Selection of Core Genes by Associations with Validated Genes}

We hypothesized that, of the candidate genes, some may have a strong correlation with uveitis. To further select core candidate genes, two criteria were designed. Candidate genes satisfying both criteria were selected for additional analysis. Candidate genes that had the strongest associations with uveitis-related genes were more likely to be novel uveitis-related genes. Thus, for each candidate gene $g$, we calculated the maximum interaction score (MIS) as follows:

$$
\operatorname{MIS}(g)=\max \left\{S\left(g, g^{\prime}\right): g^{\prime} \text { is a uveitis - related gene }\right\}
$$

A high MIS suggested that the candidate gene was closely related to at least one uveitis-related gene, indicating that it was a novel uveitis-related gene with a high probability. According to STRING, a score of 900 was the cut-off for the highest confidence level. Therefore, candidate genes with MISs larger than 900 were selected.

Validated uveitis-related genes have strong associations with specific gene ontology (GO) terms and Kyoto Encyclopedia of Genes and Genomes (KEGG) pathways. Therefore, candidate genes that had similar associations with uveitis GO terms and KEGG pathways were more likely to be novel uveitis-related genes. We performed GO term (KEGG pathway) [123-126] enrichment analysis for candidate genes and uveitis-related genes. The representation of a gene $g$ on all GO terms and KEGG pathways was encoded into a vector ES (g) using this theory. This vector can be obtained 
by an in-house program using the $\mathrm{R}$ function phyper. The $\mathrm{R}$ code used was "score $<--\log 10$ (phyper (numWdrawn-1, numW, numB, numDrawn, lower.tail = FALSE))," where numW, numB, and numDrawn are the number of genes annotated to the GO term or KEGG pathway, the number of genes not annotated to the GO term or KEGG pathway, and the number of neighbors of gene $g$ and numWdrawn is the number of neighbors of gene $g$ that are also annotated to the GO term or KEGG pathway. The relativity of the two genes $g$ and $g^{\prime}$ on GO terms and KEGG pathways was measured by

$$
\Gamma\left(g, g^{\prime}\right)=\frac{E S(g) \cdot E S\left(g^{\prime}\right)}{\|E S(g)\| \cdot\left\|E S\left(g^{\prime}\right)\right\|}
$$

A high outcome of Equation (4) indicated that $g$ and $g^{\prime}$ have a similar relationship in terms of GO terms and KEGG pathways. For any candidate gene $g$, we calculated the maximum function score (MFS) using the following equation:

$$
\operatorname{MFS}(g)=\max \left\{\Gamma\left(g, g^{\prime}\right): g^{\prime} \text { is a uveitis - related gene }\right\}
$$

Candidate genes with high MFSs were selected. In this equation, we set 0.8 as the threshold of MFS to select essential candidate genes.

\subsection{Methods for Testing RWR-Based Method}

In this study, we designed the RWR-based method to identify novel uveitis-related genes. However, it is necessary to test its effectiveness in advance. Here, the five-fold cross-validation [127] was employed. In detail, 146 Ensembl IDs of uveitis-related genes were randomly and equally divided into five parts. Then, Ensembl IDs in each part were singled out in turn and other Ensembl IDs in the rest four parts were used as the seed nodes in the RWR-based method. For each part, the results yielded by a good identification method on the rest four parts should satisfy the following conditions: (I) the results can recover a high proportion of the Ensembl IDs in the part; and (II) the results cannot contain several Ensembl IDs that are not in the part. Thus, recall and precision were employed to evaluate the results yielded by the RWR-based method, which can be calculated by

$$
\left\{\begin{array}{c}
\text { recall }=\frac{T P}{T P+F N} \\
\text { precision }=\frac{T P}{T P+F P}
\end{array}\right.
$$

where TP represented the number of Ensembl IDs in the part that can be recovered by the method, $F N$ represented the number of Ensembl IDs in the part that cannot be recovered by the method and $F P$ represented the number of Ensembl IDs that were yielded by the method and not in the part. In addition, to evaluate the predicted results on the whole, the F1-measure was also adopted, which can be computed by

$$
\mathrm{F} 1-\text { measure }=\frac{2 \times \text { recall } \times \text { precision }}{\text { recall }+ \text { precision }}
$$

It is clear that a high F1-measure means the good performance of the method.

\section{Conclusions}

This study presented a computational method to determine novel uveitis-related genes. Using the RWR algorithm and certain screening criteria, 56 putative genes were accessed. Extensive analysis of the obtained genes confirmed that several genes are associated with the pathogenesis of uveitis. We hope that the identified novel genes may be used as material to study uveitis and that the proposed method can be extended to other diseases.

Supplementary Materials: Supplementary materials can be found at www.mdpi.com/1422-0067/18/5/1045/s1. 
Acknowledgments: This study was supported by the National Natural Science Foundation of China (31371335), Natural Science Foundation of Shanghai (17ZR1412500).

Author Contributions: Lin Liu conceived and designed the experiments; Shiheng Lu, Yan Yan performed the experiments; Shiheng Lu, Zhen Li, Lei Chen and Jing Yang analyzed the data; Shiheng Lu, Yuhang Zhang, Shaopeng Wang contributed reagents/materials/analysis tools; Shiheng Lu wrote the paper.

Conflicts of Interest: The authors declare no conflict of interest.

\section{References}

1. Miserocchi, E.; Foqliato, G.; Modorati, G.; Bandello, F. Review on the worldwide epidemiology of uveitis. Eur. J. Ophthalmol. 2013, 23, 705-717. [CrossRef] [PubMed]

2. Fraga, N.A.; Oliveira, M.F.; Follador, I.; Rocha, B.O.; Rêgo, V.R. Psoriasis and uveitis: A literature review. An. Bras. Dermatol. 2012, 87, 877-883. [CrossRef] [PubMed]

3. Kulkarni, P. Review: Uveitis and immunosuppressive drugs. J. Ocul. Pharmacol. Ther. 2001, 17, 181-187. [CrossRef] [PubMed]

4. Murakami, S.; Inaba, Y.; Mochizuki, M.; Nakajima, A.; Urayama, A. A nation-wide survey on the occurrence of Vogt-Koyanagi-Harada disease in Japan. Jpn. J. Ophthalmol. 1994, 98, 389-392.

5. Moorthy, R.S.; Inomata, H.; Rao, N.A. Vogt-Koyanagi-Harada syndrome. Surv. Ophthalmol. 1995, 39, $265-292$. [CrossRef]

6. Evereklioglu, C. Current concepts in the etiology and treatment of Behcet disease. Surv. Ophthalmol. 2005, 50, 297-350. [CrossRef] [PubMed]

7. Chang, J.H.; Wakefield, D. Uveitis: A global perspective. Ocul. Immunol. Inflamm. 2002, 10, $263-279$. [CrossRef] [PubMed]

8. Priem, H.A.; Kijlstra, A.; Noens, L.; Baarsma, G.S.; de Laey, J.J.; Oosterhuis, J.A. HLA typing in birdshot chorioretinopathy. Am. J. Ophthalmol. 1988, 105, 182-185. [CrossRef]

9. Chaput, F.; Amer, R.; Baglivo, E.; Touitou, V.; Kozyreff, A.; Bron, D.; Bodaghi, B.; LeHoang, P.; Bergstrom, C.; Grossniklaus, H.E.; et al. Intraocular T-cell Lymphoma: Clinical Presentation, Diagnosis, Treatment, and Outcome. Ocul. Immunol. Inflamm. 2016, 22, 1-10. [CrossRef] [PubMed]

10. Kitazawa, K.; Nagata, K.; Yamanaka, Y.; Kuwahara, Y.; Lehara, T.; Kinoshita, S.; Sotozono, C. Diffuse Anterior Retinoblastoma with Sarcoidosis-Like Nodule. Case Rep. Ophthalmol. 2015, 6, 443-447. [CrossRef] [PubMed]

11. Catala-Mora, J.; Parareda-Salles, A.; Vicuña-Muñoz, C.G.; Medina-Zurinaga, M.; Prat-Bartomeu, J. [Uveitis masquerade syndrome as a presenting form of diffuse retinoblastoma]. Arch. Soc. Esp. Oftalmol. 2009, 84, 477-480. [PubMed]

12. All-Ericsson, C.; Economou, M.A.; Landau, I.; Seregard, S.; Träisk, F. Uveitis masquerade syndromes: Diffuse retinoblastoma in an older child. Acta Ophthalmol. Scand. 2007, 85, 569-570. [CrossRef] [PubMed]

13. Jovanovic, S.; Jovanović, Z.; Paović, J.; Teperković, V.S.; Pesić, S.; Marković, V. Two cases of uveitis masquerade syndrome caused by bilateral intraocular large B-cell lymphoma. Vojnosanit. Pregl. 2013, 70, 1151-1154. [CrossRef] [PubMed]

14. Shen, K.; Smith, S.V.; Lee, A.G. Acute myelogenous leukemia presenting with uveitis, optic disc edema, and granuloma annulare: Case report. Can. J. Ophthalmol. 2016, 51, e153-e155. [CrossRef] [PubMed]

15. Miserocchi, E.; Cimminiello, C.; Mazzola, M.; Russo, V.; Modorati, G.M. New-onset uveitis during CTLA-4 blockade therapy with ipilimumab in metastatic melanoma patient. Can. J. Ophthalmol. 2015, 50, e2-e4. [CrossRef] [PubMed]

16. Rosenbaum, J.T.; Pasadhika, S.; Crouser, E.D.; Choi, D.; Harrington, C.A.; Lewis, J.A.; Austin, C.R.; Diebel, T.N.; Vance, E.E.; Braziel, R.M.; et al. Hypothesis: Sarcoidosis is a STAT1-mediated disease. Clin. Immunol. 2009, 132, 174-183. [CrossRef] [PubMed]

17. Hou, S.; Yang, Z.; Du, L.; Jiang, Z.; Shu, Q.; Chen, Y.; Li, F.; Zhou, Q.; Ohno, S.; Chen, R.; et al. Identification of a susceptibility locus in STAT4 for Behcet's disease in Han Chinese in a genome-wide association study. Arthritis. Rheum. 2012, 64, 4104-4113. [CrossRef] [PubMed]

18. Remmers, E.F.; Cosan, F.; Kirino, Y.; Ombrello, M.J.; Abaci, N.; Satorius, C.; Le, J.M.; Yang, B.; Korman, B.D.; Cakiris, A.; et al. Genome-wide association study identifies variants in the MHC class I, IL10, and IL23R-IL12RB2 regions associated with Behcet's disease. Nat. Genet. 2010, 42, 698-702. [CrossRef] [PubMed] 
19. Mizuki, N.; Meguro, A.; Ota, M.; Ohno, S.; Shiota, T.; Kawagoe, T.; Ito, N.; Kera, J.; Okada, E.; Yatsu, K.; et al. Genome-wide association studies identify IL23R-IL12RB2 and IL10 as Behcet's disease susceptibility loci. Nat. Genet. 2010, 42, 703-706. [CrossRef] [PubMed]

20. Robinson, P.C.; Claushuis, T.A.; Cortes, A.; Martin, T.M.; Evans, D.M.; Leo, P.; Mukhopadhyay, P.; Bradbury, L.A.; Cremin, K.; Harris, J.; et al. Genetic dissection of acute anterior uveitis reveals similarities and differences in associations observed with ankylosing spondylitis. Arthritis Rheumatol. 2015, 67, 140-151. [CrossRef] [PubMed]

21. Fang, J.; Chen, L.; Tang, J.; Hou, S.; Liao, D.; Ye, Z.; Wang, C.; Cao, Q.; Kijlstra, A.; Yang, P. Association Between Copy Number Variations of TLR7 and Ocular Behcet's Disease in a Chinese Han Population. Investig. Ophthalmol. Vis. Sci. 2015, 56, 1517-1523. [CrossRef] [PubMed]

22. Kirino, Y.; Zhou, Q.; Ishigatsubo, Y.; Mizuki, N.; Tugal-Tutkun, I.; Seyahi, E.; Özyazgan, Y.; Ugurlu, S.; Erer, B.; Abaci, N. Targeted resequencing implicates the familial Mediterranean fever gene MEFV and the toll-like receptor 4 gene TLR4 in Behcet disease. Proc. Natl. Acad. Sci. USA 2013, 110, 8134-8139. [CrossRef] [PubMed]

23. Li, B.; Yang, P.; Chu, L.; Zhou, H.; Huang, X.; Zhu, L.; Kijlstra, A. T-bet expression in the iris and spleen parallels disease expression during endotoxin-induced uveitis. Graefe's Arch. Clin. Exp. Ophthalmol. 2007, 245, 407-413. [CrossRef] [PubMed]

24. Kirino, Y.; Bertsias, G.; Ishigatsubo, Y.; Mizuki, N.; Tugal-Tutkun, I.; Seyahi, E.; Ozyazgan, Y.; Sacli, F.S.; Erer, B.; Inoko, H.; et al. Genome-wide association analysis identifies new susceptibility loci for Behcet's disease and epistasis between HLA-B*51 and ERAP1. Nat. Genet. 2013, 45, 202-207. [CrossRef] [PubMed]

25. Jiang, Z.; Yang, P.; Hou, S.; Du, L.; Xie, L.; Zhou, H.; Kijlstra, A. IL-23R gene confers susceptibility to Behcet's disease in a Chinese Han population. Ann. Rheum. Dis. 2010, 69, 1325-1328. [CrossRef] [PubMed]

26. Smith, J.R.; Choi, D.; Chipps, T.J.; Pan, Y.; Zamora, D.O.; Davies, M.H.; Babra, B.; Powers, M.R.; Planck, S.R.; Rosenbaum, J.T. Unique gene expression profiles of donor-matched human retinal and choroidal vascular endothelial cells. Investig. Ophthalmol. Vis. Sci. 2007, 48, 2676-2684. [CrossRef] [PubMed]

27. Li, Z.; Liu, B.; Maminishkis, A.; Mahesh, S.P.; Yeh, S.; Lew, J.; Lim, W.K.; Sen, H.N.; Clarke, G.; Buggage, R. Gene expression profiling in autoimmune noninfectious uveitis disease. J. Immunol. 2008, 181, 5147-5157. [CrossRef] [PubMed]

28. Ohta, K.; Kikuchi, T.; Miyahara, T.; Yoshimura, N. DNA microarray analysis of gene expression in iris and ciliary body of rat eyes with endotoxin-induced uveitis. Exp. Eye Res. 2005, 80, 401-412. [CrossRef] [PubMed]

29. Li, Z.; Mzhesh, S.P.; Liu, B.; Yeh, S.; Lew, J.; Lim, W.; Levy Clarke, G.; Buggage, R.; Nussenblatt, R.B. Gene Expression Profiling of Non-infectious Uveitis Patients Using Pathway Specific cDNA Microarray Analysis. Investig. Ophthalmol. Vis. Sci. 2007, 48, 1505. [CrossRef]

30. Oliver, S. Guilt-by-association goes global. Nature 2000, 403, 601-603. [CrossRef] [PubMed]

31. Oti, M.; Snel, B.; Huynen, M.A.; Brunner, H.G. Predicting disease genes using protein-protein interactions. J. Méd. Genet. 2006, 43, 691-698. [CrossRef] [PubMed]

32. Krauthammer, M.; Kaufmann, C.A.; Conrad Gilliam, T.; Rzhetsky, A. Molecular triangulation: Bridging linkage and molecular-network information for identifying candidate genes in Alzheimer's disease. Proc. Natl. Acad. Sci. USA 2004, 101, 15148-15153. [CrossRef] [PubMed]

33. Kohler, S.; Bauer, S.; Horn, D.; Robinson, P.N. Walking the interactome for prioritization of candidate disease genes. Am. J. Hum. Genet. 2008, 82, 949-958. [CrossRef] [PubMed]

34. Li, Y.; Li, J. Disease gene identification by random walk on multigraphs merging heterogeneous genomic and phenotype data. BMC Genom. 2012, 13, S27. [CrossRef] [PubMed]

35. Jiang, R.; Gan, M.X.; He, P. Constructing a gene semantic similarity network for the inference of disease genes. BMC Syst. Biol. 2011, 5, S2. [CrossRef] [PubMed]

36. Chen, L.; Hao, X.Z.; Huang, T.; Shu, Y.; Huang, G.H.; Li, H.P. Application of the shortest path algorithm for the discovery of breast cancer related genes. Curr. Bioinform. 2016, 11, 51-58. [CrossRef]

37. Zhang, J.; Yang, J.; Yang, T.; Huang, T.; Shu, Y.; Chen, L. Identification of novel proliferative diabetic retinopathy related genes on protein-protein interaction network. Neurocomputing 2016, 217, 63-72. [CrossRef]

38. Gui, T.; Dong, X.; Li, R.; Li, Y.; Wang, Z. Identification of Hepatocellular Carcinoma-Related Genes with a Machine Learning and Network Analysis. J. Comput. Biol. 2015, 22, 63-71. [CrossRef] [PubMed]

39. Chen, L.; Yang, J.; Huang, T.; Kong, X.; Lu, L.; Cai, Y.D. Mining for novel tumor suppressor genes using a shortest path approach. J. Biomol. Struct. Dyn. 2016, 34, 664-675. [CrossRef] [PubMed] 
40. Chen, L.; Huang, T.; Zhang, Y.H.; Jiang, Y.; Zheng, M.; Cai, Y.D. Identification of novel candidate drivers connecting different dysfunctional levels for lung adenocarcinoma using protein-protein interactions and a shortest path approach. Sci. Rep. 2016, 6, 29849. [CrossRef] [PubMed]

41. Chen, L.; Wang, B.; Wang, S.; Yang, J.; Hu, J.; Xie, Z.; Wang, Y.; Huang, T.; Cai, Y.D. OPMSP: A computational method integrating protein interaction and sequence information for the identification of novel putative oncogenes. Protein Pept. Lett. 2016, 23, 1081-1094. [CrossRef] [PubMed]

42. Oguz, A.K.; Yılmaz, S.T.; Oygür, Ç.Ş.; Çandar, T.; Sayın, I.; Kılıçoğlu, S.S.; Ergün, İ.; Ateş, A.; Özdağ, H.; Akar, N. Behcet's: A Disease or a Syndrome? Answer from an Expression Profiling Study. PLoS ONE 2016, 11, e0149052. [CrossRef] [PubMed]

43. Miyazawa, I.; Abe, T.; Narikawa, K.; Feng, J.; Misu, T.; Nakashima, I.; Fujimori, J.; Tamai, M.; Fujihara, K.; Itoyama, Y. Chemokine profile in the cerebrospinal fluid and serum of Vogt-Koyanagi-Harada disease. J. Neuroimmunol. 2005, 158, 240-244. [CrossRef] [PubMed]

44. Yu, C.R.; Mahdi, R.R.; Oh, H.M.; Amadi-Obi, A.; Levy-Clarke, G.; Burton, J.; Eseonu, A.; Lee, Y.; Chan, C.C.; Egwuagu, C.E. Suppressor of cytokine signaling-1 (SOCS1) inhibits lymphocyte recruitment into the retina and protects SOCS1 transgenic rats and mice from ocular inflammation. Investig. Ophthalmol. Vis. Sci. 2011, 52, 6978-6986. [CrossRef] [PubMed]

45. Abu El-Asrar, A.M.; Berghmans, N.; Al-Obeidan, S.A.; Mousa, A.; Opdenakker, G.; van Damme, J.; Struyf, S. The Cytokine Interleukin-6 and the Chemokines CCL20 and CXCL13 Are Novel Biomarkers of Specific Endogenous Uveitic Entities. Investig. Ophthalmol. Vis. Sci. 2016, 57, 4606-4613. [CrossRef] [PubMed]

46. Hollenbaugh, D.; Grosmaire, L.S.; Kullas, C.D.; Chalupny, N.J.; Braesch-Andersen, S.; Noelle, R.J.; Stamenkovic, I.; Ledbetter, J.A.; Aruffo, A. The human T cell antigen gp39, a member of the TNF gene family, is a ligand for the CD40 receptor: Expression of a soluble form of gp39 with B cell co-stimulatory activity. EMBO J. 1992, 11, 4313-4321. [PubMed]

47. Lane, P.; Traunecker, A.; Hubele, S.; Inui, S.; Lanzavecchia, A.; Gray, D. Activated human T cells express a ligand for the human B cell-associated antigen CD40 which participates in T cell-dependent activation of B lymphocytes. Eur. J. Immunol. 1992, 22, 2573-2578. [CrossRef] [PubMed]

48. Noelle, R.J.; Ledbetter, J.A.; Aruffo, A. CD40 and its ligand, an essential ligand-receptor pair for thymus-dependent B-cell activation. Immunol. Today 1992, 13, 431-433. [CrossRef]

49. Fanslow, W.C.; Srinivasan, S.; Paxton, R.; Gibson, M.G.; Spriggs, M.K.; Armitage, R.J. Structural characteristics of CD40 ligand that determine biological function. Semin. Immunol. 1994, 6, 267-278. [CrossRef] [PubMed]

50. Howard, L.M.; Miga, A.J.; Vanderlugt, C.L.; Dal Canto, M.C.; Laman, J.D.; Noelle, R.J.; Miller, S.D. Mechanisms of immunotherapeutic intervention by anti-CD40L (CD154) antibody in an animal model of multiple sclerosis. J. Clin. Investig. 1999, 103, 281-290. [CrossRef] [PubMed]

51. Casamayor-Palleja, M.; Khan, M.; MacLennan, I.C. A subset of CD4+ memory T cells contains preformed CD40 ligand that is rapidly but transiently expressed on their surface after activation through the $\mathrm{T}$ cell receptor complex. J. Exp. Med. 1995, 181, 1293-1301. [CrossRef] [PubMed]

52. Stuber, E.; Strober, W.; Neurath, M. Blocking the CD40L-CD40 interaction in vivo specifically prevents the priming of T helper 1 cells through the inhibition of interleukin 12 secretion. J. Exp. Med. 1996, 183, 693-698. [CrossRef] [PubMed]

53. Grewal, I.S.; Flavell, R.A. CD40 and CD154 in cell-mediated immunity. Annu. Rev. Immunol. 1998, 16, 111-135. [CrossRef] [PubMed]

54. Ogard, C.; Sorensen, T.L.; Krogh, E. Increased CD40 ligand in patients with acute anterior uveitis. Acta Ophthalmol. Scand. 2005, 83, 370-373. [CrossRef] [PubMed]

55. Balashov, K.E.; Smith, D.R.; Khoury, S.J.; Hafler, D.A.; Weiner, H.L. Increased interleukin 12 production in progressive multiple sclerosis: Induction by activated CD4+ T cells via CD40 ligand. Proc. Natl. Acad. Sci. USA 1997, 94, 599-603. [CrossRef] [PubMed]

56. Ang, M.; Cheung, G.; Vania, M.; Chen, J.; Yang, H.; Li, J.; Chee, S.P. Aqueous cytokine and chemokine analysis in uveitis associated with tuberculosis. Mol. Vis. 2012, 18, 565-573. [PubMed]

57. Deng, B.; Ye, Z.; Li, L.; Zhang, D.; Zhu, Y.; He, Y.; Wang, C.; Wu, L.; Kijlstra, A.; Yang, P. Higher Expression of NOD1 and NOD2 is Associated with Vogt-Koyanagi-Harada (VKH) Syndrome But Not Behcet's Disease (BD). Curr. Mol. Med. 2016, 16, 424-435. [CrossRef] [PubMed]

58. Yang, Y.; Qi, J.; Wang, Q.; Du, L.; Zhou, Y.; Yu, H.; Kijlstra, A.; Yang, P. Berberine suppresses Th17 and dendritic cell responses. Investig. Ophthalmol. Vis. Sci. 2013, 54, 2516-2522. [CrossRef] [PubMed] 
59. Lee, Y.Y.; Wu, W.J.; Huang, C.N.; Li, C.C.; Li, W.M.; Yeh, B.W.; Liang, P.I.; Wu, T.F.; Li, C.F. CSF2 Overexpression Is Associated with STAT5 Phosphorylation and Poor Prognosis in Patients with Urothelial Carcinoma. J. Cancer 2016, 7, 711-721. [CrossRef] [PubMed]

60. Jucker, M.; Feldman, R.A. Identification of a new adapter protein that may link the common $\beta$ subunit of the receptor for granulocyte/macrophage colony-stimulating factor, interleukin (IL)-3, and IL-5 to phosphatidylinositol 3-kinase. J. Biol. Chem. 1995, 270, 27817-27822. [PubMed]

61. Bittorf, T.; Jaster, R.; Brock, J. Rapid activation of the MAP kinase pathway in hematopoietic cells by erythropoietin, granulocyte-macrophage colony-stimulating factor and interleukin-3. Cell Signal. 1994, 6, 305-311. [CrossRef]

62. Kimura, A.; Rieger, M.A.; Simone, J.M.; Chen, W.; Wickre, M.C.; Zhu, B.M.; Hoppe, P.S.; O'Shea, J.J.; Schroeder, T.; Hennighausen, L. The transcription factors STAT5A/B regulate GM-CSF-mediated granulopoiesis. Blood 2009, 114, 4721-4728. [CrossRef] [PubMed]

63. Mui, A.L.; Wakao, H.; Harada, N.; O’Farrell, A.M.; Miyajima, A. Interleukin-3, granulocyte-macrophage colony-stimulating factor, and interleukin-5 transduce signals through two forms of STAT5. J. Leukoc. Biol. 1995, 57, 799-803. [PubMed]

64. Feldman, G.M.; Rosenthal, L.A.; Liu, X.; Hayes, M.P.; Wynshaw-Boris, A.; Leonard, W.J.; Hennighausen, L.; Finbloom, D.S. STAT5A-deficient mice demonstrate a defect in granulocyte-macrophage colony-stimulating factor-induced proliferation and gene expression. Blood 1997, 90, 1768-1776. [PubMed]

65. Taheri, S.; Borlu, M.; Evereklioglu, C.; Ozdemir, S.Y.; Ozkul, Y. mRNA Expression Level of Interleukin Genes in the Determining Phases of Behcet's Disease. Ann. Dermatol. 2015, 27, 291-297. [CrossRef] [PubMed]

66. Liang, L.; Tan, X.; Zhou, Q.; Zhu, Y.; Tian, Y.; Yu, H.; Kijlstra, A.; Yang, P. IL-1ß triggered by peptidoglycan and lipopolysaccharide through TLR2/4 and ROS-NLRP3 inflammasome-dependent pathways is involved in ocular Behcet's disease. Investig. Ophthalmol. Vis. Sci. 2013, 54, 402-414. [CrossRef] [PubMed]

67. Roberge, F.G.; de Smet, M.D.; Benichou, J.; Kriete, M.F.; Raber, J.; Hakimi, J. Treatment of uveitis with recombinant human interleukin-13. Br. J. Ophthalmol. 1998, 82, 1195-1198. [CrossRef] [PubMed]

68. Marie, O.; Thillaye-Goldenberg, B.; Naud, M.C.; de Kozak, Y. Inhibition of endotoxin-induced uveitis and potentiation of local TNF- $\alpha$ and interleukin-6 mRNA expression by interleukin-13. Investig. Ophthalmol. Vis. Sci. 1999, 40, 2275-2282.

69. Lemaitre, C.; Thillaye-Goldenberg, B.; Naud, M.C.; de Kozak, Y. The effects of intraocular injection of interleukin-13 on endotoxin-induced uveitis in rats. Investig. Ophthalmol. Vis. Sci. 2001, 42, 2022-2030.

70. De Kozak, Y.; Omri, B.; Smith, J.R.; Naud, M.C.; Thillaye-Goldenberg, B.; Crisanti, P. Protein kinase C 乙 (PKC 乙) regulates ocular inflammation and apoptosis in endotoxin-induced uveitis (EIU)—Signaling molecules involved in EIU resolution by PKC $\zeta$ inhibitor and interleukin-13. Am. J. Pathol. 2007, 170, 1241-1257. [CrossRef] [PubMed]

71. Xia, Z.J.; Kong, X.L.; Zhang, P. [In vivo effect of recombined IL-15/Fc fusion protein on EAU]. Sichuan Da Хиe Хиe Bao Yi Xue Ban 2008, 39, 944-949. [PubMed]

72. Choe, J.Y.; Lee, H.; Kim, S.G.; Kim, M.J.; Park, S.H.; Kim, S.K. The distinct expressions of interleukin-15 and interleukin-15 receptor $\alpha$ in Behcet's disease. Rheumatol. Int. 2013, 33, 2109-2115. [CrossRef] [PubMed]

73. Hou, S.; Liao, D.; Zhang, J.; Fang, J.; Chen, L.; Qi, J.; Zhang, Q.; Liu, Y.; Bai, L.; Zhou, Y.; et al. Genetic variations of IL17F and IL23A show associations with Behcet's disease and Vogt-Koyanagi-Harada syndrome. Ophthalmology 2015, 122, 518-523. [CrossRef] [PubMed]

74. Lim, W.K.; Fujimoto, C.; Ursea, R.; Mahesh, S.P.; Silver, P.; Chan, C.C.; Gery, I.; Nussenblatt, R.B. Suppression of immune-mediated ocular inflammation in mice by interleukin 1 receptor antagonist administration. Arch. Ophthalmol. 2005, 123, 957-963. [CrossRef] [PubMed]

75. Lindner, E.; Weger, M.; Steinwender, G.; Griesbacher, A.; Posch, U.; Ulrich, S.; Wegscheider, B.; Ardjomand, N.; El-Shabrawi, Y. IL2RA gene polymorphism rs2104286 A>G seen in multiple sclerosis is associated with intermediate uveitis: Possible parallel pathways? Investig. Ophthalmol. Vis. Sci. 2011, 52, 8295-8299. [CrossRef] [PubMed]

76. Nussenblatt, R.B.; Fortin, E.; Schiffman, R.; Rizzo, L.; Smith, J.; van Veldhuisen, P.; Sran, P.; Yaffe, A.; Goldman, C.K.; Waldmann, T.A.; et al. Treatment of noninfectious intermediate and posterior uveitis with the humanized anti-Tac mAb: A phase I/II clinical trial. Proc. Natl. Acad. Sci. USA 1999, 96, 7462-7466. [CrossRef] [PubMed] 
77. Cenit, M.C.; Marquez, A.; Cordero-Coma, M.; Fonollosa, A.; Adan, A.; Martinez-Berriotxoa, A.; Llorenc, V.; Diaz Valle, D.; Blanco, R.; Canal, J.; et al. Evaluation of the IL2/IL21, IL2RA and IL2RB genetic variants influence on the endogenous non-anterior uveitis genetic predisposition. BMC Med. Genet. 2013, 14, 52. [CrossRef] [PubMed]

78. Niven, J.; Hoare, J.; McGowan, D.; Devarajan, G.; Itohara, S.; Gannage, M.; Teismann, P.; Crane, I. S100B Up-Regulates Macrophage Production of IL1 $\beta$ and CCL22 and Influences Severity of Retinal Inflammation. PLoS ONE 2015, 10, e0132688. [CrossRef] [PubMed]

79. Charteris, D.G.; Lightman, S.L. Comparison of the expression of interferon gamma, IL2, IL4, and lymphotoxin mRNA in experimental autoimmune uveoretinitis. Br. J. Ophthalmol. 1994, 78, 786-790. [CrossRef] [PubMed]

80. Shahram, F.; Nikoopour, E.; Rezaei, N.; Saeedfar, K.; Ziaei, N.; Davatchi, F.; Amirzargar, A. Association of interleukin-2, interleukin-4 and transforming growth factor- $\beta$ gene polymorphisms with Behcet's disease. Clin. Exp. Rheumatol. 2011, 29, S28-S31. [PubMed]

81. Chang, J.H.; McCluskey, P.; Wakefield, D. Expression of toll-like receptor 4 and its associated lipopolysaccharide receptor complex by resident antigen-presenting cells in the human uvea. Investig. Ophthalmol. Vis. Sci. 2004, 45, 1871-1878. [CrossRef]

82. Chang, J.H.; McCluskey, P.J.; Wakefield, D. Toll-like receptors in ocular immunity and the immunopathogenesis of inflammatory eye disease. Br. J. Ophthalmol. 2006, 90, 103-108. [CrossRef] [PubMed]

83. Meguro, A.; Ota, M.; Katsuyama, Y.; Oka, A.; Ohno, S.; Inoko, H.; Mizuki, N. Association of the toll-like receptor 4 gene polymorphisms with Behcet's disease. Ann. Rheum. Dis. 2008, 67, 725-727. [CrossRef] [PubMed]

84. Song, G.G.; Choi, S.J.; Ji, J.D.; Lee, Y.H. Toll-like receptor polymorphisms and vasculitis susceptibility: Meta-analysis and systematic review. Mol. Biol. Rep. 2013, 40, 1315-1323. [CrossRef] [PubMed]

85. Fang, J.; Hu, R.; Hou, S.; Ye, Z.; Xiang, Q.; Qi, J.; Zhou, Y.; Kijlstra, A.; Yang, P. Association of TLR2 gene polymorphisms with ocular Behcet's disease in a Chinese Han population. Investig. Ophthalmol. Vis. Sci. 2013, 54, 8384-8392. [CrossRef] [PubMed]

86. Liang, L.; Tan, X.; Zhou, Q.; Tian, Y.; Kijlstra, A.; Yang, P. TLR3 and TLR4 But not TLR2 are Involved in Vogt-Koyanagi-Harada Disease by Triggering Proinflammatory Cytokines Production Through Promoting the Production of Mitochondrial Reactive Oxygen Species. Curr. Mol. Med. 2015, 15, 529-542. [CrossRef] [PubMed]

87. Chen, S.; Yan, H.; Sun, B.; Zuo, A.; Liang, D. Subretinal transfection of chitosan-loaded TLR3-siRNA for the treatment of experimental autoimmune uveitis. Eur. J. Pharm. Biopharm. 2013, 85, 726-735. [CrossRef] [PubMed]

88. Fang, J.; Chen, L.; Tang, J.H.; Hou, S.P.; Liao, D.; Ye, Z.; Wang, C.K.; Cao, Q.F.; Kijlstra, A.; Yang, P.Z. Association Between Copy Number Variations of TLR7 and Ocular Behcet's Disease in a Chinese Han Population. Investig. Ophthalmol. Vis. Sci. 2015, 56, 1517-1523. [CrossRef] [PubMed]

89. Sakamoto, N.; Sekine, H.; Kobayashi, H.; Sato, Y.; Ohira, H. Association of the toll-like receptor 9 gene polymorphisms with Behcet's disease in a Japanese population. Fukushima J. Med. Sci. 2012, 58, 127-135. [CrossRef] [PubMed]

90. Cui, H.P.; Pei, Y.X.; Li, G.F.; Lou, Y.R. Effect of glucocorticoid on cytokines TLR9 and TLR7 in peripheral blood for patients with uveitis. Exp. Ther. Med. 2016, 12, 3893-3896. [CrossRef] [PubMed]

91. Shaulian, E. AP-1-The Jun proteins: Oncogenes or tumor suppressors in disguise? Cell Signal. 2010, 22, 894-899. [CrossRef] [PubMed]

92. Smeal, T.; Binetruy, B.; Mercola, D.; Grover-Bardwick, A.; Heidecker, G.; Rapp, U.R.; Karin, M. Oncoprotein-mediated signalling cascade stimulates c-Jun activity by phosphorylation of serines 63 and 73 . Mol. Cell Biol. 1992, 12, 3507-3513. [CrossRef] [PubMed]

93. Pulverer, B.J.; Kyriakis, J.M.; Avruch, J.; Nikolakaki, E.; Woodgett, J.R. Phosphorylation of c-jun mediated by MAP kinases. Nature 1991, 353, 670-674. [CrossRef] [PubMed]

94. Qing, H.; Gong, W.; Che, Y.; Wang, X.; Peng, L.; Liang, Y.; Wang, W.; Deng, Q.; Zhang, H.; Jiang, B. PAK1-dependent MAPK pathway activation is required for colorectal cancer cell proliferation. Tumour Biol. 2012, 33, 985-994. [CrossRef] [PubMed]

95. Wang, C.Y.; Chen, C.L.; Tseng, Y.L.; Fang, Y.T.; Lin, Y.S.; Su, W.C.; Chen, C.C.; Chang, K.C.; Wang, Y.C.; Lin, C.F. Annexin A2 silencing induces G2 arrest of non-small cell lung cancer cells through p53-dependent and -independent mechanisms. J. Biol. Chem. 2012, 287, 32512-32524. [CrossRef] [PubMed] 
96. Gonzalez-Villasana, V.; Gutierrez-Puente, Y.; Tari, A.M. Cyclooxygenase-2 utilizes Jun N-terminal kinases to induce invasion, but not tamoxifen resistance, in MCF-7 breast cancer cells. Oncol. Rep. 2013, 30, 1506-1510. [PubMed]

97. Gao, L.; Huang, S.; Ren, W.; Zhao, L.; Li, J.; Zhi, K.; Zhang, Y.; Qi, H.; Huang, C. Jun activation domain-binding protein 1 expression in oral squamous cell carcinomas inversely correlates with the cell cycle inhibitor p27. Med. Oncol. 2012, 29, 2499-2504. [CrossRef] [PubMed]

98. Song, X.; Tao, Y.G.; Deng, X.Y.; Jin, X.; Tan, Y.N.; Tang, M.; Wu, Q.; Lee, L.M.; Cao, Y. Heterodimer formation between c-Jun and Jun B proteins mediated by Epstein-Barr virus encoded latent membrane protein 1. Cell Signal. 2004, 16, 1153-1162. [CrossRef] [PubMed]

99. Rocha, G.; Duclos, A.; Chalifour, L.E.; Baines, M.G.; Antecka, E.; Deschenes, J. Analysis of gene expression during experimental uveitis in the rabbit. Can. J. Ophthalmol. 1996, 31, 228-233. [PubMed]

100. Turner, M.J.; DaSilva-Arnold, S.; Luo, N.; Hu, X.; West, C.C.; Sun, L.; Hall, C.; Bradish, J.; Kaplan, M.H.; Travers, J.B.; et al. STAT6-mediated keratitis and blepharitis: A novel murine model of ocular atopic dermatitis. Investig. Ophthalmol. Vis. Sci. 2014, 55, 3803-3808. [CrossRef] [PubMed]

101. Tepper, R.I.; Levinson, D.A.; Stanger, B.Z.; Campos-Torres, J.; Abbas, A.K.; Leder, P. IL-4 induces allergic-like inflammatory disease and alters $\mathrm{T}$ cell development in transgenic mice. Cell 1990, 62, 457-467. [CrossRef]

102. Amadi-Obi, A.; Yu, C.R.; Liu, X.; Mahdi, R.M.; Clarke, G.L.; Nussenblatt, R.B.; Gery, I.; Lee, Y.S.; Egwuagu, C.E. TH17 cells contribute to uveitis and scleritis and are expanded by IL-2 and inhibited by IL-27/STAT1. Nat. Med. 2007, 13, 711-718. [CrossRef] [PubMed]

103. Malla, N.; Sjoli, S.; Winberg, J.O.; Hadler-Olsen, E.; Uhlin-Hansen, L. Biological and pathobiological functions of gelatinase dimers and complexes. Connect. Tissue Res. 2008, 49, 180-184. [CrossRef] [PubMed]

104. Murphy, G.; Nagase, H. Progress in matrix metalloproteinase research. Mol. Asp. Med. 2008, 29, $290-308$. [CrossRef] [PubMed]

105. Sivak, J.M.; Fini, M.E. Mmps in the eye: Emerging roles for matrix metalloproteinases in ocular physiology. Prog. Retin. Eye Res. 2002, 21, 1-14. [CrossRef]

106. Nagase, H.; Visse, R.; Murphy, G. Structure and function of matrix metalloproteinases and TIMPs. Cardiovasc. Res. 2006, 69, 562-573. [CrossRef] [PubMed]

107. Lee, Y.J.; Kang, S.W.; Baek, H.J.; Choi, H.J.; Bae, Y.D.; Kang, E.H.; Lee, E.Y.; Lee, E.B.; Song, Y.W. Association between matrix metalloproteinase 9 promoter polymorphisms and Behcet's disease. Hum. Immunol. 2010, 71, 717-722. [CrossRef] [PubMed]

108. Quillard, T.; Coupel, S.; Coulon, F.; Fitau, J.; Chatelais, M.; Cuturi, M.C.; Chiffoleau, E.; Charreau, B. Impaired Notch4 activity elicits endothelial cell activation and apoptosis: Implication for transplant arteriosclerosis. Arterioscler. Thromb. Vasc. Biol. 2008, 28, 2258-2265. [CrossRef] [PubMed]

109. Verginelli, F.; Adesso, L.; Limon, I.; Alisi, A.; Gueguen, M.; Panera, N.; Giorda, E.; Raimondi, L.; Ciarapica, R.; Campese, A.F.; et al. Activation of an endothelial Notch1-Jagged1 circuit induces VCAM1 expression, an effect amplified by interleukin-1 $\beta$. Oncotarget 2015, 6, 43216-43229. [PubMed]

110. Crosson, J.N.; Laird, P.W.; Debiec, M.; Bergstrom, C.S.; Lawson, D.H.; Yeh, S. Vogt-Koyanagi-Harada-like syndrome after CTLA-4 inhibition with ipilimumab for metastatic melanoma. J. Immunother. 2015, 38, 80-84. [CrossRef] [PubMed]

111. Yu, C.R.; Kim, S.H.; Mahdi, R.M.; Egwuagu, C.E. SOCS3 deletion in T lymphocytes suppresses development of chronic ocular inflammation via upregulation of CTLA-4 and expansion of regulatory T cells. J. Immunol. 2013, 191, 5036-5043. [CrossRef] [PubMed]

112. Shimizu, J.; Izumi, T.; Arimitsu, N.; Fujiwara, N.; Ueda, Y.; Wakisaka, S.; Yoshikawa, H.; Kaneko, F.; Suzuki, T.; Takai, K.; et al. Skewed TGF $\beta /$ Smad signalling pathway in T cells in patients with Behcet's disease. Clin. Exp. Rheumatol. 2012, 30, S35-S39. [PubMed]

113. Li, Q.; Sun, B.; Dastgheib, K.; Chan, C.C. Suppressive effect of transforming growth factor $\beta 1$ on the recurrence of experimental melanin protein-induced uveitis: Upregulation of ocular interleukin-10. Clin. Immunol. Immunopathol. 1996, 81, 55-61. [CrossRef] [PubMed]

114. Sharma, R.K.; Gupta, A.; Kamal, S.; Bansal, R.; Singh, N.; Sharma, K.; Virk, S.; Sachdeva, N. Role of Regulatory T Cells in Tubercular Uveitis. Ocul. Immunol. Inflamm. 2016, 1-10. [CrossRef] [PubMed]

115. Fabiani, C.; Vitale, A.; Lopalco, G.; Iannone, F.; Frediani, B.; Cantarini, L. Different roles of TNF inhibitors in acute anterior uveitis associated with ankylosing spondylitis: State of the art. Clin. Rheumatol. 2016, 35, 2631-2638. [CrossRef] [PubMed] 
116. Hatemi, I.; Hatemi, G.; Pamuk, O.N.; Erzin, Y.; Celik, A.F. TNF- $\alpha$ antagonists and thalidomide for the management of gastrointestinal Behcet's syndrome refractory to the conventional treatment modalities: A case series and review of the literature. Clin. Exp. Rheumatol. 2015, 33, S129-S137. [PubMed]

117. Bharadwaj, A.S.; Schewitz-Bowers, L.P.; Wei, L.; Lee, R.W.; Smith, J.R. Intercellular adhesion molecule 1 mediates migration of Th1 and Th17 cells across human retinal vascular endothelium. Investig. Ophthalmol. Vis. Sci. 2013, 54, 6917-6925. [CrossRef] [PubMed]

118. Hu, L.; Huang, T.; Shi, X.; Lu, W.C.; Cai, Y.D.; Chou, K.C. Predicting functions of proteins in mouse based on weighted protein-protein interaction network and protein hybrid properties. PLoS ONE 2011, 6, e14556. [CrossRef] [PubMed]

119. Hu, L.L.; Huang, T.; Cai, Y.D.; Chou, K.C. Prediction of Body Fluids where Proteins are Secreted into Based on Protein Interaction Network. PLoS ONE 2011, 6, e22989. [CrossRef] [PubMed]

120. Chen, L.; Zhang, Y.H.; Huang, T.; Cai, Y.D. Identifying novel protein phenotype annotations by hybridizing protein-protein interactions and protein sequence similarities. Mol. Genet. Genom. 2016, 291, 913-934. [CrossRef] [PubMed]

121. Jensen, L.J.; Kuhn, M.; Stark, M.; Chaffron, S.; Creevey, C.; Muller, J.; Doerks, T.; Julien, P.; Roth, A.; Simonovic, M. STRING 8-a global view on proteins and their functional interactions in 630 organisms. Nucleic Acids Res. 2009, 37, D412-D416. [CrossRef] [PubMed]

122. Li, Y.; Patra, J.C. Genome-wide inferring genephenotype relationship by walking on the heterogeneous network. Bioinformatics 2010, 26, 1219-1224. [CrossRef] [PubMed]

123. Yang, J.; Chen, L.; Kong, X.; Huang, T.; Cai, Y.-D. Analysis of Tumor Suppressor Genes Based on Gene Ontology and the KEGG Pathway. PLoS ONE 2014, 9, e107202. [CrossRef] [PubMed]

124. Huang, T.; Zhang, J.; Xu, Z.P.; Hu, L.L.; Chen, L.; Shao, J.L.; Zhang, L.; Kong, X.Y.; Cai, Y.D.; Chou, K.C. Deciphering the effects of gene deletion on yeast longevity using network and machine learning approaches. Biochimie 2012, 94, 1017-1025. [CrossRef] [PubMed]

125. Zhang, J.; Xing, Z.; Ma, M.; Wang, N.; Cai, Y.-D.; Chen, L.; Xu, X. Gene Ontology and KEGG Enrichment Analyses of Genes Related to Age-Related Macular Degeneration. BioMed Res. Int. 2014, 2014, 450386. [CrossRef] [PubMed]

126. Chen, L.; Zhang, Y.-H.; Zheng, M.; Huang, T.; Cai, Y.-D. Identification of compound-protein interactions through the analysis of gene ontology, KEGG enrichment for proteins and molecular fragments of compounds. Mol. Genet. Genom. 2016, 291, 2065-2079. [CrossRef] [PubMed]

127. Kohavi, R. A study of cross-validation and bootstrap for accuracy estimation and model selection. In Proceedings of the 14th International joint Conference on artificial intelligence, Montreal, QC, Canada, 20-25 August 1995.

(C) 2017 by the authors. Licensee MDPI, Basel, Switzerland. This article is an open access article distributed under the terms and conditions of the Creative Commons Attribution (CC BY) license (http:/ / creativecommons.org/licenses/by/4.0/). 
Article

\title{
Biochemical and Computational Insights on a Novel Acid-Resistant and Thermal-Stable Glucose 1-Dehydrogenase
}

\author{
Haitao Ding ${ }^{1, *}$, Fen Gao ${ }^{2}$, Yong Yu ${ }^{1}$ and Bo Chen ${ }^{1}$ \\ 1 Key Laboratory for Polar Science of State Oceanic Administration, Polar Research Institute of China, \\ Shanghai 200136, China; yuyong@pric.org.cn (Y.Y.); chenbo@pric.org.cn (B.C.) \\ 2 East China Sea Fisheries Research Institute, Shanghai 200090, China; gaofen2011@163.com \\ * Correspondence: dinghaitao@pric.org.cn; Tel.: +86-21-5871-8663
}

Academic Editor: Quan Zou

Received: 10 May 2017; Accepted: 30 May 2017; Published: 5 June 2017

\begin{abstract}
Due to the dual cofactor specificity, glucose 1-dehydrogenase (GDH) has been considered as a promising alternative for coenzyme regeneration in biocatalysis. To mine for potential GDHs for practical applications, several genes encoding for GDH had been heterogeneously expressed in Escherichia coli BL21 (DE3) for primary screening. Of all the candidates, GDH from Bacillus sp. $\mathrm{ZJ}(\mathrm{BzGDH})$ was one of the most robust enzymes. BzGDH was then purified to homogeneity by immobilized metal affinity chromatography and characterized biochemically. It displayed maximum activity at $45^{\circ} \mathrm{C}$ and $\mathrm{pH} 9.0$, and was stable at temperatures below $50^{\circ} \mathrm{C}$. BzGDH also exhibited a broad $\mathrm{pH}$ stability, especially in the acidic region, which could maintain around $80 \%$ of its initial activity at the $\mathrm{pH}$ range of 4.0-8.5 after incubating for 1 hour. Molecular dynamics simulation was conducted for better understanding the stability feature of $\mathrm{BzGDH}$ against the structural context. The in-silico simulation shows that $\mathrm{BzGDH}$ is stable and can maintain its overall structure against heat during the simulation at $323 \mathrm{~K}$, which is consistent with the biochemical studies. In brief, the robust stability of BzGDH made it an attractive participant for cofactor regeneration on practical applications, especially for the catalysis implemented in acidic $\mathrm{pH}$ and high temperature.
\end{abstract}

Keywords: Bacillus; glucose 1-dehydrogenase; acid-resistant; thermal-stable; molecular dynamics simulation

\section{Introduction}

$\mathrm{NAD}(\mathrm{P})$-dependent glucose 1-dehydrogenase (GDH, EC 1.1.1.47) is an oxidoreductase present in various organisms and involved in glucose metabolic pathways, catalyzing the oxidation of D-glucose to D-glucono-1,5-lactone while simultaneously reducing $\mathrm{NAD}(\mathrm{P})$ to $\mathrm{NAD}(\mathrm{P}) \mathrm{H}$ [1-6]. As a member of the short-chain dehydrogenases/reductases family (SDRs), GDH is a tetrameric protein consisting of four identical subunits, which shares similar overall folding and oligomeric architecture with those of its homologous counterparts $[7,8]$. Due to the dual cofactor specificity, high activity, easy preparation, and cheap substrate, GDH has been widely used in biocatalysis [9-11], bioremediation [12], biosensors [13], and biofuel cells [14].

Biocatalysis has been considered as a powerful tool for the pharmaceutical and fine chemical synthetic processes due to the chemo-, regio-, and stereo-selectivity of enzymes [15]. However, because many kinds of industrial enzymes are cofactor-dependent, the enzymatic synthesis is limited by the considerable expenses of the cofactors. To tackle the issue of manufacturing expense on biocatalysis, several cofactor regeneration approaches have been proposed, of which the enzymatic regeneration method has been considered as an effective technique [16]. Due to the activity toward both NAD 
and NADP, GDH has been proposed as a promising candidate for coenzyme regeneration [17,18], compared with other oxidoreductases such as formate dehydrogenase [19], alcohol dehydrogenase [20], glucose-6-phosphate dehydrogenase [21], and phosphite dehydrogenase [22].

Although GDHs from various microorganisms have been employed as coenzyme regenerators for biocatalysis [9-11], new enzymes with robust stability against broad temperature and $\mathrm{pH}$ range are still preferred. In this work, a novel $\mathrm{NAD}(\mathrm{P})$-dependent glucose 1-dehydrogenase from Bacillus sp. ZJ (BzGDH), with considerable acidic tolerance and thermal stability, has been extensively characterized through biochemical experiments. In contrast to previously reported acid-resistant GDHs, including GDH from Bacillus thuringiensis M15 (BtGDH) [3], Bacillus sp. G3 (BgGDH) [23], and Bacillus cereus var. mycoides (BcGDH) [24], BzGDH exhibited superior thermal stability to its homologous counterparts. To better understand this remarkable feature that distinguishes BzGDH from other acid-resistant GDHs, molecular dynamic (MD) simulation was conducted to investigate the conformational flexibility and fluctuations of BzGDH over time and spatial scales. Analysis of the trajectory shows that $\mathrm{BzGDH}$ is stable and can maintain its overall structure against heat during the simulation at 323 Kelvin (K), which is in accordance with the biochemical studies.

\section{Results and Discussion}

\subsection{Sequence Analysis}

The gene $b z g d h$ encodes a peptide consisting of 261 amino acids with a predicted molecular weight of $28 \mathrm{kDa}$ and a theoretical isoelectric point of 5.4. Significant Pfam-A matches [25] revealed that BzGDH was affiliated to adh_short_C2 family (PF13561, Enoyl-(Acyl carrier protein) reductase), which belonged to the FAD/NAD(P)-binding Rossmann fold superfamily (CL0063), as well as other GDHs. BzGDH also shared the conserved coenzyme-binding GXXXGXG motif (14-20) and catalytic triad (Ser145/Tyr158/Lys162) with other GDHs. In addition, amino acid substitutions mostly occurred at the $\mathrm{N}$-terminus of GDHs (Figure 1), indicating that the $\mathrm{N}$-terminal sequence is less conservative than the C-terminal sequence, which played critical roles in substrate recognition. Phylogenetic analysis showed that these GDHs diverged into two clusters, and BzGDH belonged to the sub-branch consisting of BtGDH, BcGDH, and BgGDH (Figure 2), of which all exhibited acidic resistance in previous studies, suggesting that these four GDHs might originate from the same ancestral sequences.
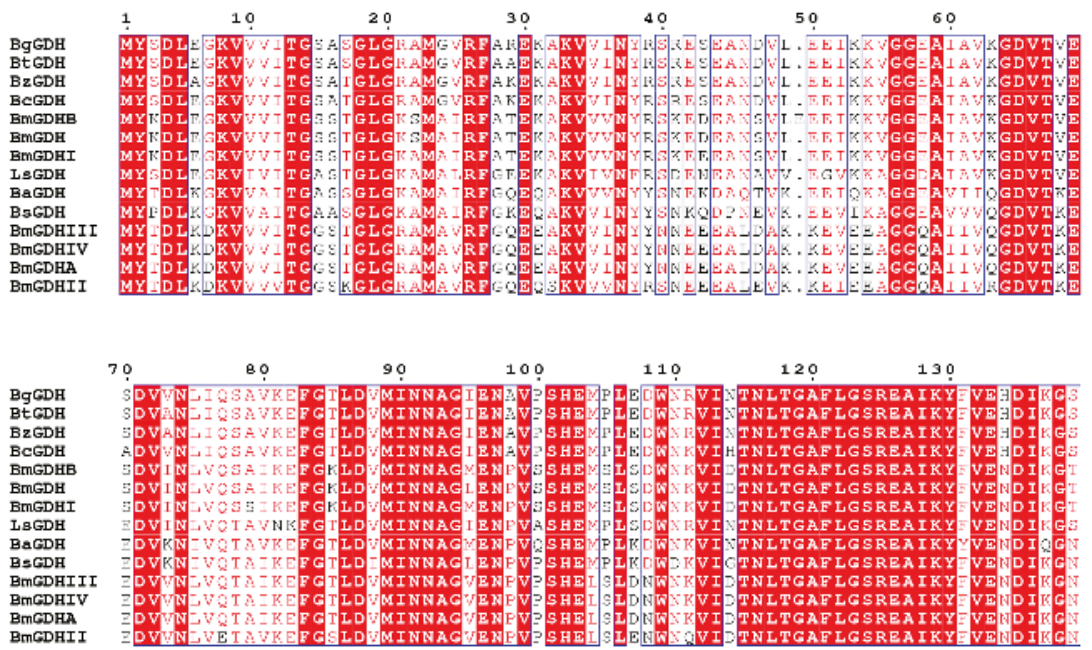

Figure 1. Cont. 

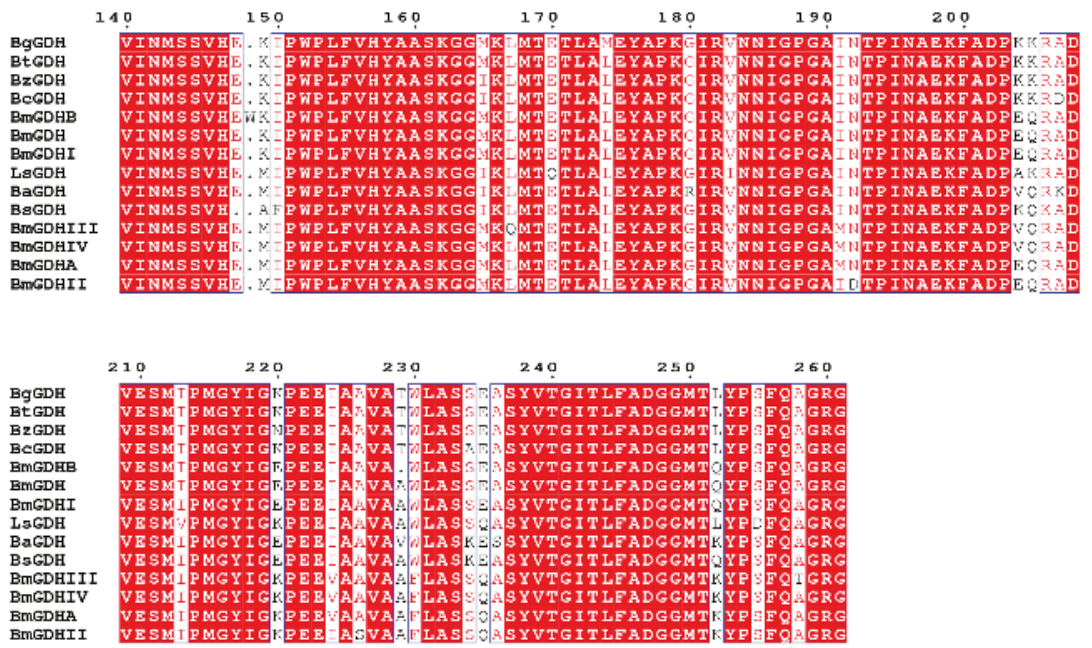

Figure 1. Multiple alignment of the primary structure of glucose 1-dehydrogenases (GDHs). Identical residues and conserved substitutions are shaded red and enveloped by rectangles, respectively. GDHs from Bacillus sp. ZJ (this study), Bacillus megaterium IWG3 [5], Lysinibacillus sphaericus G10 [2], Bacillus cereus var. mycoides [24], Bacillus sp. G3 [23], Bacillus amyloliquefaciens SB5 [1], Bacillus thuringiensis M15 [26], and Bacillus subtilis W168 [27] are abbreviated as BzGDH, BmGDH, LsGDH, BcGDH, BgGDH, BaGDH, BtGDH, and BsGDH, respectively. BmGDHA and BmGDHB are from Bacillus megaterium M1286 [6]. BmGDHI, BmGDHII, BmGDHIII, and BmGDHIV are from Bacillus megaterium IAM1030 [4,5]. Alignment of multiple protein sequences was conducted by using the Clustal X 2.0 program [28] and rendered by ESPript [29].

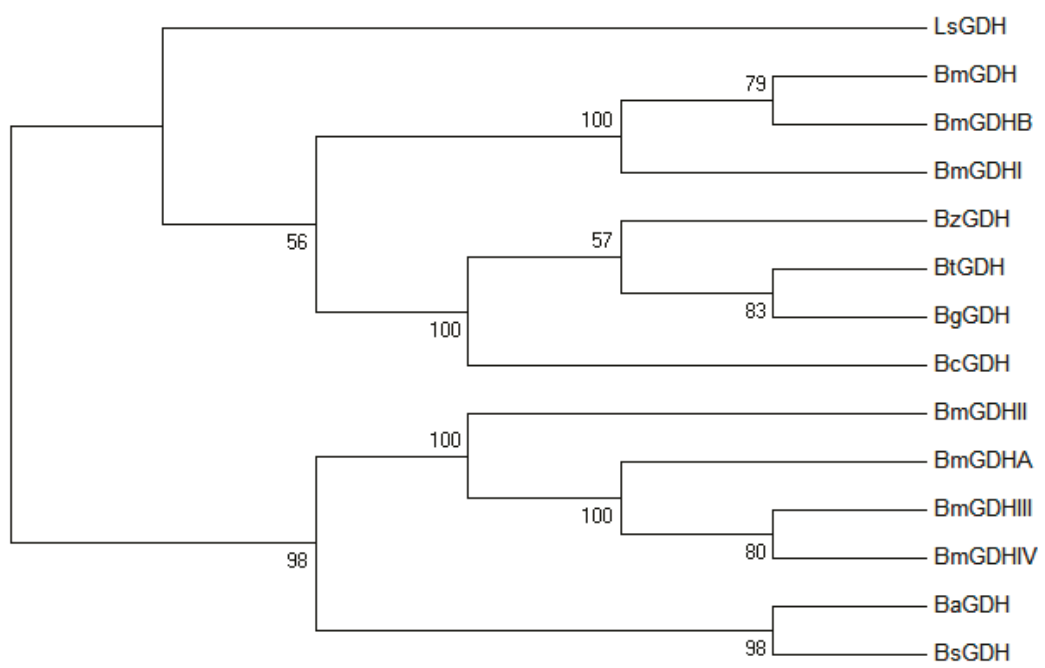

Figure 2. Unrooted phylogenetic tree of GDHs. The phylogenetic tree was constructed using the neighbor joining method [30] in MEGA7 software [31], with a bootstrap test of 1000 replicates. The evolutionary distances were computed using the Poisson correction method [32] and are in the units of the number of amino acid substitutions per site. 


\subsection{Heterologous Expression and Purification}

The specific activity of the purified BzGDH was $194 \pm 2 \mathrm{U} \cdot \mathrm{mg}^{-1}$ at $25{ }^{\circ} \mathrm{C}$ using NAD (nicotinamide adenine dinucleotide) as a cofactor. SDS-PAGE (sodium dodecyl sulfate polyacrylamide gel electrophoresis) analysis showed a homogeneous band corresponding to $30 \mathrm{kDa}$ (Figure 3). By using gel filtration chromatography through a Zorbax Bio-series GF-450 column, the molecular weight of the native BzGDH was estimated to be $120 \mathrm{kDa}$. These results indicated that BzGDH was a homo-tetramer composed of four identical subunits, as well as other NAD-dependent GDHs derived from Bacillus [1-6,23,24,26,27].

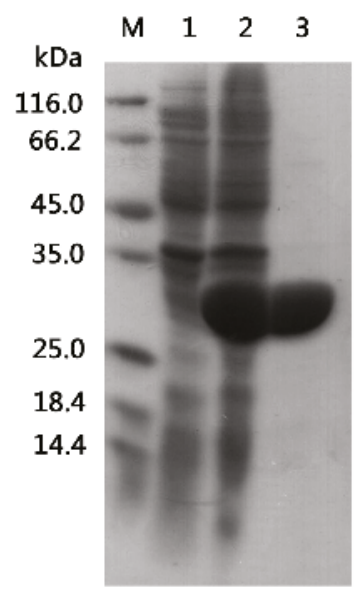

Figure 3. SDS-PAGE (sodium dodecyl sulfate polyacrylamide gel electrophoresis) analysis of total cell lysate and the purified enzyme. Lane M: protein molecular weight marker. Lane 1: uninduced total cell lysate of BzGDH. Lane 2: induced total cell lysate of BzGDH. Lane 3: purified BzGDH.

\subsection{Effects of $\mathrm{pH}$ and Temperature on the Activity and Stability}

BzGDH exhibited activity at a wide $\mathrm{pH}$ range from 4.0 to 10.5 , and displayed maximum activity at pH 9.0 in Tris- $\mathrm{HCl}$ buffer among all buffers. Actually, the chemical composition of the sodium citrate, sodium phosphate, and Tris- $\mathrm{HCl}$ buffers, showed no significant influence on the specific activity of the enzyme, and the differences in the specific activity are mainly caused by the change of the $\mathrm{pH}$ of the solution. However, a significant decrease of specific activity was observed in the glycine- $\mathrm{NaOH}$ buffer at $\mathrm{pH}$ values of 8.5 and 9.0 when compared to those of the same $\mathrm{pH}$ values of the Tris- $\mathrm{HCl}$ buffer, indicating that glycine might inhibit the activity of BzGDH. Surprisingly, the optimum $\mathrm{pH}$ was determined as 9.5 in Glycine- $\mathrm{NaOH}$ buffer (Figure 4a), which is inconsistent with the maximum activity $\mathrm{pH}$ of 9.0. A reasonable explanation for this discrepancy is that the observed activity of the enzyme is not only affected by the $\mathrm{pKa}$ of its catalytic residues which played critical roles on the activity, but is influenced by the stability of the enzyme which might be unstable at its optimum $\mathrm{pH}$ (Figure $4 b$ ), and is even sometimes affected by the chemicals in the buffer such as glycine in this case. In regards to its $\mathrm{pH}$ stability, $\mathrm{BzGDH}$ was stable over a broad $\mathrm{pH}$ range, especially in the acidic region, which could maintain around $80 \%$ of its initial activity in the $\mathrm{pH}$ range of $4.0-8.5$ after incubating for 1 hour (Figure $4 b$ ). 

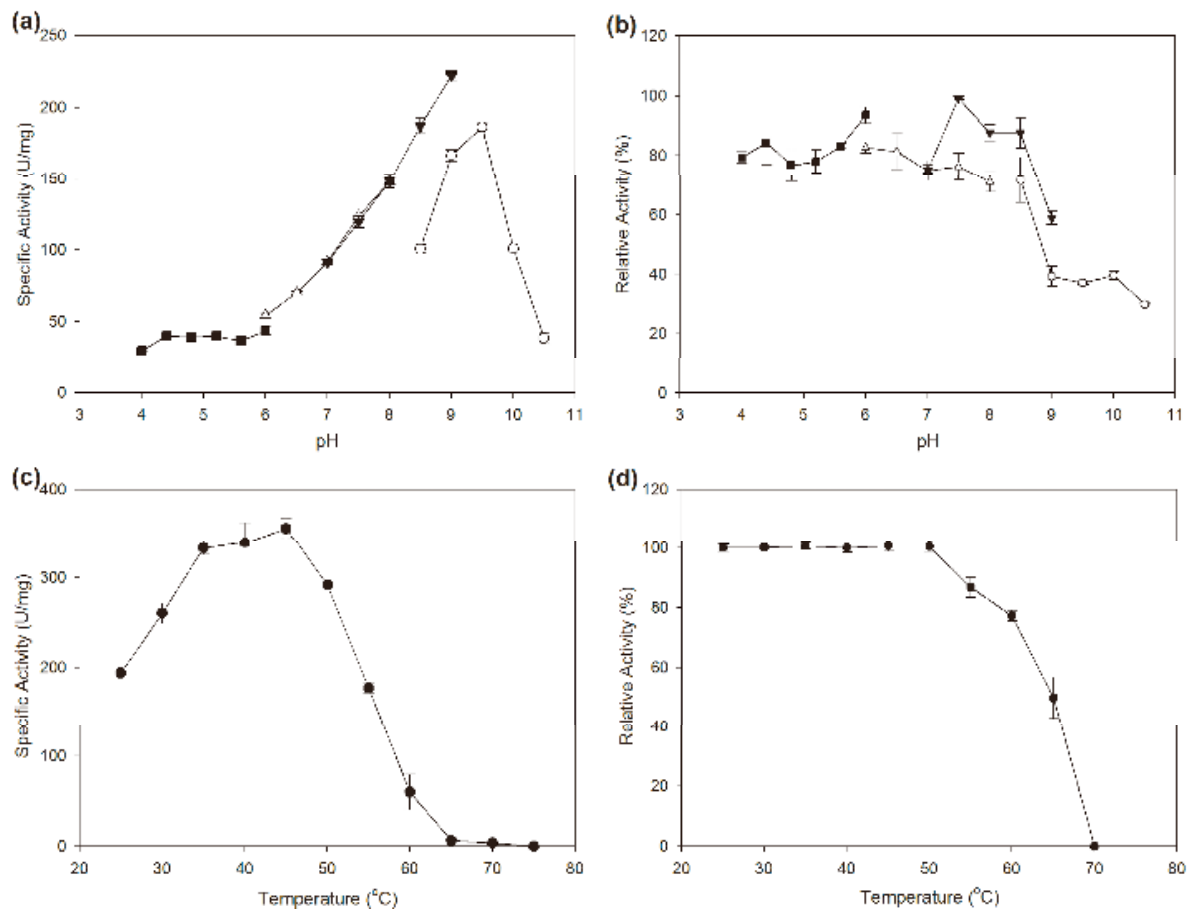

Figure 4. Effects of $\mathrm{pH}$ and temperature on the activity and stability of BzGDH. (a) Effect of $\mathrm{pH}$ on the activity of BzGDH; (b) Effect of pH on the stability of BzGDH. ( $\mathbf{\square}) \mathrm{pH}$ 4.0-6.0, $100 \mathrm{mM}$ sodium citrate buffer; $(\Delta)$ pH 6.0-8.0, 100 mM sodium phosphate buffer; ( $)$ pH 7.0-9.0, 100 mM Tris-HCl buffer; (○) $\mathrm{pH}$ 8.5-10.5, $100 \mathrm{mM}$ glycine-NaOH buffer; (c) Effect of temperature on the activity of BzGDH;

(d) Effect of temperature on the stability of BzGDH.

As demonstrated in Figure 4c, the optimum catalytic temperature of BzGDH was determined as $45^{\circ} \mathrm{C}$. The activity of BzGDH decreased linearly from 45 to $65^{\circ} \mathrm{C}$ and could not be measurable at $75{ }^{\circ} \mathrm{C}$. In consistent with its higher optimum reaction temperature, the recombinant enzyme also possessed good thermal stability, which was stable after incubation at temperatures below $50{ }^{\circ} \mathrm{C}$ for $30 \mathrm{~min}$ and still maintained $50 \%$ of its initial activity after incubation at $65^{\circ} \mathrm{C}$ for $30 \mathrm{~min}$ (Figure $4 \mathrm{~d}$ ). BzGDH exhibited superior thermal stability to its homologous counterparts, BgGDH [23] and BcGDH [24], which were almost completely inactivated after incubation at $50{ }^{\circ} \mathrm{C}$ without any protective agent.

Since stability is an indispensable characteristic for the utilization of enzymes in real life, the considerable stability of BzGDH against both heat and acid made it a very promising candidate in practical application in harsh conditions.

\subsection{Substrate Specificity and Steady-State Kinetics}

As shown in Table 1, the substrate spectrum of BzGDH was similar to that of BcGDH. However, both BzGDH and BcGDH displayed stricter substrate specificity toward various sugars than that of $\mathrm{BgGDH}$, especially for galactose and mannose, indicating that BzGDH could be a potential diagnostic reagent for blood glucose measurement as well as $\mathrm{BcGDH}$.

The steady-state kinetic constants of BzGDH were determined by using a nonlinear fitting plot (Table 2). Although BzGDH had similar $k_{\text {cat }}$ values for both NAD and NADP, the $K_{m}$ value for NADP was 5.6-fold higher than that for NAD, indicating that BzGDH preferred NAD rather than NADP as 
the cofactor. The cofactor preference of BzGDH resembled that of BmGDHIII, BmGDHIV [4], and BtGDH [3], while BmGDH, BmGDHI, BmGDHII [5], and BgGDH [23] preferred NADP.

Table 1. Substrate specificity of GDHs.

\begin{tabular}{cccc}
\hline \multirow{2}{*}{ Substrate } & \multicolumn{3}{c}{ Relative Activity (\%) } \\
\cline { 2 - 4 } & BzGDH & BgGDH [23] & BcGDH [24] \\
\hline D-glucose & 100 & 100 & 100 \\
D-galactose & 6.8 & 22.0 & 7.3 \\
D-mannose & 3.2 & 7.1 & 4.4 \\
D-fructose & 0.9 & 0.6 & 0 \\
D-xylose & 6.1 & 6.4 & 6.0 \\
D-arabinose & 0 & 0.2 & 0 \\
D-maltose & 10.0 & 13.0 & 11.0 \\
D-lactose & 3.1 & 2.6 & 5.2 \\
D-sucrose & 0.9 & 6.3 & 2.51 \\
\hline
\end{tabular}

${ }^{1}$ The activities are expressed relative to those for D-glucose.

Table 2. Kinetic constants of BzGDH.

\begin{tabular}{cccc}
\hline Substrate/Cofactor & $\boldsymbol{K}_{\boldsymbol{m}}(\mathbf{m M})$ & $\boldsymbol{k}_{\text {cat }}\left(\mathbf{s}^{-\mathbf{1}}\right)^{\mathbf{1}}$ & $\boldsymbol{k}_{\text {cat }} / \boldsymbol{K}_{\boldsymbol{m}}\left(\mathrm{mM}^{-\mathbf{1}} \cdot \mathbf{s}^{\mathbf{- 1}}\right)$ \\
\hline D-glucose & $17.126 \pm 0.946$ & $87.844 \pm 1.362$ & 5.129 \\
NAD & $0.072 \pm 0.009$ & $84.521 \pm 2.175$ & 1166.294 \\
NADP & $0.404 \pm 0.088$ & $73.960 \pm 2.677$ & 182.978 \\
\hline
\end{tabular}

\subsection{Homology Modeling and Electrostatic Potential Analysis}

The quaternary structure of BzGDH was constructed by SWISS-MODEL [33] and evaluated by ProSA-web [34] and PROCHECK [35]. Both of the Z-score and Ramachandran plot statistics indicated that the dimensional structure of BzGDH (Figure 5a) had been modeled reasonably (Table 3). To investigate the electrostatic potential of $\mathrm{BzGDH}$, the model of BzGDH was subjected to the software APBS [36] and PyMOL (The PyMOL Molecular Graphics System, Version 1.7 Schrödinger, LLC. available online: http:/ / pymol.org/), to generate the electrostatic potential molecular surface. As shown in Figure 5, the contact surfaces of subunits AB, AC, and AD circled by black ellipses are mainly constituted by non-polar amino acid residues and are surrounded by acidic amino acid residues. The non-polar areas can maintain their electrically neutral state in either acidic or alkaline solutions, whereas the acidic areas would be negatively charged in alkaline solutions, leading to the mutual repulsion between subunits. Therefore, the acid-resistance of BzGDH could be explained by the electrostatic potential of contact surfaces between subunits, as well as BcGDH [24].

Table 3. Evaluation of models generated by homology modeling.

\begin{tabular}{cccccc}
\hline \multirow{2}{*}{ Model } & Z-Score & \multicolumn{4}{c}{ Ramachandran Plot $^{2}$} \\
\cline { 3 - 5 } & & $\begin{array}{c}\text { Most Favored } \\
\mathbf{( \% )}\end{array}$ & $\begin{array}{c}\text { Additional } \\
\text { Allowed (\%) }\end{array}$ & $\begin{array}{c}\text { Generously } \\
\text { Allowed (\%) }\end{array}$ & $\begin{array}{c}\text { Disallowed } \\
(\mathbf{\%})\end{array}$ \\
\hline 1GEE & -8.87 & 91.2 & 8.0 & 0.9 & 0 \\
BzGDH & -8.80 & 89.1 & 10.5 & 0.4 & 0 \\
\hline
\end{tabular}

${ }^{1}$ Calculated by ProSA-web [34]; ${ }^{2}$ Calculated by PROCHECK [35]. 
(a)

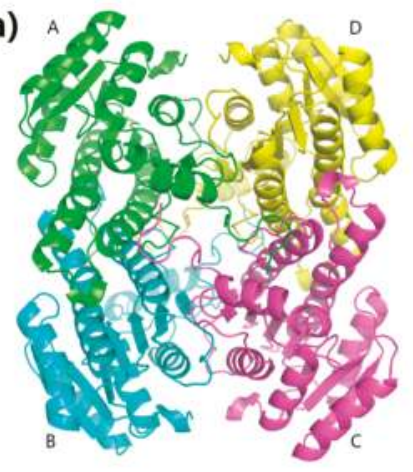

(c)

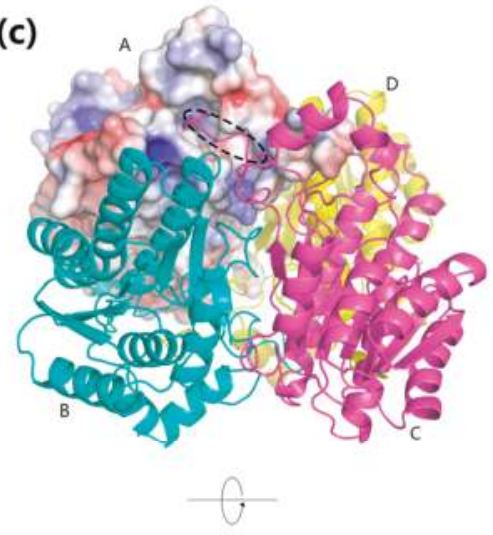

(b)

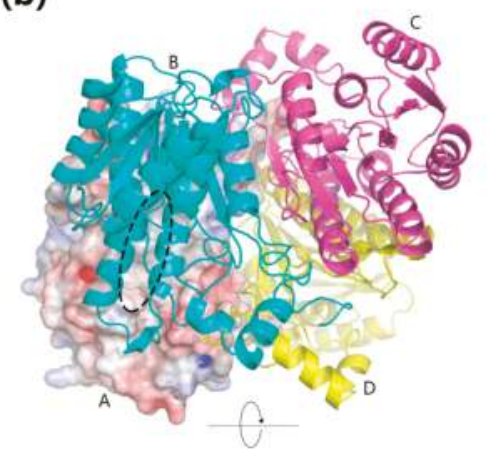

(d)

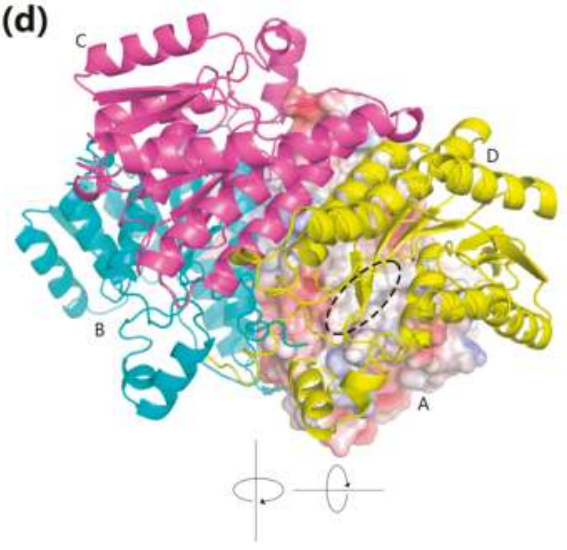

Figure 5. $\pm 5 \mathrm{kT} / \mathrm{e}$ electrostatic potential surface of BzGDH. (a) Tetrameric structure of BzGDH; (b) Electrostatic potential surface representation of the interface between subunits A and B; (c) Electrostatic potential surface representation of the interface between subunits A and C; (d) Electrostatic potential surface representation of the interface between subunits A and D. Subunits $\mathrm{ABCD}$ were labeled using the corresponding capital letters nearby, respectively. Positive, negative, and neutral electrostatic potential surfaces are rendered by blue, red, and white, respectively. The non-polar regions of the contact surfaces of subunits $\mathrm{AB}, \mathrm{AC}$, and $\mathrm{AD}$ were circled by dashed ellipses.

\subsection{Global Structure Stability}

To study the stability and mobility of $\mathrm{BzGDH}$, the model was subjected to a 20-ns MD simulation at $323 \mathrm{~K}$. The stability of BzGDH was analyzed by the all-atom and backbone-atom root mean square deviation (RMSD), respectively, both of which increased from the beginning of the simulation and reached an equilibrium state at about $10 \mathrm{~ns}$ (Figure 6a), suggesting no significant structural changes for $\mathrm{BzGDH}$ during the simulation. In addition, the radius of gyration, the hydrogen bonds of intra-protein, and the solvent accessible surface area (SASA) of BzGDH all displayed steadily dynamic changes against time (Figure $6 \mathrm{~b}-\mathrm{d}$ ), further confirming the stable global behavior of BzGDH during the simulation at $323 \mathrm{~K}$. 

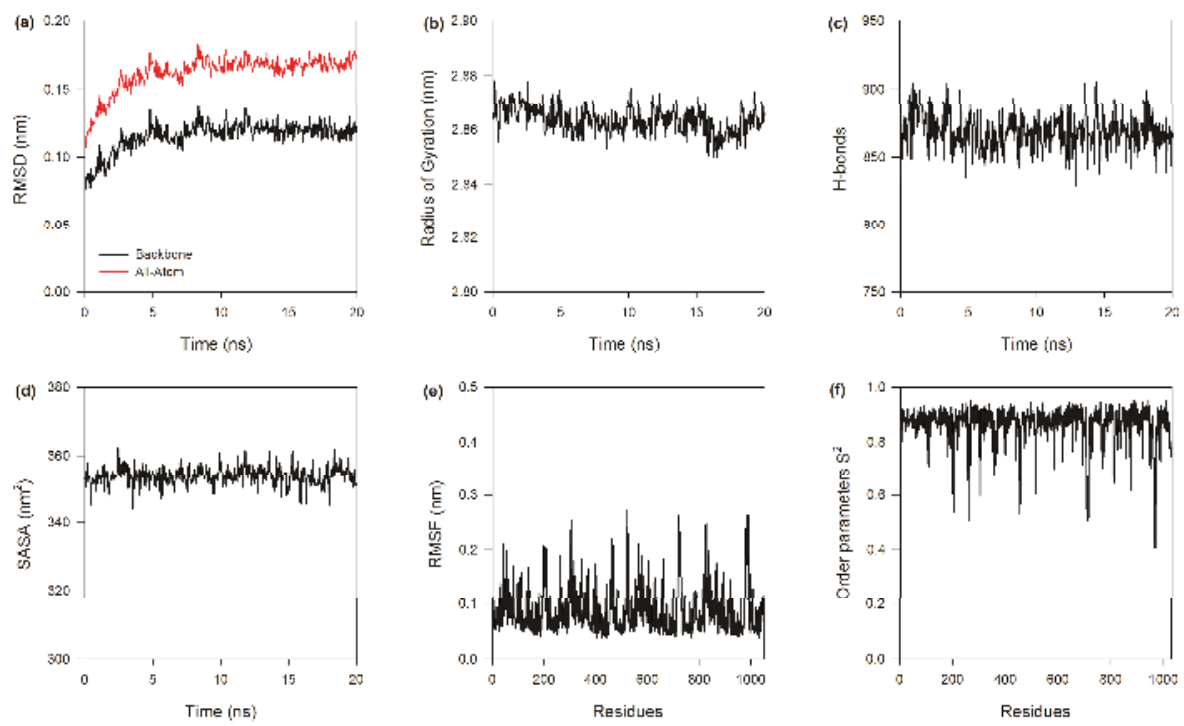

Figure 6. Dynamic changes of BzGDH in the molecular dynamics (MD) simulation. (a) All-atom and backbone-atom root mean square deviation (RMSD) as functions of time; (b) Radius of gyration as a function of time; (c) Hydrogen bonds as a function of time. Hydrogen bonds were detected by GROMACS (GROningen MAchine for Chemical Simulations) with default geometrical criterion, which defined both the donor-acceptor distance $(\leq 0.35 \mathrm{~nm})$ and the hydrogen-donor-acceptor angle $\left(\leq 30{ }^{\circ} \mathrm{C}\right)$; (d) Solvent accessible surface areas (SASA) as a function of time; (e) Root mean square fluctuation (RMSF) as a function of residue numbers; (f) N-H generalized order parameter $S^{2}$ as a function of residue numbers.

\subsection{Structure Flexibility}

The conformational flexibility of BzGDH was assessed using the root mean square fluctuation (RMSF) of C-alpha $(\mathrm{C} \alpha)$ atoms per residue. Generally, regular secondary structure regions display tiny fluctuations with small RMSF values during the simulation, whereas prominent fluctuations with large RMSF are observed for irregular secondary structure regions such as terminal or loop regions, which often bear certain function of proteins. As shown in Figure 6e, regions involved in coenzyme binding (39-55) and substrate binding (190-210) of each subunit are more flexible with large RMSF values than other regions. The RMSF values were converted to B-factors using the equation:

$$
\text { B-factor }=\left(8 \times \pi^{2} \times \operatorname{RMSF}^{2}\right) / 3
$$

to visualize global structure rigidity and flexibility of BzGDH. As shown in Figure 7, most regions of $\mathrm{BzGDH}$ are rigid, except for the aforementioned flexible regions, indicating that the enzyme is stable during the simulation at $323 \mathrm{~K}$.

In addition to the observation of RMSF, the bond-specific fluctuations in protein structure can further be captured by the Lipari-Szabo order parameter $S^{2}$ [37], which provide an intuitive description of the amplitude of spatial restriction of the internal motions of the bond vectors on a fast timescale from picosecond to nanosecond (ps-ns). More specifically, $\mathrm{S}^{2}$ represents the component of the $\mathrm{H}-\mathrm{X}$ bond vector autocorrelation function which is dissipated by global molecular tumbling, while $\left(1-S^{2}\right)$ characterizes the bond vector orientational disorder arising from internal motion occurring more rapidly than the molecular tumbling. The $S^{2}$ order parameter can range from 0 to 1 , with 1 corresponding to a rigid bond vector (completely restricted) and 0 corresponding to the highest degree 
of disorder for a bond vector (completely isotropic). Higher order parameters (0.85) were observed in the regions of secondary structure, while unstructured regions showed lower order parameters $(0.4-0.6)$.

The order parameter $\mathrm{S}^{2}$ of the main chain N-H bonds of BzGDH has been calculated based on the equilibrium MD trajectories. The average value of the order parameter $S^{2}$, over all residues, is 0.86 for BzGDH. The most flexible region that showed lower $S^{2}$ of each subunit is the substrate binding domain, with residues Lys 179, Gly 180, Arg 182, Asn 184, Asn 185, Ala 190, Asn 196, and Asp 202 involved, indicating that these residues exhibit considerable disorder on the ps-ns timescale. Similarly, residues Gln 257, Ala 258, and Gly 259 in the C-terminal region of the protein have low order parameters, also implying that this region is disordered on the ps-ns timescale. Indeed, the order parameter revealed that these regions are flexible on the ps-ns timescale, with the fluctuations functioning to allow substrate access to and release of products from the active site. The results of the computation of the order parameters are in considerable agreement with the RMSF profiles, with the greatest flexibility occurring in loop regions, while other secondary structural elements are more constrained.

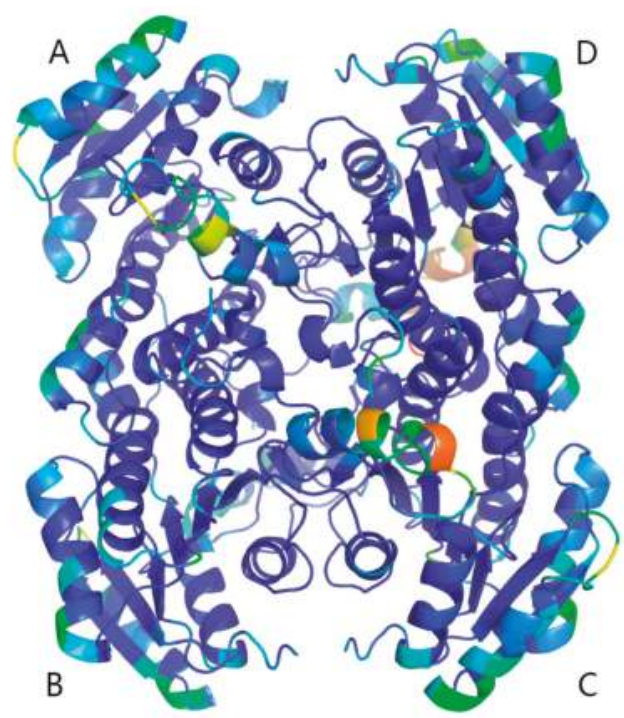

Figure 7. Cartoon representation of BzGDH shaded according to the B-factors (temperature factor) of each residue. Subunits $\mathrm{ABCD}$ were labeled using the corresponding capital letters nearby. The structure was shaded from the blue to red spectrum along with the increase of B-factor values from 3.98 to 193.74 .

\subsection{Essential Dynamics}

To reveal the concerted fluctuations of BzGDH over time and spatial scales, essential dynamics (ED) is employed to extract information from sampled conformations over the molecular dynamics trajectory [38]. Practically, the essential dynamics of a protein is obtained by performing principal component analysis (PCA), which is a multivariate statistical technique involving diagonalization of the covariance matrix (Figure 8) constructed from atomic displacements of $\mathrm{C} \alpha$ atoms, to reduce the number of dimensions required to describe protein dynamics and yield a set of eigenvectors that provide information about collective motions of the protein [39]. 


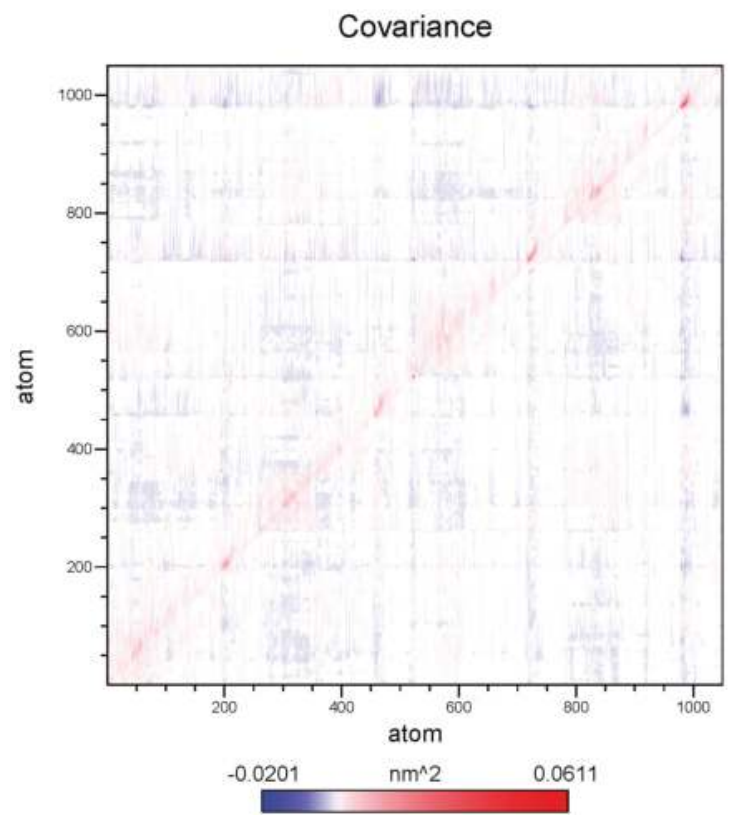

Figure 8. Covariance analysis of the atomic fluctuation of BzGDH in the MD (molecular dynamics) simulation. The correlation matrix is computed using the C- $\alpha$ Cartesian coordinates. The collective motions between pairs of residues are represented as red for correlated, white for uncorrelated, and blue for anti-correlated motions, respectively. The amplitude of fluctuation was represented by the color depth.

The eigenvectors represent the directions of motion, and the corresponding eigenvalues represent the amount of motion along each eigenvector, where larger eigenvalues describe motions on larger spatial scales. Generally, the first 10-20 eigenvectors are enough to capture the principal motions of the protein and describe more than $90 \%$ of all cumulative protein fluctuations [40]. However, it can be seen that only $14.3 \%$ of the total $\mathrm{C} \alpha$ motion can be explained by the first two eigenvectors, even the first 20 eigenvectors merely contribute for $51.2 \%$ of the total $\mathrm{C} \alpha$ motion from Figure 9a. This shows that most of the internal motions of BzGDH are not confined within a subspace of small dimension, and no obvious collective motion of the backbone of BzGDH is observed from the MD simulation performed at $323 \mathrm{~K}$, reflecting that the enzyme can maintain its overall structure against heat, which is in accordance with the biochemical experiments.

Figure $9 \mathrm{~b}$ shows the trajectory projected on the plane defined by the first two principal eigenvectors. The trajectories filled most of the expected ranges, suggesting the deficiency of a coupled force field, which leads to independent motions. The trajectories were projected onto the individual eigenvectors against time to further investigate the motion along the eigenvector directions. It is clear from Figure 10 that the fluctuations of the first six eigenvectors are relatively large, whereas those of the subsequent eigenvectors become successively flat, indicating that the motions belong to the last four eigenvectors have reached their equilibrium fluctuation, which cannot be used to describe the motions of the system. Due to the limitation of hardware, such simulations may not capture the essential motions related to function at much longer timescales. Improvements in computational power will fill the gap between reality and simulation. 

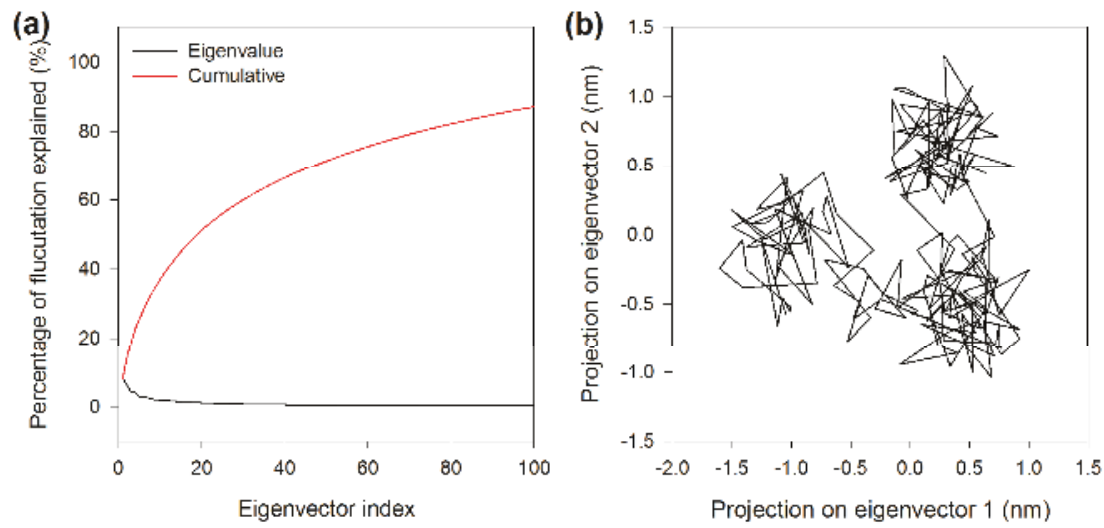

Figure 9. Principal component analysis of BzGDH in the MD simulation. (a) Relative cumulative deviation up to the first 100 eigenvectors provided by the essential dynamics analysis performed on the $\mathrm{C} \alpha$ atoms of $\mathrm{BzGDH}$; (b) Projections of the trajectory on the plane defined by the first two principal eigenvectors. Horizontal axis: atomic displacement along the first eigenvector. Vertical axis: atomic displacement along the second eigenvector.

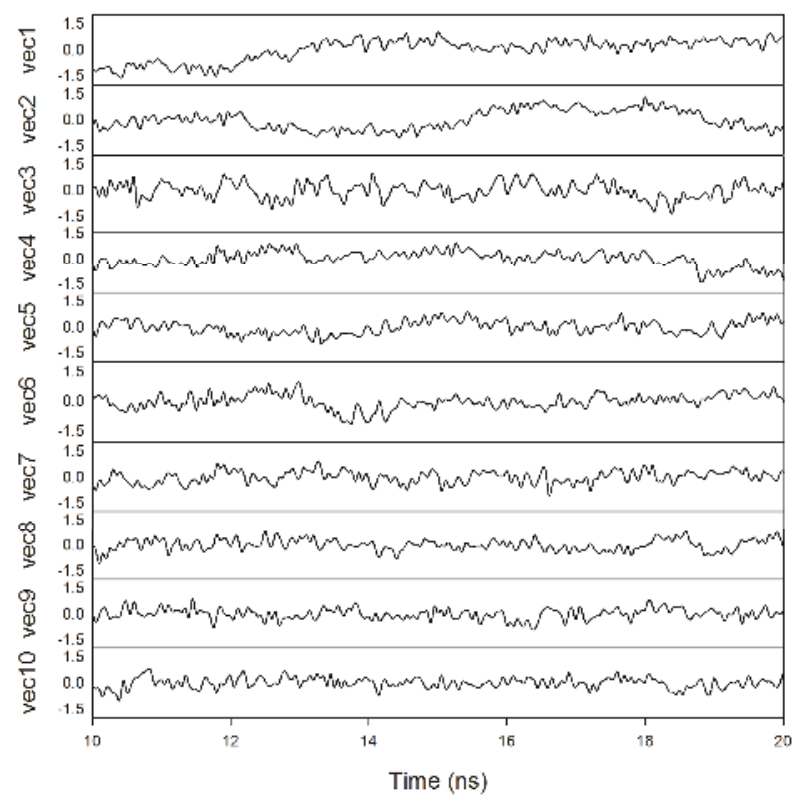

Figure 10. Motions along the first ten eigenvectors obtained from the $C \alpha$ coordinates' covariance matrix.

\section{Materials and Methods}

\subsection{Strains, Plasmids, and Chemicals}

Strain Bacillus sp. ZJ isolated from the soil near Yuhangtang River in Hangzhou, China, was used as a source for retrieving glucose 1-dehydrogenase. Escherichia coli (E. coli) DH5 $\alpha$ and BL21 (DE3), expression vector pET28a (+), and Ni-NTA resin were purchased from Invitrogen. Taq 
DNA polymerase, PrimeSTAR HS DNA Polymerase, T4 DNA ligase, NdeI, and BamHI were purchased from TaKaRa. Genomic DNA, plasmid and gel extraction kits were purchased from Axygen. All other chemicals were of analytical grade.

\subsection{Cloning of the Bzgdh Gene and Sequence Analysis}

The gene bzgdh was amplified by using genomic DNA of Bacillus sp. ZJ as a template with the forward primer 5'-GGAATTCCATATGTATAGTGATTTAGCAGG-3' and the reverse primer 5'-CGGGATCCTATTACCCACGCCCAGC-3', which carried cutting sites of NdeI and BamHI (underlined), respectively. The amplified fragments were digested with NdeI and BamHI simultaneously, then purified by using a gel extraction kit prior to ligate with the pre-digested vector pET-28a (+). The recombinant plasmid harboring gene bzgdh was transformed into competent cells of E. coli $\mathrm{DH} 5 \alpha$ for sequencing.

Homologous searches in GenBank were performed using the BLAST server (available online: http:/ / blast.ncbi.nlm.nih.gov). Alignment of multiple protein sequences was conducted by using the Clustal X 2.0 program [28] and rendered by ESPript [29]. The phylogenetic tree was constructed using the neighbor-joining method in MEGA7 [31], with a bootstrap test of 1000 replicates.

The nucleotide sequence for GDH of Bacillus sp. ZJ was deposited in GenBank under accession number KJ701281.

\subsection{Expression and Purification of Recombinant BzGDH}

The recombinant plasmid was transformed into competent cells of E. coli BL21 (DE3) for expression. The recombinant cells were cultivated in Luria-Bertani broth containing $50 \mu \mathrm{g}$ kanamycin $\cdot \mathrm{mL}^{-1}$ at $37{ }^{\circ} \mathrm{C}$ with a shaking speed of $250 \mathrm{rpm}$. The expression of recombinant protein was induced by adding $0.5 \mathrm{mM}$ of IPTG to the medium when the $\mathrm{OD}_{600}$ of the culture reached $0.5-0.8$, followed by another $12 \mathrm{~h}$ incubation at $25^{\circ} \mathrm{C}$ with a shaking speed of $200 \mathrm{rpm}$. The cells were harvested by centrifugation at $10,000 \times \mathrm{g}$ for $10 \mathrm{~min}$ at $4{ }^{\circ} \mathrm{C}$ and were washed with the binding buffer $(50 \mathrm{mM}$ $\mathrm{NaH}_{2} \mathrm{PO}_{4}, 500 \mathrm{mM} \mathrm{NaCl}, 20 \mathrm{mM}$ imidazole, $\mathrm{pH}$ 8.0), and then lysed by ultrasonication. The cell debris was removed by centrifugation at $15,000 \times g$ for $30 \mathrm{~min}$ at $4{ }^{\circ} \mathrm{C}$, and then the supernatant was loaded onto a column containing pre-equilibrated Ni-NTA resin. The column was washed with binding buffer and subsequently eluted with elution buffer $\left(50 \mathrm{mM} \mathrm{NaH}_{2} \mathrm{PO}_{4}, 500 \mathrm{mM} \mathrm{NaCl}, 250 \mathrm{mM}\right.$ imidazole, $\mathrm{pH}$ 8.0). The eluted enzyme was desalted and concentrated by ultrafiltration and stored at $-80{ }^{\circ} \mathrm{C}$ in $25 \mathrm{mM}$ sodium phosphate buffer ( $\mathrm{pH}$ 6.5) with $30 \%$ of glycerol contained. The protein concentration was determined by Bradford's method using bovine serum albumin as the reference.

Denaturing discontinuous polyacrylamide gel electrophoresis was performed on a $5 \%$ stacking gel and a $12 \%$ separating gel. The native molecular weight of GDH was determined by size-exclusion chromatography according to the protocol of the manufacture (Zorbax Bio-series GF-450, Agilent, Santa Clara, CA, USA), using lysozyme (14.3 kDa), chicken ovalbumin (45 kDa), bovine serum albumin fraction $\mathrm{V}(67 \mathrm{kDa})$, and goat $\mathrm{IgG}(150 \mathrm{kDa})$ as standards.

\subsection{Enzyme Activity Assay}

Glucose dehydrogenase activity was determined by assaying the absorbance of NADH at $340 \mathrm{~nm}$ in $100 \mathrm{mM}$ sodium phosphate ( $\mathrm{pH} 8.0$ ) containing $200 \mathrm{mM}$ glucose and $1 \mathrm{mM} \mathrm{NAD}$ at $25{ }^{\circ} \mathrm{C}$. All measurements were conducted in triplicate. One unit of enzyme activity was defined as the amount of the enzyme that catalyzed the formation of $1 \mu \mathrm{mol}$ of $\mathrm{NADH}$ per minute.

\subsection{Effects of $\mathrm{pH}$ and Temperature on the Activity and Stability of BzGDH}

The optimum $\mathrm{pH}$ of $\mathrm{BzGDH}$ was measured at $\mathrm{pH}$ ranging from 4.0 to 10.5 at $25^{\circ} \mathrm{C}$. The effect of $\mathrm{pH}$ on the stability of $\mathrm{BzGDH}$ was determined by measuring the residual activity after incubating $\mathrm{BzGDH}$ in buffers with different $\mathrm{pH}$ values for one hour at $25^{\circ} \mathrm{C}$. 
The optimal temperature of BzGDH was determined at different temperatures $\left(25-75^{\circ} \mathrm{C}\right)$ in phosphate buffer at $\mathrm{pH}$ 7.0. The thermal stability of BzGDH was assayed by measuring the residual activity after incubating $\mathrm{BzGDH}$ at different temperatures $\left(25-75^{\circ} \mathrm{C}\right)$ in phosphate buffer at $\mathrm{pH} 7.0$ for $30 \mathrm{~min}$

\subsection{Substrate Specificity of $B z G D H$}

The substrate specificity of BzGDH was determined by the aforementioned enzyme activity assay, except that glucose was replaced by sucrose, lactose, maltose, xylose, galactose, mannose, fructose, and arabinose, respectively.

\subsection{Steady-State Kinetics of BzGDH}

In order to obtain the kinetic constants for the coenzyme, $200 \mu \mathrm{M}$ of glucose was employed as the substrate and 0.01 to $0.2 \mathrm{mM}$ NAD and NADP were used as the coenzymes, respectively. For analysis of the kinetics for glucose, $1 \mathrm{mM}$ NAD was used as a cofactor, 1 to $200 \mathrm{mM}$ glucose was used as the substrate. GDH activity was measured as described above. The kinetic constants were determined by using a nonlinear fitting of the Michaelis-Menten equation:

$$
v=\left(V_{\max } \times[\mathrm{S}]\right) /\left(K_{m}+[\mathrm{S}]\right)
$$

where [S] is the concentration of the cofactor or substrate, $K_{m}$ is the Michaelis constants for the cofactor or substrate, $v$ is the reaction velocity, and $V_{\max }$ is the maximum reaction velocity. The turnover number $k_{\text {cat }}$ was calculated by the equation:

$$
V_{\max }=k_{\text {cat }} \times[\mathrm{E}]
$$

where $[\mathrm{E}]$ is the concentration of the enzyme.

\subsection{Homology Modeling and Electrostatic Potential of BzGDH}

The crystal structure of glucose 1-dehydrogenase from Bacillus megaterium IWG3 (PDB code: 1GEE, $1.60 \AA$ ) [41], which shares $88.12 \%$ identity with BzGDH, was served as the template for homology modeling of BzGDH. The three-dimensional model of BzGDH was constructed by using the SWISS-MODEL [33]. Precise evaluation of the model quality was performed using ProSA-web [34] and PROCHECK [35]. The structure for electrostatics calculations was prepared by PDB2PQR [42] using the AMBER force field and assigned protonation states at $\mathrm{pH}$ 7.0. The electrostatic potential of BzGDH was calculated by APBS [36] using the linearized Poisson-Boltzmann equation (lpbe) at $298 \mathrm{~K}$ with the monovalent ion concentration of $0.1 \mathrm{M}$. The dielectric constants of protein and solvent were set as 2.0 and 78.0, respectively. The electrostatic potential molecular surface was represented by PyMOL.

\subsection{Molecular Dynamic Simulations of BzGDH}

The constructed model of BzGDH was subjected to the software package GROMACS 5.0.2 [43], with the AMBER99SB [44] force field adopted, for molecular dynamics simulations. The model was first placed into the center of a virtual cubic box with a side length of $11.049 \mathrm{~nm}$ and solvated with 39,486 TIP3P water molecules. The $\mathrm{pH}$ condition was 7.0 according to the ionization state of the protein with a charge of -20 , and twenty $\mathrm{Na}^{+}$ions were added to the water box as counter ions to neutralize the negative charge of the entire system. Bond lengths were constrained by the LINCS algorithm to ensure covalent bonds to maintain their correct lengths during the simulation. Energy minimization of the system was conducted using the steepest descent algorithm for 5000 steps, followed by a 500-ps equilibration simulation with harmonic position restraints on the heavy protein atoms to equilibrate the solvent molecules around the protein. Subsequently, a 2-ns simulation without position restraints was conducted to equilibrate the entire system. Finally, the production simulation was performed 
for $20 \mathrm{~ns}$ at the target temperature. All simulations were performed under the NPT ensemble with periodic boundary conditions and a time step of $2 \mathrm{fs}$. The temperature of the system was kept at $323 \mathrm{~K}$ using the v-rescale method, and the pressure was kept at 1 bar using the Parrinello-Rahman method.

According to the RMSD profile of BzGDH, trajectories that reached the equilibrium state (10-20 ns) were used for further analysis. Principal component analysis was conducted to identify the direction and amplitude of the most prominent characteristics of the motions of BzGDH along the simulation trajectory. Generalized order parameters $S^{2}$, employed as a measure of the degree of spatial restriction of motion, were also calculated for the N-H bonds of BzGDH.

\section{Conclusions}

In this study, a novel NAD(P)-dependent glucose 1-dehydrogenase from Bacillus sp. ZJ has been extensively characterized, with remarkable acidic tolerance and thermal stability. To better understand the stability feature of BzGDH against the structural context, molecular dynamics simulation was conducted to investigate the conformational flexibility and fluctuations of BzGDH over time and spatial scales. Analysis of the trajectory shows that $\mathrm{BzGDH}$ is stable and can maintain its overall structure against heat during the simulation at $323 \mathrm{~K}$, which is in accordance with the biochemical studies. In brief, the robust stability of BzGDH made it a promising participant for cofactor regeneration in practical applications, especially for catalysis implemented in acidic $\mathrm{pH}$ and high temperature.

Acknowledgments: This study was supported by the Chinese Polar Environment Comprehensive Investigation and Assessment Program (CHINARE-01-05, CHINARE-04-02, CHINARE-02-01), Open Fund of Key Laboratory of Biotechnology and Bioresources Utilization of Dalian Minzu University (KF2015009), Youth Innovation Fund of Polar Science (201602), and the National Natural Science Foundation of China (31200599).

Author Contributions: Haitao Ding conceived and designed the experiments; Haitao Ding and Fen Gao performed the experiments; Haitao Ding, Fen Gao, Yong Yu, and Bo Chen analyzed the data; Haitao Ding, Yong Yu, and Bo Chen contributed reagents/materials/analysis tools; Haitao Ding wrote the paper.

Conflicts of Interest: The authors declare no conflict of interest.

\section{References}

1. Pongtharangkul, T.; Chuekitkumchorn, P.; Suwanampa, N.; Payongsri, P.; Honda, K.; Panbangred, W. Kinetic properties and stability of glucose dehydrogenase from Bacillus amyloliquefaciens SB5 and its potential for cofactor regeneration. AMB Express 2015, 5, 1-12. [CrossRef] [PubMed]

2. Ding, H.; Du, Y.; Liu, D.; Li, Z.; Chen, X.; Zhao, Y. Cloning and expression in E. coli of an organic solvent-tolerant and alkali-resistant glucose 1-dehydrogenase from Lysinibacillus sphaericus G10. Bioresour. Technol. 2011, 102, 1528-1536. [CrossRef] [PubMed]

3. Boontim, N.; Yoshimune, K.; Lumyong, S.; Moriguchi, M. Purification and characterization of D-glucose dehydrogenase from Bacillus thuringiensis M15. Ann. Microbiol. 2004, 54, 481-492.

4. Nagao, T.; Mitamura, T.; Wang, X.H.; Negoro, S.; Yomo, T.; Urabe, I.; Okada, H. Cloning, nucleotide sequences, and enzymatic properties of glucose dehydrogenase isozymes from Bacillus megaterium IAM1030. J. Bacteriol. 1992, 174, 5013-5020. [CrossRef] [PubMed]

5. Mitamura, T.; Urabe, I.; Okada, H. Enzymatic properties of isozymes and variants of glucose dehydrogenase from Bacillus megaterium. Eur. J. Biochem. 1989, 186, 389-393. [CrossRef] [PubMed]

6. Heilmann, H.J.; Magert, H.J.; Gassen, H.G. Identification and isolation of glucose dehydrogenase genes of Bacillus megaterium M1286 and their expression in Escherichia coli. Eur. J. Biochem. 1988, 174, 485-490. [CrossRef] [PubMed]

7. Nishioka, T.; Yasutake, Y.; Nishiya, Y.; Tamura, T. Structure-guided mutagenesis for the improvement of substrate specificity of Bacillus megaterium glucose 1-dehydrogenase IV. FEBS J. 2012, 279, 3264-3275. [CrossRef] [PubMed]

8. Yamamoto, K.; Kurisu, G.; Kusunoki, M.; Tabata, S.; Urabe, I.; Osaki, S. Crystal structure of glucose dehydrogenase from Bacillus megaterium IWG3 at 1.7 A resolution. J. Biochem. 2001, 129, 303-312. [CrossRef] [PubMed] 
9. Liu, Z.Q.; Ye, J.J.; Shen, Z.Y.; Hong, H.B.; Yan, J.B.; Lin, Y.; Chen, Z.X.; Zheng, Y.G.; Shen, Y.C. Upscale production of ethyl (S)-4-chloro-3-hydroxybutanoate by using carbonyl reductase coupled with glucose dehydrogenase in aqueous-organic solvent system. Appl. Microbiol. Biotechnol. 2015, 99, 2119-2129. [CrossRef] [PubMed]

10. Zhang, R.; Zhang, B.; Xu, Y.; Li, Y.; Li, M.; Liang, H.; Xiao, R. Efficicent (R)-phenylethanol production with enantioselectivity-alerted (S)-carbonyl reductase II and NADPH regeneration. PLoS ONE 2013, 8, e83586. [CrossRef] [PubMed]

11. Gao, C.; Zhang, L.; Xie, Y.; Hu, C.; Zhang, Y.; Li, L.; Wang, Y.; Ma, C.; Xu, P. Production of (3S)-acetoin from diacetyl by using stereoselective NADPH-dependent carbonyl reductase and glucose dehydrogenase. Bioresour. Technol. 2013, 137, 111-115. [CrossRef] [PubMed]

12. Gao, F.; Ding, H.; Shao, L.; Xu, X.; Zhao, Y. Molecular characterization of a novel thermal stable reductase capable of decoloration of both azo and triphenylmethane dyes. Appl. Microbiol. Biot. 2015, 99, $255-267$. [CrossRef] [PubMed]

13. Liang, B.; Lang, Q.; Tang, X.; Liu, A. Simultaneously improving stability and specificity of cell surface displayed glucose dehydrogenase mutants to construct whole-cell biocatalyst for glucose biosensor application. Bioresour. Technol. 2013, 147, 492-498. [CrossRef] [PubMed]

14. Yan, Y.M.; Yehezkeli, O.; Willner, I. Integrated, Electrically Contacted NAD(P) ${ }^{+}$-Dependent Enzyme-Carbon Nanotube Electrodes for Biosensors and Biofuel Cell Applications. Chem. A Eur. J. 2007, 13, 10168-10175. [CrossRef] [PubMed]

15. Bornscheuer, U.; Huisman, G.; Kazlauskas, R.; Lutz, S.; Moore, J.; Robins, K. Engineering the third wave of biocatalysis. Nature 2012, 485, 185-194. [CrossRef] [PubMed]

16. Liu, W.; Wang, P. Cofactor regeneration for sustainable enzymatic biosynthesis. Biotechnol. Adv. 2007, 25, 369-384. [CrossRef] [PubMed]

17. Zhao, H.; van der Donk, W.A. Regeneration of cofactors for use in biocatalysis. Curr. Opin. Biotechnol. 2003, 14, 583-589. [CrossRef] [PubMed]

18. Weckbecker, A.; Gröger, H.; Hummel, W. Regeneration of nicotinamide coenzymes: Principles and applications for the synthesis of chiral compounds. In Biosystems Engineering I; Wittmann, C., Krull, R., Eds.; Springer: Berlin, Germany, 2010; Volume 120, pp. 195-242.

19. Sheng, B.; Zheng, Z.; Lv, M.; Zhang, H.; Qin, T.; Gao, C.; Ma, C.; Xu, P. Efficient production of (R)-2-hydroxy-4-phenylbutyric acid by using a coupled reconstructed d-lactate dehydrogenase and formate dehydrogenase system. PLoS ONE 2014, 9, e104204. [CrossRef] [PubMed]

20. Lo, H.C.; Fish, R.H. Biomimetic NAD+ Models for Tandem Cofactor Regeneration, Horse Liver Alcohol Dehydrogenase Recognition of 1, 4-NADH Derivatives, and Chiral Synthesis. Angew. Chem. Int. Ed. 2002, 41, 478-481. [CrossRef]

21. Lee, W.H.; Chin, Y.W.; Han, N.S.; Kim, M.D.; Seo, J.H. Enhanced production of GDP-L-fucose by overexpression of NADPH regenerator in recombinant Escherichia coli. Appl. Microbiol. Biot. 2011, 91, 967-976. [CrossRef] [PubMed]

22. Johannes, T.W.; Woodyer, R.D.; Zhao, H.M. Efficient regeneration of NADPH using an engineered phosphite dehydrogenase. Biotechnol. Bioeng. 2007, 96, 18-26. [CrossRef] [PubMed]

23. Chen, X.; Ding, H.; Du, Y.; Lin, H.; Li, Z.; Zhao, Y. Cloning, expression and characterization of a glucose dehydrogenase from Bacillus sp. G3 in Escherichia coli. Afr. J. Microbiol. Res. 2011, 5, 5882-5888.

24. Wu, X.; Ding, H.; Ke, L.; Xin, Y.; Cheng, X. Characterization of an acid-resistant glucose 1-dehydrogenase from Bacillus cereus var. mycoides. Romanian Biotechnol. Lett. 2012, 17, 7540-7548.

25. Finn, R.D.; Coggill, P.; Eberhardt, R.Y.; Eddy, S.R.; Mistry, J.; Mitchell, A.L.; Potter, S.C.; Punta, M.; Qureshi, M.; Sangrador-Vegas, A.; et al. The Pfam protein families database: Towards a more sustainable future. Nucleic. Acids Res. 2016, 44, D279-D285. [CrossRef] [PubMed]

26. Boontim, N.; Yoshimune, K.; Lumyong, S.; Moriguchi, M. Cloning of D-glucose dehydrogenase with a narrow substrate specificity from Bacillus thuringiensis M15. Ann. Microbiol. 2006, 56, 237-240. [CrossRef]

27. Ramaley, R.F.; Vasantha, N. Glycerol protection and purification of Bacillus subtilis glucose dehydrogenase. J. Biol. Chem. 1983, 258, 12558-12565. [PubMed]

28. Larkin, M.A.; Blackshields, G.; Brown, N.P.; Chenna, R.; McGettigan, P.A.; McWilliam, H.; Valentin, F.; Wallace, I.M.; Wilm, A.; Lopez, R.; et al. Clustal W and Clustal X version 2.0. Bioinformatics 2007, 23, 2947-2948. [CrossRef] [PubMed] 
29. Gouet, P.; Robert, X.; Courcelle, E. ESPript/ENDscript: Extracting and rendering sequence and 3D information from atomic structures of proteins. Nucleic Acids Res. 2003, 31, 3320-3323. [CrossRef] [PubMed]

30. Saitou, N.; Nei, M. The neighbor-joining method: A new method for reconstructing phylogenetic trees. Mol. Biol. Evol. 1987, 4, 406-425. [PubMed]

31. Kumar, S.; Stecher, G.; Tamura, K. MEGA7: Molecular Evolutionary Genetics Analysis version 7.0 for bigger datasets. Mol. Biol. Evol. 2016, 33, 1870-1874. [CrossRef] [PubMed]

32. Zuckerkandl, E.; Pauling, L. Evolutionary divergence and convergence in proteins. Evol. Genes Proteins 1965, 97, 97-166.

33. Biasini, M.; Bienert, S.; Waterhouse, A.; Arnold, K.; Studer, G.; Schmidt, T.; Kiefer, F.; Gallo Cassarino, T.; Bertoni, M.; Bordoli, L.; et al. SWISS-MODEL: Modelling protein tertiary and quaternary structure using evolutionary information. Nucleic Acids Res. 2014, 42, W252-W258. [CrossRef] [PubMed]

34. Wiederstein, M.; Sippl, M.J. ProSA-web: Interactive web service for the recognition of errors in three-dimensional structures of proteins. Nucleic Acids Res. 2007, 35, W407-W410. [CrossRef] [PubMed]

35. Laskowski, R.A.; MacArthur, M.W.; Moss, D.S.; Thornton, J.M. PROCHECK: A program to check the stereochemical quality of protein structures. J. Appl. Crystallogr. 1993, 26, 283-291. [CrossRef]

36. Baker, N.A.; Sept, D.; Joseph, S.; Holst, M.J.; McCammon, J.A. Electrostatics of nanosystems: Application to microtubules and the ribosome. Proc. Natl. Acad. Sci. USA 2001, 98, 10037-10041. [CrossRef] [PubMed]

37. Lipari, G.; Szabo, A. Model-free approach to the interpretation of nuclear magnetic resonance relaxation in macromolecules. 1. Theory and range of validity. J. Am. Chem. Soc. 1982, 104, 4546-4559. [CrossRef]

38. Amadei, A.; Linssen, A.; Berendsen, H.J. Essential dynamics of proteins. Proteins Struct. Funct. Bioinform. 1993, 17, 412-425. [CrossRef] [PubMed]

39. Balsera, M.A.; Wriggers, W.; Oono, Y.; Schulten, K. Principal component analysis and long time protein dynamics. J. Phys. Chem. 1996, 100, 2567-2572. [CrossRef]

40. Berendsen, H.J.; Hayward, S. Collective protein dynamics in relation to function. Curr. Opin. Struc. Biol. 2000, 10, 165-169. [CrossRef]

41. Baik, S.H.; Michel, F.; Aghajari, N.; Haser, R.; Harayama, S. Cooperative effect of two surface amino acid mutations (Q252L and E170K) in glucose dehydrogenase from Bacillus megaterium IWG3 on stabilization of its oligomeric state. Appl. Environ. Microbiol. 2005, 71, 3285-3293. [CrossRef] [PubMed]

42. Dolinsky, T.J.; Nielsen, J.E.; McCammon, J.A.; Baker, N.A. PDB2PQR: An automated pipeline for the setup of Poisson-Boltzmann electrostatics calculations. Nucleic Acids Res. 2004, 32, W665-W667. [CrossRef] [PubMed]

43. Pronk, S.; Páll, S.; Schulz, R.; Larsson, P.; Bjelkmar, P.; Apostolov, R.; Shirts, M.R.; Smith, J.C.; Kasson, P.M.; van der Spoel, D. GROMACS 4.5: A high-throughput and highly parallel open source molecular simulation toolkit. Bioinformatics 2013, 29, 845-854. [CrossRef] [PubMed]

44. Hornak, V.; Abel, R.; Okur, A.; Strockbine, B.; Roitberg, A.; Simmerling, C. Comparison of multiple Amber force fields and development of improved protein backbone parameters. Proteins Struct. Funct. Bioinform. 2006, 65, 712-725. [CrossRef] [PubMed]

(C) 2017 by the authors. Licensee MDPI, Basel, Switzerland. This article is an open access article distributed under the terms and conditions of the Creative Commons Attribution (CC BY) license (http:/ / creativecommons.org/licenses/by/4.0/). 


\title{
3D-QSAR and Molecular Docking Studies on the TcPMCA1-Mediated Detoxification of Scopoletin and Coumarin Derivatives
}

\author{
Qiu-Li Hou ${ }^{\dagger}$, Jin-Xiang Luo ${ }^{\dagger}$, Bing-Chuan Zhang, Gao-Fei Jiang, Wei Ding and \\ Yong-Qiang Zhang *

\begin{abstract}
Laboratory of Natural Products Pesticides, College of Plant Protection, Southwest University, Chongqing 400715, China; houqiuli2000@126.com (Q.-L.H.); xiangxiangnx@sohu.com (J.-X.L.); zhbichting@163.com (B.-C.Z.);

Gaofei.Jiang@toulouse.inra.fr (G.-F.J.); dwing818@163.com (W.D.)

* Correspondence: zyqiang@swu.edu.cn; Tel./Fax: +86-23-6825-0218
\end{abstract} \\ † These authors contributed equally to this work.
}

Received: 20 May 2017; Accepted: 20 June 2017; Published: 27 June 2017

\begin{abstract}
The carmine spider mite, Tetranychus cinnabarinus (Boisduval), is an economically important agricultural pest that is difficult to prevent and control. Scopoletin is a botanical coumarin derivative that targets $\mathrm{Ca}^{2+}$-ATPase to exert a strong acaricidal effect on carmine spider mites. In this study, the full-length cDNA sequence of a plasma membrane $\mathrm{Ca}^{2+}$-ATPase 1 gene (TcPMCA1) was cloned. The sequence contains an open reading frame of $3750 \mathrm{bp}$ and encodes a putative protein of 1249 amino acids. The effects of scopoletin on TCPMCA1 expression were investigated. TcPMCA1 was significantly upregulated after it was exposed to $10 \%, 30 \%$, and $50 \%$ of the lethal concentration of scopoletin. Homology modeling, molecular docking, and three-dimensional quantitative structure-activity relationships were then studied to explore the relationship between scopoletin structure and TCPMCA1-inhibiting activity of scopoletin and other 30 coumarin derivatives. Results showed that scopoletin inserts into the binding cavity and interacts with amino acid residues at the binding site of the TCPMCA1 protein through the driving forces of hydrogen bonds. Furthermore, CoMFA (comparative molecular field analysis)- and CoMSIA (comparative molecular similarity index analysis)-derived models showed that the steric and H-bond fields of these compounds exert important influences on the activities of the coumarin compounds.Notably, the C3, C6, and C7 positions in the skeletal structure of the coumarins are the most suitable active sites. This work provides insights into the mechanism underlying the interaction of scopoletin with TcPMCA1. The present results can improve the understanding on plasma membrane $\mathrm{Ca}^{2+}$-ATPase-mediated (PMCA-mediated) detoxification of scopoletin and coumarin derivatives in T. cinnabarinus, as well as provide valuable information for the design of novel PMCA-inhibiting acaricides.
\end{abstract}

Keywords: Tetranychus cinnabarinus; plasma membrane $\mathrm{Ca}^{2+}$-ATPase; scopoletin; coumarin derivatives; molecular docking; three-dimensional quantitative structure activity relationship (3D-QSAR); interaction mechanism

\section{Introduction}

The plasma membrane $\mathrm{Ca}^{2+}$-ATPase (PMCA) pumps $\mathrm{Ca}^{2+}$ out of the cell to maintain cytosolic $\mathrm{Ca}^{2+}$ concentration at a level that is compatible with messenger function. The concentration of nerve membrane $\mathrm{Ca}^{2+}$ is normally higher in the cytoplasm than that in the extracellular matrix;furthermore, $\mathrm{Ca}^{2+}$ is sequestered by sarco/endoplasmic reticulum $\mathrm{Ca}^{2+}$ pumps (SERCA) or by $\mathrm{Ca}^{2+}$-binding proteins, or else extruded by $\mathrm{Na}^{+} / \mathrm{Ca}^{2+}$ exchangers or PMCAs [1-3]. PMCAs exhibit cell-specific expression patterns and play an essential role in $\mathrm{Ca}^{2+}$ homeostasis in various cell types, including sensory 
neurons [4-7]. The inhibition of PMCAs in rat and fire salamander cilia by specific drugs, such as vanadate or carboxyeosin, suggests that PMCAs play a predominant role in $\mathrm{Ca}^{2+}$ clearance [8,9]. In mammals, four genes encode PMCAs [10]. PMCA isoforms 1 and 4 are ubiquitously expressed and considered as housekeeping isoforms, whereas PMCA isoforms 2 and 3 exhibit limited expression in tissues [4-7]. Through quantitative analysis, human PMCA1 is shown to be more abundant than PMCA4 at mRNA and protein levels [11]. Numerous methods, such as transient transfection, the use of stable cell lines, and use of the vaccinia viral vector, are used to advance knowledge on the differential properties of these isoforms [12-14].

The carmine spider mite, Tetranychus cinnabarinus (Boisduval), is a global agricultural pest that parasitizes more than 100 plant species, including beans, cotton, eggplants, tomatoes, and peppers. T. cinnabarinus infestations significantly reduce the quality and yield of these crops. These mites are difficult to prevent and control given its high fecundity, short developmental duration, small individual size, limited territory, and high inbreeding rate $[15,16]$. The control and prevention of T. cinnabarinus are currently dependent on chemical insecticides and acaricides, such as spiromesifen, pyridaben, and etoxazole, which introduce a high amount of chemical residues to the environment and induce drug resistance in the target species [17]. Therefore, a novel, environmentally friendly acaricidal compound should be identified and developed to manage these problems.

Scopoletin (7-hydroxy-6-methoxychromen-2-one) is an important coumarin phytoalexin found in many herbs [18]. Scopoletin displays a wide array of pharmacological and biochemical activities [19]. In addition, scopoletin exerts insecticidal, acaridal, antibacterial, and allelopathic activities that are useful in agricultural applications [20-22]. A previous study found that scopoletin extracted from Artemisia annua L. exhibits strong acaricidal activity against carmine spider mites and inhibits oviposition [22]. Furthermore, many studies on the effects of scopoletin on various protective enzymes in the nervous system of T. cinnabarinus indicated that scopoletin inhibits $\mathrm{Ca}^{2+}$-ATPase [23]. Thus, scopoletin is has increasingly attracting interest as a potential botanical acaricide because it is more environmentally friendly compared with chemical and physical agents. However, the interaction between $\mathrm{Ca}^{2+}$-ATPase and scopoletin in T. cinnabarinus remains unclear.

The objective of this study is to investigate the PMCA-meditated detoxification mechanism of scopoletin. Molecular docking and three-dimensional quantitative structure activity relationship (3D-QSAR) analyses were performed to achieve this aim. The full-length cDNA that encodes the PMCA 1 gene (TcPMCA1) was obtained from T. cinnabarinus. The expression profiles of TcPMCA1 at the various life stages of carmine spider mites were then reported. The effects of scopoletin on TCPMCA1 expression during the adult stage of T. cinnabarinus were also investigated. The results of the molecular docking and 3D-QSAR studies were used to investigate the mechanism underlying the interaction between scopoletin and TCPMCA1, as well as the active site of coumarin compounds. This work provides an insight into the detoxification mechanism of scopoletin at the active site for future studies on the optimized structural design of scopoletin and other coumarin derivatives.

\section{Results}

\subsection{Cloning and Sequence Analysis}

The partial cDNA sequence that codes for PMCA1 was identified through the use of transcriptome data and alignment with nucleotide sequences from the genome datasets of Tetranychus urticae [24]. The remaining $5^{\prime}$ and $3^{\prime}$ ends were amplified through a RACE (rapid amplification of cDNA ends)/PCR (Polymerase Chain Reaction)-based strategy. The full-length cDNA sequence, which was designated as TcPMCA1, was deposited in the GenBank database and with the accession number of KP455490. The full-length cDNA of TcPMCA1 is $4369 \mathrm{bp}$ in length and contains a 3750-bp open reading frame (ORF), a 456-bp 5'-untranslated region (UTR), and a 163-bp 3'-UTR with a putative polyadenylation signal upstream of the poly $(A)$ (Figure 1). The ORF encodes 1249 amino acid residues with a predicted molecular mass of $137.7 \mathrm{kDa}$ and an isoelectric point of 8.10 (Figure 1). 


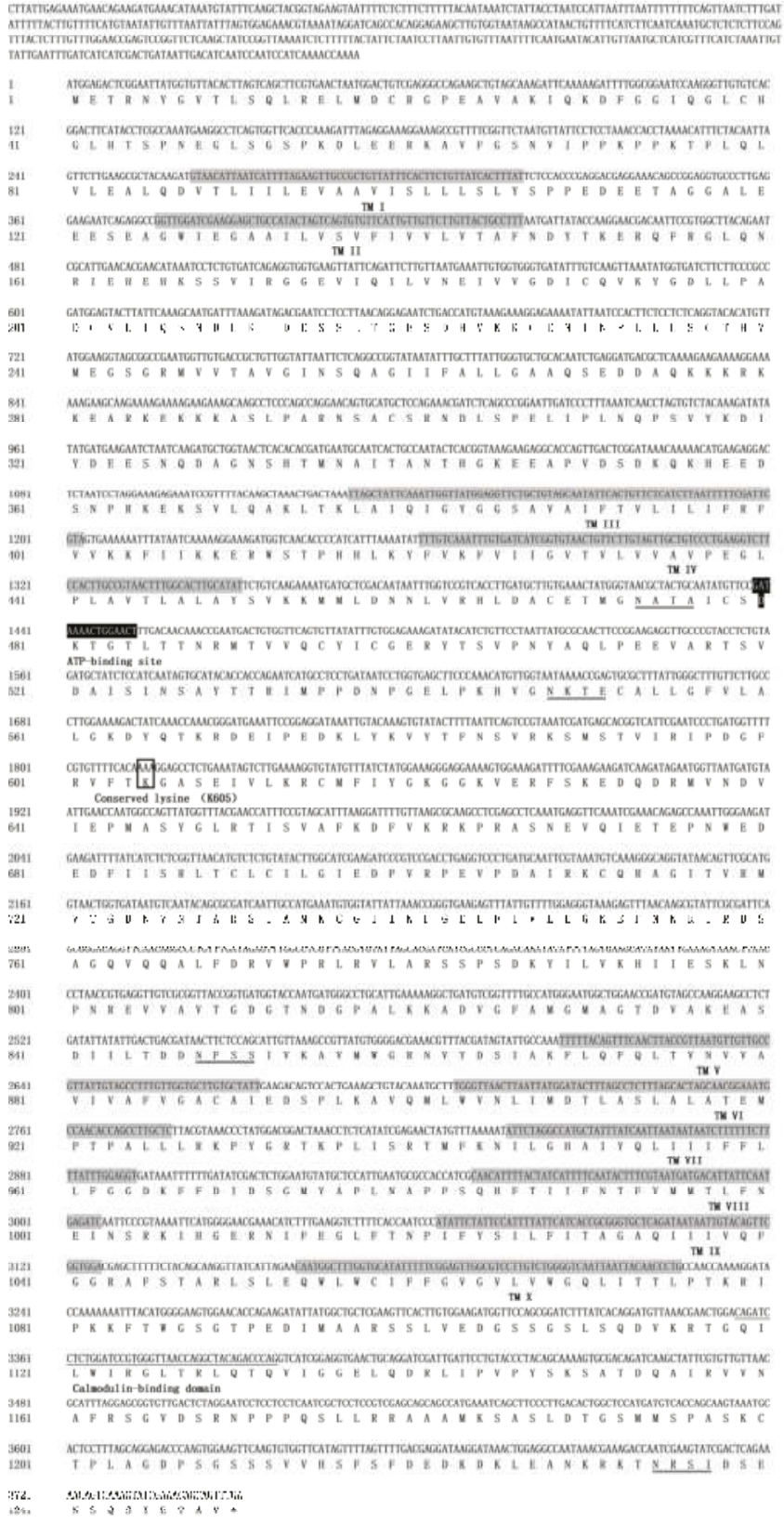

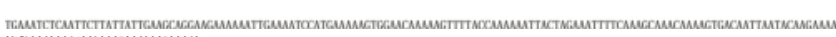

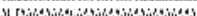

Figure 1. Nucleotide and deduced amino acid sequences of $\mathrm{Ca}^{2+}$-ATPase 1 gene (TcPMCA1) from the carmine spider mite (Tetranychus cinnabarinus (Boisduval)). Nucleotide numbers are provided on the left. The 10 transmembrane (TM) domains, which are denoted as TM I to TM X, are shaded. The ATP (Adenosine Triphosphate)-binding site, together with phosphorylable aspartate (D480), is shaded black, whereas the conserved lysine (K605) is boxed. The calmodulin-binding domain is indicated by a single line and the four $\mathrm{N}$-glycosylation sites are indicated by double lines. * represents the termination signal. 
The analysis of the deduced amino acid sequence of TCPMCA1 revealed the presence of ten membrane-spanning segments (TM), which were denoted as TM I to TM X, as well as four main cytosolic domains located between TM II and TM III, between TM IV and TM V, and at the Nand C-terminal regions. Some characteristic segments also were predicted. TcPMCA1 contains an ATP-binding site (from amino acid D480 to T484) and a calmodulin-binding domain (Q1119 to Q1130) (Figure 1).

The multiple protein alignments of the C-terminal conserved catalytic domains of the PMCAs from Arachnida and insects showed that TcPMCA1 exhibits 99.7\% amino acid sequence identity with T. urticae PMCA1. TcPMCA1 also showed nearly 70\% similarity with the PMCA genes of Ixodes scapularis, and 60-75\% similarity with the PMCA genes of insects and nematodes (Figure 2).

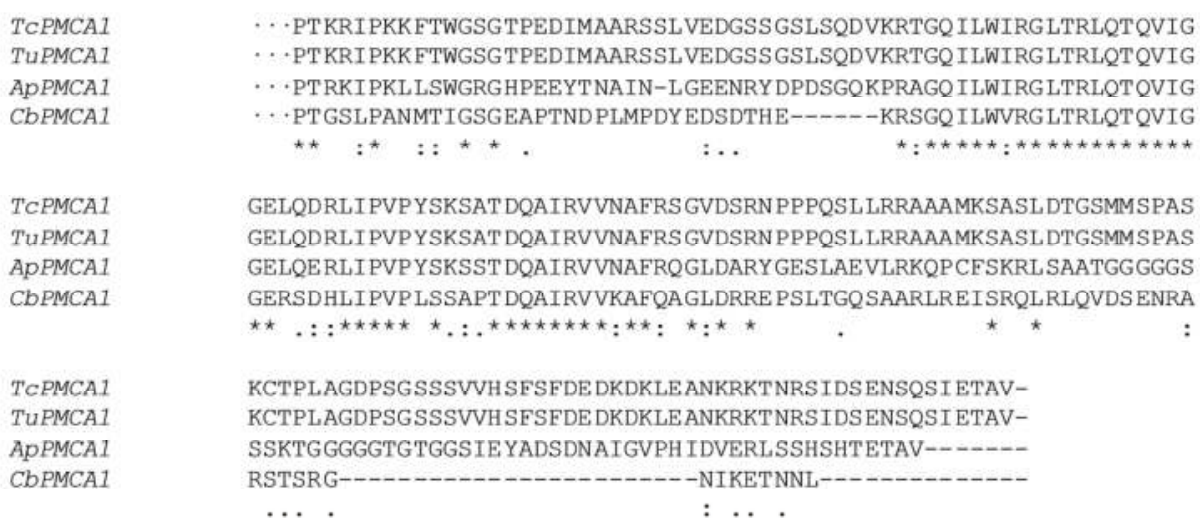

Figure 2. ClustalW alignment of the C-terminal sequence comparison of plasma membrane $\mathrm{Ca}^{2+}$-ATPase 1 (PMCA1) obtained from different species. Alignment of the sequences of the PMCAs, starting after the last (10th) putative membrane-spanning domain and ending at the last residue. Residues that are completely conserved are marked with an asterisk $\left(^{*}\right)$; those that are highly conserved are indicated by colon (:); while similar residues are indicated by a dot (.). "_" represents interval. PMCA1 sequences used in the alignment are as follows: TcPMCA1, Tetranychus cinnabarinus; TuPMCA1, Tetranychus urticae; ApPMCA1, Acythosiphon pisum; and CbPMCA1, Caenorhabditis briggsae. The PMCA2 sequences used in the alignment are as follows: TcPMCA2, Tetranychus cinnabarinus; TuPMCA2, Tetranychus urticae; IsPMCA, Ixodes scapularis; and CbPMCA1, Caenorhabditis briggsae.

\subsection{Phylogenetic Analysis}

A neighbor-joining phylogenetic tree was constructed by comparing the amino acid sequence of TcPMCA1 with those of PMCA genes from other animal species. Phylogenetic analysis showed that TcPMCA1 belongs to the cluster of Ixodes PMCA. The PMCA genes of T. cinnabarinus and T. urticae clustered into the PMCA family and apparently share a single clade. These results suggested that the PMCA genes of T. cinnabarinus and T. urticae are evolutionarily related and share similar physiological functions (Figure 3). 


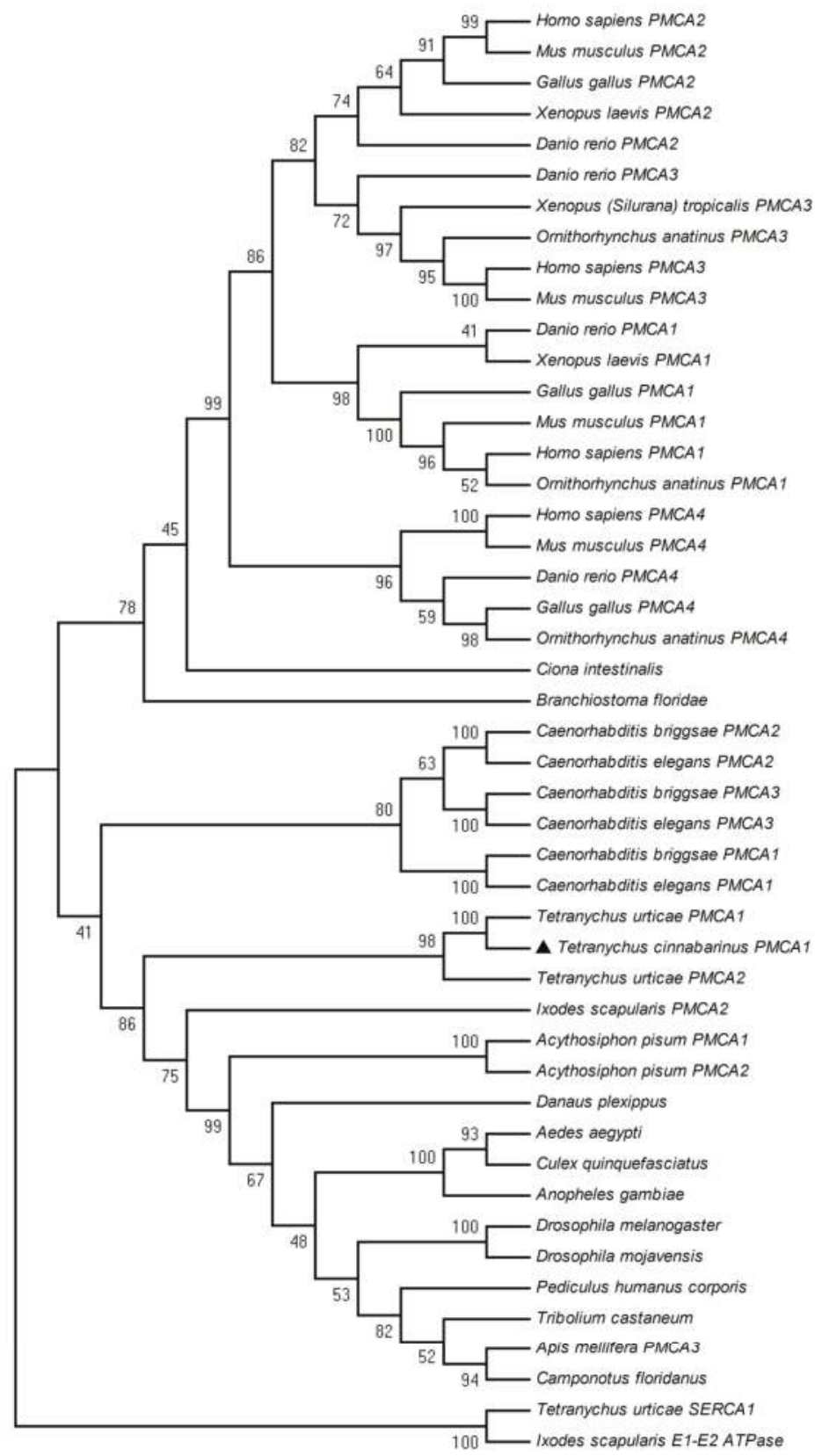

Figure 3. Phylogenetic analysis of TcPMCA1 obtained from the carmine spider mite (Tetranychus cinnabarinus (Boisduval)). The phylogenetic tree was constructed using Molecular Evolutionary Genetics Analysis (MEGA) 5.04 using the neighbor-joining method based on amino acid sequences. TcPMCA1 was indicated by " $\mathbf{\Delta}$ ". Bootstrap support values derived from 1000 replicates are shown on the branches. Sequence accession numbers are given in Electronic Supplementary Material, Table S1. 


\subsection{Developmental Expression Patterns}

To gain insights into the potential role of TcPMCA1, the expression levels of TcPMCA1 in female individuals at various life stages were quantified through Real-time Quantitative polymerase chain reaction (RT-qPCR). The results showed that TcPMCA1 mRNA was detected at all developmental stages, including the larval, nymphal, and adult stages. More specifically, the TCPMCA1 transcript was slightly detectable at the egg stage, was highly expressed at the larval, nymphal, and adult stages, and was the highest at the nymphal stage (Figure 4).

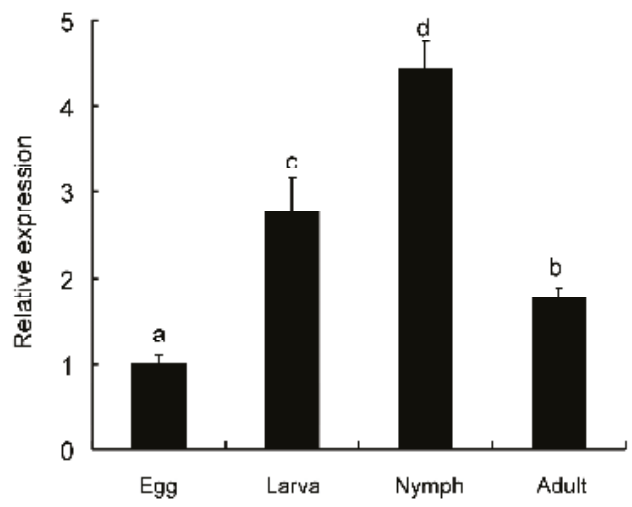

Figure 4. Expression levels of the plasma membrane $\mathrm{Ca}^{2+}$-ATPase 1 gene (TcPMCA1) at different developmental stages of Tetranychus cinnabarinus were evaluated using Real-time Quantitative polymerase chain reaction (RT-qPCR). The egg, larval, nymphal, and adult stages were analyzed. Relative expression was calculated according to the value of the lowest expression level, which was assigned with an arbitrary value of 1 . Letters above the bars indicate significant differences among different developmental stages. RPS18 was used as reference gene. Data were presented as the means $( \pm \mathrm{SE})$ of three biological replications per developmental stage. Different letters on the error bars indicate significant differences revealed by ANOVA test $(p<0.05)$.

\subsection{Effects of Scopoletin Exposure on TcPMCA Expression}

Scopoletin exposure caused spasms and high mortality among adult $T$. cinnabarinus. The results of induction showed that exposure to scopoletin significantly changed the TCPMCA1 expression. TcPMCA1 was significantly upregulated following exposure to low lethal $\left(\mathrm{LC}_{10}\right)$, sublethal $\left(\mathrm{LC}_{30}\right)$, and median lethal $\left(\mathrm{LC}_{50}\right)$ scopoletin concentrations for $12,24,36$, or $48 \mathrm{~h}$. The relative expression levels of TcPMCA1 were upregulated by more than 100-fold of that of the control following 24 or $36 \mathrm{~h}$ of exposure to scopoletin at $\mathrm{LC}_{30}$ dose. However, TcPMCA1 activation by scopoletin weakened gradually with the extension of time (Figure 5).

\subsection{Homology Modeling}

Bell Labs Layered Space-Time (BLAST) analysis revealed that the primary sequence of the target enzyme had a high sequence identity of $73 \%$ with the template 3BA6. BLAST analysis guarantees that the model structure is of a high quality. Further energy minimization was performed to remove geometric restraints prior to model construction [25]. The homology modeling of TcPMCA1 is shown in Figure 6. The 3D structure of this enzyme was further checked by Procheck to evaluate the stereo-chemical quality. Ramachandran plot analysis showed that most residues are present at the most favored regions. In particular, $90.3 \%$ of the residues were in the most favored regions, $9.0 \%$ residues in the additional allowed regions, giving a total of $99.3 \%$. Other $0.4 \%$ residues in the generously allowed regions and $0.4 \%$ residues in the disallowed regions. The results of the procheck analysis demonstrated 
that the 3D-modeling structure exhibits reasonable and reliable stereo-chemical properties and is thus appropriate for subsequent molecular docking study.

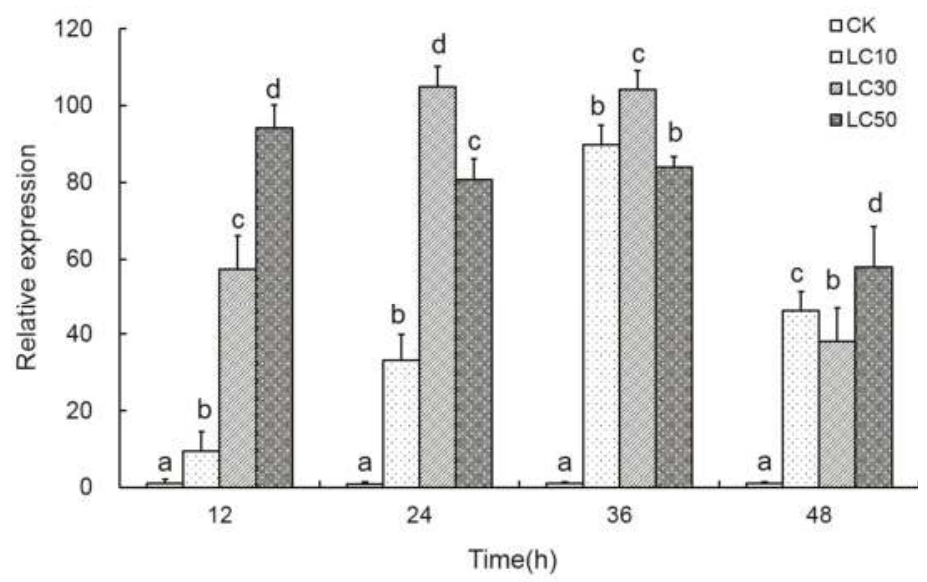

Figure 5. Relative expression levels of the TcPMCA1 gene in adult female Tetranychus cinnabarinus exposed $\mathrm{LC}_{10}\left(0.219 \mathrm{mg} \mathrm{mL}^{-1}\right), \mathrm{LC}_{30}(0.581 \mathrm{mg} \mathrm{mL}-1)$, and $\mathrm{LC}_{50}\left(1.142 \mathrm{mg} \mathrm{mL} \mathrm{mL}^{-1}\right)$ scopoletin. Expression levels were quantified using qPCR after 12, 24, 36, and $48 \mathrm{~h}$ of treatment through leaf-dip bioassay $(n=3)$. Scopoletin was mixed with acetone and Tween- 80 (scopoletin: Tween- $80=3: 1$; acetone was added until scopoletin dissolved, generally limited within 5\%). T. cinnabarinus treated with double distilled water containing $0.5 \%$ acetone and Tween- 80 were used as controls (CK). The mRNA levels in the control and in each treatment were normalized to the expression of the reference gene RPS18. The mean expression in each treatment was shown as fold change compared with the mean expression in the control, which was assigned with a basal value of 1 . Letters on the error bar indicate significant difference between scopoletin treatment and control $(p<0.05)$.

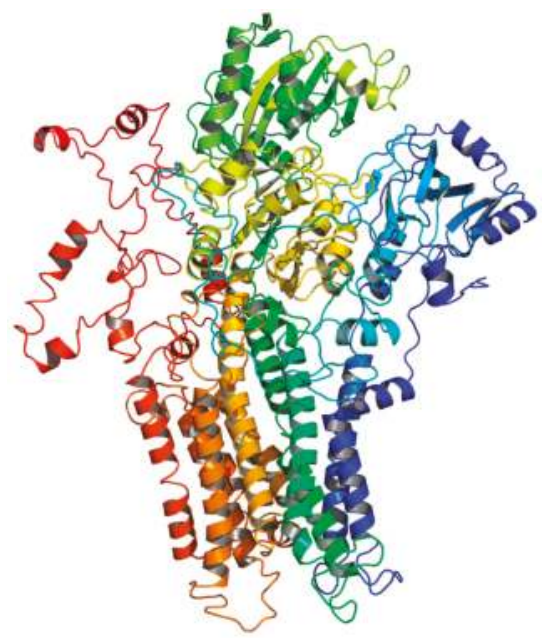

Figure 6. Homology modeling 3D-structure of TcPMCA1. 


\subsection{Molecular Docking}

To comprehend the interaction between the ligand scopoletin and TcPMCA1, molecular docking was performed to investigate the binding mode of scopoletin within the binding pocket of TcPMCA1, and to further understand their structure-activity relationship. The ligand structure of scopoletin is shown in Figure 7. The result showed that scopoletin docked with high affinity to the nucleotide-binding pocket of TcPMCA1 and amino acid residues Ser297 and 300, Thr144, Cys299, Glu83, Gln86, Asp87, and Lys301 surrounded scopoletin. Furthermore, five hydrogen bonds (the red dash lines) formed between the 7-hydroxy with Sre297, 6-methoxy with Ala298, oxygen at position 1 with Lys301, and oxygen at position 2 with Lys301 and Ser300 (Figure 8).

A<smiles>COc1cc2ccc(=O)oc2cc1O</smiles>

B

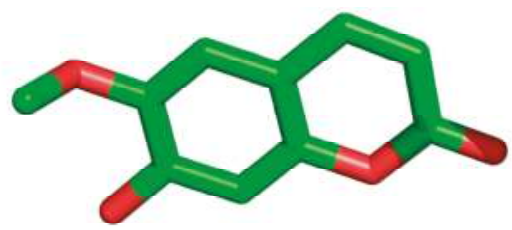

Figure 7. (A) Chemical structural formula and (B) the cartoon representation of scopoletin. Red regions represent oxygen atoms; green regions represent carbon atoms.

A

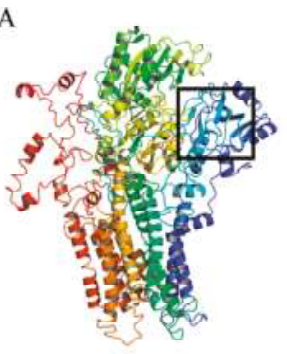

B

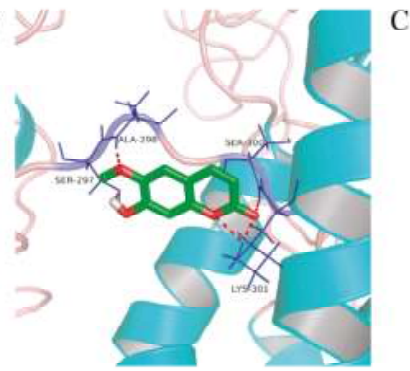

$\mathrm{C}$

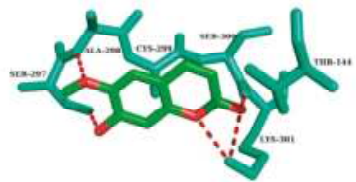

Figure 8. (A) Binding pocket of TCPMCA1 was indicated by the black frame; (B) best conformation of scopoletin docked to binding pocket of TCPMCA1; (C) cartoon representation of residues involved in the binding of scopoletin to TCPMCA1. The black box represents the binding cavity. Short, red dashed lines represent hydrogen bonds. Red regions represent oxygen atoms of scopoletin; green regions represent the carbon atoms of scopoletin; the others represent the amino acid residue of the protein.

The 30 coumarin derivatives (Table 1) were also subjected to molecular docking calculations. The derivatives all docked with high affinity to the nucleotide-binding domain (NBD). These results appeared promising and encouraged the calculation of molecular docking at the NBD for all compounds. Defined molecular docking (DMD) at the nucleotide-binding pocket revealed that all compounds showed low binding energy values. The lowest binding energy of $-6.03 \mathrm{kcal} / \mathrm{mol}$ was exhibited by compound 2 (Table 1). Therefore, compound 2 appears to be the most stable compound. 
Table 1. Docking results of coumarins with $\mathrm{Ca}^{2+}$-ATPase 1 gene of Tetranychus cinnabarinus (TcPMCA1).

\begin{tabular}{|c|c|c|c|c|c|c|c|c|c|}
\hline \multirow{2}{*}{ Compound } & \multicolumn{4}{|c|}{ AutoDock } & \multirow{2}{*}{ Compound } & \multicolumn{4}{|c|}{ AutoDock } \\
\hline & Einter & Eintra & Etors & $\Delta \mathrm{G}$ & & Einter & Eintra & Etors & $\Delta \mathrm{G}$ \\
\hline 1 & -6.87 & -0.47 & 1.19 & -5.64 & 16 & -4.64 & -0.15 & 0.3 & -4.35 \\
\hline 2 & -7.22 & -0.59 & 1.19 & -6.71 & 17 & -4.77 & -0.37 & 0.6 & -5.01 \\
\hline 3 & -4.55 & -0.56 & 0.3 & -4.65 & 18 & -5.95 & -0.86 & 0.89 & -5.03 \\
\hline 4 & -4.95 & -0.02 & 0.3 & -5.07 & 19 & -4.84 & -1.45 & 0.89 & -4.33 \\
\hline 5 & -4.65 & -0.1 & 0.3 & -4.38 & 20 & -4.67 & -1.13 & 0.6 & -4.69 \\
\hline 6 & -4.95 & 0.03 & 0.3 & -4.41 & 21 & -4.61 & -1.27 & 0.6 & -4.23 \\
\hline 7 & -6.01 & -0.55 & 0.89 & -5.14 & 22 & -4.29 & 0.02 & 0.3 & -4.32 \\
\hline 8 & -4.86 & -0.09 & 0.3 & -5.04 & 23 & -3.97 & 0 & 0 & -4.47 \\
\hline 9 & -6.56 & -1.73 & 0.89 & -5.24 & 24 & -5.89 & -0.38 & 0.89 & -6.08 \\
\hline 10 & -4.66 & 0.03 & 0.3 & -3.83 & 25 & -4.89 & -0.25 & 0.6 & -6.1 \\
\hline 11 & -4.35 & -0.06 & 0.3 & -4.59 & 26 & -4.12 & 0 & 0 & -4.59 \\
\hline 12 & -4.79 & 0.01 & 0.3 & -4.58 & 27 & -5.59 & -0.59 & 0.89 & -5.25 \\
\hline 13 & -4.56 & -0.26 & 0.6 & -4.84 & 28 & -4.91 & -0.11 & 0.3 & -5.13 \\
\hline 14 & -4.49 & 0 & 0 & -5.28 & 29 & -4.54 & -0.68 & 0.3 & -5.35 \\
\hline 15 & -4.56 & -0.14 & 0.6 & -4.36 & 30 & -4.42 & -0.89 & 0.89 & -4.6 \\
\hline
\end{tabular}

1, 3-(2-benzimidazolyl)-7-(diethylamino)coumarin; 2, 3-(2-benzothiazolyl)-7-(diethylamino)coumarin; 3, 3-Aminocoumarin; 4, 3-Acetylcoumarin; 5, 4-Methoxycoumarin; 6, 4-Hydroxycoumarin; 7, 5,7-dihydroxy-4-phenyl coumarin; 8, 6-Nitrocoumarin; 9, 7,8-dihydroxy-4-phenyl coumarin; 10, 7-amino-4-phenyl coumarin; 11, 7-methoxycoumarin(herniarin); 12, 7-mercapto-4-methyl coumarin; 13, 6,7-dimethoxy coumarin(Scoparone); 14, Psoralen; 15, 7-Hydroxy-6-methoxycoumarin(Scopoletin); 16, Xanthotoxin; 17, Pimpinellin; 18, Imperatorin; 19, Fraxetin; 20, Esculetin; 21, Daphnetin; 22, Umbelliferone; 23, Coumarin; 24, Oxypeucedanin; 25, Isopimpinellin; 26, 6-Methylcoumarin; 27, Osthole; 28, Bergapten; 29, Xanthotol; 30, Isofraxidin.

\subsection{CoMFA and CoMSIA Statistical Result}

The same training (24 compounds) and test sets (six compounds) (Table 2) were used to derive models through CoMFA and CoMSIA. The statistical details were summarized in Table 3 . The results showed that the optimal CoMFA model provided a leave-one-out $q^{2}$ of $0.75(>0.5)$ with an optimal number of principal components (ONC) of 7. A correlation coefficient $R^{2}$ of 0.993 with a low standard error of the estimate (SEE) of 0.042 , and an F-statistic value of 383.856 were also obtained. In contribution, the CoMFA steric field and electrostatic field contributed $72.6 \%$ and $27.4 \%$, respectively. The best CoMSIA model provided a $q^{2}$ of 0.71 with an ONC of 6 . An $R^{2}$ of 0.975 with a low SEE of 0.080 and an $F$ value of 124.834 were obtained. In CoMSIA model, the contributions of the steric, electrostatic, hydrophobic, H-bond donor and acceptor were $14.0 \%, 33.4 \%, 23.9 \%, 19.7 \%$ and $9.0 \%$, respectively (Table 3). Based on these field contributions, the steric field is the most important field in the CoMFA model, whereas the electrostatic field is the most important field in the CoMSIA model.

The test set (six compounds) was used to evaluate the predictive accuracy of the CoMFA and CoMSIA models. Table 4 showed the experimentally determined and predicted activitiesand the training and test sets residual values. The residual values obtained by calculating the difference between the predicted and actual $\mathrm{pLC}_{50}$ are below one logarithmic unit for all the compounds (Figure 9). Therefore, the predictive abilities of the optimal CoMFA/CoMSIA models are excellent. 
Table 2. Structures and acaricidal activities ( $\mathrm{LC}_{50}$ values) of the compounds tested in this study.

\begin{tabular}{|c|c|c|c|c|c|}
\hline Compound & Structure & $\mathrm{LC}_{50}(\mathrm{mmol} / \mathrm{L})$ & Compound & Structure & $\mathrm{LC}_{50}(\mathrm{mmol} / \mathrm{L})$ \\
\hline $1 \mathrm{a}$ & & 1.2175 & $16 a$ & & 6.0313 \\
\hline $2 a$ & & 0.8638 & $17 a$ & & 5.188 \\
\hline $3 a$ & & 2.971 & $18 a$ & & 5.3789 \\
\hline $4 a$ & & 3.52 & $19 a$ & & 6.2036 \\
\hline $5 b$ & & 2.2563 & $20 a$ & & 12.6973 \\
\hline $6 b$ & & 61.2926 & $21 b$ & & 3.8273 \\
\hline $7 a$ & & 22.784 & $22 a$ & & 20.0142 \\
\hline $8 a$ & & 3.319 & $23 a$ & & 14.1447 \\
\hline $9 b$ & & 5.4987 & $24 a$ & & 4.876 \\
\hline $10 \mathrm{~b}$ & & 14.1318 & $25 a$ & & 5.0816 \\
\hline $11 a$ & & 33.8571 & $26 a$ & & 15.4398 \\
\hline $12 b$ & & 22.269 & $27 a$ & & 1.9186 \\
\hline $13 a$ & & 1.3813 & $28 a$ & & 15.1358 \\
\hline $14 a$ & & 25.6564 & $29 a$ & & 3.8 \\
\hline $15 a$ & & 6.4698 & $30 a$ & & 2.5798 \\
\hline
\end{tabular}

a, Training compounds; b, test set compounds. The others are the same as those in Table 1 . 
Table 3. Summary of the results obtained from CoMFA (comparative molecular field analysis) and CoMSIA (comparative molecular similarity index analysis) analyses.

\begin{tabular}{ccc}
\hline Statistical Parameter & CoMFA Model & CoMSIA Model \\
\hline$q^{2}$ & 0.750 & 0.710 \\
ONC & 7 & 6 \\
$\mathrm{R}^{2}$ & 0.993 & 0.975 \\
SEE & 0.042 & 0.080 \\
$\mathrm{~F}$ & 383.856 & 124.834 \\
$\mathrm{R}^{2}$ pred & 0.6465 & 0.931 \\
& Contribution & \\
Steric & 0.726 & 0.140 \\
Electrostatic & 0.274 & 0.334 \\
Hydrophobic & & 0.239 \\
H-bond donor & & 0.197 \\
H-bond acceptor & & 0.090 \\
\hline
\end{tabular}

Table 4. Observed and predicted activities of the test compounds.

\begin{tabular}{|c|c|c|c|c|c|}
\hline \multirow{2}{*}{ Compound } & \multirow{2}{*}{$\mathrm{pLC}_{50}$} & \multirow{2}{*}{$\begin{array}{c}\text { CoMFA } \\
\text { Predicted } \mathrm{pLC}_{50}\end{array}$} & \multicolumn{3}{|c|}{ CoMSIA } \\
\hline & & & Residual & Predicted $\mathrm{pLC}_{50}$ & Residual \\
\hline 1a & 2.915 & 2.868 & 0.047 & 2.924 & -0.009 \\
\hline $2 a$ & 3.064 & 3.097 & -0.033 & 3.021 & 0.043 \\
\hline $3 a$ & 2.527 & 2.514 & 0.013 & 1.83 & 0.697 \\
\hline $4 a$ & 2.453 & 2.487 & -0.034 & 2.465 & -0.012 \\
\hline $5 b$ & 2.647 & 1.651 & 0.996 & 1.92 & 0.727 \\
\hline $6 b$ & 1.213 & 2.328 & -1.115 & 1.916 & -0.703 \\
\hline $7 a$ & 1.642 & 1.394 & 0.248 & 1.65 & -0.008 \\
\hline $8 a$ & 2.479 & 2.894 & -0.415 & 2.493 & -0.014 \\
\hline $9 b$ & 2.260 & 1.67 & 0.59 & 1.917 & 0.343 \\
\hline $10 \mathrm{~b}$ & 1.850 & 2.097 & -0.247 & 1.857 & -0.007 \\
\hline 11a & 1.470 & 2.184 & -0.714 & 1.716 & -0.246 \\
\hline $12 b$ & 1.652 & 2.245 & -0.593 & 1.756 & -0.104 \\
\hline $13 a$ & 2.860 & 2.258 & 0.602 & 2.779 & 0.081 \\
\hline $14 a$ & 1.591 & 2.271 & -0.68 & 1.739 & -0.148 \\
\hline $15 a$ & 2.189 & 1.84 & 0.349 & 2.18 & 0.009 \\
\hline $16 a$ & 2.220 & 1.703 & 0.517 & 2.127 & 0.093 \\
\hline $17 a$ & 2.285 & 1.931 & 0.354 & 2.344 & -0.059 \\
\hline $18 a$ & 2.269 & 2.304 & -0.035 & 2.263 & 0.006 \\
\hline $19 a$ & 2.207 & 2.309 & -0.102 & 2.311 & -0.104 \\
\hline $20 a$ & 1.896 & 1.947 & -0.051 & 1.769 & 0.127 \\
\hline $21 b$ & 2.417 & 2.74 & -0.323 & 2.063 & 0.354 \\
\hline $22 a$ & 1.699 & 1.841 & -0.142 & 1.641 & 0.058 \\
\hline $23 a$ & 1.849 & 2.583 & -0.734 & 1.806 & 0.043 \\
\hline $24 a$ & 2.312 & 2.008 & 0.304 & 2.298 & 0.014 \\
\hline $25 a$ & 2.294 & 1.967 & 0.327 & 2.265 & 0.029 \\
\hline $26 a$ & 1.811 & 2.122 & -0.311 & 1.765 & 0.046 \\
\hline $27 a$ & 2.717 & 1.759 & 0.958 & 2.697 & 0.02 \\
\hline $28 a$ & 1.820 & 1.697 & 0.123 & 1.683 & 0.137 \\
\hline $29 a$ & 2.420 & 2.152 & 0.268 & 1.832 & 0.588 \\
\hline $30 a$ & 2.588 & 1.681 & 0.907 & 3.694 & -1.106 \\
\hline
\end{tabular}

$\mathrm{a}$, Training compounds; $\mathrm{b}$, test set compounds. The others are the same as those in Table 1. CoMFA, comparative molecular field analysis; CoMSIA, comparative molecular similarity index analysis; $\mathrm{pLC}_{50},-\log \left(\mathrm{LC}_{50}\right)$. 
A

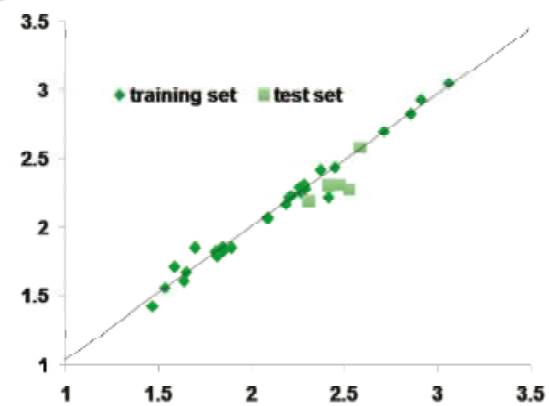

$\mathrm{B}$

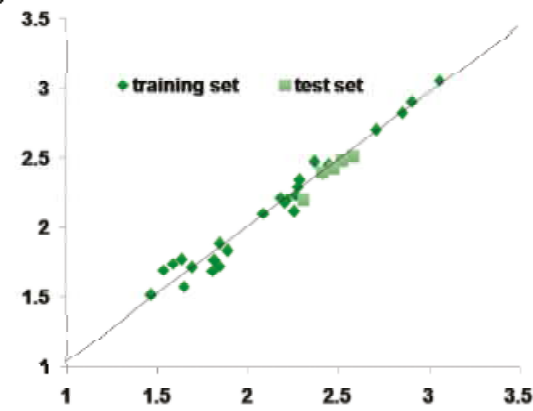

Figure 9. Plots of experimental activity $\left[\log \left(1 / \mathrm{LC}_{50}\right)\right]$ against activity as predicted using CoMFA- (A) and CoMSIA-derived (B) models.

\subsection{Contour Maps of CoMFA-Derived Models}

Stdev * Coeff contour maps were plotted on the basis of the optimal CoMFA/CoMSIA-derived models. Core structure of these test compounds were shown in Figure 10A. Compound 2 was employed as the template molecule for the analysis of contour maps (Figure 10B) because of it had the highest acaricidal effect and its lowest binding energy among all compounds. Figure 11 presents the steric and electrostatic contour maps for the optimal CoMFA-derived models. The green and yellow contours in the contour maps indicated default $80 \%$ and $20 \%$ contribution levels, respectively. From Figure 11A, a medium-sized green contour near the R5-position of ring B indicated that inhibitory activity could be improved with a bulky substituent introduced in this region. Correspondingly, other compounds have bulky substituents at this position. Another green contour occurred around the R1-position of ring $\mathrm{A}$, suggesting that inserting a bulky group into ring $\mathrm{A}$ increases inhibitory activity. By contrast, a large yellow contour near the R5-position of ring B implied that the introduction of a bulky group at this position negatively affects inhibitory activity. Another large yellow contour around the R2 and R3 positions suggested that inserting a bulky group in these positions decreases inhibitory activity. Indeed, the inhibitory activities of compounds 1-4 (with a group at R1-or R5-position) are higher than that of compound 23 (with an $\mathrm{H}$ atom at this position; Table 2).

A<smiles>[R3]c1c([R])c([R])c2c([R])c([R3])c(=O)oc2c1C</smiles>

B<smiles>CCN(CC)c1ccc2cc(-c3nc4ccccc4[nH]3)c(=O)oc2c1</smiles>

Figure 10. (A) Core structure of the test compounds and (B) the chemical structure of compound 2. 
A

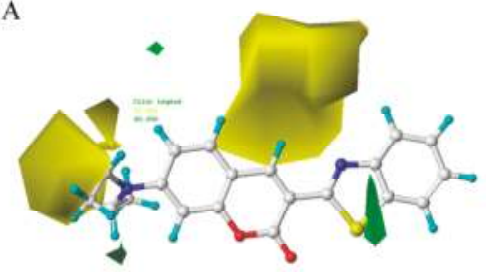

B

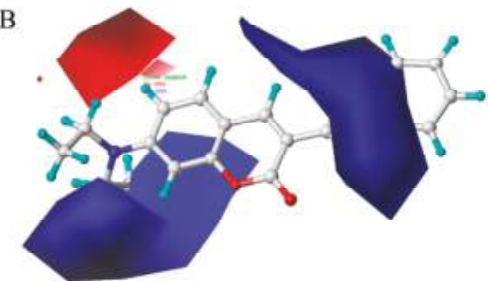

Figure 11. Steric (A) and electrostatic (B) contour maps obtained using CoMFA-derived models based on molecule 2. Green regions (A) indicates regions where the introduction of a bulky group would increase activity. Yellow regions (A) indicates regions where the introduction of a bulky group would decrease activity. Red regions (B) indicates regions where the introduction of electronegative groups is favored. Blue regions (B) indicates regions where the introduction of electropositive groups is favored. The others in Figure A and B represent the compound 2 (Red, oxygen atoms; yellow and blue, nitrogen atom; cyan, hydrogen atom; gray, carbon atoms).

Figure 11B showed the electrostatic contour maps obtained from CoMFA-derived models. Red contour indicates electronegative groups are favored; blue contour indicates electropositive groups are favored. These contours depict default contribution levels. A large blue contour near the R5 and R6 positions of ring B suggested that the introduction of electronegative groups in this position will decrease inhibitory activity. Another large blue contour near the R1-position of ring A indicated that the introduction of electropositive groupsenhances inhibitory activity. A large red contour near the R4-positions of ring B suggested that replacing the original groups with electronegative groups at these positions could improve inhibitory activity. For example, the inhibitory activities of compounds 3 (R1 $\left.=-\mathrm{NH}_{2}\right)$ and $4\left(\mathrm{R} 1=-\mathrm{COCH}_{3}\right)$ are greater than that of compound $23(\mathrm{R} 1=-\mathrm{H})$, and the inhibitory activity of compound $8\left(\mathrm{R} 4=-\mathrm{NO}_{2}\right)$ is greater than that of compound $23(\mathrm{R} 1=-\mathrm{H})($ Table 2$)$.

\subsection{Contour Maps of CoMSIA-derived Models}

The steric, electrostatic, hydrophobic, and H-bond contour maps for the optimal CoMSIA-derived models are shown in Figure 12. Figure 12A,B show the steric and electrostatic contour maps, respectively, which were obtained from the optimal CoMSIA model. The CoMSIA steric and electrostatic contour maps are similar to the corresponding CoMFA contour map. Therefore, the preceding discussion also applies to the steric and electrostatic contour maps from the CoMFA model.

Figure $12 \mathrm{C}$ shows the hydrophobic contour map of the CoMSIA model is displayed. In the CoMSIA-derived hydrophobic field, a medium-sized cyan contour near the ring B indicated that introducing hydrophilic groups to that position could improve the inhibitory activity of the molecule. Another two yellow contours around the R1-position of ring A suggested that hydrophobic groups preferentially localize at these positions. Figure 12D shows the H-bond contour map for the optimal CoMSIA model. In this figure, the cyan color indicated regions that favor H-bond donors, whereas the red color indicated regions that disfavor H-bond donors. A medium-sized cyan contour occurred at the 2-position on ring $\mathrm{A}$, thus indicating that the inhibitory activity would be improved with an $\mathrm{H}$-bond acceptor group introduced at this position. A large red contour near the 4-position of ring B implied that introducing an H-bond donor group in this position could decrease inhibitory activity.

The detailed analysis of the contour maps obtained using the optimal CoMFA- and CoMSIA-derived models may facilitate the design of a novel selective TcPMCA1 inhibitors. Introducing an electropositive, hydrophobic, or H-accepting group in region A (R1- and R2-positions of ring A) can increase inhibitory activity, and introducing a hydrophobic group in region B (R3-position of ring B) can increase activity. Meanwhile, introducing an electronegative group in region C (R4-position of ring $\mathrm{B}$ ) is favorable, and introducing a bulky, hydrophobic, or electropositive group in region D (R5and R6-position of ring B) can increase activity (Figure 13). 
A

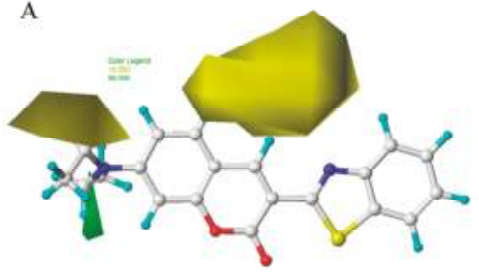

C

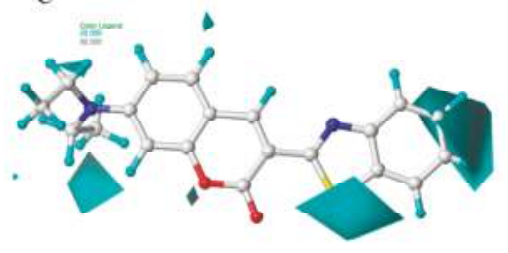

B

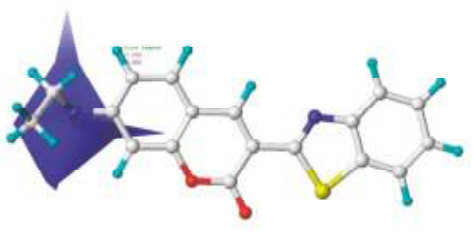

D

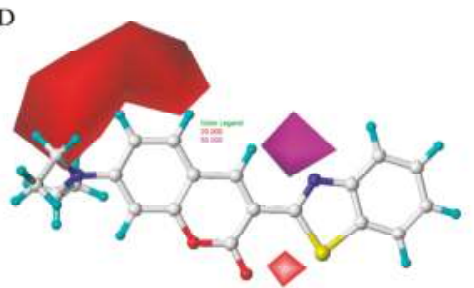

Figure 12. Steric (A), electrostatic (B), hydrophobic (C), and H-bond (D) contour maps obtained using CoMSIA-derived models based on molecule 2. Green (A) indicates regions where the introduction of a bulky group would increase activity. Yellow (A) indicates regions where the introduction of a bulky group would decrease activity. Blue (B) indicates regions where the introduction of electropositive groups is favored. Cyan (C) indicates regions where the introduction of hydrophobic is favored. Purple (D) indicates regions where the introduction of H-bond acceptors is favored. Red (D) indicates regions where the introduction of $\mathrm{H}$-bond acceptors is disfavored. The others in Figure A-D represent the compound 2 (Red, oxygen atoms; yellow and blue, nitrogen atom; cyan, hydrogen atom; gray, carbon atoms).

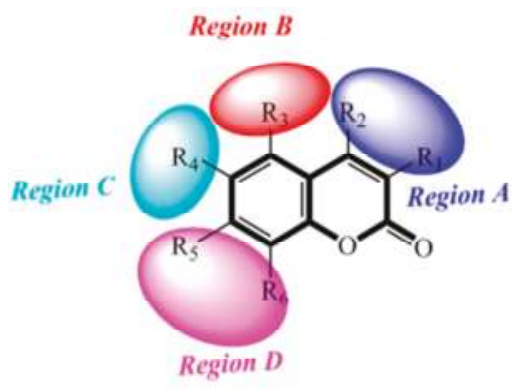

Figure 13. Diagram of structure-activity relationship based on the core structure of the tested compounds. Blue (region A) indicates regions where the introduction of electropositive group, hydrophobic group, or $\mathrm{H}$-accepting groups would increase activity. Red (region B) indicates regions where the introduction of hydrophobic group is favored. Cyan (region C) indicates regions where the introduction of electronegative group is favored. Magenta (region D) indicates regions where the introduction of a bulky group, hydrophobic group, or electropositive group would increase the activity. Dark indicates the core structure of the test compounds.

\section{Discussion}

Scopoletin is a naturally occurring, low-molecular-weight alloleochemical that is ubiquitous in the plant kingdom. Moreover, scopoletin is present in some foods and plant species used in traditional medicine. Scopoletin extracted from Artemisia annua L. exhibits strong activity against the carmine spider mite; in addition, it affects ATPase activity and is possibly a neurotoxin [22]. 
In the present study, full-length cDNA encoding PMCA1 from T. cinnabarinus was characterized and designated as TCPMCA1. The predicted amino acid sequences of TcPMCA1 consists of three major regions: the first intracellular loop region located between transmembrane segments TM II and TM III; the second large intracellular loop region located between TM IV and TM V; which possesses a putative ATP-binding site; the third part extended "tail" found next to TM X. This conformation is consistent with the structure of previously described PMCAs [26-29]. The putative CaM-binding domain of TcPMCA1 binds to the C-terminal region downstream of the last transmembrane domain and shares a common pattern with those in vertebrates [30]. Alternative splicing expands the diversity of mRNA transcripts and augments the functions of modulatory genes [31]. Previous efforts to discriminate TcPMCA1 splice variants failed, this failure was also reported in Spodoptera littoralis [32]. By contrast, mammals and Drosophila melanogaster possess a large number of splice variants [28].

The expression profiles of TCPMCA1 in T. cinnabarinus were similar to that in S. littoralis, which is present at all investigated stages and exhibits maximal expression at the nymphal stage [32]. This expression pattern is correlated to the massive synthesis of TcPMCA1 during the developmental stages, thereby confirming that TcPMCA1 is essential for the functions of T. cinnabarinus.

The reported pharmacological effects of scopoletin presuppose some interactions with membrane-bound enzymes, such as $\mathrm{Ca}^{2+}$-ATPase, which is vital in nervous signal conduction [33-35]. Oliveira [36] reported that in rats, scopoletin inhibits $\mathrm{Ca}^{2+}$-ATPase activity by inhibiting the mobilization of intracellular calcium from noradrenaline-sensitive Ca stores. $\mathrm{Ca}^{2+}$-ATPase is a major neurotransmitter, and PMCA extrudes $\mathrm{Ca}^{2+}$ from the postsynaptic region of the nerve [37]. In insects, PMCA inhibition results in internal $\mathrm{Ca}^{2+}$ flow, causing neurotransmitter accumulation [38]. In the present study, the results of scopoletin induction indicated that TcPMCA1 in T. cinnabarinus was significantly upregulated after exposure to scopoletin within $36 \mathrm{~h}$. Scopoletin also increases the expression of both peroxisome proliferator-activated receptor $\gamma 2$ and adipocyte-specific fatty acid binding protein [39]. Moreover, scopoletin inhibits the expression of cyclooxygenase in a concentration-dependent manner [40]. These results implicated TcPMCA1 in the detoxification metabolism of scopoletin in T. cinnabarinus. The inhibition of $\mathrm{Ca}^{2+}$-ATPase activity or increase in PMCA expression possibly indicates the existence of a feedback regulatory mechanism that compensates for enzyme content. The decrease of gene expression at $48 \mathrm{~h}$ may related to the organism damage caused by continuous scopoletin exposing. Basing on these results, we surmise that TcPMCA1 inhibition in T. cinnabarinus causes intra- and extracellular calcium ion imbalance and thus blocks the transmission of neural activity, causing the death of mites [41,42]. However, the influence of scopoletin on $\mathrm{Ca}^{2+}$-ATPase mechanism in the carmine spider mite requires extensive exploration because of the intricacy of PMCA-mediated detoxification.

Scopoletin is also designated as 7-hydroxy-6-methoxy coumarin and is a coumarin derivative. Coumarin is a leading molecule in biopesticides. Given the pesticidal potential of this class of compounds, the toxic effects of coumarin derivatives against mosquito species Culex quinquefasciatus and Aedes aegypti were evaluated, and the results showed that modifying the 7-OH position remarkably enhances the ovicidal activity of coumarin [43]. The antitermiticidal activity of scopoletin and coumarin derivatives were investigated against Coptotermes formosanus, and the results suggested that scopoletin has the highest activity among the tested compounds [44]. To investigate the structure-activity relationship of the methoxy and hydroxy groups at the C-6 and C-7 positions of the coumarin skeleton, 6-alkoxycoumarin derivatives and 7-alkoxycoumarins and related analogs were synthesized. The findings indicated that the presence of alkenyloxy and alkynyloxy groups at the C-6 position, as well as the cyclohexyloxy and aryloxy groups at the C-7 position, are important for the termiticidal and antifeedant activities of coumarin $[45,46]$. These results revealed that scopoletin actually inserts into the binding cavity and interacts with the active sites of TcPMCA1, suggesting that the microenvironments and conformation of the enzymes change because of these interactions [47]. Furthermore, these results indicated that the C-6 and C-7 positions of scopoletin are important for acaricidal activity. 
Molecular docking and the homology modeling of the 3D structure of the target protein were used to identify conformational protein-ligand interaction patterns [48,49]. Pharmacophore have been used to develop 3D-QSAR models over the past the decade [50]. Combined information on protein-ligand interactions from a pharmacophore and accurate binding conformations from molecular docking offers the potential for enhanced prediction accuracy [51]. In the present study, the crystallographic structure of sarco/endoplasmic reticulum $\mathrm{Ca}^{2+}$-ATPases (SERCA) was defined in rabbit [52]. The BLAST analysis performed showed that TcPMCA1 shares 73\% sequence identity with the SERCA $\mathrm{Ca}^{2+}$-ATPase of rabbit, indicating the validity of homologous protein structure [53,54]. The homologous 3D structure of TcPMCA1 allowed the evaluation of the binding energies and docking positions of scopoletin on TcPMCA1 protein. In our docking results, the hydrophobic environment of the active site is favorable for interactions with scopoletin, and the special arrangements at the C6 and C7 sites are assumed to be favorable for the acaricidal activity of scopoletin. Furthermore, the 3D-CoMFA and CoMSIA models indicating that C3, C6, and C7 regions of coumarins appear to be important acaricidal active sites of coumarins. This result is in agreement with the results of the acaricidal activity assay, which showed that coumarins substituted with methoxy at C6 or C7 have significantly better activity than coumarins substituted with other compounds at the same positions. Furthermore, coumarins with C3 substitutions also demonstrated enhanced acaricidal activity. Nakamura [55] previously investigated the structure-activity relationship between 63 natural oxycoumarin derivatives and their effects on the expression of inducible nitric oxide synthase, which showed that the C-5, C-6 and C-7 positions of oxycoumarin derivatives are essential for potent activities. In addition, the discovery and structure-activity relationship of a novel series of coumarin-based tumor necrosis factor $\alpha$ (TNF- $\alpha$ ) inhibitors showed that substitution at the C-3 and C-6 position of the coumarin ring system most dramatically influences inhibitory activity against TNF- $\alpha$ [56]. The docking results and the detailed analysis of the contour maps obtained by 3D-CoMFA and CoMSIA-derived models will encourage the design of novel, selective TcPMCA inhibitors.

\section{Materials and Methods}

\subsection{Test Mites}

The carmine spider mite culture was collected from cowpea Vigna unguiculata (L.) grown in Beibei, Chongqing, China. The mites were maintained on potted cowpea seedlings ( $30-40 \mathrm{~cm}$ tall) in a walk-in insect rearing room at $26 \pm 1{ }^{\circ} \mathrm{C}$ under 75 to $80 \% \mathrm{RH}$ and 16L:8D photoperiod. The colony was maintained for more than 12 years without any contact with insecticides/acaricides. The voucher specimens of T. cinnabarinus were deposited at the Insect Collection of Southwest University, Chongqing, China.

\subsection{Leaf-Dip Bioassay}

More than 600 leaf discs were prepared to obtain uniform individuals at different developmental stages. Fresh cowpea leaves that had not been exposed to pesticides were washed thoroughly. Leaf discs with $3 \mathrm{~cm}$ diameters were placed on a $4 \mathrm{~mm}$ water-saturated sponge in a Petri dish $(9 \mathrm{~cm}$ in diameter) [57]. Approximately 30 adult females were transferred to each leaf disc, allowed to lay eggs, and removed after $12 \mathrm{~h}$. After a batch of uniform eggs had hatched, the offspring was maintained until the progeny had developed into 3- to 5-d-old females [58].

For the leaf-dip bioassay, female adult mites were treated with scopoletin (provided by Southwest University, Beibei, Chongqing, China). The responses of TcPMCAs in mites to scopoletin were investigated by exposing the adult female mites to $10 \%$ of the lethal concentration $\left(\mathrm{LC}_{10}\right), \mathrm{LC}_{30}$, and $\mathrm{LC}_{50}$ of scopoletin for $12,24,36$, and $48 \mathrm{~h}$. The $\mathrm{LC}_{10}\left(0.219 \mathrm{mg} \cdot \mathrm{mL}^{-1}\right), \mathrm{LC}_{30}\left(0.581 \mathrm{mg} \cdot \mathrm{mL}^{-1}\right)$, and $\mathrm{LC}_{50}\left(1.142 \mathrm{mg} \cdot \mathrm{mL}^{-1}\right)$ of $T$. cinnabarinus to scopoletin were determined using leaf-dip bioassays prior to acaricide treatments. Each leaf disc, which contained 30 mites on its surface, was soaked for $5 \mathrm{~s}$ in acaricide solutions. For each treatment, more than 500 surviving mites were collected and three 
biological replicates were performed. A total of 200 mites were dipped in distilled water for $5 \mathrm{~s}$ and used as the control. All of the surviving mites were collected and stored at $-80^{\circ} \mathrm{C}$ for RNA extraction.

\subsection{RNA Isolation and Reverse Transcription}

Total RNA was isolated using RNeasy ${ }^{\circledR}$ Plus Micro Kit (Qiagen, Hilden, Germany), and genomic DNA was removed using a gDNA elimination column in accordance with the manufacturer's instructions. The quantities of total RNA were assessed at $260 \mathrm{~nm}$ using Nanovue UV-Vis spectrophotometer (GE Healthcare, Fairfield, CT, USA). RNA purities were quantified at an absorbance ratio of OD260/280. RNA integrity was evaluated via 1\% agarose gel electrophoresis. cDNA was synthesized using total RNA and the rapid amplification of cDNA ends (RACE) method. First-strand cDNA was synthesized from $0.5 \mu \mathrm{g}$ of RNA in a $10 \mu \mathrm{L}$ reaction mixture by using PrimeScript ${ }^{\circledR} 1$ st strand cDNA Synthesis Kit (TaKaRa, Dalian, China) and oligo (dT)18 primers. The synthesized samples were then stored at $-20{ }^{\circ} \mathrm{C}$.

\subsection{Sequencing and Phylogenetic Analysis}

To obtain the full-length DNA sequences of TcPMCA genes, specific primers were designed using Primer 5.0 (Available online: http://www.premierbiosoft.com/) based on the transcript unigene sequences obtained from the transcriptome (Table S2). A set of gene-specific primers and nested primers were designed to amplify the fragments. The rapid amplification of cDNA ends (RACE) methodwas amplified using the SMARTer ${ }^{\mathrm{TM}}$ RACE cDNA Amplification Kit (Clontech, Palo Alto, CA, USA). The total PCR volume was $25 \mu \mathrm{L}$ and contained $2.5 \mu \mathrm{L}$ of $10 \times$ PCR buffer $\left(\mathrm{Mg}^{2+}\right.$ free $), 2.0 \mu \mathrm{L}$ of dNTPs $(2.5 \mathrm{mM}), 2.0 \mu \mathrm{L}$ of $\mathrm{Mg}^{2+}(2.5 \mathrm{mM}), 1 \mu \mathrm{L}$ of cDNA templates, $1 \mu \mathrm{L}$ of each primer $(10 \mathrm{mM})$, $0.25 \mu \mathrm{L}$ of $\mathrm{rTaq}^{\mathrm{TM}}$ polymerase (TaKaRa), and $15.5 \mu \mathrm{L}$ of $\mathrm{ddH}_{2} \mathrm{O}$. The PCR program was performed as follows: initial denaturation for $3 \mathrm{~min}$ at $94{ }^{\circ} \mathrm{C}$, followed by 34 cycles of $94{ }^{\circ} \mathrm{C}$ for $30 \mathrm{~s}, 55$ to $60{ }^{\circ} \mathrm{C}$ (depending on gene specific primers) for $30 \mathrm{~s}$, and $72^{\circ} \mathrm{C}$ extension for $2 \mathrm{~min}$, and final extension for $10 \mathrm{~min}$ at $72{ }^{\circ} \mathrm{C}$. The PCR products were separated by agarose gel electrophoresis andpurified using Gel Extraction Mini Kit (Watson Biotechnologies, Shanghai, China). The purified PCR products were ligated into the pGEM-T vector (Promega, Fitchburg, MA, USA) and then sequenced (Invitrogen Life Technologies, Shanghai, China).

BLAST searching was performed using the NCBI BLAST website (Available online: http: //www.ncbi.nlm.nih.gov/Blast.cgi). The molecular weight and isoelectric points of the deduced protein sequences were calculated by ExPASy Proteomics Server (Available online: http://cn. expasy.org/tools/pi_tool.html) [59]. The transmembrane domain positions and protein domain were estimated using Phobius (Available online: http://phobius.sbc.su.se/), Calmodulin Target Database (Available online: http:/ / calcium.uhnres.utoronto.ca/ctdb/pub_pages/search/index.htm), and ATPint (Available online: http:/ / www.imtech.res.in/raghava/atpint/submit.html) servers. Signal peptides were predicted using Signa1P 3.0 (Available online: http://www.cbs.dtu.dk/service/ SignalP/) [60]. N-glycosylation sites were predicted by NetNGlyc 1.0 Server (Available online: http://www.cbs.dtu.dk/services/NetNGlyc/). DNAMAN 6.0 (Lynnon BioSoft, Vaudreuil, QC, Canada) was used to edit TcPMCA1 nucleotide sequences, and the corresponding phylogenetic trees were constructed using the neighbor-joining method, with 1000 bootstrap replicates, in MEGA5.01 [61].

\subsection{Real-Time Quantitative PCR ( $q P C R$ )}

Primers used for qPCR were designed by Primer 3.0 software [62]. qPCR was performed in $20 \mu \mathrm{L}$-reaction mixture that contained $10 \mu \mathrm{L}$ of qSYBR Green Supermix (BIO-RAD laboratories, Hercules, CA, USA), $1 \mu \mathrm{L}$ of cDNA template, $1 \mu \mathrm{L}$ of each primer $(0.2 \mathrm{mM})$ and $7 \mu \mathrm{L}$ of $\mathrm{ddH}_{2} \mathrm{O}$. qPCR was performed on a Stratagene Mx3000P Thermal Cycler (Stratagene, La Jolla, CA, USA) as following protocol: an initial denaturation at $95{ }^{\circ} \mathrm{C}$ for $2 \mathrm{~min}$, followed by 40 cycles at $95{ }^{\circ} \mathrm{C}$ for $15 \mathrm{~s}$, $60{ }^{\circ} \mathrm{C}$ for $30 \mathrm{~s}$, and elongation at $72{ }^{\circ} \mathrm{C}$ for $30 \mathrm{~s}$. At the end of each reaction, a melt curve analys (from 60 to $95^{\circ} \mathrm{C}$ ) was generated to rule out the possibility of primer-dimer formation. RPS18 was used as a 
stable housekeeping gene for the qPCR analysis [63]. Relative gene expression levels were calculated by $2^{-\Delta \Delta C t}$ method [64]. Three biological and two technical replicates were performed.

Expression pattern of TCPMCA1 at different developmental stages. To investigate the expression patterns of TCPMCA1 at different developmental stages, we collected mites in uniform developmental stages (2000 eggs, 1500 larvae, 1000 nymphs, and 500 adults). The samples were isolated and placed in a $1.5 \mathrm{~mL}$ diethyl pyrocarbonate (DEPC)-treated centrifuge tube containing RNA storage reagent (Tiangen, Beijing, China), immediately frozen in liquid nitrogen, and stored at $-80{ }^{\circ} \mathrm{C}$ for RNA extraction. Three independent biological replications were performed.

Expression levels of TcPMCA1 after scopoletin exposure. The differential expression levels of TcPMCA1 in response to scopoletin were investigated by exposing adult female mites to $\mathrm{LC}_{10}, \mathrm{LC}_{30}$, and $\mathrm{LC}_{50}$ scopoletin, as in leaf bioassays. After 12, 24, and $36 \mathrm{~h}$ intervals, only the surviving adults obtained from the treated and control groups (at least 500 larvae) were collected and frozen at $-80^{\circ} \mathrm{C}$ for RNA extraction. After scopoletin exposure, total RNA was isolated to analyze the expression levels of TcPMCA by TR-qPCR.

\subsection{Homology Modeling}

The homology modeling was conducted on the I-TASSER server (Available online: http:// zhanglab.ccmb.med.umich.edu/I-TASSER/) [65], and the 3D structure of TcPMCA1 protein was obtained. The details of I-TASSER protocol have been described previously [66-70]. Briefly, it consists of three steps: template identification, full-length structure assembly and structure-based function annotation. Firstly, starting from the query sequence, I-TASSER identifies homologous structure templates from the PDB library [71] using LOMETS [69,72], a meta-threading program that consists of multiple threading algorithms. Then, the topology of the full-length models is constructed by reassembling the continuously aligned fragment structures excised from the templates, where the structures of the unaligned regions are built from scratch by ab initio folding based on replica-exchange Monte Carlo simulations [73]. The low free-energy states are further identified by SPICKER [74]. To refine the structural models, a second round of structure reassembly is conducted starting from the SPICKER clusters. The low free-energy conformations refined by full-atomic simulations using FG-MD [75] and ModRefiner [76]. Finally, the biological functions of the target proteins were derived by matching the I-TASSER models with proteins in the BioLiP library [77-79].

Based on identity with the primary sequence of the target TCPMCA1, the crystal structure of the phosphoenzyme intermediate of the rabbit SERCA $\mathrm{Ca}^{2+}$-ATPase (PDB ID code: 3BA6) was retrieved from the Protein Data Bank (PDB, Available online: http://www.rcsb.org/pdb/home/home.do) and used as the template for homology modeling (the amino acid sequences of the trmplate was shown in Figure S1). The Psi/Phi Ramachandran plot obtained from Procheck analysis was used to validate the modeled 3D structure of TcPMCA1 protein [80,81].

\subsection{Dataset and Molecular Modeling}

The acaricidal activities of the 30 collected compounds (Table S3) were obtained from a previous study [82]. These 30 compounds are natural or synthetic compounds that are readily available to coumarin, which were purchased from Chengdu Aikeda Chemical Reagent Co., Ltd. and Shanghai yuanye Bio-Technology Co., Ltd. The purity of these compounds was more than $98 \%$. The structures and half-maximal inhibitory concentration $\left(\mathrm{LC}_{50}\right)$ of the compounds are shown in Table 2. These values were transformed into the corresponding $\mathrm{pLC}_{50}\left[-\log \left(\mathrm{LC}_{50}\right)\right]$ as the expression of inhibitor potency. The 30 compounds were placed in a training set of 24 compounds $(80 \%)$ and a test set of 6 compounds $(20 \%)$.

The 3D structures of these ligand compounds were constructed in Sybyl 6.9 (Tripos Software, St. Louis, MO, USA). Structures were energy minimized by using the Gasteiger-Hückel charge [83], Tripos force field [84], and Powell methods [85] with a convergence criterion of $0.005 \mathrm{kcal} /(\mathrm{mol} \AA)$. The iterations maximum number was set to 10,000, and multiple conformation search was used. Coumarin 
structure was used as the common scaffold for molecular alignment, and compound 2 with the highest acaricidal activity was used as the template molecule. All other compounds were aligned with the coumarin core using the "align database" command in Sybyl.

\subsection{Molecular Docking}

The protein model was prepared using Sybyl prior to docking simulations. All bound water molecules and ligands were removed from the protein, and hydrogen atoms and AM1-BCC charges [86] were added to the amino acid residues. The generated homology model of TcPMCA1 was used for molecular docking, and the binding pocket was defined using Discovery Studio 2.5 (Accelrys Software Inc., San Diego, CA, USA). The 3D structure of the compound was prepared as the ligand, and all of the hydrogen atoms and AM1-BCC charges were added [86]. Molecular docking was performed with AutoDock 4.0 [87]. The grid spacing was changed from $0.375 \mathrm{~nm}$, and the cubic grid map was $40 \times 40$ $\times 40 \AA$ toward the TcPMCA binding site. The docking parameters were set as follows: the number of GA Runs was set as 10, population size was set as 150, the maximum number of evaluations was set as 25,000,000, and 250 runs were performed. All other parameters were set as the default. The docking process was performed as follows: first, molecular docking was performed to evaluate the docking poses. Then, defined docking was conducted on the binding pocket. Three to six independent docking calculations were conducted. The corresponding lowest binding energies and predicted inhibition constants $\left(\mathrm{p} K_{\mathrm{i}}\right)$ were obtained from the docking log files (dlg). The mean $\pm \mathrm{SD}$ of binding energies was calculated from the dockings. AutoDock Tools and Visual Molecular Dynamics (VMD, Theoretical and Computational Biophysics group at the Beckman Institute, University of Illinois at Urbana-Champaign) $[88,89]$ was used to visualize the docking result. Surface representation images that show the binding pocket of TcPMCA1 were generated using VMD software.

\subsection{D-QSAR Study}

CoMFA and CoMSIA descriptor fields were employed in the present 3D-QSAR studies. The CoMFA fields were carried out to generate the steric and electrostatic fields with the default value of the energy cutoff at $30 \mathrm{kcal} \cdot \mathrm{mol}^{-1}$ CoMSIA fields were carried out to calculate the steric, electrostatic, hydrophobic, hydrogen-bond donor and hydrogen-acceptor donor with a default attenuation factor of 0.3 for Gaussian function. Field type "Stdev * Coeff" was used as the coefficient to analysis the contour map of each field. The partial least squares (PLS) [90] was used to construct a linear correlation by setting the biological activity ( $\mathrm{pLC}_{50}$ values) as the dependent variables and the CoMFA/CoMSIA descriptors as independent variables.

\subsection{Statistical Analysis}

All results were expressed as the mean \pm standard error. The differences among the four developmental stages and time-dependent responses to scopoletin exposure were analyzed using one-way analysis of variance (ANOVA). The level of significance of the means was then separated by Fisher's LSD multiple comparison test $(p<0.05)$. The fold change in TcPMCA gene expression was analyzed using SPSS (v.16.0, SPSS Inc., Chicago, IL, USA), and significance was determined by independent sample $t$-test $(p<0.05)$.

\section{Conclusions}

The molecular characteristics of the TcPMCA1 gene were identified and described, and the gene expression levels of TCPMCA1 after scopoletin exposure were investigated. The TCPMCA1-mediated detoxification mechanism of scopoletin in T. cinnabarinus was preliminarily explored through the integrated study of homology modeling and molecular docking. Moreover, CoMFA and CoMSIA 3D-QSAR studies have been performed to put the pharmacophoric environment that will help future structure based drug design. The results of the present study showed that scopoletin forms hydrogen bonds with the active site of TcPMCA1, and that the $\mathrm{C} 3, \mathrm{C} 6$, and $\mathrm{C} 7$ positions in the skeletal structure 
of coumarins are the most suitable active sites. These results provide a better understanding of the TcPMCA1-mediated detoxification mechanisms of scopoletin and of other coumarin derivatives. These compounds can be structurally modified to increase their acaricidal and inhibitory effects. More detailed investigations of the mechanism of action and pharmacological activities of these compounds may provide novel anti-PMCA agents for spider mite control.

Supplementary Materials: Supplementary materials can be found at www.mdpi.com/1422-0067/18/7/1380/s1.

Acknowledgments: We are grateful to Yuwei Wang in School of Pharmacy, Lanzhou University for Molecular Docking and 3D-QSAR analysis. This research was partially supported by a combination of funding from the National Science Foundation of China $(31272058,31572041$ and 31601674$)$ and Chongqing social undertakings and people's livelihood guarantee scientific and technological innovation (cstc2015shms-ztzx0129).

Author Contributions: Qiu-Li Hou, Jin-Xiang Luo, Bing-Chuan Zhang and Yong-Qiang Zhang conceived and designed the experiments; Qiu-Li Hou, Yong-Qiang Zhang, Bing-Chuan Zhang, Jin-Xiang Luo, and Gao-Fei Jiang performed the experiments and analyzed the data; Qiu-Li Hou and Yong-Qiang Zhang wrote the paper; Wei Ding, Jin-Xiang Luo and Yong-Qiang Zhang revised the paper.

Conflicts of Interest: The authors declare no conflict of interest.

\section{References}

1. Glynn, I.M. The Enzymes of Biological Membranes; Martonosi, A.N., Ed.; New York Press: New York, NY, USA, 1985; pp. 35-114.

2. Carafoli, E. The calcium pumping ATPase of the plasma membrane. Annu. Rev. Physiol. 1991, 53, 531-547. [CrossRef] [PubMed]

3. Penniston, J.T.; Enyedi, A. Modulation of the plasma membrane $\mathrm{Ca}^{2+}$ pump. J. Membr. Biol. 1998, 165, 101-109. [CrossRef]

4. Krizaj, D.; Steven, J.D.; Johnson, J.; Strehler, E.E.; Copenhagen, D.R. Cell-specific expression of plasma membrane calcium ATPase isoforms in retinal neurons. J. Comp. Neurol. 2002, 451, 1-21. [CrossRef] [PubMed]

5. Ii, W.J.P.; Thayer, S.A. Transient rise in intracellular calcium produces a long-lasting increase in plasma membrane calcium pump activity in rat sensory neurons. J. Neurochem. 2002, 83, 1002-1008.

6. Zenisek, D.; Matthews, G. The role of mitochondria in presynaptic calcium handling at a ribbon synapse. Neuron 2000, 25, 229-237. [CrossRef]

7. Street, V.A.; Mckee-Johnson, J.W.; Fonseca, R.C.; Tempel, B.L.; Noben-Trauth, K. Mutations in a plasma membrane $\mathrm{Ca}^{2+}$-ATPase gene cause deafness in deafwaddler mice. Nat. Genet. 1998, 19, 390-394. [PubMed]

8. Salome, A.; Hugh, J.R.; Matthews, R. Olfactory response termination involves $\mathrm{Ca}^{2+}$-ATPase in vertebrate olfactory receptor neuron cilia. J. Gen. Physiol. 2010, 135, 367-378.

9. Castillo, K.; Delgado, R.; Bacigalupo, J. Plasma membrane $\mathrm{Ca}^{2+}$-ATPase in the cilia of olfactory receptor neurons: Possible role in $\mathrm{Ca}^{2+}$ clearance. Eur. J. Neurosci. 2007, 26, 2524-2531. [CrossRef] [PubMed]

10. Strehler, E.E.; Filoteo, A.G.; Penniston, J.T.; Caride, A.J. Plasma membrane $\mathrm{Ca}^{2+}$-pumps: Structural diversity as basis for functional versatility. Biochem. Soc. Trans. 2007, 35, 919-922. [CrossRef] [PubMed]

11. Stauffer, T.P.; Guerini, D.; Carafoli, E. Tissue distribution of the 4 gene-products of the plasma-membrane $\mathrm{Ca}^{2+}$ pump-a study using specific antibodies. J. Biol. Chem. 1995, 270, 12184-12190. [CrossRef] [PubMed]

12. Foletti, D.; Guerini, D.; Carafoli, E. Subcellular targeting of the endoplasmic reticulum and plasma membrane $\mathrm{Ca}^{2+}$ pumps: A study using recombinant chimeras. FASEB J. 1995, 9, 670-680. [PubMed]

13. Zvaritch, E.; Vellani, F.; Guerini, D.; Carafoli, E. A signal for endoplasmic reticulum retention located at the carboxyl terminus of the plasma membrane $\mathrm{Ca}^{2+}$-ATPase isoform 4CI. J. Biol. Chem. 1995, 270, 2679-2688. [CrossRef] [PubMed]

14. Schwab, B.L.; Guerini, D.; Didszun, C.; Bano, D.; Ferrando-May, E.; Fava, E.; Tam, J.; Xu, D.; Xanthoudakis, S.; Nicholson, D.W. Cleavage of plasma membrane calcium pumps by caspases: A link between apoptosis and necrosis. Cell Death Differ. 2002, 9, 818-831. [CrossRef] [PubMed]

15. Cakmak, I.; Baspinar, H. Control of the Carmine Spider Mite Tetranychus cinnabarinus boisduval by the predatory mite Phytoseinlus persimilis (Athias-Henriot) in protected strawberries in Aydin, Turkey. Turk. J. Agric. For. 2005, 29, 259-265. 
16. Hazan, A.; Gerson, U.; Tahori, A.S. Spider mite webbing. I. The production of webbing under various environmental conditions. Acarologia 1974, 16, 68-84.

17. Bi, J.L.; Niu, Z.M.; Yu, L.; Toscano, N.C. Resistance status of the carmine spider mite, Tetranychus cinnabarinus and the twospotted spider mite, Tetranychus urticae to selected acaricides on strawberries. Insect Sci. 2016, 23, 88-93. [CrossRef] [PubMed]

18. Tal, B.; Robeson, D.J. The induction, by fungal inoculation, of ayapin and scopoletin biosynthesis in Helianthus annuus. Phytochemistry 1985, 25, 77-79. [CrossRef]

19. Gnonlonfin, G.J.B.; Sanni, A.; Brimer, L. Review Scopoletin-A coumarin phytoalexin with medicinal properties. Crit. Rev. Plant Sci. 2012, 31, 47-56. [CrossRef]

20. Rollinger, J.M.; Hornick, A.; Langer, T.; Stuppner, H.; Prast, H. Acetylcholinesterase inhibitory activity of scopolin and scopoletin discovered by virtual screening of natural products. J. Med. Chem. 2013, 47, 6248-6254. [CrossRef] [PubMed]

21. Tripathi, A.K.; Bhakuni, B.H.; Upadhyay, S.; Gaur, R. Insect feeding deterrent and growth inhibitory activities of scopoletin isolated from Artemisia annua against Spilarctia obliqua (Lepidoptera: Noctuidae). Insect Sci. 2011, 18, 189-194. [CrossRef]

22. Zhang, Y.Q.; Wei, D.; Zhao, Z.M.; Jing, W.U.; Fan, Y.H. Studies on acaricidal bioactivities of Artemisia annua L. extracts against Tetranychus cinnabarinus Bois. (Acari: Tetranychidae). Agric. Sci. China 2008, 7, 577-584. [CrossRef]

23. Hou, Q.L.; Wang, D.; Zhang, B.C.; Ding, W.; Zhang, Y.Q. Biochemical evidences for scopoletin inhibits $\mathrm{Ca}^{2+}$-ATPase activity in the Carmine spider mite, Tetranychus cinnabarinus (Boisduval). Agric. Sci. Technol. 2015, 4, 826-831.

24. Xu, Z.; Zhu, W.; Liu, Y.; Liu, X.; Chen, Q.; Peng, M.; Wang, X.; Shen, G.; He, L. Analysis of insecticide resistance-related genes of the Carmine spider mite Tetranychus cinnabarinus based on a de novo assembled transcriptome. PLoS ONE 2014, 9, e94779. [CrossRef] [PubMed]

25. Zhang, Q.Y.; Jian, W.; Xu, X.; Yang, G.F.; Ren, Y.L.; Liu, J.J.; Wang, H.; Yu, G. Structure-based rational quest for potential novel inhibitors of human HMG-CoA reductase by combining CoMFA 3D QSAR modeling and virtual screening. J. Comb. Chem. 2007, 9, 131-138. [CrossRef] [PubMed]

26. Carafoli, E.; Guerini, D. Molecular and cellular biology of plasma membrane calcium ATPase. Trends Cardiovasc. Med. 1993, 3, 177-184. [CrossRef]

27. Lnenicka, G.A.; Grizzaffi, J.; Lee, B.; Rumpal, N. $\mathrm{Ca}^{2+}$ dynamics along identified synaptic terminals in Drosophila larvae. J. Neurosci. 2006, 26, 12283-12293. [CrossRef] [PubMed]

28. Strehler, E.E.; Zacharias, D.A. Role of alternative splicing in generating isoform diversity among plasma membrane calcium pumps. Physiol. Rev. 2001, 81, 21-50. [PubMed]

29. Di, L.F.; Domi, T.; Fedrizzi, L.; Lim, D.; Carafoli, E. The plasma membrane $\mathrm{Ca}^{2+}$ ATPase of animal cells: Structure, function and regulation. Arch. Biochem. Biophys. 2008, 476, 65-74.

30. Brodin, P.; Falchetto, R.; Vorheer, T.; Carafoli, E. Identification of two domains which mediate the binding of activating phospholipids to the plasma-membrane $\mathrm{Ca}^{2+}$ pump. Eur. J. Biochem. 1992, 204, 939-946. [CrossRef] [PubMed]

31. Hicks, M.J.; Lam, B.J.; Hertel, K.J. Analyzing mechanisms of alternative pre-mRNA splicing using in vitro splicing assays. Methods 2005, 37, 306-313. [CrossRef] [PubMed]

32. François, A.; Bozzolan, F.; Demondion, E.; Montagné, N.; Lucas, P.; Debernard, S. Characterization of a plasma membrane $\mathrm{Ca}^{2+}$-ATPase expressed in olfactory receptor neurons of the moth Spodoptera littoralis. Cell Tissue Res. 2012, 350, 239-250. [CrossRef] [PubMed]

33. Ezeokonkwo, C.A.; Obidoa, O.; Eze, L.C. Effects of scopoletin and aflatoxin B 1 on bovine erythrocyte membrane Na-K-ATPase. Plant Physiol. Commun. 2010, 41, 715-719. [CrossRef]

34. Ezeokonkwo, C.A.; Obidoa, O. Effect of scopoltin on erythrocyte membrane ion motive ATPases. Niger. J. Nat. Prod. Med. 2001, 5, 37-40.

35. Ojewole, J.A.; Adesina, S.K. Cardiovascular and neuromuscular actions of scopoletin from fruit of Tetrapleura tetraptera. Planta Med. 1983, 49, 99-102. [CrossRef] [PubMed]

36. Oliveira, E.J.; Romero, M.A.; Silva, M.S.; Silva, B.A.; Medeiros, I.A. Intracellular calcium mobilization as a target for the spasmolytic action of scopoletin. Planta Med. 2001, 67, 605-608. [CrossRef] [PubMed]

37. Palmgren, M.G.; Nissen, P. P-type ATPases. Annu. Rev. Biophys. 2011, 40, 243-266. [CrossRef] [PubMed] 
38. Wang, Y.N.; Jin, Y.S.; Shi, G.L.; Bu, C.Y.; Zhao, L.; Du, J.; Liu, Y.B.; Zhao, L.L. Effects of Kochia scoparia extracts to activities of several enzymes of Tetranychus viennensis. Sci. Silvae Sin. 2008, 44, 1-5.

39. Zhang, W.Y.; Lee, J.J.; Kim, Y.; Kim, I.S.; Park, J.S.; Myung, C.S. Amelioration of insulin resistance by scopoletin in high-glucose-induced, insulin-resistant HepG2 cells. Horm. Metab. Res. 2010, 42, 930-935. [CrossRef] [PubMed]

40. Kim, H.J.; Jang, S.I.; Kim, Y.J.; Chung, H.T.; Yun, Y.G.; Kang, T.H.; Jeong, O.S.; Kim, Y.C. Scopoletin suppresses pro-inflammatory cytokines and PGE2 from LPS-stimulated cell line, RAW 264.7 cells. Fitoterapia 2004, 75, 261-266. [CrossRef] [PubMed]

41. Desaiah, D.; Cutkomp, L.K.; Koch, R.B. Inhibition of spider mite ATPases by plictran and three organochlorine acaricides. Life Sci. 1973, 13, 1693-1703. [CrossRef]

42. Jeyaprakash, A.; Hoy, M.A. The mitochondrial genome of the predatory mite Metaseiulus occidentalis (Arthropoda: Chelicerata: Acari: Phytoseiidae) is unexpectedly large and contains several novel features. Gene 2007, 391, 264-274. [CrossRef] [PubMed]

43. Deshmukh, M.; Pawar, P.; Joseph, M.; Phalgune, U.; Kashalkar, R.; Deshpande, N.R. Efficacy of 4-methyl-7-hydroxy coumarin derivatives against vectors Aedes aegypti and Culex quinquefasciatus. Indian J. Exp. Biol. 2008, 46, 788-792. [PubMed]

44. Adfa, M.; Yoshimura, T.; Komura, K.; Koketsu, M. Antitermite activities of coumarin derivatives and scopoletin from Protium javanicum Burm. f. J. Chem. Educ. 2010, 36, 720-726. [CrossRef] [PubMed]

45. Adfa, M.; Hattori, Y.; Yoshimura, T.; Komura, K.; Koketsu, M. Antifeedant and termiticidal activities of 6-alkoxycoumarins and related analogs against Coptotermes formosanus Shiraki. J. Chem. Educ. 2011, 37, 598-606. [CrossRef] [PubMed]

46. Adfa, M.; Hattori, Y.; Yoshimura, T.; Koketsu, M. Antitermite activity of 7-alkoxycoumarins and related analogs against Coptotermes formosanus Shiraki. Int. Biodeter. Biodegr. 2012, 74, 129-135. [CrossRef]

47. Lin, H.; Fang, C.; Zhu, R.; Qiang, P.; Ding, L.; Min, W. Inhibitory effect of phloretin on $\alpha$-glucosidase: Kinetics, interaction mechanism and molecular docking. Int. J. Biol. Macromol. 2017, 95, 520-527.

48. Deb, P.K.; Sharma, A.; Piplani, P.; Akkinepally, R.R. Molecular docking and receptor-specific 3D-QSAR studies of acetylcholinesterase inhibitors. Mol. Divers. 2012, 16, 803-823. [CrossRef] [PubMed]

49. Sippl, W.; Contreras, J.M.; Parrot, I.; Rival, Y.M.; Wermuth, C.G. Structure-based 3D QSAR and design of novel acetylcholinesterase inhibitors. J. Comput. Aided Mol. Des. 2001, 15, 395-410. [CrossRef] [PubMed]

50. Verma, J.; Khedkar, V.M.; Coutinho, E.C. 3D-QSAR in drug design-A review. Curr. Top. Med. Chem. 2010, 10, 95-115. [CrossRef] [PubMed]

51. Katsamakas, S.; Bermperoglou, E.; Hadjipavloulitina, D. Considering autotaxin inhibitors in terms of 2D-QSAR and 3D-mapping-review and evaluation. Curr. Med. Chem. 2015, 22, 1428-1461. [CrossRef] [PubMed]

52. Myint, W.; Gong, Q.; Ahn, J.; Ishima, R. Characterization of sarcoplasmic reticulum Ca ${ }^{2+}$ ATPase nucleotide binding domain mutants using NMR spectroscopy. Biochem. Biophys. Res. Commun. 2011, 405, 19-23. [CrossRef] [PubMed]

53. Martí-Renom, M.A.; Stuart, A.C.; Fiser, A.; Sánchez, R.; And, F.M.; Šali, A. Comparative protien structure modeling of genes and genomes. Annu. Rev. Biophys. Biomol. Struct. 2000, 29, 291-325. [CrossRef] [PubMed]

54. Min, J.; Lin, D.; Zhang, Q.; Zhang, J.; Yu, Z. Structure-based virtual screening of novel inhibitors of the uridyltransferase activity of Xanthomonas oryzae pv. oryzae GlmU. Eur. J. Med. Chem. 2012, 53, 150-158. [CrossRef] [PubMed]

55. Nakamura, T.; Kodama, N.; Oda, M.; Tsuchiya, S.; Yu, A.; Kumamoto, T.; Ishikawa, T.; Ueno, K.; Yano, S. The structure-Activity relationship between oxycoumarin derivatives showing inhibitory effects on iNOS in mouse macrophage RAW264.7 cells. J. Nat. Med. 2009, 63, 15-20. [CrossRef] [PubMed]

56. Cheng, J.F.; Chen, M.; Wallace, D.; Tith, S.; Arrhenius, T.; Kashiwagi, H.; Ono, Y.; Ishikawa, A.; Sato, H.; Kozono, T. Discovery and structure-activity relationship of coumarin derivatives as TNF- $\alpha$ inhibitors. Bioorg. Med. Chem. Lett. 2004, 14, 2411-2415. [PubMed]

57. Hu, J.; Wang, C.; Wang, J.; You, Y.; Chen, F. Monitoring of resistance to spirodiclofen and five other acaricides in Panonychus citri collected from Chinese citrus orchards. Pest Manag. Sci. 2010, 66, 1025-1030. [CrossRef] [PubMed] 
58. Michel, A.P.; Mian, M.A.R.; Davila-Olivas, N.H.; Cañas, L.A. Detached leaf and whole plant assays for Soybean aphid resistance: Differential responses among resistance sources and biotypes. J. Econ. Entomol. 2010, 103, 949-957. [CrossRef] [PubMed]

59. Bairoch, A. The PROSITE dictionary of sites and patterns in proteins, its current status. Nucleic Acids Res. 1993, 21, 3097-3103. [CrossRef] [PubMed]

60. Bendtsen, J.D.; Nielsen, H.; Von, H.G.; Brunak, S. Improved prediction of signal peptides: SignalP 3.0. J. Mol. Biol. 2004, 340, 783-795. [CrossRef] [PubMed]

61. Tamura, K.; Peterson, D.; Peterson, N.; Stecher, G.; Nei, M.; Kumar, S. MEGA5: Molecular evolutionary genetics analysis using maximum likelihood, evolutionary distance, and maximum parsimony methods. Mol. Biol. Evol. 2011, 28, 2731-2739. [CrossRef] [PubMed]

62. Rozen, S.; Skaletsky, H. Primer3 on the WWW for general users and for biologist programmers. Methods Mol. Biol. 2000, 132, 365-386. [PubMed]

63. Sun, W.; Jin, Y.; He, L.; Lu, W.; Li, M. Suitable reference gene selection for different strains and developmental stages of the carmine spider mite, Tetranychus cinnabarinus, using quantitative real-time PCR. J. Insect Sci. 2013, 10, 208. [CrossRef] [PubMed]

64. Livak, K.J.; Schmittgen, T.D. Analysis of relative gene expression data using real-time quantitative PCR and the $2^{-\Delta \Delta C t}$ Method. Methods 2001, 25, 402-408. [CrossRef] [PubMed]

65. Zhang, Y. I-TASSER server for protein 3D structure prediction. BMC Bioinform. 2008, 9, 40. [CrossRef] [PubMed]

66. Yang, J.Y.; Zhang, Y. Protein structure and function prediction using I-TASSER. Curr. Protoc. Bioinform. 2016, 52, 5.8.1-5.8.15.

67. Yang, J.; Yan, R.; Roy, A.; Xu, D.; Poisson, J.; Zhang, Y. The I-TASSER Suite: Protein structure and function prediction. Nat. Methods 2015, 12, 7-8. [CrossRef] [PubMed]

68. Roy, A.; Kucukural, A.; Zhang, Y. I-TASSER: A unified platform for automated protein structure and function prediction. Nat. Protoc. 2010, 5, 725-738. [CrossRef] [PubMed]

69. Wu, S.; Zhang, Y. LOMETS: A local meta-threading-server for protein structure prediction. Nucleic Acids Res. 2007, 35, 3375-3382. [CrossRef] [PubMed]

70. Zhang, Y. Template-based modeling and free modeling by I-TASSER in CASP7. Proteins 2007, 69, 108-117. [CrossRef] [PubMed]

71. Dutta, S.; Berman, H.M.; Bluhm, W.F. Using the tools and resources of the RCSB protein data bank. Curr. Protoc. Bioinform. 2007, 20, 1-24.

72. Zhang, Y. Progress and challenges in protein structure prediction. Curr. Opin. Struct. Biol. 2008, 18, 342-348. [CrossRef] [PubMed]

73. Zhang, Y.; Kolinski, A.; Skolnick, J. TOUCHSTONE II: A new approach to ab initio protein structure prediction. Biophys. J. 2003, 85, 1145-1164. [CrossRef]

74. Zhang, Y.; Skolnick, J. Automated structure prediction of weakly homologous proteins on a genomic scale. Proc. Natl. Acad. Sci. USA 2004, 101, 7594-7599. [CrossRef] [PubMed]

75. Zhang, J.; Liang, Y.; Zhang, Y. Atomic-level protein structure refinement using fragment-guided molecular dynamics conformation sampling. Structure 2011, 19, 1784-1795. [CrossRef] [PubMed]

76. Xu, D.; Zhang, Y. Improving the physical realism and structural accuracy of protein models by a two-step atomic-level energy minimization. Biophys. J. 2011, 101, 2525-2534. [CrossRef] [PubMed]

77. Yang, J.; Roy, A.; Zhang, Y. BioLiP: A semi-manually curated database for biologically relevant ligand-protein interactions. Nucleic Acids Res. 2013, 41, D1096-D1103. [CrossRef] [PubMed]

78. Roy, A.; Zhang, Y. Recognizing protein-ligand binding sites by global structural alignment and local geometry refinement. Structure 2012, 20, 987-997. [CrossRef] [PubMed]

79. Yang, J.; Roy, A.; Zhang, Y. Protein-ligand binding site recognition using complementary binding-specific substructure comparison and sequence profile alignment. Bioinformatics 2013, 29, 2588-2595. [CrossRef] [PubMed]

80. Laskowski, R.A.; Macarthur, M.W.; Moss, D.S.; Thornton, J.M. PROCHECK: A program to check the stereochemical quality of protein structures. J. Appl. Crystallogr. 1993, 26, 283-291. [CrossRef]

81. Porter, L.L.; Englander, S.W. Redrawing the Ramachandran plot after inclusion of hydrogen-bonding constraints. Proc. Natl. Acad. Sci. USA 2011, 108, 109-113. [CrossRef] [PubMed] 
82. Zhang, B.C.; Luo, J.X.; Lai, T.; Wang, D.; Ding, W.; Zhang, Y.Q. Study on acaricidal bioactivity and quantitative structure activity relationship of coumarin compounds against Tetranychus cinnabarinus Bois. (Acari: Tetranychidae). Chin. J. Pestic. Sci. 2016, 18, 37-48.

83. Gasteiger, J.; Marsili, M. Iterative partial equalization of orbital electronegativity-a rapid access to atomic charges. Tetrahedron 1980, 36, 3219-3228. [CrossRef]

84. Clark, M.; Cramer, R.D.; Van Opdenbosch, N. Validation of the general purpose tripos 5.2 force field. J. Comput. Chem. 1989, 10, 982-1012. [CrossRef]

85. Powell, M.J.D. Restart procedures for the conjugate gradient method. Math. Program. 1977, 12, $241-254$. [CrossRef]

86. Araz, J.; David, B.J.; Christopher, I.B. Fast, efficient generation of high-quality atomic charges. AM1-BCC model: II. Parameterization and validation. J. Comput. Chem. 2002, 23, 1623-1641.

87. Morris, G.M.; Huey, R.; Lindstrom, W.; Sanner, M.F.; Belew, R.K.; Goodsell, D.S.; Olson, A.J. AutoDock4 and AutoDockTools4: Automated docking with selective receptor flexibility. J. Comput. Chem. 2009, 30, 2785-2791. [CrossRef] [PubMed]

88. Welch, W.; Ruppert, J.; Jain, A.N. Hammerhead: Fast, fully automated docking of flexible ligands to protein binding sites. Cell Chem. Biol. 1996, 3, 449-462. [CrossRef]

89. Kadioglu, O.; Saeed, M.E.M.; Valoti, M.; Frosini, M.; Sgaragli, G.; Efferth, T. Interactions of human P-glycoprotein transport substrates and inhibitors at the drug binding domain: Functional and molecular docking analyses. Biochem. Pharmacol. 2016, 104, 42-51. [CrossRef] [PubMed]

90. Wold, S.; Geladi, P.; Esbensen, K.; Ohman, J. Multi way principal components and PLS analysis. J. Chemom. 2005, 1, 41-56. [CrossRef]

(C) 2017 by the authors. Licensee MDPI, Basel, Switzerland. This article is an open access article distributed under the terms and conditions of the Creative Commons Attribution (CC BY) license (http:/ / creativecommons.org/licenses/by/4.0/). 


\title{
Identification of Direct Activator of Adenosine Monophosphate-Activated Protein Kinase (AMPK) by Structure-Based Virtual Screening and Molecular Docking Approach
}

\author{
Tonghui Huang *, Jie Sun, Shanshan Zhou, Jian Gao and Yi Liu* \\ Jiangsu Key Laboratory of New Drug Research and Clinical Pharmacy, School of Pharmacy, \\ Xuzhou Medical University, Xuzhou 221004, China; jxbp0812@163.com (J.S.); \\ ZSS1991530@163.com (S.Z.); gaojian@xzhmu.edu.cn (J.G.) \\ * Correspondence: tonghhuang@xzhmu.edu.cn (T.H.); cbpeliuyinew@163.com (Y.L.); \\ Tel.: +86-516-8326-2137 (T.H.); +86-516-8326-2136 (Y.L.); Fax: +86-516-8326-2251 (T.H.); +86-516-8326-2136 (Y.L.)
}

Received: 11 May 2017; Accepted: 27 June 2017; Published: 30 June 2017

\begin{abstract}
Adenosine monophosphate-activated protein kinase (AMPK) plays a critical role in the regulation of energy metabolism and has been targeted for drug development of therapeutic intervention in Type II diabetes and related diseases. Recently, there has been renewed interest in the development of direct $\beta 1$-selective AMPK activators to treat patients with diabetic nephropathy. To investigate the details of AMPK domain structure, sequence alignment and structural comparison were used to identify the key amino acids involved in the interaction with activators and the structure difference between $\beta 1$ and $\beta 2$ subunits. Additionally, a series of potential $\beta 1$-selective AMPK activators were identified by virtual screening using molecular docking. The retrieved hits were filtered on the basis of Lipinski's rule of five and drug-likeness. Finally, 12 novel compounds with diverse scaffolds were obtained as potential starting points for the design of direct $\beta 1$-selective AMPK activators.
\end{abstract}

Keywords: Adenosine 5'-monophosphate-activated protein kinase; virtual screening; molecular docking; selective activator

\section{Introduction}

Kidney disease associated with diabetes is the leading cause of chronic kidney disease (CKD) and end-stage kidney disease worldwide and nearly one-third of patients with diabetes develop nephropathy [1]. As the incidence of both types 1 and 2 diabetes rises worldwide, diabetic nephropathy $(\mathrm{DN})$ is likely become a significant health and economic burden for society [2]. Current therapy for diabetic nephropathy includes glycemic optimization using antidiabetics and blood pressure control with blockade of the renin-angiotensin system [3]. However, these strategies are slow but cannot reverse or at least stop the disease progression [4]. Although several clinical trials are currently in progress, there are still no drugs approved for the treatment of DN. Among these ongoing phase 3 clinical trials, atrasentan is still in progress, while bardoxolone methyl and paricalcitol failed to meet the primary endpoint or was terminated on safety concerns [4,5]. Recently, there has been renewed interest in the development of direct $\beta 1$-selective Adenosine monophosphate-activated protein kinase (AMPK) activators that have the potential to treat diabetic nephropathy [6].

AMPK is master sensor of cellular energy and plays a critical role in the regulation of metabolic homeostasis [7]. AMPK is a heterotrimeric kinase comprised of a highly conserved catalytic $\alpha$ subunit and two regulatory subunits ( $\beta$ and $\gamma$ ) [8]. The $\alpha$ subunit possess a $\mathrm{N}$-terminal serine/threonine catalytic kinase domain (KD) that is followed by an autoinhibitory domain (AID) and a C-terminal 
$\beta$ subunit-binding domain [9]. The $\beta$ subunit serves as a scaffold to bridge $\alpha$ and $\gamma$ subunits that contains a glycogen binding domain (GBD) and a C-terminal domain [10]. The $\gamma$ subunit is composed of a $\beta$ subunit-binding region and two Bateman domains [11]. These seven subunits $(\alpha 1, \alpha 2, \beta 1, \beta 2$, $\gamma 1, \gamma 2$, and $\gamma 3$ ) are encoded by separate genes, resulting in 12 different $\alpha \beta \gamma$ AMPK heterotrimers [12]. The distinct physiological functions of each AMPK isoforms are not fully understood, but derive from differential expression patterns among different tissues [13]. For instance, the $\alpha 1$ subunit appears to be relatively evenly expressed in kidney, rat heart, liver, brain, lung and skeletal muscle tissues, while the $\alpha 2$ subunit is mainly expressed in skeletal muscle, heart, and liver tissues [14]. Among the two known $\beta$ subunits, $\beta 1$ subunit is highly abundant in kidney as suggested by mRNA levels [6].

More recently, a direct AMPK activator PF-06409577 was reported to activate $\alpha 1 \beta 1 \gamma 1$ and $\alpha 2 \beta 1 \gamma 1$ AMPK isoforms with $\mathrm{EC}_{50}$ of $7.0 \mathrm{nM}$ and $6.8 \mathrm{nM}$ but was much less active against $\alpha 1 \beta 2 \gamma 1 / \alpha 2 \beta 2 \gamma 1 / \alpha 2 \beta 2 \gamma 3$ AMPK isoforms with EC50 greater $4000 \mathrm{nM}$ [6]. Besides, compound PF-06409577 exhibited efficacy in a preclinical model of diabetic nephropathy. Compounds A-769662 and 991 possessed similar potency toward AMPK heterotrimers containing a $\beta 1$ subunit as PF-96409577 [15]. On the other hand, an allosteric site of AMPK has been named allosteric drug and metabolite site (ADaM site) [16], which was constructed by the catalytic kinase domain (KD) of $\alpha$ subunit and the regulatory carbohydrate-binding module (CBM) of $\beta$ subunit $[13,17]$. The three known direct AMPK activators (PF-06409577 [6], A-769662 [18], and 991 [19], Figure 1) all bound to the allosteric site and showed better potency for isoforms that contain the $\beta 1$ subunit. This implies that the allosteric site can be used to design the selective activators of AMPK containing the $\beta 1$ subunit.

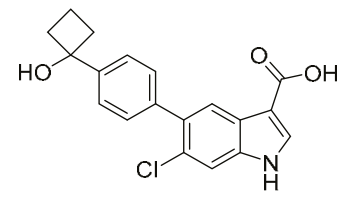

PF-06409577

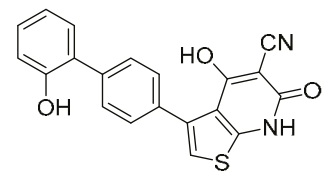

A-769662

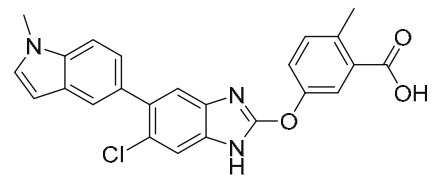

991

Figure 1. Structures of reported direct AMPK activators.

The present study aims to investigate details of the domain structure and identify new potential $\beta 1$-selective AMPK activators. Hence, sequence alignment and structural comparison were used to identify the key amino acids that are involved in the interaction with activators and structure difference between different subunits. Furthermore, molecular docking was performed for virtual screening to discover direct $\beta 1$-selective AMPK activators. The screened retrieved hits were then subjected to several filters such as estimated activity and quantitative estimation of drug-likeness (QED) [20,21]. Finally, 12 compounds with diverse scaffolds were selected as potential hit compounds for the design of novel $\beta 1$-selective AMPK activators. These findings provided a useful molecular basis for the design and development of novel $\beta 1$-selective AMPK activators.

\section{Results and Discussion}

\subsection{Sequence Alignment and Structural Comparison}

To reveal the possible molecular mechanism for the selective potency of activators against the $\beta 1$-isoform of AMPK, sequence and secondary structure elements comparison between carbohydrate-binding module of $\beta 1$ and $\beta 2$ subunits were investigated. As shown in Figure 2, the sequences that were boxed blue were located within the range of $5 \AA$ of active site. Sequence alignment reveals that $\beta 1$ and $\beta 2$ subunits shares $77.1 \%$ sequence identity. As shown in Figure 3, superposition with the two subunits reveals a deflexion of sheet1 in $\beta 2$ subunit as compared with $\beta 1$ subunit. The Phe- 82 of $\beta 1$ subunit corresponded to Ile- 81 in $\beta 2$ subunit, as well as the Thr- 85 to Ser- 84 , 
Gly-86 to Glu-85, which may account for the deflexion of sheet1 in $\beta 2$ subunit. The large aromatic Phe residues and small Thr and Gly presented a binding surface more capable of accommodating ligand.

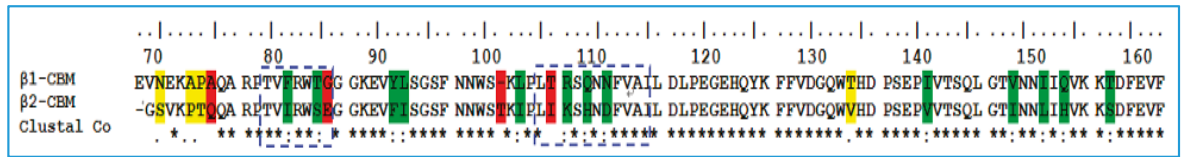

Figure 2. Sequence alignment of carbohydrate-binding module from the $\beta 1$ and $\beta 2$ subunits. Asterisks indicate positions that have a single, fully conserved residue. Colon (green) indicates conservation between groups of strongly similar properties. Period (yellow) indicates conservation between groups of weakly similar properties. Blank character (red) indicates conservation between groups of strongly different properties.

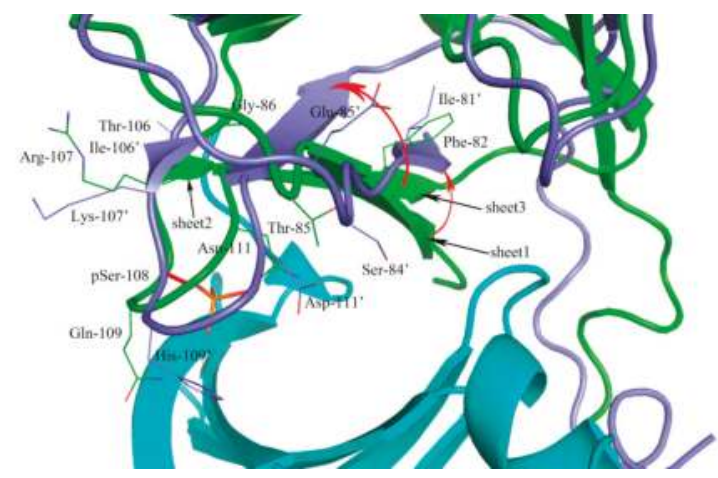

Figure 3. Structural comparison of the scope within $5 \AA$ of the active site from $\beta 1$ and $\beta 2$ subunits. The $\alpha$ subunit was shown in cartoon and colored by the cyan. The $\beta 1$ and $\beta 2$ subunits were shown in cartoon and colored by green and blue, respectively. The sites with different amino acids were shown in line. The Ser-108 was shown in stick and colored by red.

The sheet 2 torsion may attribute to the amino acid sequence differences of the sites of 106 and 107. The most notable is supposed to the Ser108 (red and stick), an autophosphorylation site, phosphorylated serine (pSer108) formed hydrogen bonds with Thr-21, Lys-29, Lys-31, His-109', and Asn- $111^{\prime}$ enhancing the ADaM site stabilization [22], and the phosphate group contributed to the binding of activators [23]. The Gln-109' and Asn- $111^{\prime}$ were mutated to His-109' and Asp-111', which abolished original hydrogen bonds and generated a large conformational change. We speculated that the above differences between $\beta 1$ and $\beta 2$ may affect the binding of activators to AMPk isoforms.

\subsection{Parameter Setting for Molecular Docking}

Docking parameters, which exert an important influence on molecular docking-based virtual screening, were optimized in advance. The crystal structure of PF-06409577 bound to the $\alpha 1 \beta 1 \gamma 1$ AMPK isoform (PDB ID: 5KQ5) and A-769662 bound to the $\alpha 2 \beta 1 \gamma 1$ AMPK isoform (PDB ID: 4CFF) were chosen as the reference, the docking parameters were adjusted until the docked poses were as close as possible to the original crystallized structures. The ring flexibility was mainly considered in final optimized docking parameters according to the default settings. The overlay of the original ligand from X-ray crystal (stick and magenta) and the conformation from Surflex-Dock results (stick and green) were shown in Figure 4, in which the indole moiety of PF-06409577 and terminal benzene ring of A-769662 generated a little deflection and there was no effect on the interaction between compounds 
and the active site. The hydrogen bond interactions appeared consistent with the original ligands and the root mean square deviation (RMSD) between these two conformations are 0.53 and $0.56 \AA$, respectively. The molecular docking results indicated that the Surflex-Dock was reliable and could be used for the further virtual screening.

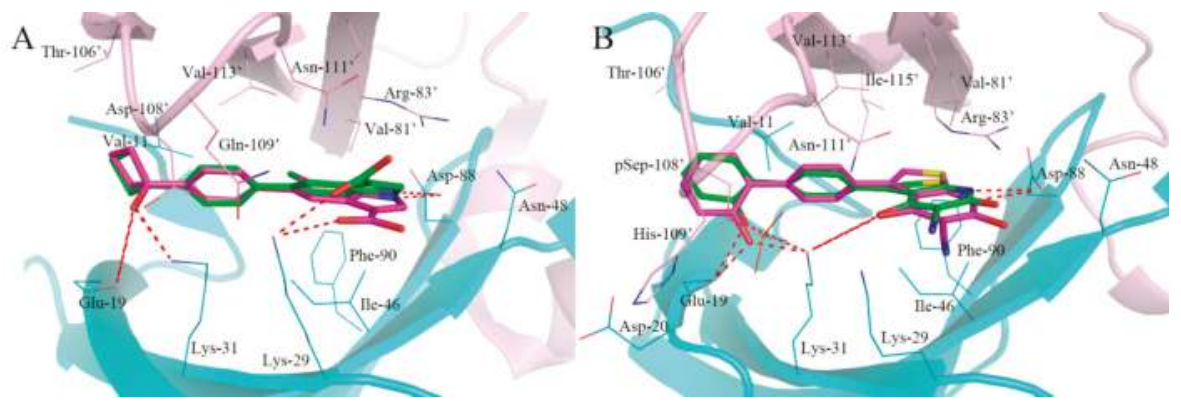

Figure 4. Conformation comparison of the original ligand from X-ray crystal (magenta and stick) and the conformation from Surflex-Dock result (green and stick). (A): PF-06409577; (B): A-769662. The indole moiety of PF-06409577 and terminal benzene ring of A-769662 generated a little deflection in compared with the original conformation. The hydrogen bond was labeled by red dashed lines.

\subsection{High-Throughput Virtual Screening Procedure}

To identify new potent activators of AMPK, virtual screening was performed on the active sites as mentioned previously. A chemical library containing with 1,500,000 commercially available compounds (ChemDiv database) was docked to the molecular models of $\alpha 1 \beta 1 \gamma 1$ and $\alpha 2 \beta 1 \gamma 1$ AMPK isoforms in silico, respectively. Prior to docking, the ChemDiv database was split into eight subsets for molecular docking. About 600 top ranked compounds with high total-scores were screened and subsequently checked for their binding modes and interactions with the active site, especially the hydrogen bonds formed with the residuals of Asp-88, Lys-29, Lys-31, and Gly-19. Then the potential hit compounds were evaluated for their drug-likeness model scores using Lipinski's rule of five (Table 1). Finally, six potential hits with new scaffolds could serve as activators for $\alpha 1 \beta 1 \gamma 1$ AMPK isoform and six for $\alpha 2 \beta 1 \gamma 1$ AMPK isoform were visually chosen from the top potential hits.

Table 1. The docking scores and drug-likeness model scores of selected activators for AMPK $(\alpha 1 \beta 1 \gamma 1$ and $\alpha 2 \beta 1 \gamma 1)$.

\begin{tabular}{|c|c|c|c|c|c|c|c|c|}
\hline Isoforms & Compound No. & Total-Score & Crash & Polar & Similarity & $\begin{array}{l}\text { Number of } \\
\text { HBA/HBD }\end{array}$ & $\begin{array}{c}\text { MolLog } \\
\text { P }\end{array}$ & $\begin{array}{c}\text { Drug-Likeness } \\
\text { Model Score }\end{array}$ \\
\hline \multirow{7}{*}{$\begin{array}{c}\text { AMPK } \\
(\alpha 1 \beta 1 \gamma 1) \\
\text { activators }\end{array}$} & F064-1335 & 10.50 & -2.35 & 3.54 & 0.44 & $6 / 1$ & 3.79 & -0.16 \\
\hline & M5653-1884 & 10.24 & -1.43 & 2.90 & 0.50 & $5 / 1$ & 6.07 & 0.22 \\
\hline & D454-0135 & 10.20 & -1.58 & 3.41 & 0.44 & $6 / 2$ & 4.03 & -0.31 \\
\hline & M8006-4303 & 10.07 & -1.83 & 4.29 & 0.58 & $6 / 1$ & 1.01 & 1.02 \\
\hline & F264-3019 & 9.93 & -1.39 & 3.19 & 0.46 & $6 / 1$ & 5.44 & 1.00 \\
\hline & F377-1213 & 10.03 & -1.43 & 1.32 & 0.44 & $6 / 1$ & 4.12 & 0.16 \\
\hline & PF-06409577 & 7.29 & -0.07 & 3.08 & 0.93 & $3 / 3$ & 3.80 & 0.71 \\
\hline \multirow{8}{*}{$\begin{array}{c}\text { AMPK } \\
(\alpha 2 \beta 1 \gamma 1) \\
\text { activators }\end{array}$} & L267-1138 & 10.96 & -2.46 & 2.78 & 0.52 & $4 / 1$ & 6.05 & -0.08 \\
\hline & F684-0053 & 10.60 & -2.77 & 4.31 & 0.54 & $7 / 3$ & 2.04 & 0.54 \\
\hline & C804-0412 & 10.15 & -3.27 & 3.39 & 0.54 & $5 / 2$ & 2.53 & 1.00 \\
\hline & M5976-1661 & 9.46 & -0.92 & 1.32 & 0.46 & $6 / 0$ & 4.84 & 0.62 \\
\hline & M039-0295 & 9.35 & -1.61 & 1.48 & 0.50 & $6 / 1$ & 2.66 & -0.20 \\
\hline & M5050-0116 & 9.27 & -1.35 & 2.82 & 0.49 & $7 / 2$ & 5.13 & 0.38 \\
\hline & A-769662 & 7.44 & -1.46 & 1.26 & 0.93 & $5 / 3$ & 3.46 & 0.30 \\
\hline & 991 & 8.38 & -0.96 & 4.08 & 0.73 & $4 / 2$ & 5.38 & 0.41 \\
\hline
\end{tabular}




\subsection{Analysis of Binding Mode of Activators for $\alpha 1 \beta 1 \gamma 1$ AMPK Isoform}

The structures of retrieved hits as activators of $\alpha 1 \beta 1 \gamma 1$ AMPK isoform are shown in Figure 5. Although these compounds possess different chemical scaffolds, they exhibit similar binding modes at the active site. Among these compounds, compounds F064-1335 and M5653-1884 possess higher docking scores, compounds M8006-4303 and F264-3019 have perfect drug-likeness model scores.

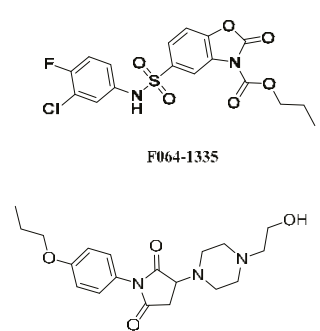

18006-4303

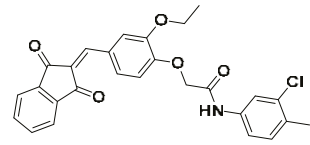

Y5653-1884

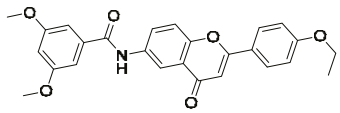

F264-0319

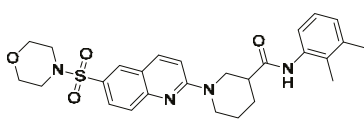

F377-1213

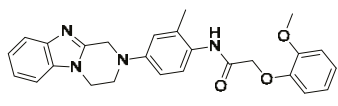

D454-013E

Figure 5. Structures of retrieved hits targeting $\alpha 1 \beta 1 \gamma 1$ AMPK isoform from ChemDiv database.

As shown in Figure 6A, the compound F064-1335 with the highest docking score (10.50) formed several hydrogen bonds with active site residues. The two oxygen atoms of sulfonamide established a hydrogen bond network with the side chain of Lys-31, Lys-29, and Asn-111'. The carbonyl oxygen atom of the ester group formed two hydrogen bonds with the main chain of Lys-29, the anther oxygen atom of the ester group was bound to the main chain of Asn- 48 by a hydrogen bond, which made the alkoxy group trend into a hydrophobic pocket formed by the Lys-51, Ile-52, Val-62, and Leu-47. In addition, the carbonyl oxygen of benzoxazolone ring formed a hydrogen bond with the side chain of Arg-83'.
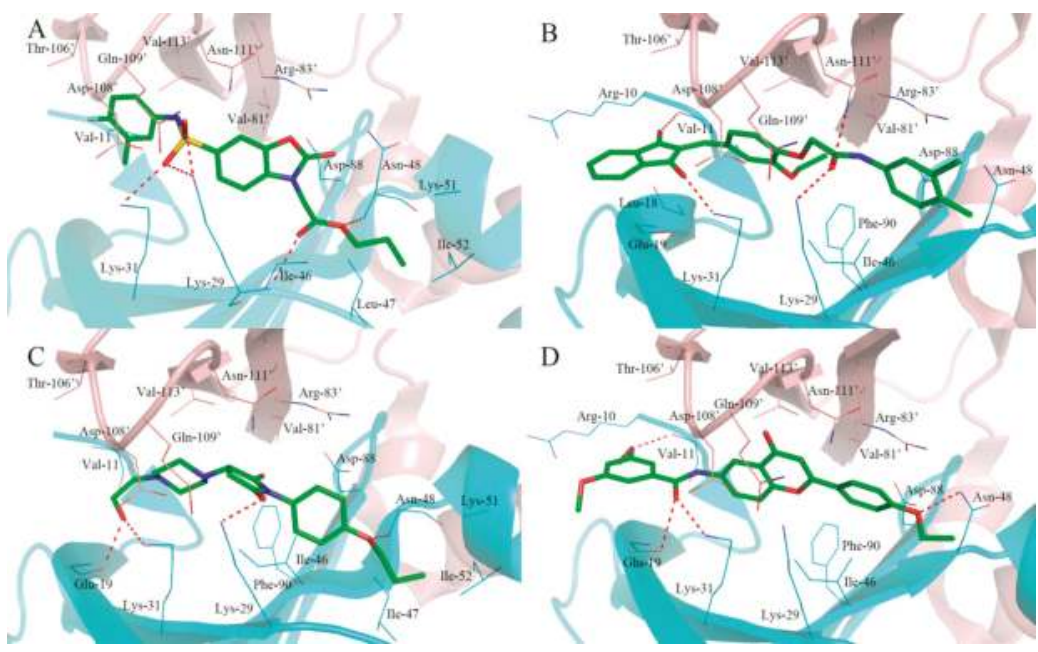

Figure 6. The binding modes of typical hit compounds for $\alpha 1 \beta 1 \gamma 1$ AMPK isoform. (A): F064-1335; (B): M5653-1884; (C): M8006-4303; (D): F264-0391. The $\alpha$ subunit was shown in cartoon and colored by cyan and the $\beta$ subunit was shown in cartoon and colored by pink. The hydrogen bonds were labeled with red dashed lines. 
The compound M5653-1884 with a considerable docking score (10.24) and the bind mode is shown in Figure 6B. Four hydrogen bonds were observed between the compound and the active site residues. One carbonyl oxygen atom of 1,3-indandione formed hydrogen bond with the side chain of Lys-31, another carbonyl oxygen atom formed a hydrogen bond with the main chain of Val-11. The carbonyl oxygen atom of the amide group showed hydrogen bond interactions with the side chain of Lys-29 and Asn-111. In addition, there was a hydrophobic effect with the side chain of Ile-46, Asn-48, Asp-88, and Phe-88.

As shown in Figure 6C, the compound of M8006-4303 exhibited similar binding mode as PF-06409577. The ethanol group attached to the piperazine group participated in two hydrogen bond interactions with the side chain of Gly-19 and Lys-31. The carbonyl oxygen atoms of pyrrolidine-2,5-dione formed a hydrogen bond interaction with the side chain of Lys-29. In addition, the oxygen atom of oxygen butyl associated with the benzene ring accepted a hydrogen bond from the main chain of Asn-48. Within the cavity of the active site, Ile-47, Asn-48, Lys-51, and Ile-52 probably generated a hydrophobic effect.

The binding mode of compound F264-3091 with a prefect drug-likeness score (1.00) was shown in Figure 6D. The oxgen atom of an oxyethyl group on the benzene ring participated in a hydrogen bond with the main chain of Val-11. The carbonyl oxygen atom of the amide group showed two hydrogen bonds with the main chain of Gln-19 and side chain of Lys-31. In addition, one hydrogen bond was formed between the side chain of Asn-48 and the oxyethyl group connected with flavone B-ring while the B-ring showed a stacked cation- $\pi$ interaction with the side chain of Val- $83^{\prime}$.

\subsection{Analysis of Binding Mode of Activators for $\alpha 2 \beta 1 \gamma 1$ AMPK Isoform}

The chemical structures of six compounds as activators of $\alpha 2 \beta 1 \gamma 1$ AMPK isoform are shown in Figure 7. The molecular docking results indicated that all the compounds possess higher docking scores than A-769662 and 991. The binding modes of the representative compound M2958-7438 and M5050-0116 in the active site of $\alpha 2 \beta 1 \gamma 1$ AMPK isoform are shown in Figure 8.
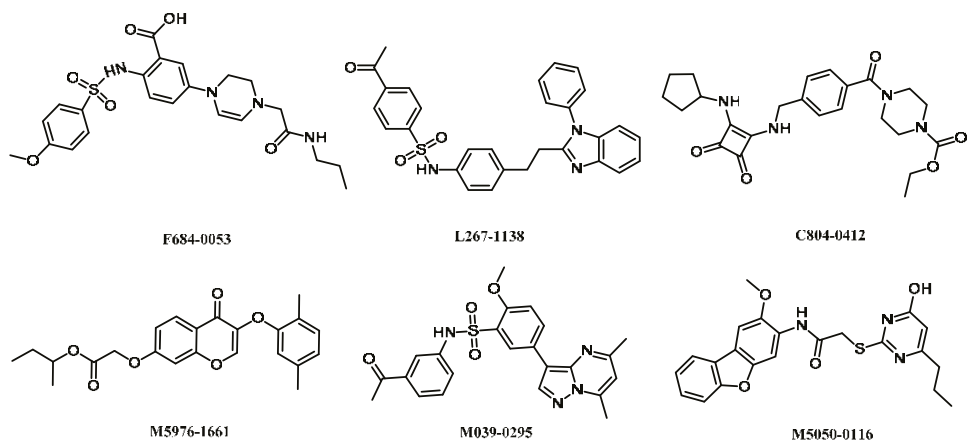

Figure 7. Structures of retrieved hits targeting $\alpha 2 \beta 1 \gamma 1$ AMPK isoform from ChemDiv database.

As shown in Figure 8A, six hydrogen bonds were formed between the compound M2958-7438 and active site residues, in which the barbituric acid ring formed three hydrogen bonds with the side chain of Asp-88, making prominent contributions to the high docking score (10.04). The oxygen atom of the anisole associated with the barbituric acid ring accepted a hydrogen bond from the side chain of Lys-29, and two oxygen atoms in the linker participated in two hydrogen bonds with the side chain of Lys-31. In addition, the barbituric acid ring generated a stacked cation- $\pi$ interaction with the side chain of Arg-83'.

The compound M5050-0116 with a docking score of 9.27 and formed four hydrogen bonds with Val-11, Leu-18, Lys-29, and Asn-111'. As shown in Figure 8B, the Lys-29 of $\alpha$ subunit and 
Asn-111' of $\beta$ subunit, simultaneously coordinated the oxygen atom of dibenzofuran with hydrogen bonds. The hydroxyl group attached on pyrimidine anchored in a suitable geometry and formed two hydrogen bonds with the main chain of Glu-19 and the side chain of Val-11. Additionally, the compound M5050-0116 exhibited hydrophobic interactions with several residues, which formed a hydrophobic pocket including Ile-46, Leu-47, Asn-48, Asp-88, and Phe-90.
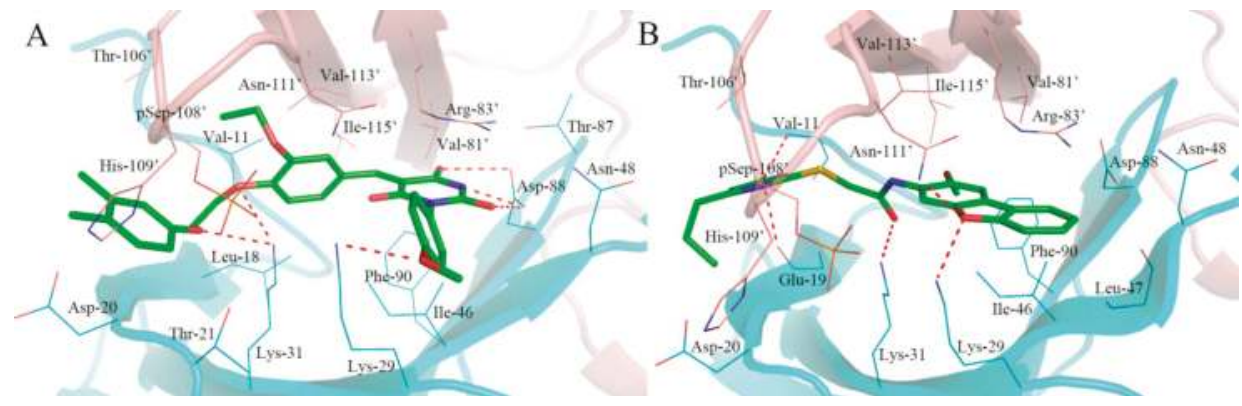

Figure 8. The binding modes of typical activators for $\alpha 2 \beta 1 \gamma 1$ AMPK isoform. (A): M2958-7438; (B): M5050-0116. The $\alpha$ subunit was shown in the illustration and colored cyan and the $\beta$ subunit was shown in the illustration and colored pink. The hydrogen bonds were labeled with red dashed lines.

\subsection{Biological Activities}

The six screened compounds based on $\alpha 1 \beta 1 \gamma 1$ AMPK isoform were evaluated for activities against AMPK ( $\alpha 1 \beta 1 \gamma 1$ isoform) at a dosages of $2 \mu \mathrm{M}$. A-769662, a known $\beta 1$-selective AMPK activator, was used as a control. The preliminary in vitro assay (Figure 9) indicated that most of the selected $\alpha 1 \beta 1 \gamma 1$ AMPK activators displayed promising activation potency against $\alpha 1 \beta 1 \gamma 1$ AMPK isoform. Compounds D454-0135 and F264-3019 displayed comparable activation activity against AMPK in the comparison with the known $\beta 1$-selective AMPK activator A-769662. Morever, compounds M8006-4303 and F264-3019 showed stronger activation activities against $\alpha 1 \beta 1 \gamma 1$ AMPK than A-769662. Compound M563-1884 with a higher $\log P$ value and showed a relatively low activation activity among the assayed compounds. This implies that the lipophilicity may play an important role in the bioavailability for the compound.

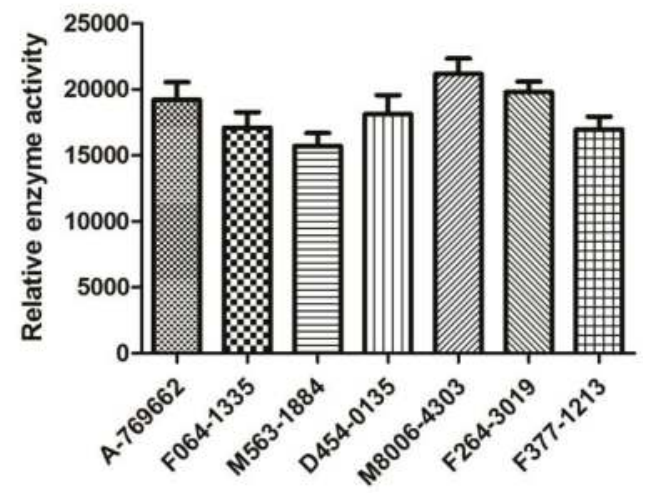

Figure 9. Activation of AMPK ( $\alpha 1 \beta 1 \gamma 1$ isoform) by the screened compounds was measured using Elisa Kit. 


\section{Materials and Methods}

\subsection{Sequence Alignment and Structural Comparison}

Sequence alignment is an essential method for similarity/dissimilarity analysis of protein, DNA, or RNA sequences [24]. The software used for sequence alignment tasks include HAlign, BioEdit, EMBL-EBI, T-Coffee, and CLUSTAL $[25,26]$. The crystal structure data of AMPK $(\alpha 1 \beta 1 \gamma 1: 5 K Q 5$, $4 Q F G ; \alpha 1 \beta 2 \gamma 1$ : 4 REW and $\alpha 2 \beta 1 \gamma 1$ : 4CFF) were obtained from RCSB Protein Data Bank $[6,8,13,19]$, as well as the amino acid sequences of carbohydrate-binding module. The amino acid sequences of carbohydrate-binding module (CBM) on $\beta$ subunits were used to study the differences. The sequence alignment between $\alpha 1 \beta 1 \gamma 1$ isoform (PDB: $4 \mathrm{QFG}$ ) and $\alpha 1 \beta 2 \gamma 1$ (PDB:4REW) isoform was performed and edited using BioEdit software (version 7.1.8) [27], which is a user-friendly biological sequence alignment editor and analysis program. The crystallographic structures of AMPK for molecular docking studying were added to the hydrogen atoms and the charge was given to the Gasteiger-Huckel. The crystal structures comparison was conducted by Sybyl X 2.1 (Tripos Associates Inc., S.H. R.: St. Louis, MO, USA.) [28] and the binding modes were generated by PyMOL V0.99 (Schrödinger, New York, NY, USA.) [29]. The polar hydrogen atoms were added to the crystal structures of the AMPK via the biopolymer module and the Gasteiger-Huckel charges were loaded on the atoms of proteins. The protein peptide backbones were shown in cartoon and colored by different colors, the side chains of the nonconservative amino acids were shown in line and colored by chain.

\subsection{Molecular Docking}

The virtual screening and molecular docking studies were performed using Surflex docking module in Sybyl X 2.1. There were still some deficiencies due to the fact that the receptor was regarded as a rigid structure. Therefore, it was essential to optimize the docking parameters, the co-crystallized ligand was extracted and re-docked into the active site of the AMPK with the varied parameters, and then the conformation of the original ligand and the re-docking ligand were compared. The binding site was defined as a sphere containing the residues that stay within $5 \AA$ from the co-ligand. The maximum conformations per fragment and maximum number of rotatable bonds per molecule were 20 and 100 , respectively. Furthermore, the options for pre-dock minimization and post-dock minimization of molecules were omitted, while other parameters were set as default options. The top 20 conformational poses were selected according to the docking score. Dock scores were evaluated by Consensus Score (CScore), which integrates the strengths of individual scoring functions combine to rank the affinity of ligands bound to the active site of a receptor.

\subsection{High-Throughput Virtual Screening}

High-throughput virtual screening was regarded as an important tool to identify novel lead compounds suitable for specific protein targets [30], and the screened compounds can be easily obtained from commercial sources for biological evaluation as well [31]. The ChemDiv database was supplied by Topscience Co. (Shanghai, China), which includes 1,500,000 compounds was employed for virtual screening through Surflex docking module in Sybyl X 2.1. To accelerate virtual screening, the maximum quantity of conformations was reduced from 20 to 10, the maximum quantity of rotatable bonds was decreased from 100 to 50, and the top six conformations were collected. The same as the molecular docking studies, the default optimization of molecules before and after the docking was canceled. Other parameters were kept as default values. Compounds PF-06409577 and A-769662 were severed as reference molecules, respectively. The compounds with the docking score $(\geq 8.0)$ were extracted for further analyzing the interactions between ligand and active site, to this end, 100 compounds were collected to calculate the drug-likeness model score. Drug-likeness model scores were computed for hit compounds using the MolSoft software (MolSoft, San Diego, CA, USA) [32]. 


\subsection{The In Vitro Activation Assay}

The in vitro preliminary kinase assays human $\alpha 1 \beta 1 \gamma 1$ AMPK were carried out according to the previous experimental method [33]. The screened compounds and $\alpha 1 \beta 1 \gamma 1$ AMPK isoform were provided by Topscience Co. (Shanghai, China) and Huawei Pharmaceutical Co. Ltd. (Shanghai, China), respectively. Generally, each of the evaluated compounds was dissolved in 10\% Dimethyl sulphoxide at $10 \mu \mathrm{M}$ and diluted to a required concentration with buffer solution. Then, $5 \mu \mathrm{L}$ of the dilution was added to a $30 \mu \mathrm{L}$ kinase assay buffer and $5 \mu \mathrm{L}$ AMPK isoform per well. The solution was mixed at $0^{\circ} \mathrm{C}$ for $30 \mathrm{~min}$. Next, $5 \mu \mathrm{L}$ of AMARA petide and $5 \mu \mathrm{L}$ Adenosine triphosphate (ATP) were added to the well. The enzymatic reactions were conducted at $30^{\circ} \mathrm{C}$ for $30 \mathrm{~min}$. The AMPK activity was determined by quantifying the amount of ATP remaining in assay solution with Kinase-Glo Plus luminescent kinase assay kit (Promega, Madison, WI, USA). The luminescent signal is correlated with the amount of ATP present, while inversely correlated with the kinase activity. The mean values from three independent experiments were used for the expression of relative activities. A-769662, a $\beta 1$-selective AMPK activator reported by Abbott laboratories, was used as a control.

\section{Conclusions}

In summary, the sequence alignment and structural comparison was performed to identify the AMPK domain structure detail, which provides a molecular basis of selective AMPK activators on ß1-containing isoforms. The key amino acid residues (Phe/Ile82, Thr/Ser85, Gly/Glu86, Thr/Ile106, Arg/Lys107, Gln/His109, Asn/Asp111) may contribute to the selectivity and provide a foundation for structure-based design of new direct $\beta 1$-selective AMPK activators. Furthermore, the structure-based virtual screening workflow for the identification of selective activators of AMPK $(\alpha 1 \beta 1 \gamma 1$ and $\alpha 2 \beta 1 \gamma 1$ ) was established and six potential hit compounds for $\alpha 1 \beta 1 \gamma 1$ isoform and $\alpha 2 \beta 1 \gamma 1$ isoform were obtained, respectively. The preliminary assay indicated that most of the selected $\alpha 1 \beta 1 \gamma 1$ AMPK activators displayed promising activation potency. Overall, these findings revealed extensive interactions of activators and AMPK for rational design of novel selective AMPK activators. Further in vitro testing of retrieved hits is still in progress in our laboratory.

Acknowledgments: This work was supported by the Postdoctoral Science Foundation funded project (2017M611916), Natural Science Foundation of Jiangsu Province (Grants No. BK20140225), Science and Technology Plan Projects of Xuzhou (Grants No. KC16SG249), Scientific Research Foundation for Talented Scholars of Xuzhou Medical College (No. D2014008), Xuzhou Medical University School of Pharmacy Graduate Student Scientific Research Innovation Projects (No. 2015YKYCX014).

Author Contributions: Tonghui Huang and Jie Sun performed the sequence alignment and structural comparison; Shanshan Zhou and Jian Gao performed virtual screening study; Yi Liu and Tonghui Huang analyzed the data; Tonghui Huang wrote the paper.

Conflicts of Interest: The authors declare no conflict of interest.

\section{References}

1. Gallagher, H.; Suckling, R.J. Diabetic nephropathy: Where are we on the journey from pathophysiology to treatment? Diabetes Obes. Metab. 2016, 18, 641-647. [CrossRef] [PubMed]

2. International Diabetes Federation. IDF Diabetes Atlas-7th Edition; IDF: Brussels, Belgium, 2015. Available online: http:/ / www.diabetesatlas.org (accessed on 9 June 2016).

3. Quiroga, B.; Arroyo, D.; de Arriba, G. Present and future in the treatment of diabetic kidney disease. J. Diabetes Res. 2015, 2015, 1-13. [CrossRef] [PubMed]

4. Chan, G.C.; Tang, S.C. Diabetic nephropathy: Landmark clinical trials and tribulations. Nephrol. Dial. Transpl. 2016, 31, 359-368. [CrossRef] [PubMed]

5. Perezgomez, M.V.; Sanchez-Niño, M.D.; Sanz, A.B.; Martin-Cleary, C.; Ruiz-Ortega, M.; Egido, J.; Navarro-González, J.F.; Ortiz, A.; Fernandez-Fernandez, B. Horizon 2020 in diabetic kidney disease: The clinical trial pipeline for add-on therapies on top of renin angiotensin system blockade. J. Clin. Med. 2015, 4, 1325-1347. [CrossRef] [PubMed] 
6. Cameron, K.O.; Kung, D.W.; Kalgutkar, A.S.; Kurumbail, R.G.; Miller, R.; Salatto, C.T.; Ward, J.; Withka, J.M.; Bhattacharya, S.K.; Boehm, M.; et al. Discovery and preclinical characterization of 6-chloro-5-[4-(1-hydroxycyclobutyl)phenyl]-1H-indole-3-carboxylic Acid (PF-06409577), a direct activator of adenosine monophosphate-activated protein kinase (AMPK), for the potential treatment of diabetic nephropathy. J. Med. Chem. 2016, 59, 8068-8081. [PubMed]

7. Hardie, D.G.; Ross, F.A.; Hawley, S.A. AMPK: A nutrient and energy sensor that maintains energy homeostasis. Nat. Rev. Mol. Cell Biol. 2012, 13, 251-262. [CrossRef] [PubMed]

8. Li, X.D.; Wang, L.L.; Zhou, X.E.; Ke, J.Y.; Waal, P.W.; Gu, X.; Tan, M.H.; Wang, D.; Wu, D.; Xu, H.E.; et al. Structural basis of AMPK regulation by adenine nucleotides and glycogen. Cell Res. 2015, 25, 50-66. [CrossRef] [PubMed]

9. Xiao, B.; Sanders, M.J.; Underwood, E.; Heath, R.; Mayer, F.V.; Carmena, D.; Jing, C.; Walker, P.A.; Eccleston, J.F.; Haire, L.F.; et al. Structure of mammalian AMPK and its regulation by ADP. Nature 2011, 472, 230-233. [CrossRef] [PubMed]

10. Rana, S.; Blowers, E.C.; Natarajan, A. Small molecule adenosine $5^{\prime}$-monophosphate activated protein kinase (AMPK) modulators and human diseases. J. Med. Chem. 2014, 58, 2-29. [CrossRef] [PubMed]

11. Miglianico, M.; Nicolaes, G.A.F.; Neumann, D. Pharmacological targeting of AMP-activated protein kinase and opportunities for computer-aided drug design: Miniperspective. J. Med. Chem. 2016, 59, 2879-2893. [CrossRef] [PubMed]

12. Ross, F.A.; MacKintosh, C.; Hardie, D.G. AMP-activated protein kinase: A cellular energy sensor that comes in 12 flavours. FEBS J. 2016, 283, 2987-3001. [CrossRef] [PubMed]

13. Calabrese, M.F.; Rajamohan, F.; Harris, M.S.; Caspers, N.L.; Magyar, R.; Withka, J.M.; Wang, H.; Borzilleri, K.A.; Sahasrabudhe, P.V.; Hoth, L.R.; et al. Structural basis for AMPK activation: Natural and synthetic ligands regulate kinase activity from opposite poles by different molecular mechanisms. Structure 2014, 22, 1161-1172. [CrossRef] [PubMed]

14. Steinberg, G.R.; Kemp, B.E. AMPK in health and disease. Physiol. Rev. 2009, 89, 1025-1078. [CrossRef] [PubMed]

15. Cameron, K.O.; Kurumbail, R.G. Recent progress in the identification of adenosine monophosphate-activated protein kinase (AMPK) activators. Bioorg. Med. Chem. Lett. 2016, 26, 5139-5148. [CrossRef] [PubMed]

16. Langendorf, C.G.; Kemp, B.E. Choreography of AMPK activation. Cell Res. 2015, 25, 5-6. [CrossRef] [PubMed]

17. Giordanetto, F.; Karis, D. Direct AMP-activated protein kinase activators: A review of evidence from the patent literature. Expert. Opin. Ther. Pat. 2012, 22, 1467-1477. [CrossRef] [PubMed]

18. Cool, B.; Zinker, B.; Chiou, W.; Kifle, L.; Cao, N.; Perham, M.; Dickinson, R.; Adler, A.; Gagne, G.; Iyengar, R.; et al. Identification and characterization of a small molecule AMPK activator that treats key components of type 2 diabetes and the metabolic syndrome. Cell Metab. 2006, 3, 403-416. [CrossRef] [PubMed]

19. Xiao, B.; Sanders, M.J.; Carmena, D.; Bright, N.J.; Haire, L.F.; Underwood, E.; Patel, B.R.; Heath, R.B.; Wlaker, P.A.; Hallen, S.; et al. Structural basis of AMPK regulation by small molecule activators. Nat. Commun. 2013, 4, 3017. [CrossRef] [PubMed]

20. Daina, A.; Michielin, O.; Zoete, V. SwissADME: A free web tool to evaluate pharmacokinetics, drug-likeness and medicinal chemistry friendliness of small molecules. Sci. Rep. 2017, 7, 42717. [CrossRef] [PubMed]

21. Zuegg, J.; Cooper, M.A. Drug-Likeness and Increased Hydrophobicity of Commercially Available Compound Libraries for Drug Screening. Curr. Top. Med. Chem. 2012, 12, 1500-1513. [CrossRef] [PubMed]

22. Scott, J.W.; Ling, N.M.; Issa, S.M.A.; Dite, T.A.; O'Brien, M.T.; Chen, Z.P.; Galic, S.; Langendorf, C.G.; Steinberg, G.R.; Kemp, B.E.; et al. Small molecule drug A-769662 and AMP synergistically activate naive AMPK independent of upstream kinase signaling. Chem. Biol. 2014, 21, 619-627. [CrossRef] [PubMed]

23. Sanders, M.J.; Ali, Z.S.; Hegarty, B.D.; Heath, R.; Snowden, M.A.; Carling, D. Defining the mechanism of activation of AMP-activated protein kinase by the small molecule A-769662, a member of the thienopyridone family. J. Biol. Chem. 2007, 282, 32539-32548. [CrossRef] [PubMed]

24. Kaya, M.; Sarhan, A.; Alhajj, R. Multiple sequence alignment with affine gap by using multi-objective genetic algorithm. Comput. Methods Progr. Biomed. 2014, 114, 38-49. [CrossRef] [PubMed]

25. Zou, Q.; Hu, Q.H.; Guo, M.Z.; Wang, G.H. HAlign: Fast multiple similar DNA/RNA sequence alignment based on the centre star strategy. Bioinformatics 2015, 31, 2475-2481. [CrossRef] [PubMed] 
26. Zou, Q.; Li, X.B.; Jiang, W.R.; Lin, Z.Y.; Li, G.L.; Chen, K. Survey of MapReduce frame operation in bioinformatics. Brief. Bioinform. 2014, 15, 637-647. [CrossRef] [PubMed]

27. Gladue, D.P.; Baker-Bransetter, R.; Holinka, L.G.; Fernandez-Sainz, I.J.; O’Donnell, V.; Fletcher, P.; Lu, Z.Q.; Borca, M.V. Interaction of CSFV E2 protein with swine host factors as detected by yeast two-hybrid system. PLoS ONE 2014, 9, e85324. [CrossRef] [PubMed]

28. Seeliger, D.; de Groot, B.L. Ligand docking and binding site analysis with PyMOL and Autodock/Vina. J. Comput. Aided Mol. Des. 2010, 24, 417-422. [CrossRef] [PubMed]

29. Asokan, R.; Nagesha, S.N.; Manamohan, M.; Krishnakumar, N.K.; Mahadevaswamy, H.M.; Rebijith, K.B.; Prakash, M.N.; Sharath Chandra, G. Molecular diversity of Helicoverpa armigera Hubner (Noctuidae: Lepidoptera) in India. Orient. Insects 2012, 46, 130-143. [CrossRef]

30. Dammganamet, K.L.; Bembenek, S.D.; Venable, J.W.; Castro, G.G.; Mangelschots, L.; Peeterst, D.C.G.; Mcallister, H.M.; Edwards, J.P.; Disepio, D.; Mirzadegan, T. A prospective virtual screening study: Enriching hit rates and designing focus libraries to find inhibitors of PI3K $\delta$ and PI3K $\gamma$. J. Med. Chem. 2016, 59, 4302-4313. [CrossRef] [PubMed]

31. Lionta, E.; Spyrou, G.; Vassilatis, D.K.; Cournia, Z. Structure-Based Virtual Screening for Drug Discovery: Principles, Applications and Recent Advances. Curr. Top. Med. Chem. 2014, 14, 1923-1938. [CrossRef] [PubMed]

32. Drug-Likeness and Molecular Property Prediction. Available online: http://www.molsoft.com/mprop/ (accessed on 9 June 2017).

33. Kashem, M.A.; Nelson, R.M.; Yingling, J.D.; Pullen, S.S.; Prokopowicz, A.S., III; Jones, J.W.; Wolak, J.P.; Rogers, G.R.; Morelock, M.M.; Snow, R.J.; et al. Three Mechanistically Distinct Kinase Assays Compared: Measurement of Intrinsic ATPase Activity Identified the Most Comprehensive Set of ITK Inhibitors. J. Biomol. Screen. 2007, 12, 70-83. [CrossRef] [PubMed]

(C) 2017 by the authors. Licensee MDPI, Basel, Switzerland. This article is an open access article distributed under the terms and conditions of the Creative Commons Attribution (CC BY) license (http:/ / creativecommons.org/licenses/by/4.0/). 


\title{
Relationship of Triamine-Biocide Tolerance of Salmonella enterica Serovar Senftenberg to Antimicrobial Susceptibility, Serum Resistance and Outer Membrane Proteins
}

\author{
Bożena Futoma-Kołoch ${ }^{1, *}$, Bartłomiej Dudek ${ }^{1}$, Katarzyna Kapczyńska ${ }^{2}$, Eva Krzyżewska ${ }^{2}$, \\ Martyna Wańczyk ${ }^{1}$, Kamila Korzekwa ${ }^{1}$, Jacek Rybka ${ }^{2}$, Elżbieta Klausa ${ }^{3}$ and \\ Gabriela Bugla-Płoskońska ${ }^{1, *}$ \\ 1 Department of Microbiology, Institute of Genetics and Microbiology, University of Wrocław, \\ 51-148 Wrocław, Poland; bartlomiej.dudek@uwr.edu.pl (B.D.); kamila.korzekwa@uwr.edu.pl (K.K.); \\ wanczyk.martyna@gmail.com (W.M.) \\ 2 Department of Immunology of Infectious Diseases, Hirszfeld Institute of Immunology and Experimental \\ Therapy, Polish Academy of Sciences, 53-114 Wrocław, Poland; \\ katarzyna.kapczynska@iitd.pan.wroc.pl (K.K.); eva.krzyzewska@iitd.pan.wroc.pl (E.K.); \\ rybka@iitd.pan.wroc.pl (J.R.) \\ 3 Regional Centre of Transfusion Medicine and Blood Bank, 50-345 Wrocław, Poland; \\ e.klausa@rckik.wroclaw.pl \\ * Correspondence: bozena.futoma-koloch@uwr.edu.pl (B.F.-K.); \\ gabriela.bugla-ploskonska@uwr.edu.pl (G.B.-P.); Tel.: +48-71-375-62-22 (B.F.-K.); +48-71-375-62-28 (G.B.-P.)
}

Received: 8 June 2017; Accepted: 30 June 2017; Published: 11 July 2017

\begin{abstract}
A new emerging phenomenon is the association between the incorrect use of biocides in the process of disinfection in farms and the emergence of cross-resistance in Salmonella populations. Adaptation of the microorganisms to the sub-inhibitory concentrations of the disinfectants is not clear, but may result in an increase of sensitivity or resistance to antibiotics, depending on the biocide used and the challenged Salmonella serovar. Exposure of five Salmonella enterica subsp. enterica serovar Senftenberg (S. Senftenberg) strains to triamine-containing disinfectant did not result in variants with resistance to antibiotics, but has changed their susceptibility to normal human serum (NHS). Three biocide variants developed reduced sensitivity to NHS in comparison to the sensitive parental strains, while two isolates lost their resistance to serum. For $S$. Senftenberg, which exhibited the highest triamine tolerance $(6 \times \mathrm{MIC})$ and intrinsic sensitivity to $22.5 \%$ and $45 \% \mathrm{NHS}$, a downregulation of flagellin and enolase has been demonstrated, which might suggest a lower adhesion and virulence of the bacteria. This is the first report demonstrating the influence of biocide tolerance on NHS resistance. In conclusion, there was a potential in S. Senftenberg to adjust to the conditions, where the biocide containing triamine was present. However, the adaptation did not result in the increase of antibiotic resistance, but manifested in changes within outer membrane proteins' patterns. The strategy of bacterial membrane proteins' analysis provides an opportunity to adjust the ways of infection treatments, especially when it is connected to the life-threating bacteremia caused by Salmonella species.
\end{abstract}

Keywords: Salmonella; biocide; serum; antimicrobial resistance; molecular biology; outer membrane protein analysis

\section{Introduction}

Cross-resistance to antibiotics of bacteria exposed to disinfectants (biocides) is an increasing problem for public health as cross-resistant phenotypes of microorganisms could potentially develop 
into life-threatening infections. The main reasons for increasing microbial resistance to disinfectants are mistakes during the disinfecting process itself, using chemicals that are not designed for specific microbiological pollution, inaccurate cleaning of surfaces with biocides (which causes high levels of organic matter and biofilm formation) or applying too low concentrations of biocides [1]. The implementation of hygiene supervision and standardization of the use of antibiotics and disinfectants seem to be a promising way to improve public safety [2]. There is still a lack of understanding of the mode of action of the biocides, especially when used at low or sub-inhibitory concentrations. A single exposure to some biocides has been found to be insufficient to select for multidrug-resistant (MDR) strains; however, repeated, sub-inhibitory exposure to biocides does result in the selection of MDR bacteria [3]. MDR is a major problem in the treatment of infections caused in humans by Salmonella isolates. It has also been noted that the drug resistance was found more frequently in the internal farm environment than in the external environment [2]. It is interesting that, although cross-resistance between biocides and antibiotics is often described for biocide-resistant mutants [2,4,5], increased susceptibility for some antimicrobials has been observed [6,7]. Moreover, resistance levels can also differ even between Salmonella serovars [7]. In two recent studies, we demonstrated that growth of Salmonella enterica subsp. enterica serovars Enteritidis, Typhimurium, Virchow and Zanzibar isolated from human fecal samples with sub-inhibitory concentrations of farm disinfectants containing dodecylamine (triamine) led to increased isolation of multiple antibiotic-resistant strains [8,9]. The antimicrobial efficacy of commercially-manufactured disinfectant substances (represented by quaternary ammonium salts (QAC) and QAC combined with other additives) were tested against Salmonella Enteritidis strains by others $[7,10]$.

QAC and triamine-containing disinfectants are effective against many Gram-positive and Gram-negative bacteria. The antibacterial effect is caused by an increase in the permeability of the bacterial cell membrane, which leads to an osmotic imbalance and an outpouring of cytoplasm [11]. A blend containing dodecylamine-based structure was designed for the cleaning and disinfection of workplaces and devices that come into contact with food and in veterinary hygiene to disinfect animal houses (manufacturer's data, Amity International). Exposure and further Salmonella adaptation to biocides may result in modification of cell envelope (an activity of efflux systems), virulence or motility [7]. It may also include various alterations of chemotaxis pathways and protein synthesis [1,12]. Several proteins have been found to be differentially expressed between biocide-tolerant variants and their parental counterparts. Recently, we have suggested that the resistance of the S. Typhimurium disinfectant (dodecylamine) variant to ciprofloxacin and cefotaxime was connected to the 55-kDa surface protein repression [8]. Moreover, S. Typhimurium and S. Enteritidis dodecylamine-tolerant isolates produced more surface proteins in the range of 30-40 kDa, which probably were porins OmpC (36 kDa), OmpF (35 kDa) and OmpD (34 kDa) [9]. Exposure of Salmonella cells to disinfectants can induce the expression of the AcrAB-TolC efflux system [13]; but after single exposure, MDR strains were not found and probably, this is not the primary mechanism of biocide tolerance generation [6]. Additionally, SugE protein is implicated in QAC resistance and is frequently found in Salmonella isolates of clinical and animal origin [14,15].

The majority of Salmonella infections result in a mild, self-limiting, gastrointestinal illness and usually do not require antimicrobial treatment. In some cases, Salmonella infection can develop to bacteremia in a minor subset of patients [16]. In the situation of severe enteric disease, or when Salmonella invades and causes bloodstream infection, therapy with antimicrobials is essential and can be life-saving. Infections with antimicrobial-resistant Salmonella strains resistant to first-line treatments, i.e., fluoroquinolones and cephalosporins, may cause treatment failure. There is a lack of studies regarding the susceptibility of biocide-tolerant bacteria to normal human serum (NHS), so the present work is the first study in which these aspects of bacterial virulence are discussed. Outer membrane proteins (OMPs) are described as surface virulence factors necessary for bacterial adaptation to human immune response [17]. Therefore, they have been analyzed in our research in the context of resistance to complement-mediated killing. The aim of the present study was to assess the in vitro antimicrobial 
effects of triamine on S. enterica subsp. enterica serovar Senftenberg sensitive to antibiotics using both MIC (minimal inhibitory concentration) and MBC (minimal bactericidal concentration) in correlation with susceptibility to NHS and OMPs patterns. Understanding the mechanisms of the individual and cross-resistance of bacteria may provide reliable clues for the design of more effective antimicrobials.

\section{Results}

\subsection{Salmonella enterica Tolerance to the Biocide Formulation}

In this paper, the biocide formulation containing active substances triamine, ethanol, cationic surfactant and nonionic surfactant (Amity International) was used in the experiments of the generation of disinfectant-tolerant bacteria. Five S. Senftenberg (Salmonella Senftenberg) strains (131, 132, 133, $134,135)$ were exposed to the disinfectant in Luria-Bertani (LB) liquid medium (Table 1). We found that the threshold for the bacterial growth was the concentration of the biocide of $8 \times$ MIC (Minimal Inhibitory Concentration) in the LB medium, which was lethal for all tested microorganisms. The strains were grown in LB supplemented with the biocide used in the concentrations of $4 \times$ MIC (131, $132,134)$ or $6 \times$ MIC $(133,135)$. After 25 days of incubation in LB containing the biocidal formulation and the following 10 days of the stability test (incubation in LB broth), the cultures were subjected to Salmonella spp. detection, because of the possible contamination with other microorganisms during extended passages. The isolates before and after the stability test were identified as Salmonella spp. on Brilliant Green agar plates as red to pink-white colonies with a red zone.

Table 1. Generation of triamine-tolerant Salmonella Senftenberg (S. Senftenberg) variants.

\begin{tabular}{|c|c|c|c|c|c|c|}
\hline \multirow{2}{*}{ Time of Incubation } & \multirow{2}{*}{ Concentration of Biocide } & \multicolumn{5}{|c|}{ S. Senftenberg Strain } \\
\hline & & 131 & 132 & 133 & 134 & 135 \\
\hline 1-day preculture in LB broth & none & + & + & + & + & + \\
\hline 7 days in Luria-Bertani (LB) broth & $0.5 \times \mathrm{MIC}$ & $\begin{array}{c}+ \\
+ \\
0.05\end{array}$ & $\begin{array}{c}+ \\
0.2\end{array}$ & $\begin{array}{c}+ \\
+ \\
0.05\end{array}$ & $\begin{array}{c}+ \\
0.05\end{array}$ & $\begin{array}{c}+ \\
0.1\end{array}$ \\
\hline \multirow{4}{*}{ Gradient $4 \times 4$ days in LB broth } & $0.75 \times \mathrm{MIC}$ & $\begin{array}{c}+ \\
0.075\end{array}$ & $\begin{array}{c}+ \\
0.3\end{array}$ & $\begin{array}{c}+ \\
0.075\end{array}$ & $\begin{array}{c}+ \\
0.075\end{array}$ & $\begin{array}{c}+ \\
+ \\
0.15\end{array}$ \\
\hline & $1.0 \times$ MIC & $\begin{array}{c}+ \\
0.1\end{array}$ & $\begin{array}{c}+ \\
0.4 \\
\end{array}$ & $\begin{array}{c}+ \\
0.1\end{array}$ & $\begin{array}{c}+ \\
0.1\end{array}$ & $\begin{array}{c}+ \\
0.2 \\
\end{array}$ \\
\hline & $1.25 \times \mathrm{MIC}$ & $\begin{array}{c}+ \\
0.125\end{array}$ & $\begin{array}{c}+ \\
0.5\end{array}$ & $\begin{array}{c}+ \\
0.125\end{array}$ & $\begin{array}{c}+ \\
0.125\end{array}$ & $\begin{array}{c}+ \\
0.25\end{array}$ \\
\hline & $1.5 \times \mathrm{MIC}$ & $\begin{array}{c}+ \\
0.15\end{array}$ & $\begin{array}{c}+ \\
0.6\end{array}$ & $\begin{array}{c}+ \\
0.15\end{array}$ & $\begin{array}{c}+ \\
0.15\end{array}$ & $\begin{array}{c}+ \\
0.3\end{array}$ \\
\hline \multirow{4}{*}{1 day in LB broth } & $2 \times \mathrm{MIC}$ & $\begin{array}{c}+ \\
0.2\end{array}$ & $\begin{array}{c}+ \\
0.8\end{array}$ & $\begin{array}{c}+ \\
0.2\end{array}$ & $\begin{array}{c}+ \\
0.2\end{array}$ & $\begin{array}{c}+ \\
0.4\end{array}$ \\
\hline & $4 \times \mathrm{MIC}$ & $\begin{array}{c}+ \\
0.4\end{array}$ & $\begin{array}{c}+ \\
1.6\end{array}$ & $\begin{array}{c}+ \\
0.4\end{array}$ & $\begin{array}{c}+ \\
0.4\end{array}$ & $\begin{array}{c}+ \\
0.8\end{array}$ \\
\hline & $6 \times \mathrm{MIC}$ & $\overline{-}-$ & $\overline{2 .}$ & $\begin{array}{c}+ \\
0.6\end{array}$ & $\overline{0}-$ & $\begin{array}{c}+ \\
1.2\end{array}$ \\
\hline & $8 \times \mathrm{MIC}$ & $\begin{array}{c}- \\
0.8\end{array}$ & $\begin{array}{c}- \\
3.2\end{array}$ & $\begin{array}{c}- \\
0.8\end{array}$ & $\begin{array}{c}- \\
0.8\end{array}$ & $\begin{array}{c}- \\
1.6\end{array}$ \\
\hline Identification on Brilliant Green & $\begin{array}{c}\text { from the highest MIC } \\
\text { (where growth was observed) }\end{array}$ & + & + & + & + & + \\
\hline Stability test 10 days in LB broth & none & + & + & + & + & + \\
\hline Identification on Brilliant Green & none & + & + & + & + & + \\
\hline
\end{tabular}

Definitions of abbreviations: $(+)$ the growth of bacteria in broth supplemented with the biocide seen as the turbidity of the tubes contents or the presence of the colonies typical for Salmonella bacteria on Brilliant Green Agar; (-) no growth; concentrations of the biocide $(\mu \mathrm{L} / \mathrm{mL})$ are also shown. MIC, minimal inhibitory concentration.

Salmonella variants were tested for MIC determination before and after the 10-day stability test (incubation in LB) to verify if the feature of tolerance to the biocide is stable or not. As can be seen in Table 2, MIC values were getting higher in the case of $S$. Senftenberg strains (131 bST, 131 aST, 132 bST, $133^{\mathrm{bST}}, 133^{\mathrm{aST}}, 134^{\mathrm{bST}}, 134^{\mathrm{aST}}, 135^{\mathrm{bST}}$ ) in comparison to their wild-type counterparts. Except for 
the $S$. Senftenberg 131 strain, in almost all tested variants cultures, MICs were decreased after the stability test, to almost the same level as it was at the beginning of the experiments. Additional MBC comparison showed that MBCs were equal to MICs for four wild tested strains: S. Senftenberg $(131,132$, $133,134)$, but not for $S$. Senftenberg 135 . It was also interesting to verify if MBC levels were maintained after the test of the stability of the tolerant phenotypes. It was demonstrated that MBC did not change (strain $132^{\mathrm{aST}}$ ) or was even slightly higher $\left(131^{\mathrm{aST}}, 133^{\mathrm{aST}}, 134^{\mathrm{aST}}, 135^{\mathrm{aST}}\right.$ ) in comparison to the MBC value estimated for the wild-type strains. In general, both parameters MIC and MBC increased as the effect of bacterial adaptation to the increasing concentration of the biocide containing triamine.

Table 2. MIC and MBC values of the triamine-containing disinfectant for Salmonella Senftenberg strains.

\begin{tabular}{|c|c|c|c|c|c|c|c|c|c|c|c|c|c|c|c|}
\hline \multirow{2}{*}{ Test } & \multicolumn{15}{|c|}{ S. Senftenberg Strains } \\
\hline & 131 & $131^{\text {bST }}$ & 131 aST & 132 & 132 bST & 132 aST & 133 & $133^{\text {bST }}$ & $133^{\text {aST }}$ & 134 & $134^{\text {bST }}$ & 134 aST & 135 & $135^{\mathrm{bST}}$ & $135^{\text {aST }}$ \\
\hline $\begin{array}{c}\mathrm{MIC} \\
(\mu \mathrm{L} / \mathrm{mL})\end{array}$ & 0.1 & 0.4 & 0.4 & 0.4 & 1.6 & 0.2 & 0.1 & 0.6 & 0.4 & 0.1 & 0.4 & 0.2 & 0.2 & 0.8 & 0.2 \\
\hline $\begin{array}{c}\mathrm{MBC} \\
(\mu \mathrm{L} / \mathrm{mL})\end{array}$ & 0.1 & $\mathrm{nt}$ & 0.4 & 0.4 & nt & 0.4 & 0.1 & nt & 0.8 & 0.1 & nt & 0.2 & 0.4 & nt & 0.8 \\
\hline
\end{tabular}

Definitions of abbreviations: MIC, minimal inhibitory concentration; MBC, minimal bactericidal concentration; nt, not tested; bST, before the test of stability; aST, after the test of stability.

\subsection{Antibiotic Susceptibility Profiling}

The obtained results showed that the passages of $S$. Senftenberg strains in medium containing disinfectant did not change the susceptibility pattern to antibiotics. The wild-type strains, as well as their biocide variants, were sensitive to ciprofloxacin (CIP, $5 \mu \mathrm{g}$ ), co-trimoxazole (STX, $25 \mu \mathrm{g}$ ), cefotaxime (CTX, $5 \mu \mathrm{g}$ ), amoxicillin/clavulanic acid (AMX 30; 20/10 $\mu \mathrm{g}$ ) and ampicillin (AMP, $10 \mu \mathrm{g}$ ).

\subsection{Bactericidal Activity of Human Serum against S. Senftenberg Variants Tolerant to the Disinfectant}

As C3 is a crucial protein in the activation of the serum complement cascade, standard analysis of C3 protein level in NHS used for experiments was performed. C3 concentration in NHS was $1470 \mathrm{mg} / \mathrm{L}$, which was in the range of standard values (970-1576 mg/L for males and 1032-1495 for females).

Bactericidal activity of diluted NHS $(22.5 \%, 45 \%)$ was performed on Salmonella wild-type strains, as well as on their biocide variants. The average number of colony forming units $(\mathrm{CFU} / \mathrm{mL})$ was calculated from the colonies grown on the agar plates from the volume of $10 \mu \mathrm{L}$ of bacterial suspensions. Between zero and 20 colonies were achieved. Two mechanisms of bacterial susceptibility to the antibacterial activity of serum were observed. Three variants of Salmonella strains $\left(131^{\mathrm{bST}}, 131^{\mathrm{aST}}, 133^{\mathrm{aST}}, 134^{\mathrm{bST}}\right.$, $134{ }^{\mathrm{aST}}$ ) were found to become resistant in $\mathrm{T}_{1}$ or $\mathrm{T}_{2}$ to NHS in comparison to the sensitive parental strains, while two biocide variants (132 bST, 132 aST, 135 bST, 135 aST $)$, lost their resistance to serum (wild-type strains were resistant) (Tables S1 and S2). In detail, the survival rate estimated for two serovars (131 and 133) increased from $10.8 \%$ (131) to $55.6 \%\left(131^{\mathrm{bST}}\right)$ at $\mathrm{T}_{1}$ in $45 \%$ NHS and to $85.3 \%$ in the case of the variant obtained after the test of stability $\left(131^{\mathrm{aST}}\right)$. Survival of bacteria increased from $0.3 \%$ (parent strain 133) to $385.7 \%$ after 15 min of incubation in $45 \%$ NHS and from $15.0-103.0 \%$ at the same time in $22.5 \%$ serum. The third strain, which exhibited resistance to NHS, was 134 bST, which multiplied before the test of stability in $22.5 \%$ at $\mathrm{T}_{2}$ (survival changed from $7.2 \%$ to $128.6 \%$ ), as well as after the test of stability ( $76.9 \%$ survival, $134 \mathrm{aST}$ ), at the same time, in comparison to the parent strain. In the higher concentration of serum of $45 \%$, the same strain became resistant, as its survival raised from $5.5-70.0 \%$ at $\mathrm{T}_{2}$. It was interesting also to compare the feature of resistance between strains before the test of stability and after that. It was helpful to determine if the phenotype of resistance in variants was stable even if the cultures did not have any contact with the disinfectant during 10 days of incubation in LB not supplemented with the biocide. It has been observed that after the test of stability, the resistance rose $\left(131^{\mathrm{aST}}, 132^{\mathrm{aST}}, 133^{\mathrm{aST}}, 134^{\mathrm{aST}}\right)$, or the resistance was maintained $\left(131^{\mathrm{aST}}, 134 \mathrm{aST}\right)$, or vanished $\left(132^{\mathrm{aST}}\right)$, depending on the time of incubation and the serum dilution. When the bactericidal activity of NHS was heat inhibited (HIS, control of experiments), bacterial cells proliferated very intensively, 
and all of the tested strains were resistant to $22.5 \%$ NHS (Table S1) and 45\% NHS (Table S2). Regarding our results and reports of other research groups, showing that resistance to the bactericidal activity of serum is determined by OMPs [17-20], the next stage of research focused on the analysis of the protein profiles of OMPs in the context of unknown OMP-dependent tolerance to the biocide.

\subsection{Analysis of the Two-Dimensional (2-DE) Profiles of Isolated Membrane Proteins}

We applied a proteomic approach using the 2-DE and mass spectrometry analysis for the identification of specific proteins that could be involved in the phenomenon of biocide and NHS resistance of the $S$. Senftenberg 133 strain. We have chosen for this analysis S. Senftenberg 133 as the only strain that was primarily sensitive to NHS and belonging to the group of the highest triamine tolerance $(6 \times \mathrm{MIC})$. Protein spots on 2-DE were visualized within the molecular weight (MW) range of 10-250 kDa and isoelectric points (pI) of 4-7. The comparative protein pattern analysis of $S$. Senftenberg strains resistant to triamine and NHS showed differences in the presence of some proteins (Figure 1), from which four were described in detail (Table 3). MWs of identified OMPs were distributed in the range of $37.49-89.52 \mathrm{kDa}$. These research spots were distributed in the range of pI of 4.85-8.48. The detailed MASCOT search results are provided as Supporting Information. It has been noted that flagellar protein FliC (Spot 1, Figure 1), as well as enolase (Spot 2) were present in lower quantities in the biocide-tolerant variant in comparison to the wild-type parent strain. In contrast, two identified molecules, chemotaxis response regulator protein-glutamate methylesterase (Spot 3), and outer membrane protein assembly factor (Spot 4 ), were overproduced in the S. Senftenberg biocide-serum-resistant isolate, although the molecular mass of Spot 4 from 2-DE does not reflect the mass of the identified protein from the database (89.252 kDa), suggesting the degradation of the protein during the preparation process.

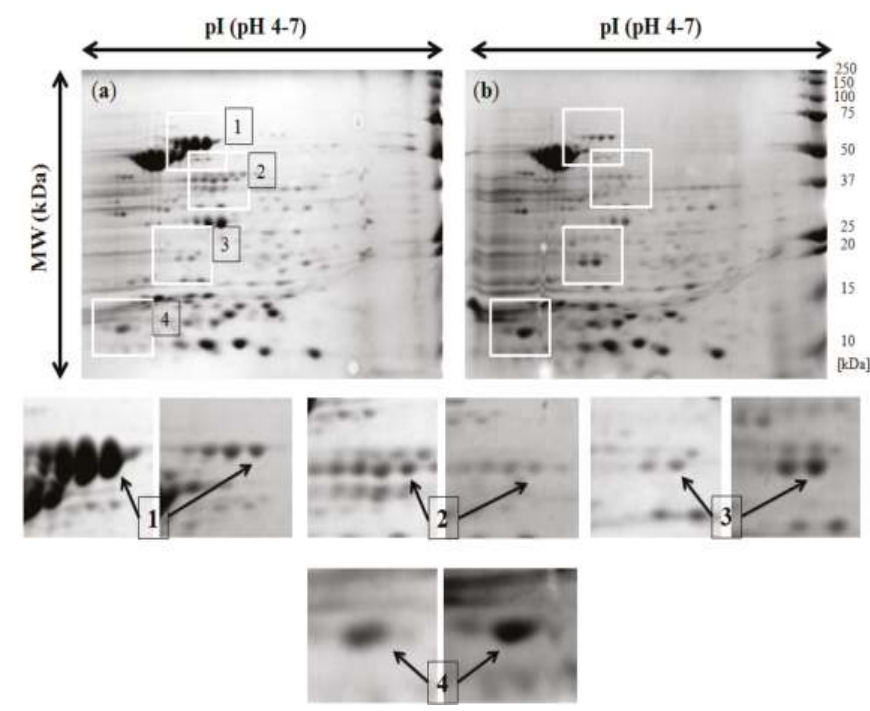

Figure 1. Comparative 2-D gel electrophoresis ( $\mathrm{pH} 4-7)$ of OMPs from Salmonella Senftenberg 133 strain without biocide exposure (a) and with simultaneous resistance to triamine-containing disinfectant and NHS (b). Identification of flagellar protein FliC (Spot 1), enolase (Spot 2), chemotaxis response regulator protein-glutamate methylesterase (Spot 3) and outer membrane protein assembly factor (Spot 4). On the right, protein marker Precision Plus Protein ${ }^{\mathrm{TM}}$ Dual Color Standards 1610374 (Bio-Rad, Hercules, CA, USA). Left arrow refers to part (a), right arrow refers to part (b). 
Table 3. Identification of isolated proteins from Salmonella Senftenberg 133 with resistance to both triamine-containing biocide $(6 \times \mathrm{MIC})$ and normal human serum (NHS).

\begin{tabular}{cccccc}
\hline Spots & Identified Proteins & Gene Symbols & Molecular Weight (kDa) & pI & Expression \\
\hline 1 & Flagellin (FliC) & fliC & 52.081 & 4.85 & downregulated \\
\hline 2 & Enolase & eno & 45.628 & 5.25 & downregulated \\
\hline 3 & $\begin{array}{c}\text { Chemotaxis response regulator } \\
\text { protein-glutamate methylesterase }\end{array}$ & cheB & 37.498 & 8.48 & upregulated \\
\hline 4 & Outer membrane protein assembly factor BamA & bamA & 89.525 & 4.92 & upregulated \\
\hline
\end{tabular}

\section{Discussion}

Salmonella enterica serovars continue to be among the most important foodborne pathogens worldwide due to the significant human rates of illness reported. Public concern for the appearance of resistant zoonotic pathogens such as Salmonella strains to many antibiotics is challenging the poultry industry to find successful means of control [21]. The increasing use of biocides in farming, food production, hospital settings and the home is contributing to the selection of antibiotic-resistant strains [3]. Within several years, it has also been documented that biocide-resistant Salmonella mutants demonstrated reduced susceptibility to antibiotics or, differently, the exposure of these microorganisms to the disinfectants has not changed their sensitivity to antimicrobials. Shengzhi et al. [2] showed that 109 Salmonella strains were co-resistant to antibiotic and disinfectant. In inquiring research, Whitehead and co-workers [3] isolated mutants able to survive challenge with "in-use" concentrations of biocides after one exposure using fluorescence-activated cell sorting (FACS). These mutants were multidrug resistant and overexpressed the AcrEF efflux pump and MarA, demonstrating that biocide exposure can select for mutants with a broad, low-level antibiotic resistance. Working on $S$. Typhimurium phage type 104 (DT104) Majtánova and Majtán indicated that isolate 5551/99 represented the multiresistant phenotype, resistant to ampicillin, chloramphenicol, streptomycin and tetracycline, but the second isolate 577/99 was sensitive to all antibiotics tested [7]. Others also observed increased susceptibility of Salmonella for some drugs. In vitro exposure to a quaternary ammonium disinfectant containing formaldehyde and glutaraldehyde (QACFG) and triclosan led to the selection of $S$. Typhimurium cells with reduced susceptibility to several antibiotics. This was associated with overexpression of the AcrAB efflux pump and accompanied with reduced invasiveness [22]. Strains used in our study, despite the tolerance to biocide, were sensitive to antibiotics, such as ciprofloxacin, co-trimoxazole, cefotaxime, amoxicillin/clavulanic acid and ampicillin. Overall, the issue of bacterial cross-resistance needs to be clarified, but in this paper, the main characteristic that was chosen for testing was Salmonella sensitivity to serum.

It has been suggested that the involvement of common general responses in biocide-tolerant mutants includes several alterations in metabolic and chemotaxis pathways, protein synthesis, cell envelope or regulation of pathogenicity islands. Unlike what has been commonly reported, overexpression of AcrAB-like pumps did not seem to be the primary mechanism involved in biocide tolerance. QACs are widely used in different settings, including the food industry as a hard-surface disinfectant, antiseptic and in foaming hand sanitizers [6]. It has been known that QACs are the membrane-active agents with the target site predominantly at the cytoplasmic membrane of bacteria. Although it was found that the antibacterial efficacy of substances containing QACs with other additives was high against $S$. Enteritidis strain 85/01, it was possible to select isolates resistant to these compounds [10]. Repeated passages of Arcobacter spp. in a medium with a low concentration of the disinfectant Incidur, containing cationic surfactant benzalkonium chloride, increased their initial resistance to 1.5-3.5 $\times$, depending on the bacterial species or origin [5]. In our study, following several rounds of in vitro variants' selection using increasing concentrations of triamine-containing disinfectant, $S$. Senftenberg isolates developed the biocide tolerance phenotype, with a four-fold or six-fold increase in the MIC. The test of stability relied on the incubation of the variants for 10 consecutive days in fresh LB medium without the addition of the biocide. Determination of MICs 
helped to conclude that the exposure of the tested strains to the triamine-containing blend resulted in increased tolerance immediately after the end of the generation of mutants that was before the stability test. However, after 10 days of incubation in non-stressful conditions, the bacteria became more sensitive to the disinfectant (Table 2). This is an optimistic phenomenon considering the public health, since tolerance to triamine was not stable. The question remains which conditions may favor stable tolerance to the biocides. The possible explanation is the presence of proteins or organic materials that reduce disinfectant activity and contribute to biofilm formation [23]. Quorum sensing is known to contribute to antibiotic resistance in Salmonella [24], but its role in biocide tolerance is not understood. In out further investigations, the growth of the bacteria was inhibited using the concentration of $0.04 \%$ (strains 131, 134), $0.16 \%$ (strain 132), $0.06 \%$ (strain 133) and $0.12 \%$ (strain 135). It is important to note that the bacteria were able to adapt to the increasing concentrations of the biocidal formulation, as has been previously shown $[8,9]$.

The ability of human pathogens to survive in serum is another feature worth determining. Salmonella infections can result in uncomplicated diarrhea in most cases, but can lead to invasive disease [25]. Unfortunately, the mechanism of bacterial survival in NHS is not entirely understood. Considering Salmonella spp. surface antigens' composition, it has been shown that long-chain LPS [26], O-antigen (O-Ag) [27], Vi capsules [28], OMPs [17,29] or the presence of fimbriae on the cell surface are virulence factors necessary for bacterial adaptation to human immune response. Recent investigations by Dudek et al. [20] revealed that sensitive $S$. Enteritidis strains possessed a high level of flagellar hook-associated protein 2 (FliD). Furthermore, others showed that O-Ag capsule-deficient mutants produced exclusively phase I flagellin (FliC) [27]. In this paper, we demonstrate that the triamine tolerant mutants displayed changes in their susceptibility profile to a diluted NHS (22.5\% and $45 \%$ ) when compared to their isogenic, wild-type parental strains. To our knowledge, this is the first report demonstrating the influence of biocide-tolerant phenotype to NHS-resistant pattern. Salmonella after repeated exposure to the biocide did not become resistant to antibiotics, but have developed resistance to NHS (Table 4). Hypothesizing, if it came to systemic infection by the bacteria with a cross-resistance to antibacterials and reduced susceptibility to serum, it would have produced treatment failure, because of an inadequate dose of a drug.

After the revision of the literature information on the role of membrane proteins in biocide or antibiotics tolerance, it can be summarized that exposure to triclosan has been associated with an upregulation of AcrAB, a major efflux system [23]. Moreover, Salmonella can survive challenge with in-use concentrations of some biocides; this is due to de-repression of the AcrEF efflux system, and these mutants were MDR [3]. They also included SugE, classically implicated in QACs resistance and frequently found in Salmonella isolates of clinical and animal origin. In this study, we compared the proteomic profile of the $S$. Senftenberg 133 variant $\left(133^{\mathrm{bST}}\right)$ with the reduced susceptibility to triamine and NHS with its isogenic biocide-tolerant counterpart. We have chosen for this analysis $S$. Senftenberg 133, because it was the only one primarily sensitive to HS, belonging to the group of the highest triamine tolerance $(6 \times \mathrm{MIC})$. Intrinsic susceptibility of the tested serovar was essential for evaluation of 2DE analysis since sensitive Salmonella enterica serovars were shown to possess higher levels of flagellar hook-associated protein 2 (FliD) [20]. In our analysis, even though the variant was tolerant to the disinfectant and was sensitive to antibiotics, we have observed four distinct changes in protein patterns related to flagellin $(\mathrm{FliC})$, outer membrane protein assembly factor, chemotaxis response regulator protein-glutamate methylesterase and enolase. Downregulation of flagellin and enolase factor might suggest a lower pathogenicity, including adhesion and invasion of the host cells. On the other hand, over-production of chemotaxis response regulator protein and outer membrane protein assembly factor in S. Senftenberg $133^{\text {bST }}$ could compensate a loss of motility. It has to be stressed that enolase is described as the multifunctional bacterial protein with the unique function of the receptor to human plasminogen. The enolase/plasminogen system is one of the mechanisms facilitating the invasiveness of pathogens, and it plays also an important role in the development of tumor tissues [30]. It seems that the tested biocide might weaken the motility-dependent virulence of $S$. Senftenberg. 


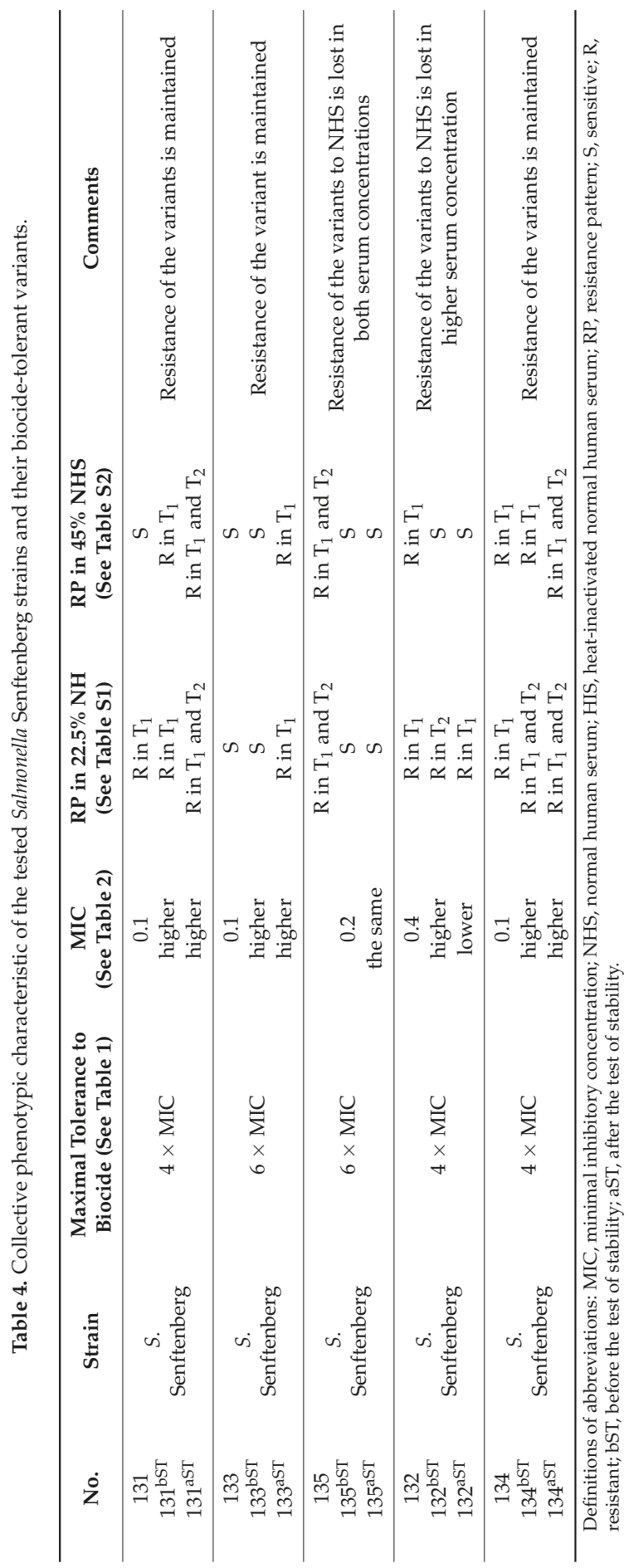


In summary, there is much potential in Salmonella spp. to adjust to hostile environments, where the biocide containing triamine is present; however, the adaptation of the bacteria to the sub-inhibitory disinfectants' concentrations does not always result in the increase of antibiotic resistance. In cases of reduced sensitivity of bacteria to antimicrobials, a good idea would be the use of different disinfectants alternately to minimize the risk of cross-resistance and developing of MDR phenotypes.

\section{Materials and Methods}

\subsection{Bacterial Strains}

Salmonella enterica subsp. enterica serovar Senftenberg strains were isolated from poultry food samples in the period of November-December 2014 at the LAB-VET Veterinary Diagnostic Laboratory (Tarnowo Podgórne, Poland) by the procedures approved by Polish Centre for Accreditation. Bacterial species were serotyped in the National Serotype Salmonella Centre (Gdańsk, Poland). Strains used in this study were as follows: S. Senftenberg 131; S. Senftenberg 132; S. Senftenberg 133; S. Senftenberg 134; S. Senftenberg 135. Strains originated from the collection of the Department of Microbiology at the University of Wrocław (Wrocław, Poland). Variants before the test of stability were marked as bST and after the test of stability as aST.

\subsection{Disinfectants and Antibiotics}

Disinfectant: commercial biocide formulation contained: triamine, 2-aminoethanol, cationic surfactants, nonionic surfactants, potassium carbonate (Amity International, Barnsley, UK) (Table 5). Antibiotics: ciprofloxacin (CIP), co-trimoxazole (STX), cefotaxime (CTX), amoxicillin/clavulanic acid (AMX 30) and ampicillin (AMP) (Thermo Fisher Scientific, Waltham, MA, USA).

\subsection{Antimicrobial Susceptibility}

Parent $S$. Senftenberg strains and their variants were tested with the broth microdilution method to determine MIC and MBC of the biocides according to Andrews et al. [31] with minor modifications. In short, biocide concentrations were prepared in Mueller-Hinton broth (Merck, Kenilworth, NJ, USA) as follows: $204.8,102.4,51.2,25.6,12.8,6.4,3.2,1.6,0.8,0.4,0.2,0.1,0.05,0.025 \mu \mathrm{L} / \mathrm{mL}$ in U-bottom microtitration plates (Medlab, Raszyn, Poland). The adjustment of the bacterial precultures suspension to the density of the $0.5 \mathrm{McF}$ arland standard was performed. Next, the inoculum was adjusted so that $10^{4} \mathrm{CFU} / \mathrm{mL}$ per spot were applied into the wells. The plates were incubated at $37^{\circ} \mathrm{C}$, and finally, MICs were estimated as the lowest concentration of biocide at which there was no visible growth. Either MBC was calculated. MBC was the lowest concentration that demonstrated a significant reduction (such as $99.9 \%$ ) in CFU/mL when compared to the MIC dilution. The testing of bacterial susceptibility to antibiotics was done using disc diffusion and the E-test method. Data interpretation was performed according to the European Committee for Antimicrobial Susceptibility Testing (EUCAST) epidemiological cut-off values and clinical breakpoints [32]. The tests were repeated three times, including appropriate controls. 
Int. J. Mol. Sci. 2017, 18, 1459

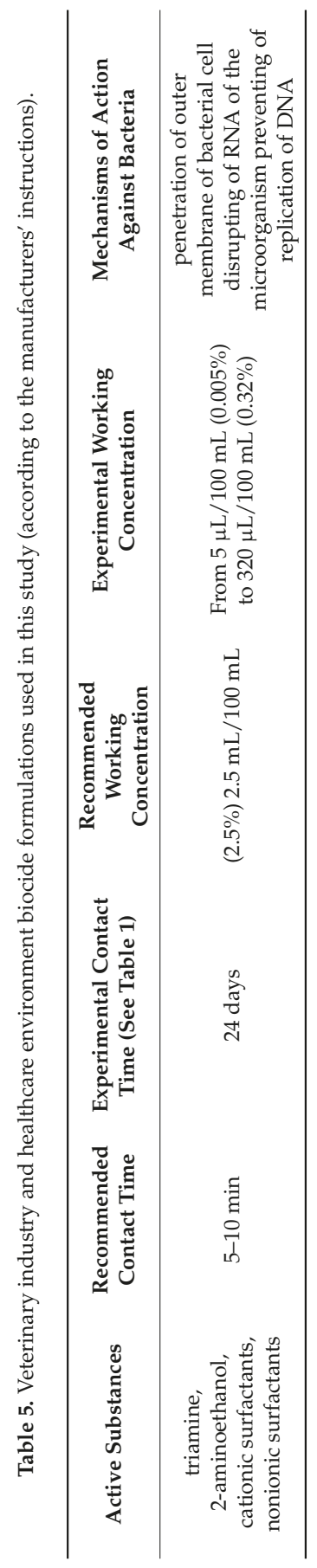




\subsection{Isolation of Biocide Tolerant Variants and the Stability of Their Phenotypes}

Isolation (generation) of variants from each culture of Salmonella was done according to Ricci et al. [33] and Karatzas et al. [22] (Table 1). The test was performed as previously described [8,9]. One-day precultures of the wild-type strains of Salmonella were exposed to subinhibitory concentrations of the disinfectant $(0.5 \times \mathrm{MIC})$ relevant to $0.05,0.1,0.2 \mu \mathrm{L} / \mathrm{mL}$ in dependence of the strain for 7 days; gradually increasing concentrations of the same substance (4 days for each concentration $0.75 \times$ MIC, $1.0 \times$ MIC, $1.25 \times$ MIC, $1.5 \times$ MIC); one-day incubation in LB broth (Merck) containing a 2-fold, 4-fold and 6-fold increase in biocides MICs; and ten days of incubation in LB broth, in the absence of the disinfectant to test the stability of the phenotypes. The tests of the stability of phenotypes were done on the cultures from the highest possible MICs. Three replicates of each concentration were used. Typical Salmonella colonies from the agar plates were transferred into sterile saline to set the density of 0.5 in McFarland standard $\left(2 \times 10^{8}\right.$ cells $)$. Then, an inoculum was created by suspending of $0.1 \mathrm{~mL}$ of the culture in $10 \mathrm{~mL}$ of saline. Next, $9.8 \mathrm{~mL}$ of LB medium, $0.1 \mathrm{~mL}$ of bacterial suspension and $0.1 \mathrm{~mL}$ of a given concentration of the biocide were pipetted into a sterile tube. The concentration of the biocide for each test depended on the value of the MIC estimated at the beginning of the experiments. The cultures were incubated at $37^{\circ} \mathrm{C}$ for $24 \mathrm{~h}$ in a shaking water bath. The cultures of the bacteria were revitalized every day through the collecting of $0.1 \mathrm{~mL}$ bacterial suspension from the previous day's incubation and transferring into fresh LB medium. The whole experiment, to obtain the variants tolerant to triamine-containing disinfectant, took 35 days. To confirm the presence of Salmonella spp. in the study, the cultures of the bacteria were inoculated onto Brilliant Green Lab-Agar (Biocorp, Warszawa, Poland).

\subsection{Serum}

NHS was obtained from Regional Centre of Transfusion Medicine and Blood Bank, Wrocław, Poland. This was conducted according to the principles expressed in the Law on public service of the blood of 20 May 2016 and in the Directive 2002/98/EC of the European Parliament and of the Council of 27 January 2003, establishing standards of quality and safety for the collection, testing, processing, storage and distribution of human blood and blood components. Blood samples were collected into aseptic tubes with clot activator and with gel for serum separation. The samples were then stored at room temperature (RT) for $30 \mathrm{~min}$. After that time, the samples were centrifuged for $5 \mathrm{~min}$ at $4000 \times \mathrm{g}$. Only the serum samples without hemolysis and lipemia were used for experiments. The serum samples were collected, pooled and kept frozen $\left(-70^{\circ} \mathrm{C}\right)$ for a period no longer than 3 months. A suitable volume of serum was thawed immediately before use. Each portion was used only once.

\subsection{Serum C3 Concentration}

The C3 concentration in the pool of NHS was quantified by a radial immunodiffusion test Human Complement C3\&C4 "Nl" Bindarid ${ }^{\mathrm{TM}}$ Radial Immunodiffusion Kit (The Binding Site, Birmingham, UK). C3 protein is thought to be the most important component of the C system [34]. NHS with the proper concentration of C3 glycoprotein (between 970 and $1576 \mathrm{mg} / \mathrm{L}$ ) was used for these studies.

\subsection{Serum Susceptibility Assay}

The bactericidal activity of normal human serum (NHS) was determined as described previously [35] with minor modifications. It was performed in sterile polystyrene U-bottom microtitration plates (Medlab, Raszyn, Polska). S. Senftenberg strains and their biocide variants before and after the test of stability were subjected to the challenge of $22.5 \%$ and $45 \%$ NHS. Serum decomplemented by heating at $56^{\circ} \mathrm{C}$ for $30 \mathrm{~min}$ (heat-inactivated normal human serum (HIS)) was used as a control [36]. After overnight incubation in LB medium (Merck, Kenilworth, NJ, USA), bacteria $(500 \mu \mathrm{L})$ in their early exponential phase were collected by centrifugation $\left(1500 \times g\right.$ for $20 \mathrm{~min}$ at $\left.4{ }^{\circ} \mathrm{C}\right)$. 
The pellet was suspended in $3 \mathrm{~mL}$ of phosphate-buffered saline (PBS) (POCH, Gliwice, Poland) and then diluted in the same saline to produce a suspension of approximately $10^{7}$ cells $/ \mathrm{mL}$. The volume of $20 \mu \mathrm{L}$ of bacterial suspension and $180 \mu \mathrm{L}$ of active or inactivated serum were transferred into the wells of the plates. Each concentration of the serum was loaded in triplicate. Finally, each well contained about $2 \times 10^{5}$ of the bacterial cells. The mixtures were incubated at $37^{\circ} \mathrm{C}$ for 0,15 and $30 \min \left(\mathrm{T}_{0}\right.$, $\mathrm{T}_{1}$ and $\mathrm{T}_{2}$, respectively) on a laboratory shaker with rotation at $20 \mathrm{rpm}$. Appropriate dilutions in the volume of $10 \mu \mathrm{L}$ were then spread in triplicate onto nutrient agar plates (Biocorp, Warszawa, Poland) and incubated at $37^{\circ} \mathrm{C}$ for $24 \mathrm{~h}$. The average number of $\mathrm{CFU} / \mathrm{mL}$ was calculated from the replicate plate counts. The survival rate for $T_{1}$ and $T_{2}$ was calculated as a percentage of the cell count for $T_{0}$ (set at $100 \%$ ). Strains with survival rates below $50 \%$ were considered susceptible to the bactericidal action of NHS, while those with survival rates above $50 \%$ were described as resistant to NHS. Each test was performed three times.

\subsection{Outer Membrane Proteins Isolation and Preparation}

The isolation of OMPs was performed according to Murphy and Bartos [37] with minor modifications [20,38]. Bacterial strains were cultured overnight at $37^{\circ} \mathrm{C}$ in $25 \mathrm{~mL}$ LB medium (Merck, Kenilworth, NJ, USA). The cells from the overnight culture were harvested $\left(1500 \times g\right.$ at $4{ }^{\circ} \mathrm{C}$ for $\left.20 \mathrm{~min}\right)$ and suspended in $1.25 \mathrm{~mL} 1 \mathrm{M}$ sodium acetate (POCH, Gliwice, Polska) with $1 \mathrm{mM} \beta$-mercaptoethanol (Merck). Subsequently, $11.25 \mathrm{~mL}$ water solution containing 5\% (w/v) Zwittergent Z 3-14 (Merck, Kenilworth, NJ, USA) and $0.5 \mathrm{M} \mathrm{CaCl}_{2}(\mathrm{POCH})$ were added. This mixture was stirred at room temperature for $1 \mathrm{~h}$. To precipitate nucleic acids, $3.13 \mathrm{~mL}$ of $96 \%(v / v)$ cold ethanol $(\mathrm{POCH})$ were added very slowly. The mixture was then centrifuged at $17,000 \times \mathrm{g}$ at $4{ }^{\circ} \mathrm{C}$ for $10 \mathrm{~min}$. The proteins were precipitated from the supernatant by the addition of $46.75 \mathrm{~mL}$ of $96 \%(v / v)$ cold ethanol and centrifuged at $17,000 \times \mathrm{g}$ at $4{ }^{\circ} \mathrm{C}$ for $20 \mathrm{~min}$. The pellet was left to dry at $\mathrm{RT}$ and then suspended in $1.5 \mathrm{~mL} 50 \mathrm{mM}$ Trizma-Base (Merck) buffer, $\mathrm{pH} 8.0$ containing $0.05 \%(w / v)$ Zwittergent Z 3-14 and $10 \mathrm{mM}$ EDTA (Merck) and stirred at room temperature for $1 \mathrm{~h}$. The solution was kept at $4{ }^{\circ} \mathrm{C}$ overnight. Insoluble material was removed by centrifugation at $12,000 \times \mathrm{g}$ at $4{ }^{\circ} \mathrm{C}$ for $10 \mathrm{~min}$, with OMPs present in the supernatant. Total protein concentration was measured using a commercial BCA Protein Assay Kit (Thermo Fisher Scientific, Waltham, MA, USA).

\subsection{Two-Dimensional Gel Electrophoresis}

The OMPs were separated with 4-7 pH immobilized gradient strips (IPG $7 \mathrm{~cm}$ ) (Bio-Rad, Hercules, CA, USA). 2-DE was carried out with the Mini-PROTEAN Tetra Cell System (Bio-Rad). Isoelectric focusing (IEF) was conducted by a stepwise increase of voltage as follows: $250 \mathrm{~V}, 20$ min (linear); $4000 \mathrm{~V}, 120 \mathrm{~min}$ (linear); and $4000 \mathrm{~V}$ (rapid); until the total volt-hours reached $14 \mathrm{kVh}$. IPG strips were then loaded onto the top of 1-mm slabs comprised of a $9 \%$ polyacrylamide stacking gel and $12.5 \%$ polyacrylamide separating gel, using $0.5 \%$ agarose (Bio-Rad) with bromophenol blue dye in the running buffer. Electrophoresis was performed at $4{ }^{\circ} \mathrm{C}$ with constant power $(3 \mathrm{~W})$ until the dye front reached the bottom [39-41]. The protein spots were visualized by Coomassie Brilliant Blue (Bio-Rad). Band patterns were visualized under white light and photographed using Gel Doc ${ }^{\mathrm{TM}}$ EZ System (Bio-Rad). Image spots of proteins were analyzed by PDQuest software 8.0.1 (Bio-Rad) [20].

\subsection{In-Gel Protein Digestion and MS Protein Identification}

After isolation, 2-DE separation and staining with the Coomassie Brilliant Blue method, protein spots of interest were excised from the gel and subjected to the in-gel tryptic digestion according to the method described by Shevchenko et al. [42]. Briefly, after destaining $\left(100 \mathrm{mM} \mathrm{NH}_{4} \mathrm{HCO}_{3}\right.$ /acetonitrile, $1: 1, v / v)$, reduction $\left(10 \mathrm{mM}\right.$ dithiothreitol in $100 \mathrm{mM} \mathrm{NH}_{4} \mathrm{HCO}_{3}$ ) and alkylation (55 mM iodoacetamide in $100 \mathrm{mM} \mathrm{NH}_{4} \mathrm{HCO}_{3}$ ), a suitable volume of $13 \mathrm{ng} / \mu \mathrm{L}$ trypsin in $10 \mathrm{mM}$ ammonium bicarbonate containing $10 \%(v / v)$ acetonitrile was added to the excised gel spot cut into cubes. The obtained peptides were extracted from the gel, concentrated and desalted with the Pierce C18 tip and 
subjected to mass spectrometry analysis using the MALDI-TOF ultrafleXtreme instrument (Bruker, Bremen, Germany). Ten milligrams per milliliter of $\alpha$-cyano-4-hydroxycinnamic acid (Bruker) in acetonitrile/0.1\% TFA in $\mathrm{H}_{2} \mathrm{O}(1: 1, v / v)$ were used as the eluent of peptides from the Pierce C18 tip directly on an MALDI plate. Spectra were acquired in positive reflector mode, averaging 2000 laser shots per MALDI-TOF spectrum. OMPs identification was achieved using a bioinformatics platform (ProteinScape 3.0., Bruker) and MASCOT (Matrix Science, 2.3.02) as a search engine to search protein sequence databases (NCBI, Swiss-Prot, date of access 10/03/2017) using the peptide mass fingerprinting method. All solvents used for digestion, MS preparation and analysis were of LC-MS grade and purchased from Merck Millipore (Billerica, MA, USA). Ammonium bicarbonate eluent additive for LC-MS, dithiothreitol and iodoacetamide were from Sigma-Aldrich (Saint Louis, MO, USA). Sequencing-grade modified trypsin was obtained from Promega (Madison, WI, USA).

Supplementary Materials: Supplementary materials can be found at www.mdpi.com/1422-0067/18/7/1459/s1.

Acknowledgments: The authors thank Jarosław Wilczyński from LAB-VET Veterinary Diagnostic Laboratory in Tarnowo Podgórne (Poland) for Salmonella strains. We also direct special thanks to Dr. Katarzyna Guz-Regner and Dr. Aleksandra Pawlak for their surveillance during performing preliminary serum bactericidal assays by Martyna Wańczyk. The present work was financed by the European Union under the framework of the European Social Fund No. BPZ.506.50.2012.MS and University of Wrocław Grant No. 1213/M/IGM/15. Publication was also supported by Wrocław Centre of Biotechnology, program The Leading National Research Centre (KNOW, Krajowy Narodowy Ośrodek Wiodacy) for the years 2014-2018. The funding agencies had no direct role in the conduct of the study, the collection, management, statistical analysis and interpretation of the data, preparation nor approval of the manuscript.

Author Contributions: Bożena Futoma-Kołoch, Jacek Rybka and Gabriela Bugla-Płoskońska conceived of and designed the experiments. Bożena Futoma-Kołoch obtained funding from The European Social Fund No. BPZ.506.50.2012.MS. Bożena Futoma-Kołoch and Gabriela Bugla-Płoskońska obtained funding from University of Wrocław Grant No. 1213/M/IGM/15. Bożena Futoma-Kołoch, Bartłomiej Dudek, Katarzyna Kapczyńska, Eva Krzyżewska, Kamila Korzekwa and Martyna Wańczyk performed the experiments. Bożena Futoma-Kołoch, Bartłomiej Dudek, Katarzyna Kapczyńska, Eva Krzyżewska and Gabriela Bugla-Płoskońska analyzed the data. Bożena Futoma-Kołoch, Barłłomiej Dudek, Jacek Rybka, Elżbieta Klausa and Gabriela Bugla-Płoskońska contributed reagents/materials/analysis tools. Bożena Futoma-Kołoch wrote the paper. Gabriela Bugla-Płoskońska and Jacek Rybka provided study supervision. All co-authors revised and approved the final manuscript.

Conflicts of Interest: The authors declare no conflict of interest.

\begin{tabular}{ll}
\multicolumn{2}{l}{ Abbreviations } \\
aST & After the test of stability \\
bST & Before the test of stability \\
CFU & Colony-forming units \\
h & Hours \\
HIS & Heat-inactivated normal human serum \\
MDR & Multidrug-resistant \\
MIC & Minimal inhibitory concentration \\
MBC & Minimal bactericidal concentration \\
NHS & Normal human serum \\
OMPs & Outer membrane proteins \\
RP & Resistance pattern \\
QAC & Quaternary ammonium salts \\
R & Resistant \\
S & Sensitive \\
2-DE & Two-dimensional gel electrophoresis
\end{tabular}




\section{References}

1. Ortega Morente, E.; Fernández-Fuentes, M.A.; Grande Burgos, M.J.; Abriouel, H.; Pérez Pulido, R.; Gálvez, A. Biocide tolerance in bacteria. Int. J. Food Microbiol. 2013, 162, 13-25. [CrossRef] [PubMed]

2. Shengzhi, Y.; Guoyan, W.; Mei, L.; Wenwen, D.; Hongning, W.; Likou, Z. Antibiotic and disinfectant resistance of Salmonella isolated from egg production chains. Yi Chuan 2016, 38, 948-956. [PubMed]

3. Whitehead, R.N.; Overton, T.W.; Kemp, C.L.; Webber, M.A. Exposure of Salmonella enterica serovar Typhimurium to high level biocide challenge can select multidrug resistant mutants in a single step. PLoS ONE 2011, 6, e22833. [CrossRef] [PubMed]

4. Gadea, R.; Glibota, N.; Pérez Pulido, R.; Gálvez, A.; Ortega, E. Adaptation to biocides cetrimide and chlorhexidine in bacteria from organic foods: Association with tolerance to other antimicrobials and physical stresses. J. Agric. Food Chem. 2017, 65, 1758-1770. [CrossRef] [PubMed]

5. Silha, D.; Silhová, L.; Vytrasová, J. Survival of selected bacteria of Arcobacter genus in disinfectants and possibility of acquired secondary resistance to disinfectants. J. Microb. Biotech. Food Sci. 2016, 5, 326-329. [CrossRef]

6. Curiao, T.; Marchi, E.; Grandgirard, D.; León-Sampedro, R.; Viti, C.; Leib, S.L.; Baquero, F.; Oggioni, M.R.; Martinez, J.L.; Coque, T.M. Multiple adaptive routes of Salmonella enterica Typhimurium to biocide and antibiotic exposure. BMC Genom. 2016, 17, 491-507. [CrossRef] [PubMed]

7. Majtánová, L.; Majtán, V. Effect of disinfectants on surface hydrophobicity and mobility in Salmonella enterica serovar Typhimurium DT104. Ceska Slov. Farm. 2003, 52, 141-147. [PubMed]

8. Futoma-Kołoch, B.; Ksiażczyk, M.; Korzekwa, K.; Migdał, I.; Pawlak, A.; Jankowska, M.; Kędziora, A.; Dorotkiewicz-Jach, A.; Bugla-Płoskońska, G. Selection and electrophoretic characterization of Salmonella enterica subsp. enterica biocide variants resistant to antibiotics. Pol. J. Vet. Sci. 2015, 18, 725-732.

9. Futoma-Kołoch, B.; Ksiażczyk, M. The risk of Salmonella resistance following exposure to common disinfectants: An emerging problem. Biol. Int. 2013, 53, 54-66.

10. Majtán, V.; Majtánová, L. Effect of disinfectants on the metabolism of Salmonella enterica serovar enteritidis. Folia Microbiol. 2003, 48, 643-648. [CrossRef]

11. Uzer Celik, E.; Tunac, A.T.; Ates, M.; Sen, B.H. Antimicrobial activity of different disinfectants against cariogenic microorganisms. Braz. Oral Res. 2016, 30, e125. [CrossRef] [PubMed]

12. Chapman, J.S. Biocide resistance mechanisms. Int. Biod. Biodegr. 2003, 51, 133-138. [CrossRef]

13. Randall, L.P.; Coles, S.W.; Coldham, N.G.; Penuela, L.G.; Mott, A.C.; Woodward, M.J.; Piddock, L.J.V.; Webber, M. Commonly used farm disinfectants can select for mutant Salmonella enterica serovar Typhimurium with decreased susceptibility to biocides and antibiotics without compromising virulence. J. Antimicrob. Chemother. 2007, 60, 1273-1280. [CrossRef] [PubMed]

14. Son, M.S.; Del Castilho, C.; Duncalf, K.A.; Carney, D.; Weiner, J.H.; Turner, R.J. Mutagenesis of SugE, a small multidrug resistance protein. Biochem. Biophys. Res. Commun. 2003, 312, 914-921. [CrossRef] [PubMed]

15. Zou, L.; Meng, J.; McDermott, P.F.; Wang, F.; Yang, Q.; Cao, G.; Hoffmann, M.; Zhao, S. Presence of disinfectant resistance genes in Escherichia coli isolated from retail meats in the USA. J. Antimicrob. Chemother. 2014, 69, 2644-2649. [CrossRef] [PubMed]

16. Kariuki, S.; Gordon, M.A.; Feasey, N.; Parry, C.M. Antimicrobial resistance and management of invasive Salmonella disease. Vaccine 2015, 33, C21-C29. [CrossRef] [PubMed]

17. Futoma-Kołoch, B.; Bugla-Płoskońska, G.; Sarowska, J. Searching for outer membrane proteins typical of serum-sensitive and serum-resistant phenotypes of Salmonella. In Salmonella-Distribution, Adaptation, Control Measures, and Molecular Technologies; Annous, B.A., Ed.; InTech: Rijeka, Croatia, 2012; pp. 265-290.

18. Riva, R.; Korhonen, T.K.; Meri, S. The outer membrane protease PgtE of Salmonella enterica interferes with the alternative complement pathway by cleaving factors B and H. Front. Microbiol. 2015, 6, 63. [CrossRef] [PubMed]

19. Futoma-Kołoch, B.; Godlewska, U.; Guz-Regner, K.; Dorotkiewicz-Jach, A.; Klausa, E.; Rybka, J.; Bugla-Płoskońska, G. Presumable role of outer membrane proteins of Salmonella containing sialylated lipopolysaccharides serovar Ngozi, sv. Isaszeg and subspecies arizonae in determining susceptibility to human serum. Gut Pathog. 2015, 7, 18. [CrossRef] [PubMed] 
20. Dudek, B.; Krzyżewska, E.; Kapczyńska, K.; Rybka, J.; Pawlak, A.; Korzekwa, K.; Klausa, E.; Bugla-Płoskońska, G. Proteomic analysis of outer membrane proteins from Salmonella Enteritidis strains with different sensitivity to human serum. PLOS ONE 2016, 11, e0164069. [CrossRef] [PubMed]

21. McDonnell, G.; Russell, A.D. Antiseptics and disinfectants: Activity, action, and resistance. Clin. Microbiol. Rev. 1999, 12, 147-179. [PubMed]

22. Karatzas, K.A.G.; Randall, L.P.; Webber, M.; Piddock, L.J.V.; Humphrey, T.J.; Woodward, M.J.; Coldham, N.G. Phenotypic and proteomic characterization of MAR variants of Salmonella enterica serovar Typhimurium selected following exposure to disinfectants. Appl. Environ. Microbiol. 2007, 74, 1508-1516. [CrossRef] [PubMed]

23. Condell, O.; Sheridan, Á.; Power, K.A.; Bonilla-Santiago, R.; Sergeant, K.; Renault, J.; Burgess, C.; Fanning, S.; Nally, J.E. Comparative proteomic analysis of Salmonella tolerance to the biocide active agent triclosan. J. Proteom. 2012, 75, 4505-4519. [CrossRef] [PubMed]

24. Roy, V.; Adams, B.L.; Bentley, W.E. Developing next generation antimicrobials by intercepting AI-2 mediated quorum sensing. Enz. Microb. Technol. 2011, 49, 113-123. [CrossRef] [PubMed]

25. Müderris, T.; Ürkmez, F.Y.; Küçüker, Ş.A.; Sağlam, M.F.; Yılmaz, G.R.; Güner, R.; Güleşen, R.; Açıkgöz, Z.C. Bacteremia caused by ciprofloxacin-resistant Salmonella serotype Kentucky: A case report and the review of literature. Mikrobiyol. Bull. 2016, 50, 598-605.

26. Bravo, D.; Silva, C.; Carter, J.A.; Hoare, A.; Alvarez, S.A.; Blondel, C.J.; Zaldívar, M.; Valvano, M.A.; Contreras, I. Growth-phase regulation of lipopolysaccharide O-antigen chain length influences serum resistance in serovars of Salmonella. J. Med. Microbiol. 2008, 57, 938-946. [CrossRef] [PubMed]

27. Marshall, J.M.; Gunn, J.S. The O-antigen capsule of Salmonella enterica serovar Typhimurium facilitates serum resistance and surface expression of FliC. Infect. Immun. 2015, 83, 3946-3959. [CrossRef] [PubMed]

28. Hart, P.J.; O'Shaughnessy, C.M.; Siggins, M.K.; Bobat, S.; Kingsley, R.A.; Goulding, D.A.; Crump, J.A.; Reyburn, H.; Micoli, F.; Dougan, G.; et al. Differential killing of Salmonella enterica serovar Typhi by antibodies targeting Vi and lipopolysaccharide O:9 antigen. PLoS ONE 2016, 11, e0145945. [CrossRef] [PubMed]

29. Wanga, W.; Jeffery, C.J. An analysis of surface proteomics results reveals novel candidates for intracellular/surface moonlighting proteins in bacteria. Mol. BioSyst. 2016, 12, 1420-1431. [CrossRef] [PubMed]

30. Seweryn, E.; Pietkiewicz, J.; Szamborska, A.; Gamian, A. Enolase on the surface of prockaryotic and eukaryotic cells is a receptor for human plasminogen. Post. Hig. Med. Dośw. 2007, 61, 672-682.

31. Andrews, J.M. Determination of minimum inhibitory concentrations. J. Antimicrob. Chemother. 2001, 48 (Suppl. 1), 5-16. [CrossRef] [PubMed]

32. European Committee on Antimicrobial Susceptibility Testing. 2015, pp. 1-78. Available online: http://www.eucast.org/.

33. Ricci, V.; Tzakas, P.; Buckley, A.; Piddock, L.J.V. Ciprofloxacin-resistant Salmonella enterica serovar Typhimurium strains are difficult to select in the absence of AcrB and TolC. Antimicrob. Agents Chemother. 2006, 50, 38-42. [CrossRef] [PubMed]

34. Futoma-Kołoch, B.; Godlewska, U.; Bugla-Płoskońska, G.; Pawlak, A. Bacterial cell surface-Place where C3 complement activation occurs. 13th Conference Molecular biology in diagnostics of infectious diseases and biotechnology. Diag. Mol. 2012, 120-123.

35. Bugla-Płoskońska, G.; Rybka, J.; Futoma-Kołoch, B.; Cisowska, A.; Gamian, A.; Doroszkiewicz, W. Sialic acid-containing lipopolysaccharides of Salmonella $\mathrm{O} 48$ strains-potential role in camouflage and susceptibility to the bactericidal effect of normal human serum. Microb. Ecol. 2010, 59, 601-613. [CrossRef] [PubMed]

36. Doroszkiewicz, W. Mechanism of antigenic variation in Shigella flexneri bacilli. IV. Role of lipopolysaccharides and their components in the sensitivity of Shigella flexneri $1 \mathrm{~b}$ and its Lac+ recombinant to killing action of serum. Arch. Immunol. Ther. Exp. 1997, 45, 235-242.

37. Murphy, T.F.; Bartos, L.C. Surface-exposed and antigenically conserved determinants of outer membrane proteins of Branhamella catarrhalis. Infect. Immun. 1989, 57, 2938-2941. [PubMed]

38. Bugla-Płoskońska, G.; Korzeniowska-Kowal, A.; Guz-Regner, K. Reptiles as a source of Salmonella O48-clinically important bacteria for children: The relationship between resistance to normal cord serum and outer membrane protein patterns. Microb. Ecol. 2011, 61, 41-51. [CrossRef] [PubMed] 
39. O'Farrell, P.H. High resolution two-dimensional electrophoresis of proteins. J. Biol. Chem. 1975, 250, 4007-4021. [PubMed]

40. Bednarz-Misa, I.; Serek, P.; Dudek, B.; Pawlak, A.; Bugla-Płoskońska, G.; Gamian, A. Application of zwitterionic detergent to the solubilization of Klebsiella pneumoniae outer membrane proteins for two-dimensional gel electrophoresis. J. Microbiol. Methods 2014, 107, 74-79. [CrossRef] [PubMed]

41. Bugla-Płoskońska, G.; Futoma-Kołoch, B.; Skwara, A.; Doroszkiewicz, W. Use of zwitterionic type of detergent in isolation of Escherichia coli O56 outer membrane proteins improves their two-dimensional electrophoresis (2-DE). Pol. J. Microbiol. 2009, 58, 205-209. [PubMed]

42. Shevchenko, A.; Tomas, H.; Havlis, J.; Olsen, J.V.; Mann, M. In-gel digestion for mass spectrometric characterization of proteins and proteomes. Nat. Protoc. 2006, 1, 2856-2860. [CrossRef] [PubMed]

(C) 2017 by the authors. Licensee MDPI, Basel, Switzerland. This article is an open access article distributed under the terms and conditions of the Creative Commons Attribution (CC BY) license (http://creativecommons.org/licenses/by/4.0/). 
Article

\title{
Prediction of Protein Hotspots from Whole Protein Sequences by a Random Projection Ensemble System
}

\author{
Jinjian Jiang ${ }^{1,2}$, Nian Wang ${ }^{1}$, Peng Chen ${ }^{3, *}$, Chunhou Zheng ${ }^{4}$ and Bing Wang ${ }^{5, *}$ \\ 1 School of Electronics and Information Engineering, Anhui University, Hefei 230601, China; \\ jiangjj@aqnu.edu.cn (J.J.); wn_xlb@ahu.edu.cn (N.W.) \\ 2 School of Computer and Information, Anqing Normal University, Anqing 246133, China \\ 3 Institute of Health Sciences, Anhui University, Hefei 230601, China \\ 4 School of Electronic Engineering \& Automation, Anhui University, Hefei 230601, China; zhengch99@126.com \\ 5 School of Electrical and Information Engineering, Anhui University of Technology, Ma'anshan 243032, China \\ * Correspondence: pchen@ahu.edu.cn (P.C.); wangbing@ustc.edu (B.W.); Tel.: +86-551-6386-1469 (P.C.)
}

Received: 7 May 2017; Accepted: 5 July 2017; Published: 18 July 2017

\begin{abstract}
Hotspot residues are important in the determination of protein-protein interactions, and they always perform specific functions in biological processes. The determination of hotspot residues is by the commonly-used method of alanine scanning mutagenesis experiments, which is always costly and time consuming. To address this issue, computational methods have been developed. Most of them are structure based, i.e., using the information of solved protein structures. However, the number of solved protein structures is extremely less than that of sequences. Moreover, almost all of the predictors identified hotspots from the interfaces of protein complexes, seldom from the whole protein sequences. Therefore, determining hotspots from whole protein sequences by sequence information alone is urgent. To address the issue of hotspot predictions from the whole sequences of proteins, we proposed an ensemble system with random projections using statistical physicochemical properties of amino acids. First, an encoding scheme involving sequence profiles of residues and physicochemical properties from the AAindex1 dataset is developed. Then, the random projection technique was adopted to project the encoding instances into a reduced space. Then, several better random projections were obtained by training an IBk classifier based on the training dataset, which were thus applied to the test dataset. The ensemble of random projection classifiers is therefore obtained. Experimental results showed that although the performance of our method is not good enough for real applications of hotspots, it is very promising in the determination of hotspot residues from whole sequences.
\end{abstract}

Keywords: random projection; hot spots; IBk; ensemble system

\section{Introduction}

Hotspot residues contribute a large portion of the binding energy of one protein in complex with another protein $[1,2]$, which are always surrounded by residues contributing less binding energy. These are not uniformly distributed for the binding energy of proteins over their interaction surfaces [1]. Hotspots are important in the binding and the stability of protein-protein interactions and thus key to perform specific functions in the protein [3,4]. Actually, hotspots are difficult to determine. A common determination method is the method of alanine scanning mutagenesis experiments, which identify a hotspot if a change in its binding free energy is larger than a predefined threshold when the residue is mutated to alanine. However, this method is costly and time consuming.

Several databases stored experimental and computational hotspot residues and the details of hotspots' properties. The first database for storing experimental hotspots was the Alanine Scanning Energetics Database (ASEdb) by the use of alanine scanning energetics experiments [5]. Another 
database is the Binding Interface Database (BID) developed by Fischer et al., which mined the primary scientific literature for detailed data about protein interfaces [6]. These databases are commonly used in previous works on hotspot identification. The Protein-protein Interactions Thermodynamic Database (PINT) is another database that mainly accumulates the thermodynamic data of interacting proteins upon binding along with all of the experimentally-measured thermodynamic data (Kd, Ka, $\Delta \mathrm{G}, \Delta \mathrm{H}$ and $\Delta \mathrm{Cp}$ ) for wild-type and mutant proteins [7]. It contains 1513 entries in 129 protein-protein complexes from 72 original research articles, where only 33 entries have complete 3D structures deposited in PDB (Protein Data Bank), in the first release of PINT. Recently, Moal et al. built the SKEMPI (Structural Kinetic and Energetic database of Mutant Protein Interactions) that has collected 3047 binding free energy changes from 85 protein-protein complexes from the literature [8].

Although some databases stored hotspot residues, few of the protein complexes were solved. Computational approaches were proposed to identify hotspot residues, and they were complementary to the experimental methods. Some methods predicted hotspots by energy function-based physical models [3,9-11], molecular dynamics simulation-based approaches [12-14], evolutionary conservation-based methods $[4,15,16]$ and docking-based methods $[17,18]$. Some methods adopted machine learning methods for the hotspot prediction, such as graph-based approaches [19], neural network [20], decision tree [3,21], SVM (Support Vector Machine) [22], random forest [23] and the consensus of different machine learning methods [24], combining features of solvent accessibility, conservation, sequence profiles and pairing potential [20,23,25-29].

All of the previous methods were developed to identify hotspots from a part of residues in the interface regions. They always worked on selected datasets containing almost the same numbers of hotspots and non-hotspots. The ratio of the number of hotspots to that of residues in whole datasets is around $20 \sim 50 \%$, for example: BID contains 54 hotspots and 58 non-hotspots; 58 hotspots and 91 non-hotspots are in the ASEdb dataset; and SKEMPI contains 196 hotspots and 777 non-hotspots [29]. However, no more than $2 \%$ of the residues in whole protein sequences are hotspots. The issue of identifying hotspots from whole protein sequences in our study is more difficult than others, but more interesting.

Most hotspot prediction methods are structure-based, which cannot be applied to protein complexes without the information of protein structures $[3,22,23]$. Therefore, identifying hotspots from the protein sequence only is important. Moreover, few works identified hotspot residues from the whole protein sequences. To address these issues, here, we propose a method that predicts hotspots from the whole protein sequences using physicochemical characteristics extracted from amino acid sequences. A random projection ensemble classifier system is developed for the hotspot predictions. The system involves an encoding scheme integrating sequence profiles of residues and the statistical physicochemical properties of amino acids from the AAindex1 (Amino Acid index1 database) dataset. Then, the random projection technique was adopted to obtain a reduced input space, but to retain the structure of the original space. Several better classifiers with the IBk algorithm are obtained after the use of random projections. The ensemble of good classifiers is therefore constructed. Experimental results showed that our method performs well in hotspot predictions for the whole protein sequences.

\section{Results}

\subsection{Performance of the Hotspot Prediction}

In the running of the random projection-based classifier, different random projections in Equation (1) construct different classifiers. After running the classifier 100 times, 100 classifiers with random projections $R$ are formed and trained on the training subset $D_{t r}^{k}$. As a result, 100 predictions are obtained. All of the classifiers are ranked in terms of the prediction measure $F 1$. The ensemble of several top $N$ classifiers is then tested on the test subset $D_{t s}^{k}$. In this work, the ASEdb0 is regarded as the training dataset, and the test dataset is BID0; while the predictions on the ASEdb0 dataset are also tested by training on the BID0 dataset. 
Table 1 shows the performance of the top individual classifiers trained by the ASEdb0 dataset and the prediction performance on the BID0 dataset. The individual classifiers are ranked in terms of the F1 measure in the training process. The top classifiers yield good predictions on the BID0 dataset. It achieves an F1 of 0.109 , as well as a precision of 0.069 at a sensitivity of 0.259 in the training process and, therefore, yields an F1 of 0.315 , as well as a precision of 0.220 at a sensitivity of 0.558 in the test process. Here, the dimensionality of the original data is reduced from 7072 to only five.

Table 2 shows the performance comparison of the ensembles of the top $N$ classifiers. In the classifier ensemble, the majority vote technique was applied to the ensemble, i.e., one residue will be identified as the hotspot if half of the $N$ classifiers predict it to be the hotspot. Here, seven ensembles of the number of top classifiers are listed, i.e., 2, 3, 5, 10, 15, 25 and 50. From Table 2, it can be seen that the ensemble of the top three classifiers with the majority vote yields a good performance compared with other classifier ensembles. It yields an MCC (Matthews Correlation Coefficient) of 0.428, as well as a precision of 0.245 at a sensitivity of 0.793 , for testing on the ASEdb0 dataset by training on the BID0 dataset; and it yields an MCC of 0.601 , as well as a precision of 0.440 at a sensitivity of 0.846 , for testing on the BID0 dataset by training on the ASEdb0 dataset. The ensemble of the top three classifiers resulted in a dramatic improvement, compared with the top three individual classifiers. The reason for the improvement is most likely in that a suitable random projection makes the classifier more diverse, where the detailed results are not shown here. Previous methods also showed that the ensemble of more diverse classifiers yielded more efficient predictions [30].

It seems that the more top classifiers the ensemble contains, the worse the ensemble performs. The ensemble with the top 50 classifiers performs the worst both for testing on the ASEdb0 and the BID0 dataset. Therefore, a suitable number of top classifiers can improve the predictions of hotspot residues. Moreover, our method on the BID0 dataset performs better than that on the ASEdb0 dataset, maybe because of the larger ratio of hotspots to the total residues in BID0 $(1.831 \%)$ than that in ASEdb0 (1.445\%).

Table 1. Prediction performance of individual classifiers with the reduced dimension of 5 on the Binding Interface Database 0 (BID0) test dataset training by Alanine Scanning Energetics Database 0 (ASEdb) dataset. There are 50 top individual classifiers listed here for a simple comparison between classifiers. Here measures of "Sen", "Prec", "F1" and "MCC" denote Sensitivity, Precision, F-Measure, and Matthews Correlation Coefficient, respectively.

\begin{tabular}{ccccccccc}
\hline \multirow{3}{*}{ No. } & \multicolumn{9}{c}{ Training } & \multicolumn{4}{c}{ Test } \\
\cline { 2 - 9 } & Sen & MCC & Prec & F1 & Sen & MCC & Prec & F1 \\
\hline 1 & 0.259 & 0.110 & 0.069 & 0.109 & 0.558 & 0.332 & 0.220 & 0.315 \\
2 & 0.069 & 0.125 & 0.250 & 0.108 & 0.558 & 0.357 & 0.250 & 0.345 \\
3 & 0.138 & 0.080 & 0.070 & 0.093 & 0.212 & 0.141 & 0.122 & 0.155 \\
4 & 0.069 & 0.085 & 0.129 & 0.090 & 0.500 & 0.274 & 0.173 & 0.257 \\
5 & 0.121 & 0.075 & 0.071 & 0.089 & 0.308 & 0.194 & 0.150 & 0.201 \\
6 & 0.069 & 0.083 & 0.125 & 0.089 & 0.096 & 0.040 & 0.044 & 0.060 \\
7 & 0.069 & 0.076 & 0.108 & 0.084 & 0.269 & 0.136 & 0.096 & 0.141 \\
8 & 0.069 & 0.076 & 0.108 & 0.084 & 0.269 & 0.129 & 0.090 & 0.135 \\
9 & 0.138 & 0.071 & 0.061 & 0.084 & 0.558 & 0.364 & 0.259 & 0.354 \\
10 & 0.138 & 0.069 & 0.058 & 0.082 & 0.346 & 0.226 & 0.173 & 0.231 \\
11 & 0.069 & 0.071 & 0.098 & 0.081 & 0.135 & 0.038 & 0.037 & 0.058 \\
12 & 0.086 & 0.066 & 0.075 & 0.080 & 0.615 & 0.337 & 0.205 & 0.308 \\
13 & 0.052 & 0.080 & 0.150 & 0.077 & 0.577 & 0.317 & 0.196 & 0.293 \\
14 & 0.052 & 0.076 & 0.136 & 0.075 & 0.404 & 0.227 & 0.153 & 0.222 \\
15 & 0.069 & 0.064 & 0.083 & 0.075 & 0.135 & 0.082 & 0.080 & 0.100 \\
16 & 0.052 & 0.074 & 0.130 & 0.074 & 0.577 & 0.323 & 0.203 & 0.300 \\
17 & 0.052 & 0.074 & 0.130 & 0.074 & 0.596 & 0.279 & 0.153 & 0.243 \\
18 & 0.069 & 0.062 & 0.080 & 0.074 & 0.404 & 0.225 & 0.151 & 0.220 \\
\hline
\end{tabular}


Table 1. Cont.

\begin{tabular}{ccccccccc}
\hline & \multicolumn{9}{c}{ Training } & & \multicolumn{4}{c}{ Test } \\
\cline { 2 - 9 } No. & MCC & Prec & F1 & Sen & MCC & Prec & F1 \\
\hline 19 & 0.069 & 0.062 & 0.080 & 0.074 & 0.308 & 0.152 & 0.102 & 0.153 \\
20 & 0.052 & 0.072 & 0.125 & 0.073 & 0.115 & 0.030 & 0.033 & 0.052 \\
21 & 0.121 & 0.058 & 0.052 & 0.073 & 0.192 & 0.135 & 0.123 & 0.150 \\
22 & 0.052 & 0.067 & 0.111 & 0.071 & 0.288 & 0.150 & 0.105 & 0.154 \\
23 & 0.190 & 0.064 & 0.044 & 0.071 & 0.577 & 0.281 & 0.159 & 0.249 \\
24 & 0.069 & 0.056 & 0.070 & 0.070 & 0.269 & 0.145 & 0.105 & 0.151 \\
25 & 0.086 & 0.054 & 0.057 & 0.069 & 0.423 & 0.171 & 0.095 & 0.155 \\
26 & 0.086 & 0.053 & 0.057 & 0.068 & 0.212 & 0.079 & 0.057 & 0.090 \\
27 & 0.086 & 0.051 & 0.054 & 0.066 & 0.365 & 0.218 & 0.156 & 0.218 \\
28 & 0.052 & 0.058 & 0.091 & 0.066 & 0.250 & 0.091 & 0.060 & 0.097 \\
29 & 0.052 & 0.057 & 0.088 & 0.065 & 0.481 & 0.237 & 0.141 & 0.218 \\
30 & 0.034 & 0.095 & 0.286 & 0.062 & 0.519 & 0.241 & 0.136 & 0.215 \\
31 & 0.034 & 0.095 & 0.286 & 0.062 & 0.346 & 0.204 & 0.146 & 0.206 \\
32 & 0.052 & 0.050 & 0.073 & 0.061 & 0.173 & 0.095 & 0.081 & 0.110 \\
33 & 0.138 & 0.048 & 0.039 & 0.061 & 0.442 & 0.271 & 0.190 & 0.266 \\
34 & 0.052 & 0.049 & 0.071 & 0.060 & 0.231 & 0.115 & 0.085 & 0.124 \\
35 & 0.224 & 0.055 & 0.035 & 0.060 & 0.346 & 0.186 & 0.127 & 0.186 \\
36 & 0.034 & 0.078 & 0.200 & 0.059 & 0.250 & 0.161 & 0.131 & 0.172 \\
37 & 0.207 & 0.052 & 0.034 & 0.059 & 0.519 & 0.273 & 0.167 & 0.252 \\
38 & 0.034 & 0.074 & 0.182 & 0.058 & 0.365 & 0.238 & 0.181 & 0.242 \\
39 & 0.034 & 0.064 & 0.143 & 0.056 & 0.192 & 0.083 & 0.064 & 0.096 \\
40 & 0.052 & 0.044 & 0.061 & 0.056 & 0.231 & 0.146 & 0.120 & 0.158 \\
41 & 0.052 & 0.042 & 0.059 & 0.055 & 0.135 & 0.070 & 0.065 & 0.088 \\
42 & 0.103 & 0.038 & 0.036 & 0.054 & 0.327 & 0.145 & 0.091 & 0.143 \\
43 & 0.103 & 0.037 & 0.036 & 0.053 & 0.192 & 0.111 & 0.093 & 0.125 \\
44 & 0.034 & 0.049 & 0.095 & 0.051 & 0.077 & 0.013 & 0.025 & 0.037 \\
45 & 0.069 & 0.035 & 0.040 & 0.051 & 0.154 & 0.054 & 0.046 & 0.071 \\
46 & 0.121 & 0.034 & 0.031 & 0.050 & 0.423 & 0.231 & 0.151 & 0.222 \\
47 & 0.224 & 0.041 & 0.028 & 0.050 & 0.288 & 0.172 & 0.129 & 0.179 \\
48 & 0.241 & 0.037 & 0.026 & 0.046 & 0.308 & 0.152 & 0.102 & 0.153 \\
49 & 0.052 & 0.030 & 0.040 & 0.045 & 0.442 & 0.210 & 0.125 & 0.195 \\
50 & 0.155 & 0.031 & 0.026 & 0.045 & 0.462 & 0.252 & 0.162 & 0.240 \\
\hline & & & & & & & &
\end{tabular}

Table 2. Prediction performance of the ensemble of the top $N$ classifiers with reduced instance dimension of 5 on the two datasets.

\begin{tabular}{cccccc}
\hline Test Set & No. Dimension & Sen & MCC & Prec & F1 \\
\hline \multirow{4}{*}{ ASEdb0 } & 2 & 0.224 & 0.322 & 0.481 & 0.306 \\
& 3 & 0.793 & 0.428 & 0.245 & 0.374 \\
& 5 & 0.897 & 0.383 & 0.177 & 0.295 \\
& 10 & 1.000 & 0.299 & 0.103 & 0.186 \\
& 15 & 1.000 & 0.219 & 0.062 & 0.116 \\
& 25 & 1.000 & 0.149 & 0.036 & 0.070 \\
BID0 & 50 & 1.000 & 0.081 & 0.021 & 0.041 \\
\hline & 2 & 0.385 & 0.260 & 0.200 & 0.263 \\
& 3 & 0.846 & 0.601 & 0.440 & 0.579 \\
& 10 & 1.000 & 0.461 & 0.226 & 0.369 \\
& 15 & 1.000 & 0.283 & 0.096 & 0.175 \\
& 25 & 1.000 & 0.222 & 0.066 & 0.124 \\
& 50 & 1.000 & 0.145 & 0.038 & 0.074 \\
& 1.000 & 0.078 & 0.024 & 0.046 \\
\hline
\end{tabular}

Furthermore, the performance comparison of ensembles with different numbers of reduced instance dimensions by the random projection technique was investigated. The ensembles of random 
projections with seven reduced dimensions were built, i.e., the dimensions of 1, 2, 5, 10, 20, 50 and 100. The ensemble with the reduced dimension of five performs the best among the seven ensembles, while the ensemble of the top three classifiers with instance dimension of one also performs well in the hotspot predictions for the whole sequences of proteins, which yields an MCC of 0.475 , as well as a precision of 0.704 at a sensitivity of 0.328 . Table 3 shows the performance comparison of the classifier ensemble with different numbers of reduced dimensions on the BID0 test dataset.

Table 3. Prediction performance of the ensemble of the top 3 classifiers with different reduced instance dimensions on the BID0 test dataset.

\begin{tabular}{ccccc}
\hline No. Dimension & Sen & MCC & Prec & F1 \\
\hline 1 & 0.328 & 0.475 & 0.704 & 0.447 \\
2 & 0.328 & 0.352 & 0.396 & 0.358 \\
5 & 0.846 & 0.601 & 0.440 & 0.579 \\
10 & 0.846 & 0.499 & 0.310 & 0.454 \\
20 & 0.481 & 0.240 & 0.144 & 0.221 \\
50 & 0.500 & 0.274 & 0.173 & 0.257 \\
100 & 0.538 & 0.252 & 0.141 & 0.224 \\
\hline
\end{tabular}

This study adopted the window length technique to encode input instances of classifiers; however, the sliding window technique makes the performance of the classifier varied. To show which window length makes the classifiers better for a specific type of dataset, several windows with different lengths were investigated. Figure 1 shows the prediction performance on different sliding windows on the BID0 dataset. Among the seven sliding windows, the window with length 13 performs the best, which yields an $F 1$ of 0.579 . It should be mentioned here that classifier ensembles with a suitable window length perform better than those with a smaller or bigger length.

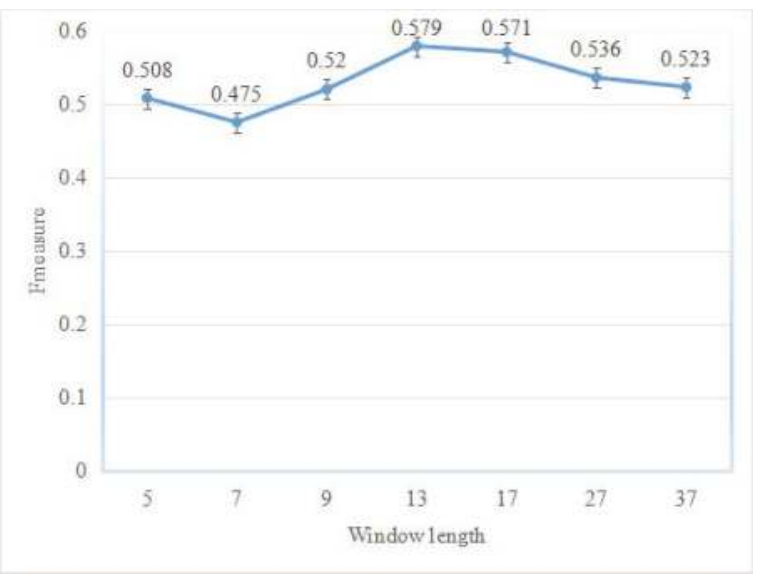

Figure 1. Prediction performance for different sliding windows in instance encoding on the BID0 dataset training by the ASEdb0 dataset. The symbol "I" for each window denotes the calculation error of prediction performance in $F 1$.

\subsection{Comparison with Other Methods}

So far, few works identified hotspots from the whole protein sequences by sequence information alone. Some top hotspot predictors did the predictions based on protein structures. Most of hotspot prediction methods predicted hotspots from protein-protein interfaces or from some benchmark datasets, such as ASEdb0 and BID0, which contained approximately the same hotspots and 
non-hotspots. Therefore, the random predictor is used to compare with our method. The random predictor was run 100 times, and the average performance was calculated. Furthermore, for prediction comparison, the tool of ISIS (Interaction Sites Identified from Sequence) [20] on the PredictProtein server [31] was adopted, which has been applied in hotspot predictions on the dataset of interface residues [20]. ISIS is a machine learning-based method that identified interacting residues from the sequence alone. Similar to our method, although the method was developed using transient protein-protein interfaces from complexes of experimentally-known 3D structures, it only used the sequence and predicted 2D information. In PredictProtein, it predicted a residue as a hotspot if the prediction score of the residue was bigger than 21, otherwise being non-hotspot residues. Since PredictProtein currently cannot process short input sequences less than 17 residues, protein sequences in PDB names "1DDMB" and "2NMBB" were removed from the BID0 test set. We tested all of the sequences of more than 17 residues on the BID0 dataset, and the performance of hotspot predictions on the dataset was obtained. The predictions of ISIS method can be referred to the Supplementary Materials.

Table 4 lists the hotspot prediction comparison in detail. Our method developed a random projection ensemble system yielding a final precision of 0.440 at a sensitivity of 0.846 by the use of sequence information only. Results showed that our method outperforms the random predictor. Furthermore, our method outperformed the ISIS method. Actually, ISIS was developed to identify protein-protein interactions. The power of ISIS for the identification of hotspot residues was poor. It can predict nine of 47 real hotspots correctly; however, 2920 non-hotspots were predicted to be hotspots in the BID0 dataset.

Table 4. Performance comparison of the three methods on the BID0 dataset by training on the ASEdb0 dataset.

\begin{tabular}{cccccc}
\hline Method & Type & Sen & MCC & Prec & F1 \\
\hline Our Method & Random Projection & 0.846 & 0.601 & 0.440 & 0.579 \\
ISIS & Neural Networks & 0.191 & 0.030 & 0.026 & 0.046 \\
Random Predictor & 0.983 & 0.000 & 0.018 & 0.035 \\
\hline
\end{tabular}

We also show the performance of classifier ensemble in several measures based on the measure of sensitivity. Figure 2 illustrates the performance of the ensemble classifier with the majority vote for the test set BID0. Although it is very difficult to identify hotspots from the whole protein sequences, our method yields a good result based on sequence information only.
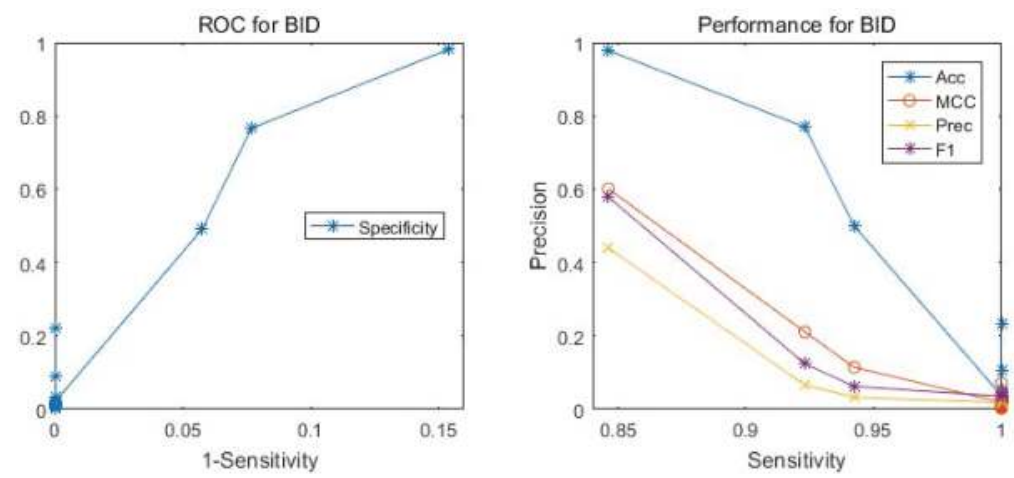

Figure 2. The performance of our method for testing on the BID0 dataset by training on the ASEdb0 dataset. The left graph illustrates the ROC (receiver operating characteristic) curve, and the right one shows the four measure curves with respect to sensitivity. 


\subsection{Case Study of Hotspot Predictions}

To show the performance of our method on a single protein chain, hotspot predictions for chain " $\mathrm{A}$ " of protein PDB:1DDM are illustrated in Figure 3. Protein 1DDM is an in vivo complex containing a phosphotyrosine-binding (PTB) domain (chain " $\mathrm{A}$ ") of the cell fate determinant Numb, which can bind a diverse array of peptide sequences in vitro, and a peptide containing an amino acid sequence "NMSF" derived from the Numb-associated kinase (Nak) (chain "B"). The Numb PTB domain is in complex with the Nak peptide. The chain "A" contains 135 amino acid residues, where only residues E144, I145, C150 and C198 are real hotspot residues in complex with the chain "B" of the protein (which contains 11 amino acid residues; see Figure 3c). Our method correctly predicted the first three true hotspots, and hotspot residue 198 was predicted as a non-hotspot, while residues 69, 112, 130 and 160 were wrongly predicted as hotspot residues. All of them are located at the surface of the protein structure. The results of ISIS are also illustrated in Figure 3b. The ISIS method cannot identify the four true hotspot residues, although most of the hotspot predictions are located at the surface of the protein.

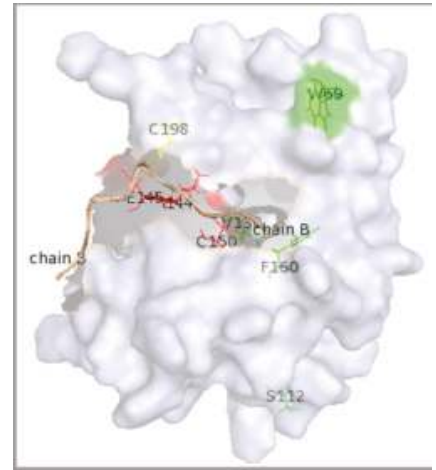

(a)Prediction of our method.

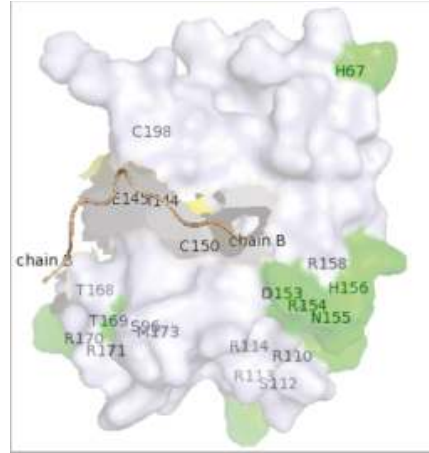

(b)Predictions of ISIS method.

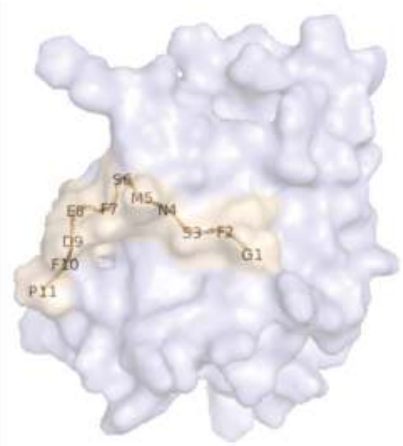

(c)Complex structure of protein PDB:1DDM.

Figure 3. Case study for the complex of protein PDB:1DDM. The subgraphs $(\mathbf{a}, \mathbf{b})$ are shown for the prediction comparison of our method and the ISIS method, respectively, where the chain B of protein 1DDM is colored in wheat. The subgraph (c) illustrates the cartoon structure of the protein complex, where the chain B of protein 1DDM is colored in green. Here, red residues are the hotspots that are predicted correctly; green residues are non-hotspots that are predicted to be hotspots; while yellow ones are real hotspots that are predicted to be non-hotspot residues. All other residues are correctly predicted as non-hotspots. 


\section{Materials and Methods}

\subsection{Hot Spot Definitions}

As we know, a residue is defined as a hotspot by the change of the binding free energy $(\Delta \Delta G)$ higher than a threshold, if mutated to alanine. Several thresholds were adopted in previous works. Many works defined residues as hotspots when their $\Delta \Delta \mathrm{Gs}$ are higher than $2.0 \mathrm{kcal} / \mathrm{mol}$, and other residues with $\Delta \Delta G$ from $0-2.0 \mathrm{kcal} / \mathrm{mol}$ were defined as non-hotspots [21-23]. Ofran et al. used another definition that defined residues with $\Delta \Delta G$ above $2.5 \mathrm{kcal} / \mathrm{mol}$ as hotspots and those with $\Delta \Delta G=0 \mathrm{kcal} / \mathrm{mol}$ (i.e., no change in binding energy) as non-hotspots [20]. Moreover, Tuncbag and colleagues defined hotspots as those with $\Delta \Delta G$ higher than $2.0 \mathrm{kcal} / \mathrm{mol}$ and non-hotspots as those with $\Delta \Delta G$ from 0-0.4 kcal/mol [24]. Previous works also investigated several definitions of hotspots $[26,29]$. They concluded that different definitions of hotspots and non-hotspots yield different ratios of the number of hotspots to that of non-hotspots and, therefore, change the performances of hotspot prediction methods [26,29]. In this paper, residues higher than $2.0 \mathrm{kcal} / \mathrm{mol}$ are defined as hotspots and all other residues in the whole protein sequences as non-hotspots, no matter if their position is in interfaces, surfaces or any other regions.

\subsection{Datasets}

Since this work addresses the issue of hotspot residue predictions for the whole sequences of proteins, the definitions of hotspot residues are the same as those of the ASEdb and BID datasets, while all of the other residues in the protein sequences are considered as non-hotspot residues.

Two commonly-used benchmark datasets are used in this work. The first dataset is ASEdb [5]. To clean the proteins in ASEdb, protein sequences in the dataset were removed when the sequence identity between any two sequences was higher than $35 \%$. Based on the hotspot definition in this study, we constructed a new ASEdb0 dataset consisting of 58 hotspots from the ASEdb dataset and 3957 non-hotspots of the other residues in whole protein sequences, totaling 4015 residues in our new ASEdb0 dataset.

The BID dataset [6] is the other one used in this work. The dataset was filtered in the same manner as the ASEdb dataset. As a result, we constructed a new BID0 dataset consisting of 54 hotspots from the BID dataset and 2895 non-hotspots from the rest of the residues in the whole protein sequences, totaling 2949 residues in our new BID0 dataset. The data in the two datasets came from different complexes and were mutually exclusive. Table 5 lists the composition of hotspots and non-hotspots.

Table 5. The details of the hotspot datasets.

\begin{tabular}{ccccc}
\hline Dataset & Hot Spots & Non-Hotspots & Total Residues & Ratio $^{\S}$ \\
\hline BID0 & 54 & 2895 & 2949 & $1.831 \%$ \\
ASEdb0 & 58 & 3957 & 4015 & $1.445 \%$ \\
\hline BID & 54 & 58 & 112 & $48.214 \%$ \\
ASEdb & 58 & 91 & 149 & $38.926 \%$ \\
\hline
\end{tabular}

$\S$ The ratio of the number of hotspots to that of total residues in the dataset.

\subsection{Feature Encoding Scheme}

The AAindex1 database [32] contained 544 numerical indices representing various physicochemical and biochemical properties of amino acids. It collected published indices with a set of 20 numerical values representing different properties of amino acids. It also contained the results of cluster analysis using the correlation coefficient as the distance between two indices. All data were derived from published literature.

The protein sequence profile of one amino acid is a set of 20 numerical values representing the evolution of the amino acid residue, where each value represents the frequency by which residue 
was mutated into another amino acid residue. It can be used to recognizing remote homologs and plays an important role in protein sequence database search, protein structure/function prediction and phylogenetic analysis. Protein sequence profiles are always obtained by a BLAST (Basic Local Alignment Search Tool) program, such as the commonly-used program of PSI-BLAST (Position-Specific Iterative Basic Local Alignment Search Tool) [33]. Therefore, for the residue $R_{i}$ of one protein sequence, the multiplication $M S K_{i}^{j}$ of the sequence profile $S P_{i}$ of residue $R_{i}$ and one physicochemical amino acid property $A A P^{j}$ can represent the statistical evolution of the amino acid property [34-36], i.e., $M S K_{i}^{j}=S P_{i} \times A A P^{j}$, where $S P_{i}$ and $A A P^{j}$ are both vectors of $1 \times 20$. The multiplication for residue $R_{i}$ results in a set of 20 numerical vectors $M S K_{i}^{j}$. The standard deviation $S T D_{i}^{j}$ of the multiplication is then obtained. For residue $R_{i}$, the 544 amino acid AAindex 1 properties yield a set of 544 standard deviations $S T D_{i}=S T D_{i}^{j}, j=1544$, which will be used for encoding residue $R_{i}$. Our previous work has shown that the standard deviations of the multiplications can reflect the evolutionary variance of the residue $R_{i}$ along with the amino acid property $A A P^{j}[29,34,35]$.

To encode the residue $R_{i}$ in one protein sequence, a sliding window involving residues centered at the residue $R_{i}$ is considered, i.e., several neighboring residues are used to represent the center residue $R_{i}$. Therefore, a set of winLen $\times 544=7072$ numerical values represents the residue $R_{i}$, where winLen $=13$ is the sliding window length in this work. A similar vector representation can be found in our previous work $[29,34,35]$. For the residue $R_{i}$, it is represented by a $1 \times 7072$ vector $V_{i}$, whose corresponding target value $T_{i}$ is 1 or 0 , denoting whether the residue is a hotspot or not. Therefore, our method is developed to learn the relationship between input vectors $V$ and the corresponding target array $T$ and tries to make its output $Y=f(V)$ as close to the target $T$ as possible.

\subsection{IBk Classifier Ensemble by the Random Mapping Technique}

The random projection technique can be traced back to the work done by Ritter and Kohonen [37], which reduced the dimensionality of the representations of the word contexts by replacing each dimension of the original space by a random direction in a smaller-dimensional space. From the literature $[37,38]$, it seems surprising that random mapping can reduce the dimensionality of the data in a manner that preserves enough structure of the original dataset to be useful. Kaski used both analytical and empirical evidence to explain the reason why the random mapping method worked well in high-dimensional spaces [39].

Given the original data, $X \in \Re^{N \times L 1}$, let the linear random projection be the multiplication of the original instances by a random matrix $R \in \Re^{L 1 \times L 2}$, where the element in the matrix ranges from $0-1$. The matrix $R$ is composed of random elements, and each column has been normalized to unity. The projection:

$$
X^{R}=X R=\sum_{i}\left(x_{i} \times r_{i}\right)
$$

yields a dimensionality-reduced instance $X^{R} \in \Re^{N \times L 2}$ from dimension $L 1$ to $L 2$, where $x_{i}$ is the $i$-th sample of the original data, $r_{i}$ is the $i$-th column of the random matrix and $L 2 \ll L 1$. In Equation (1), each original instance with dimension $L 1$ has been replaced by a random, non-orthogonal direction L2 in the reduced-dimensional space [39]. Therefore, the dimensionality of the original instance is reduced from 7072 to a rather small value.

The dimension-reduced instances are then input into the classifier with the IBk algorithm. The IBk algorithm, implementing the k-nearest neighbor algorithm, is a type of instance-based learning, where the function is only approximated locally, and all computations are deferred until classification. The simplest of the IBk algorithms among machine learning algorithms was adopted since we want to ensemble diverse classifiers and expect to yield good results. Previous results showed that the generalization error caused by one classifier can be compensated by other classifiers; therefore, the ensemble of some diverse classifiers can yield significant improvement [40].

In the hotspot prediction, the multiplication of the $k$-th random projection $R_{k}$ on the original instances $(X, Y)$ forms a set of instances $D^{k}=\left\{\left(X_{i}^{R_{k}}, Y_{i}\right)\right\}, i=1, \ldots, N$, where $N$ and $K$ denote the 
number of training instances and that of random projections, respectively. For the $k$-th random projection, the instances $D^{k}$ are generated from the original instances $(X, Y)$ as an input to an IBk classifier, and thus, it forms a classifier $I B k_{k}(x)$, where $x$ is a training instance. To train the classifier $I B k_{k}(x)$, the instance set $D^{k}$ is divided into training dataset $D_{t r}^{k}$ and test dataset $D_{t s}^{k}$ by 10 -fold cross-validation. For training the classifier, the training dataset $D_{t r}^{k}$ is divided into training subset $D_{t r}^{k-t r}$ and test subset $D_{t r}^{k-t s}$ again. The training process retains the top classifiers on some random projections, and in the test process, they are applied to test the test dataset $D_{t s}^{k}$.

After running random projection 100 times, top classifiers in the $F 1$ measure are retained for testing the test dataset $D_{t s}^{k}$. The ensemble of top classifiers yields the final predictions. The mjority vote technique was always used in classifier ensemble and often made a dramatic improvement [41]. Here, a residue is predicted as a hotspot if half of the classifiers identified it as positive Class 1, otherwise it is a non-hotspot residue.

Moreover, since the hotspot dataset is extremely imbalanced, containing only $1.4 \%$ of hotspots, balancing the dataset is necessary to avoid the overfitting of the classifier. Therefor, the training dataset $D_{t r}^{k-t r}$ is resampled and then consists of positive instances and negative instances with roughly the same number. The ensemble system can be seen i Figure 4.

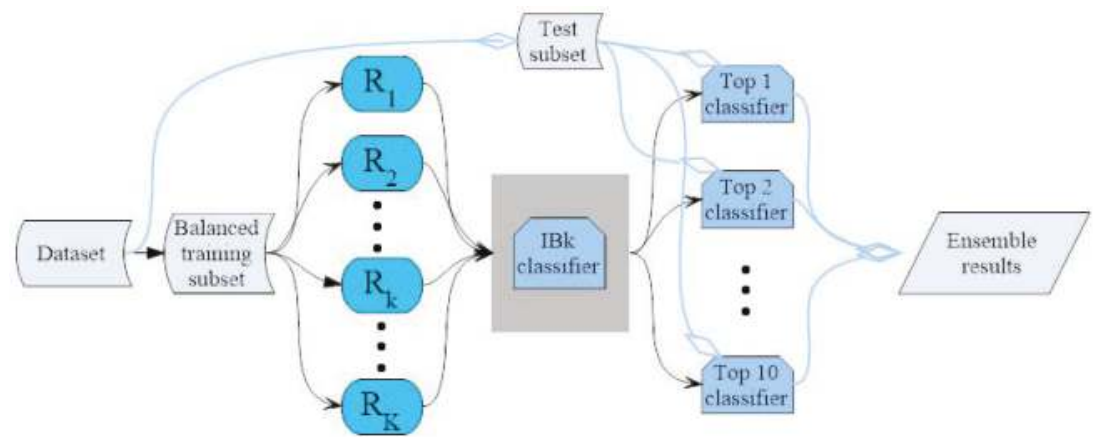

Figure 4. The flowchart of the ensemble system for the hotspot prediction. Here, $R_{k}$ means the $k$-th random projection. The IBk implements k-Nearest Neighbors (KNN) algorithm. Here the black arrows denote the flow of the training subset, while the blue ones are that of the test subset.

\subsection{Hot Spot Prediction Evaluation}

To evaluate hotspot predictions, in this work, we adopted four evaluation measures to show the ability of our model objectively. They are the criteria of sensitivity (Sen), precision (Prec), F-measure (F1) and Matthews correlation coefficient (MCC) [34,42] and shown below:

$$
\begin{aligned}
& \text { Sen }=\frac{T P}{T P+F N}, \quad \text { Prec }=\frac{T P}{T P+F P} \\
& F 1=2 \times \frac{\text { Prec } \times \text { Sen }}{\text { Prec }+ \text { Sen }} \\
& M C C=\frac{T P \times T N-F P \times F N}{\sqrt{(T P+F N)(T P+F P)(T N+F P)(T N+F N)}},
\end{aligned}
$$

where TP (true positive) is the number of correctly-predicted hotspot residues; FP (false positive) is the number of false positives (incorrectly over-predicted non-hotspot residues); TN (true negative) is the number of correctly-predicted non-hotspot residues; and FN (false negative) is false negative, i.e., incorrectly under-predicted hotspot residues. 


\section{Conclusions}

This paper proposes an ensemble method based on the random projection technique that predicts hotspots from the whole sequences of proteins, using physicochemical characteristics of amino acids. The classifier system involves an encoding scheme integrating sequence profiles of residues and statistical physicochemical properties of amino acids from the AAindex1 dataset. Then, the random projection technique was adopted to obtain a reduced input space for the original input instances, but retaining the structure of the original space. Several top classifiers are obtained after the use of random projections. The ensemble of the top classifiers is therefore constructed. The classifier with random projection ran 50 times, and 50 classifiers were sorted in the F1 measure in the training step. Applying the 50 classifiers to the test dataset yielded the final hotspot predictions. Results showed that the ensemble of the top three classifiers yields better performance in hotspot predictions. Moreover, random projections with different reduced dimensions were investigated, and the projection with the dimension of five performs the best. To select the most effective sliding window, several sliding windows were investigated for encoding instances, and a window with a length of 13 was chosen finally, which performed the best among the eight windows. It is suggested that our method is promising in computational hotspot prediction for the whole protein sequence.

Supplementary Materials: Supplementary materials can be found at www.mdpi.com/1422-0067/18/7/1543/s1.

Acknowledgments: This work was supported by the National Natural Science Foundation of China (Nos. 61672035, 61300058, 61472282 and 61271098) and the Project Foundation of Natural Science Research in Universities of Anhui Province in China (No. KJ2017A355).

Author Contributions: Jinjian Jiang and Peng Chen conceived and designed the experiments; Jinjian Jiang and Peng Chen performed the experiments; Jinjian Jiang and Nian Wang analyzed the data; Nian Wang and Bing Wang contributed reagents/materials/analysis tools; Jinjian Jiang and Peng Chen wrote the paper. All authors proved the final manuscript.

Conflicts of Interest: The authors declare no conflict of interest.

\section{Abbreviations}

$\begin{array}{ll}\text { kNN } & \text { k-Nearest Neighbor } \\ \text { Sen } & \text { Sensitivity } \\ \text { Prec } & \text { Precision } \\ \text { F1 } & \text { F-Measure } \\ \text { MCC } & \text { Matthews Correlation Coefficient } \\ \text { ASEdb } & \text { Alanine Scanning Energetics Database } \\ \text { BID } & \text { Binding Interface Database } \\ \text { SKEMPI } & \text { Structural Kinetic and Energetic Database of Mutant Protein Interactions }\end{array}$

\section{References}

1. Clackson, T.; Wells, J.A. A hot spot of binding energy in a hormone-receptor interface. Science 1995, 267, 383-386

2. Bogan, A.A.; Thorn, K.S. Anatomy of hot spots in protein interfaces. J. Mol. Biol. 1998, 280, 1-9.

3. Kortemme, T.; Baker, D. A simple physical model for binding energy hot spots in protein-protein complexes. Proc. Natl. Acad. Sci. USA 2002, 99, 14116-14121.

4. Keskin, O.; Ma, B.; Nussinov, R. Hot regions in protein-protein interactions: The organization and contribution of structurally conserved hot spot residues. J. Mol. Biol. 2005, 345, 1281-1294.

5. Thorn, K.S.; Bogan, A.A. ASEdb: A database of alanine mutations and their effects on the free energy of binding in protein interactions. Bioinformatics 2001, 17, 284-285.

6. Fischer, T.B.; Arunachalam, K.V.; Bailey, D.; Mangual, V.; Bakhru, S.; Russo, R.; Huang, D.; Paczkowski, M.; Lalchandani, V.; Ramachandra, C.; et al. The binding interface database (BID): A compilation of amino acid hot spots in protein interfaces. Bioinformatics 2003, 19, 1453-1454. 
7. Kumar, M.D.S.; Gromiha, M.M. PINT: Protein-protein interactions thermodynamic database. Nucleic Acids Res. 2006, 34, D195-D198.

8. Moal, I.H.; Fernández-Recio, J. SKEMPI: A structural kinetic and energetic database of mutant protein interactions and its use in empirical models. Bioinformatics 2012, 28, 2600-2607.

9. Guerois, R.; Nielsen, J.E.; Serrano, L. Predicting changes in the stability of proteins and protein complexes: A study of more than 1000 mutations. J. Mol. Biol. 2002, 320, 369-387.

10. Gao, Y.; Wang, R.; Lai, L. Structure-based method for analyzing protein-protein interfaces. J. Mol. Model. 2004, 10, 44-54.

11. Schymkowitz, J.; Borg, J.; Stricher, F.; Nys, R.; Rousseau, F.; Serrano, L. The FoldX web server: An online force field. Nucleic Acids Res. 2005, 33, W382-W388.

12. Huo, S.; Massova, I.; Kollman, P.A. Computational alanine scanning of the 1:1 human growth hormone-receptor complex. J. Comput. Chem. 2002, 23, 15-27.

13. Rajamani, D.; Thiel, S.; Vajda, S.; Camacho, C.J. Anchor residues in protein-protein interactions. Proc. Natl. Acad. Sci. USA 2004, 101, 11287-11292.

14. Gonzalez-Ruiz, D.; Gohlke, H. Targeting protein-protein interactions with small molecules: Challenges and perspectives for computational binding epitope detection and ligand finding. Curr. Med. Chem. 2006, 13, 2607-2625.

15. Ma, B.; Elkayam, T.; Wolfson, H.; Nussinov, R. Protein-protein interactions: Structurally conserved residues distinguish between binding sites and exposed protein surfaces. Proc. Natl. Acad. Sci. USA 2003, 100, 5772-5777.

16. Del Sol, A.; O'Meara, P. Small-world network approach to identify key residues in protein-protein interaction. Proteins 2005, 58, 672-682.

17. Guharoy, M.; Chakrabarti, P. Conservation and relative importance of residues across protein-protein interfaces. Proc. Natl. Acad. Sci. USA 2005, 102, 15447-15452.

18. Grosdidier, S.; Fernandez-Recio, J. Identification of hot-spot residues in protein-protein interactions by computational docking. BMC Bioinform. 2008, 9, 447.

19. Brinda, K.V.; Kannan, N.; Vishveshwara, S. Analysis of homodimeric protein interfaces by graph-spectral methods. Protein Eng. 2002, 15, 265-277.

20. Ofran, Y.; Rost, B. Protein-protein interaction hotspots carved into sequences. PLoS Comput. Biol. 2007, 3, e119.

21. Darnell, S.J.; Page, D.; Mitchell, J.C. An automated decision-tree approach to predicting protein interaction hot spots. Proteins 2007, 68, 813-823.

22. Lise, S.; Archambeau, C.; Pontil, M.; Jones, D.T. Prediction of hot spot residues at protein-protein interfaces by combining machine learning and energy-based methods. BMC Bioinform. 2009, 10, 365.

23. Wang, L.; Liu, Z.P.; Zhang, X.S.; Chen, L. Prediction of hot spots in protein interfaces using a random forest model with hybrid features. Protein Eng. Des. Sel. 2012, 25, 119-126.

24. Tuncbag, N.; Gursoy, A.; Keskin, O. Identification of computational hot spots in protein interfaces: Combining solvent accessibility and inter-residue potentials improves the accuracy. Bioinformatics 2009, 25, 1513-1520.

25. Guney, E.; Tuncbag, N.; Keskin, O.; Gursoy, A. HotSprint: Database of computational hot spots in protein interfaces. Nucleic Acids Res. 2008, 36, D662-D666.

26. Cho, K.I.; Kim, D.; Lee, D. A feature-based approach to modeling protein-protein interaction hot spots. Nucleic Acids Res. 2009, 37, 2672-2687.

27. Tuncbag, N.; Keskin, O.; Gursoy, A. HotPoint: Hot spot prediction server for protein interfaces. Nucleic Acids Res. 2010, 38, W402-W406.

28. Lise, S.; Buchan, D.; Pontil, M.; Jones, D.T. Predictions of hot spot residues at protein-protein interfaces using support vector machines. PLoS ONE 2011, 6, e16774.

29. Chen, P.; Li, J.; Wong, L.; Kuwahara, H.; Huang, J.Z.; Gao, X. Accurate prediction of hot spot residues through physicochemical characteristics of amino acid sequences. Proteins 2013, 81, 1351-1362.

30. Ludmila, I.; Kuncheva, C.J.W. Measures of diversity in classifier ensembles and their relationship with the ensemble accuracy. Mach. Learn. 2003, 51, 181-207. 
31. Yachdav, G.; Kloppmann, E.; Kajan, L.; Hecht, M.; Goldberg, T.; Hamp, T.; Honigschmid, P.; Schafferhans, A.; Roos, M.; Bernhofer, M.; et al. PredictProtein-An open resource for online prediction of protein structural and functional features. Nucleic Acids Res. 2014, 42, W337-W343.

32. Kawashima, S.; Pokarowski, P.; Pokarowska, M.; Kolinski, A.; Katayama, T.; Kanehisa, M. AAindex: Amino acid index database, progress report 2008. Nucleic Acids Res. 2008, 36, D202-D205.

33. Altschul, S.F.; Madden, T.L.; Schäffer, A.A.; Zhang, J.; Zhang, Z.; Miller, W.; Lipman, D.J. Gapped BLAST and PSI-BLAST: A new generation of protein database search programs. Nucleic Acids Res. 1997, 25, 3389-3402.

34. Chen, P.; Li, J. Sequence-based identification of interface residues by an integrative profile combining hydrophobic and evolutionary information. BMC Bioinform. 2010, 11, 402.

35. Chen, P.; Wong, L.; Li, J. Detection of outlier residues for improving interface prediction in protein heterocomplexes. IEEE/ACM Trans. Comput. Biol. Bioinform. 2012, 9, 1155-1165.

36. Chen, P.; Hu, S.; Zhang, J.; Gao, X.; Li, J.; Xia, J.; Wang, B. A sequence-based dynamic ensemble learning system for protein ligand-binding site prediction. IEEE/ACM Trans. Comput. Biol. Bioinform. 2016, 13, 901-912.

37. Ritter, H.; Kohonen, T. Self-organizing semantic maps. Biol. Cybern. 1989, 61, 241.

38. Papadimitriou, C.H.; Raghavan, P.; Tamaki, H.; Vempala, S. Latent semantic indexing: A probabilistic analysis. J. Comput. Syst. Sci. 2000, 61, 217-235.

39. Kaski, S. Dimensionality reduction by random mapping: Fast similarity computation for clustering. In Proceedings of the IEEE International Joint Conference on Neural Networks Proceedings, World Congress on Computational Intelligence, Anchorage, AK, USA, 4-9 May 1998; Volume 1, pp. 413-418.

40. Chen, P.; Huang, J.Z.; Gao, X. LigandRFs: Random forest ensemble to identify ligand-binding residues from sequence information alone. BMC Bioinform. 2014, 15 (Suppl. S15), S4.

41. Kuncheva, L.; Whitaker, C.; Shipp, C.; Duin, R. Limits on the majority vote accuracy in classifier fusion. Pattern Anal. Appl. 2003, 6, 22-31.

42. Wang, B.; Chen, P.; Huang, D.S.; Li, J.j.; Lok, T.M.; Lyu, M.R. Predicting protein interaction sites from residue spatial sequence profile and evolution rate. FEBS Lett. 2006, 580, 380-384.

(C) 2017 by the authors. Licensee MDPI, Basel, Switzerland. This article is an open access article distributed under the terms and conditions of the Creative Commons Attribution (CC BY) license (http:/ / creativecommons.org/licenses/by/4.0/). 


\title{
An Ameliorated Prediction of Drug-Target Interactions Based on Multi-Scale Discrete Wavelet Transform and Network Features
}

\author{
Cong Shen ${ }^{1,2}$, Yijie Ding ${ }^{1,2}$, Jijun Tang ${ }^{1,2,3, *}$, Xinying $\mathrm{Xu}^{4}$ and Fei Guo ${ }^{1,2, *}$ \\ 1 School of Computer Science and Technology, Tianjin University, Tianjin 300350, China; \\ congshen@tju.edu.cn (C.S.); wuxi_dyj@tju.edu.cn (Y.D.) \\ 2 Tianjin University Institute of Computational Biology, Tianjin University, Tianjin 300350, China \\ 3 Department of Computer Science and Engineering, University of South Carolina, Columbia, SC 29208, USA \\ 4 College of Information Engineering, Taiyuan University of Technology, Taiyuan 030024, Shanxi, China; \\ xuxinying@tyut.edu.cn \\ * Correspondence: tangjijun@tju.edu.cn (J.T.); fguo@tju.edu.cn (F.G.); \\ Tel.: +86-22-2740-6538 (J.T.); +86-182-2258-6975 (F.G.)
}

Received: 22 July 2017; Accepted: 14 August 2017; Published: 16 August 2017

\begin{abstract}
The prediction of drug-target interactions (DTIs) via computational technology plays a crucial role in reducing the experimental cost. A variety of state-of-the-art methods have been proposed to improve the accuracy of DTI predictions. In this paper, we propose a kind of drug-target interactions predictor adopting multi-scale discrete wavelet transform and network features (named as DAWN) in order to solve the DTIs prediction problem. We encode the drug molecule by a substructure fingerprint with a dictionary of substructure patterns. Simultaneously, we apply the discrete wavelet transform (DWT) to extract features from target sequences. Then, we concatenate and normalize the target, drug, and network features to construct feature vectors. The prediction model is obtained by feeding these feature vectors into the support vector machine (SVM) classifier. Extensive experimental results show that the prediction ability of DAWN has a compatibility among other DTI prediction schemes. The prediction areas under the precision-recall curves (AUPRs) of four datasets are 0.895 (Enzyme), 0.921 (Ion Channel), 0.786 (guanosine-binding protein coupled receptor, GPCR), and 0.603 (Nuclear Receptor), respectively.
\end{abstract}

Keywords: drug-target interactions; discrete wavelet transform; network property; support vector machine

\section{Introduction}

Although the PubChem database [1] has stored millions of chemical compounds, the number of compounds having target protein information are limited. Drug discovery (finding new drug-target interactions, DTIs) requires much more cost and time via biochemical experiments. Hence, some efficient computational methods for predicting potential DTIs are used to cover the shortage of traditional experimental methods. There are three categories of the DTIs prediction approaches: molecular docking, matrix-based, and feature vector-based methods. Cheng et al. [2] and Rarey et al. [3] developed molecular docking methods, which were based on the crystal structure of the target binding site (3D structures). Docking simulations quantitatively estimate the maximal affinity achievable by a drug-like molecule, and these calculated values correlate with drug discovery outcomes. However, docking simulations depend on the spatial structure of targets and are usually time-consuming because of the screening technique. In contrast to docking methods, the other two kinds of computational methods (matrix-based and feature vector-based methods) can achieve the large-scale prediction of DTIs. 
Compared with molecular docking, matrix-based methods of chemical structure similarity are more popular. Many matrix-based approaches are becoming popular in the area of DTI predicition. The bipartite graph learning (BGL) [4] model was firstly proposed by Yamanishi et al. They developed a new supervised method to infer unknown DTIs by integrating chemical space and genomic space into a unified space. Bleakley and Yamanishi et al. [5] raised the bipartite local model (BLM) to solve the DTI prediction problem in chemical and genomic spaces, and applied the bipartite model to transform prediction into a binary classification [5]. Mei et al. [6] improved the BLM with neighbor-based interaction-profile inferring (BLM-NII). The NII strategy inferred label information or training data from neighbors when there was no training data readily available from the query compound/protein itself. Laarhoven et al. designed kernel regularized least squares (RLS), in which they defined Gaussian interaction profile (GIP) kernels on the profiles of drugs and targets to predict DTIs [7]. Xia et al. raised Laplacian regularized least square based on interaction network (NetLapRLS) [8] to improve the prediction performance of RLS. Zheng et al. built a DTI predictor with collaborative matrix factorization (CMF) [9], which can incorporate multiple types of similarities from drugs and those from targets at once. Laarhoven et al. [10] also proposed weighted nearest neighbor with Gaussian interaction profile kernels (WNN-GIP) to predict DTIs. The WNN constructed an interaction score profile for a new drug compound using chemical and interaction information about known compounds in the dataset. Another matrix factorization-based method-kernelized Bayesian matrix factorization with twin kernels (KBMF2K) [11] — was proposed by Gönen, M. The novelty of KBMF2K came from the joint Bayesian formulation of projecting drug compounds and target proteins into a unified subspace using the similarities and estimating the interaction network in that subspace. Neighborhood regularized logistic matrix factorization (NRLMF) was raised by Liu et al. [12]. NRLMF focused on modeling the probability that a drug would interact with a target by logistic matrix factorization, where the properties of drugs and targets were represented by drug-specific and target-specific latent vectors, respectively. Nevertheless, the drawback of pairwise kernel method is the high computational complexity on the occasion of a large numbers of samples. In addition, matrix-based methods did not consider the physical and chemical properties of the target protein. These properties reflect some particular relationship between targets and the molecular structure of drugs.

To handle the above problem, other machine learning approaches of feature vector-based method was raised. Cao et al. firstly proposed several works to predict DTIs via drug (molecular fingerprint), target (sequence descriptors), and network information [13,14]. They used composition (C), transition (T), and distribution (D) and Molecular ACCess System (MACCS) fingerprint to describe target sequence and drug molecule, respectively. The above features were fed into random forest (RF) to detect DTIs.

In this article, we propose a new DTI predictor based on signal compression technology. The target sequence can be regarded as biomolecule signal of a cell. To further extract effective features from the target sequence, we utilize discrete wavelet transform (DWT) as a spectral analysis tool to compress the signal of the target sequence. According to Heisenberg's uncertainty principle, the velocity and location of moving quanta cannot be determined at the same time. Similarly, in a time-frequency coordinate system, the frequency and location of a signal cannot be determined at the same time. Wavelet transform can be based on the scale of the transformation and offset in different frequency bands, given different resolution. This is an effective scenario in practice. We also use MACCS fingerprint to describe the drug. Further more, network feature provides the relationship between drug-target pairs. Many models (e.g., BLM, BLM-NII, NetLapRLS, CMF, KBMF2K, NRLMF, and Cao's work [14]) were built with network information. Therefore, our feature contains sequence (DWT feature), drug (MACCS feature), and network (net feature). Moreover, we combine the above three types of features with support vector machine (SVM) and feature selection (FS) to develop a predictor of DTIs. We evaluate our method on four benchmark datasets including Enzyme, Ion Channel, guanosine-binding protein coupled receptor (GPCR), and Nuclear receptor. The result shows that our method achieves better prediction performance than outstanding approaches. 


\section{Results}

We evaluated our method (DAWN) on balanced DTI datasets, described by Cao's work [14]. We analyzed the performance of features (including MACCS, DWT, and net feature). Then, we compared DAWN with other outstanding methods, including BLM [5], RLS [7], BGL [4], NetLapRLS [8], and Cao's work [14]. In addition, we also tested DAWN on imbalanced DTI datasets, compared with NetLapRLS [8], BLM-NII [6], CMF [9], WNN-GIP [10], KBMF2K [11], and NRLMF [12]. We found that DAWN achieved better values of AUCs.

\subsection{Dataset}

To evaluate the performance and scalability of our method, we adopted enzyme, ion channels, GPCR, and nuclear receptors used by Yamanishi et al. [4] as the gold standard datasets. These datasets come from the Kyoto Encyclopedia of Genes and Genomes (KEGG) database [15]. The information of drug-target interactions comes from KEGG BRITE [15], BRENDA [16], Super Target [17], and DrugBank databases [18]. Table 1 presents some quantitative descriptors about the golden datasets, including the number of drugs $(n)$, number of targets $(m)$, number of interactions, and ratio of $n$ to $m$.

Table 1. Statistics of DTI datasets [4].

\begin{tabular}{ccccc}
\hline & Drugs $(n)$ & Targets $(m)$ & Interactions & Ratio $(n / m)$ \\
\hline Enzyme & 445 & 664 & 2926 & 0.67 \\
IC & 210 & 204 & 1476 & 1.03 \\
GPCR & 223 & 95 & 635 & 2.35 \\
Nuclear receptors & 54 & 26 & 90 & 2.08 \\
\hline
\end{tabular}

IC: ion channel; GPCR: guanosine-binding protein coupled receptor.

\subsubsection{Balanced Dataset}

In Cao's study [14], all real drug-target interaction pairs were used as the positive samples. For negative examples, they selected random, unknown interacting pairs from these drug and protein molecules. DAWN was tested on Cao's four balanced benchmark datasets (including Enzyme, Ion channels, GPCRs, and Nuclear receptors).

\subsubsection{Imbalanced Dataset}

The gold standard datasets only contain positive examples (interaction pairs). Hence, non-interaction drug-target pairs are considered as negative examples. Because the number of non-interaction pairs is larger than interaction pairs, the ratio between majority and minority examples is much greater than 1.

\subsection{Evaluation Measurements}

Three parameters were adopted as criteria: overall prediction accuracy (ACC), sensitivity (SN), and specificity (Spec).

- Accuracy:

$$
A C C=\frac{T P+T N}{T P+F P+T N+F N}
$$

- $\quad$ Sensitivity or Recall:

$$
S N=\frac{T P}{T P+F N}
$$

- Specificity:

$$
\text { Spec }=\frac{T N}{T N+F P}
$$


$T P$ represents the number of positive samples predicted correctly. Similarly, we have TN, FP and $F N$, which represent the number of negative samples predicted correctly, the number of negative samples predicted as positive, and the positive samples predicted as negative, respectively.

In signal detection theory, a receiver operating characteristic (ROC), or simply ROC curve, is a graphical plot illustrating the performance of a binary classifier system as its varied discrimination threshold. A ROC curve can be used to illustrate the relation between sensitivity and specificity.

Area under the precision-recall curve (PRC) (AUPR) is an average of the precision weighted by a given threshold probability. We employed both ROC and the area under the precision-recall curve (PRC), because the representation of PRC is more effective than ROC on highly imbalanced or skewed datasets. Area under the ROC curve (AUC) and AUPR can quantitatively describe sensitivity against specificity and precision against recall, respectively.

\subsection{Experimental Results on Balanced Datasets}

\subsubsection{Performance Analysis of Feature}

In order to analyze the performance of MACCS, DWT, and net features, we tested these features on four balanced datasets (each set contains 10 balanced subsets) through five-fold cross-validation. Results of DWT + MACCS, DWT + MACCS (with FS), DWT + NET + MACCS, and DWT + NET + MACCS (with FS) are shown in Table 2. Because the datasets are balanced, the evaluation of ACC or AUC can measure overall performance. DWT + NET + MACCS (with FS) had the best performance of ACC on Enzyme (0.938), IC (0.943), GPCR (0.890), and Nuclear receptor (0.860), respectively. The performance (AUC) of DWT + NET + MACCS (Enzyme: 0.977, IC: 0.978, GPCR: 0.934, Nuclear receptor: 0.866) was better than DWT + MACCS (Enzyme: 0.925, IC: 0.929, GPCR: 0.872, Nuclear receptor: 0.816). The feature DWT + NET + MACCS indeed improved the prediction performance by adding network information. In addition, the performance (AUC) of DWT + NET + MACCS (with FS) (Enzyme: 0.980, IC: 0.983, GPCR: 0.950, Nuclear receptor: 0.931) was better than DWT + NET + MACCS (without FS) (Enzyme: 0.977, IC: 0.978, GPCR: 0.934, Nuclear receptor: 0.866$)$.

Table 2. Comparison of the prediction performance between different features on balanced datasets.

\begin{tabular}{llcccc}
\hline Dataset & Feature & ACC & Sn & SP & AUC \\
\hline \multirow{5}{*}{ Enzyme } & DWT + MACCS & $0.867 \pm 0.002$ & $0.861 \pm 0.004$ & $0.873 \pm 0.003$ & $0.925 \pm 0.003$ \\
& DWT + MACCS (FS) & $0.895 \pm 0.001$ & $0.901 \pm 0.003$ & $0.889 \pm 0.003$ & $0.949 \pm 0.001$ \\
& DWT + NET + MACCS & $0.932 \pm 0.003$ & $0.933 \pm 0.002$ & $0.933 \pm 0.002$ & $0.977 \pm 0.002$ \\
& DWT + NET + MACCS (FS) & $0.938 \pm 0.002$ & $0.938 \pm 0.002$ & $0.939 \pm 0.004$ & $0.980 \pm 0.001$ \\
\hline \multirow{5}{*}{ IC } & DWT + MACCS & $0.864 \pm 0.003$ & $0.868 \pm 0.004$ & $0.861 \pm 0.005$ & $0.929 \pm 0.004$ \\
& DWT + MACCS (FS) & $0.879 \pm 0.004$ & $0.891 \pm 0.004$ & $0.866 \pm 0.007$ & $0.935 \pm 0.003$ \\
& DWT + NET + MACCS & $0.940 \pm 0.004$ & $0.932 \pm 0.005$ & $0.943 \pm 0.006$ & $0.978 \pm 0.003$ \\
& DWT + NET + MACCS (FS) & $0.943 \pm 0.002$ & $0.938 \pm 0.003$ & $0.949 \pm 0.003$ & $0.983 \pm 0.001$ \\
\hline \multirow{5}{*}{ GPCR } & DWT + MACCS & $0.826 \pm 0.005$ & $0.831 \pm 0.003$ & $0.822 \pm 0.007$ & $0.872 \pm 0.004$ \\
& DWT + MACCS (FS) & $0.836 \pm 0.006$ & $0.846 \pm 0.007$ & $0.827 \pm 0.009$ & $0.892 \pm 0.005$ \\
& DWT + NET + MACCS & $0.872 \pm 0.004$ & $0.872 \pm 0.005$ & $0.872 \pm 0.003$ & $0.934 \pm 0.005$ \\
& DWT + NET + MACCS (FS) & $0.890 \pm 0.005$ & $0.888 \pm 0.009$ & $0.891 \pm 0.011$ & $0.950 \pm 0.002$ \\
\hline \multirow{5}{*}{ Nuclear receptor } & DWT + MACCS & $0.750 \pm 0.011$ & $0.619 \pm 0.013$ & $0.879 \pm 0.021$ & $0.816 \pm 0.015$ \\
& DWT + MACCS (FS) & $0.791 \pm 0.017$ & $0.790 \pm 0.018$ & $0.793 \pm 0.036$ & $0.850 \pm 0.016$ \\
& DWT + NET + MACCS & $0.805 \pm 0.021$ & $0.767 \pm 0.017$ & $0.837 \pm 0.013$ & $0.866 \pm 0.011$ \\
& DWT + NET + MACCS (FS) & $0.860 \pm 0.009$ & $0.855 \pm 0.013$ & $0.867 \pm 0.024$ & $0.931 \pm 0.009$ \\
\hline
\end{tabular}

DWT: discrete wavelet transform; FS: feature selection; NET: network features; MACCS: drug features of molecular access system.

It is clear that FS plays a key role in elevating the prediction of our method. The FS can enhance generalization by reducing the overfitting. Obviously, the performance of DWT + NET + MACCS (with FS) can be seen from Figures 1 and 2. Network topology can be a useful supplement to improve prediction effect. 


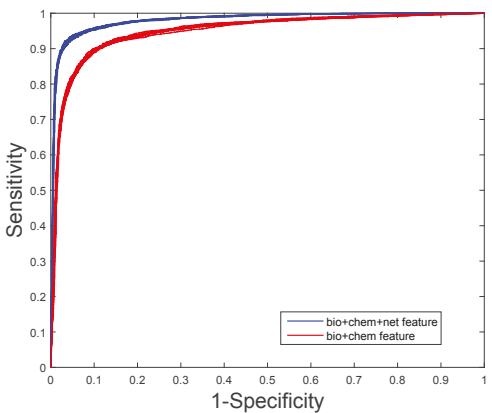

(a)

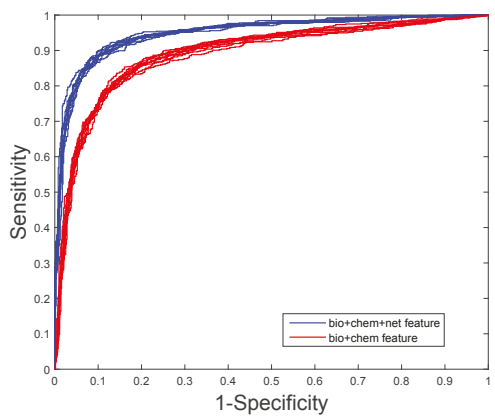

(c)

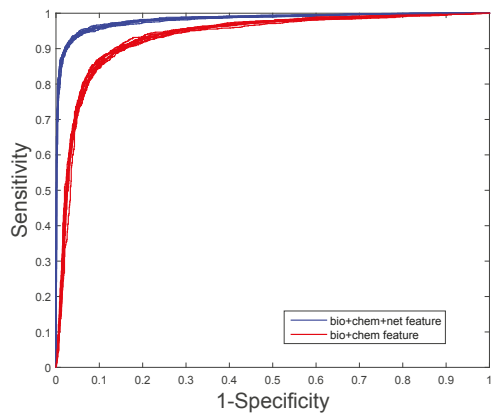

(b)

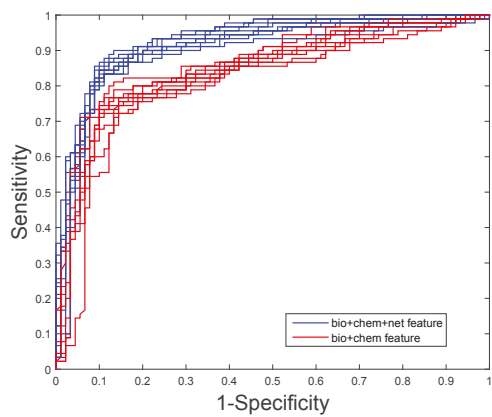

(d)

Figure 1. The area under the Receiver Operating characteristic Curve (ROC) values obtained on balanced datasets (with FS). The blue curve is the combined feature of MACCS (chem), DWT (bio), and net. The red curve is the combined feature of MACCS (chem) and DWT (bio); (a) Enzyme's ROC curve with network feature; (b) IC 's ROC curve with network feature; (c) GPCR's ROC curve with network feature; (d) Nuclear receptor's ROC curve with network feature.

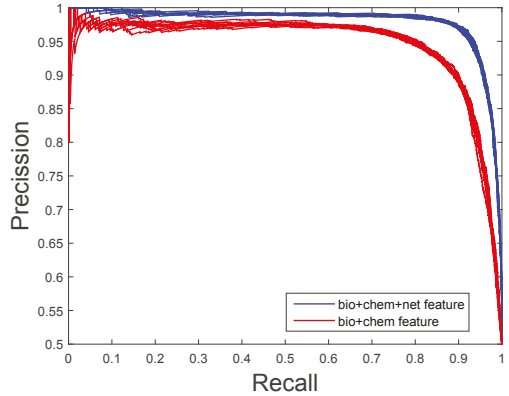

(a)

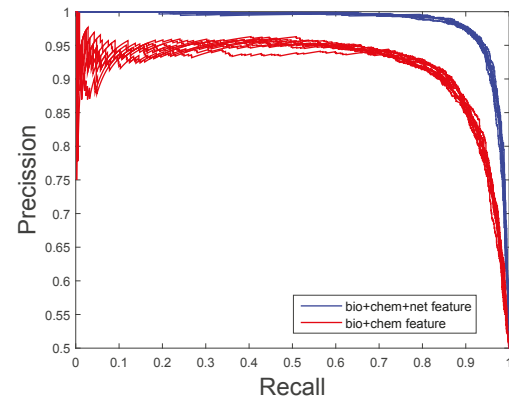

(b)

Figure 2. Cont. 


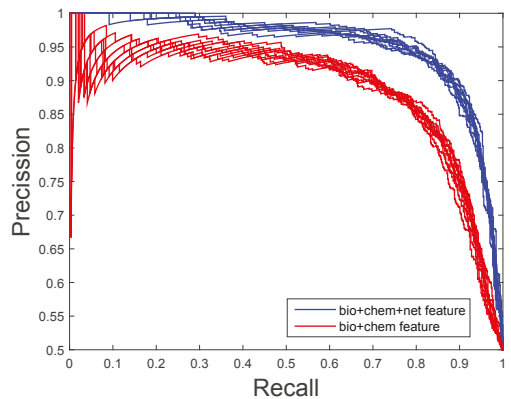

(c)

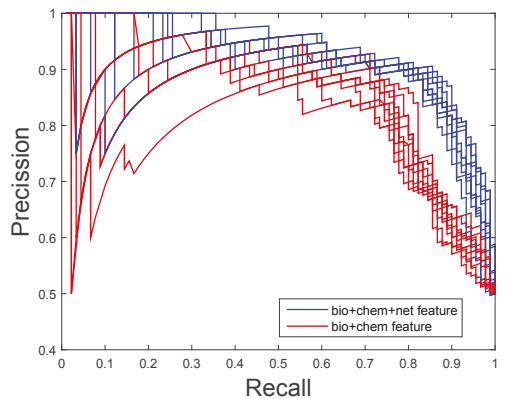

(d)

Figure 2. The area under the precision-recall (PR) curve (AUPR) values obtained on balanced datasets (with FS). The blue curve is the combined feature of MACCS (chem), DWT (bio), and net. The red curve is the combined feature of MACCS (chem) and DWT (bio); (a) Enzyme's PR curve with network feature; (b) IC's PR curve with network feature; (c) GPCR's PR curve with network feature; (d) Nuclear receptor's PR curve with network feature.

\subsubsection{Comparing with Existing Methods}

On the balanced datasets [14], we compare DAWN with other common methods by five-fold cross validation. These methods contain BLM [5], RLS [7], BGL [4], NetLapRLS [8] and Cao's work [14]. The detailed results are listed in Table 3. DAWN achieved the best values of AUCs on Enzyme (0.980) and Nuclear receptor (0.931), respectively. Although the AUC value of DAWN on Ion channel and GPCR datasets were not higher than Cao's work [14] and BLM, we still have a competitive prediction rate. Recapitulating about the aforementioned description, DAWN has a competitive ability among these works.

Table 3. The mean AUC values of five methods on balanced datasets.

\begin{tabular}{lcccc}
\hline Methods & Enzyme & IC & GPCR & Nuclear Receptor \\
\hline Cao's work [14] & 0.979 & $\mathbf{0 . 9 8 7}$ & 0.951 & 0.924 \\
BGL & 0.904 & 0.851 & 0.899 & 0.843 \\
BLM & 0.976 & 0.973 & $\mathbf{0 . 9 5 5}$ & 0.881 \\
NetLapRLS & 0.956 & 0.947 & 0.931 & 0.856 \\
RLS & 0.978 & 0.984 & 0.954 & 0.922 \\
DAWN (our method) & $\mathbf{0 . 9 8 0}$ & 0.983 & 0.950 & $\mathbf{0 . 9 3 1}$
\end{tabular}

Results excerpted from [14]. The best results in each column are in bold faces. BGL: bipartite graph learning; BLM: bipartite local model; NetLapRLS: Laplacian regularized least square based on interaction network; RLS: regularized least square. DAWN: prediction of Drug-tArget interactions based on multi-scale discrete Wavelet transform and Network features.

\subsection{Experimental Results on Imbalanced Datasets}

In order to highlight the advantage of our method, we also tested DAWN on the imbalanced datasets of DTIs by 10-fold cross validation. DAWN was compared with NetLapRLS [8], BLM-NII [6], CMF [9], WNN-GIP [10], KBMF2K [11], and NRLMF [12]. The detailed results are listed in Table 4. Because the datasets are imbalanced, the evaluation of AUC and AUPR were both used to measure overall performance. DAWN achieved average AUCs of 0.981, 0.990, 0.952, and 0.906, and the AUPR values of DAWN were 0.895, 0.921, 0.786, and 0.603 on Enzyme, Ion channel, GPCR, and Nuclear receptor, respectively. The AUC value of DAWN on the Enzyme dataset was 0.981 and AUPR was 0.895, and only the NRLMF (AUC: 0.987, AUPR: 0.892) method was comparable. On Ion channel and GPCR datasets, we also had best or second-best results. For AUPR value on Nuclear receptor, NRLMF was higher than DAWN. The Nuclear receptor dataset is smaller than the other three datasets. The size of 
the dataset might be a reason for DAWN's performance. Therefore, the DAWN method that adopted the mean of DWT was not as effective as larger datasets. However, among methods in Table 4, none could give markedly higher prediction performance on all four datasets in both AUC and AUPR. Therefore, it is fair to claim that our strategy has comparable performance. Further, Figures 3 and 4 show the curves of AUC and AUPR on imbalanced datasets through 10-fold cross validation. Related datasets, codes, and figures of our algorithm are available at https://github.com/6gbluewind/DTI_DWT.

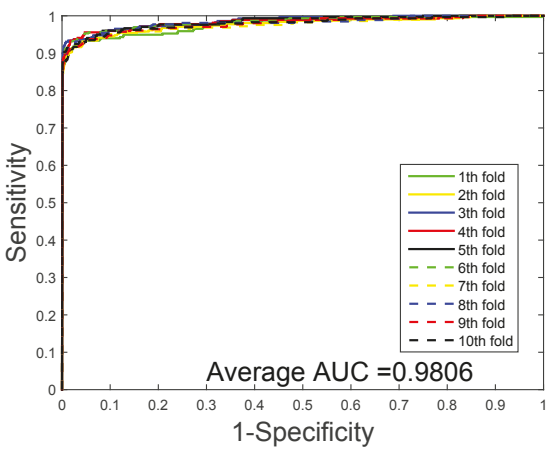

(a)

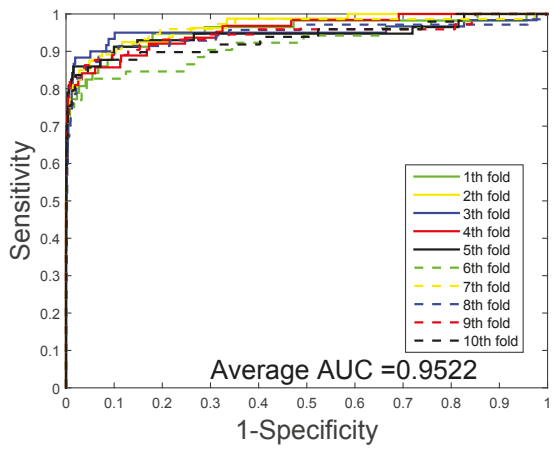

(c)

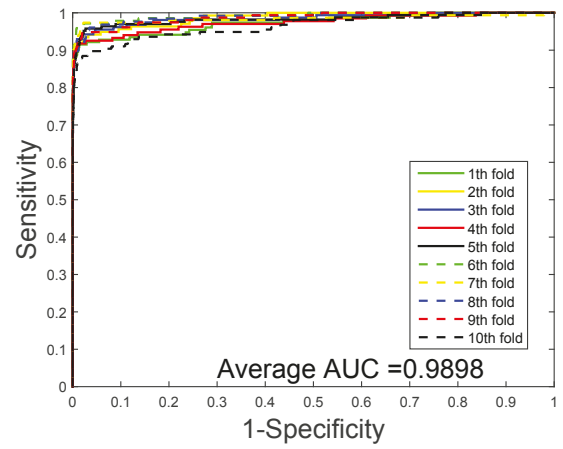

(b)

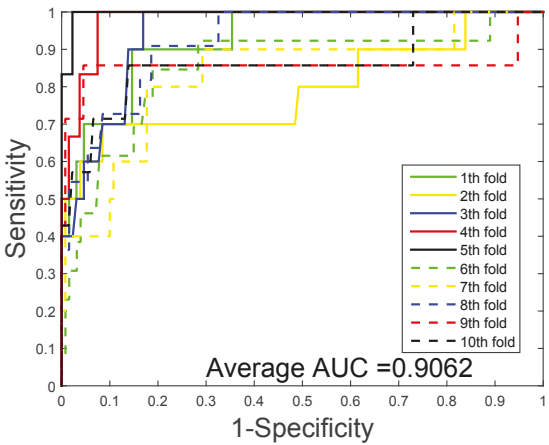

(d)

Figure 3. ROC of imbalanced datasets by 10-fold cross-validation; (a) Enzyme's ROC curve with network feature; (b) IC's ROC curve with network feature; (c) GPCR's ROC curve with network feature; (d) Nuclear receptor's ROC curve with network feature. 


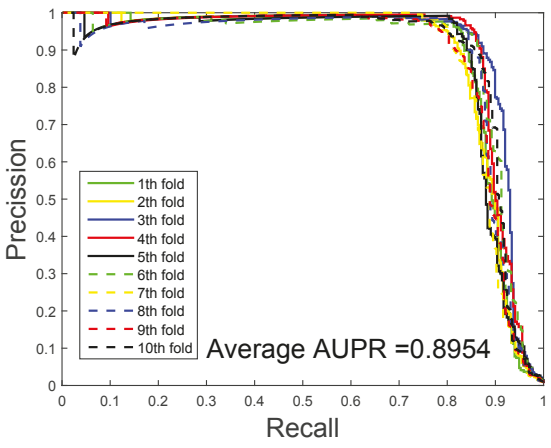

(a)

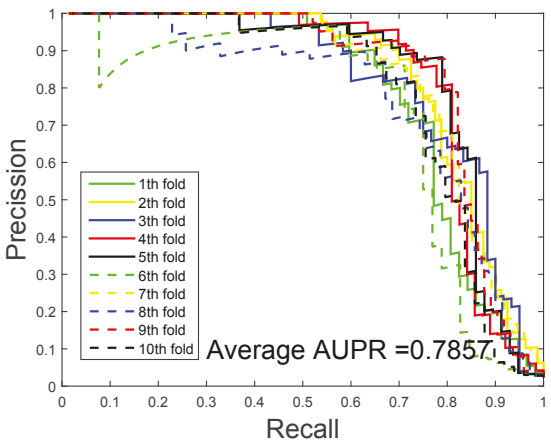

(c)

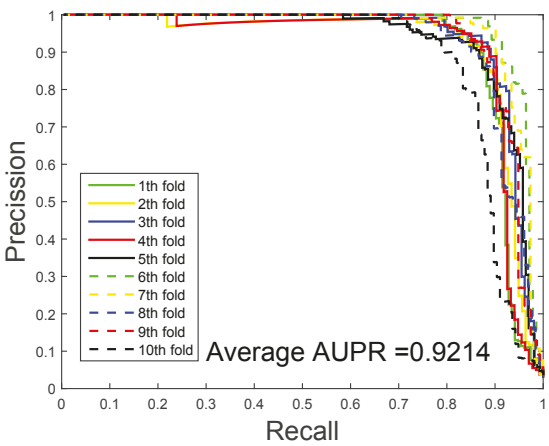

(b)

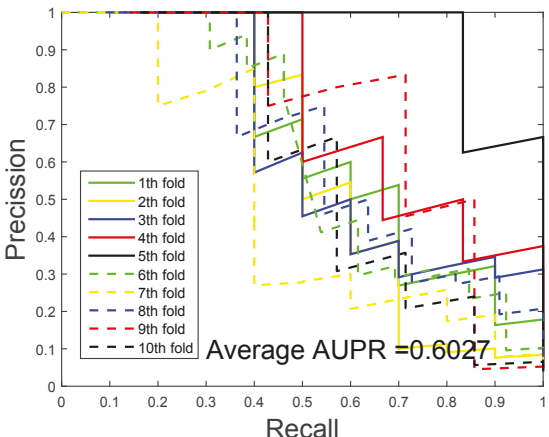

(d)

Figure 4. AUPR of imbalanced datasets by 10-fold cross-validation. (a) Enzyme's PR curve with network feature. (b) IC's PR curve with network feature. (c) GPCR's PR curve with network feature. (d) Nuclear receptor's PR curve with network feature.

Table 4. Overall AUC and AUPR values of different methods on imbalanced dataset for four species.

\begin{tabular}{cccccc}
\hline Evaluation & Method & Enzyme & Ion Channel & GPCR & Nuclear Receptor \\
\hline \multirow{4}{*}{ NetLapRLS } & $0.972 \pm 0.002$ & $0.969 \pm 0.003$ & $0.915 \pm 0.006$ & $0.850 \pm 0.021$ \\
BLM-NII & $0.978 \pm 0.002$ & $0.981 \pm 0.002$ & $0.950 \pm 0.006$ & $0.905 \pm 0.023$ \\
WNN-GIP & $0.964 \pm 0.003$ & $0.959 \pm 0.003$ & $0.944 \pm 0.005$ & $0.901 \pm 0.017$ \\
& KBMF2K & $0.905 \pm 0.003$ & $0.961 \pm 0.003$ & $0.926 \pm 0.006$ & $0.877 \pm 0.023$ \\
CMF & $0.969 \pm 0.002$ & $0.981 \pm 0.002$ & $0.940 \pm 0.007$ & $0.864 \pm 0.026$ \\
& NRLMF & $\mathbf{0 . 9 8 7} \pm 0.001$ & $\underline{0.989} \pm 0.001$ & $\mathbf{0 . 9 6 9} \pm 0.004$ & $\mathbf{0 . 9 5 0} \pm 0.011$ \\
DAWN & $\underline{0.981} \pm 0.004$ & $\mathbf{0 . 9 9 0} \pm 0.014$ & $\underline{0.952} \pm 0.009$ & $\underline{0.906} \pm 0.067$ \\
\hline NetLapRLS & $0.789 \pm 0.005$ & $0.837 \pm 0.009$ & $0.616 \pm 0.015$ & $0.465 \pm 0.044$ \\
BLM-NII & $0.752 \pm 0.011$ & $0.821 \pm 0.012$ & $0.524 \pm 0.024$ & $\underline{0.659} \pm 0.039$ \\
& WNN-GIP & $0.706 \pm 0.017$ & $0.717 \pm 0.020$ & $0.520 \pm 0.021$ & $0.589 \pm 0.034$ \\
KBMF2K & $0.654 \pm 0.008$ & $0.771 \pm 0.009$ & $0.578 \pm 0.018$ & $0.534 \pm 0.050$ \\
CMF & $0.877 \pm 0.005$ & $\mathbf{0 . 9 2 3} \pm 0.006$ & $0.745 \pm 0.013$ & $0.584 \pm 0.042$ \\
& NRLMF & $\underline{0.892} \pm 0.006$ & $0.906 \pm 0.008$ & $\underline{0.749} \pm 0.015$ & $\mathbf{0 . 7 2 8} \pm 0.041$ \\
DAWN & $\mathbf{0 . 8 9 5} \pm 0.011$ & $\underline{0.921} \pm 0.036$ & $\mathbf{0 . 7 8 6} \pm 0.023$ & $0.603 \pm 0.087$ \\
\hline
\end{tabular}

Results excerpted from [12]. The best results in each column are in bold faces and the second best results are underlined. BLM-NII: improved BLM with neighbor-based interaction-profile inferring; CMF: collaborative matrix factorization; KBMF2K: kernelized Bayesian matrix factorization with twin kernels; NRLMF: neighborhood regularized logistic matrix factorization; WNN-GIP: weighted nearest neighbor with Gaussian interaction profile kernels. 


\subsection{Predicting New DTIs}

In this experiment, the balanced DTIs were set as training data sets. We ranked the remaining non-interacting pairs and selected the top five non-interacting pairs as predicted interactions. We utilized four well-known biological databases (including ChEMBL (C) [19], DrugBank (D) [18], KEGG (K) [15] and Matador (M) [20]) as references to verify whether or not the predicted new DTIs are true. The predicted novel interactions by DAWN can be ranked based on the interaction probabilities, which are shown in Table 5. The potential DTIs may be present in one or several databases. For example, the secondly ranked DTI of GPCR (D00563: hsa3269) belongs to DrugBank and Matador databases. In addition, the DTI databases (the above four databases) are still being updated, and the accuracy of identifying new DTIs by DAWN may be increased.

Table 5. Top five new DTIs predicted by DAWN on four data sets.

\begin{tabular}{lcccc}
\hline Dataset & Rank & Drug & Target & Databases \\
\hline \multirow{4}{*}{ Enzyme } & 1 & D00545 & hsa1571 & \\
& 2 & D03365 & hsa1571 & \\
& 3 & D00437 & hsa1559 & M \\
4 & D00546 & hsa1571 & \\
& 5 & D00184 & hsa5478 & D \\
\hline \multirow{5}{*}{ Ion channel } & 1 & D00542 & hsa6262 & \\
& 2 & D00542 & hsa6263 & M \\
& 3 & D00349 & hsa6263 & \\
& 4 & D00477 & hsa6336 & C \\
& 5 & D01448 & hsa3782 & \\
\hline \multirow{5}{*}{ GPCR } & 1 & D01051 & hsa3269 & \\
& 2 & D00563 & hsa3269 & D, M \\
& 3 & D00563 & hsa1812 & D \\
& 4 & D00715 & hsa1129 & D, K \\
& 5 & D00563 & hsa1129 & \\
\hline \multirow{5}{*}{ Nuclear receptor } & 1 & D01689 & hsa5241 & \\
& 2 & D01115 & hsa5241 & \\
& 4 & D00443 & hsa5241 & D \\
& 5 & D00443 & hsa367 & D \\
& 5 & D00187 & hsa2099 & \\
\hline
\end{tabular}

C: ChEMBL; D: DrugBank; K: KEGG; M: Matador.

\section{Discussion}

In this paper, we proposed a new DTIs predictor based on signal compression technology. We encoded the drug molecule by a substructure fingerprint with a dictionary of substructure patterns. Moreover, we applied the DWT to extract features from target sequences. At last, we concatenated the target, drug, and network features to construct predictive model of DTIs.

To evaluate the performance of our method, the DTIs model was compared to other state-of-the-art DTIs prediction methods on four benchmark datasets. DAWN achieved average AUCs of 0.981, 0.990, 0.952, and 0.906, and the AUPR values of DAWN were 0.895, 0.921, 0.786, and 0.603 on Enzyme, Ion channel, GPCR, and Nuclear receptor, respectively. Although our result using feature selection could be a kind of ameliorated prediction, the imbalanced problem of DTIs prediction is not solved very well. SVM is poor on imbalanced data. The AUPR value of DAWN is low on the Nuclear receptor dataset.

\section{Materials and Methods}

To predict DTIs by machine learning methods, one challenge is to extract effective features from the target protein, drug, and the relationship between drug-target pairs. Considering that DTIs depend on the molecular properties of the drug and the physicochemical properties of target, we use MACCS fingerprints (Open Babel 2.4.0 Released, OpenEye Scientific Software, Inc., Santa Fe, New Mexico, United States) to represent the drug, and extract biological features from the target via DWT. 
In addition, the net feature describes the topology information of the DTIs network. We utilize the above features to train the SVM predictor (LIBSVM Version 3.22, National Taiwan University, Taiwan, China) for detecting DTIs.

\subsection{Molecular Substructure Fingerprint of Drug}

To encode the chemical structure of the drug, we utilize MACCS fingerprints with 166 common chemical substructures. These substructures are defined in the Molecular Design Limited (MDL) system, which can be found from OpenBabel (http:/ / openbabel.org). The MACCS feature is encoded by a binary bits vector, which shows the presence (1) or absence (0) of some specific substructures in a molecule. Please refer to the relevant literature $[13,14]$ for details.

\subsection{Biological Feature of Target}

\subsubsection{Six Physicochemical Properties of Amino Acids}

The target sequence can be denoted by seq $=\left\{r_{1}, r_{2}, \cdots, r_{i}, \cdots, r_{L}\right\}$, where $1 \leq i \leq L . r_{i}$ is the $i$-th residue of sequence seq, and $L$ is the length of sequence seq. In addition, for ease of calculation about feature representation, we select six kinds of physicochemical properties for 20 amino acid types as original target features [21-24]. More specifically, they are hydrophobicity (H), volumes of side chains of amino acids (VSC), polarity (P1), polarizability (P2), solvent-accessible surface area (SASA) and net charge index of side chains (NCISC), respectively. Values of all kinds of amino acid are shown in Table 6.

Table 6. Six physicochemical properties of 20 amino acid types.

\begin{tabular}{ccccccc}
\hline Amino Acid & H & VSC & P1 & P2 & SASA & NCISC \\
\hline A & 0.62 & 27.5 & 8.1 & 0.046 & 1.181 & 0.007187 \\
C & 0.29 & 44.6 & 5.5 & 0.128 & 1.461 & -0.03661 \\
D & -0.9 & 40 & 13 & 0.105 & 1.587 & -0.02382 \\
E & -0.74 & 62 & 12.3 & 0.151 & 1.862 & 0.006802 \\
F & 1.19 & 115.5 & 5.2 & 0.29 & 2.228 & 0.037552 \\
G & 0.48 & 0 & 9 & 0 & 0.881 & 0.179052 \\
H & -0.4 & 79 & 10.4 & 0.23 & 2.025 & -0.01069 \\
I & 1.38 & 93.5 & 5.2 & 0.186 & 1.81 & 0.021631 \\
K & -1.5 & 100 & 11.3 & 0.219 & 2.258 & 0.017708 \\
L & 1.06 & 93.5 & 4.9 & 0.186 & 1.931 & 0.051672 \\
M & 0.64 & 94.1 & 5.7 & 0.221 & 2.034 & 0.002683 \\
N & -0.78 & 58.7 & 11.6 & 0.134 & 1.655 & 0.005392 \\
P & 0.12 & 41.9 & 8 & 0.131 & 1.468 & 0.239531 \\
Q & -0.85 & 80.7 & 10.5 & 0.18 & 1.932 & 0.049211 \\
R & -2.53 & 105 & 10.5 & 0.291 & 2.56 & 0.043587 \\
S & -0.18 & 29.3 & 9.2 & 0.062 & 1.298 & 0.004627 \\
T & -0.05 & 51.3 & 8.6 & 0.108 & 1.525 & 0.003352 \\
V & 1.08 & 71.5 & 5.9 & 0.14 & 1.645 & 0.057004 \\
W & 0.81 & 145.5 & 5.4 & 0.409 & 2.663 & 0.037977 \\
Y & 0.26 & 117.3 & 6.2 & 0.298 & 2.368 & 0.023599
\end{tabular}

H: hydrophobicity; VSC: volumes of side chains of amino acids; P1: polarity; P2: polarizability; SASA: solvent-accessible surface area; NCISC: net charge index of side chains.

For the sake of facilitating the dealing with the datasets, the amino acid residues are translated and normalized according to Equation (4).

$$
P_{i j}^{\prime}=\frac{P_{i j}-P_{j}}{S_{j}}(j=1,2, \ldots, 6 ; i=1,2, \ldots, 20)
$$

where $P_{i, j}$ and $P_{j}$ indicate the value of the $j$-th descriptor of amino acid type $i$ and the mean of 20 amino acid types of descriptor value $j$, respectively, standard deviation (SD) corresponding to $S_{j}$.

Each target sequence can be translated into six vectors with each amino acid represented by normalized values of six descriptors. Thus, the seq can be represented as physicochemical matrix $X=\left[x_{1}, \ldots, x_{c h}, \ldots, x_{6}\right], X \in R^{L \times 6}, x_{c h} \in R^{L \times 1}, c h=1,2, \ldots, 6$. 


\subsubsection{Discrete Wavelet Transform}

Discrete wavelet transform (DWT) with its inversion formula was established by physical intuition and practical experience of signal processing [25].

If a signal or a function can be represented as Equation (5), then the signal or function has a linear decomposition. If the formula of expansion is unique, then the set of expansion can be said as a group of basis. If this group of basis is orthogonal or represented as Equation (6), then the coefficient can be computed by inner product as Equation (7).

$$
\begin{gathered}
f(t)=\sum_{\ell} a_{\ell} \psi_{\ell}(t), \\
\left(\psi_{k}(t), \psi_{\ell}(t)\right)=\int \psi_{k}(t) \psi_{\ell}(t) d t=0, k \neq \ell, \\
a_{k}=\left(f(t), \psi_{k}(t)\right)=\int f(t) \psi_{k}(t) d t,
\end{gathered}
$$

where $\ell$ and $k$ are the finite or infinite integer indexes, $a_{\ell}$ and $a_{k}$ are the real coefficients of the expansion, and $\psi_{\ell}(t)$ and $\psi_{k}(t)$ are the set of real functions.

For wavelet expansion, we can construct a system with two parameters, then the formula can be transferred as Equation (8):

$$
f(t)=\sum_{k} \sum_{j} a_{j, k} \psi_{j, k}(t),
$$

where $j$ and $k$ are integer index, and $\psi_{j, k}(t)$ is wavelet function, which generally forms a group of orthogonal basis.

The expansion coefficient set $a_{j, k}$ is known as the discrete wavelet transform (DWT) of $f(t)$. Nanni et al. proposed an efficient algorithm to perform DWT by assuming that the discrete signal $f(t)$ is $x_{c h}(n)$.

$$
\begin{aligned}
& y_{l, \text { high,ch }}(n)=\sum_{k=1}^{L}\left[x_{c h}(k) \cdot h(2 n-k)\right] \\
& y_{l, \text { low }, \text { ch }}(n)=\sum_{k=1}^{L}\left[x_{c h}(k) \cdot g(2 n-k)\right]
\end{aligned}
$$

where $h$ and $g$ refer to high-pass filter and low-pass filter, $L$ is the length of discrete signal, $y_{l, l o w, c h}(n)$ is the approximate coefficient (low-frequency components) of the signal, $l(l=1,2,3,4)$ is the decomposition level of DWT, $\operatorname{ch}(c h=1,2,3,4,5,6)$ is the physicochemical index, and $y_{l, l o w, c h}(n)$ is the detailed coefficient (high-frequency components).

DWT can decompose discrete sequences into high- and low-frequency coefficients. Nanni et al. [26] substituted each amino acid of the protein sequence with a physicochemical property. Then, the protein sequence was encoded as a numerical sequence. DWT compresses discrete sequence and removes noise from the origin sequence. Different decomposition scales with discrete wavelet have different results for representing the sequence of the target protein. They used 4-level DWT and calculated the maximum, minimum, mean, and standard deviation values of different scales (four levels of both low- and high-frequency coefficients). In addition, high-frequency components are more noisy while low-frequency components are more critical. Therefore, they extracted the beginning of the first five Discrete Cosine Transform (DCT) coefficients from the approximation coefficients. We utilize Nanni's method to describe the sequence of the target protein. The schematic diagram of a 4-level DWT is shown in Figure 5. 


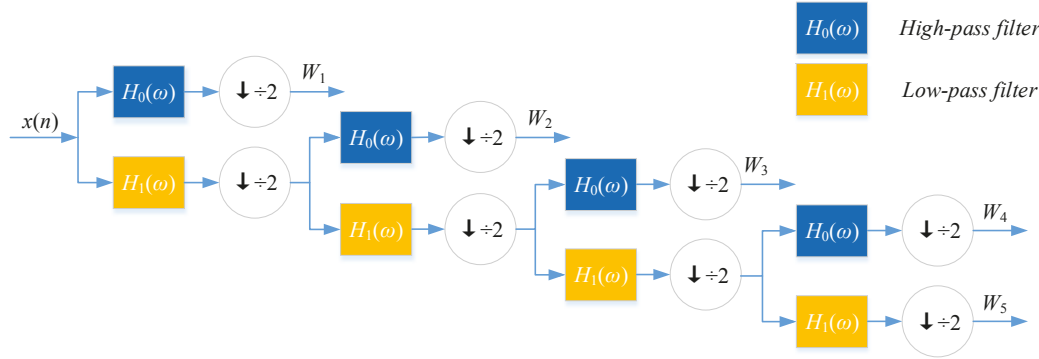

Figure 5. Wavelet decomposition tree.

\subsection{Drug-Target Associations from Network}

State-of-the-art works such as BLM [5], BLM-NII [6], NetLapRLS [8], CMF [9], KBMF2K [11], NRLMF [12], and Cao's work [14] used DTI network topology information to improve the prediction performance. Therefore, we also consider utilizing net feature to build a DTI predictor.

The DTI network can be conveniently regarded as a bipartite graph. In the network, each drug is associated with $n_{t}$ targets, and each target is associated with $n_{d}$ drugs. Excluding target $T_{j}$ itself, we make a binary vector of all other known targets of $D_{i}$ in the bipartite network, as well as a separate list of targets not known to be targeted by $D_{i}$. Known and unknown targets are labeled by 1 and 0 , respectively. For drug $D_{i}$, we get $\left(n_{t}-1\right)$-dimensional binary vector. Similarly, we also get $\left(n_{d}-1\right)$-dimensional binary vector of target $T_{j}$. Thus, we can get a $\left[\left(n_{d}-1\right)+\left(n_{t}-1\right)\right]$-dimensional vector for describing net feature.

\subsection{Feature Selection and Training SVM Model}

Not all features are useful for DTIs prediction. Therefore, we apply support vector machine recursive feature elimination and correlation bias reduction (SVM-RFE+CBR) $[27,28]$ to select the important features of DTIs. The SVM-RFE+CBR can estimate the score of importance for each dimensional feature. We rank these features (including MACCS feature, DWT feature, and net feature) by the scores in descending order. Then, we select an optimal feature subset in top $k$ ranked manner to predict DTIs.

Support vector machine (SVM) was originally developed by Vapnik [29] and coworkers, and has shown a promising capability to solve a number of chemical or biological classification problems. SVM and other machine learning algorithms (e.g., random forest, RF, k-nearest neighbor, kNN, etc.) are widely used in computational biology [30-33]. SVM performs classification tasks by constructing a hyperplane in a multidimensional space to differentiate two classes with a maximum margin. The input data of SVM is defined as $\left\{x_{i}, y_{i}\right\}, i=1,2, \ldots, N$, feature vector $x_{i} \in R^{n}$ and labels $y_{i} \in\{+1,-1\}$.

The classification decision function implemented by SVM is shown as Equation (10).

$$
f(\mathbf{x})=\operatorname{sgn}\left\{\sum_{i=1}^{N} y_{i} \alpha_{i} \cdot K\left(\mathbf{x}, \mathbf{x}_{\mathbf{i}}\right)+b\right\}
$$

where the coefficient $\alpha_{i}$ is obtained by solving a convex quadratic programming problem, and $K\left(\mathbf{x}, \mathbf{x}_{\mathbf{i}}\right)$ is called a kernel function.

Here, we focus on choosing a radial basis function (RBF) kernel [34], because it not only has better boundary response but can also make most high-dimensional data approximate a Gaussian-like distribution. The architecture of our proposed method is shown in Figures 6 and 7 . 


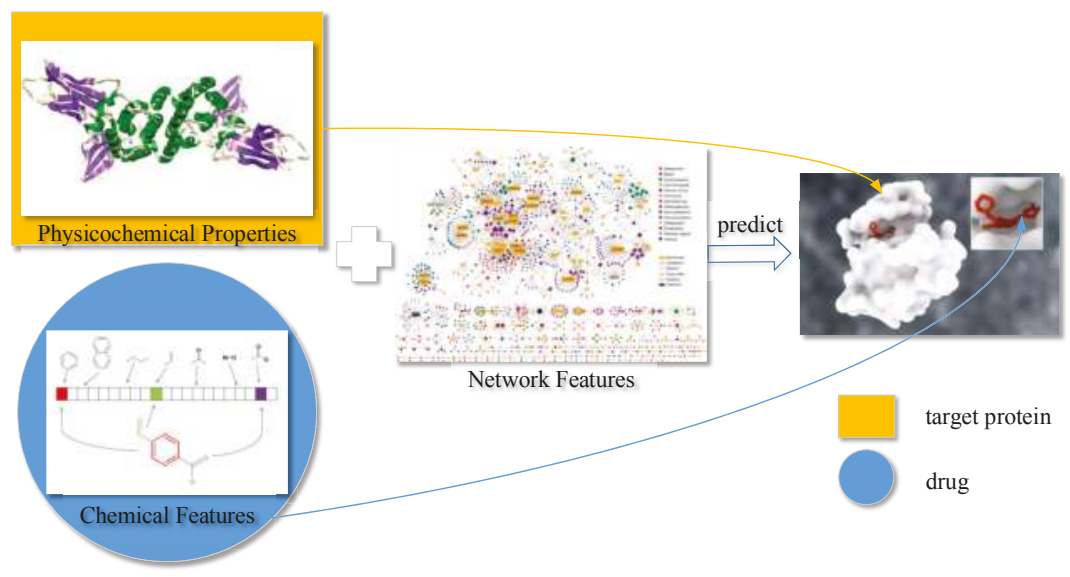

Figure 6. Overview of the drug-target interaction (DTI) prediction.

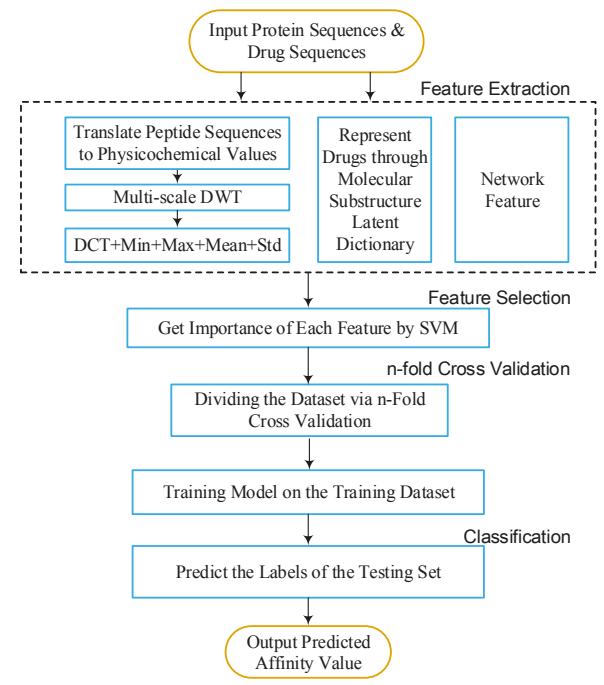

Figure 7. Flow chart. DWT: discrete wavelet transform; DCT: discrete cosine transform; Std: standard deviation; SVM: support vector machine.

\section{Conclusions}

In this paper, we present a DTI prediction method by using multi-scale discrete wavelet transform and network features. We employ a DWT algorithm to extract target features, and combine them with drug fingerprint and network feature. Our method can achieve satisfactory prediction performances, and our prediction can be a kind of ameliorated prediction by comparing with other existing methods after feature selection. However, the imbalanced problem of DTIs prediction is not solved very well. SVM is poor on imbalanced data. The AUPR value of DAWN is low on the Nuclear receptor dataset.

The prediction accuracy may be further enhanced with the further expansion of more refined representation of the structural and physicochemical properties or a better machine learning model 
(such as sparse representation and gradient boosting decision tree) for predicting drug-target interactions. In the future, we will build the classification by the strategy of bootstrap sampling and weighting sub-classifiers.

Acknowledgments: This research and this article's publication costs are supported by a grant from the National Science Foundation of China (NSFC 61,772,362, 61,402,326), Peiyang Scholar Program of Tianjin University (no. 2016XRG-0009), and the Tianjin Research Program of Application Foundation and Advanced Technology (16JCQNJC00200).

Author Contributions: Cong Shen, Yijie Ding and Fei Guo conceived the study. Cong Shen and Yijie Ding performed the experiments and analyzed the data. Cong Shen, Yijie Ding and Fei Guo drafted the manuscript. All authors read and approved the manuscript.

Conflicts of Interest: The authors declare that they have no competing interests.

\section{References}

1. Sayers, E.W.; Barrett, T.; Benson, D.A.; Bryant, S.H.; Canese, K.; Chetvernin, V.; Church, D.M.; DiCuccio, M.; Edgar, R.; Federhen, S.; et al. Database resources of the national center for biotechnology information. Nucleic Acids Res. 2009, 37, 5-15.

2. Cheng, A.C.; Coleman, R.G.; Smyth, K.T.; Cao, Q.; Soulard, P.; Caffrey, D.R.; Salzberg, A.C.; Huang, E.S. Structure-based maximal affinity model predicts small-molecule druggability. Nat. Biotechnol. 2007, 25, 71-75.

3. Rarey, M.; Kramer, B.; Lengauer, T.; Klebe, G. A fast flexible docking method using an incremental construction algorithm. J. Mol. Biol. 1996, 261, 470-489.

4. Yamanishi, Y.; Araki, M.; Gutteridge, A.; Honda, W.; Kanehisa, M. Prediction of drug-target interaction networks from the integration of chemical and genomic spaces. Bioinformatics 2008, 24, i232-i240.

5. Bleakley, K.; Yamanishi, Y. Supervised prediction of drug-target interactions using bipartite local models. Bioinformatics 2009, 25, 2397-2403.

6. Mei, J.P.; Kwoh, C.K.; Yang, P.; Li, X.L.; Zheng, J. Drug-target interaction prediction by learning from local information and neighbors. Bioinformatics 2013, 29, 238-245.

7. Van, L.T.; Nabuurs, S.B.; Marchiori, E. Gaussian interaction profile kernels for predicting drug-target interaction. Bioinformatics 2011, 27, 3036-3043.

8. Xia, Z.; Wu, L.Y.; Zhou, X.; Wong, S.T. Semi-supervised drug-protein interaction prediction from heterogeneous biological spaces. BMC Syst. Biol. 2010, 4, 6-17.

9. Zheng, X.; Ding, H.; Mamitsuka, H.; Zhu, S. Collaborative matrix factorization with multiple similarities for predicting drug-target interactions. In Proceedings of the 19th ACM SIGKDD International Conference on Knowledge Discovery and Data Mining, Chicago, IL, USA , 11-14 August 2013; pp. 1025-1033.

10. Van, L.T.; Marchiori, E. Predicting Drug-Target Interactions for New Drug Compounds Using a Weighted Nearest Neighbor Profile. PLoS ONE 2013, 8, e66952.

11. Gönen, M. Predicting drug-target interactions from chemical and genomic kernels using Bayesian matrix factorization. Bioinformatics 2012, 28, 2304-2310.

12. Liu, Y.; Wu, M.; Miao, C.; Zhao, P.; Li, X.L. Neighborhood Regularized Logistic Matrix Factorization for Drug-Target Interaction Prediction. PLoS Comput. Biol. 2016, 12, e1004760.

13. Cao, D.S.; Liu, S.; Xu, Q.S.; Lu, H.M.; Huang, J.H.; Hu, Q.N.; Liang, Y.Z. Large-scale prediction of drug-target interactions using protein sequences and drug topological structures. Anal. Chim. Acta 2012, 752, 1-10.

14. Cao, D.S.; Zhang, L.X.; Tan, G.S.; Xiang, Z.; Zeng, W.B.; Xu, Q.S.; Chen, A.F. Computational Prediction of DrugTarget Interactions Using Chemical, Biological, and Network Features. Mol. Inform. 2014, 33, 669-681.

15. Kanehisa, M.; Goto, S.; Hattori, M.; Aoki-Kinoshita, K.F.; Itoh, M.; Kawashima, S.; Katayama, T.; Araki, M.; Hirakawa, M. From genomics to chemical genomics: New developments in KEGG. Nucleic Acids Res. 2006, 34, 354-357.

16. Schomburg, I.; Chang, A.; Placzek, S.; Söhngen, C.; Rother, M.; Lang, M.; Munaretto, C.; Ulas, S.; Stelzer, M.; Grote, A.; et al. BRENDA in 2013: Integrated reactions, kinetic data, enzyme function data, improved disease classification: New options and contents in BRENDA. Nucleic Acids Res. 2013, 41, 764-772.

17. Hecker, N.; Ahmed, J.; Eichborn, J.V.; Dunkel, M.; Macha, K.; Eckert, A.; Gilson, M.K.; Bourne, P.E.; Preissner, R. SuperTarget goes quantitative: Update on drug-target interactions. Nucleic Acids Res. 2012, 40, 1113-1117. 
18. Law, V.; Knox, C.; Djoumbou, Y.; Jewison, T.; Guo, A.C.; Liu, Y.; Maciejewski, A.; Arndt, D.; Wilson, M.; Neveu, V.; et al. DrugBank 4.0: Shedding new light on drug metabolism. Nucleic Acids Res. 2014, 42, 1091-1097.

19. Gaulton, A.; Bellis, L.J.; Bento, A.P.; Chambers, J.; Davies, M.; Hersey, A.; Light, Y.; McGlinchey, S.; Michalovich, D.; Al-Lazikani, B.; et al. Chembl: A large-scale bioactivity database for drug discovery. Nucleic Acids Res. 2012, 40, D1100-D1107.

20. Günther, S.; Kuhn, M.; Dunkel, M.; Campillos, M.; Senger, C.; Petsalaki, E.; Ahmed, J.; Urdiales, E.G.; Gewiess, A.; Jensen, L.J.; et al. Supertarget and matador: Resources for exploring drug-target relationships. Nucleic Acids Res. 2008, 36, 919-922.

21. Ding, Y.; Tang, J.; Guo, F. Predicting protein-protein interactions via multivariate mutual information of protein sequences. BMC Bioinform. 2016, 17, 389-410.

22. Ding, Y.; Tang, J.; Guo, F. Identification of Protein-Protein Interactions via a Novel Matrix-Based Sequence Representation Model with Amino Acid Contact Information. Int. J. Mol. Sci. 2016, 17, 1623.

23. Li, Z.; Tang, J.; Guo, F. Learning from real imbalanced data of 14-3-3 proteins binding specificity. Neurocomputing 2016, 217, 83-91.

24. You, Z.H.; Lei, Y K.; Zhu, L.; Xia, J.F.; Wang, B. Prediction of protein-protein interactions from amino acid sequences with ensemble extreme learning machines and principal component analysis. BMC Bioinform. 2013, 14, doi:10.1186/1471-2105-14-S8-S10.

25. Mallat, S.G. A Theory for Multiresolution Signal Decomposition: The Wavelet Representation. IEEE Trans. Pattern Anal. Mach. Intell. 1989, 11, 674-693.

26. Nanni, L.; Brahnam, S.; Lumini, A. Wavelet images and Chou's pseudo amino acid composition for protein classification. Amino Acids 2012, 43, 657-665.

27. Guyon, I.; Weston, J.; Barnhill, S.; Vapnik, V. Gene selection for cancer classification using support vector machines. Mach. Learn. 2002, 46, 389-422.

28. Yan, K.; Zhang, D. Feature selection and analysis on correlated gas sensor data with recursive feature elimination. Sens. Actuators B Chem. 2015, 212, 353-363.

29. Cortes, C.; Vapnik, V. Support-vector networks. Mach. Learn. 1995, 20, 273-297.

30. Zou, Q.; Zeng, J.C.; Cao, L.J.; Ji, R.R. A Novel Features Ranking Metric with Application to Scalable Visual and Bioinformatics Data Classification. Neurocomputing 2016, 173, 346-354.

31. Zou, Q.; Wan, S.X.; Ju, Y.; Tang, J.J; Zeng, X.X. Pretata: Predicting TATA binding proteins with novel features and dimensionality reduction strategy. BMC Syst. Biol. 2016, 10 (Suppl. 4), 114.

32. Wei, L.Y.; Tang, J.J.; Zou, Q. Local-DPP: An Improved DNA-binding Protein Prediction Method by Exploring Local Evolutionary Information. Inf. Sci. 2017, 384, 135-144.

33. Zou, Q.; Li, J.J.; Hong, Q.Q.; Lin, Z.Y.; Wu, Y.; Shi, H.; Ju, Y. Prediction of microRNA-disease associations based on social network analysis methods. BioMed Res. Int. 2015, 2015, 810514.

34. Chang, C.C.; Lin, C.J. LIBSVM: A Library for support vector machines. ACM Trans. Intell. Syst. Technol. 2011, 2, 389-396.

(C) 2017 by the authors. Licensee MDPI, Basel, Switzerland. This article is an open access article distributed under the terms and conditions of the Creative Commons Attribution (CC BY) license (http:/ / creativecommons.org/licenses/by/4.0/). 


\title{
Understanding Insulin Endocrinology in Decapod Crustacea: Molecular Modelling Characterization of an Insulin-Binding Protein and Insulin-Like Peptides in the Eastern Spiny Lobster, Sagmariasus verreauxi
}

\author{
Jennifer C. Chandler ${ }^{1, *}$, Neha S. Gandhi ${ }^{2,3}$, Ricardo L. Mancera ${ }^{3}$, Greg Smith ${ }^{4}$, Abigail Elizur ${ }^{1}$ \\ and Tomer Ventura ${ }^{1, *}$ \\ 1 GenEcology Research Centre, Faculty of Science, Health, Education and Engineering, \\ University of the Sunshine Coast, 4 Locked Bag, Maroochydore, Queensland 4556, Australia; \\ aelizur@usc.edu.au \\ 2 School of Mathematical Sciences, Queensland University of Technology, 2 George Street, Brisbane, \\ Queensland 4000, Australia; neha.gandhi@qut.edu.au \\ 3 School of Biomedical Sciences, Curtin Health Innovation Research Institute and Curtin Institute for \\ Computation, Curtin University, GPO Box U1987, Perth, Western Australia 6845, Australia; \\ r.mancera@curtin.edu.au \\ 4 Fisheries and Aquaculture Centre, Institute for Marine and Antarctic Studies (IMAS), \\ University of Tasmania, Private Bag 49, Hobart, Tasmania 7001, Australia; gregory.smith@utas.edu.au \\ * Correspondence: jennifer.chandler@research.usc.edu.au (J.C.C.); tventura@usc.edu.au (T.V.); \\ Tel.: +61-(0)-754565984 (J.C.C. \& T.V.)
}

Received: 24 July 2017; Accepted: 19 August 2017; Published: 23 August 2017

\begin{abstract}
The insulin signalling system is one of the most conserved endocrine systems of Animalia from mollusc to man. In decapod Crustacea, such as the Eastern spiny lobster, Sagmariasus verreauxi $(\mathrm{Sv})$ and the red-claw crayfish, Cherax quadricarinatus (Cq), insulin endocrinology governs male sexual differentiation through the action of a male-specific, insulin-like androgenic gland peptide (IAG). To understand the bioactivity of IAG it is necessary to consider its bio-regulators such as the insulin-like growth factor binding protein (IGFBP). This work has employed various molecular modelling approaches to represent $S$. verreauxi IGFBP and IAG, along with additional Sv-ILP ligands, in order to characterise their binding interactions. Firstly, we present Sv- and Cq-ILP2: neuroendocrine factors that share closest homology with Drosophila ILP8 (Dilp8). We then describe the binding interaction of the N-terminal domain of Sv-IGFBP and each ILP through a synergy of computational analyses. In-depth interaction mapping and computational alanine scanning of IGFBP_N' highlight the conserved involvement of the hotspot residues $Q_{67}, G_{70}, D_{71}, S_{72}, G_{91}, G_{92}$, $\mathrm{T}_{93}$ and $\mathrm{D}_{94}$. The significance of the negatively charged residues $\mathrm{D}_{71}$ and $\mathrm{D}_{94}$ was then further exemplified by structural electrostatics. The functional importance of the negative surface charge of IGFBP is exemplified in the complementary electropositive charge on the reciprocal binding interface of all three ILP ligands. When examined, this electrostatic complementarity is the inverse of vertebrate homologues; such physicochemical divergences elucidate towards ligand-binding specificity between Phyla.
\end{abstract}

Keywords: insulin-like growth factor binding protein (IGFBP); insulin-like androgenic gland peptide (IAG); insulin-like peptides (ILP1; ILP2); molecular modelling; binding interaction; alanine scanning; hotspot residue; electrostatics; decapod 


\section{Introduction}

The binding interaction of insulin-like growth factor binding proteins (IGFBPs) and their insulin-like growth factor (IGF) ligands has been a significant focus of IGF endocrinology for the past two decades [1-3]. This is reflective of the central function of the high-affinity IGFBP subgroup (IGFBP1-6) in mediating the bioavailability and activity of IGFI and II at their receptor(s) [1-3]. In doing so, IGFBPs not only facilitate the translocation of their binding partners but they also provide proteolytic protection, extending the half-life and maintaining a functionally viable reservoir of the hormone in circulation [1-3].

The structure of the IGFBP is central to this function. Although domain specifics of the superfamily vary, most notably across the low-affinity IGFBP-related subgroup (IGFBP-rP1-9) [1], the family conforms to a common architecture: a highly structured, globular $\mathrm{N}$ terminal $\left(\mathrm{N}^{\prime}\right)$ insulin-binding domain; a flexible linking domain; and a flexi-folded $\mathrm{C}$ terminal $\left(\mathrm{C}^{\prime}\right)$ domain, which is the most variable domain across subgroups and species $[1,3]$. The highly structured $N^{\prime}$ insulin-binding domain (the only domain conserved across the entire IGFBP superfamily) provides the primary binding interface for the ligand and is capable of binding in isolation [4]. In the case of IGFBP1-6, the $C^{\prime}$ domain functions to maximise binding affinity, encapsulating the ligand to stabilise binding by interacting with the $\mathrm{N}^{\prime}$ domain $[3,5,6]$. In doing so, the $C^{\prime}$ shields some of the key residues involved in the interaction of IGF with its receptor, increasing the antagonistic action of the IGFBP [5]. This synergistic binding of $\mathrm{N}^{\prime}$ and $\mathrm{C}^{\prime}$ domains is coordinated through the flexible linking domain [1].

We have identified an IGFBP homologue in the decapod crustacean Sagmariasus verreauxi, commonly referred to as the Eastern spiny lobster [7], prior to which a similar protein was identified in the red-claw crayfish (Cherax quadricarinatus) [8]. Additional homologues have since been found in a prawn, Macrobrachium nipponense [9], and two crab species, Scylla paramamosain [10] and Callinectes sapidus [11]. These decapod IGFBPs share closest homology with the human IGFBP-rP1 (known as MAC25) from the low-affinity IGF-binding subgroup. They all share a kazal-type serine proteinase inhibitor as the linking domain and an immunoglobulin-like domain as the $C^{\prime}$ domain (rather than the thyroglobulin-type I domain of human IGFBP1-6 [1]). Unlike IGFBP1-6, the binding capacity of IGFBP-rP1 is more diverse, enabling it to bind insulin with a similar affinity as the IGFs, although with a reduced affinity compared to its specialised (IGFBP1-6) counterparts [12]. This is thought to be achieved through the substituted $C^{\prime}$ immunoglobulin domain [1], which is proposed to reduce the synergistic $\mathrm{N}^{\prime}$ and $\mathrm{C}^{\prime}$ domain high-affinity binding for IGFs, whilst also better exposing the insulin binding site $[13,14]$. In addition, it has been recognised that although they share the same overall fold, the $\mathrm{N}^{\prime}$ insulin-binding domain of this IGFBP-r subgroup contains notable structural variations from IGFBP1-6, resulting in a decreased IGF binding affinity [15]. Thus some suggest that the IGF binding of the IGFBP-r subgroup is biologically irrelevant $[15,16]$, advising of a primary function unrelated to IGF binding [17]. Even so, the general consensus appears to be that the IGFBP-r subgroup functions in both IGF-dependent and independent roles [1,17].

In the context of the decapod IGFBPs, the homology with the less IGF-specific IGFBP-rP1 is likely to have functional significance, relating to the ligands with which these decapod homologues bind. The IGFs comprise one subgroup of the insulin-like superfamily, with the insulin-like peptides (ILP) encompassing the other $[7,18]$. The structural distinction centres around the pre-prohormone structure and processing. IGFs tend to retain their truncated C-domain and have additional D (uncleaved) and $\mathrm{E}$ (cleaved) domains after the A-chain $[2,19,20]$. ILPs undergo cleavage of the C-peptide and terminate after the A-chain $[7,18]$. However, both IGFs and ILPs share the same disulfide bond topology, with two inter (B to A) and one intra (A)-chain bonds [18,20].

IGF homologues have not yet been identified in decapods, but the Crustacean class Malocastraca (which includes the Order Decapoda) is known for an ILP termed the insulin-like androgenic gland peptide (IAG). This hormone, only found in males (with noted exceptions [21,22]), is specifically produced and secreted from a male-specific endocrine gland known as the androgenic gland (AG) [23-25]. Upon secretion, IAG stimulates and maintains the broad tissue effects of male 
sexual differentiation and maturation [26-30], reviewed in [31]. More recently, the prevalence of ILPs in these species has diversified with the first identification of a DILP7/relaxin-like ILP in S. verreauxi (Sv-ILP1) [7], since identified across the Order [32].

Work in C. quadricarinatus has already demonstrated the capacity of Cq-IGFBP to bind Cq-IAG through a pull-down assay with AG homogenate, where the IGFBP was shown to bind residues within the A-chain, B-chain and C-peptide; highlighting the ability of the IGFBP to also bind the IAG pre-prohormone [8]. The IGF/ILP receptor signalling system, as characterised in mammals [2,3] and Drosophila [33], is conserved in decapods (as evidenced by the identification of an active tyrosine kinase insulin receptor (TKIR) [34,35] and an inactive decoy (TKIR_decoy) [34]). Consequently, it seems highly likely that the IGFBP will adopt a similarly conserved role within the system. Thus, to realistically interpret the bioactivity of IAG in mediating male sexual development, we must integrate the regulatory influences of the IGFBP. Furthermore, the identification of additional ILPs [7] and the broad tissue distribution of the IGFBP $[7,8,10,11]$ in the decapods may suggest a multi-ligand binding role.

In light of the dramatic advancements that have been made in the field of computational protein-modelling and interaction studies [36,37], this work employed an in silico approach to study the IGFBP_N'-ILP ligand interaction in decapod Crustacea. Firstly, we present Sv-ILP2 and Cq-ILP2, which are novel to the Order. We then model the $\mathrm{N}^{\prime}$ domain of Sv-IGFBP and each ligand (Sv-IAG, ILP1 and ILP2) in order to characterise the binding interaction of each. In doing so we determine a subset of consistently interacting residues at the IGFBP interface, involved in binding all three ligands, which are further suggested to be hotspots based on computational alanine scanning. Electrostatic potential surface calculations illustrate the significance of the negatively charged hotspots, suggesting them to be a fundamental feature of complex formation. Together, these analyses emphasise the consequence of amino-acid variations in determining the physicochemical structure and consequential binding interactions of the seemingly conserved $\mathrm{N}^{\prime}$ insulin-binding domain of the IGFBP.

\section{Results}

\subsection{Identification of Sv-ILP2}

This work is the first to describe the identification of a second insulin-like peptide in S. verreauxi, Sv-ILP2 (KP006646). Sv-ILP2 conforms to the ILP superfamily structure exhibiting all of its conserved features: a signal peptide ( 20 amino acids (aa)); followed by a B-chain ( $28 \mathrm{aa})$ containing two cysteines; a C-peptide (71 aa), flanked by RR cleavage sites; and an A-chain (17 aa) containing a double and two single cysteines (Figure 1a). Interestingly, unlike Sv-IAG and Sv-ILP1, Sv-ILP2 contains multiple RR cleavage sites throughout the C-peptide. These additional chains are somewhat reminiscent of the additional $\mathrm{D}$ and $\mathrm{E}$ domains of the IGF-like pre-prohormone structure. However, as the CCxxxC cysteine signature is located in the $C^{\prime}$ domain of the open reading frame it was considered to be the A-chain, suggesting these additional cleavage sites constitute an elongated C-peptide; we therefore classified Sv-ILP2 as an ILP rather than an IGF.

Spatial expression analyses of Sv-ILP2 (Figure 1b) show it to be predominantly expressed in the neuroendocrine tissues (brain and eyestalk) of males and females, although all RPKMs are low (RPKMs as follows: female eyestalk 1.15; male eyestalk 0.8; male brain 0.39; immature AG 0.2). Temporal RT-PCR analyses were also conducted (from phyllosoma instar 16 to puerulus) but Sv-ILP2 expression was not identified (data not shown). When blasted at NCBI (https:/ / blast.ncbi.nlm.nih.gov/Blast.cgi), neither the pre-pro nor mature hormone gave any significant hits, although our phylogenetic analyses (with a range of model ILPs) shows that Sv-ILP2 clusters with Dilp8 (Figure 1c). Thus, Sv-ILP2 is the first of a new subclass of ILPs to be described in the decapods.

This work also presents two homologues of Sv-ILP1 and ILP2 (S. verreauxi, Suborder Achelata) in the red-claw crayfish, C. quadricarinatus (Suborder Astacidea); we have therefore named these peptides Cq-ILP1 (KP006644) and Cq-ILP2 (KP006645) (Figure 1c). Cq-ILP1 is comprised of: a signal peptide 
(25 amino acids (aa)); a B-chain (37 aa); a C peptide (115 aa); and an A-chain (37 aa). Cq-ILP2 is comprised of a: signal peptide (25 aa); a B-chain (29 aa); a C-peptide (159 aa); and an A-chain (17 aa). When assessed by RT-PCR, the Cq homologues display similar spatial expression to that described in $S$. verreauxi, with Cq-ILP1 present in the male and female brain, antennal gland, gonads, and the female, but not male hepatopancreas, while Cq-ILP2 expression is specific to the male and female brain and thoracic ganglia (no expression in the eyestalk) (data not shown).

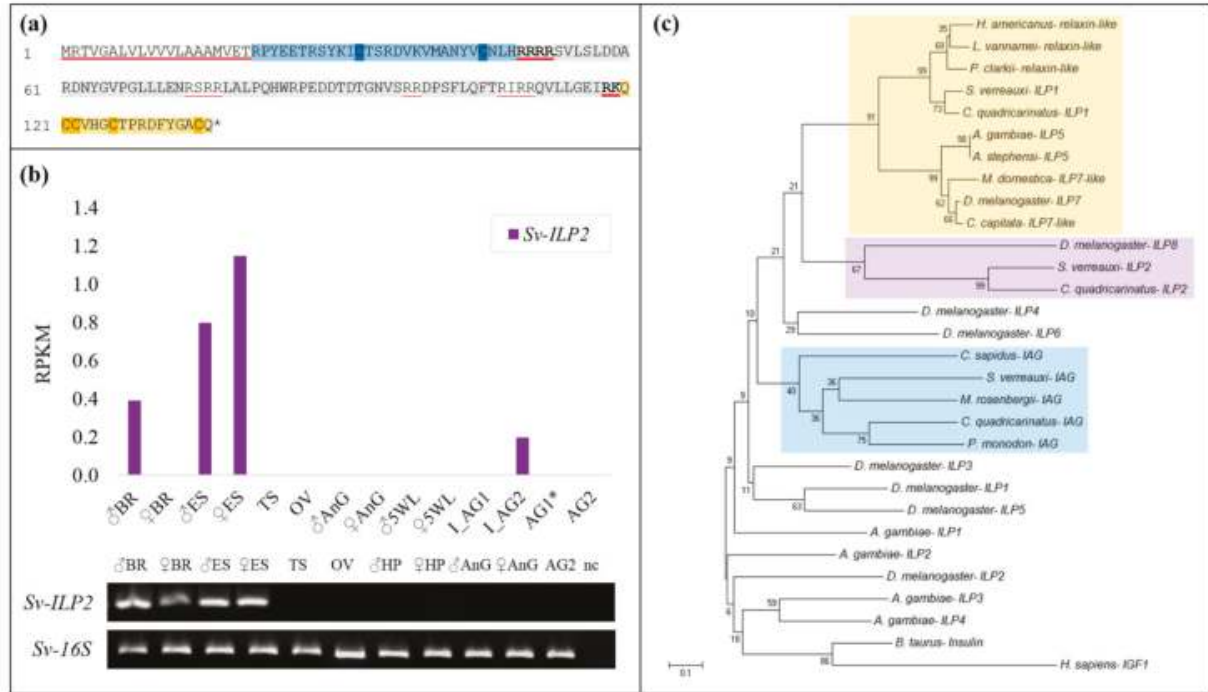

Figure 1. Spatial expression and phylogeny of Sv-ILP2: (a) sequence of Sv-ILP2, the signal peptide is underlined in red and the B-(blue) and A-(orange) chains boxed with the cysteine core of each highlighted. The C-peptide is shown in grey with the predicted Arg-C cleavage sites shown, with those predicted to generate the mature hormone underlined in red. (b) Transcriptomic spatial expression of Sv-ILP2 quantified as reads per kilobase per million reads (RPKM) for male and female brain (BR), eyestalk (ES), gonads (TS and OV), antennal gland (AnG), and fifth walking leg (5WL), immature androgenic glands (I_AG1 and I_AG2), and mature androgenic glands (AG1* and AG2, where * indicates a hypertrophied gland). Validated with spatial RT-PCR analyses, with the removal of the immature AGs, 5WL, and AG1*, and the addition of male and female hepatopancreas (HP). Negative control (NC) in the fifteenth lane, $16 S$ as positive control. (c) Neighbour-joining phylogram of Sv- and Cq-ILP1 and ILP2 with a range of ILPs from model species: Anopheles ILP1-5, Drosophila Dilp1-8, decapod insulin-like androgenic gland peptides (IAGs), and bovine insulin and human IGFI. Bootstrap values are shown at each node and were performed with 1000 replicates. Scale bar indicates number of amino acid substitutions per site. IAG cluster boxed in blue, Dilp7/relaxin-like cluster in yellow, and novel decapod ILP2s in purple.

\subsection{Sequence Analyses of IGFBPs and ILP Ligands}

We conducted pairwise alignment of the IGFBP, IAG, ILP1, and ILP2 peptides from S. verreauxi and C. quadricarinatus to assess physicochemical conservation. The IGFBP sequences share a pairwise identity score of $68.9 \%$ and significant conservation in physicochemical properties (Figure 2a). With regard to the ligands, the IAG peptides share the lowest identity score of the three ILPs, at $32.2 \%$ across the pre-prohormone and 35.5\% across the mature hormone (consisting of only the $\mathrm{A}$ and B-chains) (Figure 2b). The ILP1 homologues share the highest conservation at $64.6 \%$ across the pre-prohormone and $83.1 \%$ across the mature hormone (Figure $2 \mathrm{c}$ ). This high identity score is 
fitting with the strong conservation of this relaxin-like ILP subclass across species (also known as the Dilp7-likes characterized in Drosophila).

The ILP2 homologues share an identity score of $44.6 \%$ at the pre-prohormone level, which increases to $57.8 \%$ for the mature peptide (Figure $2 \mathrm{~d}$ ). As previously mentioned, we surmise that the final cleavage site indicates the beginning of the A-chain, based on the structural placement of the cysteines but also reflective of the increased conservation observed between the Sv- and Cq-ILP2 homologues in this $\mathrm{C}^{\prime}$ domain. These sequence alignments exemplify the minimized evolutionary restraint within the $\mathrm{C}$-peptide, showing far higher rates of divergence than that observed in the $\mathrm{A}$ and B-chains which ultimately form the mature and active hormone.

(a)

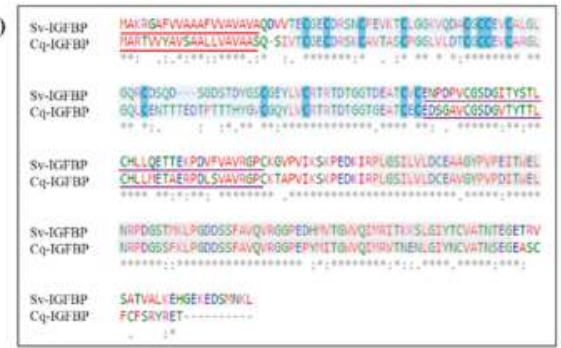

(b)

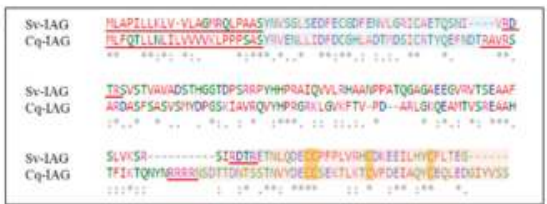

(c)

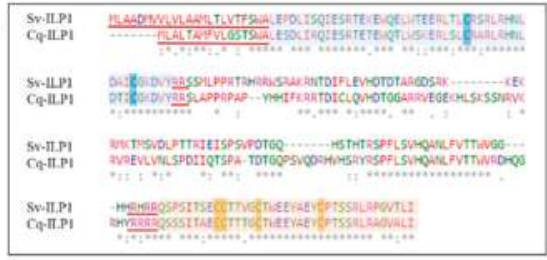

(d)

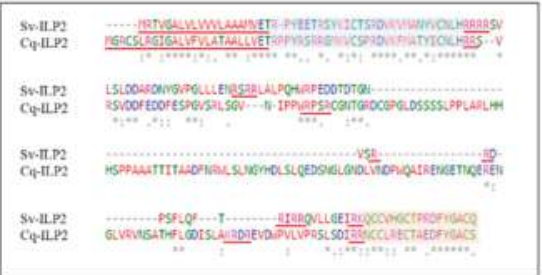

Figure 2. Sequence alignment of Sagmariasus verreauxi and Cherax quadricarinatus IGFBP and ILP homologues, with emphasis on the conservation of physicochemical properties. Residues are colored in accordance with properties: red-defines small, hydrophobic residues; blue-negatively charged/acidic; magenta-positively charged/basic; and green—polar and amine groups. An asterisk $\left(^{*}\right)$ indicates a conserved amino acid, a colon (:) those with conserved physicochemical properties, and a full stop (.) those with weakly similar properties. (a) Compares Cq-IGFBP and Sv-IGFBP, the signal peptide is underlined in red, the insulin-binding domain boxed in blue with the cysteine core highlighted, the kazal domain underlined in purple, and the $\mathrm{C}^{\prime}$ immunoglobulin domain boxed in grey. (b) Compares Cq-IAG and Sv-IAG; (c) Sv-ILP1 and the novel Cq-ILP1; and (d) the novel Sv-ILP2 and Cq-ILP2. In each case the signal peptide is underlined in red and the mature hormone is highlighted as the B-(blue) and A-(orange) chains with the cysteine core of each highlighted; C-peptide Arg-C proteinase cleavage sites are underlined in red.

To provide a more cohesive understanding of the insulin-signaling system in S. verreauxi, we generated a spatial expression profile summarizing all of the insulin factors identified in the species to date (Figure 3), namely, Sv-IAG [38]; Sv-IGFBP and Sv-ILP1 [7]; Sv-TKIR and Sv-TKIR_decoy [34,39]; and Sv-ILP2, presented in this work (all RPKMs have been validated with independent RT-PCR, some with additional in situ analyses; related references given above after each gene). Of note is the broad tissue distribution of Sv-IGFBP and the dramatically higher expression of IAG relative to all other endocrine factors. However, this expression must be considered in the context of localization, as IAG is secreted by a relatively small subset of cells, all of which are accounted for in this one gland. 


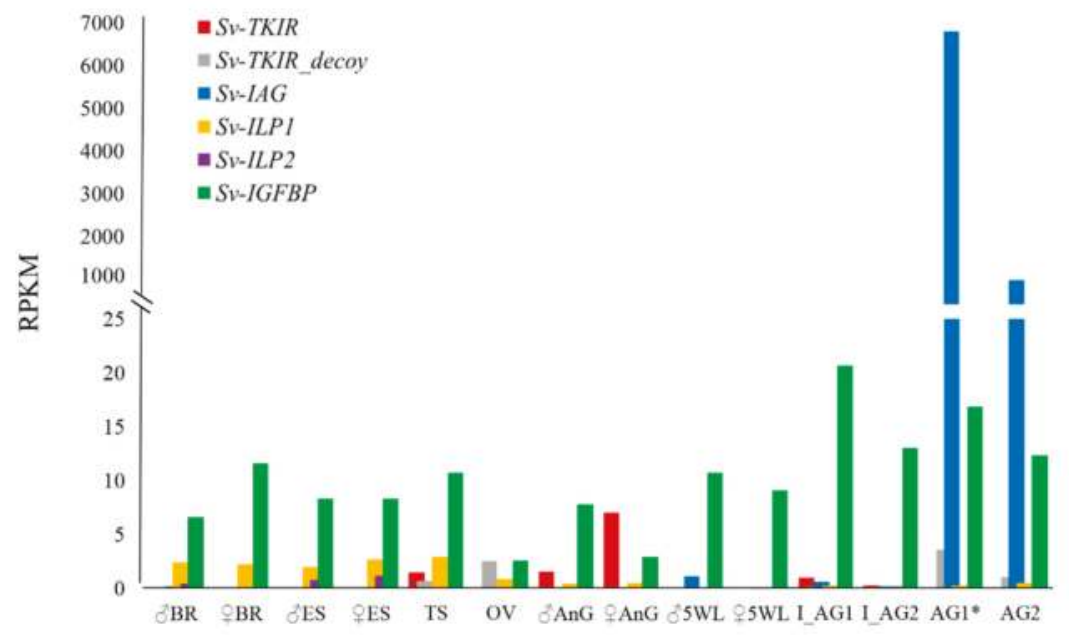

Figure 3. Spatial expression profile summarising all insulin factors identified in Sagmariasus verreauxi for a cohesive depiction of our current understanding of the insulin endocrine system: IAG [38]; ILP1 and IGFBP [7]; the active (TKIR) and decoy (TKIR_decoy) tyrosine kinase insulin receptors [34,39]; and Sv-ILP2. Quantified as RPKM; tissue abbreviations as previously described.

\subsection{Structural Modelling}

Structures of Sv-IGFBP (3TJQ and 2CQV) and Sv-IAG, Sv-ILP1 and Sv-ILP2 (2KQP) were predicted by homology modelling using the described PDB templates. The predicted structure of Sv-IGFBP provides clear visualization of the domain architecture of the molecule (Figure 4a). The highly structured $\mathrm{N}^{\prime}$ insulin-binding domain contains seven disulfide bonds, ensuring accurate folding of the binding interface. The kazal domain, defined as the flexible linking region which connects the $\mathrm{N}^{\prime}$ and $\mathrm{C}^{\prime}$ domains, contains an alpha helical region and one disulfide bond. The $\mathrm{C}^{\prime}$ immunoglobulin domain also contains a disulfide bond, as well as a cis-peptide bond, but lacks any other significant orienting features and is mainly comprised of beta pleated sheets and random coils.

Each ILP ligand conforms to the characteristic tertiary structure of the ILP family with two inter and one intra (A-chain) disulfide bonds that determine the overall fold of the molecule (Figure $4 \mathrm{~b}-\mathrm{d}$ ). Within the confines of the generic ILP fold, each protein displays unique features. The most prominent of these include the elongated A-chain of Sv-ILP1, which does not form the usual alpha-helix but instead a random coil (Figure 4c; Figure S1). Conversely, Sv-ILP2 has a truncated A-chain, where the residues ${ }_{1} \mathrm{QCCV}_{4}$ result in an alpha turn rather than a complete helix (Figure $4 \mathrm{~d}$; Figure S1). Refer to M\&M and Figure S1 for full modelling procedures. It is these features that determine the specifics of the interactive interface shared between the A- and B-chains, dictating the molecular structure of each ligand. This is best illustrated by the inter-chain interactions: the A- and B-chains of IAG, ILP1, and ILP2 are predicted to share one, three, and one hydrogen bonds and 153, 109, and 74 non-bonded contacts, respectively, in addition to the two disulfide bonds common to all three (predicted by PDBsum) (Figure S2). 


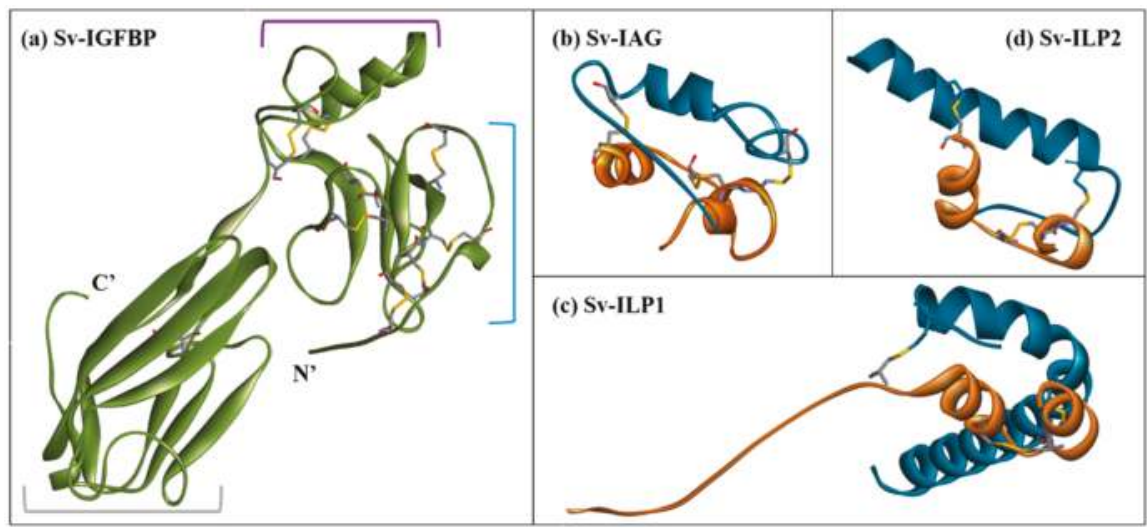

Figure 4. Molecular structure models of (a) Sv-IGFBP, the domains of which are indicated with: a blue bracket for the highly structured $\mathrm{N}^{\prime}$ insulin-binding domain; a purple bracket for the linking kazal domain; and grey bracket for the $C^{\prime}$ immunoglobulin domain. (b) Sv-IAG, (c) Sv-ILP1, and (d) Sv-ILP2 are shown as previously described with the A-chain in orange and B-chain in blue. All structures are shown in secondary structure ribbon format with disulfide bonds highlighted as sticks.

\subsection{Complex Formation}

Complex formation and protein-protein interactions were then investigated through both manual structural alignment and predictive binding analyses. Due to the reduced reliability of modelling the bound $C^{\prime}$ immunoglobulin domain, Sv-IGFBP was truncated after the kazal domain (Sv-IGFBP_N') (as is common in IGFBP binding studies [40,41]; PDB: 1H59 [42]; PDB: 1WQJ [5]). Sv-IGFBP_N' was used for all further interaction studies. We collated manual alignment analyses, with predicted binding interactions generated through PDBsum [43] and PRODIGY [44] to generate an interaction map of all the residues involved in complex formation (Figure 5). Visual comparison of the three Sv-IGFBP_N' complexes (Figure 5a) clearly demonstrates the binding interface of the $\mathrm{N}^{\prime}$ insulin-binding domain (supported by HADDOCK2.2 simulations: Figure S3), with all highlighted interacting residues predicted by both PDBsum and PRODIGY (shown in Figure 5b; additional residues predicted by PRODIGY presented in Table S1). Of all the predicted interacting residues presented in Figure 5b, we have highlighted those amino acids of IGFBP_N' that show conserved interaction contacts with all three ligands $(*)$, namely: the negatively charged $\operatorname{Asp}(D)_{71}$ and $\operatorname{Asp}(D)_{94}$; supported by the polarGln $(\mathrm{Q})_{67}$ (where proton acceptor properties enable it to form two hydrogen bonds, stabilizing the overall negative charge); the neutrally charged $\operatorname{Ser}(\mathrm{S})_{72}$ and $\operatorname{Thr}(\mathrm{T})_{93}$; and $\operatorname{Gly}(\mathrm{G})_{70}, \operatorname{Gly}(\mathrm{G})_{91}$, and $\operatorname{Gly}(\mathrm{G})_{92}$. In addition to these eight consistent contacts of IGFBP_N', PRODIGY predicts a further nine (Table S1). The physicochemical nature of each interaction as predicted by PRODIGY suggests that a relatively even contribution of charged, polar, and non-polar interactions contribute to binding, with charged forces slightly dominating with IAG and ILP1 and hydrophobic forces being slightly dominant with ILP2 (Table S1b). 

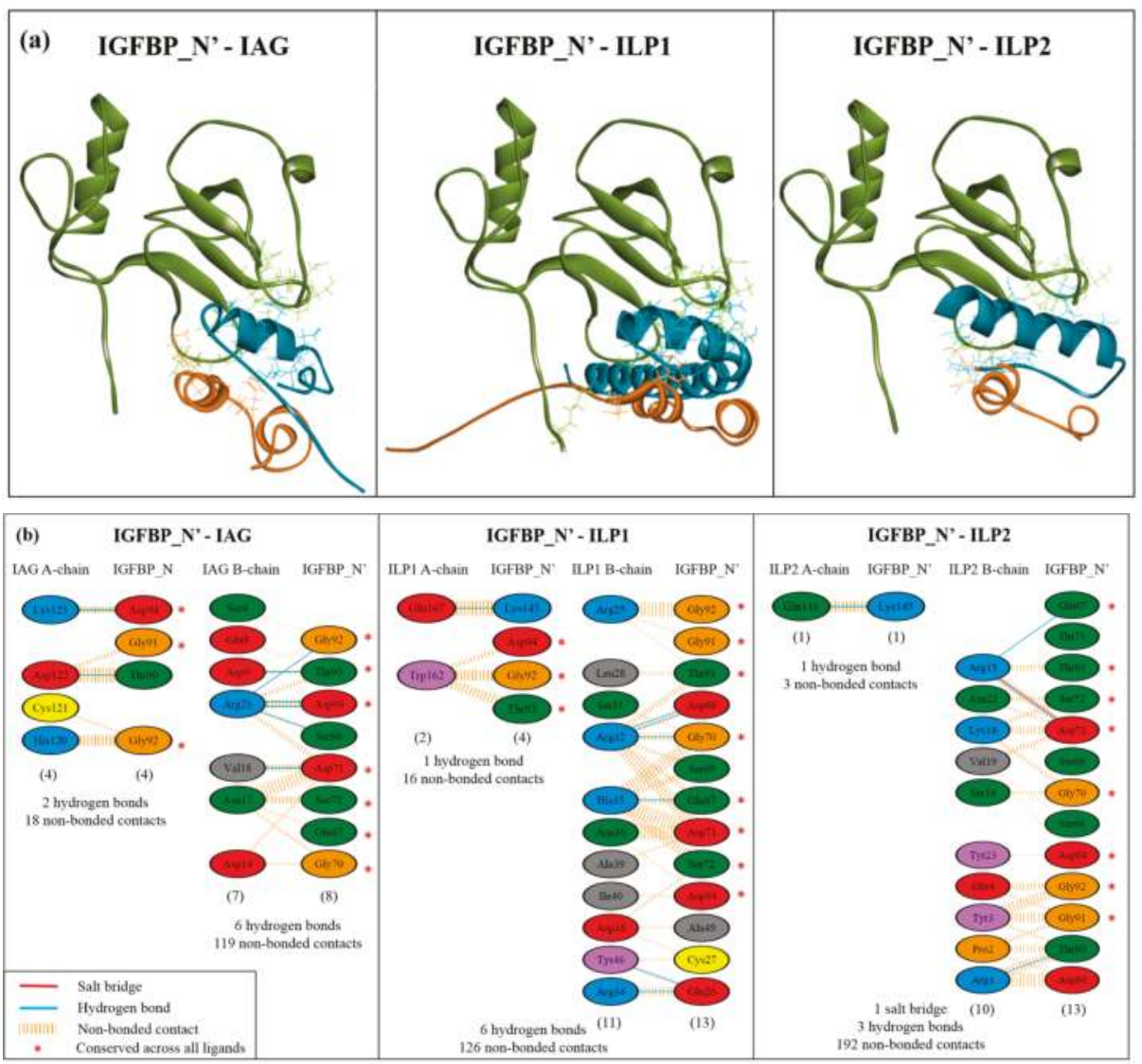

Figure 5. Molecular structure models of bound complexes and interaction map: (a) Complex of Sv-IGFBP_N' with each ligand. In each case the IGFBP_N' is in green and the ligand in orange (A-chain) and blue (B-chain). All residues involved at the binding interface are indicated in stick, with colouring as previously described. (b) Interaction map describing residue-specific interactions between Sv-IGFBP_N' and each ligand. Standard amino acid abbreviations are used, with colours indicating physicochemical properties as follows: blue-positive, red-negative, green-neutral, grey—aliphatic, mauve-aromatic, orange—proline and glycine, and yellow-cysteine. Number of interacting residues is given in brackets; an asterisk denotes those residues that show a conserved binding interaction with all three ligands $(n=8)$.

\subsection{Interaction Hotspots}

As the IGFBP_N' is predicted to contain a subset of consistently interacting residues when in complex with all three ligands, the energy contributions of these residues were investigated using computational alanine scanning to highlight binding hotspots. A hotspot is defined as an amino acid that significantly decreases the binding free-energy of complex formation. As a rule: hotspot potential increases as the degree of buriedness of a residue decreases (due to shape complementarity); it increases if a given residue forms a salt bridge, and hotspot residues tend to show structural and interactive complementarity to that of their binding partners. In brief, alanine scanning is conducted by mutating each residue in the complex to alanine, removing the sidechains that are fundamental to its 
physicochemical and interaction properties. The binding free-energy is then calculated and compared to the wild-type. In doing so, those residues that contribute most significantly to achieving the binding energetics of complex formation are determined. This work used five independent algorithms to generate a meta-style analysis. These hotspot predictions are in strong support of our predicted interactions, with all the consistently interacting residues predicted by both PDBsum and PRODIGY being identified as hotspots (Figure 6a). Furthermore, of the additional predicted hotspots, four of the seven residues were predicted as consistently interacting residues by PRODIGY (denoted with a ${ }^{\mathrm{P}}$ in Table S1). Together, this allows confident prediction that these residues are vital to the binding capacity of IGFBP_N', suggesting a synergistic physicochemical influence of negative charge, polar neutral residues, and glycine. Figure $6 \mathrm{~b}$ shows the sequence positioning of these residues, which appear to cluster into two defined regions as hotspot pockets, which, at the structural level, orientate across the exposed binding interface (Figure 6c).

(a)

\begin{tabular}{|c|c|c|c|}
\hline Software & LAG-IGFBP_N" & ILPI-IGFBP_N & ILP2-IGFBP_N' \\
\hline Cpeliab & D71 $>$ Das & 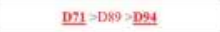 & D94 \\
\hline Rabetra & $\underline{\mathbf{s} 2} \times \underline{\mathbf{p} 71}>\mathrm{D} 65$ & T8s & $\underline{992}>\mathrm{T} 86>\underline{\mathrm{T} 93}$ \\
\hline KFC & 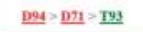 & 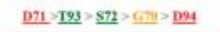 & $\underline{\mathrm{D} 71}>\underline{\mathrm{S} 72}>\mathrm{S} 66>\underline{\mathrm{D} 94}>\underline{\mathrm{CO} 2}$ \\
\hline ANCHOR & D24 $>\$ 66, \mathrm{~T} 90>192$ & $\mathrm{D} 68>126 \mathrm{G}>\mathrm{G}, \mathrm{711}>\mathrm{D} 74>\mathrm{D} 65, \mathrm{D71}$ & 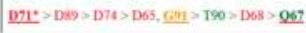 \\
\hline Hat-Pviat & men & $\underline{971}, 572$ & $566, \underline{572} \underline{194}$ \\
\hline Concensus & D71 and D94 & D71 (\$72 and 1094) & prix and pys \\
\hline \multicolumn{4}{|c|}{ - _ECGECDRSNCPEVKTCLCGKVQDACCCCEVCALGLOQRCDSQDS SDSTDYGSCGEYLVCATRTDTGOTDEATCVC } \\
\hline \multicolumn{4}{|c|}{ ENPDPVCGSDGITYSTLCHLLQETTEKPDVFVAVRGFCKGVVVIKSKPEDKIR } \\
\hline \multicolumn{4}{|c|}{ Conserved interacting residves and perdicted hotspots. } \\
\hline \multicolumn{4}{|c|}{ 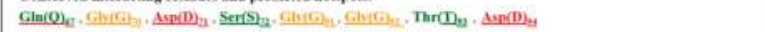 } \\
\hline \multicolumn{4}{|c|}{ Additional hosesoess } \\
\hline \multicolumn{4}{|c|}{ 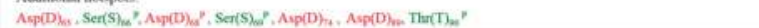 } \\
\hline
\end{tabular}

(c)

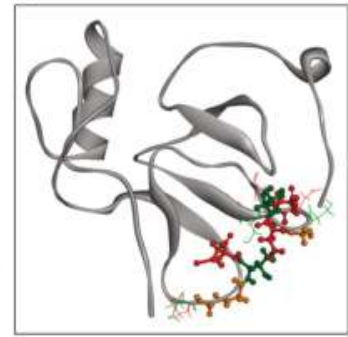

Figure 6. Identification of hotspot residues: (a) determined by five independent alanine scanning algorithms. Amino acids are shown in standard letter notation. Other notation is as follows: an asterisk $\left({ }^{*}\right)$ indicates the presence of a salt bridge predicted by PDBsum; a dash (') a salt bridge predicted by Cpclab; bold underlined indicates residues that show a conserved binding interaction with all three ligands (see Figure 5b). (b) Illustration of Sv-IGFBP_N' sequence (insulin-binding domain boxed in blue and kazal domain underlined in purple) highlighting the positioning of predicted hotspots. The eight consistently interacting hotspot residues are in bold underline. Additional predicted hotspots are also listed, with a ${ }^{\mathrm{P}}$ highlighting those residues that were predicted as consistently interacting residues by PRODIGY. (c) Structural illustration of Sv-IGFBP_N' with conserved interacting hotspot residues highlighted in ball and stick and additional hotspots in stick. Throughout, red indicates negatively charged residues; green, neutrally charged; and orange, glycine. Note the neutral $G \ln (Q)$ has been underlined in red in (b) sequence and coloured red in (c) structure due to its role in stabilizing the overall negative charge through the formation of hydrogen bonds.

\subsection{Electrostatic Potential Molecular Surfaces}

Considering the conserved prevalence of negatively charged residues throughout the hotspot predictions, we conducted an electrostatic potential surface analysis to further investigate the significance of these negatively charged residues. Figure 7a shows the electrostatic potential surface of the IGFBP_N'-IAG complex and the binding interfaces of each individual molecule, as well as the additional ligands, ILP1 (Figure 7b) and ILP2 (Figure 7c). The binding interface of Sv-IGFBP_N' is indeed characterized by a strong negative electrostatic potential. The complementary positive electrostatic potential that exists on the reciprocal interface of all three ligands is in support of an electrostatic interaction. Considering the sequence conservation of Sv-IGFBP and Cq-IGFBP (Figure 2a), we performed similar analyses on Cq-IGFBP_N'. Indeed, the negative electrostatic potential 
of the predicted binding interface is conserved to Cq-IGFBP_N' ${ }^{\prime}$, as is the broader physicochemical nature of 13 of the 15 interaction hotspots characterized in Sv (Figure 8). Of note is the Glu(E) 70 substitution in $\mathrm{Cq}$ (replacing the $\mathrm{Gln}(\mathrm{Q})_{67}$ of $\mathrm{Sv}$ ) emphasizing the suggested negative-centered properties of this residue in Sv. This is indicative of conserved interactive properties and the resulting binding mechanism of the two IGFBP_N' of these species.

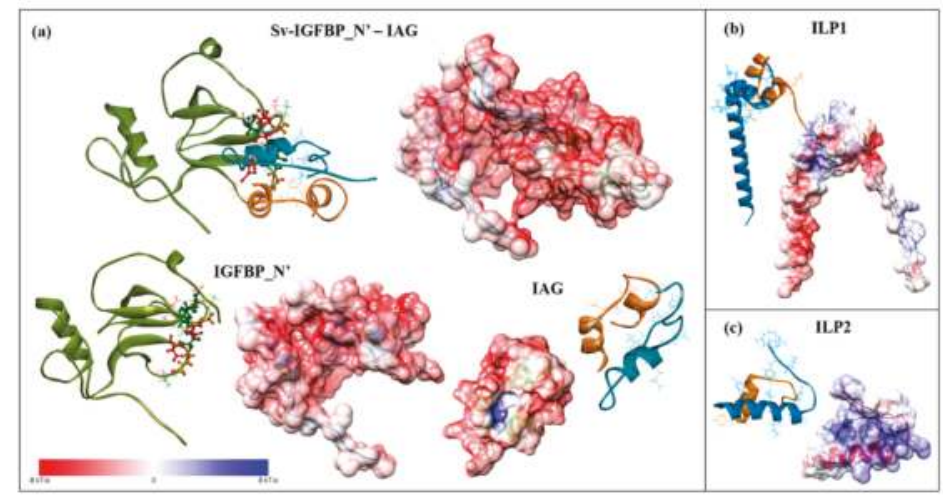

Figure 7. Electrostatic potential surface of: (a) Sv-IGFBP_N'-IAG complex and the individual binding partners; (b) ILP1; and (c) ILP2. IGFBP_N' is coloured in green and the ligands in blue and orange; the interacting hotspot residues of IGFBP_N' are highlighted as described in Figure 6c; ligands have been orientated to display the binding interface. Surfaces are colored by potential on the solvent accessible surface on a scale of $-\mathrm{kT} / \mathrm{e}$ (red) to $+\mathrm{kT} / \mathrm{e}$ (blue), as indicated by the scale bar.

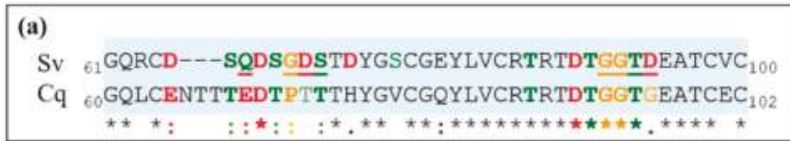

(b) Cq-IGFBP_N'

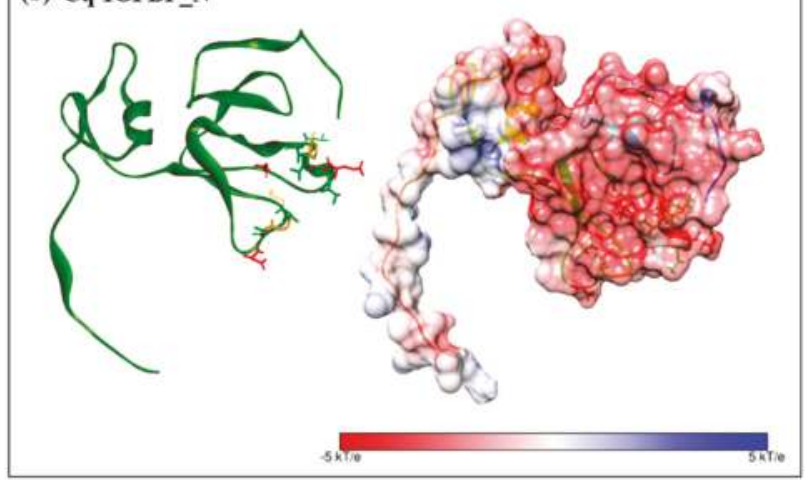

Figure 8. Comparison of Sv-IGFBP_N' and Cq-IGFBP_N': (a) sequence alignment of the focal region of Sv and Cq insulin-binding domains (boxed in blue); (b) Electrostatic potential surface of Cq-IGFBP_N'. All physicochemical conserved residues (predicted as interaction contacts in Sv) are highlighted in bold in sequence and as sticks in structure, the two non-conserved residues ( $\mathrm{Thr}_{75}$ and Gly97) are shown in line. Throughout, red indicates negatively charged residues; green, neutrally charged; and orange, proline and glycine. Surfaces are colored by potential on the solvent accessible surface on a scale of $-\mathrm{kT} / \mathrm{e}$ (red) to $+\mathrm{kT} / \mathrm{e}$ (blue), as indicated by the scale bar. 
For evolutionary comparison, we conducted electrostatic potential surface calculations on the template complexes IGFBP4_N' and IGFI (PDB: 1WQJ) (Figure 9a), and IGFBP5_N' and IGFI (PDB: 1H59) (Figure 9b), as well as IGFII (PDB: 2L29) (Figure S4), and found that these human complexes (positive electrostatic potential on the IGFBP and negative on the ligand) display inverse complementary electrostatic potentials to those described in S. verreauxi and C. quadricarinatus (negative electrostatic potential on the IGFBP and positive on the ligand). We then proceeded to conduct electrostatic potential surface calculations on a range of publicly available vertebrate IGFBP1_N $\mathrm{N}^{\prime}$ and IGFI structures. All of those analyzed (namely rat, cow, chicken, for which IGFBP2_N' replaced IGFBP1 and salmon) display a similar electrostatic potential complementarity to that observed in human, with a positive (IGFBP) and negative (IGF) electrostatic potential (data not shown). Thus, the complementarity common to the vertebrate IGFBP-ligand examples differs from that described in these decapod Crustacea.
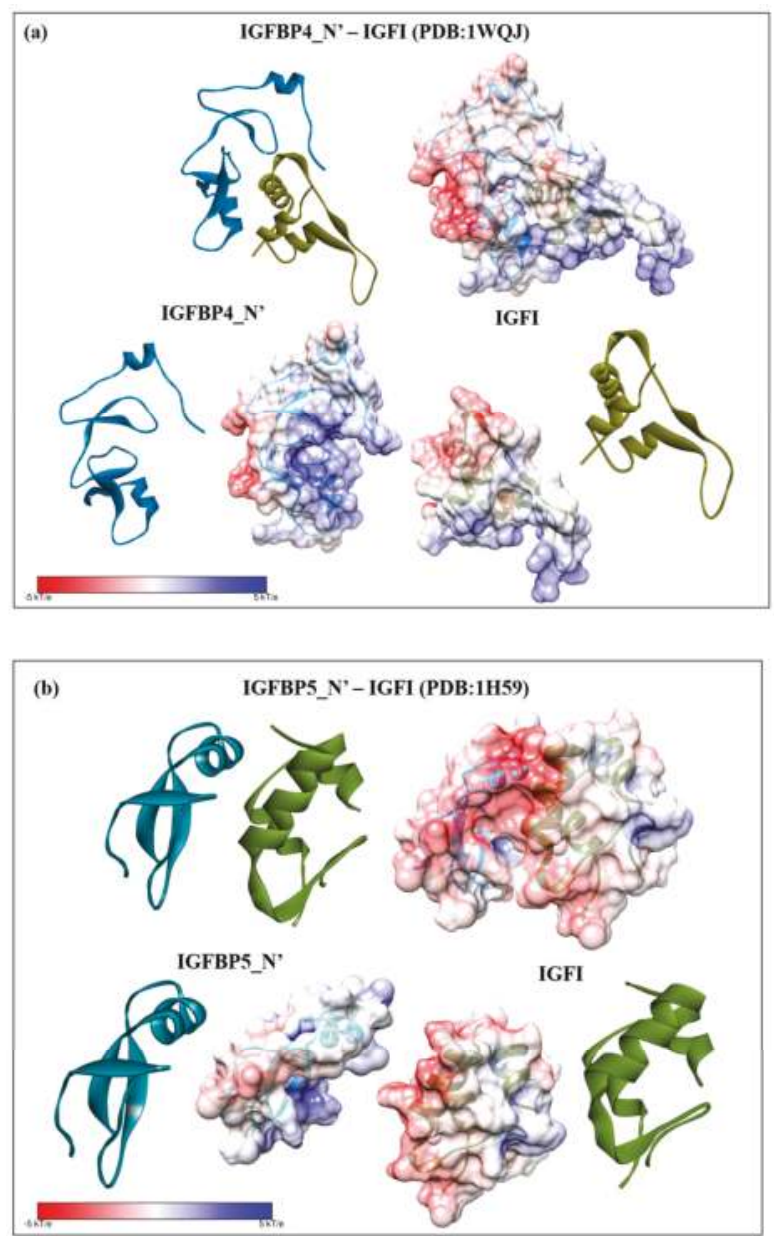

Figure 9. Electrostatic potential surface in vertebrates: (a) the bound complex and individual binding partners of human IGFBP4_N'-IGFI (PDB: 1WQJ); and (b) human IGFBP5_N'-IGFI (PDB: 1H59). In both cases the IGFBP_N' is coloured in blue and the IGFI in green. Surfaces are colored by potential on the solvent accessible surface on a scale of $-\mathrm{kT} / \mathrm{e}$ (red) to $+\mathrm{kT} / \mathrm{e}$ (blue), as indicated by the scale bar. 


\section{Discussion}

The interaction studies conducted in this work provide structural and physicochemical evidence for the capacity of IGFBP to bind the ILP ligands identified in S. verreauxi. By comparing Sv002D-IGFBP with a homologous IGFBP from C. quadricarinatus (a member of the sister Suborder Astacidea), we highlight the conservation of these physicochemical properties, suggesting a similar binding interaction to be conserved. These structural studies are indicative of a conserved function of the IGFBP in the insulin endocrine system of these decapods.

The contacts that occur at the binding interface are the fundamental features that dictate a binding interaction and, critically, its stability [45]. This makes in silico analyses such as these a highly suitable method to investigate and visualise molecular binding. Furthermore, the development of computational alanine substitution has provided significant insight into the energetic contributions of the binding interface $[45,46]$, most significantly highlighting that only a few key residuesthe hotspots-are those that contribute most significantly to the binding free-energy of complex formation [45]. Our analyses of Sv-IGFBP_N' agree with this, firstly identifying those residues that are involved in interaction contacts with all three ligands (Figure $5 \mathrm{~b}$ ) and then verifying their significance as interaction hotspots. Hotspot residues tend to cluster in pockets within the centre of the exposed binding interface [45]. This is true of our predicted hotspots, which show close structural orientation across the exposed centre of the $\mathrm{N}^{\prime}$ domain (Figure 6c).

The physicochemical nature of these conserved interacting hotspots $\left(\mathrm{Q}_{67}, \mathrm{G}_{70}, \mathrm{D}_{71}, \mathrm{~S}_{72}, \mathrm{G}_{91}, \mathrm{G}_{92}\right.$, $\mathrm{T}_{93}$ and $\mathrm{D}_{94}$ ) suggests that a range of contact properties exist at the binding interface (supported by the PRODIGY contact predictions; Table S1b). In particular, we illustrate the significance of the negatively charged hotspots, providing a structural illustration of the negative charge of the entire Sv-IGFBP_N' binding interface. Taken with the complementary positive charge of the reciprocal interface of each ILP (Figure 7), it appears that complex formation occurs, at least in part through an electrostatic interaction. Electrostatic interactions promote complex affinity through hydrogen bonding and in certain cases, such as that predicted for Asp $(\mathrm{D})_{71}$ in complex with ILP2 (Figure $5 \mathrm{~b}$ ), salt bridge formation, adding significant stability to the bound complex.

However, any binding interface is achieved through a complex synergy of molecular interactions [45]. For example, hydrophobicity has been repeatedly described as the interactive force in IGFBP_N'-IGF complexes. Studies with human IGFBP5_N' [42] and IGFBP4_N' [6] highlight the conserved importance of the hydrophobic residues $\left(\mathrm{Val}_{49 / 48}, \mathrm{Leu}_{70 / 69}, \mathrm{Leu}_{74 / 72}\right)$. This hydrophobic patch is conserved across all six IGFBP_N $\mathrm{N}^{\prime}$ [47] and has been mutated in IGFBP3-6_N', resulting in a 1000 fold decrease in binding affinity [40,41,48-50]. The solved complex of IGFI with IGFBP5_N' further verified the hydrophobic interaction, evident through the interwoven hydrophobic contacts of protruding side chains [42], also described for IGFBP4_N' $[5,6]$. Of the above, the only mention of electrostatic properties comes from IGFBP3_N' and 5_N', where the electropositive residues Lys $_{68} / \operatorname{Arg}_{69}$ were also highlighted as critical for high-affinity binding [40,41]. It is only more recently that the role of electrostatic interactions has been established. Chen et al., (2014) [51], conducted computational alanine scanning of IGFI to select mutation hotspots in order to conduct comparative molecular dynamic simulations. Five of the six determined hotspots were negatively charged (three Glu(E) and two Asp(D)) and electrostatic interactions were determined to be the dominant driving force behind the IGF-IGFBP interaction [51]. These simulations are in strong support of our electrostatic potential surface analyses, which describe an electropositive (IGFBP) to electronegative (IGF) complementarity in vertebrates (Figures 9 and S4).

It follows that residues across the binding interface coevolve, acquiring binding pockets enriched with amino acids that ensure an interdependent binding interaction [45]. Such coevolution is evident in the significant sequence conservation between Sv and Cq-IGFBP, and further still by the inverse electrostatic complementarity that we have observed between the crustacean (Figures 7 and 8 ) and vertebrate (Figures 9 and S4) IGFBP_N'-ligand complexes. Rosen et al., (2013) [8], found that Cq-IGFBP was not able to bind human insulin and could only weakly bind human IGFI. This emphasises that 
although the conserved cysteine architecture of the IGFBP_N' family coordinates the same overall fold $[1,15]$, it is the specific properties of amino acids, particularly the interaction hotspots, that govern side-chain interactions [15] and thus the binding capacity for any given ligand. Indeed, the same has been noted with the evolutionary conservation of the insulin receptor and its interactions with insulin [52].

A structural comparison of the bound Sv-IGFBP_N' complexes suggests that a similar interaction is shared across all three ligands. Binding affinity predictions varied, with PRODIGY [43,44] and PYDOCK [53] showing no significant distinction and ROSETTADOCK [54] indicating an ILP1 > IAG > ILP2 affinity pattern and FIREDOCK [55] an ILP2 > ILP1 > IAG pattern. Thus, no reliable affinity prediction can be determined; however, taken with our structural studies, it can be stated that Sv-IGFBP_N' appears to lack a selective affinity for any of these ligands, similar to that described for IGFBP-rP1 with insulin, IGFI, and IGFII [12]. The inability to generate consistent predictions of relative binding affinity is partly due to the intrinsic error in the computational prediction of binding affinities but also reflects the use of a molecular model of the isolated IGFBP_N'. Indeed, this is a common problem in IGFBP structural studies. Although well aware of the interactive nature of the IGFBP_N $\mathrm{N}^{\prime}$ and $\mathrm{C}^{\prime}$ domains in the mediation of binding affinity, the $\mathrm{N}^{\prime}$ insulin-binding domain remains the focus of structural and affinity studies, mainly due to the poor ability to solve the flexible linking domain [51]. Yet, as we are well aware of the synergistic function of the $\mathrm{N}^{\prime}$ and $\mathrm{C}^{\prime}$ domains in mediating affinity $[3,6]$, we must strive to generate interaction studies of the entire protein [37] in order to gain accurate in silico quantification of IGFBP binding affinity across ligands.

In a practical context, these in silico proof-of-binding studies suggest that these decapod IGFBPs may offer a distinct mode to regulate the bioavailability and consequential activity of IAG. The use of RNAi biotechnologies employing IAG to induce sex-reversal for the monosex population culture has been highly successful in the commercial decapod $M$. rosenbergii [56,57], with similar research practices occurring across commercial species. Molecular evidence for the interconnected nature of the IGFBP and IAG was demonstrated by Li et al., (2015) [9], who showed that the silencing of IAG in M. nipponense caused a $\sim 50 \%$ reduction in the expression of the IGFBP (so named IAGBP). However, although this is evidence of a transcriptional interaction, when interpreted in the context of this work these conclusions may be somewhat misleading. This is most evident in the naming of the IGFBP as an IAGBP, suggesting specificity. This study clearly demonstrates the capacity of the IGFBP to bind non-IAG ILPs. Indeed, the finding that IAG silencing only resulted in a significant decline of IAGBP in the AG, testis, muscle, and hepatopancreas, but not in the neuroendocrine tissues of brain, eyestalk, and nerve cord is evidence for a maintained function of the IAGBP in these tissues. As we show both ILP1 [7] and ILP2 (this study) to express in neuroendocrine tissues, perhaps the maintained expression of "IAGBP" in the neuroendocrine tissues of $M$. nipponense is evidence of an unaffected interaction with additional ILPs. This is further supported by the increasing evidence of additional ILP1/relaxin-like ILPs in the decapods [32], which are likely to share a similar binding interaction with their IGFBPs. Thus, we caution against employing IGFBP as a target for IAG manipulation, as it is likely to induce off-target effects across the broader insulin endocrinology of these species. In the context of IAG regulation, additional bio-regulators may provide a more specific anti-protease action, such as the family of AG enriched $\alpha 2$-macroglobulins [58] identified through their $>5 \times$ higher expression in the AG relative to all other tissues [59].

Moreover, the IGF-independent action of the IGFBP superfamily (most significantly the IGFBP-r subgroup) is not to be ignored [3,17]. Thus, an ILP-independent functionality of these decapod IGFBPs is very probable; an example being the immunological function investigated by Huang et al., (2016) [11]. When one considers the unspecified IGF/insulin binding capacity of IGFBP-rP1, as well as its ligand independent functions [1], perhaps these homologous decapod IGFBPs [7-11] (which are the only IGFBP subtype to be identified in the Order) are the multi-functional, unspecified ancestors of the superfamily described in vertebrates. Multiple modes for the evolution of the IGFBP family have been suggested, but establishing the evolutionary trajectory of this diverse superfamily is complex, 
with the only pronounced feature being the early emergence and conservation of the $\mathrm{N}^{\prime}$ insulin-binding domain [1].

In summary, this work has added novel evolutionary perspectives to the IGFBP superfamily, demonstrating the conserved functionality of an IGFBP-rP1 homologue in binding multiple insulin-like ligands in a decapod crustacean. This constitutes further evidence for the conserved nature of the insulin-signalling system in decapod species. By employing molecular modelling approaches we have assessed the structural, but more importantly, the physicochemical nature of the IGFBP_N'. In doing so, we suggest that these physicochemical characteristics are at the core of the IGFBP_N' divergences across species. The inverse electrostatic complementarity that we illustrate to exist between the decapod and vertebrate IGFBP_N'-ligand complexes is evidence of such. These dramatic divergences likely justify the specificity of ligand binding between Phyla.

\section{Materials and Methods}

\subsection{Sequences}

In addition to the previously described sequences for Sv-IAG (KF220491.1), Sv-ILP1 (KP006643), and Sv-IGFBP (KU195720), this work is the first to describe a second insulin-like peptide in S.-verreauxi, Sv-ILP2 (KP006646), as well two homologues in C. quadricarinatus, so named Cq-ILP1 (KP006644) and Cq-ILP2 (KP006645). Sequences were mined from transcriptomic data using a Java script for the conserved cysteine residue motif (using CLC (v7.5.1)). All sequences have been submitted to NCBI Genbank (Accession Numbers given in brackets). Phylogenetic analyses were conducted with mature ILP sequences (removal of signal and C-peptide) in Mega (7.0.21), aligned by Muscle and trees constructed using the neighbour-joining method with 1000 bootstrap replicates. This work also uses the C. quadricarinatus IGFBP, Cq-IGFBP (KC952011.1), and Cq-IAG (ABH07705.1). Sequence alignment and physicochemical analyses were conducted using Clustal2.1 (http://www.ebi.ac.uk/Tools/msa/clustalo/).

\subsection{Protein Structure Modelling}

Sequences for Sv-IGFBP, Cq-IGFBP, Sv-IAG, Sv-ILP1, and Sv-ILP2 were submitted to LOMETS http:/ / zhanglab.ccmb.med.umich.edu/LOMETS [60] to select the closest resolved structures available from the Protein Data Bank (PDB) to serve as structural templates. Models were based on the following templates: Sv- and Cq-IGFBP insulin binding and kazal domains on IGFBP-rP5, also known as HTRA1 (PDB: 3TJQ_A), and the immunoglobulin domain on a myosin light chain kinase (PDB: 2CQV_A). Sv-IAG, Sv-ILP1, and Sv-ILP2 were constructed based on insulin (PDB: 2KQP_A). In addition, each sequence was analysed using Network Protein Sequence Analysis, Consensus Secondary Structure Prediction meta-server (https:/ / npsa-prabi.ibcp.fr/cgi-bin/npsa_automat.pl?page=/NPSA/npsa_ seccons.html), to detect any structural variances from the chosen template, which were then specifically applied to each sequence (refer to Figure S1 for full details on modelling procedure).

The sequence alignments were imported into Discovery Studio 4.0 (Biovia; Accelrys Inc., San Diego, CA, USA) for model construction. Each protein model was generated using the "Build Homology Model" by MODELER [61], implementing the disulfide bond criteria and any secondary structure restraints (refer to Figure S1). In the case of Sv-IGFBP, an additional cis-peptide bond was defined in the $\mathrm{C}^{\prime}$ immunoglobulin domain. In each case, the optimal model was selected via its lowest energy and associated DOPE score [62] (Sv-IGFBP, -14117.9; Cq-IGFBP, -8248.2; IAG, -7841.97, ILP1, -10224.5 , ILP2, -6622.85). For the ILPs the C-peptide was kept intact for modelling (as it is likely to be involved with orientation and folding) and later removed. Due to the flexible structure of the IGFBP C' immunoglobulin domain, truncated models (IGFBP_N') consisting of the insulin-binding and kazal domains were generated for $\mathrm{Sv}$ (truncated at $\mathrm{R}_{153}$ ) and $\mathrm{Cq}$ (truncated at $\mathrm{R}_{155}$ ) and used for subsequent analyses. 


\subsection{Molecular Docking and Binding Studies}

For interaction studies, Sv-IGFBP_N' and each ligand were imported to the Matchmaker module in UCSF Chimera (http:/ / www.rbvi.ucsf.edu/chimera) and aligned to the resolved, bound structure of IGFBP4_N' and IGFI (PDB: 1WQJ). The resulting bound models were saved relative to the template and reimported to Discovery Studio. Each complex was then individually refined by energy minimisation (using the CHARMm force field) to reduce steric clashes. For interaction assessment, refined complexes were then submitted to PDBsum Generate, (http:/ /www.ebi.ac.uk/thornton-srv/databases/pdbsum/ Generate.html), PRODIGY [43,44] (http://milou.science.uu.nl/services/PRODIGY/), CCharPPI [63] (https:/ / life.bsc.es/pid/ccharppi), and then reanalysed manually in Chimera to validate interacting residues. To assess the reliability of our modelled complexes, structures were also submitted to HADDOCK2.2: Easy interface (http://milou.science.uu.nl/services/HADDOCK2.2) [64,65] to generate comparative docked complexes.

\subsection{Alanine Scanning and Hotspot Residues}

We employed computational alanine scanning to determine the hotspot residues of Sv-IGFBP_N'. This was done by replacing each residue in turn with alanine (the smallest most inert amino acid) and assessing for a significant decrease in the binding free-energy [46]. We submitted each refined bound complex to five software platforms to gain a meta-style output: Cpclab (http://cpclab.uni-duesseldorf.de/dsppi/) [66]; Robetta (http://www.robetta.org/ alascansubmit.jsp) [67]; KFC (http://kfc.mitchell-lab.org/) [68]; ANCHOR (http://structure.pitt. edu/anchor/upload/); and HotRegion (http://prism.ccbb.ku.edu.tr/hotregion/) [69]. In addition, the electrostatic interaction of each Sv complex, Cq-IGFBP_N' and a range of vertebrate structures was determined by performing electrostatic potential surface calculations using PDB2PQR [70] and APBS [71] programmes within UCSF Chimera (with protonation states at physiological pH and $298 \mathrm{~K}$ and parse charges). The surface potential representation is shown in each figure, with charge levels ranging from $-\mathrm{kT} / \mathrm{e}$ (red) to $+\mathrm{kT} / \mathrm{e}$ (blue), as indicated by the scale bar.

Supplementary Materials: Supplementary materials can be found at www.mdpi.com/1422-0067/18/9/1832/s1.

Acknowledgments: This work was supported by the Australian Research Council via a Discovery Early Career Research Award [DE130101089 to Tomer Ventura], Industrial Transformation Research Hub [IH120100032 to Greg Smith] and Discovery [DP160103320 to Tomer Ventura], a University of the Sunshine Coast (USC) Collaborative Research Networks (CRN) grant program, a USC International Research Scholarship to Jennifer C. Chandler, and a Curtin Research Fellowship to Neha S. Gandhi.

Author Contributions: Jennifer C. Chandler, Neha S. Gandhi, Ricardo L. Mancera, Abigail Elizur, and Tomer Ventura designed and coordinated the study. Jennifer C. Chandler and Neha S. Gandhi conducted analyses. Greg Smith provided the animals that facilitated the study. Jennifer C. Chandler wrote the manuscript. All authors reviewed and approved the final version of the manuscript.

Conflicts of Interest: The authors declare no conflict of interest.

\section{Abbreviations}

$\begin{array}{ll}\text { AG } & \text { Androgenic gland } \\ \mathrm{C}^{\prime} & \text { C terminal } \\ \text { Cq } & \text { Cherax quadricarinatus (red-claw crayfish) } \\ \text { Dilp7 } & \text { Drosophila ILP7 } \\ \text { Dilp8 } & \text { Drosophila ILP8 } \\ \text { IAG } & \text { Insulin-like androgenic gland peptide } \\ \text { IGF } & \text { Insulin-like growth factor } \\ \text { IGFBP } & \text { Insulin-like growth factor binding protein } \\ \text { IGFBP-rP1 } & \text { Insulin-like growth factor binding- related protein } \\ \text { ILP } & \text { Insulin-like peptide } \\ \mathrm{N}^{\prime} & \text { N terminal }\end{array}$


RPKM Reads per kilobase per million reads

Sv Sagmariasus verreauxi (Eastern spiny lobster)

TKIR Tyrosine kinase insulin receptor

\section{References}

1. Hwa, V.; Oh, Y.; Rosenfeld, R.G. The Insulin-Like Growth Factor-Binding Protein (IGFBP) Superfamily. Endocr. Rev. 1999, 20, 761-787. [CrossRef] [PubMed]

2. Denley, A.; Cosgrove, L.J.; Booker, G.W.; Wallace, J.C.; Forbes, B.E. Molecular interactions of the IGF system. Cytokine Growth Factor Rev. 2005, 16, 421-439. [CrossRef] [PubMed]

3. Forbes, B.; McCarthy, P.; Norton, R. Insulin-like growth factor binding proteins: A structural perspective. Front. Endocrinol. 2012, 3. [CrossRef] [PubMed]

4. Weiss, I.M.; Göhring, W.; Fritz, M.; Mann, K. Perlustrin, a Haliotis laevigata (Abalone) Nacre Protein, Is Homologous to the Insulin-like Growth Factor Binding Protein N-Terminal Module of Vertebrates. Biochem. Biophys. Res. Commun. 2001, 285, 244-249. [CrossRef] [PubMed]

5. Siwanowicz, I.; Popowicz, G.M.; Wisniewska, M.; Huber, R.; Kuenkele, K.-P.; Lang, K.; Engh, R.A.; Holak, T.A. Structural Basis for the Regulation of Insulin-like Growth Factors by IGF Binding Proteins. Structure 2005, 13, 155-167. [CrossRef] [PubMed]

6. Sitar, T.; Popowicz, G.M.; Siwanowicz, I.; Huber, R.; Holak, T.A. Structural basis for the inhibition of insulin-like growth factors by insulin-like growth factor-binding proteins. Proc. Natl. Acad. Sci. USA 2006, 103, 13028-13033. [CrossRef] [PubMed]

7. Chandler, J.C.; Aizen, J.; Elizur, A.; Hollander-Cohen, L.; Battaglene, S.C.; Ventura, T. Discovery of a novel insulin-like peptide and insulin binding proteins in the Eastern rock lobster Sagmariasus verreauxi. Gen. Comp. Endocrinol. 2015, 215, 76-87. [CrossRef] [PubMed]

8. Rosen, O.; Weil, S.; Manor, R.; Roth, Z.; Khalaila, I.; Sagi, A. A crayfish insulin-like-binding protein: Another piece in the androgenic gland insulin-like hormone puzzle is revealed. J. Biol. Chem. 2013, 288, 22289-22298. [CrossRef] [PubMed]

9. Li, F.; Bai, H.; Xiong, Y.; Fu, H.; Jiang, S.; Jiang, F.; Jin, S.; Sun, S.; Qiao, H.; Zhang, W. Molecular characterization of insulin-like androgenic gland hormone-binding protein gene from the oriental river prawn Macrobrachium nipponense and investigation of its transcriptional relationship with the insulin-like androgenic gland hormone gene. Gen. Comp. Endocrinol. 2015, 216, 152-160. [CrossRef] [PubMed]

10. Huang, X.; Ye, H.; Feng, B.; Huang, H. Insights into insulin-like peptide system in invertebrates from studies on IGF binding domain-containing proteins in the female mud crab, Scylla paramamosain. Mol. Cell. Endocrinol. 2015, 416, 36-45. [CrossRef] [PubMed]

11. Huang, X.; Bae, S.H.; Bachvaroff, T.R.; Schott, E.J.; Ye, H.; Chung, J.S. Does a blue crab putative insulin-like peptide binding protein (ILPBP) play a role in a virus infection? Fish Shellfish Immunol. 2016, 58, 340-348. [CrossRef] [PubMed]

12. Oh, Y.; Nagalla, S.R.; Yamanaka, Y.; Kim, H.-S.; Wilson, E.; Rosenfeld, R.G. Synthesis and Characterization of Insulin-like Growth Factor-binding Protein (IGFBP)-7: Recombinant Human MAC25 protein specifically binds IGF-I and -II. J. Biol. Chem. 1996, 271, 30322-30325. [CrossRef] [PubMed]

13. Yamanaka, Y.; Wilson, E.M.; Rosenfeld, R.G.; Oh, Y. Inhibition of Insulin Receptor Activation by Insulin-like Growth Factor Binding Proteins. J. Biol. Chem. 1997, 272, 30729-30734. [CrossRef] [PubMed]

14. Akaogi, K.; Sato, J.; Okabe, Y.; Sakamoto, Y.; Yasumitsu, H.; Miyazaki, K. Synergistic growth stimulation of mouse fibroblasts by tumor-derived adhesion factor with insulin-like growth factors and insulin. Cell Growth Differ. 1996, 7, 1671-1677. [PubMed]

15. Eigenbrot, C.; Ultsch, M.; Lipari, M.T.; Moran, P.; Lin, S.J.; Ganesan, R.; Quan, C.; Tom, J.; Sandoval, W.; van Lookeren Campagne, M.; et al. Structural and Functional Analysis of HtrA1 and its Subdomains. Structure 2012, 20, 1040-1050. [CrossRef] [PubMed]

16. Vorwerk, P.; Hohmann, B.; Oh, Y.; Rosenfeld, R.G.; Shymko, R.M. Binding properties of insulin-like growth factor binding protein-3 (IGFBP-3), IGFBP-3 N- and C-terminal fragments, and structurally related proteins MAC25 and connective tissue growth factor measured using a biosensor. Endocrinology 2002, 143, 1677-1685. [CrossRef] [PubMed] 
17. Walker, G.E.; Kim, H.-S.; Yang, Y.-F.; Oh, Y. IGF-independent effects of the IGFBP superfamily. In Insulin-Like Growth Factor Receptor Signalling; Leroith, D., Zumkeller, W., Baxter, R.C., Eds.; Plenum Publishers: New York, NY, USA, 2003; pp. 262-274.

18. Wu, Q.; Brown, M.R. Signaling and function of insulin-like peptides in insects. Annu. Rev. Entomol. 2006, 51, 1-24. [CrossRef] [PubMed]

19. Brogiolo, W.; Stocker, H.; Ikeya, T.; Rintelen, F.; Fernandez, R.; Hafen, E. An evolutionarily conserved function of the Drosophila insulin receptor and insulin-like peptides in growth control. Curr. Biol. 2001, 11, 213-221. [CrossRef]

20. Mizoguchi, A.; Okamoto, N. Insulin-like and IGF-like peptides in the silkmoth Bombyx mori: Discovery, structure, secretion and function. Front. Physiol. 2013, 4, 217. [CrossRef] [PubMed]

21. Chung, J.S. An insulin-like growth factor found in hepatopancreas implicates carbohydrate metabolism of the blue crab Callinectes sapidus. Gen. Comp. Endocrinol. 2014, 199, 56-64. [CrossRef] [PubMed]

22. Huang, X.; Ye, H.; Huang, H.; Yang, Y.; Gong, J. An insulin-like androgenic gland hormone gene in the mud crab, Scylla paramamosain, extensively expressed and involved in the processes of growth and female reproduction. Gen. Comp. Endocrinol. 2014, 204, 229-238. [CrossRef] [PubMed]

23. Charniaux-Cotton, H. Discovery in, an amphipod crustacean (Orchestia gammarella) of an endocrine gland responsible for the differentiation of primary and secondary male sex characteristics. C. R. Hebd. Seances Acad. Sci. 1954, 239, 780-782. [PubMed]

24. Charniaux-Cotton, $\mathrm{H}$. The androgenic gland of some decapodal crustaceans and particularly of Lysmata seticaudata, a species with functional protandrous hermaphroditism. C. R. Hebd. Seances Acad. Sci. 1958, 246, 2814-2817. [PubMed]

25. Sagi, A.; Snir, E.; Khalaila, I. Sexual differentiation in decapod crustaceans: Role of the androgenic gland. Invertebr. Reprod. Dev. 1997, 31, 55-61. [CrossRef]

26. Martin, G.; Sorokine, O.; Moniatte, M.; Bulet, P.; Hetru, C.; Van Dorsselaer, A. The structure of a glycosylated protein hormone responsible for sex determination in the isopod, Armadillidium vulgare. Eur. J. Biochem. 1999, 262, 727-736. [CrossRef] [PubMed]

27. Okuno, A.; Hasegawa, Y.; Ohira, T.; Katakura, Y.; Nagasawa, H. Characterization and cDNA cloning of androgenic gland hormone of the terrestrial isopod Armadillidium vulgare. Biochem. Biophys. Res. Commun. 1999, 264, 419-423. [CrossRef] [PubMed]

28. Manor, R.; Weil, S.; Oren, S.; Glazer, L.; Aflalo, E.D.; Ventura, T.; Chalifa-Caspi, V.; Lapidot, M.; Sagi, A. Insulin and gender: An insulin-like gene expressed exclusively in the androgenic gland of the male crayfish. Gen. Comp. Endocrinol. 2007, 150, 326-336. [CrossRef] [PubMed]

29. Ventura, T.; Manor, R.; Aflalo, E.D.; Weil, S.; Raviv, S.; Glazer, L.; Sagi, A. Temporal silencing of an androgenic gland-specific insulin-like gene affecting phenotypical gender differences and spermatogenesis. Endocrinology 2009, 150, 1278-1286. [CrossRef] [PubMed]

30. Rosen, O.; Manor, R.; Weil, S.; Gafni, O.; Linial, A.; Aflalo, E.D.; Ventura, T.; Sagi, A. A sexual shift induced by silencing of a single insulin-like gene in crayfish: Ovarian upregulation and testicular degeneration. PLoS ONE 2010, 5, e15281. [CrossRef] [PubMed]

31. Ventura, T.; Rosen, O.; Sagi, A. From the discovery of the crustacean androgenic gland to the insulin-like hormone in six decades. Gen. Comp. Endocrinol. 2011, 173, 381-388. [CrossRef] [PubMed]

32. Veenstra, J.A. Similarities between decapod and insect neuropeptidomes. Peer J. 2016, 4, e2043. [CrossRef] [PubMed]

33. Okamoto, N.; Nakamori, R.; Murai, T.; Yamauchi, Y.; Masuda, A.; Nishimura, T. A secreted decoy of InR antagonizes insulin/IGF signaling to restrict body growth in Drosophila. Genes Dev. 2013, 27, 87-97. [CrossRef] [PubMed]

34. Aizen, J.; Chandler, J.C.; Fitzgibbon, Q.P.; Sagi, A.; Battaglene, S.C.; Elizur, A.; Ventura, T. Production of recombinant insulin-like androgenic gland hormones from three decapod species: In vitro testicular phosphorylation and activation of a newly identified tyrosine kinase receptor from the Eastern spiny lobster, Sagmariasus verreauxi. Gen. Comp. Endocrinol. 2016, 229, 8-18. [CrossRef] [PubMed]

35. Sharabi, O.; Manor, R.; Weil, S.; Aflalo, E.D.; Lezer, Y.; Levy, T.; Aizen, J.; Ventura, T.; Mather, P.B.; Khalaila, I.; et al. Identification and characterization of an insulin-like receptor involved in crustacean reproduction. Endocrinology 2016, 157, 928-941. [CrossRef] [PubMed] 
36. Lee, D.; Redfern, O.; Orengo, C. Predicting protein function from sequence and structure. Nat. Rev. Mol. Cell Biol. 2007, 8, 995-1005. [CrossRef] [PubMed]

37. Gupta, C.L.; Akhtar, S.; Bajpai, P. In silico protein modeling: Possibilities and limitations. EXCLI J. 2014, 13, 513-515. [PubMed]

38. Ventura, T.; Fitzgibbon, Q.P.; Battaglene, S.C.; Sagi, A.; Elizur, A. Identification and characterization of androgenic gland specific insulin-like peptide-encoding transcripts in two spiny lobster species: Sagmariasus verreauxi and Jasus edwardsii. Gen. Comp. Endocrinol. 2014, 214, 126-133. [CrossRef] [PubMed]

39. Chandler, J.C.; Aizen, J.; Fitzgibbon, Q.P.; Elizur, A.; Ventura, T. Applying the Power of Transcriptomics: Understanding Male Sexual Development in Decapod Crustacea. Integr. Comp. Biol. 2016, 56, 1144-1156. [CrossRef] [PubMed]

40. Imai, Y.; Moralez, A.; Andag, U.; Clarke, J.B.; Busby, W.H.; Clemmons, D.R. Substitutions for Hydrophobic Amino Acids in the N-terminal Domains of IGFBP-3 and -5 Markedly Reduce IGF-I Binding and Alter Their Biologic Actions. J. Biol. Chem. 2000, 275, 18188-18194. [CrossRef] [PubMed]

41. Hong, J.; Zhang, G.; Dong, F.; Rechler, M.M. Insulin-like Growth Factor (IGF)-binding Protein-3 Mutants That Do Not Bind IGF-I or IGF-II Stimulate Apoptosis in Human Prostate Cancer Cells. J. Biol. Chem. 2002, 277, 10489-10497. [CrossRef] [PubMed]

42. Żesławski, W.; Beisel, H.-G.; Kamionka, M.; Kalus, W.; Engh, R.A.; Huber, R.; Lang, K.; Holak, T.A. The interaction of insulin-like growth factor-I with the N-terminal domain of IGFBP-5. EMBO J. 2001, 20, 3638-3644. [CrossRef] [PubMed]

43. Vangone, A.; Bonvin, A.M.J.J. Contacts-based prediction of binding affinity in protein-protein complexes. eLife 2015, 4, e07454. [CrossRef] [PubMed]

44. Xue, L.C.; Rodrigues, J.P.; Kastritis, P.L.; Bonvin, A.M.; Vangone, A. PRODIGY: A web server for predicting the binding affinity of protein-protein complexes. Bioinformatics 2016, 32, 3676-3678. [CrossRef] [PubMed]

45. Moreira, I.S.; Fernandes, P.A.; Ramos, M.J. Hot spots-A review of the protein-Protein interface determinant amino-acid residues. Proteins Struct. Funct. Bioinf. 2007, 68, 803-812. [CrossRef] [PubMed]

46. Moreira, I.S.; Fernandes, P.A.; Ramos, M.J. Computational alanine scanning mutagenesis—An improved methodological approach. J. Comput. Chem. 2006, 28, 644-654. [CrossRef] [PubMed]

47. Kalus, W.; Zweckstetter, M.; Renner, C.; Sanchez, Y.; Georgescu, J.; Grol, M.; Demuth, D.; Schumacher, R.; Dony, C.; Lang, K. Structure of the IGF-binding domain of the insulin-like growth factor-binding protein-5 (IGFBP-5): Implications for IGF and IGF-I receptor interactions. EMBO J. 1998, 17, 6558-6572. [CrossRef] [PubMed]

48. Qin, X.; Strong, D.D.; Baylink, D.J.; Mohan, S. Structure-Function Analysis of the Human Insulin-like Growth Factor Binding Protein-4. J. Biol. Chem. 1998, 273, 23509-23516. [CrossRef] [PubMed]

49. Yan, X.; Forbes, B.E.; McNeil, K.A.; Baxter, R.C.; Firth, S.M. Role of N- and C-terminal Residues of Insulin-like Growth Factor (IGF)-binding Protein-3 in Regulating IGF Complex Formation and Receptor Activation. J. Biol. Chem. 2004, 279, 53232-53240. [CrossRef] [PubMed]

50. Fu, P.; Thompson, J.A.; Bach, L.A. Promotion of cancer cell migration: An insulin-like growth factor (IGF)-independent action of IGF-binding protein-6. J. Biol. Chem. 2007, 282, 22298-22306. [CrossRef] [PubMed]

51. Chen, X.; Duan, D.; Zhu, S.; Zhang, J. Investigation of alanine mutations affecting insulin-like growth factor (IGF) I binding to IGF binding proteins. Growth Factors 2015, 33, 40-49. [CrossRef] [PubMed]

52. Renteria, M.E.; Gandhi, N.S.; Vinuesa, P.; Helmerhorst, E.; Mancera, R.L. A comparative structural bioinformatics analysis of the insulin receptor family ectodomain based on phylogenetic information. PLOS ONE 2008, 3, e3667. [CrossRef] [PubMed]

53. Cheng, T.M.; Blundell, T.L.; Fernandez-Recio, J. pyDock: Electrostatics and desolvation for effective scoring of rigid-body protein-protein docking. Proteins 2007, 68, 503-515. [CrossRef] [PubMed]

54. Chaudhury, S.; Lyskov, S.; Gray, J.J. PyRosetta: A script-based interface for implementing molecular modeling algorithms using Rosetta. Bioinformatics 2010, 26, 689-691. [CrossRef] [PubMed]

55. Andrusier, N.; Nussinov, R.; Wolfson, H.J. FireDock: Fast interaction refinement in molecular docking. Proteins 2007, 69, 139-159. [CrossRef] [PubMed]

56. Ventura, T.; Manor, R.; Aflalo, E.D.; Weil, S.; Rosen, O.; Sagi, A. Timing sexual differentiation: Full functional sex reversal achieved through silencing of a single insulin-like gene in the prawn, Macrobrachium rosenbergii. Biol. Reprod. 2012, 86, 1-6. [CrossRef] [PubMed] 
57. Lezer, Y.; Aflalo, E.D.; Manor, R.; Sharabi, O.; Abilevich, L.K.; Sagi, A. On the safety of RNAi usage in aquaculture: The case of all-male prawn stocks generated through manipulation of the insulin-like androgenic gland hormone. Aquaculture 2015, 435, 157-166. [CrossRef]

58. Borth, W. Alpha 2-macroglobulin, a multifunctional binding protein with targeting characteristics. FASEB J. 1992, 6, 3345-3353. [PubMed]

59. Chandler, J.C.; Aizen, J.; Elizur, A.; Battaglene, S.C.; Ventura, T. Male Sexual Development and the Androgenic Gland: Novel Insights through the de novo Assembled Transcriptome of the Eastern Spiny Lobster, Sagmariasus verreauxi. Sex. Dev. 2016, 9, 338-354. [CrossRef] [PubMed]

60. Wu, S.; Zhang, Y. LOMETS: A local meta-threading-server for protein structure prediction. Nucleic Acids Res. 2007, 35, 3375-3382. [CrossRef] [PubMed]

61. Fiser, A.; Sali, A. Modeller: Generation and refinement of homology-based protein structure models. Methods Enzymol. 2003, 374, 461-491. [PubMed]

62. Shen, M.-Y.; Sali, A. Statistical potential for assessment and prediction of protein structures. Protein Sci. 2006, 15, 2507-2524. [CrossRef] [PubMed]

63. Moal, I.H.; Jimenez-Garcia, B.; Fernandez-Recio, J. CCharPPI web server: Computational characterization of protein-protein interactions from structure. Bioinformatics 2015, 31, 123-125. [CrossRef] [PubMed]

64. Dominguez, C.; Boelens, R.; Bonvin, A.M. HADDOCK: A protein-protein docking approach based on biochemical or biophysical information. J. Am. Chem. Soc. 2003, 125, 1731-1737. [CrossRef] [PubMed]

65. De Vries, S.J.; van Dijk, M.; Bonvin, A.M.J.J. The HADDOCK web server for data-driven biomolecular docking. Nat. Protoc. 2010, 5, 883-897. [CrossRef] [PubMed]

66. Kruger, D.M.; Gohlke, H. DrugScorePPI webserver: Fast and accurate in silico alanine scanning for scoring protein-protein interactions. Nucleic Acids Res. 2010, 38, W480-W486. [CrossRef] [PubMed]

67. Kortemme, T.; Kim, D.E.; Baker, D. Computational alanine scanning of protein-protein interfaces. Sci. STKE 2004, 219, pl2. [CrossRef] [PubMed]

68. Zhu, X.; Mitchell, J.C. KFC2: A knowledge-based hot spot prediction method based on interface solvation, atomic density, and plasticity features. Proteins 2011, 79, 2671-2683. [CrossRef] [PubMed]

69. Cukuroglu, E.; Gursoy, A.; Keskin, O. HotRegion: A database of predicted hot spot clusters. Nucleic Acids Res. 2012, 40, D829-D833. [CrossRef] [PubMed]

70. Dolinsky, T.J.; Nielsen, J.E.; McCammon, J.A.; Baker, N.A. PDB2PQR: An automated pipeline for the setup of Poisson-Boltzmann electrostatics calculations. Nucleic Acids Res. 2004, 32, W665-W667. [CrossRef] [PubMed]

71. Baker, N.A.; Sept, D.; Joseph, S.; Holst, M.J.; McCammon, J.A. Electrostatics of nanosystems: Application to microtubules and the ribosome. Proc. Natl. Acad. Sci. USA 2001, 98, 10037-10041. [CrossRef] [PubMed]

(C) 2017 by the authors. Licensee MDPI, Basel, Switzerland. This article is an open access article distributed under the terms and conditions of the Creative Commons Attribution (CC BY) license (http:/ / creativecommons.org/licenses/by/4.0/). 


\title{
IonchanPred 2.0: A Tool to Predict Ion Channels and Their Types
}

\author{
Ya-Wei Zhao ${ }^{1}$, Zhen-Dong Su ${ }^{1}$, Wuritu Yang ${ }^{1,2}$, Hao Lin ${ }^{1, *}$, Wei Chen ${ }^{1,3, *}$ and Hua Tang ${ }^{4, *}$ \\ 1 Key Laboratory for Neuro-Information of Ministry of Education, School of Life Science and Technology, \\ Center for Informational Biology, University of Electronic Science and Technology of China, \\ Chengdu 610054, China; lianyingteng@hotmail.com (Y.-W.Z.); zhendong_su@163.com (Z.-D.S.); \\ wyang@imu.edu.cn (W.Y.) \\ 2 Development and Planning Department, Inner Mongolia University, Hohhot 010021, China \\ 3 Department of Physics, School of Sciences, and Center for Genomics and Computational Biology, \\ North China University of Science and Technology, Tangshan 063000, China \\ 4 Department of Pathophysiology, Southwest Medical University, Luzhou 646000, China \\ * Correspondence: hlin@uestc.edu.cn (H.L.); greatchen@ncst.edu.cn (W.C.); \\ tanghua771211@aliyun.com (H.T.); Tel.: +86-28-8320-2351 (H.L. \& W.C. \& H.T.)
}

Received: 7 August 2017; Accepted: 21 August 2017; Published: 24 August 2017

\begin{abstract}
Ion channels (IC) are ion-permeable protein pores located in the lipid membranes of all cells. Different ion channels have unique functions in different biological processes. Due to the rapid development of high-throughput mass spectrometry, proteomic data are rapidly accumulating and provide us an opportunity to systematically investigate and predict ion channels and their types. In this paper, we constructed a support vector machine (SVM)-based model to quickly predict ion channels and their types. By considering the residue sequence information and their physicochemical properties, a novel feature-extracted method which combined dipeptide composition with the physicochemical correlation between two residues was employed. A feature selection strategy was used to improve the performance of the model. Comparison results of in jackknife cross-validation demonstrated that our method was superior to other methods for predicting ion channels and their types. Based on the model, we built a web server called IonchanPred which can be freely accessed from http://lin.uestc.edu.cn/server/IonchanPredv2.0.
\end{abstract}

Keywords: ion channels; pseudo-dipeptide composition; machine learning method

\section{Introduction}

Ion channels are pore-forming membrane proteins for the transmembrane exchange of inorganic ions (as shown in Figure 1). Ion channels exist in the membranes of all cells and are required in numerous physiological and pathological processes, such as regulating neuronal and cardiac excitability, muscle contraction, hormone secretion, fluid movement, and immune cell activation [1]. Due to their important role in biological processes, ion channels are often used as targets for disease diagnosis and drug development. There are over 300 types of ion channels in living cells [2], and they differ in their structure and function. According to the different gating mechanisms, the ion channels can be mainly divided into two categories, namely voltage-gated ion channels (VGIC) and ligand-gated ion channels (LGIC) [3]. The opening and closing of the voltage-gated ion channels depends on the change of the membrane potential, whereas the state of the ligand channels is closely related to the binding of the ligand. The voltage-gated ion channels can be further classified into the following four subclasses: potassium $\left(\mathrm{K}^{+}\right)$, sodium $\left(\mathrm{Na}^{+}\right)$, calcium $\left(\mathrm{Ca}^{2+}\right)$, and anion channels. 


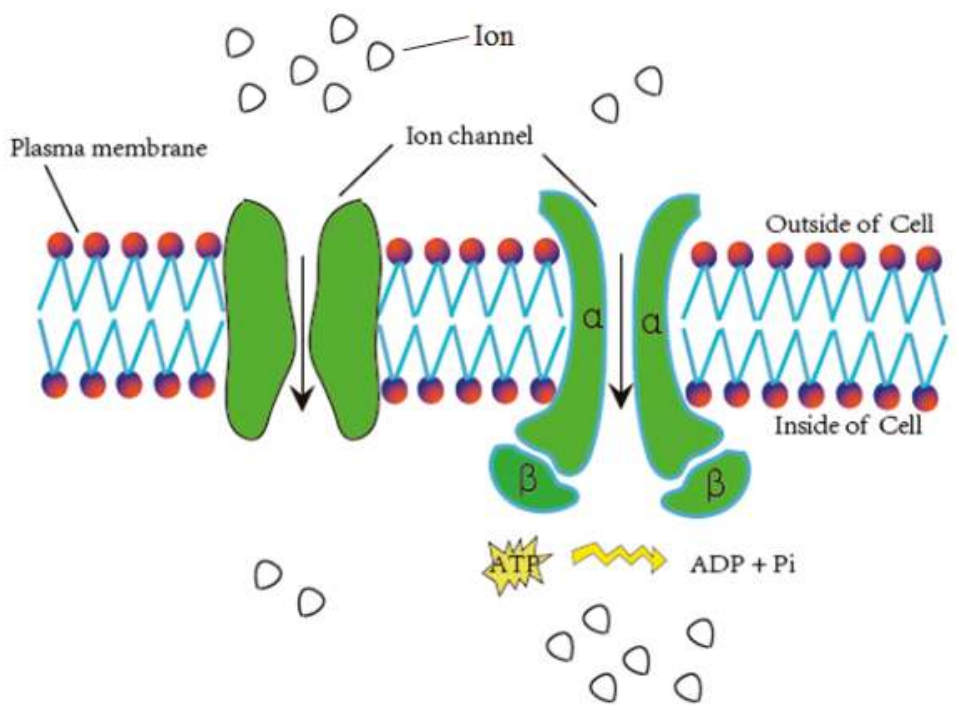

Figure 1. Schematic diagram of material exchange through ion channels.

In view of the important role and multiple types of ion channels, the structures and functions of ion channels have continued to attract the attention of numerous researchers in recent years [4-10]. Due to the rapid growth of proteomic data, it is particularly important to develop bioinformatics tools to quickly predict and identify ion channels and their types. Consequently, many computational methods based on machine learning algorithm have been developed in the last 10 years [11-17]. Liu et al. [11] proposed a method to identify voltage-gated potassium channels, and indicated that the local sequence information-based method was better than the global sequence information-based method. Saha et al. [12] developed a support vector machine (SVM)-based method by using amino acid composition and dipeptide composition to predict voltage-gated ion channels and their subtypes. In 2011, our group [13] developed a more generalized predictive tool, called IonchanPred, and identified ion channels and their types accurately. Recently, Tiwari et al. [16] proposed a random forest based methods and Gao et al. [17] proposed a model to predict ion channels and their subfamilies by combining a SVM-based model with BLAST sequence similarity search. Although many predictors for identifying ion channels are available, three essential issues remain elusive. Firstly, the use of high similarity sequences may overestimate the performance of a model. Secondly, the long-range effect is lost in most published models. Thirdly, web servers should be improved.

In this paper, a support vector machine-based model was constructed to quickly identify ion channels and their types. In this model, a novel feature extraction method called pseudo-dipeptide composition was employed. The analysis of variance (ANOVA) [18] was introduced to rank features. The incremental feature selection (IFS) was employed to find an optimized feature set which can produce the maximum accuracy. Finally, a web server called IonchanPred 2.0 was established. The flow chart is shown in Figure 2. 


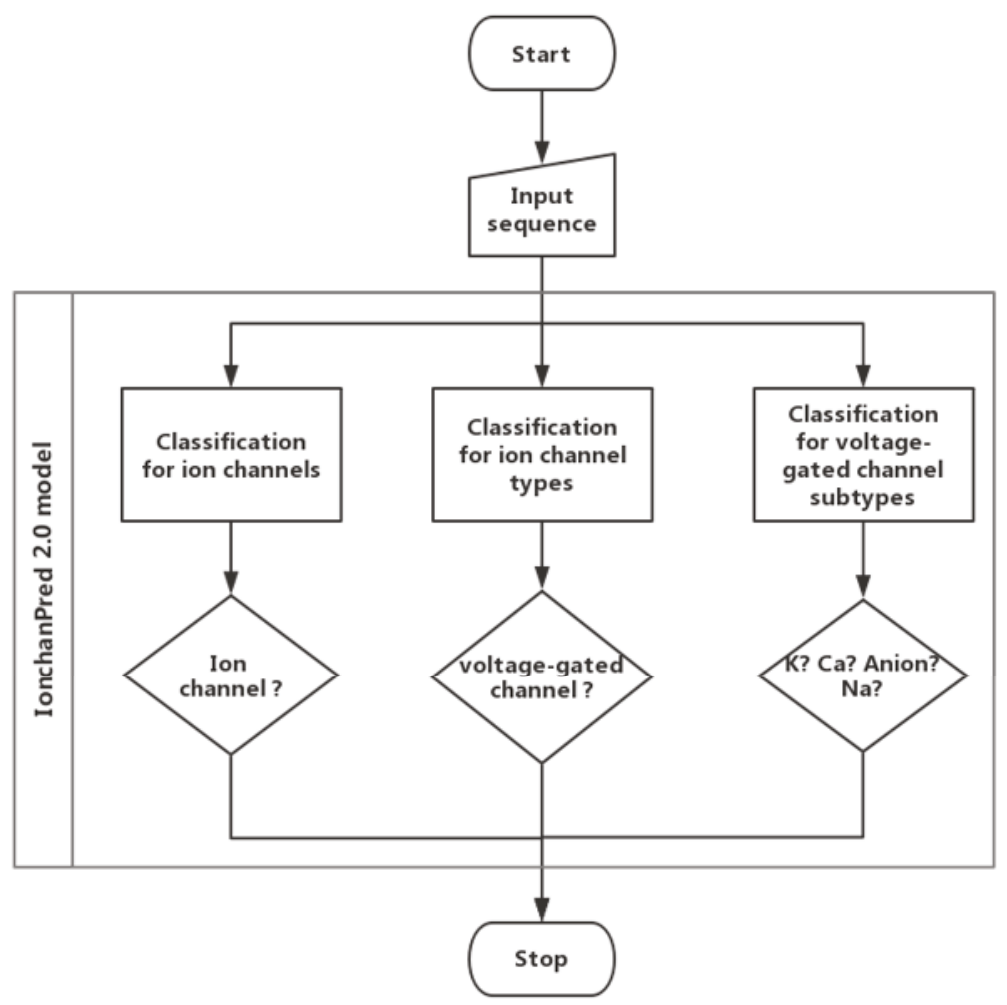

Figure 2. Workflow of the IonchanPred 2.0 model.

\section{Results and Discussion}

\subsection{Parameter Optimization}

The establishment of our proposed model depends on two important parameters: $\lambda$ and $\omega . \lambda$ factor denotes the rank of correlation and the larger $\lambda$ may contain more global sequence-order information. $\omega$ represents the weight of the correlation of residues' physiochemical properties compared to the traditional dipeptide component. To obtain the optimal value for the two parameters, a serial of experiments was performed according to the following standard:

$$
\left\{\begin{array}{l}
1 \leq \lambda \leq 30 \text { with step } \Delta=1 \\
0.05 \leq \omega \leq 0.70 \text { with step } \Delta=0.05
\end{array}\right.
$$

In view of this, a total of $30 \times 14=420$ individual combinations were obtained. Then, we can investigate the accuracy of SVM with the jackknife test. The optimal parameter combinations corresponding to the three individual datasets are shown in Table 1. It shows that the highest overall accuracy can be up to $87.5 \%$ when $\lambda=21$ and $\omega=0.20$ for the dataset including ion channels and non-ion channels (NIC). For the benchmark dataset VGIC vs. LGIC, the maximum accuracy is $93.9 \%$ when $\lambda=7$ and $\omega=0.30$. The best model for four types of VGIC prediction can produce overall accuracy of $89.1 \%$. After the parameters are optimized, the samples for the three individual datasets can be respectively formulated as follows: a 589-dimensional vector involving 400 dimensions for traditional dipeptide composition and $9 \times 21=189$ dimensions for correlation information for IC vs. 
NIC prediction, a vector involving $400+9 \times 7=463$ dimensions for VGIC vs. LGIC, and a vector involving $400+9 \times 9=481$ dimensions for four types of voltage-gated ion channels datasets.

Table 1. Optimal parameters for the three datasets.

\begin{tabular}{cccc}
\hline Database & $\lambda$ & $\omega$ & $\boldsymbol{O A}(\mathbf{\%})$ \\
\hline IC vs. NIC & 21 & 0.20 & 87.5 \\
VGIC vs. LGIC & 7 & 0.30 & 93.9 \\
four types of VGIC & 9 & 0.15 & 89.1 \\
\hline
\end{tabular}

IC: ion channels; NIC: non-ion channels; VGIC: voltage-gated ion channels; LGIC: ligand-gated ion channels; $O A$ : overall accuracy.

\subsection{Model Establishment}

In order to further improve the accuracy, we used ANOVA to exclude noise or redundant information. After the feature selection, the features were sorted according to the decreasing order of the $F$ values described in Section 3.3 Feature Selection to obtain the feature list. Then, we used the IFS to determine the optimal number of features, as described below. The feature subset starts from a feature ranking first in the feature list. A new feature subset was composed when the second feature of this list was added. We repeated this process until all candidate features were added. In this case, we obtained 589, 463, and 535 feature subsets, respectively, for the three benchmark datasets mentioned above. The performance of each feature subset was examined by using SVM with the jackknife test. We plotted the relationship between the overall accuracy and the numbers of features in Figure 3. We noticed that the prediction performances were the best when the top ranked 527, 460, and 147 features were used for the three datasets, respectively.
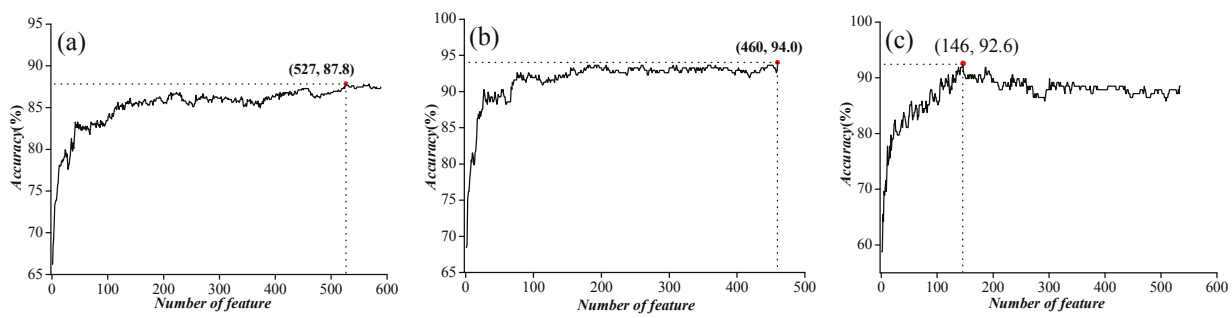

Figure 3. The feature selection results for three independent datasets. (a) Incremental feature selection (IFS) curve for ion channel (IC) vs. non-ion channel (NIC) prediction; (b) IFS curve for voltage-gated ion channels (VGIC) vs. ligand-gated ion channels (LGIC) prediction; (c) IFS curve for four types of VGIC prediction.

In order to further evaluate the predictive performance of our model, we also calculated the average accuracies for the three datasets. A comparison of the results with the previous model [13] are shown in Table 2. It is clear that the predictive performance of our proposed model is better than the previous model. 
Table 2. Performance evaluation parameters of our proposed model and a previous model.

\begin{tabular}{|c|c|c|c|c|c|c|c|}
\hline \multicolumn{2}{|c|}{ Datasets } & \multicolumn{3}{|c|}{ Our Model } & \multicolumn{3}{|c|}{ Previous Model [13] } \\
\hline & & Sn & $O A$ & $A A$ & Sn & $O A$ & $A A$ \\
\hline IC vs. NIC & IC & 80.2 & \multirow{2}{*}{87.8} & \multirow{2}{*}{87.8} & 85.9 & \multirow{2}{*}{86.6} & \multirow{2}{*}{86.6} \\
\hline & & & & & & & \\
\hline \multirow{2}{*}{ VGIC vs. LGIC } & VGIC & 94.7 & \multirow{2}{*}{94.0} & \multirow{2}{*}{94.0} & 94.6 & \multirow{2}{*}{92.6} & \multirow{2}{*}{92.7} \\
\hline & LGIC & 93.2 & & & 90.7 & & \\
\hline \multirow{4}{*}{ Types of VGIC } & $\mathrm{K}^{+}$ & 97.5 & \multirow{4}{*}{92.6} & \multirow{4}{*}{87.7} & 92.6 & \multirow{4}{*}{87.8} & \multirow{4}{*}{83.7} \\
\hline & $\mathrm{Ca}^{2+}$ & 89.7 & & & 82.8 & & \\
\hline & $\mathrm{Na}^{+}$ & 75.0 & & & 75.0 & & \\
\hline & $\mathrm{An}^{-}$ & 88.5 & & & 84.6 & & \\
\hline
\end{tabular}

Sn: sensitivity; $A A$ : average accuracy; $O A$ : overall accuracy; IC: ion channels; NIC: non-ion channels; VGIC: voltage-gated ion channels; LGIC: ligand-gated ion channels.

\section{Materials and Methods}

\subsection{Benchmark Databases}

The data used to establish the prediction model in this paper were collected from Lin et al. [13]. The sequences of ion channels were collected from the Universal Protein Resource (UniProt) [19] and the Ligand-Gated Ion channel database [20]. To construct a high-quality benchmark dataset, some sequences were removed according to three characteristics. Firstly, a sequence that contained some ambiguous residues (such as " $\mathrm{X}$ ", "B", "Z"). Secondly, a sequence that was the fragment of other proteins. Thirdly, a sequence that was annotated based on homology or prediction. Then, redundant sequences were removed by using the CD-HIT [21] program with a sequence identity threshold of $40 \%$, which has been widely used to filter out redundant samples in genomics and proteomics [22-26].

After the raw data were preprocessed, we finally obtained 298 ion channels including 148 voltage-gated ion channels and 150 ligand-gated ion channels. These voltage-gated ion channels can be classified into four subtypes as follows: 81 potassium $\left(\mathrm{K}^{+}\right), 29$ calcium $\left(\mathrm{Ca}^{2+}\right), 12$ sodium $\left(\mathrm{Na}^{+}\right)$, and 26 voltage-gated anion channels. Here, all the 300 non-ion channel proteins were randomly selected from the membrane proteins which were not marked as ion channels in the UniProt database. Moreover, any two sequences in these non-ion channels should guarantee that the identity between them is less than $40 \%$.

\subsection{Feature Extraction of Samples}

In order to characterize each protein sequence as accurately as possible, the order effect of sequence was usually selected as a method for generating effective feature vectors. Therefore, PseAAC $[27,28]$ incorporating dipeptide composition was selected as the method for feature extraction of protein samples in this paper.

Assuming that there is a protein sequence of $L$ amino acid residues:

$$
\mathbf{P}=\mathrm{R}_{1} \mathrm{R}_{2} \mathrm{R}_{3} \mathrm{R}_{4} \mathrm{R}_{5} \mathrm{R}_{6} \mathrm{R}_{7} \ldots \mathrm{R}_{L}
$$

where $\mathrm{R}_{i}(i=1,2,3 \ldots L)$ represents the amino acid residue at $i$-th sequence position. Therefore, we can get a set of feature vectors with the dimension of $400+n \lambda$ from any sequence like Equation (1)

$$
\mathbf{P}=\left[P_{1}, P_{2}, \ldots, P_{400}, P_{401}, \ldots, P_{400+n \lambda}\right]^{\mathrm{T}}
$$

where the first 400 features $P_{1}, P_{2}, \ldots, P_{400}$ represent the effect of the classical dipeptide composition; the $n \lambda$ elements $P_{400+1}, P_{400+2}, \ldots, P_{400+n \lambda}$ in addition to the 400 components represent the sequence 
order effect of protein samples, namely the first tier to $\lambda$-th tier correlation factors of protein sequence. These features can be calculated by:

$$
P_{u}=\left\{\begin{array}{l}
\frac{f_{u}}{\sum_{i=1}^{400} f_{i}+\omega \sum_{j=1}^{n \lambda} \tau_{j}}(1 \leq u \leq 400) \\
\frac{\omega \tau_{u}}{\sum_{i=1}^{400} f_{i}+\omega \sum_{j=1}^{n \lambda} \tau_{j}}(400+1 \leq u \leq 400+n \lambda)
\end{array}\right.
$$

where $f_{i}(i=1,2, \ldots, 400)$ is the normalized occurrence frequencies of the 400 dipeptides in protein $\mathbf{P}$; $\omega$ is the weight factor; $\tau_{j}(j=1,2, \ldots, n \lambda)$ is the $j$-tier sequence-correlation factor computed by:

$$
\left\{\begin{array}{c}
\tau_{1}=\frac{1}{L-1} \sum_{i=1}^{L-1} H_{i, i+1}^{1} \\
\tau_{2}=\frac{1}{L-1} \sum_{i=1}^{L-1} H_{i, i+1}^{2} \\
\cdots \\
\tau_{n}=\frac{1}{L-1} \sum_{i=1}^{L-1} H_{i, i+1}^{n} \\
\tau_{n+1}=\frac{1}{L-2} \sum_{i=1}^{L-2} H_{i, i+2}^{1} \\
\tau_{n+2}=\frac{1}{L-2} \sum_{i=1}^{L-2} H_{i, i+2}^{2} \\
\cdots \\
\tau_{2 n}=\frac{1}{L-2} \sum_{i=1}^{L-2} H_{i, i+2}^{n} \\
\cdots \\
\tau_{n \lambda-1}=\frac{1}{L-\lambda} \sum_{i=1}^{L-\lambda} H_{i, i+\lambda}^{n-1} \\
\tau_{n \lambda}=\frac{1}{L-\lambda} \sum_{i=1}^{L-\lambda} H_{i, i+\lambda}^{n}
\end{array}\right.
$$

where $H_{i, j}^{n}$ is the correlation function of physicochemical properties and can be calculated as:

$$
H_{i, j}^{n}=h^{n}\left(\mathrm{R}_{i}\right) \cdot h^{n}\left(\mathrm{R}_{j}\right)
$$

where $h^{n}\left(\mathrm{R}_{i}\right)$ denotes the value of $n$-th kind physicochemical property of $\mathrm{R}_{i} ; h^{n}\left(\mathrm{R}_{j}\right)$ is similar. To obtain the high-quality feature set, all the data of physicochemical properties must be subjected to a standard conversion as below:

$$
h^{k}\left(\mathrm{R}_{i}\right)=\frac{h_{0}^{k}\left(\mathrm{R}_{i}\right)-\sum_{\alpha=1}^{20} h_{0}^{k}\left(\mathrm{R}_{\alpha}\right) / 20}{\sqrt{\sum_{u=1}^{20}\left[h_{0}^{k}\left(\mathrm{R}_{i}\right)-\sum_{\alpha=1}^{20} h_{0}^{k}\left(\mathrm{R}_{\alpha}\right) / 20\right]^{2}}}
$$

where $\mathrm{R}_{i}(i=1,2, \ldots, 20)$ represents the 20-native amino acid according to the alphabetical order of their single-letter codes: A, C, D, E, F, G, H, I, K, L, M, N, P, Q, R, S, T, V, W, and Y. $h_{0}^{k}\left(R_{i}\right)$ denotes the original value of the $k$-th physicochemical property for residue $\mathrm{R}_{i}$. The values of each physicochemical property obtained after the standard conversion have two advantages. These values will have a zero-mean over the 20 native amino acids and remain unchanged if they are subjected to the same conversion procedure again. The values of the nine kinds of physicochemical properties used in this paper are from previous results [29]. 


\subsection{Feature Selection}

Generally, all features do not equally contribute to an ion channel prediction system. Some features make key contributions, whereas some others make minor contributions [30,31]. Therefore, the selection of features is an important step for establishing an effective prediction model. To analyze these feature vectors, ANOVA was used to choose the optimal feature sets in this paper.

In order to assess the contribution of each feature to the predictive system, the $F$ value was defined as follows:

$$
F(\lambda)=\frac{S_{B}^{2}(\lambda)}{S_{W}^{2}(\lambda)}
$$

where $S_{B}^{2}(\lambda)$ and $S_{W}^{2}(\lambda)$ respectively denote the sample variance between groups (also called means square between, MSB) and the sample variable within groups (also called means square within, MSW), and are expressed as:

$$
\left\{\begin{array}{l}
S_{B}^{2}(\lambda)=\frac{\sum_{i=1}^{K} n_{i}\left(\sum_{j=1}^{n_{i}} f_{i j}(\lambda) / n_{i}-\sum_{i=1}^{K} \sum_{j=1}^{n_{i}} f_{i j}(\lambda) / \sum_{i=1}^{K} n_{i}\right)^{2}}{K-1} \\
S_{W}^{2}(\lambda)=\frac{\sum_{i=1}^{K} \sum_{j=1}^{n_{i}}\left(f_{i j}(\lambda)-\sum_{i=1}^{K} \sum_{j=1}^{n_{i}} f_{i j}(\lambda) / \sum_{i=1}^{K} n_{i}\right)^{2}}{N-K}
\end{array}\right.
$$

where $K$ and $N$ respectively denote the number of groups and the total number of samples. $f_{i j}(\lambda)$ represents the frequency of the $\lambda$-th feature of the $j$-th sample in the $i$-th group. $n_{i}$ denotes the total number of samples in the $i$-th group. Thus, each feature corresponds to an $F$ score.

Obviously, the larger $F$ value means the greater contribution of the corresponding feature to the classification. Thus, according to their $F$ values, we may rank all features. Subsequently, we used the incremental feature selection (IFS) to determine the optimal number of features [32]. Firstly, we examined the accuracy of the first feature subset including a feature with the highest $F$ value in the ranked feature set. Secondly, we investigated the accuracy of the second feature subset which was produced by adding the feature with the second highest $F$ value. This process was repeated from the higher $F$ to the lower $F$ value until all candidate features were added. The performances of all feature subsets were evaluated. Then, we were able to obtain the best feature subset which was capable of producing the maximum accuracy.

\subsection{Support Vector Machine}

SVM is a kind of classification algorithm that can improve the generalization ability of machine learning and achieve the minimization of experience risk and confidence scope by minimizing the structural risk. Therefore, a good statistical result can be usually achieved even using a small sample. SVM, as a powerful supervised learning method, has been widely used in various fields including bioinformatics [33-38]. In this paper, we used LIBSVM 3.21 [39] which could be freely downloaded from http://www.csie.ntu.edu.tw/ cjlin/libsvm/. The radial basis function (RBF) kernel was selected as kernel function and one vs. one (OVO) strategy was used for multiclass classification. For achieving the optimal model, the penalty constant $C$ and the kernel width parameter $\lambda$ were tuned by an optimization procedure with a grid search method [39]. The search spaces for $C$ and $\lambda$ were $\left[2^{-5}, 2^{15}\right]$ and $\left[2^{5}, 2^{-15}\right]$ with steps being 2 and $2^{-1}$, respectively.

\subsection{Performance Evaluation}

A cross-validation technique is generally employed to estimate the accuracy of a predictive model. Three cross-validation methods including the independent dataset test, subsampling test, and jackknife test can be used [40-43]. Among them, the jackknife test is considered to be the most objective and rigorous one. Therefore, the jackknife test was employed to assess the performance of our methods. 
In addition, we also used other assessment criteria to evaluate the effectiveness of our predictive model in this paper. These assessment criteria, including sensitivity $(S n)$, overall accuracy $(O A)$, and average accuracy $(A A)$, are defined as follows:

$$
\begin{gathered}
S_{n}(i)=\frac{T P_{i}}{T P_{i}+F N_{i}} \\
O A=\sum_{i=1}^{n} \frac{T P_{i}}{N} \\
A A=\sum_{i=1}^{n} \frac{S_{n}(i)}{n}
\end{gathered}
$$

where $T P_{i}$ and $F N_{i}$ respectively denote true positives and false negatives of the $i$-th class. $N$ and $n$ represent the total number of samples and number of classes, respectively.

\section{Conclusions}

We constructed an SVM-based model for the accurate prediction of ion channel proteins and their types. In this model, a pseudo-dipeptide composition was adopted to extract features. The ANOVA was used to exclude noise or redundant information of feature vectors and then IFS was employed to determine the optimal number of features. High accuracies indicated that the proposed method was an effective tool for predicting ion channels and their types. A free web server based on the proposed method presented in this paper has been constructed and is accessible at the website (http://lin.uestc.edu.cn/server/IonchanPredv2.0).

Acknowledgments: This work was supported by the Applied Basic Research Program of Sichuan Province (No. 2015JY0100 and 14JC0121), the Fundamental Research Funds for the Central Universities of China (Nos. ZYGX2015J144; ZYGX2015Z006; ZYGX2016J118; ZYGX2016J125; ZYGX2016J126), Program for the Top Young Innovative Talents of Higher Learning Institutions of Hebei Province (No. BJ2014028), the Outstanding Youth Foundation of North China University of Science and Technology (No. JP201502), China Postdoctoral Science Foundation (No.2015M582533), and the Scientific Research Foundation of the Education Department of Sichuan Province (11ZB122).

Author Contributions: Hao Lin, Wei Chen, and Hua Tang conceived and designed the experiments; Ya-Wei Zhao performed the experiments; Ya-Wei Zhao analyzed the data; Ya-Wei Zhao and Zhen-Dong Su contributed reagents/materials/analysis tools; Ya-Wei Zhao, Wuritu Yang, and Hao Lin wrote the paper.

Conflicts of Interest: The authors declare no conflict of interest.

\section{References}

1. Wulff, H.; Christophersen, P. Recent developments in ion channel pharmacology. Channels 2015, 9, 335. [CrossRef] [PubMed]

2. Gabashvili, I.S.; Sokolowski, B.H.; Morton, C.C.; Giersch, A.B. Ion channel gene expression in the inner ear. J. Assoc. Res. Otolaryngol. 2007, 8, 305-328. [CrossRef] [PubMed]

3. Ger, M.F.; Rendon, G.; Tilson, J.L.; Jakobsson, E. Domain-based identification and analysis of glutamate receptor ion channels and their relatives in prokaryotes. PLoS ONE 2010, 5, e12827. [CrossRef] [PubMed]

4. Wei, F.; Yan, L.M.; Su, T.; He, N.; Lin, Z.J.; Wang, J.; Shi, Y.W.; Yi, Y.H.; Liao, W.P. Ion Channel Genes and Epilepsy: Functional Alteration, Pathogenic Potential, and Mechanism of Epilepsy. Neurosci. Bull. 2017, 33, 455-477. [CrossRef] [PubMed]

5. Wang, F.; Knutson, K.; Alcaino, C.; Linden, D.R.; Gibbons, S.J.; Kashyap, P.; Grover, M.; Oeckler, R.; Gottlieb, P.A.; Li, H.J.; et al. Mechanosensitive ion channel Piezo2 is important for enterochromaffin cell response to mechanical forces. J. Phys. 2017, 595, 79-91. [CrossRef] [PubMed]

6. Nguyen, T.H.; Huang, S.; Meynard, D.; Chaine, C.; Michel, R.; Roelfsema, M.R.G.; Guiderdoni, E.; Sentenac, H.; Very, A.A. A Dual Role for the OsK5.2 Ion Channel in Stomatal Movements and $\mathrm{K}^{+}$Loading into Xylem Sap. Plant Phys. 2017, 174, 2409-2418. [CrossRef] [PubMed]

7. Zubcevic, L.; Herzik, M.A., Jr.; Chung, B.C.; Liu, Z.; Lander, G.C.; Lee, S.Y. Cryo-electron microscopy structure of the TRPV2 ion channel. Nat. Struct. Mol. Biol. 2016, 23, 180-186. [CrossRef] [PubMed] 
8. Linsdell, P. Metal bridges to probe membrane ion channel structure and function. Biomol. Concepts 2015, 6, 191-203. [CrossRef] [PubMed]

9. Prindle, A.; Liu, J.; Asally, M.; Ly, S.; Garcia-Ojalvo, J.; Suel, G.M. Ion channels enable electrical communication in bacterial communities. Nature 2015, 527, 59-63. [CrossRef] [PubMed]

10. Hille, B.; Dickson, E.J.; Kruse, M.; Vivas, O.; Suh, B.C. Phosphoinositides regulate ion channels. Biochim. Biophys. Acta 2015, 1851, 844-856. [CrossRef] [PubMed]

11. Liu, L.X.; Li, M.L.; Tan, F.Y.; Lu, M.C.; Wang, K.L.; Guo, Y.Z.; Wen, Z.N.; Jiang, L. Local sequence information-based support vector machine to classify voltage-gated potassium channels. Acta Biochim. Biophys. Sin. 2006, 38, 363-371. [CrossRef] [PubMed]

12. Saha, S.; Zack, J.; Singh, B.; Raghava, G.P. VGIchan: Prediction and classification of voltage-gated ion channels. Genom. Proteom. Bioinform. 2006, 4, 253-258. [CrossRef]

13. Lin, H.; Ding, H. Predicting ion channels and their types by the dipeptide mode of pseudo amino acid composition. J. Theor. Biol. 2011, 269, 64-69. [CrossRef] [PubMed]

14. Chen, W.; Lin, H. Identification of voltage-gated potassium channel subfamilies from sequence information using support vector machine. Comput. Biol. Med. 2012, 42, 504-507. [CrossRef] [PubMed]

15. Liu, W.X.; Deng, E.Z.; Chen, W.; Lin, H. Identifying the subfamilies of voltage-gated potassium channels using feature selection technique. Int. J. Mol. Sci. 2014, 15, 12940-12951. [CrossRef] [PubMed]

16. Tiwari, A.K.; Srivastava, R. An efficient approach for the prediction of ion channels and their subfamilies. Comput. Biol. Chem. 2015, 58, 205-221. [CrossRef] [PubMed]

17. Gao, J.; Cui, W.; Sheng, Y.; Ruan, J.; Kurgan, L. PSIONplus: Accurate Sequence-Based Predictor of Ion Channels and Their Types. PLoS ONE 2016, 11, e0152964. [CrossRef] [PubMed]

18. Lin, H.; Liu, W.X.; He, J.; Liu, X.H.; Ding, H.; Chen, W. Predicting cancerlectins by the optimal g-gap dipeptides. Sci. Rep. 2015, 5, 16964. [CrossRef] [PubMed]

19. The UniProt, C. UniProt: The universal protein knowledgebase. Nucleic Acids Res. 2017, 45, D158-D169. [CrossRef]

20. Donizelli, M.; Djite, M.A.; le Novere, N. LGICdb: A manually curated sequence database after the genomes. Nucleic Acids Res. 2006, 34, 267-269. [CrossRef] [PubMed]

21. Li, W.; Godzik, A. Cd-hit: A fast program for clustering and comparing large sets of protein or nucleotide sequences. Bioinformatics 2006, 22, 1658-1659. [CrossRef] [PubMed]

22. Chen, W.; Feng, P.; Tang, H.; Ding, H.; Lin, H. Identifying 2'-O-methylationation sites by integrating nucleotide chemical properties and nucleotide compositions. Genomics 2016, 107, 255-258. [CrossRef] [PubMed]

23. Chen, W.; Feng, P.; Yang, H.; Ding, H.; Lin, H.; Chou, K.C. iRNA-AI: Identifying the adenosine to inosine editing sites in RNA sequences. Oncotarget 2017, 8, 4208-4217. [CrossRef] [PubMed]

24. Zou, Q.; Mao, Y.; Hu, L.; Wu, Y.; Ji, Z. miRClassify: An advanced web server for miRNA family classification and annotation. Comput. Biol. Med. 2014, 45, 157-160. [CrossRef] [PubMed]

25. Chen, W.; Lin, H. Prediction of midbody, centrosome and kinetochore proteins based on gene ontology information. Biochem. Biophys. Res. Commun. 2010, 401, 382-384. [CrossRef] [PubMed]

26. Chen, W.; Feng, P.; Lin, H. Prediction of ketoacyl synthase family using reduced amino acid alphabets. J. Ind. Microbiol. Biotechnol. 2012, 39, 579-584. [CrossRef] [PubMed]

27. Shen, H.B.; Chou, K.C. PseAAC: A flexible web server for generating various kinds of protein pseudo amino acid composition. Anal. Biochem. 2008, 373, 386-388. [CrossRef] [PubMed]

28. Liu, B.; Liu, F.; Wang, X.; Chen, J.; Fang, L.; Chou, K.-C. Pse-in-One: A web server for generating various modes of pseudo components of DNA, RNA, and protein sequences. Nucleic Acids Res. 2015, 43, W65-W71. [CrossRef] [PubMed]

29. Tang, H.; Chen, W.; Lin, H. Identification of immunoglobulins using Chou's pseudo amino acid composition with feature selection technique. Mol. BioSyst. 2016, 12, 1269-1275. [CrossRef] [PubMed]

30. Zhao, Y.W.; Lai, H.Y.; Tang, H.; Chen, W.; Lin, H. Prediction of phosphothreonine sites in human proteins by fusing different features. Sci. Rep. 2016, 6, 34817. [CrossRef] [PubMed]

31. Liu, B.; Chen, J.; Wang, X. Protein remote homology detection by combining Chou's distance-pair pseudo amino acid composition and principal component analysis. Mol. Genet. Genom. 2015, 290, 1919-1931. [CrossRef] [PubMed] 
32. Ding, H.; Feng, P.M.; Chen, W.; Lin, H. Identification of bacteriophage virion proteins by the ANOVA feature selection and analysis. Mol. BioSyst. 2014, 10, 2229-2235. [CrossRef] [PubMed]

33. Liao, Z.; Ju, Y.; Zou, Q. Prediction of G-protein-coupled receptors with SVM-Prot features and random forest. Scientifica 2016, 2016, 8309253. [CrossRef] [PubMed]

34. Li, D.; Ju, Y.; Zou, Q. Protein Folds Prediction with Hierarchical Structured SVM. Curr. Proteom. 2016, 13, 79-85. [CrossRef]

35. Chen, W.; Xing, P.; Zou, Q. Detecting N6-methyladenosine sites from RNA transcriptomes using ensemble Support Vector Machines. Sci. Rep. 2017, 7, 40242. [CrossRef] [PubMed]

36. Liu, B.; Zhang, D.; Xu, R.; Xu, J.; Wang, X.; Chen, Q.; Dong, Q.; Chou, K.-C. Combining evolutionary information extracted from frequency profiles with sequence-based kernels for protein remote homology detection. Bioinformatics 2014, 30, 472-479. [CrossRef] [PubMed]

37. Lai, H.Y.; Chen, X.X.; Chen, W.; Tang, H.; Lin, H. Sequence-based predictive modeling to identify cancerlectins. Oncotarget 2017, 8, 28169-28175. [CrossRef] [PubMed]

38. Feng, P.; Ding, H.; Yang, H.; Chen, W.; Lin, H.; Chou, K.C. iRNA-PseColl: Identifying the Occurrence Sites of Different RNA Modifications by Incorporating Collective Effects of Nucleotides into PseKNC. Mol. Ther. Nucleic Acids 2017, 7, 155-163. [CrossRef] [PubMed]

39. Chang, C.C.; Lin, C.J. LIBSVM: A Library for Support Vector Machines. Acm Trans. Intell. Syst. Technol. 2011, 2, 27. [CrossRef]

40. Chou, K.C.; Zhang, C.T. Prediction Of Protein Structural Classes. Crit. Rev. Biochem. Mol. Biol. 1995, 30, 275-349. [CrossRef] [PubMed]

41. Liu, B.; Wu, H.; Wang, X.; Chou, K.-C. Pse-Analysis a python package for DNA, RNA and protein peptide sequence analysis based on pseudo components and kernel methods. Oncotarget 2017, 8, 13338-13343. [CrossRef] [PubMed]

42. Lin, H.; Liang, Z.Y.; Tang, H.; Chen, W. Identifying $\sigma 70$ promoters with novel pseudo nucleotide composition. IEEE/ACM Trans. Comput. Biol. Bioinform. 2017. [CrossRef] [PubMed]

43. Zhang, C.J.; Tang, H.; Li, W.C.; Lin, H.; Chen, W.; Chou, K.C. iOri-Human: Identify human origin of replication by incorporating dinucleotide physicochemical properties into pseudo nucleotide composition. Oncotarget 2016, 7, 69783-69793. [CrossRef] [PubMed]

(C) 2017 by the authors. Licensee MDPI, Basel, Switzerland. This article is an open access article distributed under the terms and conditions of the Creative Commons Attribution (CC BY) license (http:/ / creativecommons.org/licenses/by/4.0/). 


\title{
PSFM-DBT: Identifying DNA-Binding Proteins by Combing Position Specific Frequency Matrix and Distance-Bigram Transformation
}

\author{
Jun Zhang and Bin Liu * \\ School of Computer Science and Technology, Harbin Institute of Technology Shenzhen Graduate School, \\ Shenzhen 518055, China; junzhangcs@foxmail.com \\ * Correspondence: bliu@hit.edu.cn; Tel.: +86-0755-8601-1630
}

Received: 28 July 2017; Accepted: 22 August 2017; Published: 25 August 2017

\begin{abstract}
DNA-binding proteins play crucial roles in various biological processes, such as DNA replication and repair, transcriptional regulation and many other biological activities associated with DNA. Experimental recognition techniques for DNA-binding proteins identification are both time consuming and expensive. Effective methods for identifying these proteins only based on protein sequences are highly required. The key for sequence-based methods is to effectively represent protein sequences. It has been reported by various previous studies that evolutionary information is crucial for DNA-binding protein identification. In this study, we employed four methods to extract the evolutionary information from Position Specific Frequency Matrix (PSFM), including Residue Probing Transformation (RPT), Evolutionary Difference Transformation (EDT), Distance-Bigram Transformation (DBT), and Trigram Transformation (TT). The PSFMs were converted into fixed length feature vectors by these four methods, and then respectively combined with Support Vector Machines (SVMs); four predictors for identifying these proteins were constructed, including PSFM-RPT, PSFM-EDT, PSFM-DBT, and PSFM-TT. Experimental results on a widely used benchmark dataset PDB1075 and an independent dataset PDB186 showed that these four methods achieved state-of-the-art-performance, and PSFM-DBT outperformed other existing methods in this field. For practical applications, a user-friendly webserver of PSFM-DBT was established, which is available at http://bioinformatics.hitsz.edu.cn/PSFM-DBT/.
\end{abstract}

Keywords: PSFM-DBT; DNA binding protein; distance bigram transformation; PSFM

\section{Introduction}

DNA-binding proteins play crucial roles in various biological processes, such as DNA replication and repair, transcriptional regulation, the combination and separation of single-stranded DNA and other biological activities associated with DNA. Therefore, effective methods for identifying DNA-binding proteins are highly required.

There are some experimental recognition techniques for DNA-binding protein identification, such as filter binding assays, genetic analysis, chromatin immune precipitation on microarrays, and X-ray crystallography. However, these methods are both time consuming and expensive [1]. With the development of genomic and proteomic sequencing techniques, the number of protein sequences is growing rapidly. It is highly desired to develop fast and effective computational methods to identify the DNA binding proteins based on the protein sequences. In this regard, some computational methods based on machine learning algorithms have been proposed. These methods can be roughly divided into two groups: structure-based methods [2-8] and sequence-based methods. Stawiski et al. [7] analyzed the positive electrostatic patches in protein surface, and represented proteins with 12 features including the patch size, percent helix in patch, 
average surface area, hydrogen-bonding potential, three conserved special residues, and other features of the protein. These features were then inputted into a Neural Network (NN) for identifying DNA-binding proteins.

A webserver for the identification of DNA binding proteins (iDBPs) [9] recently was constructed for DNA binding protein identification, in which a random forest (RF) classifier was trained based on multiple structural features, such as electrostatic potential, cluster-based amino acid conservation patterns, secondary structure content of the patches, dipole moment and hydrogen-bonding potential. Song et al developed nDNA-Prot, which employed an imbalanced classifier [10]. Bhardwaj et al. [11] examined the sizes of positively charged patches on the surface of proteins, and used generated structural features to train a support vector machine (SVM) classifier. These structure-based methods achieved state-of-the-art performance. However, they require the structure information of proteins, which is not always available. In contrast, the sequence-based methods identify the DNA binding proteins only based on the sequence information of proteins, for example, Cai and Lin [12] proposed a method representing proteins employing pseudo amino acid composition (PseAAC) [13], in which amino acid composition, limited range correlation of hydrophobicity and solvent accessible surface area were taken into account. In method DNA-Prot [14], proteins was represented by various sequence properties, including frequency of amino acid, physical chemical properties, secondary structure, neutral amino acids, etc. Fang et al. [15] extracted protein features by using autocross-covariance (ACC) transform, pseudo amino acid composition, and dipeptide composition. Evolutionary profiles were introduced into this field by Kumar et al. [16]; they also developed a SVM-based predictor based on generated features. Recently, evolutionary profile was widely used in this field. Position specific score matrix distance transformation (PSSM-DT) [17] combined PSSM distance transformation with SVM. An improved DNA-binding protein prediction method (Local-DPP) [18] extracted local evolutionary information from some equally sized sub-PSSMs to represent proteins. Zhang et al. [19] proposed a new method in which feature vectors were extracted from PSSM, secondary structure, and physicochemical properties. They further improved the performance by using an improved Binary Firefly Algorithm (BFA) to filter noisy features and select optimal parameters for the classifier. Waris et al. [20] combined three different protein representations (dipeptide composition, split amino acid composition, and PSSM), and three machine learning algorithms ( $k$ Nearest Neighbor (KNN), SVM, and RF).

All these aforementioned methods have made great contributions to the development of this important field; the profile-based methods especially achieved state-of-the-art performance by incorporating evolutionary information into the predictors. Almost all of the machine-learning-based classifiers require fixed length feature vectors as inputs [21]. However, it is not an easy task to convert the profiles into feature vectors because a profile such as PSSM is a matrix with different dimensions. In this study, we employed four methods to extract the evolutionary information from Position Specific Frequency Matrix (PSFM), including Residue Probing Transformation (RPT) [22], Evolutionary Difference Transformation (EDT) [3], Distance-Bigram Transformation (DBT) [17,23,24], and Trigram Transformation (TT) [25]. The PSFMs were converted into fixed length feature vectors by these four methods, and then respectively combined with SVMs; four predictors for DNA binding protein identification were constructed, including PSFM-RPT, PSFM-EDT, PSFM-DBT and PSFM-TT. Experimental results on a widely used benchmark dataset and an independent dataset showed that these four methods achieved state-of-the-art-performance, and outperformed other existing methods in this field.

\section{Result and Discussion}

\subsection{Impact of the Maximum Distance D}

In order to evaluate the performance of the proposed methods, and select the optimized parameter, we explored the effect of the parameter $D$ (see Equations (9) and (12)) in methods PSFM-EDT and PSFM-DBT. Taking into account the time cost, the predictive results were obtained by using 5 -fold 
cross validation on benchmark dataset. The results of PSFM-EDT and PSFM-DBT with different values of $D$ are shown in Figure 1a,b, respectively, from which we can see that PSFM-EDT and PSFM-DBT can achieve stable performance with different $D$ values, and they achieved best performance when $D$ $=7$ and $D=4$ respectively. Therefore, the parameter $D$ of PSFM-EDT was set as 7 and the parameter $D$ of PSFM-DBT was set as 4 .

(a)

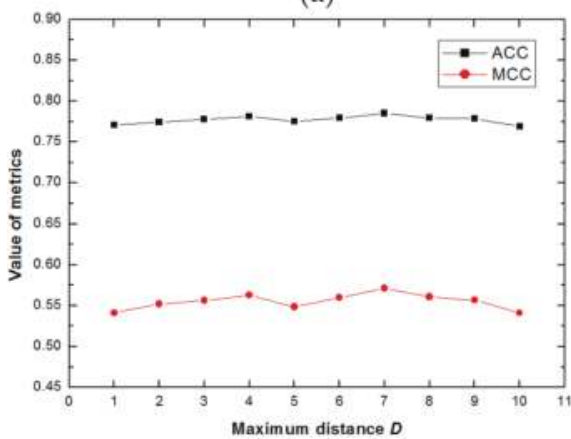

(b)

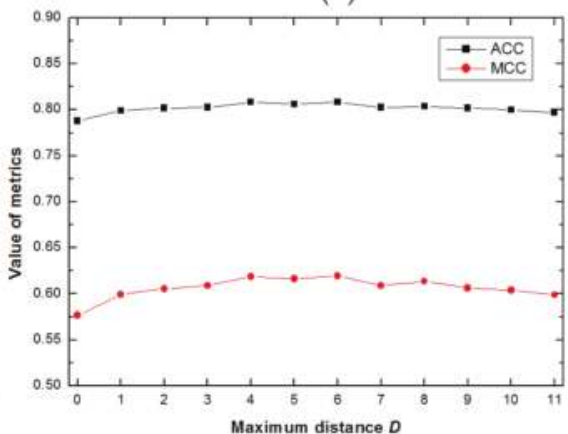

Figure 1. (a) The performance of Position Specific Frequency Matrix-Evolutionary Difference Transformation (PSFM-EDT) with different D on the benchmark dataset via five-cross validation.

(b) The performance of Position Specific Frequency Matrix-Distance-Bigram Transformation (PSFM-DBT) with different $D$ on the benchmark dataset via five-cross validation.

\subsection{Comparison of the Four PSFM-Based Methods}

The performance of the four proposed PSFM-based methods was shown in Table 1 by using jackknife test on benchmark dataset, and the corresponding ROC curves of these methods were shown in Figure 2a. From Table 1 and Figure 2a we can see that the PSFM-DBT is better than all the other methods. The reason is that PSFM-DBT incorporates more sequence-order effects by considering bigrams separated by different distances, which is more efficient than the other three approaches. Furthermore, a recent study showed that these sequence-order effects are critical for DNA binding protein identification [23].

(a)

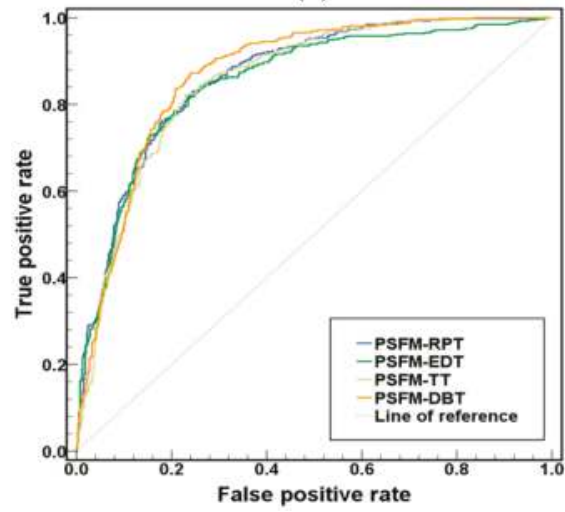

(b)

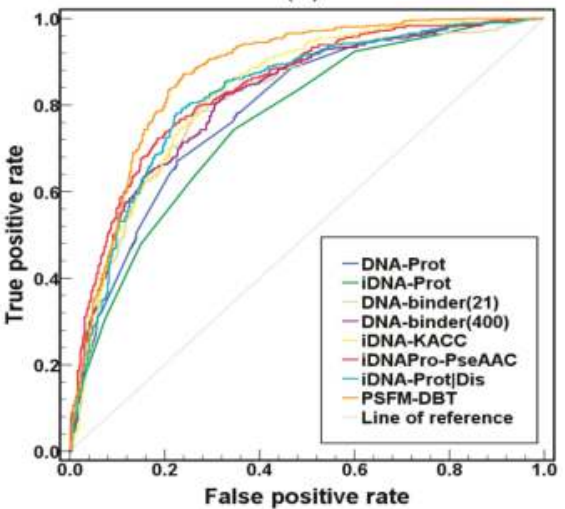

Figure 2. (a) The Receiver Operating Characteristic (ROC) curves of the four PSFM-based methods on the benchmark dataset using the jackknife tests. (b) The ROC curves of various methods on the benchmark dataset using the jackknife tests. 
Table 1. The results of the four Position Specific Frequency Matrix (PSFM)-based methods on the benchmark dataset.

\begin{tabular}{cccccc}
\hline Method & ACC (\%) & MCC & AUC (\%) & SN (\%) & SP (\%) \\
\hline PSFM-RPT $^{\text {a }}$ & 78.88 & 0.5785 & 86.35 & 80.76 & 77.09 \\
PSFM-EDT $^{\mathrm{b}}$ & 79.35 & 0.5868 & 84.49 & 78.86 & $\mathbf{7 9 . 8 2}$ \\
PSFM-DBT $^{\mathrm{c}}$ & $\mathbf{8 1 . 0 2}$ & $\mathbf{0 . 6 2 2 4}$ & $\mathbf{8 7 . 1 2}$ & $\mathbf{8 4 . 1 9}$ & 78.00 \\
PSFM-TT $^{\mathrm{d}}$ & $\mathbf{7 9 . 1 6}$ & 0.5840 & 85.54 & 80.95 & 77.45 \\
\hline
\end{tabular}

The results were obtained by jackknife test on benchmark dataset with SVM algorithm. The bold numbers represent the best values of the corresponding evaluation criteria in this table. ${ }^{a}$ The parameters were: $c=2^{4}, g=2^{6}$; b The parameters were: $D=7, c=2^{9}, g=2^{-2}$; ${ }^{\mathrm{C}}$ The parameters were: $D=4, c=2^{3}, g=2^{5}$; ${ }^{\mathrm{d}}$ The parameters were: $c=2^{5}, g=2^{-9}$.

\subsection{Comparison with Existing Methods}

The performance of PSFM-DBT was compared with other existing methods on the benchmark dataset, including DNAbinder [16], DNA-Prot [14], iDNA-Prot [26], iDNA-KACC [27], PseDNA-Pro [17], iDNA-Prot I dis [23], iDNAPro-PseAAC [28], PSSM-DT [17] and Local-DPP [18]. Among these nine methods, DNAbinder, iDNAPro-PseAAC, PSSM-DT and Local-DPP are profile-based methods, and the other five methods are sequence-based methods. The performance of various methods was shown in Table 2 and Figure 2b, from which we can see that the profile-based methods achieved higher performance than other sequence-based methods, and PSFM-DBT obviously outperformed other methods, indicating that evolutionary information is critical for DNA binding protein identification, and PSFM-DBT is an efficient method. ACC represents the percentage of the samples which are correctly predicted among all samples; MCC explains the reliability of models; Sensitivity (SN) is an important measure, it presents the accuracy of predicting positive samples; Specificity (SP) denotes the percentage of true negative samples among negative samples; AUC is the area under ROC curve which gives a measure of the quality of binary classification methods, the larger AUC is, the better its predictive quality is.

Table 2. The performance of various methods on benchmark dataset.

\begin{tabular}{cccccc}
\hline Method & ACC (\%) & MCC & AUC (\%) & SN (\%) & SP (\%) \\
\hline DNA-Prot & 72.55 & 0.44 & 78.90 & 82.67 & 59.75 \\
iDNA-Prot & 75.40 & 0.50 & 76.10 & 83.81 & 64.73 \\
DNAbinder (dimension 400) & 73.58 & 0.47 & 81.50 & 66.47 & 80.36 \\
DNAbinder (dimension 21) & 73.95 & 0.48 & 81.40 & 68.57 & 79.09 \\
PseDNA-Pro & 76.55 & 0.53 & N/A & 79.61 & 73.63 \\
iDNA-Prot I dis & 77.30 & 0.54 & 82.60 & 79.40 & 75.27 \\
iDNAPro-PseAAC & 76.56 & 0.53 & 83.92 & 75.62 & 77.45 \\
iDNA-KACC & 75.16 & 0.50 & 83.00 & 77.52 & 72.90 \\
PSSM-DT & 79.96 & 0.62 & 86.50 & 78.00 & $\mathbf{8 1 . 9 1}$ \\
Local-DPP & 79.10 & 0.59 & N/A & $\mathbf{8 4 . 8 0}$ & 73.60 \\
PSFM-DBT a & $\mathbf{8 1 . 0 2}$ & $\mathbf{0 . 6 2}$ & $\mathbf{8 7 . 1 2}$ & 84.19 & 78.00 \\
\hline
\end{tabular}

The results of all methods in the table were obtained by jackknife validation on benchmark dataset. The bold numbers represent the best values of the corresponding evaluation criteria in this table. ${ }^{\text {a }}$ See the footnote of Table 1.

\subsection{Independent Test}

In this study, the four proposed PSFM-based methods were further evaluated on an independent dataset PDB186 constructed by Lou et al. [1]. It contains 93 DNA-binding proteins and 93 non-DNA-binding proteins selected from PDB. Because there are some proteins in benchmark dataset share more than $25 \%$ sequence identity with some proteins in independent dataset, this will lead to homology bias. In order to avoid this problem, the NCBI's BLASTCLUST [29] was employed to filter those proteins from the benchmark dataset which have more than $25 \%$ sequence identity to any 
protein in a same subset of the PDB186 dataset. Then we retrained the four proposed PSFM-based methods on such a reduced benchmark dataset, based on which the proteins in the independent dataset were predicted, and the results were shown in Table 3 and Figure 3a. PSFM-DBT achieved the top performance, which further demonstrates that it is a useful predictor for DNA binding protein identification.

Table 3. Performance of various methods on the independent dataset.

\begin{tabular}{cccccc}
\hline Method & ACC (\%) & MCC & AUC (\%) & SN (\%) & SP (\%) \\
\hline DNA-Prot & 61.80 & 0.240 & N/A & 69.90 & 53.80 \\
iDNA-Prot & 67.20 & 0.344 & N/A & 67.70 & 66.70 \\
DNAbinder & 60.80 & 0.216 & 60.70 & 57.00 & 64.50 \\
DNABIND & 67.70 & 0.355 & 69.40 & 66.70 & 68.80 \\
DBPPred & 76.90 & 0.538 & 79.10 & 79.60 & 74.20 \\
iDNA-Prot I dis & 72.00 & 0.445 & 78.60 & 79.50 & 64.50 \\
iDNAPro-PseAAC-EL & 71.50 & 0.442 & 77.80 & 82.80 & 60.2 \\
iDNA-KACC-EL & 79.03 & 0.611 & 81.40 & $\mathbf{9 4 . 6 2}$ & 63.44 \\
PSSM-DT & 80.00 & $\mathbf{0 . 6 4 7}$ & 87.40 & 87.09 & 72.83 \\
Local-DPP & 79.00 & 0.625 & N/A & 92.50 & 65.60 \\
PSFM-TT & 78.49 & 0.580 & 86.63 & 88.17 & 68.82 \\
PSFM-RPT & 79.57 & 0.594 & 85.67 & 84.95 & 74.19 \\
PSFM-EDT & 79.57 & 0.600 & 86.88 & 88.17 & 70.97 \\
PSFM-DBT & $\mathbf{8 0 . 6 5}$ & 0.624 & $\mathbf{8 8 . 0 3}$ & 90.32 & 70.97 \\
\hline
\end{tabular}

The bold numbers represent the best values of the corresponding evaluation criteria in this table.

The number of DNA-binding proteins is much lower than that of the non DNA-binding proteins in the real world. In order to simulate real world applications, we evaluated the performance of PSFM-DBT on this independent dataset with different ratios of positive and negative samples, and the results were shown in Figure 3b, from which we can see that the ACC increases slightly as the ratio of positive samples increases, indicating that the PSFM-DBT can achieve stable performance and it is suitable for DNA binding protein prediction.

(a)

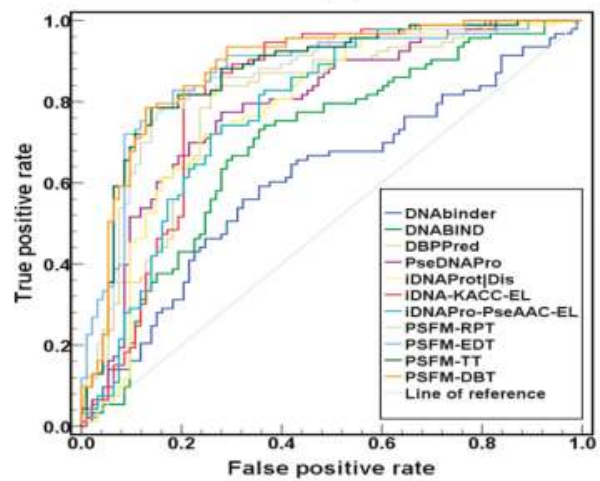

(b)

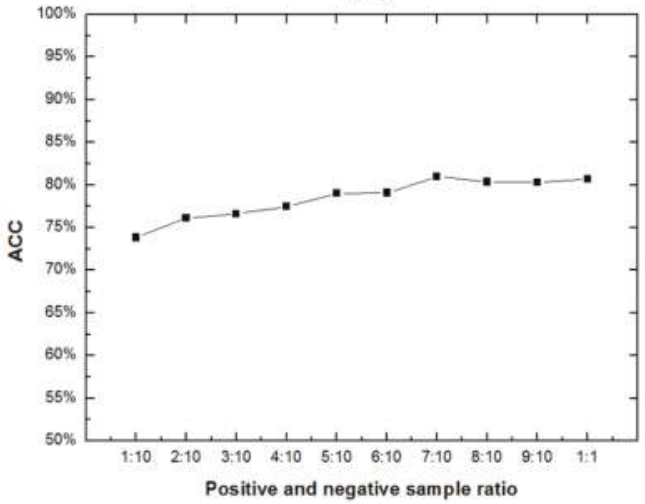

Figure 3. (a) The ROC curves of various methods on the independent dataset PDB186. (b) The performance of PSFM-DBT on the independent dataset with different ratios of positive samples.

\subsection{Feature Analysis}

To further investigate the importance of the features and to reveal the biological meaning of the features in proposed PSFM-DBT, we followed some previous studies $[30,31]$ to calculate the 
discriminant weight vector in the feature space. The sequence-specific weight obtained from the SVM training process can be used to calculate the discriminant weight of each feature to measure the importance of the features. Given the weight vectors of the training set with $N$ samples obtained from the kernel-based training $\mathbf{A}=\left[a_{1}, a_{2}, a_{3}, \ldots, a_{N}\right]$, the feature discriminant weight vector $\mathbf{W}$ in the feature space can be calculated by the following equation:

$$
\mathbf{W}=\mathbf{A} \cdot \mathbf{M}=\left[\begin{array}{c}
a_{1} \\
a_{2} \\
\vdots \\
a_{N}
\end{array}\right]^{\mathbf{T}}\left[\begin{array}{cccc}
m_{11} & m_{12} & \cdots & m_{1 j} \\
m_{21} & m_{22} & \cdots & m_{2 j} \\
\vdots & \vdots & \ddots & \vdots \\
m_{N 1} & m_{N 2} & \cdots & m_{N j}
\end{array}\right]
$$

where $\mathbf{M}$ is the matrix of sequence representatives; $\mathbf{A}$ is the weight vectors of the training samples; $N$ is the number of training samples; $j$ is the dimension of the feature vector. The element in $\mathbf{W}$ represents the discriminative power of the corresponding feature.

In this study, the feature analysis was based on the predictor PSFM-DBT $(D=4)$. The discriminative weights of the 2000 features were calculated by Equation (1). Then we analyzed the features of amino acid composition and the features of amino acid bigrams respectively. The discriminant weights of the 400 features with $d=0$ were visualized by a heatmap shown in Figure $4 \mathrm{a}$. The 20 elements in the diagonal represent the 20 features of amino acids composition, from which we can see that the amino acid K (Lys) has the highest weight value among all the 20 features, indicating that amino acid $\mathrm{K}$ is critical for predicting the DNA binding proteins. For further exploration, all the discriminant weights of all the 20 features of amino acid composition were shown in Figure $4 \mathrm{~b}$. We can see that 10 amino acids show positive discriminative weights, while the other 10 amino acids show negative discriminative weights. The top five most discriminative amino acids are K (Lys), R (Arg), L (Leu), E (Glu) and T (Thr). It has been reported that the positively charged amino acids (such as Arg and Lys) and the polar amino acids (such as Thr and Ser) are important for a protein binding with a DNA sequence, and the acidic amino acids, such as D (Asp) and E (Glu), show low propensity for the interaction of protein and DNA [32,33]. However, amino acid Glu show positive discriminative weights in Figure 4b indicating that the bigram composition is more accurate than the amino acid composition.

(a)

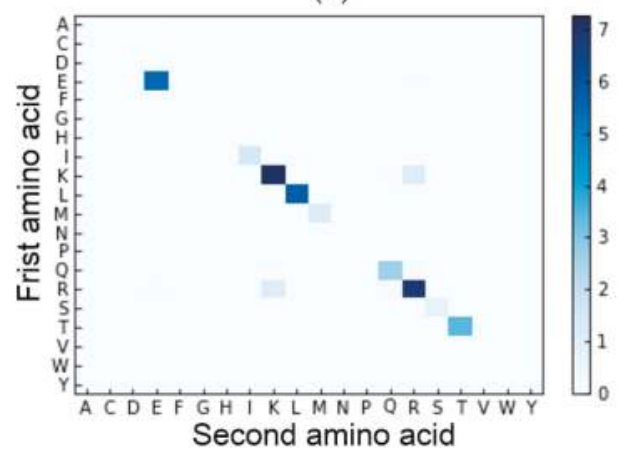

(b)

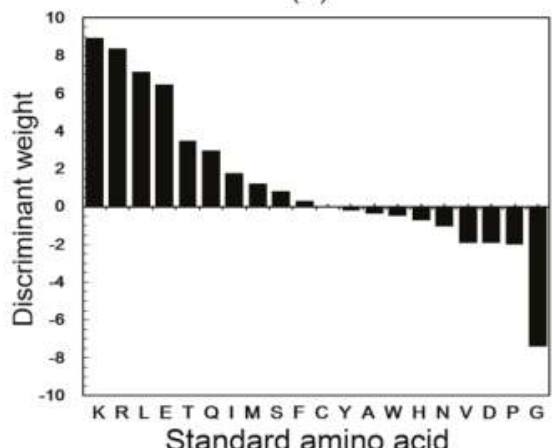

Figure 4. Cont. 
(c)

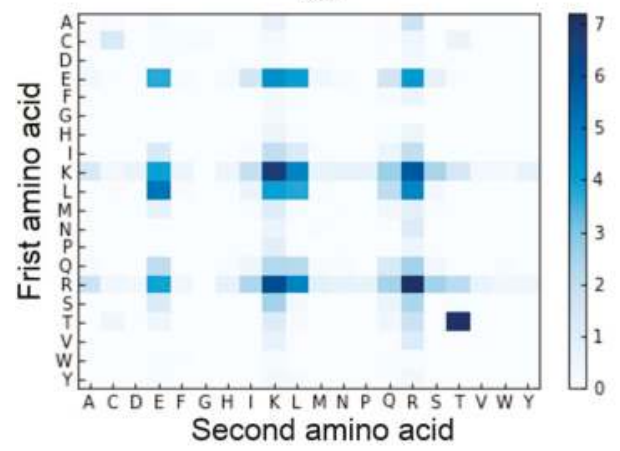

(d)

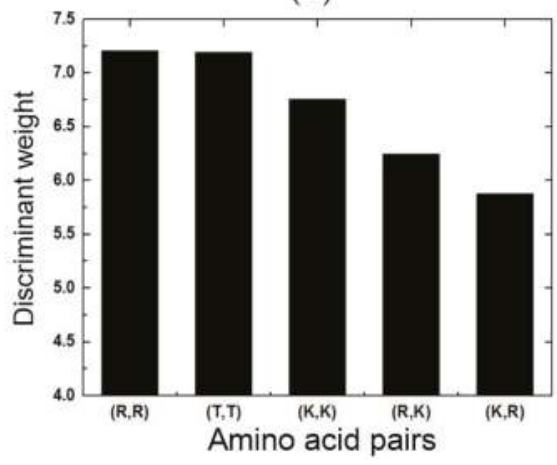

Figure 4. Feature analysis based on the features generated by PSFM-DBT. (a) The discriminant weights of the 400 features with $d=0$. Each element in the figure represents the discriminant weight of the corresponding feature. The diagonal elements represent 20 features of amino acid composition. (b) The discriminant weights of the 20 amino acids according to amino acid composition. (c) The discriminant weights of the 400 standard amino acid pairs $(d=1,2,3,4)$. Each element in the figure represents the sum of the discriminant weights of the corresponding bigrams, for example, the discriminant weight of bigrams $(R, R)$ is $W_{(R, R)}=W_{(R R)}+W_{\left(R^{*} R\right)}+W_{\left(R^{* *} R\right)}+W_{\left(R^{* * *} R\right)}$, where * represents mismatch. The x-axis and $y$-axis represent the second amino acid and first amino acid in a bigram, respectively. (d) The discriminant weights of the top five most discriminant bigrams, including $(R, R),(T, T),(K, K),(R, K)$ and $(K, R)$.

Then we analyzed the rest of the 1600 features of amino acid bigrams obtained by PSFM-DBT with $d=1,2,3,4$. The weight values of the same kinds of bigrams with different $d$ values were summed, and the results are shown in Figure $4 \mathrm{c}$. We can see from this figure, the top five most discriminative amino acid bigrams are $(R, R),(T, T),(K, K),(R, K)$ and $(K, R)$, whose discriminant weights were shown in Figure $4 \mathrm{~d}$. These results further confirmed that the importance of amino acid R (Arg), $T$ (Thr) and $\mathrm{K}$ (Lys). Interestingly, this conclusion is fully consistent with previous studies [32-35]. A specific DNA-binding protein 1IGN chain B was selected as an example to further explore the importance of the features in PSFM-DBT. 1IGNB is known as the yeast RAP1, a multifunctional protein binding with the telomeric DNA in the yeast $S$. cerevisiae via a sequence-specific manner, it is also involved in transcriptional regulation [36]. As shown in Figure 4d, bigrams (R, R) have the highest weight values among all the four bigrams. There are four kinds of $(R, R)$ bigrams, including $R R, R^{*} R, R^{* *} R$ and $R^{* * *} R$ (* represents mismatch) with distance $d=1,2,3,4$ respectively. The distributions of these bigrams in the protein sequence 1IGNB and its 3D structure were shown in Figure 5a,c, respectively, from which we can see that most of the $(R, R)$ bigrams were located in the DNA binding regions, except that two occurred in the structural disordered regions, and all (R, R) bigrams occurred in the area close to DNA major grooves. Previous studies reported $[23,34]$ that the arginine rich region is indeed critical for the protein helix, and DNA major groove interaction by a mechanism known as 'phosphate bridging by an arginine-rich helix'. Moreover, we counted the numbers of these amino acid residues interacting with DNA in protein 1IGNB, the corresponding histogram is shown in Figure 5b, from which we can see that the positively charged amino acids (Arg, Lys and His) and the polar amino acids (Thr, Ser and Asn) are more likely to bind to DNA. This proved the correctness of the above conclusion, and explained the reason why the proposed PSFM-DBT predictor works well for DNA binding protein identification. 

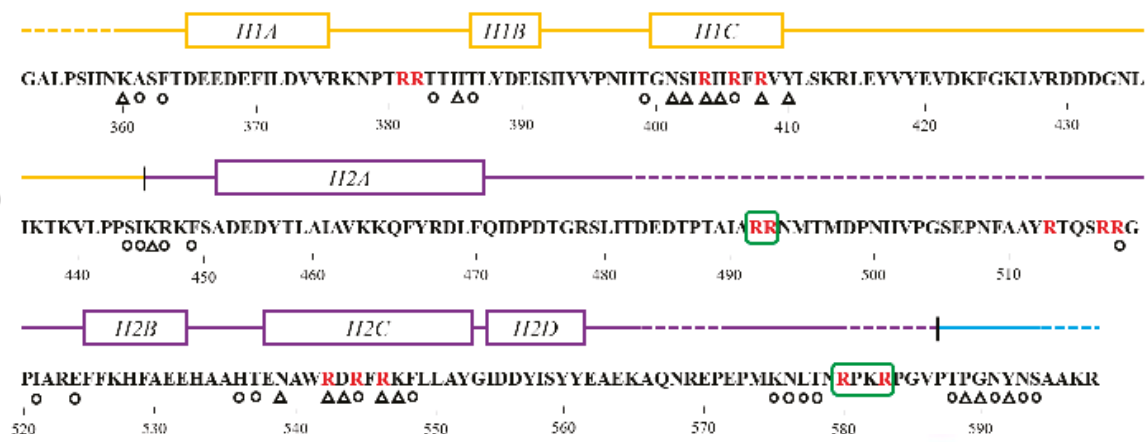

(b)

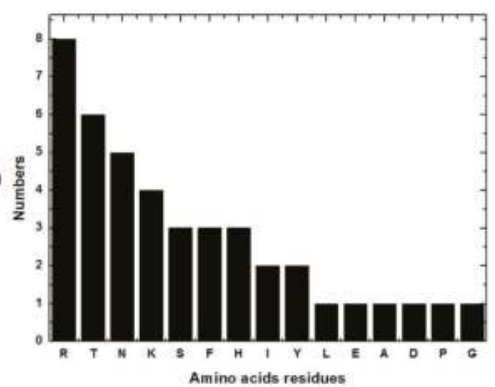

(c)

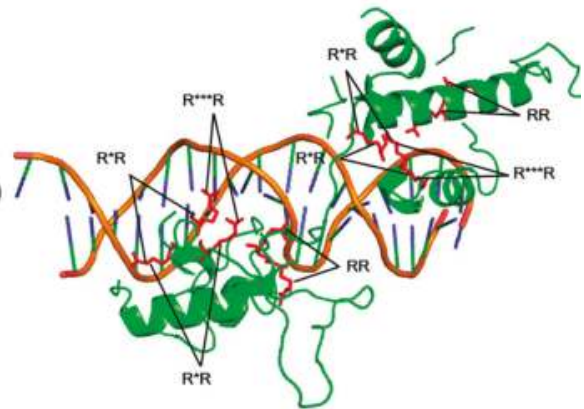

Figure 5. (a) The distributions of bigrams ( $R, R)$ in protein 1IGNB. The structural domains of this protein are color coded (orange represents domain 1, purple represents domain 2, and C-terminal tail is shown in blue). The open rectangles indicate the positions of helices, and broken lines mark regions of structural disorder. Residues interacting with DNA bases are indicated by triangles, and those contacting the phosphate backbone are indicated by circles. The two $(R, R)$ bigrams shown in green rectangles are the two bigrams occurring in non-DNA-binding regions. (b) Histogram of the number of amino acid residues which binding with DNA in protein 1IGNB. (c) The distributions of bigrams $(R, R)$ with different distances in the 3D structure of protein 1IGNB. The 3D structures of protein and DNA are shown in green and brown, respectively.

\subsection{Web-Server Guide}

We established an accessible web-server for the proposed PSFM-DBT predictor. Furthermore, for the convenience of the vast majority of experimental scientists, a step-by-step guide about how to use the web-server without the need to carefully understand the mathematical details was stated as follows.

Step 1. Open the web-server at http:/ /bioinformatics.hitsz.edu.cn/PSFM-DBT/ and you will see the home page of PSFM-DBT, as shown in Figure 6. Click on the "ReadMe" button to see a brief introduction of the server and the caveat when using it.

Step 2. You can input the query sequences into the input box or directly upload your input data via the "Browse" button. The input sequence should be in the FASTA format. The examples of sequences in the FASTA format could be shown in the input box by clicking the Example button right above the input box.

Step 3. Click on the "Submit" button to execute the recognition program, then the predicted results will be shown in a new page. For example, if you use the four example protein sequences as the input, you will see on your computer screen that the first and second query sequences are DNA-binding proteins. The third and fourth are non-DNA-binding proteins. 


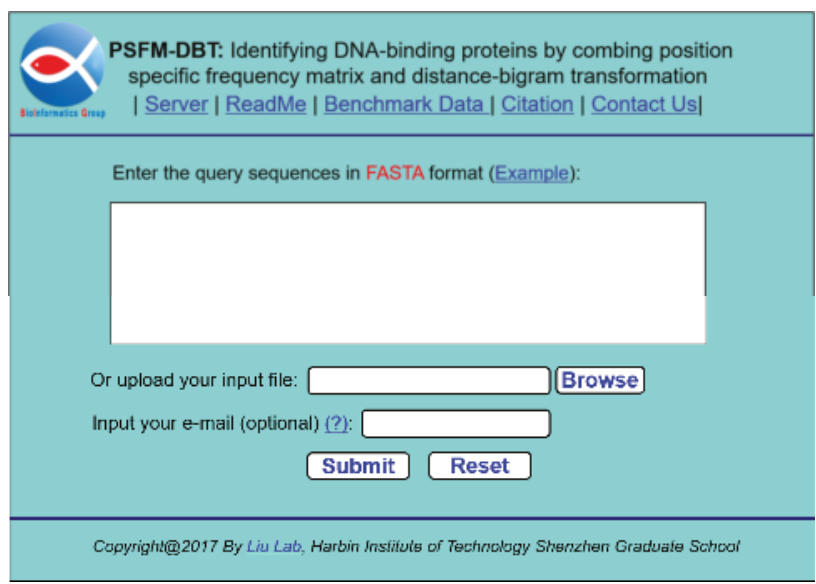

Figure 6. A semi-screenshot to show the home page of the web-server PSFM-DBT, which is available at http://bioinformatics.hitsz.edu.cn/PSFM-DBT/.

\section{Methods and Materials}

\subsection{Dataset}

The quality of the data set determines the quality of the research results. In the current study, we selected a widely used dataset PDB1075 [23] as the benchmark dataset. PDB1075 was constructed by Liu et al., which can be formulated as

$$
\mathbb{S}=\mathbb{S}^{+} \cup \mathbb{S}^{-}
$$

where $\mathbb{S}^{+}$is the subset of positive samples, $\mathbb{S}^{-}$is the subset of negative samples and the symbol $\cup$ represents the "union" in the set theory. These proteins were all extracted from Protein Data Bank (PDB) released at December 2013, where DNA-binding proteins were obtained by searching the mmCIF keyword of 'DNA binding protein' through the advanced search interface and non-DNA-binding proteins were obtained by randomly extracting from PDB. To construct a high quality and non-redundant benchmark dataset, these proteins were filtered strictly according to the following criteria. (1) Remove all the sequences which have less than 50 amino acids or contain character of ' $X$ '. (2) Using PISCES [37] to filter those sequences that have $\geq 25 \%$ pairwise sequence similarity to any other in the same subset. Finally, the subset $\mathbb{S}^{+}$consist of 525 DNA-binding proteins and the subset $\mathbb{S}^{-}$consists of 550 non-DNA-binding proteins.

\subsection{Protein Representation}

One of the most challenging problems in machine learning-based methods for computational biology is how to effectively represent a biological sequence with a discrete model [38-40], because all the existing machine learning algorithms [41], such as NN, SVM, RF, and KNN can only handle vector rather than protein sequences with different lengths. To solve this problem, many researchers have proposed various methods. Previous experimental results showed that evolutionary information can obviously improve the performance of predictors for identifying DNA-binding proteins. In order to incorporate the evolutionary information into the predictors, we employed four feature extraction methods to extract the evolutionary information from the Position Specific Frequency Matrix (PSFM) [42]. PSFM and the four methods will be introduced in more detail in the following sections. 


\subsubsection{Position Specific Frequency Matrix}

PSFM has been widely used in the field of predicting the structure and function of proteins $[42,43]$. Therefore, in this study, we employed the PSFM, which was generated by using PSI-BLAST [29] to search the target proteins against the non-redundant database NRDB90 [44] with default parameters, except the iteration and $e$-value were set as 10 and 0.001 , respectively.

Given a protein sequence $\mathbf{P}$ with $L$ amino acids, it can be formulated as:

$$
\mathbf{P}=\mathrm{R}_{1} \mathrm{R}_{2} \mathrm{R}_{3} \mathrm{R}_{4} \mathrm{R}_{5} \cdots \mathrm{R}_{L}
$$

where $R_{1}$ represents the 1 st residue, $R_{2}$ the 2 nd residue, and so forth.

The PSFM profile can be represented as a matrix with dimensions of $20 \times L$ as follows:

$$
\text { PSFM }=\left[\begin{array}{cccc}
P_{1,1} & P_{1,2} & \cdots & P_{1,20} \\
P_{2,1} & P_{2,2} & \cdots & P_{2,20} \\
\vdots & \vdots & \ddots & \vdots \\
P_{L, 1} & P_{L, 2} & \cdots & P_{L, 20}
\end{array}\right]
$$

where 20 represents the number of standard amino acids, and $L$ is the length of the query protein sequence. The element $P_{i, j}$ represents the occurrence probability of amino acid $j$ at position $i$ of the protein sequence, the rows of matrix represent the positions of the sequence, and the columns of the matrix represent the 20 standard amino acids. The sum of elements in each row is 1.

\subsubsection{Residue Probing Transformation}

RPT, first proposed by Jeong et al. [22], focuses on domains with similar conservation rates by grouping domain families based on their conservation scores in PSSM profiles. Because the idea is similar to the probe concept used in microarray technologies, it was called RPT. Each probe is a standard amino acid, and corresponds to a particular column in the PSFM profiles.

Given a PSFM (Equation (4)), it was divided into 20 groups according to 20 different standard amino acids, and for each group, we calculated the sum of the PSFM values in every column, leading to a feature vector of 20 dimension. Iteratively, for the 20 groups, the PSFM was translated into a Matrix $\mathbf{M}$ with $20 \times 20$ dimension, as follows:

$$
\mathbf{M}=\left[\begin{array}{cccc}
e_{1,1} & e_{1,2} & \cdots & e_{1,20} \\
e_{2,1} & e_{2,2} & \cdots & e_{2,20} \\
\vdots & \vdots & \ddots & \vdots \\
e_{20,1} & e_{20,2} & \cdots & e_{20,20}
\end{array}\right]
$$

The $\mathbf{M}$ was then transferred into a feature vector of 400 dimension, as follows:

$$
\mathbf{P}=\left[f\left(e_{1,1}\right) f\left(e_{1,2}\right) \cdots f\left(e_{i, j}\right) \cdots f\left(e_{20,20}\right)\right]
$$

where $f\left(e_{i, j}\right)$ was calculated by the following equation:

$$
f\left(e_{i, j}\right)=\frac{e_{i, j}}{L}(i, j=1,2, \cdots, 20)
$$

In this study, the amino acid composition of the 20 standard amino acids in PSFM was also incorporated into the RPT approach. As a result, the dimension of the corresponding feature vector is $400+20=420$. 


\subsubsection{Evolutionary Difference Transformation}

EDT [3] is able to extract the information of the non-co-occurrence probability of two amino acids separated by a certain distance $d$ in protein during the evolutionary process of the protein. The $d$ is the distance between these two amino acids $\left(d=1,2, \ldots, L_{\min }-1\right.$, where $L_{\min }$ is the length of the shortest proteins in the benchmark dataset (Equation (2)). For example, $d=1$ means the two amino acids are adjacent; $d=2$ means there is one amino acid between the two amino acids; $d=3$ means there are two amino acids between the two amino acids, and so forth.

For a given PSFM (Equation (4)), it can be transferred into a feature vector, as follows:

$$
\mathbf{P}=\left[\psi_{1} \psi_{2} \cdots \psi_{k} \cdots \psi_{\Omega}\right]
$$

where $\Omega$ is an integer reflecting the vector's dimension, its value is $D \times 400$; where $D$ is the maximum value of $d$. The non-co-occurrence probability of two amino acids separated by distance $d$ can be calculated by:

$$
f\left(\mathrm{~A}_{x}, \mathrm{~A}_{y} \mid d\right)=\frac{1}{L-d} \sum_{i=1}^{L-d}\left(P_{i, x}-P_{i+d, y}\right)^{2}
$$

where $P_{i, x}\left(P_{i+d, y}\right)$ is the element in PSFM; $\mathrm{A}_{x}$ and $\mathrm{A}_{y}$ can be any of the 20 standard amino acids in the protein (Equation (3)).

Thus, each element in feature vector (Equation (8)) is obtained by

$$
\left\{\begin{array}{c}
\psi_{1}=f\left(\mathrm{~A}_{1}, \mathrm{~A}_{1} \mid 1\right) \\
\psi_{2}=f\left(\mathrm{~A}_{1}, \mathrm{~A}_{2} \mid 1\right) \\
\cdots \\
\psi_{400}=f\left(\mathrm{~A}_{20}, \mathrm{~A}_{20} \mid 1\right),(1 \leq d \leq D) \\
\cdots \\
\psi_{k}=f\left(\mathrm{~A}_{x}, \mathrm{~A}_{y} \mid d\right) \\
\cdots \\
\psi_{\Omega}=f\left(\mathrm{~A}_{20}, \mathrm{~A}_{20} \mid D\right)
\end{array}\right.
$$

\subsubsection{Distance-Bigram Transformation}

DBT $[17,23,24]$ calculate the occurrence frequency of a combination of two amino acids separated by a certain distance along the protein sequence. The distance $d$ is determined by the number of amino acids between the two amino acids of bigram. Some previous studies $[17,23,24]$ have reported that the occurrence frequencies of amino acid pairs can well capture characteristics of proteins and they worked well for protein functionality annotation. To capture the characteristics of DNA-binding proteins, we represented proteins by combining PSFM with distance-bigram transformation, which can transform PSFM into fixed length feature vector.

For a given PSFM (Equation (4)), it can be transferred into a feature vector, as follows:

$$
\mathbf{P}=\left[\psi_{1} \psi_{2} \cdots \psi_{k} \cdots \psi_{\Omega}\right]
$$

where $\Omega$ is an integer to reflect the vector's dimension, its value is determined by $D$ the maximum value of $d$. In order to incorporate the amino acid composition of the 20 standard amino acids in PSFM into the DBT approach, in this method, $d=0$ was taken into account, therefore, $\Omega=400 \times D+400$.

The detail of DBT can be summarized mathematically as in the below equation.

$$
f\left(\mathrm{~A}_{x}, \mathrm{~A}_{y} \mid d\right)=\frac{1}{L-d} \sum_{i=1}^{L-d} P_{i, x} P_{i+d, y}
$$


where $P_{i, x}\left(P_{i+d, y}\right)$ is the element of the PSFM matrix; $f\left(\mathrm{~A}_{x}, \mathrm{~A}_{y} \mid d\right)$ represents the occurrence frequency of a bigram (standard amino acids $\mathrm{A}_{x}$ and $\mathrm{A}_{y}$ separated by a certain distance $d$ ) in evolutionary process.

Accordingly, each element in the feature vector (Equation (11)) is obtained by

$$
\left\{\begin{array}{l}
\psi_{1}=f\left(\mathrm{~A}_{1}, \mathrm{~A}_{1} \mid 0\right) \\
\psi_{2}=f\left(\mathrm{~A}_{1}, \mathrm{~A}_{2} \mid 0\right) \\
\cdots \\
\psi_{400}=f\left(\mathrm{~A}_{20}, \mathrm{~A}_{20} \mid 0\right),(0 \leq d \leq D) \\
\cdots \\
\psi_{k}=f\left(\mathrm{~A}_{x}, \mathrm{~A}_{y} \mid d\right) \\
\cdots \\
\psi_{\Omega}=f\left(\mathrm{~A}_{20}, \mathrm{~A}_{20} \mid D\right)
\end{array}\right.
$$

\subsubsection{Trigram Transformation}

TT [25] is able to consider the local and global sequence-order effects by considering the trigrams along the protein sequences, the resulting feature vectors can be represented as:

$$
\mathbf{P}=\left[\psi_{1} \psi_{2} \cdots \psi_{k} \cdots \psi_{8000}\right]
$$

This technique can be summarized mathematically as shown in the below equation.

$$
f\left(\mathrm{~A}_{x}, \mathrm{~A}_{y}, \mathrm{~A}_{z}\right)=\sum_{i=1}^{L-2} P_{i, x} P_{i+1, y} P_{i+2, z}
$$

where $P_{i, x}, P_{i+1, y}$ and $P_{i+2, z}$ represent the corresponding elements in PSFM (Equation (4)); $\mathrm{A}_{x}, \mathrm{~A}_{y}$ and $\mathrm{A}_{z}$ can be any of the 20 standard amino acids in the protein (Equation (3)); $f\left(\mathrm{~A}_{x}, \mathrm{~A}_{y}, \mathrm{~A}_{z}\right)$ represents the occurrence frequency of trigram $\left(\mathrm{A}_{x} \mathrm{~A}_{y} \mathrm{~A}_{z}\right)$ in evolutionary process.

Accordingly, each element in the feature vector (Equation (14)) is obtained by

$$
\left\{\begin{array}{c}
\psi_{1}=f\left(\mathrm{~A}_{1}, \mathrm{~A}_{1}, \mathrm{~A}_{1}\right) \\
\psi_{2}=f\left(\mathrm{~A}_{1}, \mathrm{~A}_{1}, \mathrm{~A}_{2}\right) \\
\cdots \\
\psi_{k}=f\left(\mathrm{~A}_{x}, \mathrm{~A}_{y}, \mathrm{~A}_{z}\right) \quad,(x, y, x=1,2, \cdots, 20) \\
\cdots \\
\psi_{8000}=f\left(\mathrm{~A}_{20}, \mathrm{~A}_{20}, \mathrm{~A}_{20}\right)
\end{array}\right.
$$

\subsection{Support Vector Machine}

SVM is a machine learning algorithm based on the structural-risk minimization principle of statistical learning theory. It was first presented by Vapnik [45] and has been widely used in bioinformatics. SVM is not only suitable for linear data, but also suitable for non-linear data. For linear data, SVM seek for an optimal hyper-plane to maximize the separation boundary between the positive instance and the negative instance, thereby separating the two instances. The nearest two points to the hyper-plane are called support vectors. For a non-linear model, SVM uses a non-linear transformation to map the input feature space to a high dimensional feature space where the samples can be well separated by an optimal hyper-plane. Kernel function is the most vital part for SVM; it determines the final performance of the SVM algorithm. There are some commonly used kernel functions for SVM, including Linear Function, Polynomial Function, Gaussian Function, Laplacian Function, Sigmoid Function and Radial Basis Function (RBF). SVM also can be used in the hierarchical classification [46]. Ensemble SVM may improve performance, too [47-49]. In the current study, an available SVM algorithm package called LIBSVM [50] was used to implement SVM algorithm, in which the RBF was 
chosen as the kernel function and the two parameters $c$ and $g$ were optimized by 5 -fold cross validation on the benchmark.

\subsection{Evaluation of Performance}

In the current study, three commonly used methods were used to evaluate the performance of the proposed methods, including $k$-fold cross-validation, jackknife test and independent test. Moreover, sensitivity (SN), specificity (SP), accuracy (ACC), Matthew's correlation coefficient (MCC), the Receiver Operating Characteristic (ROC) curve [51] and the area under ROC curve (AUC) were selected as evaluation criteria. These criteria have been widely used in various studies for biological sequence annotation. They can be mathematically defined as follows:

$$
\left\{\begin{array}{l}
\mathrm{SN}=\frac{\mathrm{TP}}{\mathrm{TP}+\mathrm{HN}} \\
\mathrm{SP}=\frac{\mathrm{TN}}{\mathrm{TN}+\mathrm{FP}} \\
\mathrm{ACC}=\mathrm{TP}+\mathrm{TP}+\mathrm{TN} \\
\mathrm{MCC}=\frac{\mathrm{TN}}{\sqrt{(\mathrm{TP}+\mathrm{FN}) \times(\mathrm{TP}+\mathrm{FP}) \times(\mathrm{TN}+\mathrm{FP}) \times(\mathrm{TN}+\mathrm{FN})}}
\end{array}\right.
$$

where TP is the number of true positive samples; TN is the number of true negative samples; FP is the number of false positive samples; and FN is the number of false negative samples. SN denote percentage of true positive samples among positive samples and SP denote percentage of true negative samples among negative samples. ACC represent the percentage of the samples which were correctly predicted among all samples. MCC explains the reliability of models, and its values range from -1 to 1 , when $\mathrm{MCC}=-1$ if all predictions are incorrect and when $\mathrm{MCC}=1$ if all predictions are correct. For MCC $=0$, the prediction is no better than random. The ROC curve is a plot which is usually used to evaluate the performance of predictors. The AUC is the area under ROC curve which gives a measure of the quality of binary classification methods; the larger AUC, the better the predictive quality is.

\section{Conclusions}

To further improve the prediction accuracy and understand the binding regular patterns of DNA binding proteins, we explored and compared the performance of four feature extraction methods, including Residue Probing Transformation (RPT), Evolutionary Difference Transformation (EDT), Distance-Bigram Transformation (DBT), and Trigram Transformation (TT). Experimental results showed that PSFM-DBT achieved the best performance, and outperformed other existing methods in this field. This method was further evaluated on an independent dataset. Furthermore, some interesting patterns were discovered by analyzing the features generated PSFM-DBT, fully consistent with previous studies. Finally, a web server of the proposed PSFM-DBT predictor was established in order to help the users to use this method, which would be a useful tool for protein sequence analysis, especially for studying the structure and function of proteins. Future studies will focus on exploring advanced machine learning techniques to improve the performance of DNA binding protein prediction [52,53].

Supplementary Materials: Supplementary materials can be found at www.mdpi.com/1422-0067/18/9/1856/s1 . The benchmark dataset PDB1075 contains 525 DNA-binding proteins (positive samples) and 550 non-DNA-binding proteins (negative samples) (See Equation (2)), which is available at http:/ / bioinformatics.hitsz.edu.cn/PSFM$\mathrm{DBT} /$ data/.

Acknowledgments: This work was supported by the National Natural Science Foundation of China (No. 61672184), the Natural Science Foundation of Guangdong Province (2014A030313695), Guangdong Natural Science Funds for Distinguished Young Scholars (2016A030306008), Scientific Research Foundation in Shenzhen (Grant No. JCYJ20150626110425228, JCYJ20170307152201596), and Guangdong Special Support Program of Technology Young talents (2016TQ03X618).

Author Contributions: Bin Liu conceived and designed the experiments; Jun Zhang performed the experiments; Bin Liu analyzed the data; Jun Zhang wrote the paper.

Conflicts of Interest: The authors declare no conflict of interest. 


\section{References}

1. Lou, W.; Wang, X.; Chen, F.; Chen, Y.; Jiang, B.; Zhang, H. Sequence Based Prediction of DNA-Binding Proteins Based on Hybrid Feature Selection Using Random Forest and Gaussian Naive Bayes. PLoS ONE 2014, 9, e86703. [CrossRef] [PubMed]

2. Zhao, H.; Yang, Y.; Zhou, Y. Structure-based prediction of DNA-binding proteins by structural alignment and a volume-fraction corrected DFIRE-based energy function. Bioinforma 2010, 26, 1857-1863. [CrossRef] [PubMed]

3. Zhang, L.; Zhao, X.; Kong, L. Predict protein structural class for low-similarity sequences by evolutionary difference information into the general form of Chou's pseudo amino acid composition. J. Theor. Biol. 2014, 355, 105-110. [CrossRef] [PubMed]

4. Yu, X.; Cao, J.; Cai, Y.; Shi, T.; Li, Y. Predicting rRNA-, RNA-, and DNA-binding proteins from primary structure with support vector machines. J. Theor. Biol. 2006, 240, 175-184. [CrossRef] [PubMed]

5. Xia, J.; Zhao, X.; Huang, D. Predicting protein-protein interactions from protein sequences using meta predictor. Amino Acids 2010, 39, 1595-1599. [CrossRef] [PubMed]

6. Tjong, H.; Zhou, H. DISPLAR: An accurate method for predicting DNA-binding sites on protein surfaces. Nucleic Acids Res. 2007, 35, 1465-1477. [CrossRef] [PubMed]

7. Stawiski, E.W.; Gregoret, L.M.; Mandelgutfreund, Y. Annotating Nucleic Acid-Binding Function Based on Protein Structure. J. Mol. Biol. 2003, 326, 1065-1079. [CrossRef]

8. Shanahan, H.P.; Garcia, M.A.; Jones, S.; Thornton, J.M. Identifying DNA-binding proteins using structural motifs and the electrostatic potential. Nucleic Acids Res. 2004, 32, 4732-4741. [CrossRef] [PubMed]

9. Nimrod, G.; Schushan, M.; Szilagyi, A.; Leslie, C.; Bental, N. iDBPs: A web server for the identification of DNA binding proteins. Bioinformatics 2010, 26, 692-693. [CrossRef] [PubMed]

10. Song, L.; Li, D.; Zeng, X.; Wu, Y.; Guo, L.; Zou, Q. nDNA-prot: Identification of DNA-binding Proteins Based on Unbalanced Classification. BMC Bioinform. 2014, 15, 298. [CrossRef] [PubMed]

11. Bhardwaj, N.; Langlois, R.; Zhao, G.; Lu, H. Kernel-based machine learning protocol for predicting DNA-binding proteins. Nucleic Acids Res. 2005, 33, 6486-6493. [CrossRef]

12. Cai, Y.; Zhou, G.; Chou, K.-C. Support Vector Machines for Predicting Membrane Protein Types by Using Functional Domain Composition. Biophys. J. 2003, 84, 3257-3263. [CrossRef]

13. Chou, K.C. Prediction of protein cellular attributes using pseudo amino acid composition. Proteins 2001, 43, 246-255. [CrossRef] [PubMed]

14. Kumar, K.K.; Pugalenthi, G.; Suganthan, P.N. DNA-Prot: Identification of DNA binding proteins from protein sequence information using random forest. J. Biomol. Struct. Dyn. 2009, 26, 679-686. [CrossRef] [PubMed]

15. Fang, Y.; Guo, Y.; Feng, Y.; Li, M. Predicting DNA-binding proteins: Approached from Chou's pseudo amino acid composition and other specific sequence features. Amino Acids 2007, 34, 103-109. [CrossRef] [PubMed]

16. Kumar, M.; Gromiha, M.M.; Raghava, G.P. Identification of DNA-binding proteins using support vector machines and evolutionary profiles. BMC Bioinform. 2007, 8, 463. [CrossRef] [PubMed]

17. Liu, B.; Xu, J.; Fan, S.; Xu, R.; Zhou, J.; Wang, X. PseDNA-Pro: DNA-Binding Protein Identification by Combining Chou's PseAAC and Physicochemical Distance Transformation. Mol. Inform. 2015, 34, 8-17. [CrossRef]

18. Wei, L.; Tang, J.; Zou, Q. Local-DPP: An improved DNA-binding protein prediction method by exploring local evolutionary information. Inf. Sci. 2016, 384, 135-144. [CrossRef]

19. Zhang, J.; Gao, B.; Chai, H.; Ma, Z.; Yang, G. Identification of DNA-binding proteins using multi-features fusion and binary firefly optimization algorithm. BMC Bioinform. 2016, 17, 323. [CrossRef] [PubMed]

20. Waris, M.; Ahmad, K.; Kabir, M.; Hayat, M. Identification of DNA binding proteins using evolutionary profiles position specific scoring matrix. Neurocomputing 2016, 199, 154-162. [CrossRef]

21. Liu, S.; Wang, S.; Ding, H. Protein sub-nuclear location by fusing AAC and PSSM features based on sequence information. In Proceedings of the International Conference on Electronics Information and Emergency Communication, Beijing, China, 14 May 2015.

22. Jeong, J.C.; Lin, X.; Chen, X.-W. On Position-Specific Scoring Matrix for Protein Function Prediction. IEEE/ACM Trans. Comput. Biol. Bioinform. 2011, 8, 308-315. [CrossRef] [PubMed] 
23. Liu, B.; Xu, J.; Lan, X.; Xu, R.; Zhou, J.; Wang, X.; Chou, K.-C. iDNA-Prot I dis: Identifying DNA-Binding Proteins by Incorporating Amino Acid Distance-Pairs and Reduced Alphabet Profile into the General Pseudo Amino Acid Composition. PLoS ONE 2014, 9, e106691. [CrossRef] [PubMed]

24. Saini, H.; Raicar, G.; Lal, S.P.; Dehzangi, A.; Imoto, S.; Sharma, A. Protein Fold Recognition Using Genetic Algorithm Optimized Voting Scheme and Profile Bigram. J. Softw. 2016, 11, 756-767. [CrossRef]

25. Paliwal, K.K.; Sharma, A.; Lyons, J.; Dehzangi, A. A tri-gram based feature extraction technique using linear probabilities of position specific scoring matrix for protein fold recognition. IEEE Trans. Nanobiosci. 2014, 13, 44-50. [CrossRef] [PubMed]

26. Lin, W.; Fang, J.; Xiao, X.; Chou, K.-C. iDNA-Prot: Identification of DNA binding proteins using random forest with grey model. PLoS ONE 2011, 6, e24756. [CrossRef] [PubMed]

27. Liu, B.; Wang, S.; Dong, Q.; Li, S.; Liu, X. Identification of DNA-binding proteins by combining auto-cross covariance transformation and ensemble learning. IEEE Trans. NanoBiosci. 2016, 15, 328-334. [CrossRef] [PubMed]

28. Liu, B.; Wang, S.; Wang, X. DNA binding protein identifcation by combining pseudo amino acid composition and profle-based protein representation. Sci. Rep. 2015, 5, 15497.

29. Altschul, S.F.; Madden, T.L.; Schaffer, A.A.; Zhang, J.; Zhang, Z.; Miller, W.; Lipman, D. J. Gapped BLAST and PSI-BLAST: A new generation of protein database search programs. Nucleic Acids Res. 1997, 25, 3389-3402. [CrossRef] [PubMed]

30. Liu, B.; Zhang, D.; Xu, R.; Xu, J.; Wang, X.; Chen, Q.; Dong, Q.; Chou, K.-C. Combining evolutionary information extracted from frequency profiles with sequence-based kernels for protein remote homology detection. Bioinformatics 2014, 30, 472-479. [CrossRef] [PubMed]

31. Liu, B.; Wang, X.; Lin, L.; Dong, Q.; Wang, X. A Discriminative Method for Protein Remote Homology Detection and Fold Recognition Combining Top-n-grams and Latent Semantic Analysis. BMC Bioinform. 2008, 9, 510. [CrossRef] [PubMed]

32. Mandelgutfreund, Y.; Schueler, O.; Margalit, H. Comprehensive Analysis of Hydrogen Bonds in Regulatory Protein DNA-Complexes: In Search of Common Principles. J. Mol. Biol. 1995, 253, 370-382. [CrossRef] [PubMed]

33. Jones, S.; Van Heyningen, P.; Berman, H.M.; Thornton, J.M. Protein-DNA interactions: A structural analysis. J. Mol. Biol. 1999, 287, 877-896. [CrossRef] [PubMed]

34. Tanaka, Y.; Nureki, O.; Kurumizaka, H.; Fukai, S.; Kawaguchi, S.; Ikuta, M.; Iwahara, J.; Okazaki, T.; Yokoyama, S. Crystal structure of the CENP-B protein-DNA complex: The DNA-binding domains of CENP-B induce kinks in the CENP-B box DNA. EMBO J. 2001, 20, 6612-6618. [CrossRef] [PubMed]

35. Szabóová, A.; Kuželka, O.; Železný, F.; Tolar, J. Prediction of DNA-binding propensity of proteins by the ball-histogram method using automatic template search. BMC Bioinform. 2012, 13, 1-11. [CrossRef] [PubMed]

36. Konig, P.; Giraldo, R.; Chapman, L.; Rhodes, D. The crystal structure of the DNA-binding domain of yeast RAP1 in complex with telomeric DNA. Cell 1996, 85, 125. [CrossRef]

37. Wang, G.; Dunbrack, R.L. PISCES: Recent improvements to a PDB sequence culling server. Nucleic Acids Res. 2005, 33, W94-W98. [CrossRef] [PubMed]

38. Liu, B.; Liu, F.; Fang, L.; Wang, X.; Chou, K.-C. repRNA: A web server for generating various feature vectors of RNA sequences. Mol. Genet. Genom. 2016, 291, 473-481. [CrossRef]

39. Zhu, L.; Deng, S.-P.; Huang, D.-S. A Two-Stage Geometric Method for Pruning Unreliable Links in Protein-Protein Networks. IEEE Trans. Nanobiosci. 2015, 14, 528-534.

40. Deng, S.-P.; Huang, D.-S. SFAPS: An R package for structure/function analysis of protein sequences based on informational spectrum method. Methods 2014, 69, 207-212. [CrossRef] [PubMed]

41. Zhao, Z.-Q.; Huang, D.-S.; Sun, B.-Y. Human face recognition based on multi-features using neural networks committee. Pattern Recognit. Lett. 2004, 25, 1351-1358. [CrossRef]

42. Liu, B.; Wang, X.; Chen, Q.; Dong, Q.; Lan, X. Using Amino Acid Physicochemical Distance Transformation for Fast Protein Remote Homology Detection. PLoS ONE 2012, 7, e46633. [CrossRef] [PubMed]

43. Wang, B.; Chen, P.; Huang, D.-S.; Li, J.-J.; Lok, T.-M.; Lyu, M.R. Predicting protein interaction sites from residue spatial sequence profile and evolution rate. FEBS Lett. 2006, 580, 380-384. [CrossRef]

44. Holm, L.; Sander, C. Removing near-neighbour redundancy from large protein sequence collections. Bioinformatics 1998, 14, 423-429. [CrossRef] [PubMed] 
45. Cortes, C.; Vapnik, V. Support-Vector Networks. Mach. Learn. 1995, 20, 273-297. [CrossRef]

46. Li, D.; Ju, Y.; Zou, Q. Protein Folds Prediction with Hierarchical Structured SVM. Curr. Proteom. 2016, 13, 79-85. [CrossRef]

47. Chen, W.; Xing, P.; Zou, Q. Detecting N6-methyladenosine sites from RNA transcriptomes using ensemble Support Vector Machines. Sci. Rep. 2017, 7, 40242. [CrossRef] [PubMed]

48. Zou, Q.; Guo, J.; Ju, Y.; Wu, M.; Zeng, X.; Hong, Z. Improving tRNAscan-SE annotation results via ensemble classifiers. Mol. Inform. 2015, 34, 761-770. [CrossRef]

49. Zhu, L.; You, Z.-H.; Huang, D.-S. Increasing the reliability of protein-protein interaction networks via non-convex semantic embedding. Neurocomputing 2013, 121, 99-107. [CrossRef]

50. Chang, C.; Lin, C. LIBSVM: A library for support vector machines. ACM Trans. Intell. Syst. Technol. 2011, 2, 27. [CrossRef]

51. Sonego, P.; Kocsor, A.; Pongor, S. ROC analysis: Applications to the classification of biological sequences and 3D structures. Brief. Bioinform. 2008, 9, 198-209. [CrossRef] [PubMed]

52. Huang, D.-S. Radial basis probabilistic neural networks: Model and application. Int. J. Pattern Recognit. Artif. Int. 1999, 13, 1083-1101. [CrossRef]

53. Huang, D.S.; Du, J.-X. A constructive hybrid structure optimization methodology for radial basis probabilistic neural networks. IEEE Trans. Neural Netw. 2008, 19, 2099-2115. [CrossRef]

(c) 2017 by the authors. Licensee MDPI, Basel, Switzerland. This article is an open access article distributed under the terms and conditions of the Creative Commons Attribution (CC BY) license (http:/ / creativecommons.org/licenses/by/4.0/). 


\title{
CytoCluster: A Cytoscape Plugin for Cluster Analysis and Visualization of Biological Networks
}

\author{
Min $\mathrm{Li}^{1}$, Dongyan $\mathrm{Li}^{2}$, Yu Tang ${ }^{1}$, Fangxiang $\mathrm{Wu}^{1,3}$ and Jianxin Wang ${ }^{1, *}$ \\ 1 School of Information Science and Engineering, Central South University, Changsha 410083, China; \\ limin@csu.edu.cn (M.L.); tangyu@csu.edu.cn (Y.T.); faw341@mail.usask.ca (F.X.W.) \\ 2 School of software, Central South University, Changsha 410083, China; dongyanli@csu.edu.cn \\ 3 Department of Mechanical Engineering and Division of Biomedical Engineering, \\ University of Saskatchewan, Saskatoon, SK S7N 5A9, Canada \\ * Correspondence: jxwang@mail.csu.edu.cn; Tel.: +86-731-888-30212
}

Received: 7 August 2017; Accepted: 23 August 2017; Published: 31 August 2017

\begin{abstract}
Nowadays, cluster analysis of biological networks has become one of the most important approaches to identifying functional modules as well as predicting protein complexes and network biomarkers. Furthermore, the visualization of clustering results is crucial to display the structure of biological networks. Here we present CytoCluster, a cytoscape plugin integrating six clustering algorithms, HC-PIN (Hierarchical Clustering algorithm in Protein Interaction Networks), OH-PIN (identifying Overlapping and Hierarchical modules in Protein Interaction Networks), IPCA (Identifying Protein Complex Algorithm), ClusterONE (Clustering with Overlapping Neighborhood Expansion), DCU (Detecting Complexes based on Uncertain graph model), IPC-MCE (Identifying Protein Complexes based on Maximal Complex Extension), and BinGO (the Biological networks Gene Ontology) function. Users can select different clustering algorithms according to their requirements. The main function of these six clustering algorithms is to detect protein complexes or functional modules. In addition, BinGO is used to determine which Gene Ontology (GO) categories are statistically overrepresented in a set of genes or a subgraph of a biological network. CytoCluster can be easily expanded, so that more clustering algorithms and functions can be added to this plugin. Since it was created in July 2013, CytoCluster has been downloaded more than 9700 times in the Cytoscape App store and has already been applied to the analysis of different biological networks. CytoCluster is available from http://apps.cytoscape.org/apps/cytocluster.
\end{abstract}

Keywords: biological networks; cluster analysis; cytoscape; visualization

\section{Introduction}

In recent years, people have paid more and more attention to recognizing life activities within a cell by protein interactions and protein complexes [1-3] in the field of systems biology. Proteins are one of the most important biological molecules in a cell. Within a cell, a protein cannot work alone, but rather works together with other proteins to perform cellular functions. Proteins are involved in a life process through protein complexes. Protein complexes can help us to understand certain biological processes and to predict the functions of proteins. Also, they can realize the cell signaling regulation functions by allosteric, competitive binding, interaction, and post-translational modification [4]. Protein-protein interaction (PPI) networks are powerful models that represent the pairwise protein interactions of organisms. Clustering PPI networks can be useful for isolating groups of interacting proteins that participate in the same biological processes or that, together, perform specific biological functions.

Up to now, many clustering algorithms, which are used to predict protein complexes from proteomics data, have been proposed and applied to biological networks. Out of these methods, 
the graph-based approaches are the most popular, which includes the partition-based clustering method, the density-based clustering method, the hierarchical-based clustering method and the spectral-based clustering method.

The partition-based clustering algorithms detect protein complexes by finding an optimal network partition, and making sure that the divided objects in the same cluster are as close as possible and the objects in different clusters are as far away as possible, such as HCS (Highly Connected Subgraph) [5], RNSC (Restricted Neighborhood Search Clustering) [6], MSCF (Minimal Seed Cover for Finding protein complexes) [7]. These partition-based clustering algorithms need to know the partition number, which is albeit generally unknown to us. What is more, partition-based methods cannot predict overlapping clusters.

The density-based clustering algorithms identify protein complexes by mining dense subgraphs from biological networks, such as MCL (Markov CLuster) [8], MCODE (Molecular COmplex DEtection) [9], CPM (Clique Percolation Method) [10], LCMA (Local Clique Merging Algorithm) [11], Dpclus (Density-periphery based clustering) [12], IPCA (Identifying Protein Complex Algorithm) [13], CMC (Clustering based on Maximal Cliques) [14], MCL-Caw (a refinement of MCL for detecting yeast complexes) [15], ClusterONE (Clustering with Overlapping Neighborhood Expansion) [16], and so on. These clustering algorithms have the advantage of recognizing dense subgraphs. However, it is difficult to predict the clusters which are non-dense subgraphs with these methods, such as the subgraph of "star" and "cycle."

The basic idea of the hierarchical clustering method is measuring the possibility that any two proteins are located in the same cluster according to their similarity or the distance between them. Hierarchical clustering methods can be further divided into divisive methods and agglomerative methods. A divisive method is a top-down approach, whose main action regards the total PPI network as a cluster first, then divides the network according to a rule until all nodes belong to different clusters. An agglomerative method is a bottom-up approach, whose main action regards each protein in the PPI network as a cluster first, then merges any two clusters according to their similarity value until all nodes are assigned to clusters. For example, G-N (Girvan-Newman) [17], MoNet (Modular organization of protein interaction Networks) [18], FAG-EC (Fast AGglomerate algorithm for mining functional modules based on the Edge Clustering coefficients) [19], EAGLE (agglomerativE hierarchicAl clusterinG based on maximaL cliquE) [20], HC-PIN (Hierarchical Clustering algorithm in Protein Interaction Networks) [21] are all hierarchical clustering algorithms. Hierarchical clustering methods can be used for mining arbitrary shape clusters, and can render the hierarchical organization of the entire PPI network based on a tree structure. However, this type of method is very sensitive to noise data and cannot obtain overlapping clusters. Some researchers extend the hierarchical clustering method to detect overlapping clusters by initializing a triangle with three interacting proteins instead of a single protein, such as OH-PIN (identifying Overlapping and Hierarchical modules in Protein Interaction Networks) [22].

The spectral-based clustering algorithms predict protein complexes based on the spectrum theory, such as QCUT (Combines spectral graph partitioning and a local search to optimize the modularity Q) [23], ADMSC (Adjustable Diffusion Matrix-based Spectral Clustering) [24], and SSCC (Semi-Supervised Consensus Clustering) [25]. These spectral-based clustering methods can be a simple and fast approach to a certain extent. These clustering algorithms depend on the feature vector, which determines the final clustering results. In addition, many other kinds clustering algorithms can be found in survey papers [26,27].

With the developments of clustering methods, the visualization of clusters becomes more and more important. Several tools [28-33] have been developed to help researchers to better recognize positive protein complexes. Cytoscape [34] is a friendly and open bioinformatics platform, which shows an exceptional performance both in virtualizations and manipulation of biological networks. Cytoscape also has the advantage of formidable extensibility of integrating a vast amount of plugins with diverse functions over other platforms. There are 33 apps concerning clustering based on Cytoscape described 
in our supplement, many of which aim to find meaningful pathways, or visualize networks by semantic similarities, or construct dynamic networks. Among all of the apps, there are several apps, such as ClusterViz [35], clusterMake [36], and ClusterONE [16], which are used to detect and visualize protein complexes in PPI networks. They are all useful tools with different clustering methods, which have been used in different areas of life sciences in recent years. However, a great deal of newly developed clustering algorithms has lost favor with the Cytoscape platform and do not implement visualization. Also, several plugins with old versions cannot work on the new Cytoscape platform any more. In order to solve the above limitations, we developed a new plugin named CytoCluster, which integrates six new clustering algorithms in total. In our plugin, five new approaches named IPCA, OH-PIN, HC-PIN, DCU (Detecting Complexes based on Uncertain graph model) [37], IPC-MCE (Identifying Protein Complexes based on Maximal Complex Extension) [38] were added, which are not integrated in any existing apps, but are important methods used to predict protein complexes. Our CytoCluster plugin also contains the BinGO function, which is used to determine which Gene Ontology (GO) categories are statistically overrepresented in a set of genes or a subgraph of a biological network. So, our app becomes a versatile tool that offers such comprehensive clustering algorithms, in addition to the BinGO function for biological networks.

\section{Architecture}

In this paper, we adopt Cytoscape 3.x to develop our app. Cytoscape 3.x has notable advantages over Cytoscape 2.x, which can be described in the following two aspects. First, the platform of Cytoscape 3.x adopts the OSGI (Open Service Gateway Initiative) framework, which allows developers to dynamically install, load, update, unload, and uninstall the newly developed bundles in an easy way. Second, Cytoscape 3.x employs Maven, which can help developers manage many jar files. In Cytoscape 3.x, both core modules and apps are called OSGI bundles, and they can significantly reduce complexity in app development to some extent. Also, two methods can be used for developing apps in Cytoscape 3.x. The first way is to develop apps as bundles, which can both register a service in the OSGI framework and withdraw its service from the registry. The second way is to implement the apps with Simplified CyApp API (Application Programming Interface), just like in Cytoscape 2.x.

The architecture of CytoCluster is shown in Figure 1, which includes three main bundles: the interface of CytoCluster bundles, the cluster algorithm bundles, and the visualization, BinGO, and export bundles. The interface of CytoCluster bundles is made up of a graphic user interface and a data exchange system, which allows the users to obtain different forms of bioinformatics networks including .txt and .csv files, and send the clustering results to Cytoscape. The six clustering algorithms bundles play an important role in our plugin CytoCluster, and we have defined the abstract Java class named clustering algorithms, making it is easy for us to integrate more clustering algorithms in CytoCluster. The BinGO bundles are the core functionality in analyzing the GO terms, which can be used to determine which GO categories are statistically overrepresented in a set of genes or a subgraph of a biological network. The visualization of BinGO and export bundles provide a way to intuitively visualize the clustering results in Cytoscape, determine which GO categories are statistically overrepresented, and export the clustering results to .txt or .cvs files. 


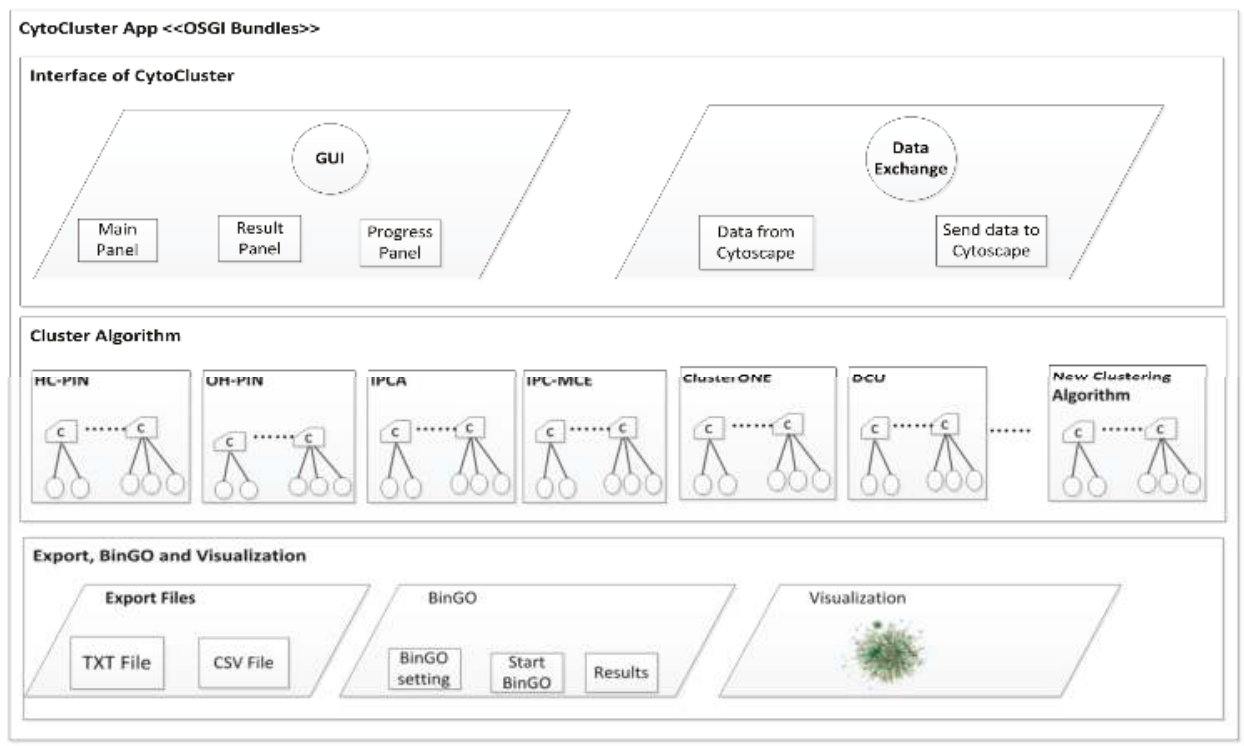

Figure 1. Architecture of CytoCluster.

\section{Implementation}

A user-friendly clustering software system to detect clusters is very important for biologists. By running the software, users can easily detect and analyze the protein complexes participating in the different life activities. Based on this basic idea, we developed our plugin CytoCluster by adopting the OSGI framework and the Cytoscape Maven archetypes. These frameworks and archetypes can create a maven-based project that builds an initial OSGI bundle-based Cytoscape app. The design is guided by the following three goals: first, to extend new clustering algorithms and add more functions; second, to dispatch the interface of CytoCluster and the algorithms; third, to respond quickly when the user operates the GUI (Graphical User Interface).

CyActivator class is an abstract class, which plays an important role in connecting Cytoscape with CytoCluster. All of the functions of CyActivator start to work as soon as you install the CytoCluster.jar for Cytoscape. The Analyze Action, as one of the service bundles, is the most important function in CytoCluster. Once the network is imported into Cytoscape, then our plugin CytoCluster is able to obtain these data from Cytoscape for further analysis. Two parts can be seen in the main panel. The top part mainly contains the two kinds of the clustering algorithms, overlap clustering algorithms and nonoverlap clustering algorithms. The bottom panel mainly provides six clustering algorithm panels, which are the IPCA panel, HC-PIN panel, OH-PIN panel, DCU panel, ClusterONE panel, and IPC-MCE panel. The user can choose different parameters according to their needs from these clustering algorithm panels. The result panel and the "export to .txt" function must be contained in CytoCluster, which provides an easy way to further analyze the results produced by different clustering algorithms. In addition, the progress panel is included in our app, which is used to visualize the progression of the running clustering algorithms.

Finally, we constructed this CytoCluster app containing four parts: Open, Close, About, and BinGO. Each part has its own function. Six clustering algorithms are included in the Open part. When users want to terminate this app, they should select the Close part. Here, BinGO plays an important role in determining which GO categories are statistically overrepresented in biological networks. Lastly, if you want to learn more information about the app, you cannot miss the About part. 


\subsection{Calculation and Basic Analysis}

When users open the CytoCluster plugin, six clustering algorithms are provided, which are HC-PIN, OH-PIN, IPCA, IPC-MCE, ClusterONE, and DCU. In the following, these six clustering algorithms are briefly described.

\subsubsection{HC-PIN (Hierarchical Clustering Algorithm in Protein Interaction Networks)}

The HC-PIN algorithm [21] is a fast, hierarchical clustering algorithm, which can be used in a weighted graph or an unweighted graph. The main processes can be described as follows. First, all vertices in the PPI network are regarded as singleton clusters. Then, HC-PIN [21] calculates the clustering value of each edge and queues all of the edges into a queue Sq in non-increasing order according to their clustering values. The higher clustering value the edge has, the more likely its two vertices will be in the same module. In the process of adding edges in the queue Sq to cluster, $\lambda$-modules are formed. Finally, $\lambda$-modules can be outputted when the number of its proteins is no less than a threshold $s$.

\subsubsection{OH-PIN (Identifying Overlapping and Hierarchical Modules in Protein Interaction Networks)}

The OH-PIN algorithm [22] is an improved hierarchical clustering method, which can identify overlapping clusters. The basic idea of OH-PIN can be summarized as follows. At the beginning, the cluster set C_set is empty. For each edge in the protein interaction network, its B_Cluster is generated and the B_Cluster is added to the C_set, if B_Cluster is not already included in the C_set, until every B_Cluster is included. Then, OH-PIN [22] merges all highly overlapping cluster pairs in the C_set in terms of the threshold overlapping value. After the above step, OH-PIN assembles all of the clusters in the $C \_$set into $\lambda$-modules by gradually merging the cluster pair with the maximum clustering coefficient.

\subsubsection{IPCA (Identifying Protein Complex Algorithm)}

The IPCA algorithm [13] is a density-based clustering algorithm, which can identify dense subgraphs in protein interaction networks. IPCA has four major sub-algorithms: weighting vertex, selecting weed, extending cluster, and extend-judgment. First, IPCA [13] calculates the weight of each edge by counting the common neighbors of its connected two nodes and computes the weight of each node by summing up the weights of its incident edges. The higher weight one node has, the more likely the node is regarded as the seed. At the beginning, a seed is initialed as a cluster. IPCA extends a cluster by adding vertices recursively from its neighbors in terms of the nodes' priority. Whether a node can be added to a cluster is determined by two conditions: its interaction probability and the shortest path between it and the nodes in the cluster.

\subsubsection{IPC-MCE (Identifying Protein Complexes based on Maximal Complex Extension)}

The IPC-MCE algorithm [38] is a maximal clique-based clustering algorithm. The basic idea of IPC-MCE can be described as follows. First, IPC-MCE removes all the nodes which have only one neighbor. Then IPC-MCE enumerates all the maximal cliques in the remained PPI network and puts them into the set MCS (Maximal Clique Sets). For each neighborhood vertex $v$ of the maximal clique $K$ in set MCS, if $\mathrm{IP}_{\mathrm{vk}}$ is no less than the threshold $t$, the vertex $v$ can be added to the maximal clique $K$. The definition of $\mathrm{IPv}_{\mathrm{k}}$ is as follows:

$$
\mathrm{IP}_{\mathrm{vk}}=\frac{\left|E_{\mathrm{vk}}\right|}{\left|V_{k}\right|}
$$

$E_{\mathrm{VK}}$ is the number of the edges between the vertex $v$ and $K$, and $\left|V_{k}\right|$ is the number of nodes in $K$. Finally, IPC-MCE [38] filters the repeated maximal clique according to a pre-defined overlapping value. 


\subsubsection{ClusterONE (Clustering with Overlapping Neighborhood Expansion)}

The ClusterONE algorithm [16] mainly contains three steps. First, groups are grown by adding or removing vertices with high cohesiveness from selected seed proteins. At the beginning, the protein with the highest degree is regarded as the first seed and grows a cohesive group from it using a greedy procedure. ClusterONE repeats this grown process to form overlapping complexes until there are no proteins remaining in the PPI network. Then ClusterONE merges the highly overlapping pairs of locally optimal cohesive groups according to a pre-defined overlapping score. Finally, ClusterONE outputs protein complexes that contain no less than three proteins or whose density is larger than a given threshold $\partial$ (its default value is 0.8 ).

\subsubsection{DCU (Detecting Complexes Based on Uncertain Graph Model)}

The DCU algorithm [37] is a clustering algorithm, which detects protein complexes based on an uncertain graph model. First, DCU [37] starts from a seed vertex and adds other vertices by using a greedy procedure to form a candidate core with high cohesion and low coupling. Then, DCU uses a core-attachment strategy to add attachments to core sets to form complexes. Specifically, for each protein of a candidate set, if its internal absolute degree is less than its external absolute degree, which consists of neighbors of protein vertices in the candidate set, the protein must be removed from the candidate set. Finally, DCU needs to solve the problem of the repeated protein complexes by controlling their overlapping value. Users can select any kind of clustering algorithms they want in the main panel and input the parameters of the algorithm, which decide the creation of a specific clustering algorithm object in memory. Our CytoCluster plugin also provides the visualization of clustering results after running each of these six clustering algorithms, which can be seen in the result panel in the form of a thumbnail list. They can be sorted by the score, the size, or the modularity. In the result panel, the "Export" button and "Discard Result" button are included. The "Export" button is used for exporting results to a .txt file, including the name of algorithm, the parameters, and the clusters, while the "Discard Result" button is used for closing the result panel. Users can close the visualization of clustering results after running these six clustering algorithms with default parameters. In addition, users can see the visualization of cluster results after running a clustering algorithm. Therefore, CytoCluster is a convenient and fast app to obtain smaller networks from a large network.

\subsection{BinGO}

Here, we integrate the BinGO function to be the part of the CytoCluster. All this is done for the convenience of the users. When they install a cytocluster jar, users can not only choose different clustering algorithms, but also use BinGO. Once the BinGO part is opened, a panel will appear in the center of the computer monitor. Users can make a choice from this setting panel according to their need. The main function of BinGO is to determine the overrepresentation of Gene Ontology (GO) categories in a subgraph of a biological network or a set of genes. Once given a set of genes or a subgraph of a network on the GO hierarchy, BinGO can map the predominant functional themes and output this map in the form of a Cytoscape graph. The BinGO function has the same features as the BiNGO [39] plugin. These features contain graphs or genes list inputs; make and use custom annotations, ontologies, and reference sets; save the extensive results in a tab-delimited text file format; and so on. Selecting the "Start BiNGO" button is required after users have chosen their basic parameters. Then, the visualization of GO can be seen from a chosen network. The result can also be saved in a .bgo, which can be used for further studies.

In the BinGO part, two modes are included for selecting the set of genes to be functionally recommended. One is the default mode, and the other is the flexible mode. In the default mode, nodes can be chosen from a Cytoscape network, either manually or by other plugins. In the flexible mode, nodes can be selected from other sources, for example a set of nodes that are obtained from an experiment and pasted in a text input box. Here, the relevant GO annotations can be retrieved and 
propagated upwards through the GO hierarchy; namely, any genes related to a certain GO category can be predicted explicitly and included in all parental categories. Two statistical tests are also concerned so as to assess the enrichment of a GO term better. The most important characteristic of the BinGO part is its interactive use for molecular interaction networks, such as protein interaction networks. Furthermore, it is very flexible for BinGO to use ontologies and annotations. Both the traditional GO ontologies and the GOSlim ontologies are supported by BinGO. Then, the Cytoscape graph produced by BinGO can be seen, altered, and saved in a variety of ways.

\section{Cases Studies}

CytoCluster integrates different types of clustering algorithms including density-based clustering algorithms, hierarchical clustering algorithms, and maximal clique-based methods. Many researchers have downloaded and used the plugin since CytoCluster was released. So far, CytoCluster has been downloaded more than 9700 times since it was released in July 2013. Several important scientific articles indicated that CytoCluster can help scholars with their studies on the mechanisms of biological networks. There are several generic stages of how to run the clustering algorithms in our CytoCluster plugin, which include installing the CytoCluster app, loading the network, setting the data scope and parameters of clustering algorithms, running the cluster algorithm, and receiving or exporting the information of clustering results. The "CytoCluster" menu appears in the "App" menu, after installing the CytoCluster app. In this paper, we present a case to illustrate the use of our plugin. In addition, more cases on these six clustering algorithms can be seen in Table 1 . 


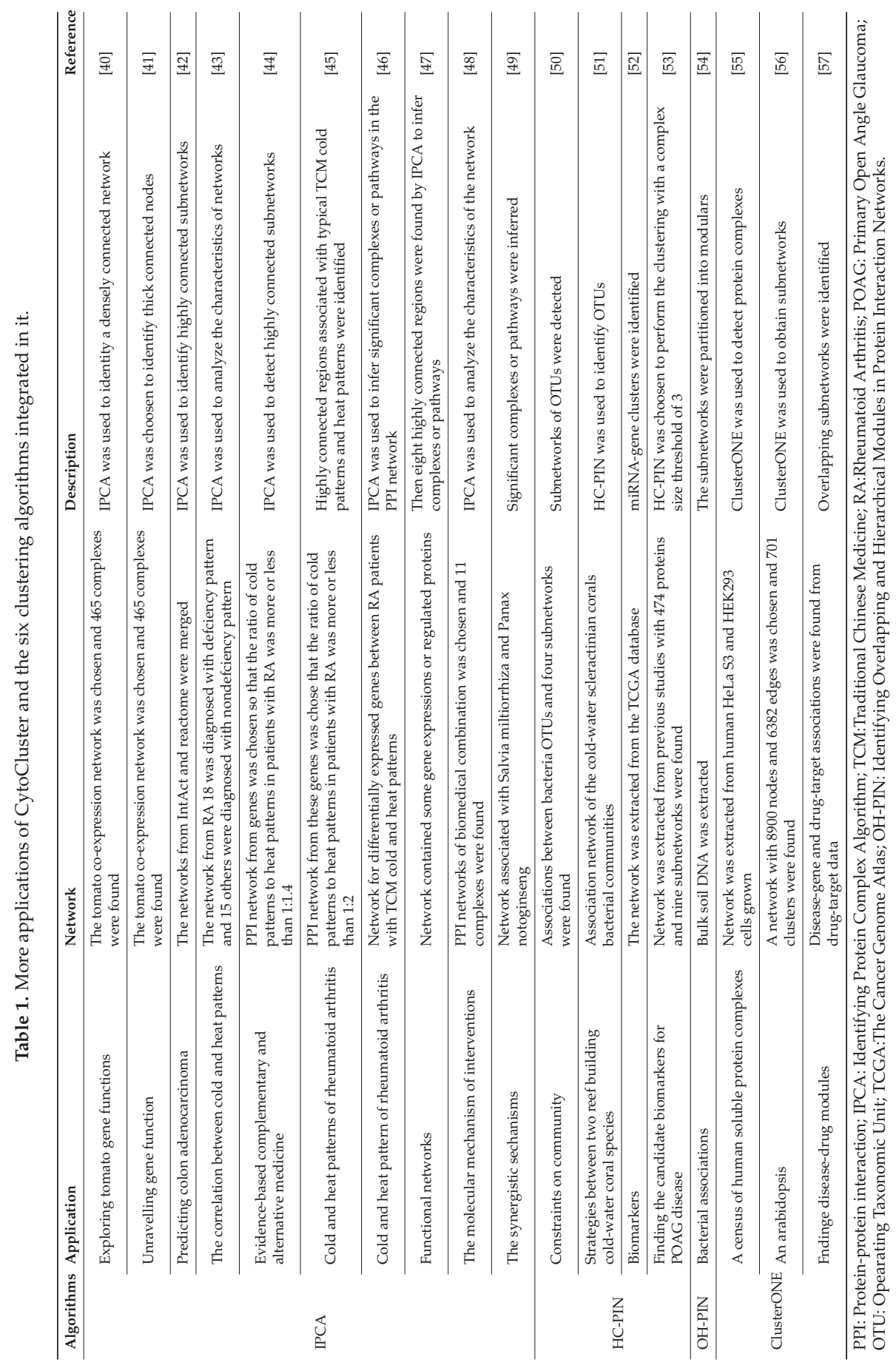


The case of CytoCluster was applied in botany [58]. This paper was published in Plant Physiology by Baute et al. The co-expression network was generated by Cytoscape 3.2.0 [59] according to the nodes and edges $[60,61]$ at first. Then, the newly co-expression network was loaded, which incorporated 185 genes and 943 edges. Third, the main panel of the CytoCluster was opened and the HC-PIN clustering algorithm was chosen with standard settings and a complex size threshold of 10 . In this case, 185 genes and 943 edges were included after dealing with the whole network. The identified subnetworks were further filtered, so as to only include the co-expression networks based on PCCs (Pearson Correlation Coefficients) of 0.7 and higher, as well as protein-protein interactions between query genes based on both experimental and predicted data from CORNET, when the users clicked on the "Analysis" button. Then, four subnetworks were formed after using our plugin for analysis, which can be seen from Figure 2. Each circle in Figure 2 shows a subnetwork. What is more, the generated co-expression network achieved by the HC-PIN algorithm can be seen in the result panel or exported to a .txt, so users can output the results from the different algorithms for further analysis. The table panel can list proprieties of clustering results when users select the corresponding clustering. The progress panel is used to visualize the progression of a specific cluster algorithm.

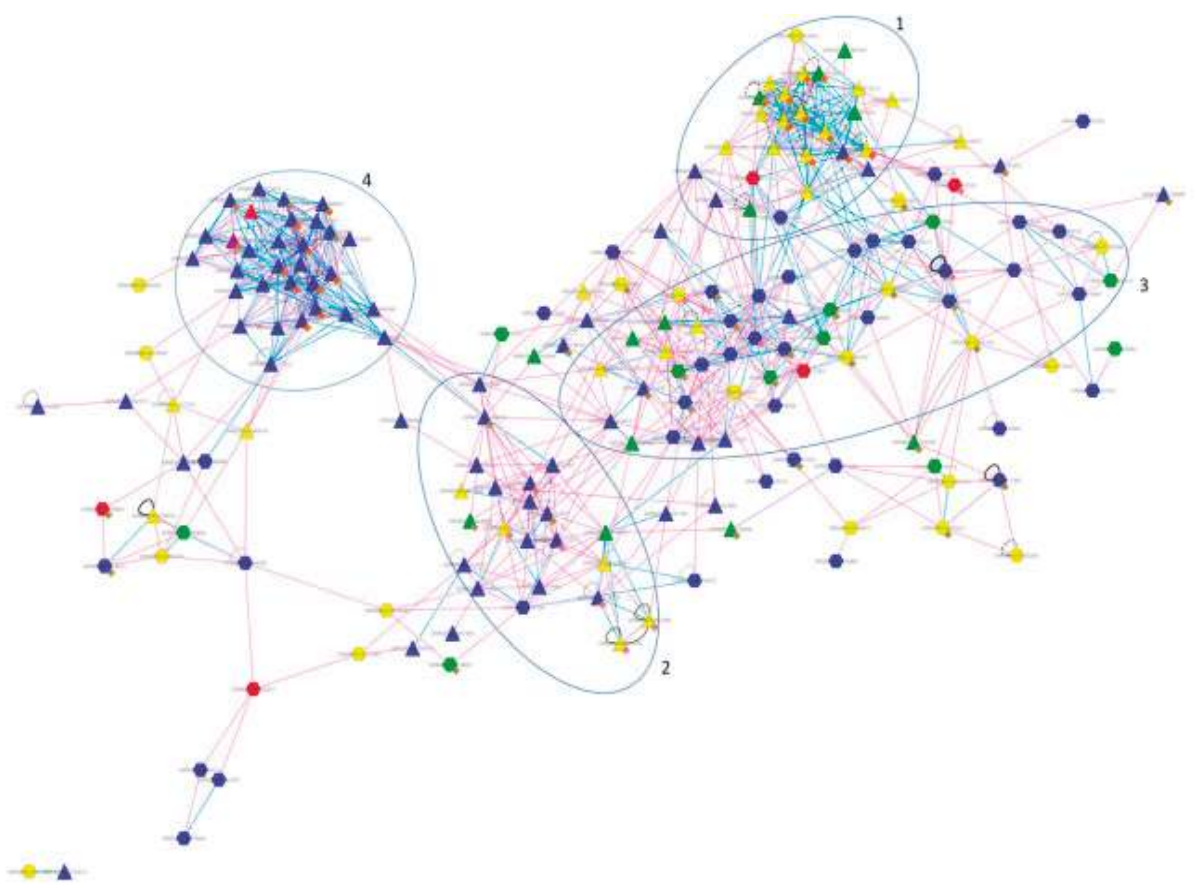

Figure 2. Four subnetworks achieved in the first case [58].

\section{Conclusions}

Our CytoCluster plugin is a platform-independent app for Cytoscape, which is also a functional diversity tool to offer different types of clustering algorithms, including IPCA, DCU, HC-PIN, OH-PIN, IPC-MCE, and ClusterONE. OH-PIN and HC-PIN are both hierarchical-based clustering algorithms, HC-PIN generates non-overlapping clusters, and on the contrary, OH-PIN produces overlapping clusters. IPCA, DCU, IPC-MCE, and ClusterONE are all density-based clustering algorithms, but the clusters generated by them also have some differences. Moreover, the same method will produce different results by changing the values of parameters. Users can both choose different clustering 
algorithms and analyze which GO categories are statistically overrepresented in a set of genes or a subgraph of a biological network. Our CytoCluster plugin is not only convenient for researchers to use, but also renders the investigated biological process easy to understand. Because our app has the advantage of expandability, more clustering algorithms such as those reported in References [62-65] as well as modules can be added to CytoCluster. Owing to such features, we firmly believe our app will be of great help in biology research.

Acknowledgments: This work was supported in part by the National Natural Science Foundation of China under Grants (No. 61622213, No. 61370024 and No. 61420106009).

Author Contributions: Min Li, Dongyan Li and Yu Tang conceived and designed the software, test and experiments; Dongyan Li and Yu Tang implemented the software and performed the experiments; Min Li and Dongyan Li wrote the paper. Min Li, Dongyan Li, Yu Tang, FangXiang Wu and Jianxin Wang revised the manuscript.

Conflicts of Interest: The authors declare no conflict of interest.

\section{References}

1. Wang, J.; Liang, J.; Zheng, W. A graph clustering method for detecting protein complexes. J. Comput. Res. Dev. 2015, 52, 1784-1793.

2. Alberts, B. The cell as a collection of protein machines: Preparing the next generation of molecular biologists. Cell 1998, 92, 291-294. [CrossRef]

3. Lasserre, J.P.; Beyne, E.; Pyndiah, S.; Pyndiah, S.; Lapaillerie, D.; Claverol, S.; Bonneu, M. A complexomic study of Escherichia coli using two-dimensional blue native/SDS polyacrylamide gel electrophoresis. Electrophoresis 2006, 27, 3306-3321. [CrossRef] [PubMed]

4. Gibson, T.J. Cell regulation: Determined to signal discrete cooperation. Trends Biochem. Sci. 2009, 3410, 471-482. [CrossRef] [PubMed]

5. Pržulj, N.; Wigle, D.A.; Jurisica, I. Functional topology in a network of protein interactions. Bioinformatics 2004, 203, 340-348. [CrossRef] [PubMed]

6. King, A.D.; Pržulj, N.; Jurisica, I. Protein complex prediction via cost-based clustering. Bioinformatics 2004, 2017, 3013-3020. [CrossRef] [PubMed]

7. Ding, X.; Wang, W.; Peng, X.; Wang, J. Mining protein complexes from PPI networks using the minimum vertex cut. Tsinghua Sci. Technol. 2012, 176, 674-681. [CrossRef]

8. Enright, A.J.; Dongen, S.V.; Ouzounis, C.A. An efficient algorithm for large-scale detection of protein families. Nucleic Acids Res. 2002, 30, 1575-1584. [CrossRef] [PubMed]

9. Bader, G.D.; Hogue, C.W.V. An automated method for finding molecular complexes in large protein interaction networks. BMC Bioinform. 2003, 41, 2.

10. Palla, G.; Derényi, I.; Farkas, I.; Vicsek, T. Uncovering the overlapping community structure of complex networks in nature and society. Nature 2005, 435, 814-818. [CrossRef] [PubMed]

11. Li, X.L.; Foo, C.S.; Tan, S.H.; Ng, S.K. Interaction graph mining for protein complexes using local clique merging. Genome Inform. 2005, 16, 260-269. [PubMed]

12. Altaf-Ul-Amin, M.; Shinbo, Y.; Mihara, K.; Kurokawa, K.; Kanaya, S. Development and implementation of an algorithm for detection of protein complexes in large interaction networks. BMC Bioinform. 2006, 7, 207. [CrossRef] [PubMed]

13. Li, M.; Chen, J.; Wang, J.; Hu, B.; Chen, G. Modifying the DPClus algorithm for identifying protein complexes based on new topological structures. BMC Bioinform. 2008, 9, 398. [CrossRef] [PubMed]

14. Liu, G.; Wong, L.; Chua, H.N. Complex discovery from weighted PPI networks. Bioinformatics 2009, 25, 1891-1897. [CrossRef] [PubMed]

15. Srihari, S.; Ning, K.; Leong, H.W. MCL-CAw: A refinement of MCL for detecting yeast complexes from weighted PPI networks by incorporating core-attachment structure. BMC Bioinform. 2010, 11, 504. [CrossRef] [PubMed]

16. Nepusz, T.; Yu, H.; Paccanaro, A. Detecting overlapping protein complexes in protein-protein interaction networks. Nat. Methods 2012, 9, 471-472. [CrossRef] [PubMed] 
17. Girvan, M.; Newman, M.E.J. Community structure in social and biological networks. Proc. Natl. Acad. Sci. USA 2002, 99, 7821-7826. [CrossRef] [PubMed]

18. Luo, F.; Yang, Y.; Chen, C.F.; Chang, R.; Zhou, J.; Scheuermann, R.H. Modular organization of protein interaction networks. Bioinformatics 2007, 23, 207-214. [CrossRef] [PubMed]

19. Li, M.; Wang, J.; Chen, J. A fast hierarchical clustering algorithm for functional modules in protein interaction networks. In Proceedings of the IEEE 2008 International Conference on BioMedical Engineering and Informatics (BMEI), Sanya, China, 27-30 May 2008; Volume 1, pp. 3-7.

20. Shen, H.; Cheng, X.; Cai, K.; Hu, M.B. Detect overlapping and hierarchical community structure in networks. Phys. A Stat. Mech. Appl. 2009, 388, 1706-1712. [CrossRef]

21. Wang, J.; Li, M.; Chen, J.; Pan, Y. A fast hierarchical clustering algorithm for functional modules discovery in protein interaction networks. IEEE/ACM Trans. Comput. Biol. Bioinform. 2011, 8, 607-620. [CrossRef] [PubMed]

22. Wang, J.; Ren, J.; Li, M.; Wu, F.X. Identification of hierarchical and overlapping functional modules in PPI networks. IEEE Trans. Nanobiosci. 2012, 11, 386-393. [CrossRef] [PubMed]

23. Chen, D.; Fu, Y.; Shang, M. A fast and efficient heuristic algorithm for detecting community structures in complex networks. Phys. A Stat. Mech. Appl. 2009, 388, 2741-2749. [CrossRef]

24. Inoue, K.; Li, W.; Kurata, H. Diffusion model based spectral clustering for protein-protein interaction networks. PLoS ONE 2010, 5, e12623. [CrossRef] [PubMed]

25. Wang, Y.; Pan, Y. Semi-supervised consensus clustering for gene expression data analysis. BioData Min. 2014, 7, 1-13. [CrossRef] [PubMed]

26. Li, M.; Wu, X.; Wang, J.; Pan, Y. Progress on graph-based clustering methods for the analysis of protein-protein interaction networks. Comput. Eng. Sci. 2012, 34, 124-136.

27. Ji, J.; Liu, Z.; Liu, H.; Liu, C. An overview of research on functional module detection for protein-protein interaction networks. Acta Autom. Sin. 2014, 40, 577-593.

28. Protein-Protein Interaction Networks Co-Clustering. Available online: http://wwwinfo.deis.unical.it/ rombo/co-clustering/ (accessed on 21 April 2017).

29. Batagelj, V.; Mrvar, A. Pajek-program for large network analysis. Connections 1998, 21, 47-57.

30. Adamcsek, B.; Palla, G.; Farkas, I.J.; Derényi, I.; Vicsek, T. CFinder: Locating cliques and overlapping modules in biological networks. Bioinformatics 2006, 22, 1021-1023. [CrossRef] [PubMed]

31. Moschopoulos, C.N.; Pavlopoulos, G.A.; Schneider, R.; Likothanassis, S.D.; Kossida, S. GIBA: A clustering tool for detecting protein complexes. BMC Bioinform. 2009, 10, S11. [CrossRef] [PubMed]

32. Zheng, G.; Xu, Y.; Zhang, X.; Liu, Z.P.; Wang, Z.; Chen, L.; Zhu, X.G. CMIP: A software package capable of reconstructing genome-wide regulatory networks using gene expression data. BMC Bioinform. 2016, 17, 137. [CrossRef] [PubMed]

33. Li, M.; Tang, Y.; Wu, X.; Wang, J.; Wu, F.X.; Pan, Y. C-DEVA: Detection, evaluation, visualization and annotation of clusters from biological networks. Biosystems 2016, 150, 78-86. [CrossRef] [PubMed]

34. Shannon, P.; Markiel, A.; Ozier, O.; Baliga, N.S.; Wang, J.T.; Ramage, D.; Amin, N.; Schwikowski, B.; Ideker, T. Cytoscape: A software environment for integrated models of biomolecular interaction networks. Genome Res. 2003, 13, 2498-2504. [CrossRef] [PubMed]

35. Wang, J.; Zhong, J.; Chen, G.; Li, M.; Wu, F.X.; Pan, Y. ClusterViz: A Cytoscape APP for cluster analysis of biological network. IEEE/ACM Trans. Comput. Biol. Bioinform. 2015, 12, 815-822. [CrossRef] [PubMed]

36. Morris, J.H.; Apeltsin, L.; Newman, A.M.; Baumbach, J.; Wittkop, T.; Su, G.; Bader, G.D.; Ferrin, T.E. clusterMaker: A multi-algorithm clustering plugin for Cytoscape. BMC Bioinform. 2011, 12, 436. [CrossRef] [PubMed]

37. Zhao, B.; Wang, J.; Li, M.; Wu, F.X.; Pan, Y. Detecting protein complexes based on uncertain graph model. IEEE/ACM Trans. Comput. Biol. Bioinform. 2014, 11, 486-497. [CrossRef] [PubMed]

38. Li, M.; Wang, J.X.; Liu, B.B.; Chen, J.E. An algorithm for identifying protein complexes based on maximal clique extension. J. Cent. South Univ. 2010, 41, 560-565.

39. Maere, S.; Heymans, K.; Kuiper, M. BiNGO: A Cytoscape plugin to assess overrepresentation of gene ontology categories in biological networks. Bioinformatics 2005, 21, 3448-3449. [CrossRef] [PubMed]

40. Fukushima, A.; Nishizawa, T.; Hayakumo, M.; Hikosaka, S.; Saito, K.; Goto, E.; Kusano, M. Exploring tomato gene functions based on coexpression modules using graph clustering and differential coexpression approaches. Plant Physiol. 2012, 158, 1487-1502. [CrossRef] [PubMed] 
41. Schaefer, R.J.; Michno, J.M.; Myers, C.L. Unraveling gene function in agricultural species using gene co-expression networks. Biochim. Biophys. Acta (BBA)-Gene Regul. Mech. 2017, 1860, 53-63. [CrossRef] [PubMed]

42. Wang, Y.; Zhang, J.; Li, L.; Xu, X.; Zhang, Y.; Teng, Z.; Wu, F. Identification of molecular targets for Predicting Colon Adenocarcinoma. Med. Sci. Monit. Int. Med. J. Exp. Clin. Res. 2016, 22, 460-468. [CrossRef]

43. Wang, M.; Chen, G.; Lu, C.; Xiao, C.; Li, L.; Niu, X.; He, X.; Jiang, M.; Lu, A. Rheumatoid arthritis with deficiency pattern in traditional Chinese medicine shows correlation with cold and hot patterns in gene expression profiles. Evid.-Based Complement. Altern. Med. 2013, 2013, 248650. [CrossRef] [PubMed]

44. Lu, C.; Niu, X.; Xiao, C.; Chen, G.; Zha, Q.; Guo, H.; Jiang, M.; Lu, A. Network-based gene expression biomarkers for cold and heat patterns of rheumatoid arthritis in traditional Chinese medicine. Evid.-Based Complement. Altern. Med. 2012, 2012, 203043. [CrossRef] [PubMed]

45. Lu, C.; Xiao, C.; Chen, G.; Jiang, M.; Zha, Q.; Yan, X.; Kong, W.; Lu, A. Cold and heat pattern of rheumatoid arthritis in traditional Chinese medicine: Distinct molecular signatures indentified by microarray expression profiles in CD4-positive T cell. Rheumatol. Int. 2012, 32, 61-68. [CrossRef] [PubMed]

46. Chen, G.; Lu, C.; Zha, Q.; Xiao, C.; Xu, S.; Ju, D.; Zhou, Y.; Jia, W.; Lu, A. A network-based analysis of traditional Chinese medicine cold and hot patterns in rheumatoid arthritis. Complement. Ther. Med. 2012, 20, 23-30. [CrossRef] [PubMed]

47. Chen, G.; Liu, B.; Jiang, M.; Tan, Y.; Lu, A.P. Functional networks for Salvia miltiorrhiza and Panax notoginseng in combination explored with text mining and bioinformatical approach. J. Med. Plants Res. 2011, 5, 4030-4040.

48. Jiang, M.; Lu, C.; Chen, G.; Xiao, C.; Zha, Q.; Niu, X.; Chen, S.; Lu, A. Understanding the molecular mechanism of interventions in treating rheumatoid arthritis patients with corresponding traditional Chinese medicine patterns based on bioinformatics approach. Evid.-Based Complement. Altern. Med. 2012, 2012, 129452. [CrossRef] [PubMed]

49. Chen, G.; Liu, B.; Jiang, M.; Aiping, L. System Analysis of the Synergistic Mechanisms between Salvia Miltiorrhiza and Panax Notoginseng in Combination. World Sci. Technol. 2010, 12, 566-570.

50. Kalenitchenko, D.; Fagervold, S.K.; Pruski, A.M.; Vétion, G.; Yücel, M.; Le Bris, N.; Galand, P.E. Temporal and spatial constraints on community assembly during microbial colonization of wood in seawater. ISME J. 2015, 9, 2657-2670. [CrossRef] [PubMed]

51. Meistertzheim, A.L.; Lartaud, F.; Arnaud-Haond, S.; Kalenitchenko, D.; Bessalam, M.; Le Bris, N.; Galand, P.E. Patterns of bacteria-host associations suggest different ecological strategies between two reef building cold-water coral species. Deep Sea Res. Part I Oceanogr. Res. Pap. 2016, 114, 12-22. [CrossRef]

52. Guo, H.; Chen, J.; Meng, F. Identification of novel diagnosis biomarkers for lung adenocarcinoma from the cancer genome atlas. Orig. Artic. 2016, 9, 7908-7918.

53. Atan, N.A.D.; Yekta, R.F.; Nejad, M.R.; Nikzamir, A. Pathway and network analysis in primary open angle glaucoma. J. Paramed. Sci. 2014, 5. [CrossRef]

54. Wang, H.; Wei, Z.; Mei, L.; Gu, J.; Yin, S.; Faust, K.; Raes, J.; Deng, Y.; Wang, Y.; Shen, Q.; Yin, S. Combined use of network inference tools identifies ecologically meaningful bacterial associations in a paddy soil. Soil Biol. Biochem. 2017, 105, 227-235. [CrossRef]

55. Havugimana, P.C.; Hart, G.T.; Nepusz, T.; Yang, H.; Turinsky, A.L.; Li, Z.; Wang, P.I.; Boutz, D.R.; Fong, V.; Phanse, S.; et al. A census of human soluble protein complexes. Cell 2012, 150, 1068. [CrossRef] [PubMed]

56. Van Landeghem, S.; de Bodt, S.; Drebert, Z.J.; Inzé, D.; van de Peer, Y. The potential of text mining in data integration and network biology for plant research: A case study on Arabidopsis. Plant Cell 2013, 25, 794-807. [CrossRef] [PubMed]

57. Wu, C.; Gudivada, R.C.; Aronow, B.J.; Jegga, A.G. Computational drug repositioning through heterogeneous network clustering. BMC Syst. Biol. 2013, 7, S6. [CrossRef] [PubMed]

58. Baute, J.; Herman, D.; Coppens, F.; de Block, J.; Slabbinck, B.; dell'Aqcua, M.; Pè, M.E.; Maere, S.; Nelissen, H.; Inzé, D. Combined large-scale phenotyping and transcriptomics in maize reveals a robust growth regulatory network. Plant Physiol. 2016, 170, 1848-1867. [CrossRef] [PubMed]

59. Czerwinska, U.; Calzone, L.; Barillot, E.; Zinovyev, A. DeDaL: Cytoscape 3 app for producing and morphing data-driven and structure-driven network layouts. BMC Syst. Biol. 2015, 9, 46. [CrossRef] [PubMed] 
60. Kerrien, S.; Aranda, B.; Breuza, L.; Bridge, A.; Broackes-Carter, F.; Chen, C.; Duesbury, M.; Dumousseau, M.; Feuermann, M.; Hinz, U.; et al. The IntAct molecular interaction database in 2012. Nucleic Acids Res. 2012, 40, D841-D846. [CrossRef] [PubMed]

61. Croft, D.; O’Kelly, G.; Wu, G.; Haw, R.; Gillespie, M.; Matthews, L.; Caudy, M.; Garapati, P.; Gopinath, G.; Jassal, B.; et al. Reactome: A database of reactions, pathways and biological processes. Nucleic Acids Res. 2010, 39, D691-D697. [CrossRef] [PubMed]

62. Li, M.; Wang, J.; Chen, J.; Cai, Z.; Chen, G. Identifying the overlapping complexes in protein interaction networks. Int. J. Data Min. Bioinform. 2010, 4, 91-108. [CrossRef] [PubMed]

63. Li, X.; Wang, J.; Zhao, B.; Wu, F.X.; Pan, Y. Identification of protein complexes from multi-relationship protein interaction networks. Hum. Genom. 2016, 10, 17. [CrossRef] [PubMed]

64. Lei, X.; Ding, Y.; Wu, F.X. Detecting protein complexes from DPINs by density based clustering with Pigeon-Inspired Optimization Algorithm. Sci. China Inf. Sci. 2016, 59, 070103. [CrossRef]

65. Zhao, B.; Wang, J.; Li, M.; Li, X.; Li, Y.; Wu, F.X.; Pan, Y. A new method for predicting protein functions from dynamic weighted interactome networks. IEEE Trans. Nanobiosci. 2016, 15, 131-139. [CrossRef] [PubMed]

(C) 2017 by the authors. Licensee MDPI, Basel, Switzerland. This article is an open access article distributed under the terms and conditions of the Creative Commons Attribution (CC BY) license (http:/ / creativecommons.org/licenses/by/4.0/). 
Article

\title{
Protein Complexes Prediction Method Based on Core-Attachment Structure and Functional Annotations
}

\author{
Bo $\mathrm{Li}^{*,+}$ and Bo Liao ${ }^{+}$ \\ College of Computer Science and Electronic Engineering, Hunan University, Changsha 410082, China; \\ dragonbw@163.com \\ * Correspondence: nonegenius@hnu.edu.cn; Tel.: +731-8882-1907 \\ + These authors contributed equally to this work.
}

Received: 25 August 2017; Accepted: 1 September 2017; Published: 6 September 2017

\begin{abstract}
Recent advances in high-throughput laboratory techniques captured large-scale protein-protein interaction (PPI) data, making it possible to create a detailed map of protein interaction networks, and thus enable us to detect protein complexes from these PPI networks. However, most of the current state-of-the-art studies still have some problems, for instance, incapability of identifying overlapping clusters, without considering the inherent organization within protein complexes, and overlooking the biological meaning of complexes. Therefore, we present a novel overlapping protein complexes prediction method based on core-attachment structure and function annotations (CFOCM), which performs in two stages: first, it detects protein complex cores with the maximum value of our defined cluster closeness function, in which the proteins are also closely related to at least one common function. Then it appends attach proteins into these detected cores to form the returned complexes. For performance evaluation, CFOCM and six classical methods have been used to identify protein complexes on three different yeast PPI networks, and three sets of real complexes including the Munich Information Center for Protein Sequences (MIPS), the Saccharomyces Genome Database (SGD) and the Catalogues of Yeast protein Complexes (CYC2008) are selected as benchmark sets, and the results show that CFOCM is indeed effective and robust for achieving the highest F-measure values in all tests.
\end{abstract}

Keywords: protein-protein interaction network; overlapping; clustering

\section{Introduction}

Most proteins in living organisms, performing their biological functions or involving with cellular processes, barely serve as single isolated entities, but rather via molecular interactions with other partners to form complexes [1]. In fact, protein complexes are the key molecular entities to perform cellular functions, such as signal transduction, post-translational modification, DNA transcription, and mRNA translation. Moreover, the damage of protein complexes is one of the main factors inducing severe diseases [2]. Identification of protein complexes, therefore, becomes a fundamental task in better understanding the biological functions in different cellular systems, uncovering regularities of cellular activities and contributing to interpreting the causes, diagnosis, and even the treatments of complex diseases. As a result, lots of techniques including laboratory-based and computational-based have been proposed to address this issue.

Up to now, significant progress in high-throughput laboratory techniques involving Tandem Affinity Purification (TAP) [3] and Mass Spectrometry (MS) [4] has been made to discover protein complexes on a large scale. However, laboratory experiments are expensive and time-consuming, resulting in poor coverage of the complete protein complexes. Fortunately, the genomic-scale 
protein-protein interaction (PPI) networks created from pairwise protein-protein interactions make it possible to automatically and computationally detect protein complexes. Given a PPI network, as the protein complexes are formed by physical aggregations of several binding proteins, they are assumed to be the functionally and structurally cohesive substructures, and thus graph clustering methods have been put forward to search densely connected regions in PPI networks as protein complexes.

Since some proteins have multiple functions, in other words, they may belong to more than one protein complex, so the ideal approaches need to be able to detect overlapping complexes. However, several types of graph clustering methods don't allow overlaps between detected protein complexes due to the confinements of the rationales behind them. For example, the partition-based clustering methods such as the Restricted Neighborhood Search Clustering algorithm (RNSC) [5], the Bayesian Nonnegative Matrix Factorization(NMF)-based weighted Ensemble Clustering algorithm (EC-BNMF) [6], obtain, however, some highly reliable protein complexes, since they need prior knowledge of the exact number of clusters that thus cannot detect overlapping functional modules, and, in addition, most of the hierarchy-based clustering methods [7-9] utilize hierarchical trees to represent the hierarchical module organization for a PPI network, but it is difficult to detect overlapping complexes as well. In addition, although some algorithms are capable of finding overlapping complexes, they still have some distinct shortcomings-for instance, the Molecular Complex Detection (MCODE) [10] predicts only quite a small number of protein complexes. CFinder [11] first discovers k-cliques by using the clique percolation method (CPM) [12], and then combines the adjacent k-cliques to get the functional modules, but may fail to detect some regular complexes. ClusterONE [13] requires one pre-determined parameter, which is depended on the quality of PPI network, and it is difficult to determine.

Furthermore, the aforementioned methods still have a common fatal weakness-ignorance of the inherent organization of the complexes-but actually experimental analysis has already reported that a protein complex generally consists of a core, in which proteins share similar functions and tend to be highly co-expressed, and other attach proteins surrounding to the core [14]. Based on these, several core-attachment based algorithms have been presented, and experimental results indicate that they can acquire better performance compared to traditional methods neglecting inherent organization. Among them, CORE [15] first calculates the probability of each pairwise proteins to be in the same core and then uses it to detect cores. $\mathrm{COACH}$ [16] detects cores from neighborhood graphs of the selected seed proteins, and then applies an outward growing strategy to generate protein complexes. Compared with CORE, $\mathrm{COACH}$ can find overlapping cores. Other methods including [17] predict complexes based on multi-structures in PPI network, and achieve significant performance. The complexes predicted by structure-based methods, in general, have been verified more in accordance with the known complexes.

In addition, to precisely predict more biological explainable complexes, some methods of fusing various types of prior knowledge including functional annotations [18-20], gene expression data [21-23], as well as sub-cellular location of proteins [24], are presented and have already been proved that can help to improve the performance to some extent. However, these kinds of valuable information are either used in data preprocessing or post-processing, such as filtering low-confidence edges, weighting edges, discarding some biological meaningless complexes, but seldom helps mining cores with better biological meaning, in which most proteins are co-subcellular or co-expression or with similar functions. Furthermore, since these data are undeniably incomplete and imprecise, how to generate a impartial and efficient model incorporating different types of data is still a hot topic in complex prediction [25-27].

In summary, we may come to the conclusion that a comparatively well-designed protein complexes identification method may need to meet the following conditions: capable of detecting overlapping complexes, fewer parameters, being easy to be determine, consideration of the inherent organization of protein complexes, particularly finding topological and biological meaningful cores, properly incorporating prior information as much as possible into the predicting model, and robust to PPI networks with false positives and false negatives. Unfortunately, even though many effective 
techniques have been proposed, as far as we know, few of them satisfy most of the above-mentioned requirements, which results in impeding further practical applications, and thus there is still urgent need for new approaches.

In this manuscript, we introduced a novel core-attachment based method to predict protein complexes, and the proteins in our detected cores are closely linked, share high similar topology that is highly connected to internal vertexes and relatively sparsely connected to outsides, and are more biologically significant, namely more likely to participate in one or more biological processes with the appliance of GO functional annotation. Furthermore, the detected complexes can be overlapping. We applied our algorithm to two PPI networks of yeast, and validated our predicted complexes using benchmark complexes collected from several public databases. The experimental results indicated that our algorithm is efficient and outperforms other existing classical methods.

\section{Results}

We have applied our CFOCM method on the Database of Interacting Proteins (DIP) data and Gavin data. In this section, we will first discuss parameter $t$ affecting the performance of CFOCM. Next, we perform comprehensive comparisons with various existing classical methods and analyse the results in detail. Finally, we explore the functional definition of the complex-core as a whole, contributing to the biological significance of the detected complexes.

\subsection{Evaluation Metrics}

The neighborhood affinity score $N S(p, r)$ can also be devoted to measure the overall similarity between a predicted complex $p$ and a real complex $r$, and if $N S(p, r) \geq \omega, p$ and $r$ are considered to be matching. On the one hand, the greater setting value of $\omega$ means the more stringent matching of between the predicted complex and the real complex in the benchmark, probably resulting in a sharp decline in all the prediction measure values; on the other, the smaller value could not only lead to identify the low-confidence predicted complex as the real complex, which is also not reasonable. In our experiments, we set $\omega$ to 0.2 the same as most literatures do $[5,7,11,13,15,28]$, which provides easy and fair comparisons between results of various algorithms.

Let $P$ and $R$ represent the set of predicted complexes and the real complexes in benchmarks, respectively. $N_{c p}=\{p \in P \mid \exists r \in B, \mathrm{NS}(p, r) \geq \omega\}$ denotes the predicted complexes matching at least one real complex, and $N_{c r}=\{r \in R \mid \exists p \in P, \mathrm{NS}(r, p) \geq \omega\}$ denotes the real complexes matching at least one predicted complex. In addition, then the performance of a clustering algorithm can be measured using precision, recall, and F-measure, which can be calculated as follows:

$$
\begin{gathered}
\text { Precison }=\frac{\left|N_{c p}\right|}{|P|}, \\
\text { Recall }=\frac{\left|N_{c r}\right|}{|R|}, \\
\text { F-measure }=2 \times \text { Precison } \times \text { Recall } / \text { Precision }+ \text { Recall }),
\end{gathered}
$$

where Precision means the ratio of predicted protein complexes that are matched with the real complexes, Recall means the rate of real complexes that are successfully detected and F-measure evaluates the overall performance.

\subsection{Optimization of the Parameter $t$}

Recall that the process of mining cores from PPI network in Algorithm 1 of CFOCM employs a user-defined parameter $t$ calculated by $\mathrm{NS}\left(m c_{i}, m c_{j}\right)$ to decide whether a certain candidate core $m c_{j}$ should be merged into the family of the current candidate core $m c_{i}$. In general, CFOCM can predict more complexes with the bigger value of $t$; nevertheless, this may lead to compromise on the quality 
of the predicted complexes, and thus how to choose a relatively appropriate $t$ to achieve a balance between the predicted complexes' quality and quantity needs to be probed. Here, varying $t$ from 0.2 to 0.6 with the interval 0.01 , the F-measure values of each predicted complex set are computed, and help us to intuitively observe that the variation of $t$ affects the performance of our CFOCM method and selects the relatively suitable $t$ as well (see Figure 1).

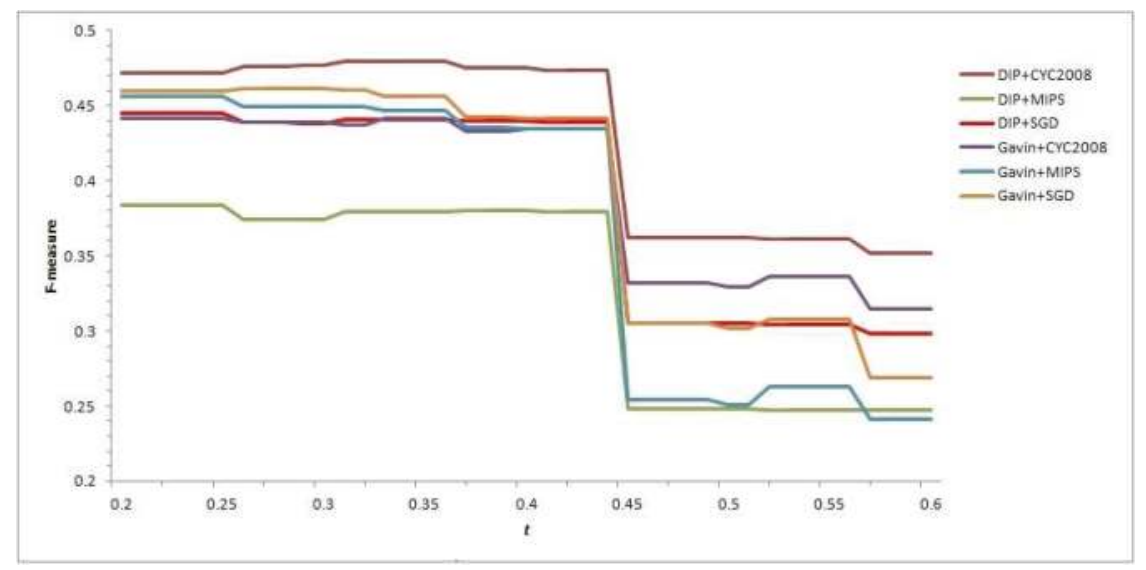

Figure 1. The effect of $t$, showing how the variation of parameter $t$ affects the performance of our proposed overlapping protein complexes prediction method based on core-attachment structure and function annotations (CFOCM) in terms of F-measure.

In Figure 1, all the curves of different CFOCMs, based on DIP data or Gavin data, validated in benchmark set MIPS or SGD or CYC2008, are comparably smooth and steady when the $t$ varies from 0.2 to 0.44 . However, the curves change abruptly near $t=0.45$, and the causation of this phenomenon can be rationally explained with the NS score of two candidate cores being $4 / 9(\approx 0.44)$ in which the number of proteins are both three and two of them are the overlapping; that is to say, these two cores can not be put into the same family if $t$ is larger than $4 / 9$, resulting in a rapid increase of low-confidence detected cores with size 3 and a sharp decease of recall value and F-measure score as well. For example, under $t=0.44$, CFOCM based on DIP and Gavin network generates 751,453 complexes respectively, while under $t=0.45$ generates 2629, 1703 complexes respectively, conforming to the above analysis and interpretation.

As stated above, $t$ should definitely not be set to larger than 0.44 as increasing abundant low-confidence three-size cores, and actually the performance of CFOCM does not change significantly when $t \in[0.2,0.44]$. Still, demand for more complexes shows a preference to a larger $t$; otherwise, if there is demand for a fewer number of complexes, a preference is shown for a smaller $t$. For example, CFOCM predicts 545 complexes with average matching of 156 real complexes in MIPS when $t=0.2$, while predicting 751 complexes matching 205 real complexes in MIPS when $t=0.44$. In the following part, either in DIP data or Gavin data, the $t$ of our CFOCM algorithm is set to 0.4 .

\subsection{Comparison Experiments on Different Datasets}

For performance evaluation, the comparison experiments between CFOCM and six representative algorithms including MCL, MCODE, RNSC, CORE, COACH and ClusterONE are performed on both DIP data , Gavin data and Srihari data. Note that the parameters of these six comparative methods are set to the default values. Figure 2, Table 1, Figure 3, Table 2 ,Figure 4, and Table 3 exhibit the overall comparison results in terms of Precision, Recall and F-measure on DIP data, Gavin data and Srihari data, respectively. 


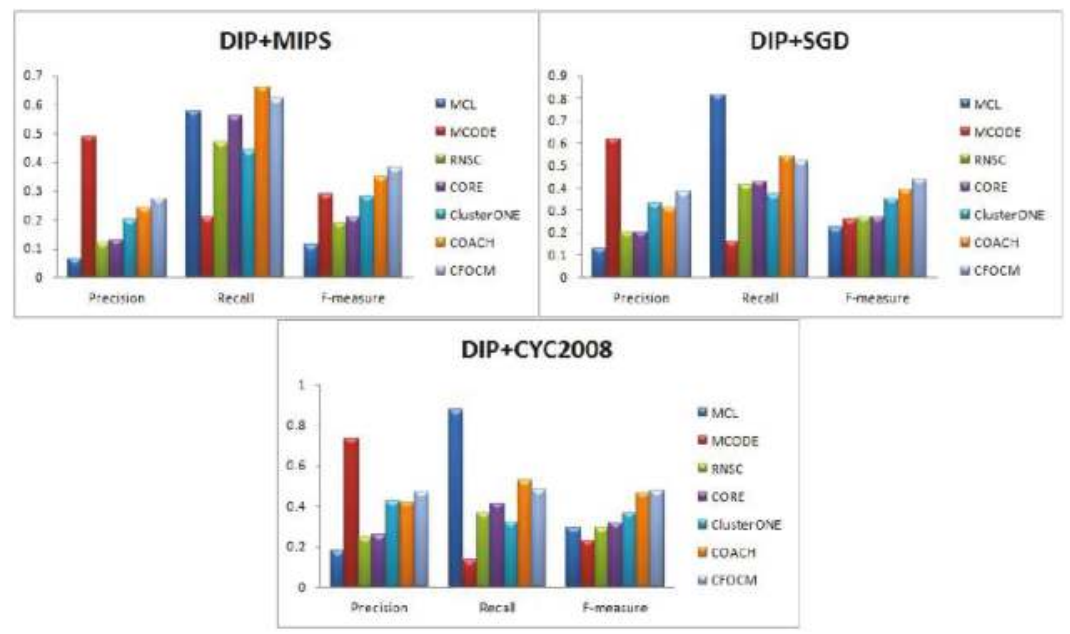

Figure 2. Comparative performance of CFOCM and the other six methods in DIP data using benchmark MIPS, SGD, CYC2008, respectively.

Table 1. Results of various approaches using DIP data.

\begin{tabular}{cccccccc}
\hline Algorithms & MCL & MCODE & RNSC & COER & ClusterONE & COACH & CFOCM \\
\hline \# complexes & 4838 & 63 & 543 & 592 & 341 & 746 & 748 \\
$N_{p}$ (MIPS) & 305 & 31 & 65 & 78 & 69 & 179 & 205 \\
$N_{b}$ (MIPS) & 117 & 42 & 96 & 113 & 89 & 134 & 126 \\
$N_{p}$ (SGD) & 621 & 39 & 106 & 117 & 112 & 231 & 285 \\
$N_{b}$ (SGD) & 262 & 53 & 134 & 138 & 121 & 176 & 168 \\
$N_{p}$ (CYC2008) & 853 & 46 & 134 & 153 & 145 & 311 & 351 \\
$N_{b}$ (CYC2008) & 358 & 55 & 149 & 168 & 132 & 215 & 196 \\
\hline
\end{tabular}

In Figure 2, no matter whether benchmarks MIPS or SGD or CASP2008 are used, MCODE achieves the highest precision that is far beyond other methods. However, since the number of predicted protein complexes is very limited and also matches with fewer real complexes, resulting in much low recall and F-measure values. In addition, CORE, RNSC, and ClusterONE are observed to attain high recall values, but, nevertheless, the F-measure values of them merely end up with relatively lower F-measure value due to their very low precision values. In fact, CFOCM and COACH demonstrate their distinctive competitive advantages in F-measure as a result of balanced precisions and recalls. Moreover, it is obvious that CFOCM remarkably outperforms COACH in F-measure when using benchmark MIPS and SGD. Meanwhile, both CFOCM and COACH are based on core-attach structure, it may indicate that the protein complex detection method seems more appropriate when taking consideration of the inherent organization of complex. As Table 1 shows, CFOCM detects moderate number of complexes, many of which correctly match with the real complexes and have a high coverage rate of real complexes as well.

In order to evaluate the robustness of algorithm CFOCM, comparison experiments are also carried on Gavin network, which is different from the DIP network for containing much fewer and more densely connected proteins. Figure 3 illustrates the results for Gavin data, CFOCM shows even better performance for Gavin data, which achieves the highest precision values when using benchmark MIPS and CYC2008, and, apparently, CFOCM obtains the best F-measure value for every benchmark. This may suggest that CFOCM indeed works on dense network as well. For each method, the total number of identified complexes, the number of correct predictions $N_{p}$ matching at least a real complex, and the 
number of real complexes $N_{b}$ matching at least a predicted one are listed in Table 2, reaching similar conclusions that are consistent with DIP data.

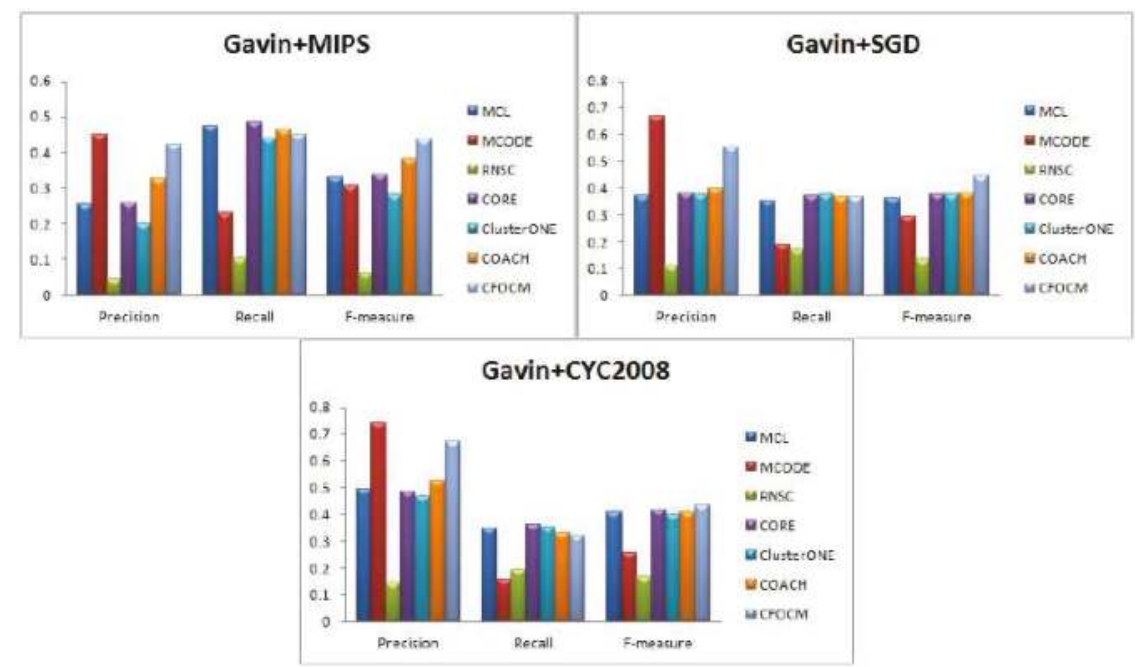

Figure 3. Comparative performance of CFOCM and the other six methods in Gavin data using benchmarks MIPS, SGD, CYC2008, respectively.

Table 2. Results of various approaches using Gavin data.

\begin{tabular}{cccccccc}
\hline Algorithms & MCL & MCODE & RNSC & COER & ClusterONE & COACH & CFOCM \\
\hline \# complexes & 232 & 69 & 476 & 267 & 292 & 326 & 453 \\
$N_{p}$ (MIPS) & 59 & 31 & 22 & 69 & 65 & 106 & 191 \\
$N_{b}$ (MIPS) & 96 & 47 & 21 & 98 & 80 & 94 & 91 \\
$N_{p}$ (SGD) & 86 & 46 & 53 & 101 & 109 & 130 & 250 \\
$N_{b}$ (SGD) & 114 & 61 & 55 & 120 & 121 & 118 & 119 \\
$N_{p}$ (CYC2008) & 115 & 51 & 68 & 130 & 136 & 171 & 305 \\
$N_{b}$ (CYC2008) & 142 & 63 & 79 & 148 & 143 & 135 & 131 \\
\hline
\end{tabular}

For further evaluation, Srihari data derived from three different repositories are also used for comparison, and the results are showed in Figure 4 and Table 3. Similar conclusions can be reached as in DIP and Gavin data, except that both the Precision value and Recall value of CFOCM are better than $\mathrm{COACH}$, and this may indicate that CFOCM has more potential on composite data.

Table 3. Results of various approaches using Srihari data.

\begin{tabular}{cccccccc}
\hline Algorithms & MCL & MCODE & RNSC & COER & ClusterONE & COACH & CFOCM \\
\hline \# complexes & 4732 & 88 & 552 & 525 & 773 & 726 & 758 \\
$N_{p}$ (MIPS) & 325 & 26 & 78 & 92 & 117 & 219 & 225 \\
$N_{b}$ (MIPS) & 168 & 42 & 102 & 111 & 131 & 150 & 152 \\
$N_{p}$ (SGD) & 654 & 36 & 108 & 176 & 224 & 299 & 322 \\
$N_{b}$ (SGD) & 292 & 44 & 184 & 189 & 217 & 231 & 240 \\
$N_{p}$ (CYC2008) & 846 & 46 & 138 & 218 & 275 & 397 & 452 \\
$N_{b}$ (CYC2008) & 362 & 57 & 154 & 236 & 272 & 281 & 290 \\
\hline
\end{tabular}




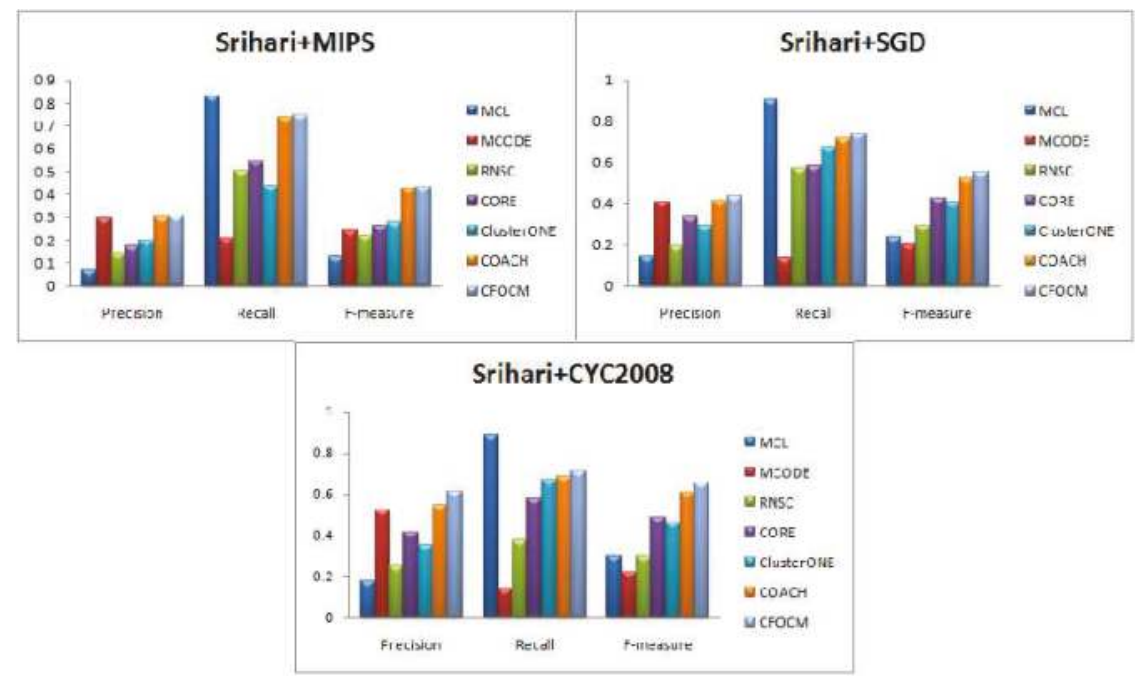

Figure 4. Comparative performance of CFOCM and the other six methods in Srihari data using benchmarks MIPS, SGD, CYC2008, respectively.

In a word, either in relatively sparse DIP networks or in relatively dense Gavin data even using a composite data set, CFOCM is able to identify a suitable number of protein complexes, and, meanwhile, the predicted complexes are also biologically meaningful as a consequence of cooperating the protein function annotations into our model, so it compellingly performs better than other existing methods in term of F-measure. Thus, we can come to the conclusion that CFOCM is efficient and has strong adaptability and robustness to different types of data.

\section{Discussion}

\subsection{The Effectiveness of Functional Annotation}

As the assumption of the complex-core described before, the proteins in each CFOCM detected core must be functional related to a certain common GO item, namely either annotated with that GO item or annotated with a GO item that is functionally interdependent with that GO item. To estimate the contribution of this, comparison experiments between CFOCM and CFOCM without use (unCFOCM) are conducted. As the results listed in Table 4 (DIP) and Table 5 (Gavin), unCFOCM in all the tests predicts much more biological meaningless complexes on account of not using GO annotation, leading to lower F-measure values. In other words, owing to the requirement of functional relevance within the discovered cores, CFOCM is capable of filtering abundant low-confidence protein complexes, and the detected protein complexes are supposed to be more biologically significant. Therefore, the cores detected by CFOCM should share some common functions, which is more in conformity with the original definition of the complex core, and it is greatly obliged to help finding more accurate protein complexes.

\subsection{Case Studies}

This section illustrates two predicted protein complexes, namely the Glycine decarboxylase complex and the RNA polymerase I complex as Figure 5. The Glycine decarboxylase complex is a small-sized complex responsible for the oxidation of glycine by mitochondria, and it consists of four proteins including YDR019C, YMR18W, YAL044C and YFL018C. As showed, CFOCM successfully identified these four proteins, in which YDR019C, YMR18W, and YAL044C are recognized as core 
proteins and YFL018C is detected as an attachment to the core. In another case, the RNA polymerase I complex is a larger complex comprised of 14 proteins, and CFOCM could also completely identify all the proteins in this complex with 100\% precision, in which all proteins except YHR143W-A are detected as members of the core having more dense connections with each other and sharing more functional relevance as well.

Table 4. Results of CFOCM and CFOCM without using Gene Ontology (GO) (unCFOCM) on DIP data.

\begin{tabular}{ccccccc}
\hline Algorithms + Benchmark & \# Complexes & $\boldsymbol{N}_{\boldsymbol{p}}$ & $\boldsymbol{N}_{\boldsymbol{b}}$ & Precision & Recall & F-Measure \\
\hline CFOCM + MIPS & 748 & 205 & 126 & 0.2741 & 0.6207 & 0.3802 \\
unCFOCM + MIPS & 862 & 213 & 130 & 0.2471 & 0.6404 & 0.3566 \\
CFOCM + SGD & 748 & 285 & 168 & 0.381 & 0.5201 & 0.4398 \\
unCFOCM + SGD & 862 & 297 & 175 & 0.3445 & 0.5418 & 0.4212 \\
CFOCM + CYC2008 & 748 & 351 & 196 & 0.4693 & 0.4804 & 0.4748 \\
unCFOCM + CYC2008 & 862 & 363 & 201 & 0.4211 & 0.4926 & 0.4541 \\
\hline
\end{tabular}

Table 5. Results of CFOCM and CFOCM without using Gene Ontology (GO) (unCFOCM) on Gavin data.

\begin{tabular}{ccccccc}
\hline Algorithms + Benchmark & \# Complexes & $\boldsymbol{N}_{\boldsymbol{p}}$ & $\boldsymbol{N}_{\boldsymbol{b}}$ & Precision & Recall & F-Measure \\
\hline CFOCM + MIPS & 453 & 191 & 91 & 0.4216 & 0.4483 & 0.4345 \\
unCFOCM + MIPS & 551 & 197 & 92 & 0.3575 & 0.4532 & 0.3997 \\
CFOCM + SGD & 453 & 250 & 119 & 0.5519 & 0.3684 & 0.4419 \\
unCFOCM + SGD & 551 & 262 & 124 & 0.4755 & 0.3839 & 0.4248 \\
CFOCM + CYC2008 & 453 & 305 & 131 & 0.6733 & 0.3211 & 0.4348 \\
unCFOCM + CYC2008 & 551 & 321 & 138 & 0.5826 & 0.3382 & 0.4280 \\
\hline
\end{tabular}

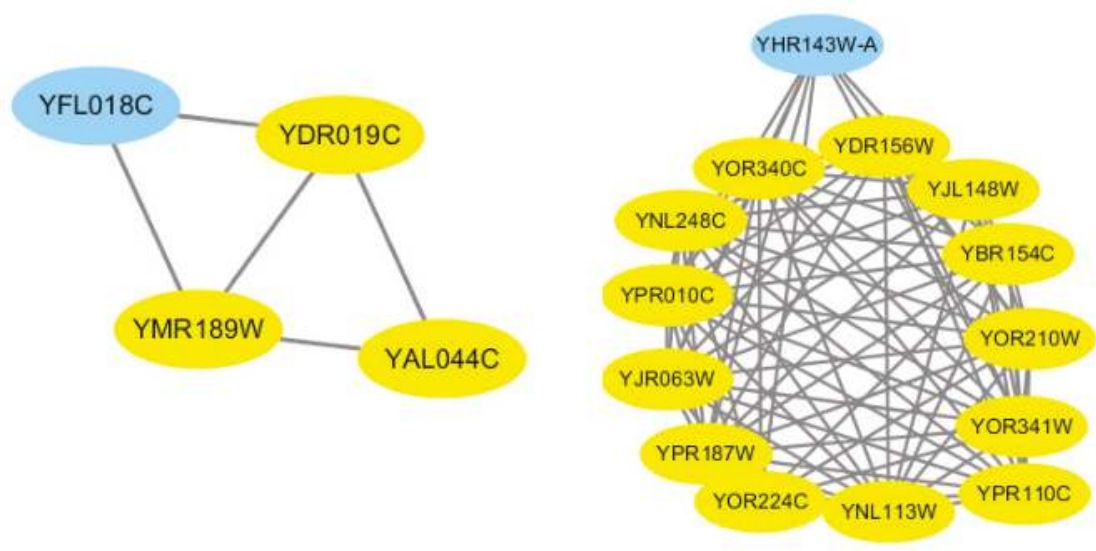

Figure 5. The Glycine decarboxylase complex and the RNA polymerase I complex as detected by CFOCM. The yellow nodes represent proteins within the complex core, while the blue node proteins represent proteins that are attachments.

\section{Materials and Methods}

\subsection{Terminologies}

A PPI Network typically can be represented as an undirected graph $G=(V, E)$, where $V$ and $E=\{(u, v) \mid u, v \in V)$ represent proteins and protein-protein interactions, respectively. A graph $G^{\prime}=\left(V^{\prime}, E^{\prime}\right)$ is regarded as a subgraph of $G$ if $V^{\prime} \subseteq V$ and $E^{\prime} \subseteq E$. $v^{\prime}$ s direct interacting neighbors in 
graph $G$ is denoted as $N_{v}=\{u \mid(u, v) \in E, u \in V\}$, and $N_{v}^{G^{\prime}}=\left\{u \mid(u, v) \in E, u \in V^{\prime}\right\}$ is $v^{\prime}$ s neighbors in subgraph $G^{\prime}$. Subgraph $G^{\prime}$ external boundary nodes are defined as $V_{o b}\left(G^{\prime}\right)=\{v \mid<v, w>\in E(G)$, $\left.v \in V(G) \backslash V\left(G^{\prime}\right), w \in V\left(G^{\prime}\right)\right\}$.

A neighborhood affinity score metric [25], denoted as NS $\left(G^{\prime}, G^{\prime \prime}\right)$, is imported to measure the similarity between two overlapping graphs $G^{\prime}=\left(V^{\prime}, E^{\prime}\right)$ and $G^{\prime \prime}=\left(V^{\prime \prime}, E^{\prime \prime}\right)$,

$$
\mathrm{NS}\left(G^{\prime}, G^{\prime \prime}\right)=\frac{\left|V_{G^{\prime}} \cap V_{G^{\prime \prime}}\right|^{2}}{\left|V_{G^{\prime}}\right| \times\left|V_{G^{\prime \prime}}\right|},
$$

where, if $\mathrm{NS}\left(G^{\prime}, G^{\prime \prime}\right)>=t\left(t\right.$ is a predefined threshold), we may declare cluster $G^{\prime}=\left(V^{\prime}, E^{\prime}\right)$ and cluster $G^{\prime \prime}=\left(V^{\prime \prime}, E^{\prime \prime}\right)$ can be further merged as a result of their high topological similarity.

As is well known, GO is composed of three orthogonal ontologies capturing knowledge about biological process, molecular function and cellular component, and each ontology consists of controlled and structured biological terms that can be used to annotate genes and proteins. Some GO item pairs are highly functionally related-for example, sharing a common parent node, or one is just a near ancestor of the other, while other GO item pairs may possess much weaker relationships or even be functionally independent. Therefore, the urgent need is to design a metric to quantify the functional interdependence between two GO items. Fortunately, Ref. [18] has done what we want (see the formula below):

$$
f r_{i, j}=\frac{r e_{i, j}-e e_{i, j}}{\sqrt{e e_{i, j}\left(1-\left(\sum_{k \in G I} e e_{i, k} /|E|\right)\right)\left(1-\left(\sum_{k \in G I} e e_{k, j} /|E|\right)\right)}},
$$

where $r e_{i, j}$ represents the real number of edges in $G$ connecting one protein annotated with GO item $i$ and the other annotated with item $j, e e_{i, j}$ represents the expected number of edges that one protein is annotated with item $i$ and the other annotated with item $j$ in $G$, hence it equals (Number of edges in $G$ with one protein annotated with $i$ )*(Number of edges in $G$ with one protein annotated with $j$ to the others) $/|E|$, and GI represents the whole GO items set. Ref. [18] also indicates that item $i$ and $j$ are functionally interdependent if $f r_{i, j}>1.96$; otherwise, they are considered to be functionally independent.

A protein complex is pervasively modeled as an induced subgraph of PPI network $G$, the proteins in which have dense intra-connections and are sparely connected to the rest of the network, thus we introduce a new and effective closeness function to quantify the probability that $G^{\prime}$ is complex based on network topology:

$$
\operatorname{cf}\left(G^{\prime}\right)=\operatorname{density}\left(G^{\prime}\right) \times\left(\frac{1}{\left|G^{\prime}\right|} * \sum_{v \in G^{\prime}} \frac{\left|N_{v}^{G^{\prime}}\right|}{\left|N_{v}\right|}\right),
$$

where $\operatorname{density}\left(G^{\prime}\right)=\frac{2 \times\left|\mathrm{E}^{\prime}\right|}{\left|\mathrm{V}^{\prime}\right| \times\left(\left|\mathrm{V}^{\prime}\right|-1\right)}$ is the density of graph $G^{\prime}$, depicted to quantify the richness of edges in $G^{\prime}$, and $\frac{\left|N_{v}^{G^{\prime}}\right|}{\left|N_{v}\right|}$ corresponds to the percentage of $v^{\prime}$ s direct neighbors located within $G^{\prime}$. If $\frac{\left|N_{v}^{G^{\prime}}\right|}{\left|N_{v}\right|}$ equals 1 , all the neighbors of $v$ are in $G^{\prime}$, so there is a high tendency that $v$ should be a member of $G^{\prime}$. If equals 0 , $v$ has little chance to be a member of $G^{\prime}$. Consequently, the expression in the bracket represents the mean possibility of each node being retained in $G^{\prime}$. Compared with previous closeness function based on the density of $G^{\prime}$, cf not only assesses the inner denseness of $G^{\prime}$, but also takes the ratio of $G^{\prime}$ inner edges and outer edges into consideration, hence manifesting superiority in appraising the likelihood of $G^{\prime}$ to be a real complex.

\subsection{Description of CFOCM Algorithm}

Most of the protein complexes contain core-attachment structure, and the proteins in the core share similar topology and are highly functionally related, while the attach proteins are usually located in the periphery of the core [14]. As the differences between core proteins and attach proteins, therefore, 
our core-attachment based algorithm CFOCM for protein complexes identification, comprised of two necessary phases, which first detects the protein complexes' cores and then selects attach proteins to the discovered cores.

\subsubsection{The Complex Cores Detection}

Protein-complex core plays a key role for complex performing biological function, and determines the cellular role and significance of the complex in the context to a large extent [14]. The results of biological analysis also indicate that most protein complex cores own some significant distinguishing features: including a small group of proteins which are densely intra-connected and sparsely to outsides, allowing overlaps between cores, possession of some common functions, showing an altitudinal mRNA co-expression patterns. In this paper, however, only the former three features are used to portray the cores discovered by CFOCM, and our detected cores satisfy the following assumption.

Assumption 1. A subgraph $G^{\prime}=\left(V^{\prime}, E^{\prime}\right)$ is a protein-complex core unless if satisfying the followed conditions:

1. The topology of $G^{\prime}$ meets: $\left|G^{\prime}\right|>=3, G^{\prime}$ reaches the local optimum that there does not exist any neighbor node $v$ that satisfies $\operatorname{cf}\left(G^{\prime}+\{v\}\right)>\operatorname{cf}\left(G^{\prime}\right)$ or $\operatorname{cf}\left(G^{\prime}-\{v\}\right)>\operatorname{cf}\left(G^{\prime}\right)$, and no such $G^{\prime \prime}$ exists if $G^{\prime} \subseteq G^{\prime \prime}$ and $G^{\prime \prime}$ is a complex core.

2. If $G^{\prime}$ has overlaps with $G^{\prime \prime}$, then $\mathrm{NS}\left(G^{\prime}, G^{\prime \prime}\right)<t$ must be satisfied; otherwise, $G^{\prime}$ and $G^{\prime \prime}$ could combine together.

3. $G^{\prime}$ needs to be biologically significant: $m x$ is defined as the the maximum common GO item annotating a maximum number of nodes in $G^{\prime}, \forall v \in V^{\prime}, v$ is either annotated by $m x$ or annotated by a GO item gi interdependent with $m x$, which satisfies $\operatorname{fr}(g i, m x)>1.96$.

Different from traditional methods exploring each core protein separately, our above complex-core assumption is more plausible for considering all proteins in the core as a whole. Benefiting from this renovation ensures that each protein in the core owns similar topology and contributes to the enforcement of core's biological functions. Conditions 1, 2, and 3 guarantees the maximizes closeness function value of core, the nearest distance can be retained between different cores, and participation of at least one common biological functions, respectively. Specifically, most traditional literature is mainly focused on the assurance of highly functional similarity between each protein pair in the core, which will result in neglecting that the core as a whole should perform some common functions, while this flaw is certainly renovated by our integrated global view of the core.

Algorithm 1 illustrates that the overall framework to detect protein-complex cores, and, without question, the discovered cores comply with definitions in Assumption 1. We first compute the functional interdependence between each GO items pair by the definition $\mathrm{fr}$ in line 1 . Then, in line 2, we identify all cliques that are fully connected subgraphs by using a complete enumeration method [29], based on the fact that a k-clique can be obtained by adding a vertex to the clique with k-1 vertices and the 2-cliques can be initialized as the edges in the graph, but only the maximal cliques are reserved at last, and a k-clique is regarded as a maximal k-clique only in the case that it cannot be enlarged by adding any vertex. After that, lines 4-19 mining complex cores by a iteration process on the basis of the two aforementioned pretreatment works. Here, a concept of candidate-core family is presented, containing the core itself and its similar candidate-cores with the neighborhood affinity score NS less than a predefined threshold $t$. For each certain candidate-core, its family set is obtained in lines 8-13, and a more reasonable combined candidate-core comes into being through algorithm Merge_Similar_Cores in line 14. The details of Merge_Similar_Cores algorithm are described in Algorithm 2. Still, in lines 15-17, if the current generated candidate-core already exists in the generated candidate-core set, we simply discard it; otherwise, we add it to the candidate-core set. After these steps, though, there unavoidably exist some incorrect manipulations, excessive overlapping and biological meaningless candidate cores are substantially removed, and the overwhelming majority of 
the vertexes in retained cores are densely connected internally, possess similar topology and attend to share at least one common GO annotated function.

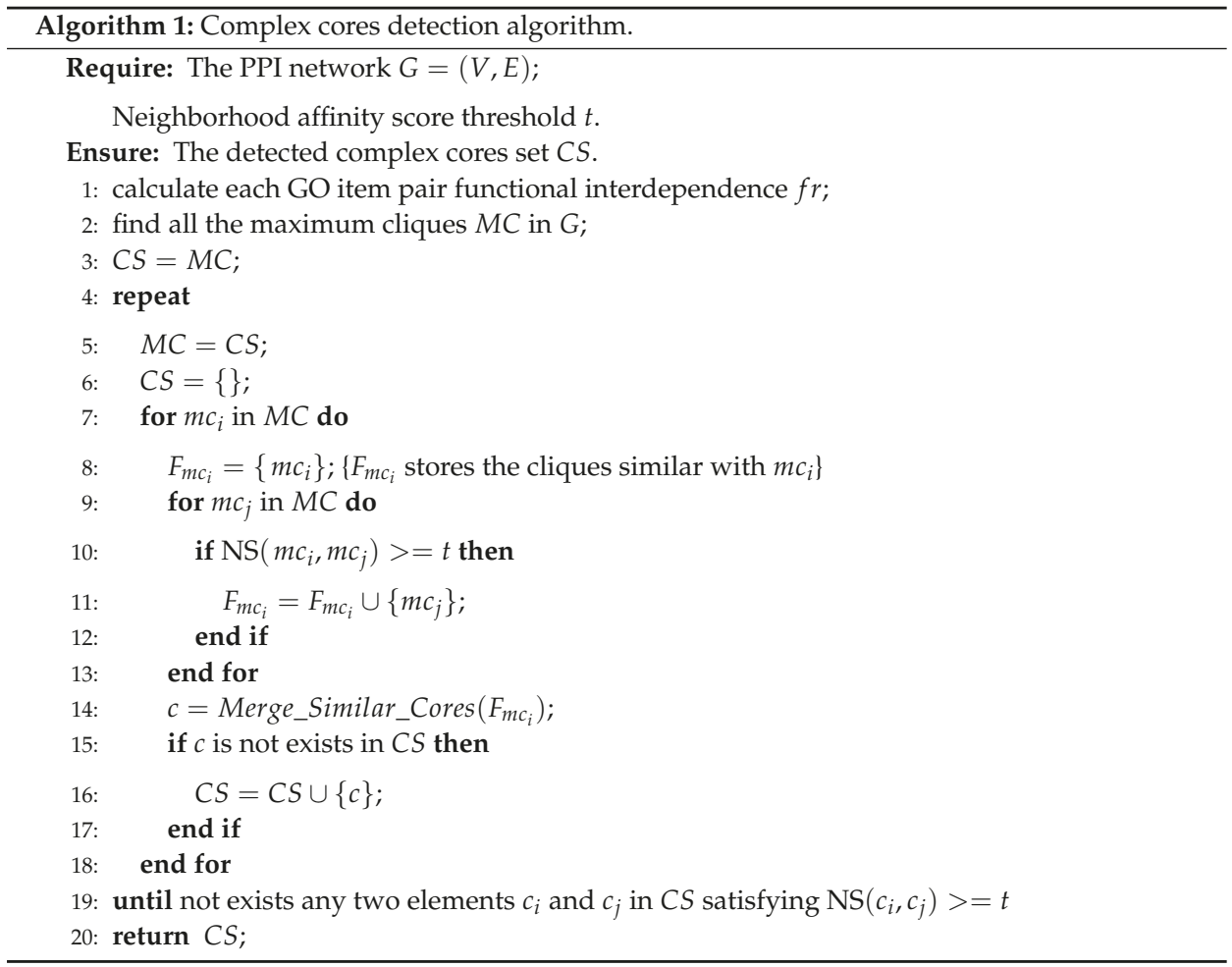

A crucial artifice, not described in Algorithm 1, is applied in the process of detecting cores. First, for each maximal cliques set with the same number of vertexes, we generate their corresponding new candidate cores by executing steps in lines 4-19, and then form the final detected cores via the same steps on these different-sized generated cores. Without using this, the smaller cliques may be annexed by the larger similar cliques so that they barely contribute to the generation of the new candidate core. Actually, this artifice is proved to be an effective means of improving the predicting performance.

\subsubsection{Similar Complex Cores Merge}

Given the family $F_{m c}$ of the candidate core $m c$, the Merge_Similar_Cores algorithm will filter the proteins that can not help to preserve the topology of the core or are functionally independent with other proteins in the core and return a new candidate core.

Our Merge_Similar_Cores algorithm works as follows. To begin with, we extract the proteins PS from the input family of a candidate-core in line 1, and find the GO item $m$ disappeared in the GO annotations of maximal proteins in line 2. Afterwards, in lines 3-7, we remove proteins that are neither annotated by the common item $m$ nor have a GO item functional interdependent with item $m$, and this procedure ensures that the returned candidate-core has a high probability of owning at least one common GO function because the proteins in the returned candidate-core either have the common GO item $m$ or a GO item $j$ exists that is functionally interdependent with $m$. Finally, in lines 8-10, we iteratively delete a protein $p$ from the PS until no such protein $p$ exists, satisfying 
$\operatorname{cf}(P S-\{p\})>\operatorname{cf}(P S)$, and ensuring that the remaining proteins reach the local optimum, which is relatively richly inner-connected and sparsely connected to the outside.

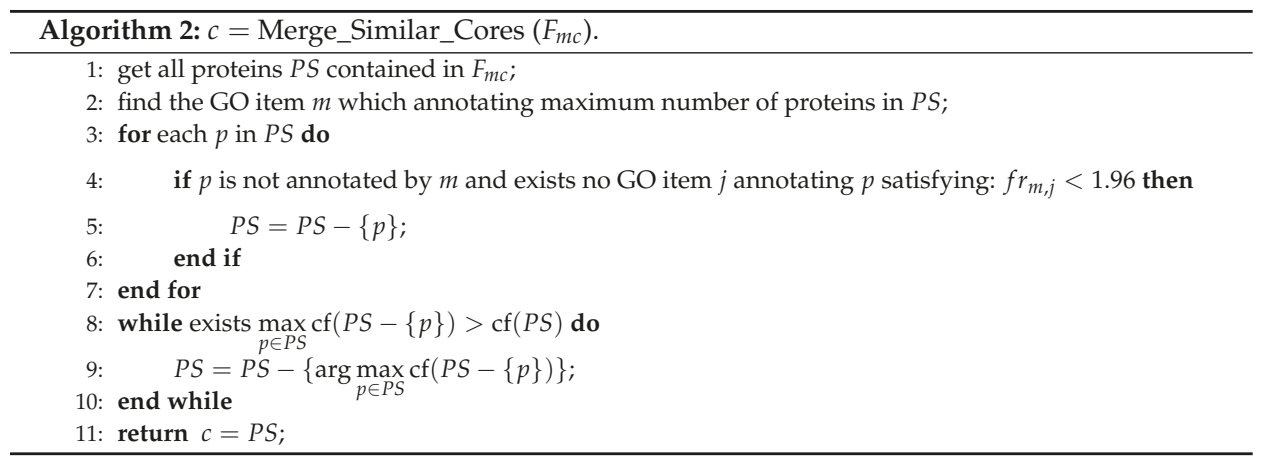

Each input candidate-core family goes through these steps, and a newer candidate-core has been formed. In addition, Figure 6 also provides an example to illustrate the process of our proposed Merge_Similar_Cores algorithm.

\subsubsection{Attach-Proteins Screening}

After the foregoing phase of our CFOCM method, the protein-complex cores have already been mined from PPI network $G=(V, E)$. In the second phase, we will form the final predicted complex by appending reliable peripheral proteins to the discovered cores. Given a protein complex core $c$, for each external boundary protein $p$ of current core $c$, the following Assumption 2 presents whether $p$ should be an attachment to the core $c$ or not.

Assumption 2. A external boundary protein $p$ is affirmed as an attachment to the complex core $c$ if satisfying $\operatorname{cf}(c+\{p\})>\operatorname{cf}(c)$.

From the above assumption, the external boundary protein $p$ improves the closeness function cf of the current cluster selected as an attachment. Through appending some attachment proteins to the current core, the topology of core can still be reserved, and thus all the proteins in each final predicted complex are densely connected and sparsely connected to the outside. Algorithm 3 is the pseudo code description.

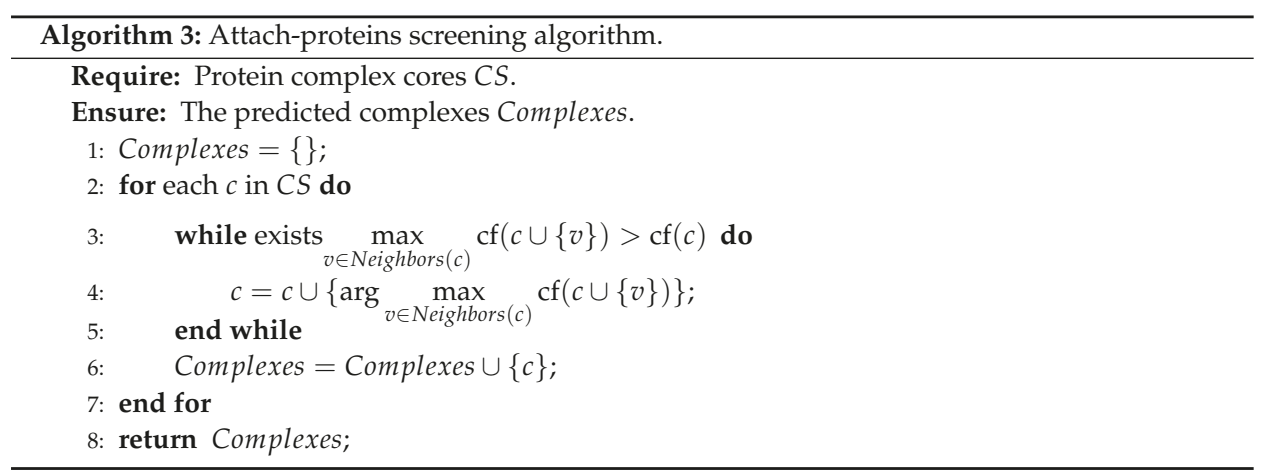




\begin{tabular}{|l|l||c|c|c|c|c|c|c|}
\hline ID & \multicolumn{1}{|c|}{ G0 } \\
\hline A & GO:01, GO:02, GO:04 \\
\hline B & GO:02, GO:04 & fr & G0:01 & G0:02 & G0:03 & G0:04 & G0:05 & G0:06 \\
\hline C & GO:02 \\
\hline D & GO:02 & INF & 0.87 & 1.23 & 1.28 & 0.21 & 0 \\
\hline E & GO:03, GO:04 & G0:02 & 0.87 & INF & 1.34 & 3.26 & 0 & 0.04 \\
\hline F & GO:05 & G0:03 & 1.23 & 1.34 & INF & 0.76 & 0 & 0.03 \\
\hline G & GO:02, GO:06 & G0:04 & 1.28 & 3.26 & 0.76 & INF & 0 & 0.02 \\
\hline H $0: 05$ & 0.21 & 0 & 0 & 0 & INF & 0.01 \\
\hline G0:06 & GO:02 & 0.05 & 0.04 & 0.03 & 0.02 & 0.01 & INF \\
\hline
\end{tabular}

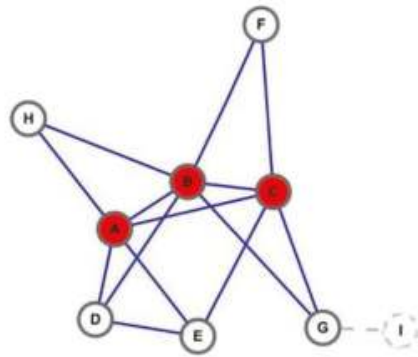

(A)

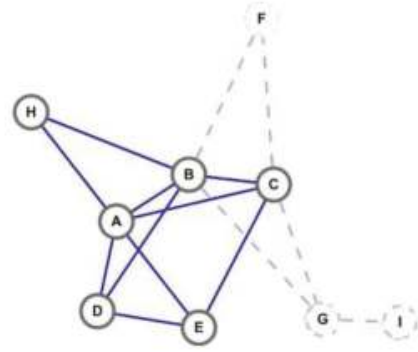

(C)

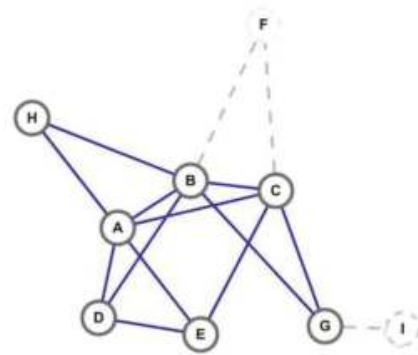

(B)

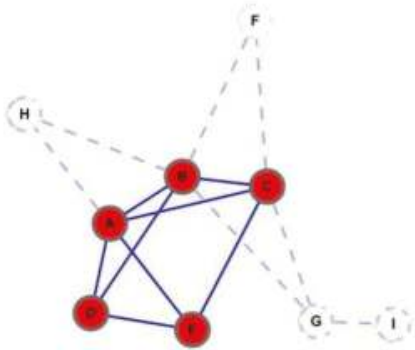

(D)

Figure 6. The diagram of Merge_Similar_Cores algorithm. In the example, $(A)$ is the family graph of clique $\{A, B, C\}$, including cliques $\{\{A, B, C\},\{A, B, D\},\{A, B, H\},\{A, C, E\},\{B, C, F\},\{B, C, G\}\}$, and the proteins set is $\{A, B, C, D, E, F, G, H\}$. In (B), the common Gene Ontology (GO) item is GO:02, and reserve vertex $\mathrm{E}$ as $\mathrm{fr}_{\mathrm{GO}: 02, \mathrm{GO}: 04}>1.96$, while drop vertex $\mathrm{F}$ is $f r_{G O: 02, G O: 05}<1.96$. In (C), drop vertex $\mathrm{G}$ is $\arg \max _{p \in P S} \operatorname{cf}(P S-\{p\})=G$. In (D), drop vertex $\mathrm{H}$ is arg $\max _{p \in P S} \operatorname{cf}(P S-\{p\})=H$, and returns the next candidate-core $\mathrm{A}, \mathrm{B}, \mathrm{C}, \mathrm{D}, \mathrm{E}$, as no remove operation can improve the cf.

\subsection{Data Sources}

Three publicly available yeast PPI networks, namely the Database of Interacting Proteins (DIP) data [30], Gavin data [14] and Srihari data collected by Srihari et al. [31], are used to evaluate the performance of our method CFOCM in protein complex prediction. DIP consists of 17,203 PPIs involving 4930 proteins, while Gavin data contains fewer proteins but is more densely connected, which consists of 6531 high-quality interactions among 1430 proteins. Srihiri data contains 20,000 interactions covering 3680 proteins derived from the BioGRID, IntAct, and MINT repositories. 
Table 6. Three protein-protein interaction (PPI) networks used in the experiments.

\begin{tabular}{cccc}
\hline Dataset & \#Proteins & \#Interactions & Average Node Degree \\
\hline DIP & 4930 & 17203 & 6.98 \\
Gavin & 1430 & 6531 & 9.13 \\
Srihari & 3680 & 20,000 & 10.87 \\
\hline
\end{tabular}

To validate our predicted complexes, three reference sets of real complexes, denoted as the Munich Information Center for Protein Sequence (MIPS) [32], Saccharomyces Genome Database (SGD) [33], and CYC2008 [34], are selected as benchmarks. MIPS consists of 203 protein complexes manually curated from the literature, SGD contains 323 complexes derived from Gene Ontology-based complex annotations, and CYC2008 consists of 408 hand-curated complexes reliably backed by small-scale experiments.

The yeast GO annotation dataset is downloaded from the SGD database, and the submission data is February 2014.

\section{Conclusions}

In this paper, we have proposed a novel algorithm CFOCM for protein complex identification from the protein-protein interaction network. According to the fact that there some proteins involved in more than one biological function or cellular processes, CFOCM implements allowing overlaps between detected complexes. Meanwhile, CFOCM also takes into account the inherent core-attachment structure in the protein complexes. Moreover, CFOCM ensures topological similarity and functional interdependence between each pair of proteins within detected cores.

Comparison experiments between CFOCM and the other six state-of-the-art methods are carried out in DIP networks, Gavin networks and Srihari data, and the results of all tests show that CFOCM significantly outperforms the others. Moreover, CFOCM has been demonstrated to be capable of filtering the low-confidence or biological insignificant protein complexes via comparing with unCFOCM without consideration that the proteins in a complex core should occupy some common functions. In a word, CFOCM is efficient, robust, and it is applicable for helping biologists search for new biological meaningful protein complexes.

The follow-up works are ongoing. For instance, since some proteins still have not been functionally annotated, and we intend to find a more suitable strategy to handle this data problem, and design a parallel version of CFOCM to accelerate the operating speed. In addition, how to extend CFOCM to detect protein complexes and functional modules in dynamic PPI networks, which can be constructed by incorporating gene expression data, is also a promising direction.

Acknowledgments: This work is supported by the Program for New Century Excellent Talents in University (Grant NCET-10-0365), the National Nature Science Foundation of China (Grant 60973082, 11171369, 61272395, 61370171, 61300128), the National Nature Science Foundation of Hunan Province (Grant 12JJ2041), the Planned Science and Technology Project of Hunan Province (Grant 2009FJ3195, 2011FJ3123) and supported by the Fundamental Research Funds for the Central Universities, Hunan University.

Author Contributions: Bo Li and Bo Liao conceived and designed the experiments; Bo Li performed the experiments; Bo Li and Bo Liao analyzed the data; Bo Li contributed reagents/materials/analysis tools; $\mathrm{Bo} \mathrm{Li}$ wrote the paper.

Conflicts of Interest: The authors declare no conflict of interest.

\section{References}

1. Gavin, A.C.; Basche, M.; Krause, R.; Grandi, P.; Marzioch, M.; Bauer, A.; Schultz, J.; Rick, J.M.; Michon, A.M.; Cruciat, C.M.; et al. Functional organization of the yeast proteome by systematic analysis of protein complexes. Nature 2002, 415, 141-147.

2. Eichler, E.E.; Flint, J.; Gibson, G.; Kong, A.; Leal, S.M.; Moore, J.H.; Nadeau, J.H. Missing heritability and strategies for finding the underlying causes of complex disease. Nat. Rev. Genet. 2010, 11, 446-450. 
3. Rigaut, G.; Shevchenko, A.; Rutz, B.; Wilm, M.; Mann, M.; Séraphin, B. A generic protein purification method for protein complex characterization and proteome exploration. Nat. Biotechnol. 1999, 17, 1030-1032.

4. Ho, Y.; Gruhler, A.; Heilbut, A.; Bader, G.D.; Moore, L.; Adams, S.L.; Millar, A.; Taylor, P.; Bennett, K.; Boutilier, K.; et al. Systematic identification of protein complexes in Saccharomyces cerevisiae by mass spectrometry. Nature 2002, 415, 180-183.

5. King, A.D.; Preulj, N.; Jurisica, I. Protein complex prediction via cost-based clustering. Bioinformatics 2004, 20, 3013-3020.

6. Ou-Yang, L.; Dai, D.Q.; Zhang, X.F. Protein complex detection via weighted ensemble clustering based on Bayesian nonnegative matrix factorization. PLOS ONE 2013, 8, e62158.

7. Aldecoa, R.; Priulj, I. Jerarca: Efficient analysis of complex networks using hierarchical clustering. PLoS ONE 2010, 5, e11585.

8. Wang, J.; Li, M.; Chen, J.; Pan, Y. A fast hierarchical clustering algorithm for functional modules discovery in protein interaction networks. IEEE/ACM Trans. Comput. Biol. Bioinform. 2011, 8, 607-620.

9. Pizzuti, C.; Rombo, S.E. A Coclustering Approach for Mining Large Protein-Protein Interaction Networks. IEEE/ACM Trans. Comput. Biol. Bioinform. 2012, 9, 717-730.

10. Bader, G.D.; Hogue, C.W.V. An automated method for finding molecular complexes in large protein interaction networks. BMC Bioinform. 2003, 4, 2.

11. Adamcsek, B.; Palla, G.; Farkas, I.J.; Derényi, I.; Vicsek, T. CFinder: Locating cliques and overlapping modules in biological networks. Bioinformatics 2006, 22, 1021-1023.

12. Palla, G.; Derinyi, I.; Farkas, I.; Vicsek, T. Uncovering the overlapping community structure of complex networks in nature and society. Nature 2005, 435, 814-818.

13. Nepusz, T.; Yu, H.; Paccanaro, A. Detecting overlapping protein complexes in protein-protein interaction networks. Nat. Methods 2012, 9, 471-472.

14. Gavin, A.C.; Aloy, P.; Grandi, P.; Krause, R.; Boesche, M.; Marzioch, M.; Rau, C.; Jensen, L.J.; Bastuck, S.; Dümpelfeld, B.; et al. Proteome survey reveals modularity of the yeast cell machinery. Nature 2006, 440, 631-636.

15. Leung, H.C.M.; Xiang, Q.; Yiu, S.M.; Chin, F.Y. Predicting protein complexes from PPI data: A core-attachment approach. J. Comput. Biol. 2009, 16, 133-144.

16. Wu, M.; Li, X.; Kwoh, C.K.; Ng, S.K. A core-attachment based method to detect protein complexes in PPI networks. BMC Bioinform. 2009, 10, 169.

17. Chen, B.; Wu, F.X. Identifying Protein Complexes Based on Multiple Topological Structures in PPI Networks. IEEE Trans. NanoBiosci. 2013, 12, 165-172.

18. Lam, W.W.M.; Chan, K.C.C. Discovering functional interdependence relationship in PPI networks for protein complex identification. IEEE Trans. Biomed. Eng. 2012, 59, 899-908.

19. Zhang Y, Lin H, Yang Z, Wang J. Construction of Ontology Augmented Networks for Protein Complex Prediction. PLoS ONE 2013, 8, e62077.

20. Hu, A.L.; Chan, K.C.C. Utilizing Both Topological and Attribute Information for Protein Coplex Identification in PPI Networks. IEEE/ACM Trans. Comput. Biol. Bioinform. 2013, 10, 780-792.

21. Li, M.; Wu, X.; Wang, J.; Pan, Y. Towards the identification of protein complexes and functional modules by integrating PPI network and gene expression data. BMC Bioinform. 2012, 13, 109.

22. Chen, B.; Fan, W.; Liu, J.; Wu, F.X. Identifying protein complexes and functional modules from static PPI networks to dynamic PPI networks. Brief. Bioinform. 2014, 15, 177-194.

23. Bernigen, D.; Pers, T.H.; Thorrez, L.; Huttenhower, C.; Moreau, Y.; Brunak, S. Concordance of gene expression in human protein complexes reveals tissue specificity and pathology. Nucleic Acids Res. 2013, 41, e171.

24. Babu, M.; Vlasblom, J.; Pu, S.; Guo, X.; Graham, C.; Bean, B.D.; Burston, H.E.; Vizeacoumar, F.J.; Snider, J.; Phanse, S.; et al. Interaction landscape of membrane-protein complexes in Saccharomyces cerevisiae. Nature 2012, 489, 585-589.

25. Li, X.; Wu, M.; Kwoh, C.K.; Ng, S.K. Computational approaches for detecting protein complexes from protein interaction networks: A survey. BMC Genom. 2010, 11 (Suppl. 1), S3.

26. Srihari, S.; Leong, H.W. A survey of computational methods for protein complex prediction from protein interaction networks. J. Bioinform. Comput. Biol. 2013, 11, 1230002.

27. Ji, J.; Zhang, A.; Liu, C.; Quan, X.; Liu, Z. Survey: Functional module detection from protein-protein interaction networks. IEEE Trans. Knowl. Data Eng. 2013, 26, 261-277. 
28. Dongen, S.M.V. Graph Clustering by Flow Simulation. Utrecht University Repository, 2000. Available online: https://dspace.library.uu.nl/bitstream/handle/1874/848/full.pdf?sequence=1\&isAllowed=y (accessed on 6 September 2017).

29. Spirin, V.; Mirny, L.A. Protein complexes and functional modules in molecular networks. Proc. Natl. Acad. Sci. USA 2003, 100, 12123-12128.

30. Salwinski, L.; Miller, C.S.; Smith, A.J.; Pettit, F.K.; Bowie, J.U.; Eisenberg, D. The database of interacting proteins: 2004 update. Nucleic Acids Res. 2004, 32 (Suppl. 1), D449-D451.

31. Srihari, S.; Yong, C.H.; Patil, A.; Wong, L. Methods for protein complex prediction and their contributions towards understanding the organisation, function and dynamics of complexes. FEBS Lett. 2015, 589, 2590-2602. doi:10.1016/j.febslet.2015.04.026.

32. Mewes, H.W.; Frishman, D.; Gmldener, U.; Mannhaupt, G.; Mayer, K.; Mokrejs, M.; Morgenstern, B.; Münsterkötter, M.; Rudd, S.; Weil, B. MIPS: A database for genomes and protein sequences. Nucleic Acids Res. 2002, 30, 31-34.

33. Cherry, J.M.; Adler, C.; Ball, C.; Chervitz, S.A.; Dwight, S.S.; Hester, E.T.; Jia, Y.; Juvik, G.; Roe, T.; Schroeder, M.; et al. SGD: Saccharomyces genome database. Nucleic Acids Res. 1998, 26, 73-79.

34. Pu, S.; Wong, J.; Turner, B.; Cho, E.; Wodak, S.J. Up-to-date catalogues of yeast protein complexes. Nucleic Acids Res. 2009, 37, 825-831.

(C) 2017 by the authors. Licensee MDPI, Basel, Switzerland. This article is an open access article distributed under the terms and conditions of the Creative Commons Attribution (CC BY) license (http:/ / creativecommons.org/licenses/by/4.0/). 


\title{
Predicting Amyloidogenic Proteins in the Proteomes of Plants
}

\author{
Kirill S. Antonets ${ }^{1,2}$ and Anton A. Nizhnikov ${ }^{1,2, *}$ \\ 1 Laboratory for Proteomics of Supra-Organismal Systems, All-Russia Research Institute for Agricultural \\ Microbiology, Podbelskogo sh., 3, Pushkin, St. Petersburg 196608, Russia; kirantonez@gmail.com \\ 2 Department of Genetics and Biotechnology, St. Petersburg State University, Universitetskaya nab., 7/9, \\ St. Petersburg 199034, Russia \\ * Correspondence: ant.nizhnikov@gmail.com; Tel.: +7-812-470-5100
}

Received: 25 August 2017; Accepted: 13 October 2017; Published: 16 October 2017

\begin{abstract}
Amyloids are protein fibrils with characteristic spatial structure. Though amyloids were long perceived to be pathogens that cause dozens of incurable pathologies in humans and mammals, it is currently clear that amyloids also represent a functionally important form of protein structure implicated in a variety of biological processes in organisms ranging from archaea and bacteria to fungi and animals. Despite their social significance, plants remain the most poorly studied group of organisms in the field of amyloid biology. To date, amyloid properties have only been demonstrated in vitro or in heterologous systems for a small number of plant proteins. Here, for the first time, we performed a comprehensive analysis of the distribution of potentially amyloidogenic proteins in the proteomes of approximately 70 species of land plants using the Waltz and SARP (Sequence Analysis based on the Ranking of Probabilities) bioinformatic algorithms. We analyzed more than 2.9 million protein sequences and found that potentially amyloidogenic proteins are abundant in plant proteomes. We found that such proteins are overrepresented among membrane as well as DNA- and RNA-binding proteins of plants. Moreover, seed storage and defense proteins of most plant species are rich in amyloidogenic regions. Taken together, our data demonstrate the diversity of potentially amyloidogenic proteins in plant proteomes and suggest biological processes where formation of amyloids might be functionally important.
\end{abstract}

Keywords: amyloid; Waltz; SARP; plant; prion; seed storage protein; proteomics; compositionally biased region; amyloidogenic region

\section{Introduction}

Amyloids represent protein fibrils consisting of monomers that form intermolecular $\beta$-sheets located along the axis of a fibril and are stabilized by numerous hydrogen bonds. Such a spatial structure is called "cross- $\beta$ " [1]. The term "cross- $\beta$ " refers to the common pattern of amyloids in $X$-ray diffraction analysis with two scattering signals of approximately 4.7 and $10 \AA$ corresponding to the distances between $\beta$-strands comprising $\beta$-sheets and between intermolecular $\beta$-sheets, respectively [2,3]. Their highly ordered structure gives amyloids unusual properties including resistance to treatment with ionic detergents [4], other protein denaturants [5] and proteinases [6].

Initially, amyloids were described as lethal pathogens causing incurable diseases (amyloidoses) of humans and animals [7]. The term "amyloid" was proposed in 1854 by Rudolf Virchow, who was the first to stain pathological amyloid deposits in human tissues with iodine [8]. Though "amyloid" is a derivative from "amylon" and "amylum" (starch-like in Greek and Latin, respectively), the key components of amyloid deposits are protein fibrils [7,9]. Nevertheless, such deposits additionally contain a significant number of proteoglycans and glycosaminoglycans that were initially detected by iodine and led to an incorrect interpretation of the chemical nature of amyloids [10]. Amyloidoses 
occur primarily due to mutations that change the structure of the corresponding amyloid-forming proteins or lead to their overproduction [11]. To date, more than 30 human proteins have been shown to adopt pathological amyloid states [12].

Another aspect of these proteins was revealed over the last two decades, when amyloids that were not associated with pathogenesis were found. These amyloids, which are formed under native conditions and are implicated in cellular processes, were named "functional amyloids" [13,14]. In bacteria, functional amyloids are important for biofilm formation [15], toxin metabolism [16], and overcoming surface tension by aerial hyphae [17]. In archaea, such amyloids not only participate in the formation of biofilms [18] but also act as the structural components of the cell sheaths [19]. Functional amyloids of fungi regulate heterokaryon incompatibility [20] as well as facultative multicellularity [21] and, similar to bacterial amyloids, contribute to the formation of aerial hyphae [22]. Amyloids forming under native conditions in animals (including humans) are involved in long-term memory formation [23,24], melanin polymerization [25], hormone storage [26], tooth enamel polymerization, programmed necrosis [27], and antiviral responses [28]. Taken together, amyloids represent not only pathogenic but also widespread functionally important variants of the quaternary protein structure and are vital for many species.

The propensity of a protein to form amyloid fibrils is determined by the presence in its amino acid sequence of so-called "amyloidogenic regions" (ARs) that drive amyloidogenesis [29-31] acting as a "trigger" for polymerization [32]. Amyloid-forming proteins may contain one or multiple ARs [33,34], which are relatively short [35] and predominantly composed of hydrophobic residues, especially aromatics (W, F, Y) and aliphatics (V, I, L) [36]. ARs can be predicted using a wide range of algorithms, one of the most efficient of which is Waltz [37], which is based on a position-specific scoring matrix [36,37]. Another type of AR is represented by compositionally biased regions (CBRs) that are rich in glutamine (Q) and/or asparagine (N) [38]. The key role of QN-rich CBRs in amyloid formation was initially demonstrated on the human poly-Q expanded Huntingtin protein [39] and further deepened by the data obtained on the yeast amyloid-forming proteins [40]. In addition to QN, CBRs rich in E are also amyloid-prone [41]. Compositionally biased regions rich in Q, N or E can be efficiently predicted by different existing bioinformatic algorithms, including LPS (Lower Probability Subsequences) [42] and SARP (Sequence Analysis based on the Ranking of Probabilities) [43]. Hereafter, short amyloidogenic regions predicted with Waltz are referred to as ARs, while potentially amyloidogenic compositionally biased regions are referred to as CBRs. Currently, bioinformatic prediction is widely used for the detection of potentially amyloidogenic (i.e., containing amyloidogenic regions) proteins in the proteomes of different species $[42,44,45]$ as well as for the identification of amyloidogenic regions in particular proteins to analyze their amyloid properties in vitro and in vivo [46-48].

Despite the fact that plants are one of the most economically important groups of organisms, they remain the least studied in the field of amyloid biology. To date, amyloid properties have been demonstrated for several plant proteins or their fragments only in vitro $[49,50]$ or in heterologous systems in vivo [46] (for a review, see [51]). Here, we present a large-scale analysis of the distribution of potentially amyloidogenic proteins in the proteomes of land plants reported to date. We screened the proteomes of 75 species comprising more than 2.9 million proteins for the presence of amyloidogenic regions using the SARP and Waltz algorithms. We analyzed the molecular functions of potentially amyloidogenic plant proteins along with their subcellular localization and molecular process involvement. We found plant-specific groups of proteins in which amyloidogenic regions are overrepresented and discuss the analysis of amyloid properties of such proteins and their potential significance.

\section{Results}

\subsection{Abundance of Potentially Amyloidogenic Proteins in the Proteomes of Plants}

To assess the abundance of potentially amyloidogenic proteins in plant proteomes, the proteins of 75 plant species available in the Uniprot Proteomes database (available at http:/ / www.uniprot.org/ 
proteomes /) were analyzed for the presence of amyloidogenic regions with two different bioinformatic approaches: Waltz, which predicts short amyloidogenic regions (ARs) based on a position-specific scoring matrix [37], and SARP, which searches for compositionally biased potentially amyloidogenic regions (CBRs) rich in particular residues [43]. For each proteome, we calculated the following: (i) fraction of potentially amyloidogenic proteins in the proteome; and (ii) the coverage of total proteome length with ARs and QN-rich CBRs (Figure 1, Table S1a).

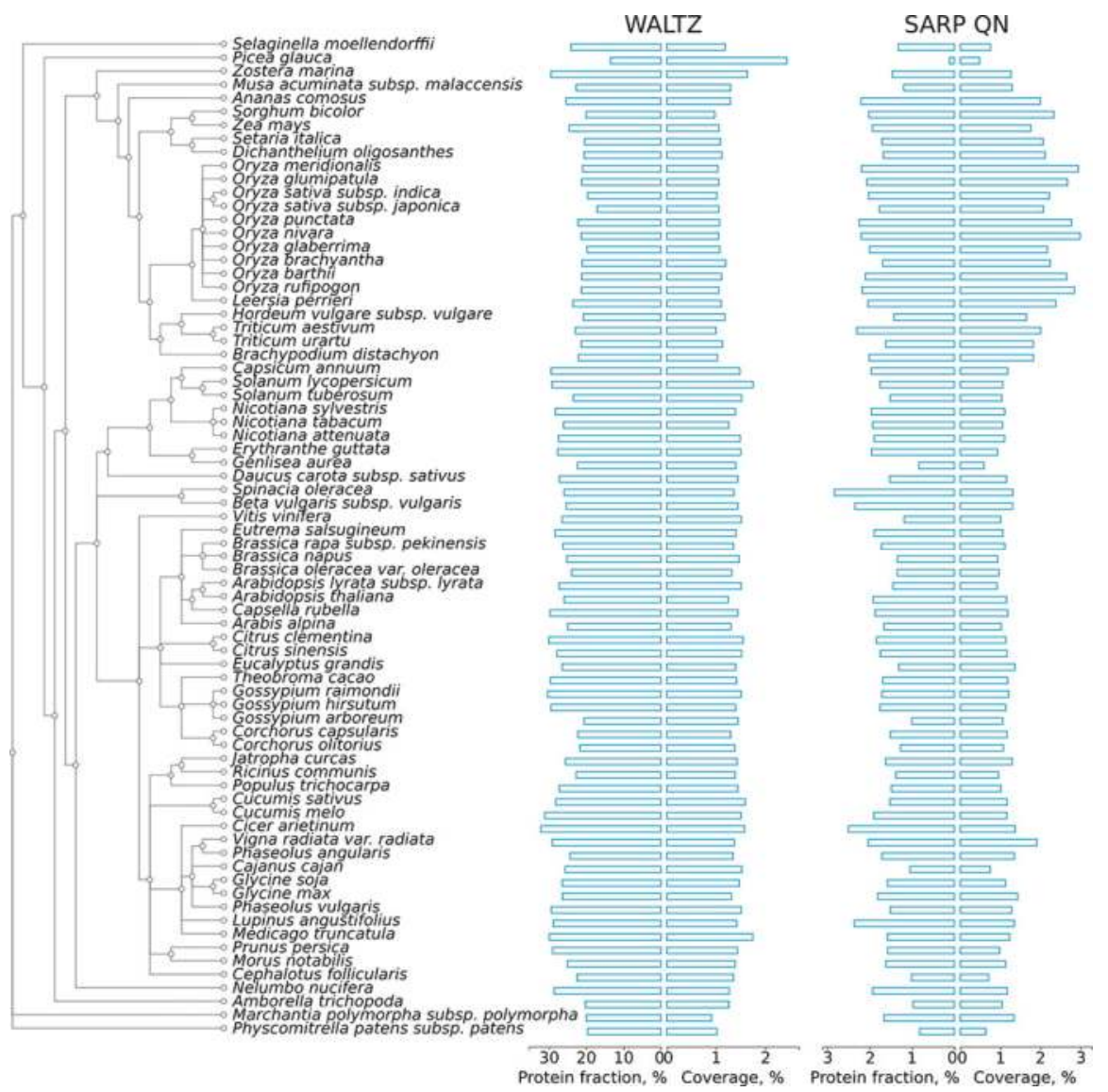

Figure 1. Distribution of amyloidogenic regions in the proteomes of land plants. A phylogenetic tree of plant species is shown according to the Uniprot Taxonomy. The results for proteins bearing ARs predicted by Waltz and QN-rich CBRs found with SARP are shown. For each type of amyloidogenic region, the percentage of proteins harboring these regions (\%) and the coverage of the total proteome length with these regions (\%) are shown. ARs, amyloidogenic regions; $\mathrm{Q}$, glutamine; $\mathrm{N}$, asparagine; CBRs, compositionally biased regions; SARP, Sequence Analysis based on the Ranking of Probabilities.

Amyloidogenic regions (ARs) predicted by Waltz are abundant in the proteomes of plants. More than half of all proteins in each proteome contained at least one such region (Figure S1). Most ARs are very short at approximately 6-9 amino acids long, with a modal length of seven residues (Figure S2). Though such regions are amyloid-prone themselves [37], they may not contribute to amyloid-forming properties of the full-length proteins due to their short lengths. Therefore, to enhance the specificity of the predictions, we excluded from the Waltz analysis all ARs shorter than 10 amino acids. 
After this filtering, the median percentage of plant proteins that contained ARs predicted by Waltz was $25.41 \%$ (Table S1a). Potentially amyloidogenic compositionally biased regions (CBRs) predicted by SARP were significantly less abundant than ARs predicted by Waltz: approximately $1.38 \%$ of plant proteins contain QN-rich CBRs. The median length of CBRs predicted by SARP in plant proteomes was 203 residues for QN-rich CBRs (Table S1a). In contrast to potentially amyloidogenic proteins predicted by Waltz, most of the potentially amyloidogenic proteins predicted by SARP contained only one potentially amyloidogenic compositionally biased region. Notably, though amyloidogenic region predictions by Waltz and SARP were completely different, ARs predicted by Waltz were associated with CBRs rich in hydrophobic residues I, W, Y, F predicted by SARP (Figure S3). This result corresponds with the previous observation that amino acids with hydrophobic side chains have the highest amyloidogenic potential (i.e., propensity to form amyloid structure) [36].

The AR contents predicted by Waltz and SARP varied broadly in the proteomes of different plant species and may be significantly different even in closely related species (Figure 1, Table S1a). For example, Gossypium arboreum has many fewer proteins containing ARs predicted by Waltz (20.6\%) compared to Gossypium hirsutum (29.5\%) (Figure 1), which originated as a hybrid of Gossypium arboreum and Gossypium raimondii [52]. Species of Oryza spp. significantly differ from one another in the content of proteins with QN-rich CBRs (Figure 1). We excluded Ipomeae nil from analysis because its proteome, available at Uniprot (Table S2), contained only proteins encoded by the chloroplast or mitochondrial genomes. The only conifer species, Picea glauca, drastically differed from other species in AR and QN-rich CBR contents (Figure 1), but this could be associated with an incomplete proteome available at Uniprot (Table S2). Despite variability in the content of ARs and QN-rich CBRs in the proteomes of land plants, there is a common tendency of the proteomes of grasses to have a lower percentage of proteins with ARs predicted by Waltz and to be more abundant in QN-rich proteins (Figure 1). It should be noted that the proteomes of plants have similar contents of potentially amyloidogenic proteins compared with the Escherichia coli, Saccharomyces cerevisiae and Homo sapiens proteomes (Table S1b), in which experimentally verified amyloid proteins have been previously reported $[22,53,54]$. Moreover, since plants have very large proteomes, the total number of potentially amyloidogenic proteins in several species of plants is greater even than the corresponding number in the human proteome (Table S1a,b).

\subsection{Molecular Functions of Potentially Amyloidogenic Proteins of Plants}

Functional amyloids participate in diverse molecular functions in a wide spectrum of prokaryotic and eukaryotic species $[13,54,55]$. Functional amyloids may be active in the amyloid state $[23-25,28]$ or act as protein or peptide storage reservoirs [26]. Thus, it was important to analyze the molecular functions of the predicted potentially amyloidogenic plant proteins to reveal functions that could be associated with amyloid formation. We searched for Gene Ontology (GO) terms related to molecular functions where potentially amyloidogenic proteins detected by Waltz and SARP are overrepresented. We found that GO terms enriched in proteins harboring ARs predicted by Waltz were drastically different from the terms associated with QN-rich proteins predicted by SARP. For instance, amyloidogenic regions predicted by Waltz were found mostly in transmembrane proteins with transporter activity as well as proteins with motor and kinase activities (Figure 2, Table S1c). Conversely, proteins harboring QN-rich CBRs were mostly associated with transcription, DNA- and RNA-binding activities, and protein oligomerization (Figure 3, Table S1d). Both ARs and QN-rich CBRs-containing proteins shared kinase activity as a function (Figures 2 and 3). Several molecular functions were specific to particular systematic groups. For example, microtubule motor and actin-binding activities were characteristic of Poaceae QN-rich proteins (Figure 3). Notably, QN-rich proteins of approximately two-thirds of the analyzed species were associated with nutrient reservoir activity. Proteins with this function belong mostly to seed storage proteins that are known to be rich in $Q$ and $E$ in several species $[56,57]$. 


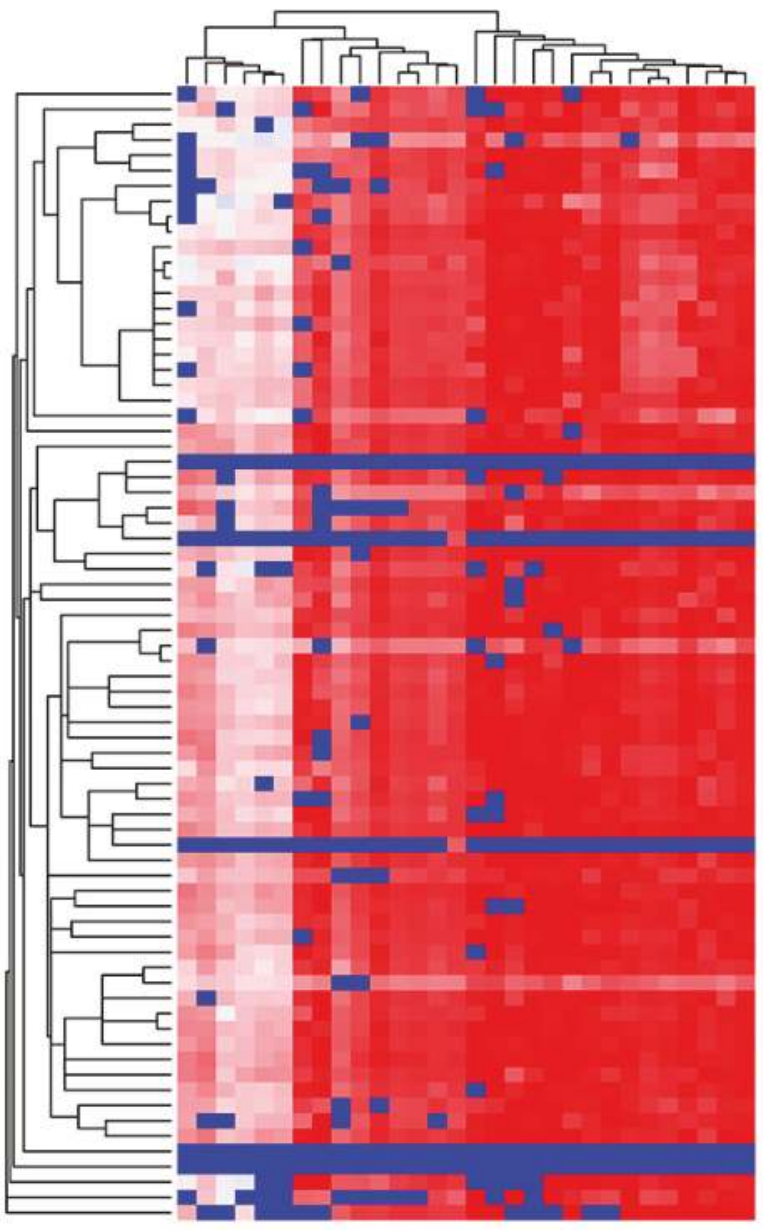

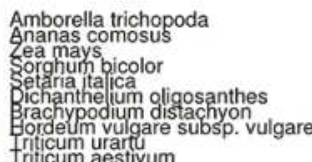

ryza rufipogon

8ryza sativa subsp. jagonica

8 rza barthi

8ryza glaberrima

8ryza meridionalis

8ryza gumipatt

Uusa acuminata subsp. malaccensis

fostera marina aucus carota subsp. sativus

Raucus carota subsp

Nicotiana attenuata

solanum lycopersicum

colanum tuberosum

cripstranthe guttat

spinacia oleracea
sta vulgaris subsp. vulgaris

Eucalyptus grandis

Arabicoosis thaliana

Arabido osis lyrata subsp. lyrata

rrassica oleracea var. oracea

rassca rapa subsp. pekinensis

rassicanapus

Ertrema salsugineum

Citrus clementina

Eitrus sinensis

corchorus olitorius
\&orchous capsularis

cossypium arporeum

Gossypium hirsutum

theobroma cacao

cucumis melo

punus persica

Morus notabilis icularis

coppalotus to

列

chaseolus ann

Yjgna radiata var, radiata

Ciceolus vulgan

Medicago truncatula

upinus angustifolius

populus trichocarp

Natiopha curcas

Sicea glauca

Siyscommirella patens subsp, patens
Marchantia polymorpha subsp. polymorpha
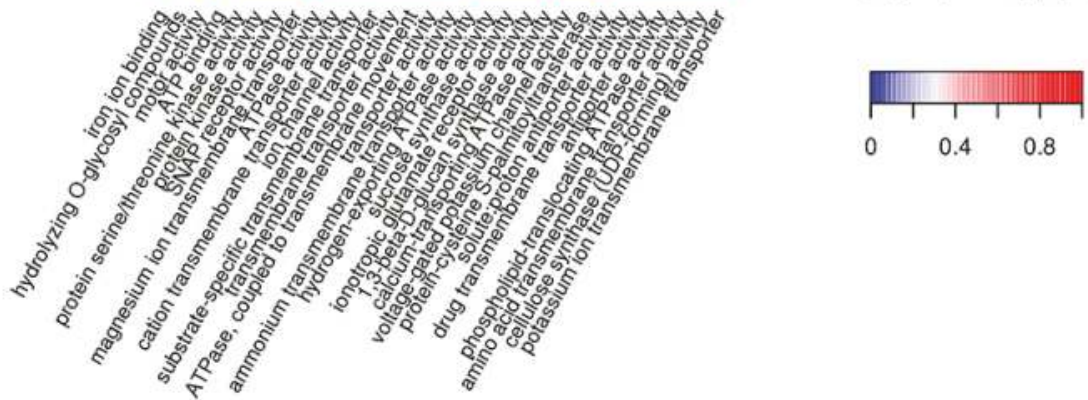

$\begin{array}{lll}0 & 0.4 & 0.8\end{array}$

Figure 2. Heat map of GO molecular functions in which potentially amyloidogenic proteins predicted by Waltz are overrepresented. For such proteins, the top $30 \mathrm{GO}$ terms from the molecular function ontology are shown. The color of the cells denotes the fraction of potentially amyloidogenic proteins predicted by Waltz among all proteins annotated with this term. All cells with $p$-values greater than 0.01 have values of 0 (dark blue). The dendrogram of plant species corresponds to their phylogenetic tree. GO, Gene Ontology. 

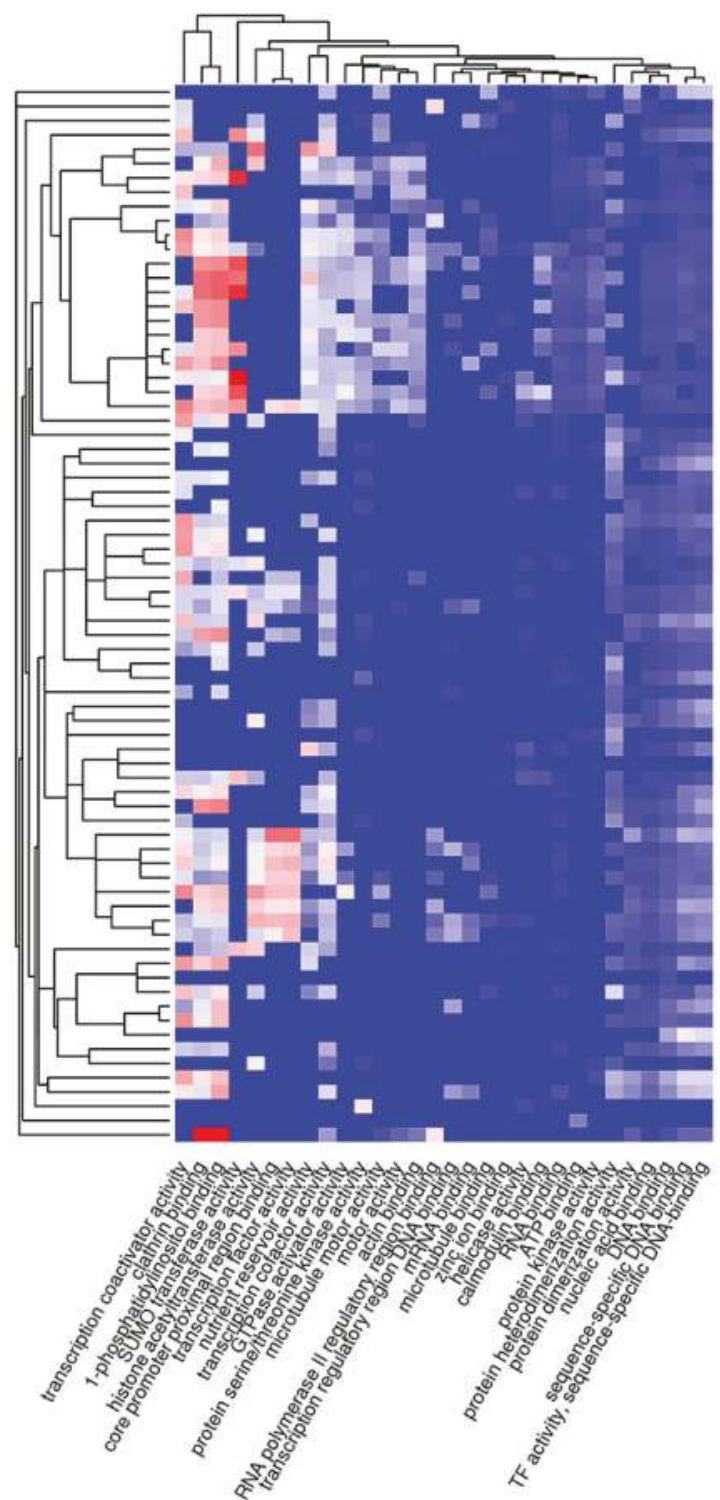

Marchantia polymorpha subsp. polymorpha

Amborella trichopoda

Ananas comosus

Sorghum bicolor

Bichanthe lium oligosanthes

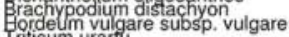

torticum urartí

oryzam olumipatula

8 ryza ufinogon

8ryza gaberima

8ryza nivara subsp. japonica
oryza sativa sua subsp: Indica

oryza punctata

chersia perrienta subsp. malaccensis

ostera marina

ceons notabilitilicularis

cucumis melo

Cucumis sativus

Hiaseolus angtharis

女igna radiata var. radiata

of cine sojan

cy cen anietinum

Medicago truncatula

Ricinus communis

Jatropha curcas

citrus clemeentina

Cirrus csinensis
Eucalyptus grandis

Gossypium arboreum

cossypum hirsutum

corctiorus olitoriys
Corchorus capsularis

theobroma cacao

rassica oleracea var. oleracea

rassca rapa subsp. pekinensis

Crassica napus

Cansella rubella

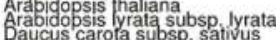

Nicoliana atienuala
Nicotiana sylvestris

Nicotiana tabacum

solanum ycopersicum

Capsicum annuum

Erythranthe guttata

spinacia oleracea

Relumiguaris subs

selaginela mo moellendorffii

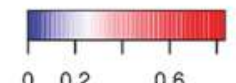

$\begin{array}{lll}0 & 0.2 & 0.6\end{array}$

Figure 3. Heat map of GO molecular functions in which potentially amyloidogenic proteins containing QN-rich CBRs predicted by SARP are overrepresented. For such proteins, the top 30 GO terms from the molecular function ontology are shown. The color of the cells denotes the fraction of potentially amyloidogenic proteins predicted by SARP among all proteins annotated with this term. All cells with $p$-values greater than 0.01 have values of 0 (dark blue). The dendrogram of plant species corresponds to their phylogenetic tree.

Since E-rich proteins are also potentially amyloidogenic, we analyzed GO molecular functions associated with plant proteins containing E-rich CBRs predicted by SARP (Figure S4, Table S1e). Several functions of E-rich proteins were found to be similar to those observed for QN-rich proteins including nucleic acid and clathrin binding. In contrast to QN-rich proteins, in which microtubule 
motor and actin binding activities were typical only for Poaceae proteins, E-rich proteins harboring these functions were characteristic of most plant species analyzed (Figure S4, Table S1e). Some functions, including translation-associated activities and unfolded protein binding, were specific to E-rich proteins (Figure S4). Finally, E-rich proteins with nutrient reservoir activity were abundant in fewer plant species compared to QN-rich proteins (Figure 3 and Figure S4). Thus, the molecular functions of potentially amyloidogenic proteins predicted by Waltz drastically differ from the functions of potentially amyloidogenic QN- and E-rich proteins that are partially similar.

\subsection{Subcellular Localization of Potentially Amyloidogenic Proteins of Plants}

We analyzed distribution of amyloidogenic proteins over different cellular components according to the Gene Ontology database (available at http:/ / www.geneontology.org/). Potentially amyloidogenic proteins harboring ARs predicted by Waltz were found to be associated with different membranes, membrane organelles, myosin and V-type ATPase complexes (Figure S5, Table S1f). Potentially amyloidogenic proteins with QN-rich CBRs were associated with the RNA polymerase II transcription complex, nucleus, RNA-processing complexes, cytoskeleton and clathrin-coated vesicles (Figure S6, Table S1g). Interestingly, QN-rich proteins were abundant among proteins of P-bodies of only Asian species of rice, but not in the African species (Figure S6, Table S1g). Potentially amyloidogenic proteins with E-rich CBRs were associated with the translation machinery complex, cytoskeleton and chromosomes (Figure S7, Table S1h). Overall, the cellular components where different types of potentially amyloidogenic proteins predominate correspond to the molecular functions of these proteins. The general tendency is that potentially amyloidogenic proteins predicted by Waltz have membrane localization, while potentially amyloidogenic proteins with QN- and E-rich CBRs predicted by SARP are mainly cytoplasmic or intranuclear.

\subsection{Biological Processes Implementing Potentially Amyloidogenic Proteins of Plants}

We characterized the molecular functions and subcellular localization of potentially amyloidogenic proteins of different plant species. As a next step, we analyzed biological processes in which potentially amyloidogenic proteins participate. We found that proteins with ARs predicted by Waltz are overrepresented in biological processes associated with transmembrane transport, such as regulation of $\mathrm{pH}$ and ion (sodium, potassium, phosphate) and carbohydrate transport (Figure 4, Table S1i). Among these, there are several processes related to biosynthesis (cellulose and lipid biosynthesis, cell wall modifications) or associated with responses to outer factors (recognition of pollen and defense response). Interestingly, the defense response is a biological process in which Waltz-predicted potentially amyloidogenic proteins are abundant in the majority of plant species, with the exception of most grasses (Figure 4).

The biological processes in which QN-rich potentially amyloidogenic proteins are abundant are mostly related to transcription, cytoskeleton organization and clathrin vesicle formation (Figure 5, Table S1j). Some are connected with the regulation of development, such as the negative regulation of long-day photoperiodism, seed and flower development, auxin, jasmonic and abscisic acid pathways (Figure 5, Table S1j). Overrepresentation of potentially amyloidogenic proteins in some of these processes can only occur in a few species. For example, the flower development process is only associated with QN-rich proteins in several very distant plant species: Arabidopsis spp., Teobroma cacao, Vitis vinifera, Amborella trichopoda and some grasses. Similar to QN-rich proteins, potentially amyloidogenic E-rich proteins are associated with the cytoskeleton and genome organization, as well as RNA processing (Figure S8, Table S1k). However, E-rich proteins are also overrepresented among the translation initiation and folding machinery components (Figure S8, Table S1k). Taken together, QN-rich proteins are similar to E-rich proteins for subcellular localizations, but each of the three groups of potentially amyloidogenic proteins (Waltz-predicted, QN-rich and E-rich) is involved in specific molecular functions and biological processes that only partially overlap. 


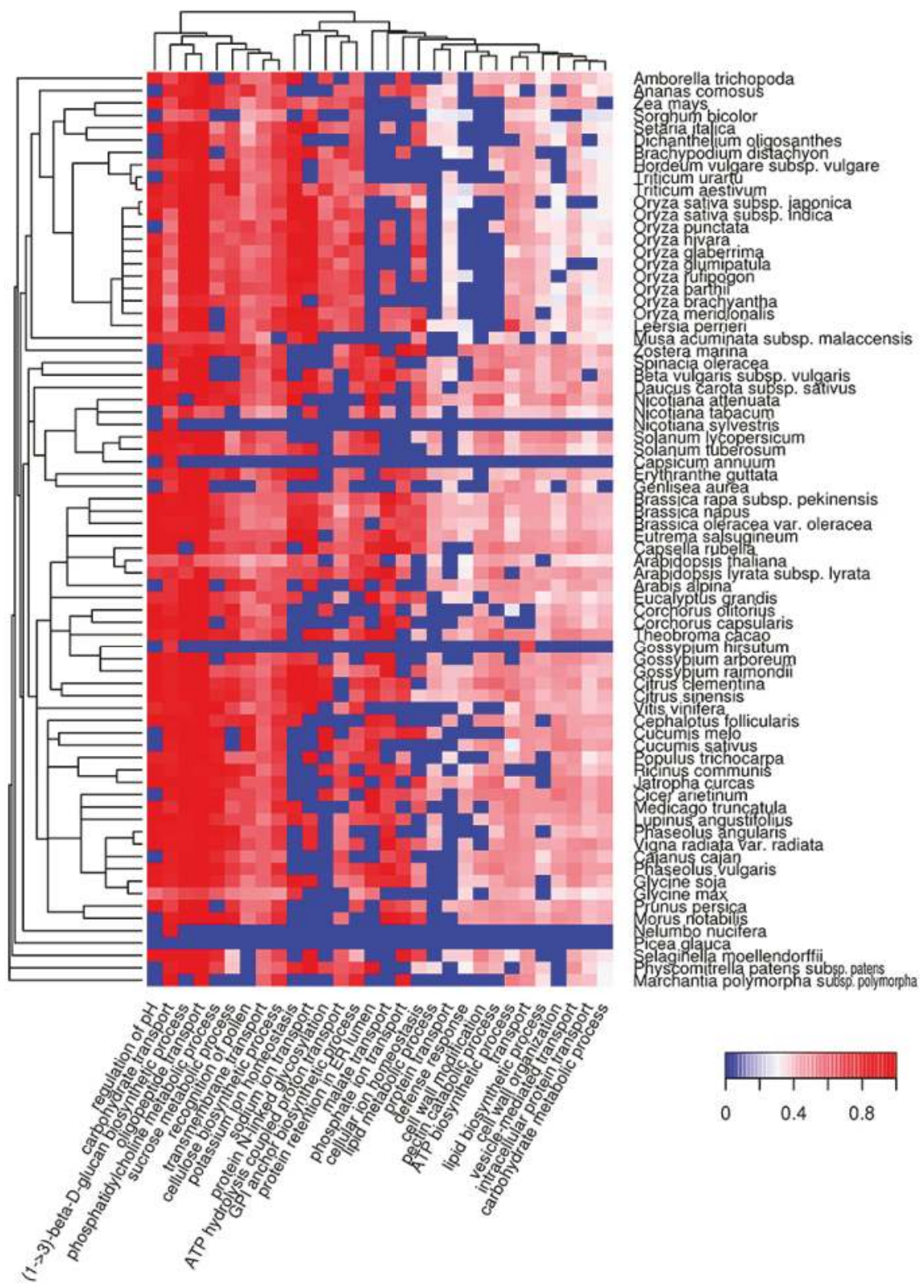

Figure 4. Heat map of GO biological processes in which potentially amyloidogenic proteins predicted by Waltz are overrepresented. For such proteins, the top $30 \mathrm{GO}$ terms from the molecular function ontology are shown. The color of the cells denotes the fraction of potentially amyloidogenic proteins predicted by Waltz among all proteins annotated with this term. All cells with $p$-values greater than 0.01 have values of 0 (dark blue). The dendrogram of plant species corresponds to their phylogenetic tree. 


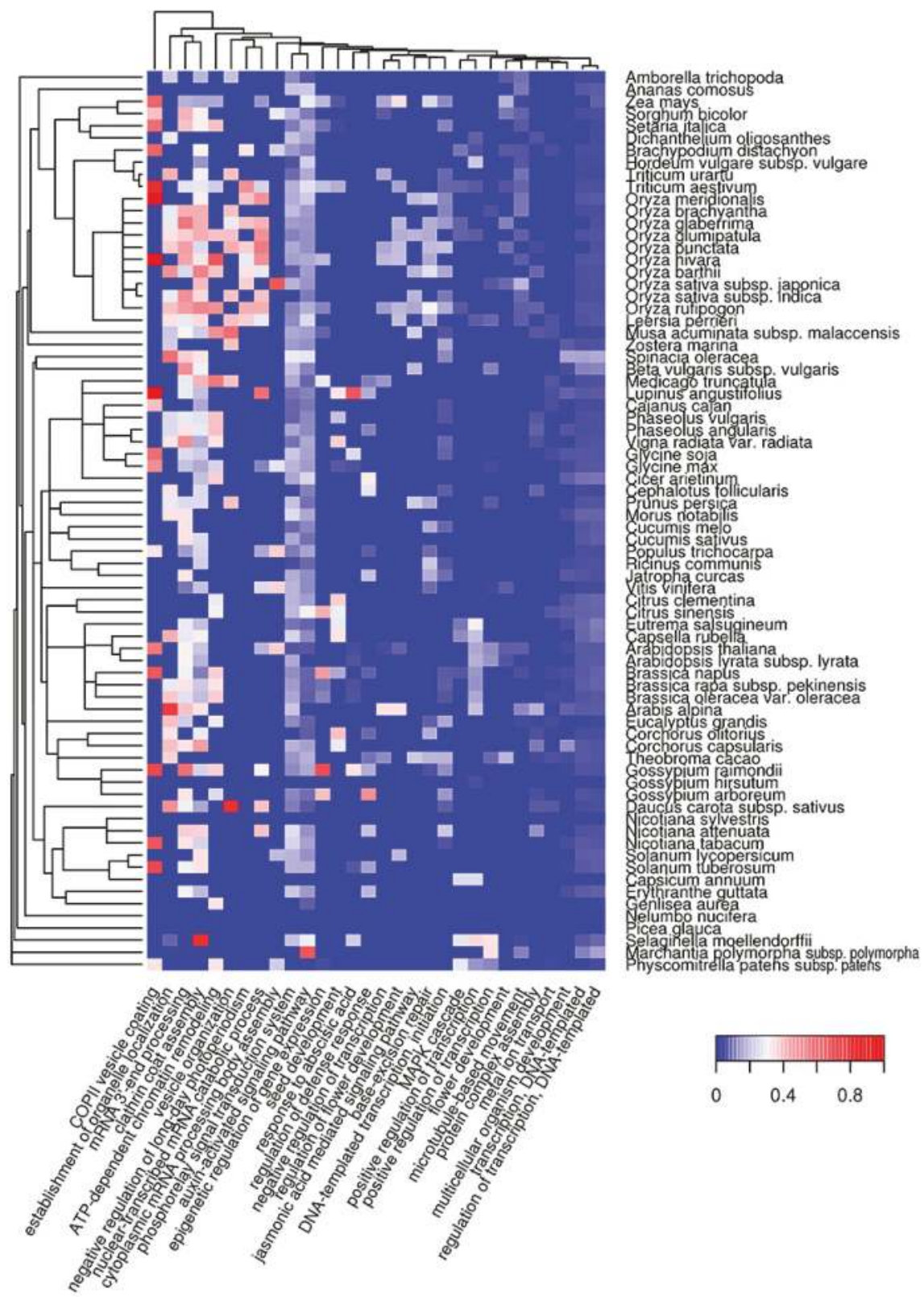

Figure 5. Heat map of GO biological processes in which QN-rich potentially amyloidogenic proteins predicted by SARP are overrepresented. For such proteins, the top $30 \mathrm{GO}$ terms from the molecular function ontology are shown. The color of the cells denotes the fraction of QN-rich potentially amyloidogenic proteins predicted by SARP among all proteins annotated with this term. All cells with $p$-values greater than 0.01 have values of 0 (dark blue). The dendrogram of plant species corresponds to their phylogenetic tree. 


\subsection{Amyloidogenic Proteins in the Chloroplast and Mitochondrial Proteomes of Different Plant Species}

Proteins encoded in the organellar genomes might be very different from proteins encoded in the nuclear genome. Therefore, we separately analyzed the distribution of potentially amyloidogenic proteins among the proteins encoded by the chloroplast and mitochondrial genomes. We found that proteins encoded in the organellar genomes have more regions predicted by Waltz in both the chloroplast and mitochondrion proteomes (Figure $6 a, b$ ) compared to the nuclear genome encoded proteins of the same species (Figure 1). At the same time, only three chloroplast proteins (Figure 6a) and no mitochondrial proteins contained QN-rich regions. These three proteins encoded in the chloroplast genome demonstrate interesting variability in the presence of QN-rich regions. The first is TIC214, the only component of the translocon at the chloroplast inner envelope [58]. It is present in most land plant species with the exception of grasses [59] (Figure 6a) and has a long QN-rich region in its C-terminus. The second chloroplast protein, Ycf2, has a QN-rich region only in Bryophyta (spreading earth moss, Psycomitrella patens) and Pinophyta (white spruce, Picea glauca) species, but not in the flowering plants. The third protein, an omnipresent ribosomal protein of the small subunit, rps18, has a short QN-rich region only in grasses. The QN-rich region of rps18 in many species of grasses was too short to be detected with SARP, but it was validated manually. Taken together, proteins encoded in the organellar genomes are enriched with potentially amyloidogenic proteins predicted by Waltz, while chloroplast QN-rich proteins show evolutionary conservation of their amyloidogenic regions.

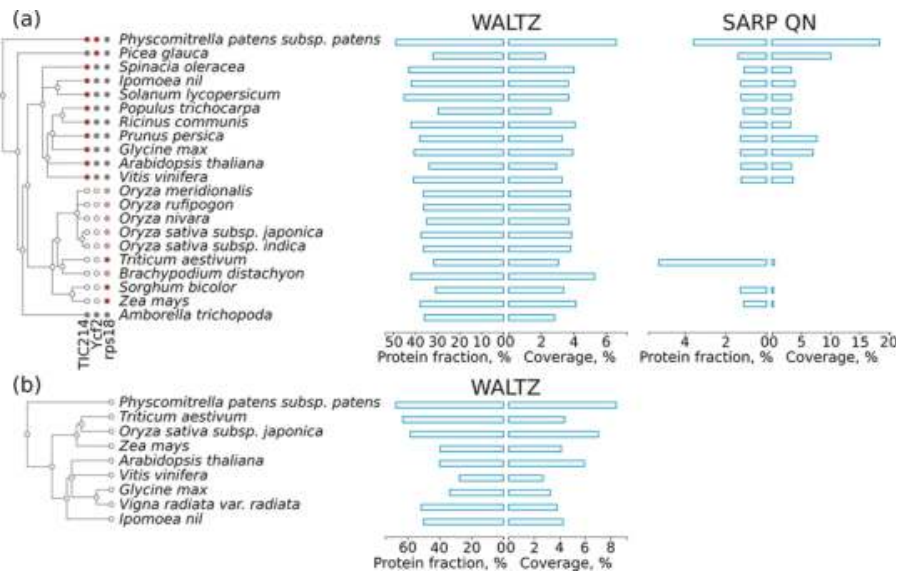

Figure 6. (a) Distribution of chloroplast sequences potentially capable of forming amyloids across land plant proteins. A taxonomic tree of plant species is shown according to the Uniprot Taxonomy. The results for amyloidogenic regions predicted by Waltz and QN-rich sequences found with SARP are shown. For each type of amyloidogenic region, the fraction of proteins harboring these regions and the coverage of the total proteome length with these regions are shown. For the TIC214, Ycf2 and rps18 proteins: (i) a red circle means that the protein is present in the proteome and has a QN-rich region; (ii) a gray circle denotes that the protein is encoded by the chloroplast genome but lacks a QN-rich region; (iii) a white circle denotes that there is no corresponding gene in the chloroplast genome; and (iv) a pink circle denotes that the rps18 protein has a small, manually verified QN-rich region. (b) Distribution of potentially amyloidogenic regions across higher plant proteins encoded by the mitochondrion genome. A taxonomic tree of plant species is shown according to the Uniprot Taxonomy. The results for Waltz-predicted regions are shown. For each type of amyloidogenic region, the fraction of proteins harboring these regions and the coverage of the total proteome length with these regions are shown. The results for QN-rich proteins predicted by SARP are not shown since such proteins are absent in the proteome of the mitochondrion. 


\subsection{Co-Occurrence of Potentially Amyloidogenic Regions with the Structural Features of Proteins}

Potentially amyloidogenic regions have specific amino acid compositions and physical properties, and thus they might tend to be incorporated into certain structural features of proteins. We analyzed co-occurrence of QN-rich regions and regions predicted with Waltz with different types of protein domains. We found that QN-rich regions tend to co-occur with different DNA- (HTH Myb-type) and RNA-binding (YTH, RRM, PUM-HD), kinase (FAT), lipase (GDSL), and cytoskeleton-related domains (Dilute, Myosin, Kinesin) (Figure 7). QN-rich regions were also found to be associated with the LRRNT domain, which is mostly responsible for protein-protein interactions [60]. Importantly, in many plant species, the QN-rich regions overlap with the conserved barrel domain, Cupin1, of the $11 S$ and $7 S$ plant seed storage proteins. For deeper analysis of the association between seed storage protein domains and QN-rich regions, we used PFAM database (see Section 4.7) [61]. We found that 302 storage proteins with Cupin1 were Q/N-rich in 54 of 75 plant species analyzed (Table 1). Q/N-rich storage proteins containing other domains were less abundant. For example, we detected 119 Q/N-rich proteins with Zein domain in three plant species; 121 with Gliadin domain in 15 species; 13 with Vicilin domain in nine species; and seven with high molecular weight Glutenin in two plant species analyzed (Table 1). Taken together, our data show that different seed storage proteins in various plant species are associated with the presence of potentially amyloidogenic Q/N-rich regions.

Similar to QN-rich regions, E-rich regions of plant proteins were mainly enriched with DNA-binding (HMG, SMC) and cytoskeleton-associated (NAB, Kinesin) domains (Figure S9). Additionally, E-rich regions were associated with Helicase and Cactin domains as well as with GTD and FF domains, which are likely responsible for protein-protein interactions (Figure S9). In contrast to QN- and E-rich regions, amyloidogenic regions predicted with Waltz tend to be inside transmembrane domains (EamA, TPT, PBPe, MFS, ABC transmembrane Type-1, etc.) in all plant species analyzed except for P. glauca (Figure 8), which is likely because of incomplete proteome annotation for this species. Signal peptides were strongly associated with ARs predicted by Waltz in all species except grasses (Figure 8). Notably, both QN-rich regions and ARs predicted by Waltz are associated with protein kinase domains (Figures 7 and 8). Thus, amyloidogenic regions occupy specific protein domains (Figures 7 and 8 and Figure S9), which might reflect the involvement of ARs in the functioning of these domains. 


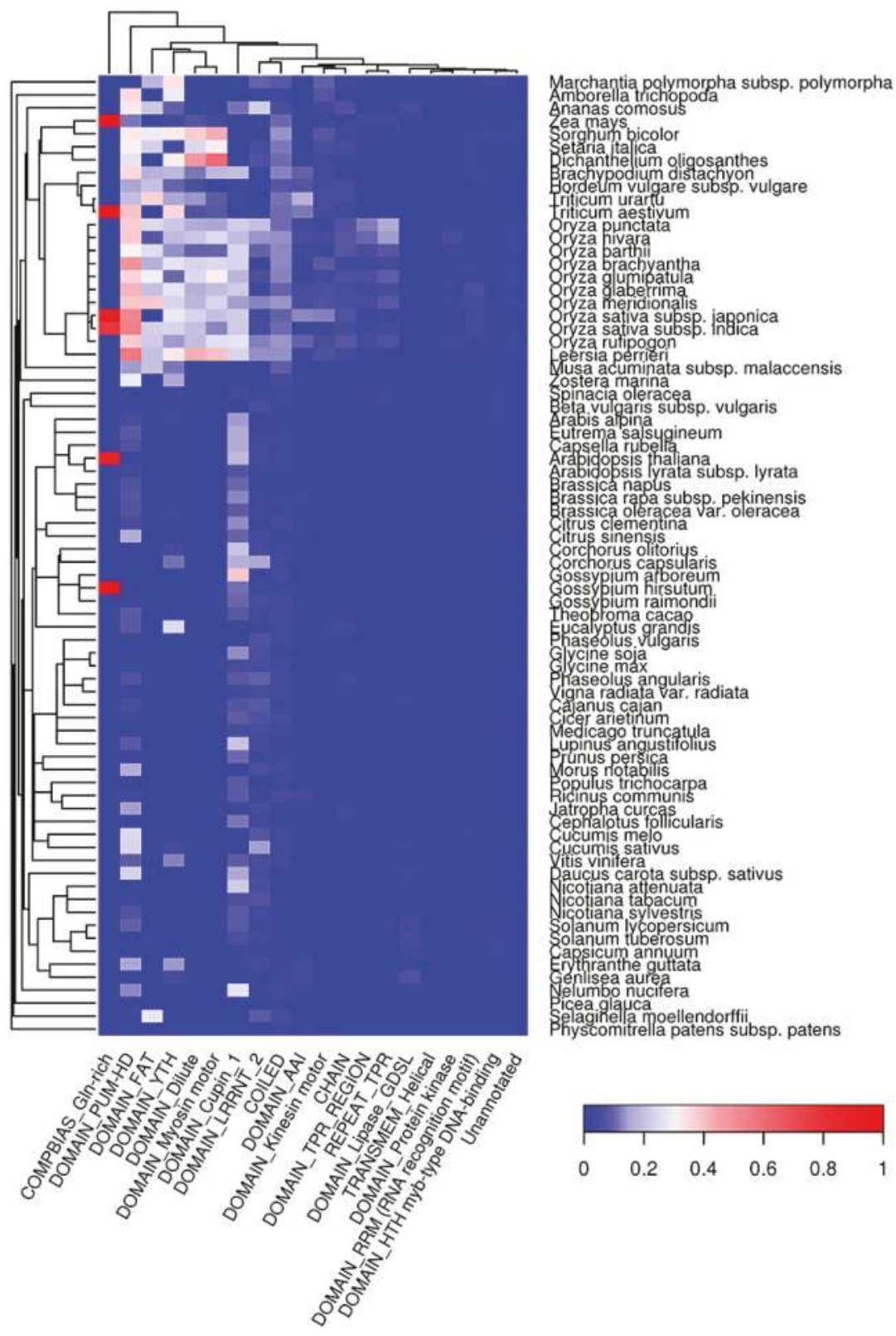

Figure 7. Top 20 protein features that are overrepresented in QN-rich regions predicted with SARP. The color of the cells denotes the fraction of proteins with amyloidogenic regions among all proteins with this feature. The dendrogram of plant species corresponds to their phylogenetic tree. 


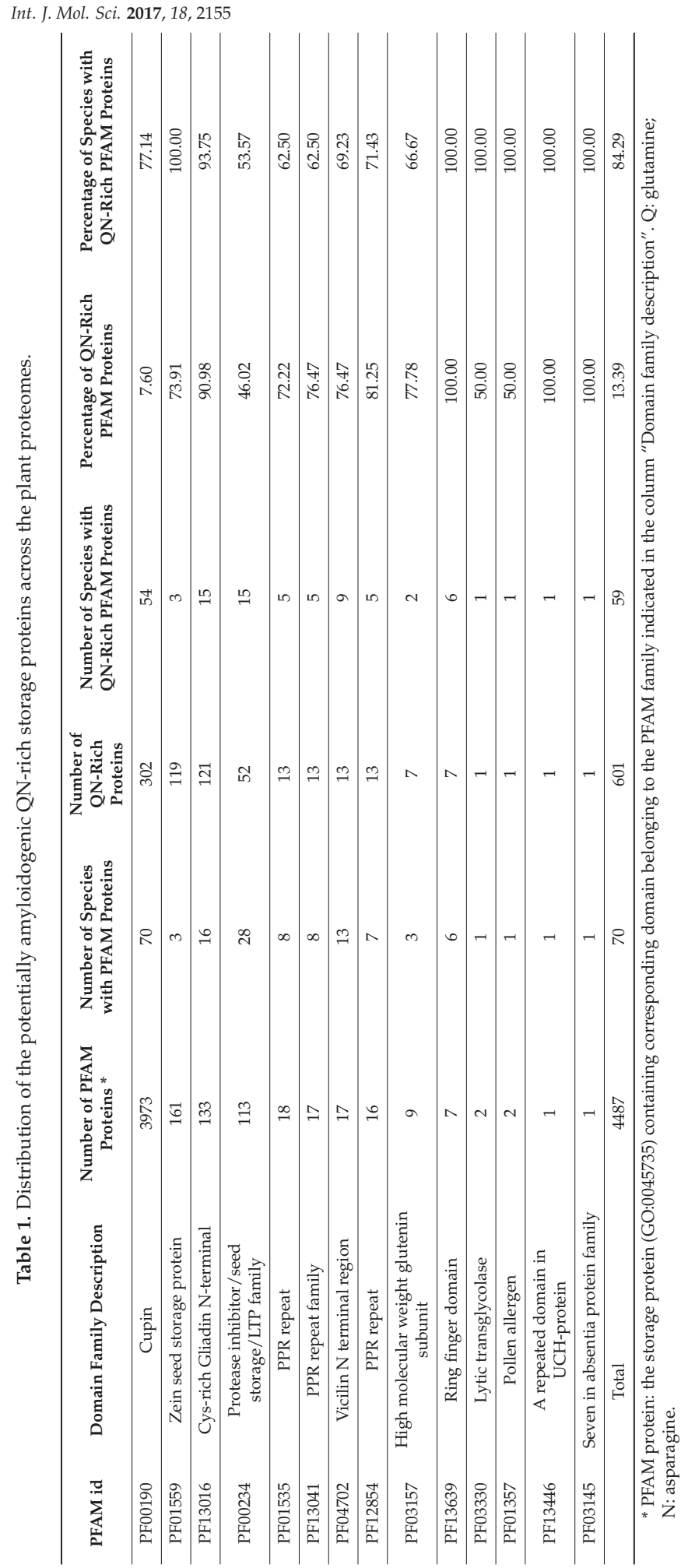




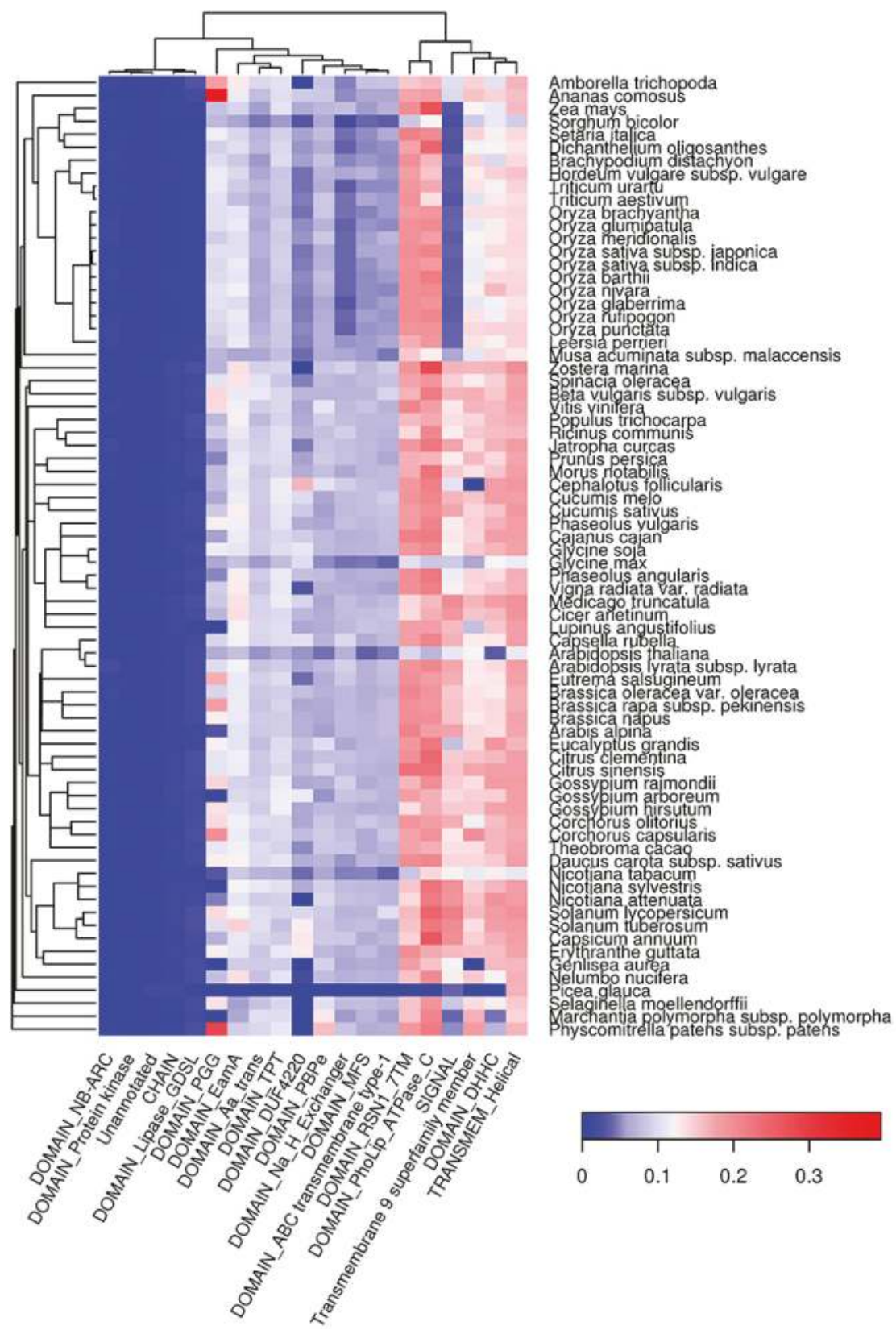

Figure 8. Top 20 protein features that are overrepresented in Waltz-predicted amyloidogenic regions. The color of the cells denotes the fraction of proteins with amyloidogenic regions among all proteins with this feature. The dendrogram of plant species corresponds to their phylogenetic tree. 


\section{Discussion}

The bioinformatic analysis performed in this study revealed that potentially amyloidogenic proteins are abundant in the proteomes of land plants (Figure 1). These proteins exhibit various molecular functions, cellular localizations and biological processes (Figures 2-5). Two algorithms used in our study, Waltz and SARP, revealed different groups of potentially amyloidogenic plant proteins based on their primary structure. Some of these proteins are related to amyloid-forming proteins in other groups of organisms identified in vivo or plant proteins whose amyloid properties were partially characterized in vitro and in heterologous systems.

Most groups of plant proteins predicted by Waltz are transmembrane proteins acting as transporters of different compounds. Such proteins can potentially have amyloid properties. For example, porins OmpA and OmpC of the bacteria Escherichia coli were shown to have amyloid properties $[62,63]$. Thus, we cannot exclude that several membrane proteins of plants could also adopt amyloid structures. The second group of amyloidogenic proteins predicted by Waltz to be abundant in most of the species analyzed were defense proteins. These proteins represent a large and heterogeneous group, many representatives of which are hydrophobic [64]. Interestingly, several plant defense proteins and peptides were shown to have amyloid-like properties in vitro [49,50,65]. Amyloid formation by such plant proteins could stabilize them and enhance their survival during interactions with pathogens, since amyloids are extremely stable [66].

Amyloidogenic proteins of plants predicted with SARP were mainly localized in the nucleus and cytoplasm. In the case of QN-rich plant proteins, DNA- and RNA-binding activities including transcriptional regulation are the most common. There are numerous examples of $Q$ and/or $\mathrm{N}$-rich transcriptional factors among human and yeast amyloid-forming proteins [38]. Moreover, Luminidependens, a QN-rich transcriptional regulator of flowering in Arabidopsis thaliana, was recently shown to have amyloid- and prion-like properties in a heterologous yeast system [46]. We also found that QN-rich proteins are overrepresented among floral regulators, but only in several species including A. thaliana (Figure 5). Overall, according to bioinformatic data, DNA- and RNA-binding QN-rich proteins of plants represent a promising group to search for novel amyloid-forming proteins. The second group of potentially amyloidogenic proteins predicted by SARP was E-rich, which were similar to QN-rich in function and localization, but additionally included translation- and folding-related proteins (Figure S8) that could be involved in amyloid formation.

One of the most important findings of this study was the overrepresentation in different plant species of potentially amyloidogenic proteins among proteins acting as nutrient reservoirs (Figure 3 and Figure S4), including seed storage proteins, which constitute an important part of the human diet. Moreover, the evolutionarily conserved Cupin1 as well as Zein, Gliadin, Vicilin and high molecular weight Glutenin domains of seed storage proteins tend to have potentially amyloidogenic QN-rich regions (Figure 7, Table 1). Previously, proteolytic peptides of seed storage proteins of leguminous plants were shown to form fibrils with several properties of amyloids in vitro [67-69]. Based on these observations, we hypothesized that storage proteins might adopt amyloid states in seeds to accumulate and stabilize their molecules during dehydration that naturally occurs as a result of seed maturation [51]. The data obtained in this study strongly support our hypothesis. We may expect that the process of accumulation of storage proteins in the seeds could be similar to the accumulation of human hormones in the amyloid state [26] or dehydration-dependent amyloid formation by the proteins of egg envelop of "annual killfish" Austrofundulus limnaeus [51,70].

We found that QN-rich proteins were absent in the mitochondria and that few chloroplast proteins contained QN-rich regions (Figure 6). One such protein is TIC214, which harbors a QN-rich region in its C-terminus in all investigated plant species (see Section 2.5). It should be noted that TIC214 is the only translocon component on the inner envelope of chloroplasts that is encoded in the chloroplast genome [59]. Though it is omnipresent in most species of plants (except grasses), the C-terminal region is highly variable. The only common feature of the C-terminal region of TIC214 in different species is the presence of charged motifs [59]. Possibly, an increased QN content might be important for 
interspersing these motifs. Another chloroplast protein, Ycf2, contains a QN-rich region, but not in the flowering plants (Figure 6). The changes in Ycf2 composition coincide with its gene duplication in the flowering plants lineage [71]. The Poaceae species have lost the Ycf1/TIC214 protein, but they have a small QN-rich region in the C-terminal region of the rps18 protein (Figure 6). These examples suggest that the composition of QN-rich regions might correspond with the evolution of species, even when the sequence of such regions is highly variable. Additionally, such a conservation of amino acid composition suggests that CBRs may be functionally important.

Undoubtedly, the presence of bioinformatically predicted amyloidogenic regions does not indicate that the corresponding full-length proteins have amyloid properties in vivo. Nevertheless, resistance of proteins to treatment with ionic detergents, which is one of the key properties of amyloids, correlates with the presence of ARs predicted by WALTZ and CBRs predicted by SARP [72], and the most of experimentally analyzed amyloidogenic plant proteins (LD, FPA, FCA, TGZ, monellin, pro-hevein) [51] bear such regions. Thus, predictions of potentially amyloidogenic proteins with these algorithms are useful not only to analyze molecular functions, subcellular functions, and domain structure of such proteins but also to reveal candidates in plant proteomes for experimental analysis of their amyloid-forming properties. Identification of novel amyloid proteins is laborious and time-consuming, but bioinformatic predictions in combination with recently developed proteomic approaches [72-75] are useful in this regard. In addition, future development of novel, more efficient bioinformatic algorithms based on the machine learning, which is actively using now for protein analysis [76,77], could also contribute to the progress in the proteomics of amyloids.

Overall, in this study, we have investigated the diversity of amyloidogenic proteins in plant species, analyzed their functions and localization, and, based on the obtained bioinformatic data, suggested possible roles of amyloid formation in different biological processes including defense from pathogens and storage of proteins in seeds.

\section{Materials and Methods}

\subsection{Datasets}

All protein sequences of 75 plant species were downloaded with their annotations from the Uniprot Proteomes database (available at http:/ /www.uniprot.org/proteomes/). We used the sequences listed in the reference proteomes for these species in June of 2017. To fetch the data, we used the Proteins REST API (available at http:/ / www.ebi.ac.uk/proteins/api/doc) [78]. Phylogenetic trees of plant species were obtained according to the Uniprot Taxonomy (available at http://www.uniprot. org/taxonomy/). IDs of the proteomes and taxonomies used are listed in Table S2.

\subsection{Prediction of Amyloidogenic Regions}

Prediction of amyloidogenic regions was performed using the Waltz algorithm [37], with parameters set as follows: threshold-best overall selectivity and pH 7.0. Protein sequences that did not match the Waltz requirements (sequence should not contain uncanonical amino acid letters and should not be longer ten thousand residues) were excluded. Proteins harboring at least one region predicted with Waltz longer than 9 amino acids were marked as potentially amyloidogenic proteins. Coverages of Waltz-predicted regions were calculated as follows: total length of all regions predicted by WALTZ divided by sum of lengths of all proteins in the corresponding proteome. A comparison of different species by the portion of potentially amyloidogenic proteins in the proteomes was performed with Fisher's exact test [79] with a Benjamini and Hochberg $p$-value adjustment [80].

\subsection{Prediction of Compositionally Biased Regions}

Prediction of compositionally biased regions (CBRs) in proteins for E, Q and $\mathrm{N}$ amino acids was performed with the SARP algorithm [43]. The threshold of probability was set to $10^{-8}$. Calculations of coverage of CBRs and comparisons of different species by their proportion of compositionally biased 
regions in proteomes were performed as for ARs (see Section 4.1). The proteins were considered potentially amyloidogenic if they harbor at least one CBR rich in $\mathrm{E}$ or $\mathrm{Q}$ and $\mathrm{N}$.

\subsection{GO Term Enrichment Test}

GO term enrichment tests were performed with the topGO R package [81]. Only terms with $p$-values less than 0.01 and at least five proteins in the list of interest were selected. All proteins in the proteome for each species were used as the protein universe, and only proteins with predicted amyloidogenic regions or compositionally biased regions were included in the list of proteins of interest. The heatmap. 2 function from the gplots package was used to draw heat maps with default clustering functions.

\subsection{Identification of Potentially Amyloidogenic Proteins in the Proteomes of Organelles}

Data on whether proteins were encoded by mitochondrion or chloroplast genomes were obtained from the proteome annotation in the Uniport database. For each set of proteins, amyloidogenic regions were predicted with Waltz (see Section 4.2), and QN-rich CBRs were found with SARP (see Section 4.3). Statistics for the ARs and CBRs were calculated for each set separately, as described in Sections 4.2 and 4.3.

\subsection{Analysis of the Association between Amyloidogenic Regions and Different Protein Features}

Feature annotation was obtained from the Uniprot database. All sequence regions that were not assigned to any feature were marked as unannotated. For each type of feature, the sum of the length of overlaps of all amyloidogenic regions, and amyloidogenic CBRs rich in QN or E with these features were calculated and divided by the total length of features of that type. The distribution of ARs predicted by Waltz over different CBRs was calculated the same way (summing the lengths of all ARs overlapping with CBRs of a given type and dividing by the total length of all CBRs of this type). The heatmap.2 function from the gplots package was used to draw heat maps with default clustering functions.

\subsection{Analysis of the Abundance of the PFAM Domains among Proteins Containing CBRs}

We used PFAM annotation for proteins from Uniprot database (available at http:/ /www.uniprot. org/). The descriptions for PFAM families were fetched from PFAM database [61] (available at http://pfam.xfam.org/). To calculate the abundance of the PFAM domains among proteins with nutrient reservoir activity, we obtained the list of PFAM accessions associated with the proteins with GO:0045735 and calculate the number of proteins from this subset for each PFAM accession. The abundance of the PFAM domains among QN-rich proteins was calculated in the same way, but only proteins with GO:0045735 containing QN-rich regions predicted by SARP were selected. For each PFAM accession, we calculated the number of species in which proteomes proteins with corresponding PFAM domains from given subsets were present.

Supplementary Materials: Supplementary materials can be found at www.mdpi.com/1422-0067/18/10/2155/s1.

Acknowledgments: This work was supported by the Russian Science Foundation (Grant No 17-16-01100).

Author Contributions: Kirill S. Antonets and Anton A. Nizhnikov conceived of and designed the experiments; Kirill S. Antonets and Anton A. Nizhnikov performed the experiments; Kirill S. Antonets and Anton A. Nizhnikov analyzed the data; and Kirill S. Antonets and Anton A. Nizhnikov wrote the paper.

Conflicts of Interest: The authors declare no conflicts of interest. The founding sponsors had no role in the design of the study, in the collection, analyses, or interpretation of data, or in the writing of the manuscript and the decision to publish the results. 


\section{Abbreviations}

AR Amyloidogenic region

SARP Sequence Analysis Based on the Ranking of Probabilities

CBR compositionally biased region

\section{References}

1. Sipe, J.D.; Cohen, A.S. Review: History of the amyloid fibril. J. Struct. Biol. 2000, 130, 88-98. [CrossRef] [PubMed]

2. Eanes, E.D.; Glenner, G.G. X-ray diffraction studies on amyloid filaments. J. Histochem. Cytochem. 1968, 16, 673-677. [CrossRef] [PubMed]

3. Tycko, R.; Wickner, R.B. Molecular structures of amyloid and prion fibrils: Consensus versus controversy. Acc. Chem. Res. 2013, 46, 1487-1496. [CrossRef] [PubMed]

4. Selkoe, D.J.; Ihara, Y.; Salazar, F.J. Alzheimer's disease: Insolubility of partially purified paired helical filaments in sodium dodecyl sulfate and urea. Science 1982, 215, 1243-1245. [CrossRef] [PubMed]

5. Hazeki, N.; Tukamoto, T.; Goto, J.; Kanazawa, I. Formic acid dissolves aggregates of an N-terminal huntingtin fragment containing an expanded polyglutamine tract: Applying to quantification of protein components of the aggregates. Biochem. Biophys. Res. Commun. 2000, 277, 386-393. [CrossRef] [PubMed]

6. Bolton, D.C.; McKinley, M.P.; Prusiner, S.B. Identification of a protein that purifies with the scrapie prion. Science 1982, 218, 1309-1311. [CrossRef] [PubMed]

7. Kyle, R.A. Amyloidosis: A convoluted story. Br. J. Haematol. 2001, 114, 529-538. [CrossRef] [PubMed]

8. Virchow, R. Ueber eine im Gehirn und Ruckenmark des Menschen aufgefunde Substanz mit der chemishen Reaction der Cellulose. Virchows Arch. Path. Anat. Physiol. 1854, 6, 135-138. [CrossRef]

9. Friedreich, N.; Kekule, F.A. Zur Amyloidfrage. Virchows Arch. Path. Anat. Physiol. 1859, 16, 50-65. [CrossRef]

10. Buxbaum, J.N.; Linke, R.P. A molecular history of the amyloidoses. J. Mol. Biol. 2012, 421, 142-159. [CrossRef] [PubMed]

11. Chiti, F.; Dobson, C.M. Protein Misfolding, Amyloid Formation, and Human Disease: A Summary of Progress Over the Last Decade. Annu. Rev. Biochem. 2017, 86, 27-68. [CrossRef] [PubMed]

12. Sipe, J.D.; Benson, M.D.; Buxbaum, J.N.; Ikeda, S.; Merlini, G.; Saraiva, M.J.; Westermark, P. Nomenclature 2014: Amyloid fibril proteins and clinical classification of the amyloidosis. Amyloid 2014, 21, 221-224. [CrossRef] [PubMed]

13. Pham, C.L.L.; Kwan, A.H.; Sunde, M. Functional amyloid: Widespread in nature, diverse in purpose. Essays Biochem. 2014, 56, 207-219. [CrossRef] [PubMed]

14. Kelly, J.W.; Balch, W.E. Amyloid as a natural product. J. Cell Biol. 2003, 161, 461-462. [CrossRef] [PubMed]

15. Chapman, M.R.; Robinson, L.S.; Pinkner, J.S.; Roth, R.; Heuser, J.; Hammar, M.; Normark, S.; Hultgren, S.J. Role of Escherichia coli curli operons in directing amyloid fiber formation. Science 2002, 295, 851-855. [CrossRef] [PubMed]

16. Bieler, S.; Estrada, L.; Lagos, R.; Baeza, M.; Castilla, J.; Soto, C. Amyloid formation modulates the biological activity of a bacterial protein. J. Biol. Chem. 2005, 280, 26880-26885. [CrossRef] [PubMed]

17. Claessen, D.; Rink, R.; De Jong, W.; Siebring, J.; De Vreugd, P.; Boersma, F.G.H.; Dijkhuizen, L.; Wosten, H.A.B. A novel class of secreted hydrophobic proteins is involved in aerial hyphae formation in Streptomyces coelicolor by forming amyloid-like fibrils. Genes Dev. 2003, 17, 1714-1726. [CrossRef] [PubMed]

18. Chimileski, S.; Franklin, M.J.; Papke, R.T. Biofilms formed by the archaeon Haloferax volcanii exhibit cellular differentiation and social motility, and facilitate horizontal gene transfer. BMC Biol. 2014, 12, 65. [CrossRef] [PubMed]

19. Dueholm, M.S.; Larsen, P.; Finster, K.; Stenvang, M.R.; Christiansen, G.; Vad, B.S.; Boggild, A.; Otzen, D.E.; Nielsen, P.H. The tubular sheaths encasing methanosaeta thermophila filaments are functional amyloids. J. Biol. Chem. 2015, 290, 20590-20600. [CrossRef] [PubMed]

20. Coustou, V.; Deleu, C.; Saupe, S.; Begueret, J. The protein product of the het-s heterokaryon incompatibility gene of the fungus Podospora anserina behaves as a prion analog. Proc. Natl. Acad. Sci. USA 1997, 94, 9773-9778. [CrossRef] [PubMed]

21. Holmes, D.L.; Lancaster, A.K.; Lindquist, S.; Halfmann, R. Heritable remodeling of yeast multicellularity by an environmentally responsive prion. Cell 2013, 153, 153-165. [CrossRef] [PubMed] 
22. Gebbink, M.F.B.G.; Claessen, D.; Bouma, B.; Dijkhuizen, L.; Wösten, H.A.B. Amyloids-A functional coat for microorganisms. Nat. Rev. Microbiol. 2005, 3, 333-341. [CrossRef] [PubMed]

23. Si, K.; Giustetto, M.; Etkin, A.; Hsu, R.; Janisiewicz, A.M.; Miniaci, M.C.; Kim, J.H.; Zhu, H.; Kandel, E.R. A neuronal isoform of cpeb regulates local protein synthesis and stabilizes synapse-specific long-term facilitation in aplysia. Cell 2003, 115, 893-904. [CrossRef]

24. Majumdar, A.; Cesario, W.C.; White-Grindley, E.; Jiang, H.; Ren, F.; Khan, M.R.; Li, L.; Choi, E.M.L.; Kannan, K.; Guo, F.; et al. Critical role of amyloid-like oligomers of Drosophila Orb2 in the persistence of memory. Cell 2012, 148, 515-529. [CrossRef] [PubMed]

25. Fowler, D.M.; Koulov, A.V.; Alory-Jost, C.; Marks, M.S.; Balch, W.E.; Kelly, J.W. Functional amyloid formation within mammalian tissue. PLoS Biol. 2006, 4, e6. [CrossRef] [PubMed]

26. Maji, S.K.; Perrin, M.H.; Sawaya, M.R.; Jessberger, S.; Vadodaria, K.; Rissman, R.A.; Singru, P.S.; Nilsson, K.P.; Simon, R.; Schubert, D.; et al. Functional amyloids as natural storage of peptide hormones in pituitary secretory granules. Science 2009, 325, 328-332. [CrossRef] [PubMed]

27. Carneiro, K.M.M.; Zhai, H.; Zhu, L.; Horst, J.A.; Sitlin, M.; Nguyen, M.; Wagner, M.; Simpliciano, C.; Milder, M.; Chen, C.-L.; et al. Amyloid-like ribbons of amelogenins in enamel mineralization. Sci. Rep. 2016, 6, 23105. [CrossRef] [PubMed]

28. Cai, X.; Chen, J.; Xu, H.; Liu, S.; Jiang, Q.X.; Halfmann, R.; Chen, Z.J. Prion-like polymerization underlies signal transduction in antiviral immune defense and inflammasome activation. Cell 2014, 156, 1207-1222. [CrossRef] [PubMed]

29. Teng, P.K.; Eisenberg, D. Short protein segments can drive a non-fibrillizing protein into the amyloid state. Protein Eng. Des. Sel. 2009, 22, 531-536. [CrossRef] [PubMed]

30. Von Bergen, M.; Friedhoff, P.; Biernat, J.; Heberle, J.; Mandelkow, E.M.; Mandelkow, E. Assembly of tau protein into Alzheimer paired helical filaments depends on a local sequence motif ((306)VQIVYK(311)) forming $\beta$ structure. Proc. Natl. Acad. Sci. USA 2000, 97, 5129-5134. [CrossRef] [PubMed]

31. López de la Paz, M.; Serrano, L. Sequence determinants of amyloid fibril formation. Proc. Natl. Acad. Sci. USA 2004, 101, 87-92. [CrossRef] [PubMed]

32. Esteras-Chopo, A.; Serrano, L.; López de la Paz, M. The amyloid stretch hypothesis: Recruiting proteins toward the dark side. Proc. Natl. Acad. Sci. USA 2005, 102, 16672-16677. [CrossRef] [PubMed]

33. Kadnar, M.L.; Articov, G.; Derkatch, I.L. Distinct type of transmission barrier revealed by study of multiple prion determinants of Rnq1. PLoS Genet. 2010, 6, e1000824. [CrossRef] [PubMed]

34. Das, S.; Pal, U.; Das, S.; Bagga, K.; Roy, A.; Mrigwani, A.; Maiti, N.C. Sequence complexity of amyloidogenic regions in intrinsically disordered human proteins. PLoS ONE 2014, 9. [CrossRef] [PubMed]

35. Das, A.K.; Pandit, R.; Maiti, S. Effect of amyloids on the vesicular machinery: Implications for somatic neurotransmission. Philos. Trans. R. Soc. Lond. B Biol. Sci. 2015, 370. [CrossRef] [PubMed]

36. Ahmed, A.B.; Kajava, A.V. Breaking the amyloidogenicity code: Methods to predict amyloids from amino acid sequence. FEBS Lett. 2013, 587, 1089-1095. [CrossRef] [PubMed]

37. Maurer-Stroh, S.; Debulpaep, M.; Kuemmerer, N.; Lopez de la Paz, M.; Martins, I.C.; Reumers, J.; Morris, K.L.; Copland, A.; Serpell, L.; Serrano, L.; et al. Exploring the sequence determinants of amyloid structure using position-specific scoring matrices. Nat. Methods 2010, 7, 237-242. [CrossRef] [PubMed]

38. Nizhnikov, A.A.; Antonets, K.S.; Bondarev, S.A.; Inge-Vechtomov, S.G.; Derkatch, I.L. Prions, amyloids, and RNA: Pieces of a puzzle. Prion 2016, 10, 182-206. [CrossRef] [PubMed]

39. Scherzinger, E.; Lurz, R.; Turmaine, M.; Mangiarini, L.; Hollenbach, B.; Hasenbank, R.; Bates, G.P.; Davies, S.W.; Lehrach, H.; Wanker, E.E. Huntingtin-encoded polyglutamine expansions form amyloid-like protein aggregates in vitro and in vivo. Cell 1997, 90, 549-558. [CrossRef]

40. Michelitsch, M.D.; Weissman, J.S. A census of glutamine/asparagine-rich regions: Implications for their conserved function and the prediction of novel prions. Proc. Natl. Acad. Sci. USA 2000, 97, 11910-11915. [CrossRef] [PubMed]

41. Colaco, M.; Park, J.; Blanch, H. The kinetics of aggregation of poly-glutamic acid based polypeptides. Biophys. Chem. 2008, 136, 74-86. [CrossRef] [PubMed]

42. Harrison, P.M.; Gerstein, M. A method to assess compositional bias in biological sequences and its application to prion-like glutamine/asparagine-rich domains in eukaryotic proteomes. Genome Biol. 2003, 4, R40. [CrossRef] [PubMed] 
43. Antonets, K.S.; Nizhnikov, A.A. SARP: A novel algorithm to assess compositional biases in protein sequences. Evol. Bioinform. Online 2013, 9, 263-273. [CrossRef] [PubMed]

44. Beerten, J.; Van Durme, J.; Gallardo, R.; Capriotti, E.; Serpell, L.; Rousseau, F.; Schymkowitz, J. WALTZ-DB: A benchmark database of amyloidogenic hexapeptides. Bioinformatics 2014, 31, 1698-1700. [CrossRef] [PubMed]

45. Alberti, S.; Halfmann, R.; King, O.; Kapila, A.; Lindquist, S. A systematic survey identifies prions and illuminates sequence features of prionogenic proteins. Cell 2009, 137, 146-158. [CrossRef] [PubMed]

46. Chakrabortee, S.; Kayatekin, C.; Newby, G.A.; Mendillo, M.L.; Lancaster, A.; Lindquist, S. Luminidependens (LD) is an Arabidopsis protein with prion behavior. Proc. Natl. Acad. Sci. USA 2016, 113, 6065-6070. [CrossRef] [PubMed]

47. Yang, W.; Willemse, J.; Sawyer, E.B.; Lou, F.; Gong, W.; Zhang, H.; Gras, S.L.; Claessen, D.; Perrett, S. The propensity of the bacterial rodlin protein RdlB to form amyloid fibrils determines its function in Streptomyces coelicolor. Sci. Rep. 2017, 7, 42867. [CrossRef] [PubMed]

48. Macindoe, I.; Kwan, A.H.; Ren, Q.; Morris, V.K.; Yang, W.; Mackay, J.P.; Sunde, M. Self-assembly of functional, amphipathic amyloid monolayers by the fungal hydrophobin EAS. Proc. Natl. Acad. Sci. USA 2012, 109, 5152-5153. [CrossRef] [PubMed]

49. Gour, S.; Kaushik, V.; Kumar, V.; Bhat, P.; Yadav, S.C.; Yadav, J.K. Antimicrobial peptide (Cn-AMP2) from liquid endosperm of Cocos nucifera forms amyloid-like fibrillar structure. J. Pept. Sci. 2016, 22, 201-207. [CrossRef] [PubMed]

50. Berthelot, K.; Lecomte, S.; Coulary-Salin, B.; Bentaleb, A.; Peruch, F. Hevea brasiliensis prohevein possesses a conserved C-terminal domain with amyloid-like properties in vitro. Biochim. Biophys. Acta 2016, 1864, 388-399. [CrossRef] [PubMed]

51. Antonets, K.S.; Nizhnikov, A.A. Amyloids and prions in plants: Facts and perspectives. Prion 2017, 11, 300-312. [CrossRef] [PubMed]

52. Wendel, J.F. New World tetraploid cottons contain Old World cytoplasm. Proc. Natl. Acad. Sci. USA 1989, 86, 4132-4136. [CrossRef] [PubMed]

53. Wickner, R.B.; Shewmaker, F.P.; Bateman, D.A.; Edskes, H.K.; Gorkovskiy, A.; Dayani, Y.; Bezsonov, E.E. Yeast prions: Structure, biology, and prion-handling systems. Microbiol. Mol. Biol. Rev. 2015, 79, 1-17. [CrossRef] [PubMed]

54. Nizhnikov, A.A.; Antonets, K.S.; Inge-Vechtomov, S.G. Amyloids: From pathogenesis to function. Biochemistry 2015, 80, 1127-1144. [CrossRef] [PubMed]

55. Fowler, D.M.; Koulov, A.V.; Balch, W.E.; Kelly, J.W. Functional amyloid-From bacteria to humans. Trends Biochem. Sci. 2007, 32, 217-224. [CrossRef] [PubMed]

56. Balakireva, A.V.; Zamyatnin, A.A. Properties of gluten intolerance: Gluten structure, evolution, pathogenicity and detoxification capabilities. Nutrients 2016, 8, 644. [CrossRef] [PubMed]

57. Jackson, P.; Boulter, D.; Thurman, D.A. A comparison of some properties of vicilin and legumin isolated from seeds of Pisum sativum, Vicia faba and Cicer arietinum. New Phytol. 1969, 68, 25-33. [CrossRef]

58. Kikuchi, S.; Bédard, J.; Hirano, M.; Hirabayashi, Y.; Oishi, M.; Imai, M.; Takase, M.; Ide, T.; Nakai, M. Uncovering the protein translocon at the chloroplast inner envelope membrane. Science 2013, 339, 571-574. [CrossRef] [PubMed]

59. De Vries, J.; Sousa, F.L.; Bölter, B.; Soll, J.; Gould, S.B. YCF1: A Green TIC? Plant Cell 2015, 27, 1827-1833. [CrossRef] [PubMed]

60. Kobe, B.; Kajava, A.V. The leucine-rich repeat as a protein recognition motif. Curr. Opin. Struct. Biol. 2001, 11, 725-732. [CrossRef]

61. Finn, R.D.; Coggill, P.; Eberhardt, R.Y.; Eddy, S.R.; Mistry, J.; Mitchell, A.L.; Potter, S.C.; Punta, M.; Qureshi, M.; Sangrador-Vegas, A.; et al. The Pfam protein families database: Towards a more sustainable future. Nucleic Acids Res. 2016, 44, D279-D285. [CrossRef] [PubMed]

62. Danoff, E.J.; Fleming, K.G. Aqueous, Unfolded OmpA forms amyloid-like fibrils upon self-association. PLoS ONE 2015, 10. [CrossRef] [PubMed]

63. Joseph Sahaya Rajan, J.; Chinnappan Santiago, T.; Singaravel, R.; Ignacimuthu, S. Outer membrane protein C $(\mathrm{OmpC})$ of Escherichia coli induces neurodegeneration in mice by acting as an amyloid. Biotechnol. Lett. 2016, 38, 689-700. [CrossRef] [PubMed] 
64. Nawrot, R.; Barylski, J.; Nowicki, G.; Broniarczyk, J.; Buchwald, W.; Goździcka-Józefiak, A. Plant antimicrobial peptides. Folia Microbiol. 2014, 59, 181-196. [CrossRef] [PubMed]

65. Garvey, M.; Meehan, S.; Gras, S.L.; Schirra, H.J.; Craik, D.J.; van der Weerden, N.L.; Anderson, M.A.; Gerrard, J.A.; Carver, J.A. A radish seed antifungal peptide with a high amyloid fibril-forming propensity. Biochim. Biophys. Acta Proteins Proteom. 2013, 1834, 1615-1623. [CrossRef] [PubMed]

66. Wiggins, R.C. Prion Stability and infectivity in the environment. Neurochem. Res. 2009, 34, 158-168. [CrossRef] [PubMed]

67. Munialo, C.D.; Martin, A.H.; van der Linden, E.; de Jongh, H.H.J. Fibril formation from pea protein and subsequent gel formation. J. Agric. Food Chem. 2014, 62, 2418-2427. [CrossRef] [PubMed]

68. Tang, C.H.; Wang, C.S. Formation and characterization of amyloid-like fibrils from soy $\beta$-conglycinin and glycinin. J. Agric. Food Chem. 2010, 58, 11058-11066. [CrossRef] [PubMed]

69. Ridgley, D.M.; Ebanks, K.C.; Barone, J.R. Peptide mixtures can self-assemble into large amyloid fibers of varying size and morphology. Biomacromolecules 2011, 12, 3770-3779. [CrossRef] [PubMed]

70. Podrabsky, J.E.; Carpenter, J.F.; Hand, S.C. Survival of water stress in annual fish embryos: Dehydration avoidance and egg envelope amyloid fibers. Am. J. Physiol. Regul. Integr. Comp. Physiol. 2001, 280, R123-R131. [PubMed]

71. Wolf, P.G.; Der, J.P.; Duffy, A.M.; Davidson, J.B.; Grusz, A.L.; Pryer, K.M. The evolution of chloroplast genes and genomes in ferns. Plant Mol. Biol. 2011, 76, 251-261. [CrossRef] [PubMed]

72. Antonets, K.S.; Volkov, K.V.; Maltseva, A.L.; Arshakian, L.M.; Galkin, A.P.; Nizhnikov, A.A. Proteomic analysis of Escherichia coli protein fractions resistant to solubilization by ionic detergents. Biochemistry 2016, 81, 34-46. [CrossRef] [PubMed]

73. Nizhnikov, A.A.; Alexandrov, A.I.; Ryzhova, T.A.; Mitkevich, O.V.; Dergalev, A.A.; Ter-Avanesyan, M.D.; Galkin, A.P. Proteomic screening for amyloid proteins. PLoS ONE 2014, 9, e116003. [CrossRef] [PubMed]

74. Nizhnikov, A.A.; Ryzhova, T.A.; Volkov, K.V.; Zadorsky, S.P.; Sopova, J.V.; Inge-Vechtomov, S.G.; Galkin, A.P. Interaction of Prions Causes Heritable Traits in Saccharomyces cerevisiae. PLOS Genet. 2016, 12, e1006504. [CrossRef] [PubMed]

75. Kryndushkin, D.; Pripuzova, N.; Burnett, B.G.; Shewmaker, F. Non-targeted identification of prions and amyloid-forming proteins from yeast and mammalian cells. J. Biol. Chem. 2013, 288, 27100-27111. [CrossRef] [PubMed]

76. Wan, S.; Duan, Y.; Zou, Q. HPSLPred: An Ensemble Multi-Label Classifier for Human Protein Subcellular Location Prediction with Imbalanced Source. Proteomics 2017, 17. [CrossRef] [PubMed]

77. Liao, Z.; Wang, X.; Zeng, Y.; Zou, Q. Identification of DEP domain-containing proteins by a machine learning method and experimental analysis of their expression in human HCC tissues. Sci. Rep. 2016, 6, 39655. [CrossRef] [PubMed]

78. Nightingale, A.; Antunes, R.; Alpi, E.; Bursteinas, B.; Gonzales, L.; Liu, W.; Luo, J.; Qi, G.; Turner, E.; Martin, M. The Proteins API: Accessing key integrated protein and genome information. Nucleic Acids Res. 2017, 45, W539-W544. [CrossRef] [PubMed]

79. Fisher, R.A. The logic of inductive inference. J. R. Stat. Soc. 1932, 98, 39-82. [CrossRef]

80. Benjamini, Y.; Hochberg, Y. Controlling the False Discovery Rate: A Practical and Powerful Approach to Multiple Testing. J. R. Stat. Soc. Ser. B 1995, 57, 289-300.

81. Alexa, A.; Rahnenfuhrer, J. topGO: Enrichment Analysis for Gene Ontology. R package version 2.28.0. Bioconductor 2016. [CrossRef]

(C) 2017 by the authors. Licensee MDPI, Basel, Switzerland. This article is an open access article distributed under the terms and conditions of the Creative Commons Attribution (CC BY) license (http:/ / creativecommons.org/licenses/by/4.0/). 


\title{
Protein-Protein Interactions Prediction Using a Novel Local Conjoint Triad Descriptor of Amino Acid Sequences
}

\author{
Jun Wang ${ }^{1}$, Long Zhang ${ }^{1}$, Lianyin Jia ${ }^{2}$, Yazhou Ren ${ }^{3}$ and Guoxian Yu ${ }^{1, *}$ \\ 1 College of Computer and Information Science, Southwest University, Chongqing 400715, China; \\ kingjun@swu.edu.cn (J.W.); 18234031968@163.com (L.Z.) \\ 2 College of Information Engineering and Automation, Kunming University of Science and Technology, \\ Kunming 650000, China; jlianyin@163.com \\ 3 SMILE (Statistical Machine Intelligence \& Learning) Lab and Big Data Research Center, School of Computer \\ Science and Engineering, University of Electronic Science and Technology of China, Chengdu 610000, China; \\ yazhou.ren@uestc.edu.cn \\ * Correspondence: gxyu@swu.edu.cn; Tel.: +86-23-6825-4396
}

Received: 9 October 2017; Accepted: 4 November 2017; Published: 8 November 2017

\begin{abstract}
Protein-protein interactions (PPIs) play crucial roles in almost all cellular processes. Although a large amount of PPIs have been verified by high-throughput techniques in the past decades, currently known PPIs pairs are still far from complete. Furthermore, the wet-lab experiments based techniques for detecting PPIs are time-consuming and expensive. Hence, it is urgent and essential to develop automatic computational methods to efficiently and accurately predict PPIs. In this paper, a sequence-based approach called DNN-LCTD is developed by combining deep neural networks (DNNs) and a novel local conjoint triad description (LCTD) feature representation. LCTD incorporates the advantage of local description and conjoint triad, thus, it is capable to account for the interactions between residues in both continuous and discontinuous regions of amino acid sequences. DNNs can not only learn suitable features from the data by themselves, but also learn and discover hierarchical representations of data. When performing on the PPIs data of Saccharomyces cerevisiae, DNN-LCTD achieves superior performance with accuracy as $93.12 \%$, precision as $93.75 \%$, sensitivity as $93.83 \%$, area under the receiver operating characteristic curve (AUC) as $97.92 \%$, and it only needs $718 \mathrm{~s}$. These results indicate DNN-LCTD is very promising for predicting PPIs. DNN-LCTD can be a useful supplementary tool for future proteomics study.
\end{abstract}

Keywords: protein-protein interactions; amino acid sequences; local conjoint triad descriptor; deep neural networks

\section{Introduction}

Protein-protein interactions (PPIs) play critical roles in virtually all cellular processes, including immune response [1], DNA transcription and replication [2], and signal transduction [3]. Therefore, correctly identifying PPIs can not only better elucidate protein functions but also further understand the various biological processes in cells [4-6]. In recent years, biologists take advantage of high-throughput technologies to detect PPIs, such as mass spectrometric (MS), tandem affinity purification (TAP) [7], yeast two-hybrid system ( $\mathrm{Y} 2 \mathrm{H})[8,9]$, and so on. Unfortunately, these wet-lab experiments are costly and labor-intensive, and have a high rate of both false positive and false negative, and limited coverage. Hence, it is extremely imperative to develop reliable computational models to predict PPIs in large scale [10].

So far, a number of computational methods have been developed for the detection of PPIs. Most of these methods are based on the genomic information, such as Gene Ontology and 
annotations [11], phylogenetic profile, and gene fusion [12]. Methods employ 3D structural information of proteins $[13,14]$ and the sequence conservation between interacting proteins [15] also have been reported. However, these methods are heavily dependent on the pre-knowledge of the proteins, such as protein functional domains, structure information of proteins, and physicochemical properties of proteins [16,17]. In other words, all these methods are hardly implementable unless the pre-knowledge about proteins is available. Compared to the abundant data of protein sequences, other types of data including 3D structure, Gene Ontology annotations, and domain-domain interactions of proteins are still limited.

Many researchers have innovated sequence-based methods for detecting PPIs [18-24], and experimental results have shown that the information of the amino acid sequences alone is sufficient to identify new PPIs. Among them, Shen et al. [18] achieved an excellent effect based on support vector machine (SVM). They grouped 20 standard amino acids into 7 classes according to their dipoles, volumes of the side chains, and then employed conjoint triad (CT) method to extract the features information of amino acid sequences based on the classification of amino acids. Next, SVM predictor is used to predict PPIs. Their method yields a high prediction accuracy of $89.3 \%$ on human PPIs. However, it does not consider the neighboring effect and PPIs are almost always occurring in the non-continuous segments of amino acid sequences. Guo et al. [19] developed SVM-based method by using auto covariance (AC) to abstract the feature information in the discontinuous amino acid segments in the sequence, and obtained a perfect result with accuracy as $86.55 \%$ on Saccharomyces cerevisiae (S. cerevisiae). Yang et al. [20] introduced local descriptor (LD) to encode amino acid sequences based on $k$-nearest neighbor $(k N N)$. In this study, they grouped 20 standard amino acids into 7 classes as done by Shen et al. [18]. Then they divided an entire protein sequence into ten segments with varying length and extracted information of each segment. Finally, they applied $k \mathrm{NN}$ to predict PPIs. This $k \mathrm{NN}$ based method achieves prediction accuracy as $86.15 \%$ on S. cerevisiae. You et al. [21] innovated a novel multi-scale continuous and discontinuous (MCD) descriptor based on the LD [20]. In order to discover more information from amino acid sequences, MCD descriptor applies the binary coding scheme to construct varying length segments and abstracts the feature vectors from these segments. Then the minimum redundancy maximum relevancy criterion [25], which can reduce the feature abundance and computation complexity, is used to select an optimal feature subset. Finally, SVM is employed to predict new PPIs. This solution obtains a high accuracy as $91.36 \%$ on $S$. cerevisiae. Recently, Du et al. [22] employed deep neural networks (DNNs), a recently famous and popular machine learning technique, and amphiphilic pseudo amino acid composition (APAAC) [26] to predict new PPIs. They firstly extracted the feature information from two respective amino acid sequences by APAAC, then they took APAAC features of two respective proteins as inputs of two separate DNNs and fused the two DNNs to predict PPIs. Their method obtains an accuracy of $92.5 \%$ on PPIs of S. cerevisiae.

LD descriptor [20] only considers the neighboring effect of adjacent two types of amino acids. Hence, it cannot sufficiently abstract information of neighboring amino acids but can sufficiently discover information of discontinuous segments of the amino acid sequences. On the other hand, CT [18] considers the neighboring effect of adjacent three types of amino acids but ignores the discontinuous information. Given these observations, we combine the advantage of local descriptor [20] and conjoint triad method [18], and introduce a novel feature representation method called local conjoint triad descriptor (LCTD). LCTD can better account for the interactions between sequentially distant but spatially close amino acid residues than LD [20] and CT [18]. DNNs, a recently powerful machine learning technique, can not only reduce the impact of noise in the raw data and automatically extract high-level abstractions, but also have better performance than traditional models $[27,28]$. Inspired by these characteristics of DNNs, we employ DNNs to detect the PPIs based LCTD feature representation of amino acid sequences and introduce an approach called DNN-LCTD. Particularly, DNN-LCTD extracts the feature information of the amino acid sequences by LCTD, then it trains a 3-hidden layers neural network by taking feature sets derived from LCTD as inputs 
and accelerates training by graphics processing unit (GPU). Finally, the learned network is employed to predict new PPIs. We perform experiments on PPIs of S. cerevisiae, DNN-LCTD achieves $93.12 \%$ accuracy, $93.83 \%$ sensitivity, $93.75 \%$ precision, and area under the receiver operating characteristic curve (AUC) as $97.92 \%$, and only uses 718 s. Experimental results on other five independent datasets: Caenorhabditis elegans (4013 interacting pairs), Escherichia coli (6954 interacting pairs), Helicobacter pylori (1420 interacting pairs), Homo sapiens (1412 interacting pairs), and Mus musculus (313 interacting pairs), further demonstrate the effectiveness of DNN-LCTD.

\section{Results and Discussion}

In this section, we briefly introduce the evaluation metrics employed in performance comparison. Then, we provide the recommended configuration of experiments. Finally, we analyze and discuss the experimental results and compare our results with those of other related work.

\subsection{Evaluation Metrics}

To reasonably evaluate the performance of DNN-LCTD, five-fold cross validation is adopted. Cross validation can avoid the overfitting and enhance the generalization performance [29]. Six evaluation metrics are used to quantitatively measure the prediction performance of DNN-LCTD, including overall prediction accuracy (ACC), precision (PE), recall (RE), specificity (SPE), matthews correlation coefficient (MCC), $\mathrm{F}_{1}$ score values, and area under the receiver operating characteristic curve (AUC). They (except AUC) are defined as follows:

$$
\begin{gathered}
\mathrm{ACC}=\frac{\mathrm{TP}+\mathrm{TN}}{\mathrm{TP}+\mathrm{FP}+\mathrm{TN}+\mathrm{FN}} \\
\mathrm{PE}=\frac{\mathrm{TP}}{\mathrm{TP}+\mathrm{FP}} \\
\mathrm{RE}=\frac{\mathrm{TP}}{\mathrm{TP}+\mathrm{FN}} \\
\mathrm{SPE}=\frac{\mathrm{TN}}{\mathrm{TN}+\mathrm{FP}} \\
\mathrm{MCC}=\frac{\mathrm{TP} \times \mathrm{TN}-\mathrm{FP} \times \mathrm{FN}}{\sqrt{(\mathrm{TP}+\mathrm{FP})(\mathrm{TP}+\mathrm{FN})(\mathrm{TN}+\mathrm{FP})(\mathrm{TN}+\mathrm{FN})}} \\
\mathrm{F}_{1}=\frac{2 \mathrm{TP}}{2 \mathrm{TP}+\mathrm{FP}+\mathrm{FN}}
\end{gathered}
$$

where TP (true positive) is the number of the true PPIs that are correctly predicted, the FN (false negative) is the number of the true interacting pairs that are failed to be predicted, TN (true negative) is the number of the true non-interactions protein pairs of that are correctly predicted, FP (false positive) is the number of true non-interactions pairs that are failed to be predicted. MCC is a measure for the quality of binary classification. MCC equal to 0 means completely random prediction, -1 means completely wrong prediction and 1 means perfect prediction. $F_{1}$ score is a harmonic average of precision and recall. A larger $F_{1}$ denotes a better performance. Receiver operating characteristic curve (ROC) can elucidate the diagnostic ability of a binary classifier system by graphical plot. This curve is produced by plotting the true positive rate versus the false positive rate under different thresholds [30,31]. AUC is the area under the ROC curve and its value is widely employed to compare predictors. The larger the value of AUC, the better the predictor is. 


\subsection{Experimental Setup}

DNN-LCTD is implemented on Tensorlfow platform https://www.tensorflow.org. The flowchart of DNN-LCTD is shown in Figure 1. DNN-LCTD firstly encodes the amino acid sequences using the novel LCTD. After that, we train a 3-hidden layers neural network with GPU based on the encoded feature sets. Finally, we apply the learned DNN to predict new PPIs. Hyper-parameters of the DNN model heavily impact the experimental results. Deep learning algorithms have ten or more hyper-parameters to be properly specified, trying all of them is impossible in practice [32]. We summarize the recommended configuration of DNN-LCTD in Table 1. As to the parameters setup of the comparing methods, we use the grid search approach to obtain the optimal parameters. The optimal parameters is shown in Table 2. The details of the parameters of comparing methods are available at http://scikit-learn.org. For Du et al. work [22], there are too many parameters need to be set, the information of parameters can be accessed via http://ailab.ahu.edu.cn:8087/DeepPPI/index.html. All the experiments are carried out on a server with configuration: CentOS 7.3, 256 GB RAM, and Intel Exon E5-2678 v3. DNN-LCTD uses NVIDIA Corporation GK110BGL [Tesla K40c] to accelerate training of DNNs.

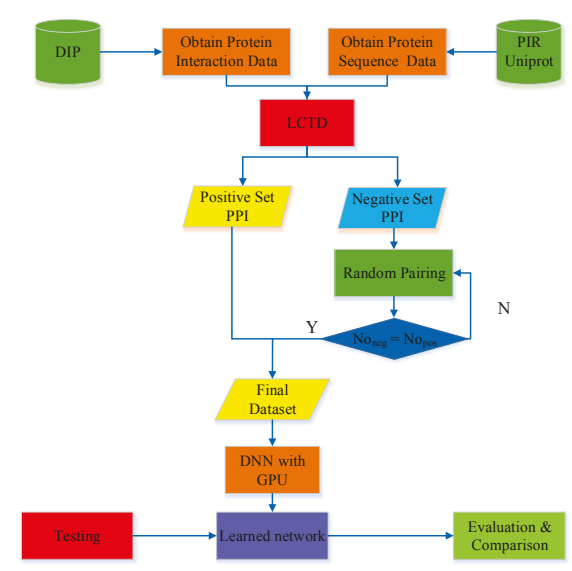

Figure 1. The flowchart of DNN-LCTD for predicting protein-protein interactions. There are some abbreviations in this figure, including database of interacting proteins (DIP), protein information resource, local conjoint triad descriptor (LCTD), protein-protein interactions (PPIs), and graphics processing unit (GPU). The Noneg is the number of non-interacting protein pairs, Nopos is the number of interacting protein pairs. $\mathrm{Y} / \mathrm{N}$ means yes/no.

\subsection{Results on PPIs of S. cerevisiae}

In order to achieve good experimental results, the corresponding hyper-parameters for deep neural network are firstly optimized. Table 1 provides the recommended hyper-parameters that are chosen by a large number of experiments. Considering the numerous samples used in this work, five-fold cross validation is adopted to reduce the impact of data dependency and to minimize the risk of over-fitting. Thus, five models are generated for the five sets of data. Table 3 reports the results of DNN-LCTD on five individual folds (fold 1-5) and the overall average results of five folds. From Table 3, we can observe that all the prediction accuracies are nearly $\geq 93.1 \%$, the precisions are $\geq 93.35 \%$, all the recalls are almost $\geq 93.4 \%$, the specificities are $\geq 92.75 \%$, and the $F_{1}$ are $\geq 92.4 \%$. In order to comprehensively evaluate the performance of DNN-LCTD, the MCC and AUC are also calculated. DNN-LCTD achieves superior prediction performance with an average accuracy as $93.11 \%$, precision as $93.75 \%$, recall as $92.40 \%$, specificity as $92.75 \%$, MCC as $86.24 \%, \mathrm{~F}_{1}$ as $93.06 \%$, and AUC as $97.95 \%$. 
Table 1. Recommended parameters of DNN-LCTD in the experiments.

\begin{tabular}{lll}
\hline Name & Range & Recommendation \\
\hline Learning rate & $1,0.1,0.001,0.002,0.003,0.0001$ & 0.002 \\
\hline Batch size & $32,64,128,256,512,1024$ & 512,1024 \\
\hline Weight initialization & $\begin{array}{l}\text { uniform, normal, lecun_uniform, } \\
\text { glorot_normal, glorot_uniform }\end{array}$ & glorot_normal \\
\hline Per-parameter adaptive learning rate & $\begin{array}{l}\text { SGD, RMSprop, Adagrad, } \\
\text { Adadelta, Adam, Adamax, Nadam }\end{array}$ & Adam \\
\hline Activation function & relu, tanh, sigmoid, softmax, softplus & relu, sigmoid \\
\hline Dropout rate & $0.5,0.6,0.7$ & 0.6 \\
\hline Depth & $2,3,4,5,6,7,8,9$ & 3 \\
\hline Width & $16,32,64,128,256,1024,2048,4096$ & $2048,512,32$ \\
\hline GPU & Yes, No & Yes \\
\hline
\end{tabular}

Table 2. Optimal parameters of comparing methods.

\begin{tabular}{cccccc}
\hline Method & Name & \multicolumn{4}{c}{ Parameters } \\
\hline \multirow{2}{*}{ Guo's work [19] } & SVM + AC & C & \multicolumn{2}{c}{$\gamma$} & kernel \\
& & n2768.0 & \multicolumn{2}{c}{0.074325444687670064} & poly \\
Yang's work [20] & kNN + LD & n_nighbors & weights & algorithm & $\mathrm{p}$ \\
& & 3 & distance & auto & 1 \\
Zhou's work [33] & SVM + LD & C.1748021 & 0.07432544468767006 & rbf \\
& & n_estimators & max_features & criterion & bootstrap \\
You's work [21] & RF + MCD & 5000 & auto & gini & True \\
\hline
\end{tabular}

SVM: support vector machine, $k \mathrm{NN}$ : $k$-nearest neighbor, RF: random forest, AC: auto covariance, LD: local descriptor, MCD: multi-scale continuous and discontinuous, rbf: radical basis function, gini: gini index.

Plenty sequence-based methods have been employed to predict PPIs. We compare the prediction performance of DNN-LCTD with the other existing approaches on S. cerevisiae, including Guo et al. [19], Yang et al. [20], Zhou et al. [33], You et al. [21], and Du et al. [22]. The details of these approaches were introduced in Section 1. From Table 3, we can observe that DeepPPI [22] achieves the best performance among comparing methods (except DNN-LCTD). DeepPPI firstly uses APAAC descriptor to encode the amino acid sequence for each protein and takes the APAAC features as separate inputs for two individual DNNs to extract high-level features of these two proteins, it finally fuses the extracted features to predict PPIs. Its average prediction accuracy is $92.58 \% \pm 0.38 \%$, precision is $94.21 \% \pm 0.45 \%$, recall is $90.95 \% \pm 0.41 \%$, MCC is $85.41 \% \pm 0.76 \%, \mathrm{~F}_{1}$ is $92.55 \% \pm 0.39 \%$, and AUC is $97.55 \% \pm 0.16 \%$. This result mean that DeepPPI [22] is indeed successful for predicting new PPIs using DNNs with APAAC [26]. DNN-LCTD encodes the amino acid sequences of each protein via LCTD descriptor, it then concatenates the LCTD features of two proteins into a longer feature vector and takes the concatenated features as inputs of DNN for prediction. The average accuracy, recall, MCC, $\mathrm{F}_{1}$ and AUC of DNN-LCTD are $0.53 \%, 1.45 \%, 0.83 \%, 1.05 \%$ and $0.4 \%$ higher than those of DeepPPI, respectively. The reason is that LCTD can discover more feature information from amino acid sequences than APAAC. The DNN-LCTD is far greater than other comparing approaches can be attributed to the merits of DNNs and of LCTD. The contributions of LCTD and DNNs will be further investigated in Sections 2.4 and 2.5. The S. cerevisiae dataset contains tremendous samples, hence, a little improvement in prediction performance still has a great effect. Based on these experimental results, we can conclude that DNN-LCTD can more effectively predict PPIs than other comparing methods, and the proposed LCTD descriptor can explore more patterns from continuous and discontinuous amino acid segments. 


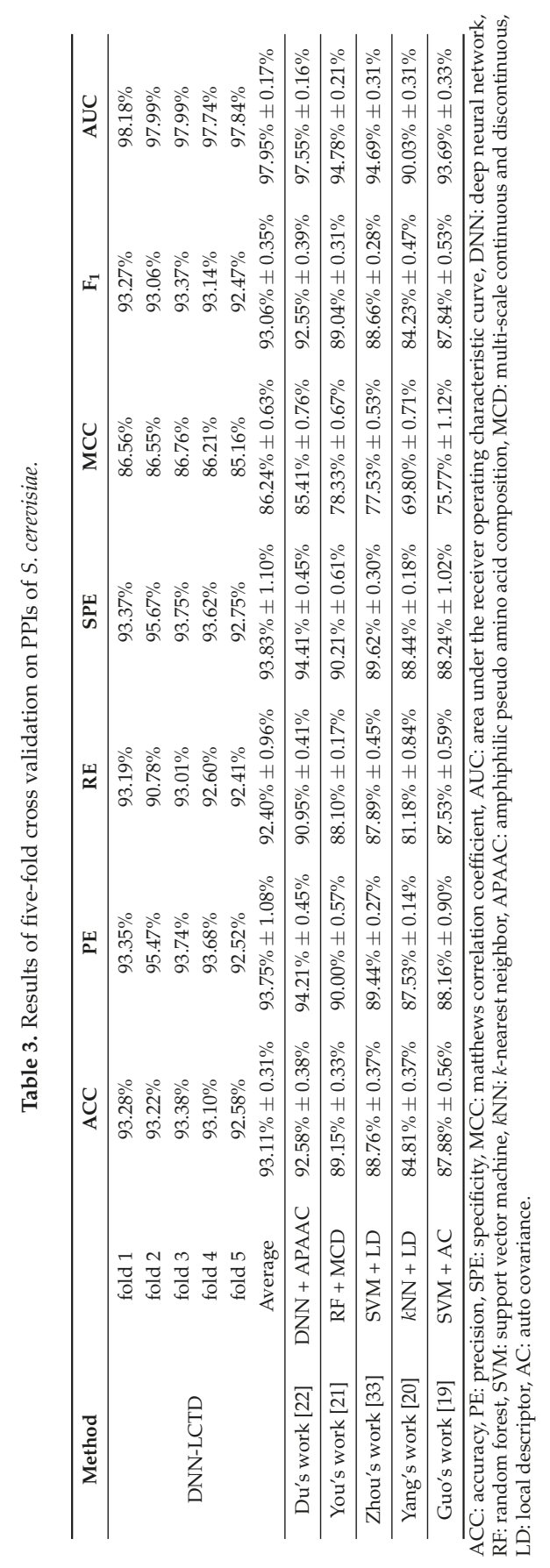


The adopted negative PPIs set may lead to a biased estimation of prediction performance [34]. To prove the rationality of a negative set generated by selecting non-interacting pairs of non-co-localized proteins [19], we perform additional testing on a simulated dataset of S. cerevisiae. Particularly, we firstly construct the negative PPIs set by pairing proteins whose subcellular localizations are different, and we randomly select 17,257 protein pairs as the negative set of the simulated dataset. Next, we construct the positive PPIs set by pairing proteins whose subcellular localizations are the same, regardless of being interacting pairs or not. We then randomly select 17,257 protein pairs as the positive set. As a result, the simulated testing dataset includes 34,514 protein pairs for testing, where half are positives and the other half are negatives. After that, we randomly divide these testing PPIs into five folds, and apply the same DNN as trained on the dataset in Table 3 to predict PPIs in each fold. Table 4 reports the evaluation results on this simulated dataset. From Table 4, we can see that the values of accuracy, recall, MCC, and $F_{1}$ are much lower than the corresponding values reported in Table 3. The reason for the high specificity in Table 4 is that the way of constructing negative dataset in the training dataset (used in Table 3) and simulated testing dataset is the same. These results indicate that the constructed negative set is reasonable.

Table 4. Results on simulated S. cerevisiae dataset.

\begin{tabular}{cccccccc}
\hline & ACC & PE & RE & SPE & MCC & F & AUC \\
\hline fold 1 & $82.53 \%$ & $92.24 \%$ & $71.01 \%$ & $94.04 \%$ & $66.85 \%$ & $80.24 \%$ & $92.47 \%$ \\
fold 2 & $82.89 \%$ & $93.57 \%$ & $70.71 \%$ & $95.12 \%$ & $67.86 \%$ & $80.55 \%$ & $93.52 \%$ \\
fold 3 & $82.56 \%$ & $93.25 \%$ & $70.30 \%$ & $94.89 \%$ & $67.22 \%$ & $80.16 \%$ & $92.52 \%$ \\
fold 4 & $82.09 \%$ & $94.02 \%$ & $68.95 \%$ & $95.52 \%$ & $66.74 \%$ & $79.56 \%$ & $93.08 \%$ \\
fold 5 & $82.24 \%$ & $91.74 \%$ & $70.26 \%$ & $93.86 \%$ & $66.14 \%$ & $79.58 \%$ & $92.85 \%$ \\
Average & $82.46 \% \pm 0.31 \%$ & $92.97 \% \pm 0.95 \%$ & $70.25 \% \pm 0.79 \%$ & $94.68 \% \pm 0.71 \%$ & $66.96 \% \pm 0.64 \%$ & $80.02 \% \pm 0.44 \%$ & $92.89 \% \pm 0.43 \%$ \\
\hline
\end{tabular}

\subsection{Comparison with Different Descriptors}

To further investigate the contribution of the novel local conjoint triad descriptor, we separately train DNNs based on CT [18], AC [19], LD [20,33], MCD [21], APAAC [22], and LCTD. After that we use pairwise $t$-test at 95\% significance level to check the statistical significance between LCTD and LD, MCD, AC, CT, APAAC in five-fold cross validation and report the results in Figure 2 and Table 5. In Table 5, • means that LCTD is statistically significant better than other descriptors on a particular evaluation metric. From Figure 2 and Table 5, we can observe that the prediction performance using LCTD outperforms other descriptors across nearly all evaluation metrics. The ACC, MCC, $\mathrm{F}_{1}$ and AUC of DNN-LCTD are $1.76 \%, 3.48 \%, 1.86 \%$, and $2.85 \%$ higher than those of DNN-MCD; $2.92 \%, 5.81 \%$, $3.05 \%$ and $1.62 \%$ higher than those of DNN-LD; $3.62 \%, 7.25 \%, 3.56 \%$ and $2.06 \%$ than those of DNN-AC; $1.27 \%, 7.74 \%, 9.41 \%$ and $1.99 \%$ than those of DNN-CT; $3.02 \%, 5.99 \%, 3.03 \%$ and $2.06 \%$ than those of DNN-APAAC, respectively. These improvements can be attributed to that LCTD can extract more useful feature information of amino acid sequences by incorporating the advantage of LD [20,33] and conjoint triad (CT) descriptor [18]. From these results, we can conclude that the novel LCTD can more sufficiently capture the feature information of amino acid sequences for PPIs prediction. 


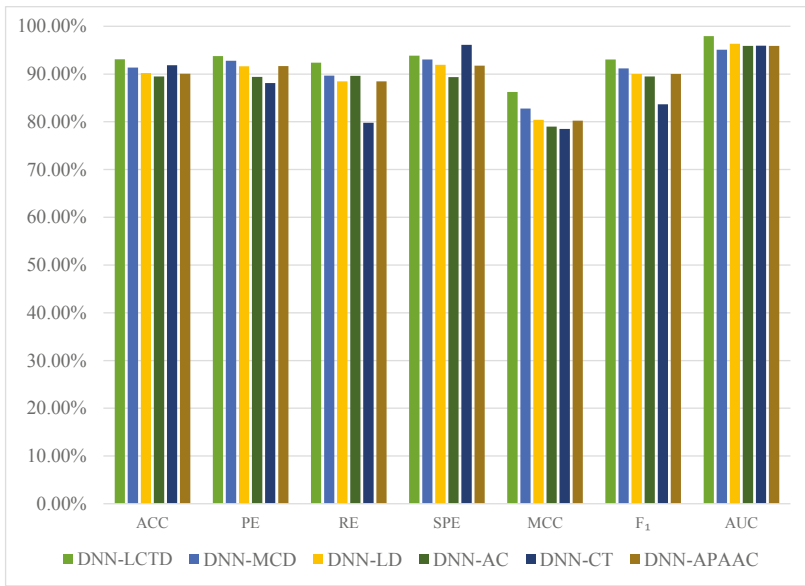

Figure 2. Performance comparison based on DNNs with AC, LD, MCD, LCTD, CT, or APAAC on S. cerevisiae dataset.

Table 5. Results based on DNNs with AC, LD, MCD, LCTD, CT, and APAAC on S. cerevisiae dataset. - indicates LCTD is statistically (according to pairwise $t$-test at $95 \%$ significance level) superior to the other descriptor.

\begin{tabular}{lccccccc}
\hline & ACC (\%) & PE (\%) & RE (\%) & SPE (\%) & MCC (\%) & F $($ (\%) & AUC (\%) \\
\hline DNN-LCTD & $93.11 \pm 0.33$ & $93.75 \pm 0.88$ & $92.40 \pm 0.81$ & $93.83 \pm 0.85$ & $86.24 \pm 0.66$ & $93.06 \pm 0.39$ & $97.95 \pm 0.16$ \\
DNN-MCD & $91.35 \pm 0.31 \bullet$ & $92.80 \pm 1.08$ & $89.67 \pm 0.96 \bullet$ & $93.03 \pm 1.10$ & $82.76 \pm 0.64 \bullet$ & $91.20 \pm 0.35 \bullet$ & $95.10 \pm 0.17 \bullet$ \\
DNN-LD & $90.19 \pm 0.26 \bullet$ & $91.63 \pm 0.77 \bullet$ & $88.46 \pm 0.42 \bullet$ & $91.92 \pm 0.72 \bullet$ & $80.43 \pm 0.55 \bullet$ & $90.01 \pm 0.27 \bullet$ & $96.33 \pm 0.18 \bullet$ \\
DNN-AC & $89.49 \pm 0.36 \bullet$ & $89.40 \pm 3.06 \bullet$ & $89.61 \pm 3.92 \bullet$ & $89.38 \pm 1.25 \bullet$ & $78.99 \pm 1.19 \bullet$ & $89.50 \pm 1.15 \bullet$ & $95.89 \pm 0.31 \bullet$ \\
DNN-CT & $91.84 \pm 0.31 \bullet$ & $88.12 \pm 0.27 \bullet$ & $79.81 \pm 1.08 \bullet$ & $96.12 \pm 0.44$ & $78.50 \pm 0.59 \bullet$ & $83.65 \pm 0.46 \bullet$ & $95.96 \pm 0.34 \bullet$ \\
DNN-APAAC & $90.09 \pm 0.20 \bullet$ & $91.66 \pm 0.27 \bullet$ & $88.45 \pm 0.56 \bullet$ & $91.77 \pm 0.33 \bullet$ & $80.25 \pm 0.39 \bullet$ & $90.03 \pm 0.23 \bullet$ & $95.89 \pm 0.03 \bullet$ \\
\hline
\end{tabular}

\subsection{Comparison with Existing Methods}

Meanwhile, in order to further investigate the effective of DNNs, we separately train the different state-of-the-art predictors on S. cerevisiae dataset using LCTD to encode amino acid sequences, these predictors include support vector machine (SVM) [35], $k$ neighbor nearest $(k N N)$ [36], random forest (RF) [37], and adaboost [38]. Then, we compare the prediction performance based on the six already introduced evaluation metrics. In this study, five-fold cross validation is employed to reduce the impact of data dependency and enhance the reliability of the experiments. The results are shown in Figure 3. From Figure 3 we can see that a high average accuracy of $93.11 \%$ is obtained by DNN-LCTD. The average accuracy of adaboost, $k \mathrm{NN}$, random forest, and $\mathrm{SVM}$ are $92.83 \%, 86.87 \%, 92.28 \%, 92.76 \%$, respectively. DNNs have the highest prediction performance across all evaluation metrics except in RE and SPE. In practice, grid search is used to seek the optimal parameters of these comparing algorithms. We also show the training speed of different comparing methods in Table 6. We can observe that DNN-LCTD with central processing unit (CPU) is separately 2, 25 and 39 times faster than random forest, adaboost and SVM. In order to speed up training of DNN-LCTD, GPU is employed. We can see that the training time of DNN-LCTD with GPU is 3 times faster than that with CPU, 4, 9.5, 97.5 and 148 times than $k$ neighbor nearest, random forest, adaboost and SVM. According to these experimental results, we can conclude that DNN-LCTD can accurately and efficiently predict PPIs from amino acid sequences. 


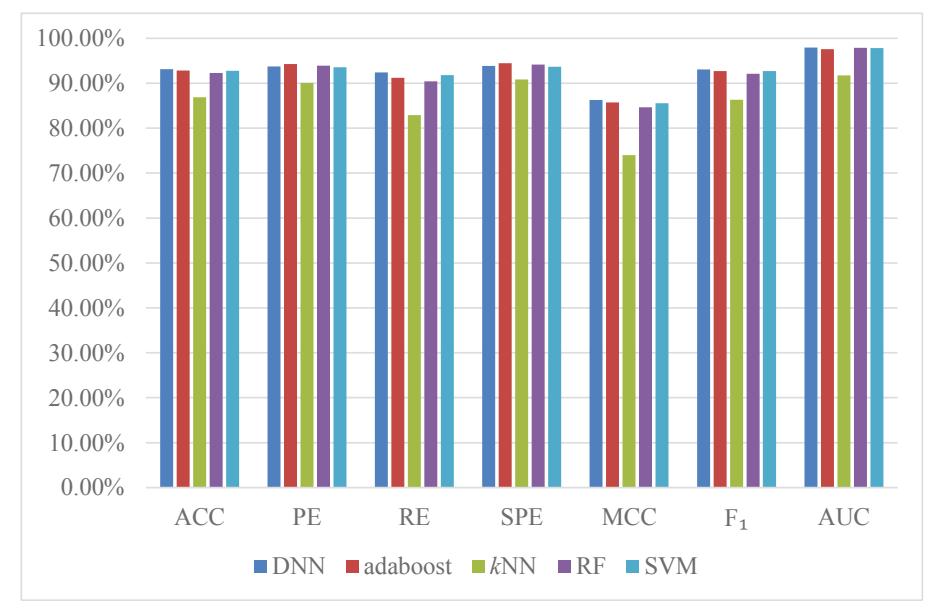

Figure 3. Performance comparison of other algorithms with LCTD descriptor on S. cerevisiae dataset.

Table 6. Comparison of training times of different comparing algorithms.

\begin{tabular}{ccccccc}
\hline Method & DNN-LCTD (GPU) & DNN-LCTD (CPU) & SVM & $k$ NN & Random Forest & Adaboost \\
\hline Times (s) & 718 & 2680 & 106,347 & 2814 & 6906 & 70,026 \\
\hline
\end{tabular}

\subsection{Results on Independent Datasets}

To further assess the practical prediction ability of DNN-LCTD and other comparing methods, we firstly train different models with optimal configurations (details in Section 2.2) using PPIs of S. cerevisiae dataset (34,514 protein pairs). After that, five independent datasets that only contain the samples of interactions, including Caenorhabditis elegans (4013 interacting pairs), Escherichia coli (6954 interacting pairs), Helicobacter pylori (1420 interact-ing pairs), Homo sapiens (1412 interacting pairs), and Mus musculus (313 interacting pairs), are used as test sets to evaluate the prediction performance of these trained models. The prediction results are shown in Table 7. From Table 7, we can observe that the accuracy of DNN-LCTD on C. elegans, E. coli, H. pylori, H. sapiens, and M. musculus are $93.17 \%$, $94.62 \%, 87.38 \%, 94.18 \%$, and $92.65 \%$, respectively. DNN-LCTD has a higher accuracy than DeepPPI [22] and SVM + LD [33] on E. coil, H. sapiens, and M. musculus. The accuracy of SVM + LD [33] is far lower than DNN-LCTD on C. elegans and H. pylori. These prediction accuracies are satisfying except on $H$. pylori. The reason is that we use $S$. cerevisiae as the training set to train models, the trained model is inclined to species that are closer to S. cerevisiae. In reality, S. cerevisiae has closer relationship with other four datasets than with H. pylori. These prediction results indicate that DNN-LCTD has a good generalization ability for predicting PPIs.

Table 7. Prediction results on five independent PPIs datasets, PPIs of S. cerevisiae are used as the training set.

\begin{tabular}{ccccc}
\hline \multirow{2}{*}{ Species } & \multirow{2}{*}{ Test Pairs } & \multicolumn{3}{c}{ ACC } \\
\cline { 3 - 5 } & & DNN-LCTD & Du's Work [22] & Zhou's Work [33] \\
\hline C. elegans & 4013 & $93.17 \%$ & $94.84 \%$ & $75.73 \%$ \\
E. coli & 6984 & $94.62 \%$ & $92.19 \%$ & $71.24 \%$ \\
H. sapiens & 1412 & $94.18 \%$ & $93.77 \%$ & $76.27 \%$ \\
H. pylori & 1420 & $87.38 \%$ & $93.66 \%$ & $75.87 \%$ \\
M. musculus & 313 & $92.65 \%$ & $91.37 \%$ & $76.68 \%$ \\
\hline
\end{tabular}




\section{Materials and Methods}

In this section, we briefly introduce the datasets we used for experiments, including $S$. cerevisiae and other five independent datasets. Then, we introduce the details of LCTD, a novel feature representation descriptor. Finally, we present a brief introduction of deep neural networks (DNNs), including characteristics and skills.

\subsection{PPIs Datasets}

To reliably evaluate the performance of DNN-LCTD, a validation benchmark dataset is necessary. We adopt the S. cerevisiae dataset used by Du et al. [22] for experiments. This dataset was collected from the database of interacting proteins (DIP; version 20160731) [39]. The protein pairs of this dataset exclude proteins with fewer than 50 amino acids and $\geq 40 \%$ sequence identity [19]. Finally, this dataset contains 17,257 positive protein pairs. Negative examples impact the prediction results of PPIs. The common approach is based on annotations of cellular localization [40,41]. The negative set is obtained by pairing proteins whose subcellular localizations are different. The strategy must meet the following requirements [18,19]: (1) the non-interaction pairs cannot appear in the positive dataset, and (2) the contribution of proteins in the negative set should be as harmonious as possible, which means that proteins without subcellular localization information, or denoted as 'putative', 'hypothetical' are excluded for constructing the negative set. Finally, 48,594 negative pairs are generated via this strategy. In the end, S. cerevisiae contains 34,514 protein pairs, where half are from positive dataset and the other (17,257 negative pairs) are randomly selected from the whole negative set. Other five independent PPIs datasets, including Caenorhabditis elegans (4013 interacting pairs), Escherichia coli (6954 interacting pairs), Helicobacter pylori (1420 interacting pairs), Homo sapiens (1412 interacting pairs), and Mus musculus (313 interacting pairs) [33], are used as independent test datasets to assess the generalization ability of DNN-LCTD. These datasets are available at http://ailab.ahu.edu.cn:8087/DeepPPI/index.html.

\subsection{Feature Vector Extraction}

Whether the encoded features are reliable or not can heavily affect the performance of PPIs prediction. The main challenge is how to effectively describe and represent an interacting protein pairs by a fixed length feature vector, in which the essential information content of interacting proteins is fully encoded. Various sequence-based methods are proposed to predict new PPIs, but one flaw of them is that they cannot adequately capture interaction information from continuous and discontinuous amino acid segments at the same time. To overcome this problem, we introduce a novel local conjoint triad descriptor (LCTD), which incorporates the advantage of local descriptor (LD) $[20,33]$ and conjoint triad (CT) [18] sequence representation approach. To clearly introduce the LCTD, we first briefly introduce the feature representation methods of CT $[18]$ and LD $[20,33]$ in the following two subsections.

\subsubsection{Conjoint Triad (CT) Method}

Shen et al. [18] introduced the conjoint triad (CT). In order to conveniently represent the 20 standard amino acids and to suit synonymous mutation, they firstly divided these 20 standard amino acids into 7 groups based on the dipoles and volumes of the side chains as shown in Table 8 . After that, the conjoint triad method is introduced to extract the sequence information, which includes the properties of one amino acid and its vicinal amino acids and regards any three continuous amino acids as a unit [18]. The process of generating descriptor vectors is described as follows.

Table 8. Division of amino acids into seven groups based on the dipoles and volumes of the side chains.

\begin{tabular}{ccccccc}
\hline Group 0 & Group 1 & Group 2 & Group 3 & Group 4 & Group 5 & Group 6 \\
\hline A, G, V & C & F, I, L, P & M, S, T, Y & H, N, Q, W & K, R & D, E \\
\hline
\end{tabular}


Firstly, they replaced each amino acid in the protein sequence by the index depending on its grouping. For instance, protein sequence "VCCPPVCVVCPPVCVPVPPCCV" is replaced by 0112201001220102022110 . Then, binary space $(\mathbf{V}, \mathbf{F})$ stands for a protein sequence. Here, $\mathbf{V}$ is the vector space of the sequence features, and each feature $\mathbf{v}_{i}$ represents a kind of triad type [18]. For example, $\mathbf{v}_{1}, \mathbf{v}_{7}$, and $\mathbf{v}_{10}$ are separately representing the triad unit of $100,010,310 . \mathbf{F}$ is the frequency vector corresponding to $\mathbf{V}$, and the value of the $i$ th dimension of $\mathbf{F}\left(\mathbf{f}_{i}\right)$ is the frequency of type $\mathbf{v}_{i}$ appearing in amino acid sequence [18]. As the amino acids grouped into seven classes, the size $\mathbf{V}$ should be $7 \times 7 \times 7$; therefore, $i=0,1, \cdots, 342$. The detailed definition and description is shown in Figure 4 . Clearly, each protein has a corresponding $\mathbf{F}$ vector. Nevertheless, the value of $\mathbf{f}_{i}$ relates to the length of amino acid sequence. A longer amino acid sequence generally have a larger value of $\mathbf{f}_{i}$, which complicates the comparison between two heterogeneous proteins. As such they employed the normalization to solve this problem as follows:

$$
\mathbf{d}_{i}=\left(\mathbf{f}_{i}-\min \left\{\mathbf{f}_{0}, \mathbf{f}_{1}, \cdots, \mathbf{f}_{342}\right\}\right) / \max \left\{\mathbf{f}_{0}, \mathbf{f}_{1}, \cdots, \mathbf{f}_{342}\right\}
$$

where the value of $\mathbf{d}_{i}$ is normalized in the range [0,1]. $\mathbf{f}_{i}$ is the frequency of conjoint triad unit $\mathbf{v}_{i}$ appearing in the protein sequence. Finally, they connected the vector spaces of two proteins to present the interaction features. Thus, a 686-dimensional vector (343 for each protein) is generated for each pair of proteins.

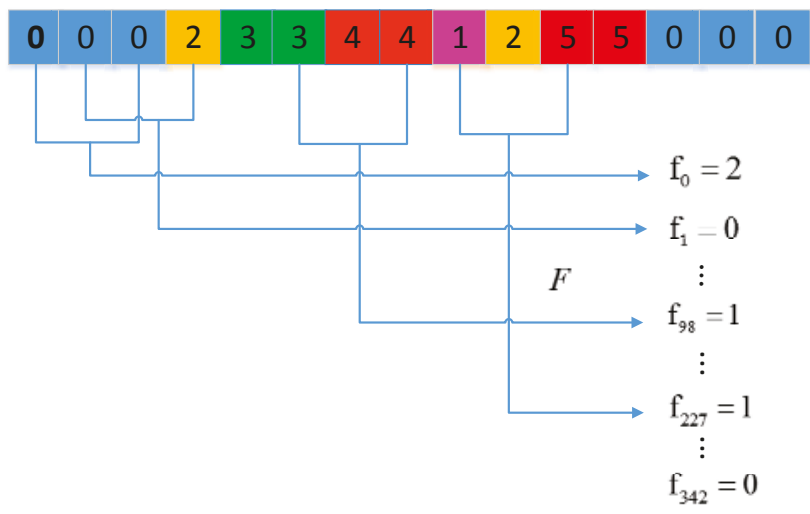

Figure 4. Schematic diagram for conjoint triad. The number is the classes grouped by the dipoles and volumes of the side chains. $\mathbf{f}_{i}$ is the frequency that triad type appears in the protein sequence. $\mathbf{F}$ is the vector set for all $\mathbf{f}_{i}$.

\subsubsection{Local Descriptor (LD)}

Local descriptor (LD) is an alignment-free approach previously used to classify several proteins families [42,43]. Yang et al. [20] and Zhou et al. [33] employed this method to extract the interactions information from amino acid sequences. 20 standard amino acids are grouped into 7 groups based on the dipoles and volumes of the side chains at first, as shown in Table 8. Then each entire protein sequence is divided into 10 segments as shown in Figure 5. For each local region, three local descriptors including composition (C), transition (T) and distribution (D) are employed to extract the feature information. $\mathrm{C}$ represents the composition of each amino acid group. $\mathrm{T}$ stands for the frequency from a type of amino acids to another type. $\mathrm{D}$ describes the distribution pattern along the entire region by measuring the location of the first $25 \%, 50 \%, 75 \%$ and $100 \%$ of residues of a given group [33,44]. 
Protein Sequence

\section{0}

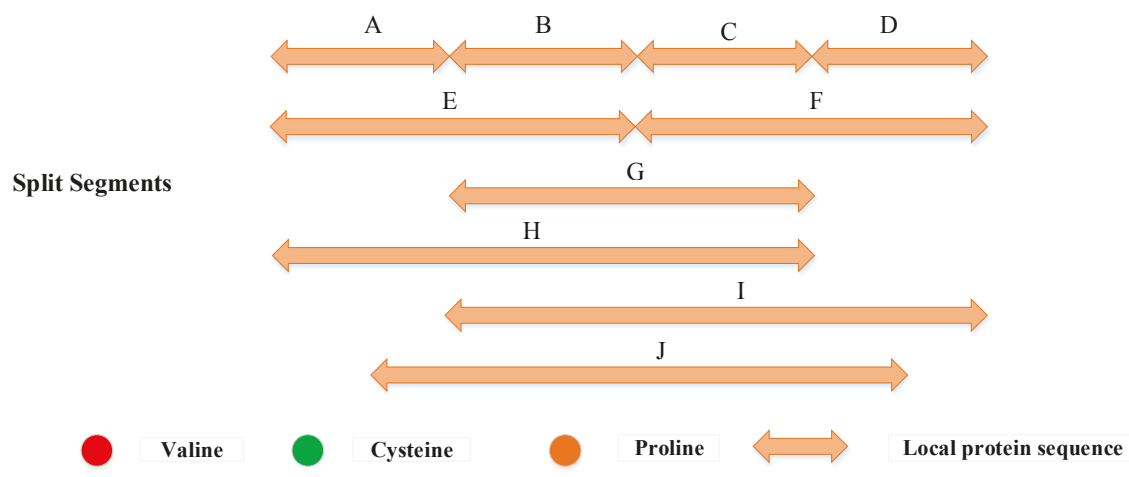

Figure 5. The 10 descriptor regions (A-J) are split for a hypothetical protein sequence. The regions $\mathrm{A}-\mathrm{D}$ and $\mathrm{E}-\mathrm{F}$ are obtained by dividing the entire amino acid sequence into four equal regions and two equal regions [20,33], respectively. $\mathrm{G}$ stands for the central $50 \%$ of the amino acid sequence. Regions $\mathrm{H}$, I, and J represent the first, final and central $75 \%$ of the amino acid sequence, respectively.

Then, each local region split is replaced by the index depending on the classification of amino acids. For example, protein sequence "VCCPPVCVVCPPVCVPVPPCCV" is replaced by 0112201001220102022110 based on classification of amino acids as shown in Figure 6. There have eight ' 0 ', seven ' 1 ', and seven ' 2 ' in the protein sequence. The composition for these three symbols is $8 \times 100 \% /(8+7+7)=36.36 \%, 7 \times 100 \% /(8+7+7)=31.82 \%$, and $6 \times 100 \% /(8+7+7)=31.82 \%$, respectively. There are 7 transitions from ' 0 ' to ' 1 ' or from ' 1 ' to ' 0 ' in this sequence, and the percentage frequency of these transitions is $(7 / 21) \times 100 \%=33.33 \%$. Similarly, the transitions from ' 0 ' to ' 2 ' or ' 2 ' to ' 0 ' and transitions from ' 1 ' to ' 2 ' or ' 2 ' to ' 1 ' are respectively calculated as $(3 / 21) \times 100 \%=14.29 \%$ and $(4 / 21) \times 100 \%=19.05 \%$. For distribution $D$, there are 8 residues encoded as ' 0 ' in the example of Figure 6 , the position of the first residue ' 0 ', the second residue ' 0 ' $(25 \% \times 8=2)$, the fourth residue ' 0 ' $(50 \% \times 8=4)$, the sixth ' 0 ' residue $(75 \% \times 8=6)$, and the eight residue ' 0 ' $(100 \% \times 8=8)$ in the encoded sequence are $1,6,9,15$, and 22 , respectively. Thus $\mathrm{D}$ descriptor for ' 0 ' is: $(1 / 22 \times 100 \%=4.55 \%)$, $(2 / 22 \times 100 \%=9.09 \%),(4 / 22 \times 100 \%=18.18 \%),(6 / 22 \times 100 \%=27.27 \%)$ and $(8 / 22 \times 100 \%=36.36 \%)$, respectively. Similarly, the D descriptor for ' 1 ' and ' 2 ' is $(9.09 \%, 13.64 \%, 45.45 \%, 63.64 \%, 95.45 \%)$ and $(18.18 \%, 22.73 \%, 54.55 \%, 72.73 \%, 86.36 \%)$, respectively.

For each local region, three descriptors $(C, T, D)$ are computed and concatenated into a 63-dimensional feature vector, 7 for $C, 21(7 \times 6 / 2)$ for $\mathrm{T}$ and $35(7 \times 5)$ for $\mathrm{D}$. Then all descriptors from 10 regions are concatenated into an 630-dimensional vector. Finally, LD concatenates the vectors of two individual amino acid sequences. Thus, a 1260-dimensional vector is constructed to characterize each protein pair. 


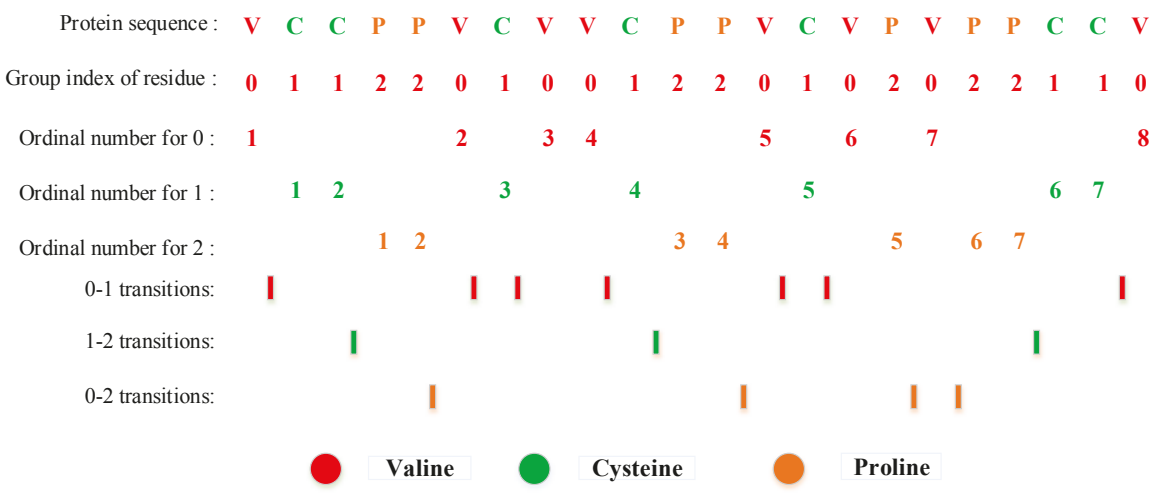

Figure 6. A hypothetical protein sequence figuring the structure of composition, transition and distribution pattern of a protein region.

\subsubsection{Local Conjoint Triad Descriptor (LCTD)}

From the process of LD descriptor [20,33], we can find that it only considers the neighboring effect of adjacent two types of amino acids. Therefore, it cannot sufficiently extract information of neighbor amino acids, but can sufficiently discover information of discontinuous segments of the amino acid sequence. Meanwhile, we observe that the conjoint triad method [18] considers the neighboring effect of adjacent three types of amino acid, but ignores the discontinuous information. Thus, we advocate to integrate the merits of $\mathrm{LD}[20,33]$ and conjoint triad (CT) [18] to introduce a novel feature representation of amino acid sequence called LCTD. LCTD groups the 20 standard amino acids into 7 groups on the dipoles and volumes of the side chains at first as shown in Table 8. Then it divides the entire protein sequence into 10 segments as done by LD [20,33]. Next, for each local region, we calculate four descriptors, composition (C), transition (T) and distribution (D), and conjoint triad (CT). C represents the composition of each amino acid group. $\mathrm{T}$ stands for the frequency from a type of amino acid to another type. D describes the distribution pattern along the entire region by measuring the location of the first $25 \%, 50 \%, 75 \%$ and $100 \%$ of residues of a given group [33,44]. Conjoint triad considers the properties of one amino acid and its vicinal amino acids, it regards any three continuous amino acids as a unit [18]. These descriptors are introduced in Sections 3.2.1 and 3.2.2. For each local region, the four descriptors (C, T, D, CT) are calculated and concatenated, and a total of $63+343$ descriptors are generated: 7 for $C, 21(7 \times 6 / 2)$ for T and $35(7 \times 5)$ for D, and 343 for CT. After that, all descriptors from 10 regions are concatenated into an 4060-dimensional vector. Finally, LCTD concatenates the vectors of two individual proteins. Thus, a 8120-dimensional vector is constructed to encode each protein pair. The corresponding equations are shown as follows:

$$
\begin{gathered}
D_{A i}=C \oplus T \oplus D \oplus C T(i=1,2, \cdots, 10) \\
D_{B i}=C \oplus T \oplus D \oplus C T(i=1,2, \cdots, 10) \\
D_{A}=D_{A 1} \oplus D_{A 2} \oplus \cdots \oplus D_{A 10} \\
D_{B}=D_{B 1} \oplus D_{B 2} \oplus \cdots \oplus D_{B 10}
\end{gathered}
$$




$$
D_{A B}=D_{A} \oplus D_{B}
$$

where $A$ and $B$ are a pair of proteins, $\oplus$ is the vector concatenating operator. $D_{A}, D_{B}$ is the extracted feature vector from $A$ and $B$, respectively. $i$ refers to any segment in 10 split segments. $D_{A B}$ is the extracted feature of two amino acid sequences. These 8120 -dimensional feature vectors are used as input of DNNs for training and prediction.

\subsection{Deep Neural Network}

Deep learning, a popular type of machine learning algorithms, consists with an artificial neural network of multiple nonlinear layers. It is inspired by the biological neural network that constitutes animal brains. The characteristics of deep learning are that it can learn suitable features from the original data without designed by human engineers, and discover hierarchical representations of data [45]. The depth of a neural network corresponds to the number of hidden layers, and the width is the maximum number of neurons in one of its layers [27]. Neural network with a large number of hidden layers (three or more hidden layers) is called deep neural network [27].

The basic structure of DNN consists of an input layer, multiple hidden layers, and an output layer, the special configuration of our neural network is shown in Figure 7. In general, input data $(x)$ are given to the DNN, the output values are sequentially computed along the layers of the network. Neurons of a hidden layer or output layer are connected to all neurons of the previous layer [27]. Each neuron computes a weighted sum of its inputs and applies a nonlinear activation function to calculate its outputs $f(x)$ [27]. The representations in the layer below are transformed into slightly more abstract representations by the computation in each layer [46]. In general, the nonlinear activation function including sigmoid, hyperbolic tangent, or rectified linear unit (ReLU) [47]. The sigmoid and ReLU are used in this study.

In this work, we use the mini-batch gradient descent [48] and Adam algorithm [49] to reduce the sensitivity to the specific choice of learning rate [27], and speed up training using GPU. The dropout technique is employed to avoid the overfitting, which the activation of some neurons is randomly set to zero during training in each forward pass as shown in Figure 7 [27]. The dotted line means this neuron will not be activated and calculated. The activation function of ReLU [47] and the loss of cross entropy is employed because they can both accelerate the model training and obtain better prediction results [50]. Batch normalization approach is also employed to reduce the dependency of training with the parameter initialization, speed up training and minimize the risk of over-fitting. The following equations are used to calculate the loss:

$$
\begin{gathered}
\mathbf{H}_{i 1}=\sigma_{1}\left(\mathbf{W}_{i 1} \mathbf{X}_{i 1}+\mathbf{b}_{i 1}\right)(i=1, \cdots, n) \\
\mathbf{H}_{i(j+1)}=\sigma_{1}\left(\mathbf{W}_{i j} \mathbf{H}_{i j}+\mathbf{b}_{i j}\right)(i=2, \cdots, n, \quad j=1, \cdots, h) \\
L=-\frac{1}{n} \sum_{i=1}^{n}\left[\mathbf{y}_{i} \ln \left(\sigma_{2}\left(\mathbf{W}_{i h} \mathbf{H}_{i h}+\mathbf{b}_{i h}\right)+\left(1-\mathbf{y}_{i}\right) \ln \left(1-\sigma_{2}\left(\mathbf{W}_{i h} \mathbf{H}_{i h}+\mathbf{b}_{i h}\right)\right)\right]\right.
\end{gathered}
$$

where $n$ is the number of PPIs for batch training. $\sigma_{1}$ is the activation function of ReLU, $\sigma_{2}$ is the activation function of the output layer with sigmoid, $\mathbf{X}$ is the batch training inputs, $\mathbf{H}$ is the outputs of hidden layer, and $\mathbf{y}$ is the corresponding desired outputs. $h$ is the depth of the DNN, $\mathbf{W}$ is the weight matrix between the input layer and the output layer and $\mathbf{b}$ is the bias. 


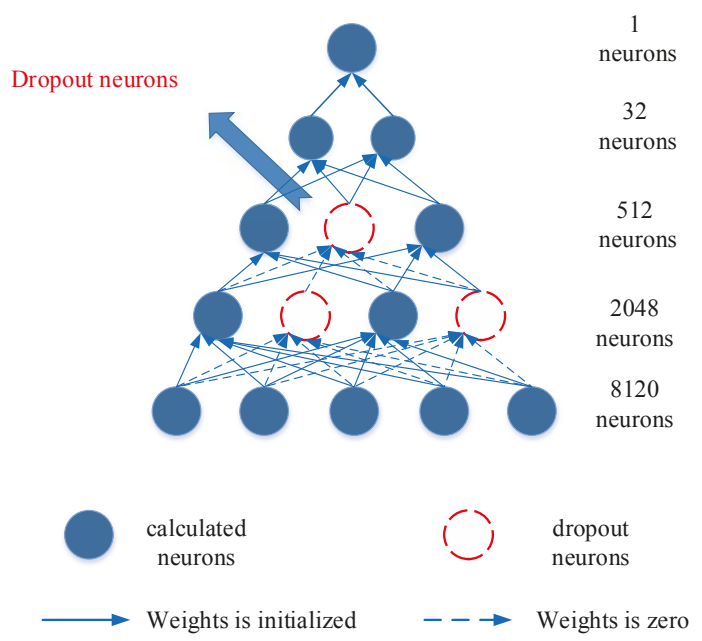

Figure 7. The structure of the adopted DNN with LCTD features and the dropout technique.

\section{Conclusions}

In this article, we propose an efficient approach for predicting PPIs from protein primary sequences by a novel local conjoint triad feature representation with DNNs. The LCTD takes PPIs of continuous segments and discontinuous segments in protein sequence into account at the same time. The feature sets, characterized by LCTD, are capable of capturing more essential interactions information from the continuous and discontinuous binding patterns within a protein sequence. We then train a DNN with LCTD feature sets as inputs. Finally, the trained DNN is employed to predict the new PPIs. The experimental results indicate that DNN-LCTD is very promising for predicting PPIs and can be an available supplementary tool to other approaches.

The high prediction accuracy can be partially attributed to a biased selection of positive/negative training data. In practice, the available PPIs are incomplete and have a high rate of false positives and false negative. Furthermore, constructing the negative data set by subcellular localization information may also result in bias. How to construct a high quality negative set and how to reduce the impact of noisy and bias of PPIs data are future pursues. Another possible reason for the high accuracy is that DNN can model complex relationship between molecules by hidden layers and reduce the impact of noisy and bias of PPIs data.

Acknowledgments: This work is supported by Natural Science Foundation of China (61402378 and 61562054), Natural Science Foundation of Chongqing Science and Technology Commission (cstc2016jcyjA0351), Fundamental Research Funds for the Central Universities of China (2362015XK07 and XDJK2016B009).

Author Contributions: Jun Wang and Guoxian Yu proposed the idea and conceived the whole program. Long Zhang and Jun Wang implemented the experiments and drafted the manuscript. Lianyin Jia, Yazhou Ren and Guoxian Yu participated in analyzing the experimental data and revising the manuscript. All the authors read and approved the final manuscript.

Conflicts of Interest: The authors declare no conflict of interest.

\section{References}

1. Williams, N.E. Immunoprecipitation procedures. Methods Cell Biol. 2000, 62, 449-453.

2. Santoro, C.; Mermod, N.; Andrews, P.C.; Tjian, R. A family of human CCAAT-box-binding proteins active in transcription and DNA replication: Cloning and expression of multiple cDNAs. Nature 1988, 334, 218-224. 
3. Zhao, X.M.; Wang, R.S.; Chen, L.; Aihara, K. Uncovering signal transduction networks from high-throughput data by integer linear programming. Nucleic Acids Res. 2008, 36, e48.

4. Zhang, Z.; Zhang, J.; Fan, C.; Tang, Y.; Deng, L. KATZLGO: Large-scale Prediction of LncRNA Functions by Using the KATZ Measure Based on Multiple Networks. IEEE/ACM Trans. Comput. Biol. Bioinform. 2017, doi:10.1109/TCBB.2017.2704587.

5. Zhang, J.; Zhang, Z.; Chen, Z.; Deng, L. Integrating Multiple Heterogeneous Networks for Novel LncRNA-disease Association Inference. IEEE/ACM Trans. Comput. Biol. Bioinform. 2017, doi:10.1109/TCBB.2017.2701379.

6. Yu, G.; Fu, G.; Wang, J.; Zhao, Y. NewGOA: Predicting new GO annotations of proteins by bi-random walks on a hybrid graph. IEEE/ACM Trans. Comput. Biol. Bioinform. 2017, doi:10.1109/TCBB.2017.2715842.

7. Huang, H.; Alvarez, S.; Nusinow, D.A. Data on the identification of protein interactors with the Evening Complex and PCH1 in Arabidopsis using tandem affinity purification and mass spectrometry (TAP-MS). Data Brief 2016, 8, 56-60.

8. Mehla, J.; Caufield, J.H.; Uetz, P. Mapping protein-protein interactions using yeast two-hybrid assays. Cold Spring Harb. Protoc. 2015, 2015, 442-452.

9. Gavin, A.C.; Bösche, M.; Krause, R.; Grandi, P.; Marzioch, M.; Bauer, A.; Schultz, J.; Rick, J.M.; Michon, A.M.; Cruciat, C.M.; et al. Functional organization of the yeast proteome by systematic analysis of protein complexes. Nature 2002, 415, 141-147.

10. Skrabanek, L.; Saini, H.K.; Bader, G.D.; Enright, A.J. Computational prediction of protein-protein interactions. Mol. Biotechnol. 2008, 38,1-17.

11. Lee, H.; Deng, M.; Sun, F.; Chen, T. An integrated approach to the prediction of domain-domain interactions. BMC Bioinform. 2006, 7, 1-15.

12. Enright, A.J.; Iliopoulos, I.; Kyrpides, N.C.; Ouzounis, C.A. Protein interaction maps for complete genomes based on gene fusion events. Nature 1999, 402, 86-90.

13. Aloy, P.; Russell, R.B. Interrogating protein interaction networks through structural biology. Proc. Natl. Acad. Sci. USA 2002, 99, 5896-5901.

14. Aloy, P.; Russell, R.B. InterPreTS: Protein Inter action Pre diction through T ertiary S tructure. Bioinformatics 2003, 19, 161-162.

15. Huang, T.W.; Tien, A.C.; Huang, W.S.; Lee, Y.C.G.; Peng, C.L.; Tseng, H.H.; Kao, C.Y.; Huang, C.Y.F. POINT: A database for the prediction of protein-protein interactions based on the orthologous interactome. Bioinformatics 2004, 20, 3273-3276.

16. Du, T. Predicting Protein-Protein Interactions, Interaction Sites and Residue-Residue Contact Matrices with Machine Learning Techniques; University of Delaware: Newark, DE, USA, 2015.

17. Bock, J.R.; Gough, D.A. Predicting protein-protein interactions from primary structure. Bioinformatics 2001, 17, 455-460.

18. Shen, J.; Zhang, J.; Luo, X.; Zhu, W.; Yu, K.; Chen, K.; Li, Y.; Jiang, H. Predicting protein-protein interactions based only on sequences information. Proc. Natl. Acad. Sci. USA 2007, 104, 4337-4341.

19. Guo, Y.; Yu, L.; Wen, Z.; Li, M. Using support vector machine combined with auto covariance to predict protein-protein interactions from protein sequences. Nucleic Acids Res. 2008, 36, 3025-3030.

20. Yang, L.; Xia, J.F.; Gui, J. Prediction of protein-protein interactions from protein sequence using local descriptors. Protein Pept. Lett. 2010, 17, 1085-1090.

21. You, Z.H.; Zhu, L.; Zheng, C.H.; Yu, H.J.; Deng, S.P.; Ji, Z. Prediction of protein-protein interactions from amino acid sequences using a novel multi-scale continuous and discontinuous feature set. BMC Bioinform. 2014, 15, S9.

22. Du, X.; Sun, S.; Hu, C.; Yao, Y.; Yan, Y.; Zhang, Y. DeepPPI: Boosting Prediction of Protein-Protein Interactions with Deep Neural Networks. J. Chem. Inform. Model. 2017, 57, 1499-1510.

23. Wang, Y.; You, Z.; Li, X.; Chen, X.; Jiang, T.; Zhang, J. PCVMZM: Using the Probabilistic Classification Vector Machines Model Combined with a Zernike Moments Descriptor to Predict Protein-Protein Interactions from Protein Sequences. Int. J. Mol. Sci. 2017, 18, 1029.

24. Zeng, J.; Li, D.; Wu, Y.; Zou, Q.; Liu, X. An empirical study of features fusion techniques for protein-protein interaction prediction. Curr. Bioinform. 2016, 27, 899-901.

25. Peng, H.; Long, F.; Ding, C. Feature selection based on mutual information criteria of max-dependency, max-relevance, and min-redundancy. IEEE Trans. Pattern Anal. Mach. Intell. 2005, 27, 1226-1238. 
26. Chou, K.C. Using amphiphilic pseudo amino acid composition to predict enzyme subfamily classes. Bioinformatics 2004, 21, 10-19.

27. Angermueller, C.; Pärnamaa, T.; Parts, L.; Stegle, O. Deep learning for computational biology. Mol. Syst. Biol. 2016, 12, 878 .

28. Asgari, E.; Mofrad, M.R.K. Continuous Distributed Representation of Biological Sequences for Deep Proteomics and Genomics. PLoS ONE 2015, 10, e0141287.

29. Browne, M.W. Cross-validation methods. J. Math. Psychol. 2000, 44, 108-132.

30. Bewick, V.; Cheek, L.; Ball, J. Statistics review 13: Receiver operating characteristic curves. Crit. Care 2004, 8, 508-512.

31. Akobeng, A.K. Understanding diagnostic tests 3: Receiver operating characteristic curves. Acta Paediatr. 2007, 96, 644-647.

32. Bengio, Y. Practical recommendations for gradient-based training of deep architectures. In Neural Networks: Tricks of The Trade; Springer: Berlin, Germany, 2012; pp. 437-478.

33. Zhou, Y.Z.; Gao, Y.; Zheng, Y.Y. Prediction of protein-protein interactions using local description of amino acid sequence. Adv. Comput. Sci. Edu. Appl. 2011, 202, 254-262.

34. Ben-Hur, A.; Noble, W.S. Choosing negative examples for the prediction of protein-protein interactions. BMC Bioinform. 2006, 7, 1-6.

35. Cortes, C.; Vapnik, V. Support-vector networks. Mach. Learn. 1995, 20, 273-297.

36. Cover, T.; Hart, P. Nearest neighbor pattern classification. IEEE Trans. Inf. Theory 1967, 13, 21-27.

37. Breiman, L. Random Forests. Mach. Learn. 2001, 45, 5-32.

38. Collins, M.; Schapire, R.E.; Singer, Y. Logistic Regression, AdaBoost and Bregman Distances. Mach. Learn. 2002, 48, 253-285.

39. Xenarios, I.; Rice, D.W.; Salwinski, L.; Baron, M.K.; Marcotte, E.M.; Eisenberg, D. DIP: The database of interacting proteins. Nucleic Acids Res. 2000, 28, 289-291.

40. Shin, C.J.; Wong, S.; Davis, M.J.; Ragan, M.A. Protein-protein interaction as a predictor of subcellular location. BMC Syst. Biol. 2009, 3, 28.

41. Wei, L.; Ding, Y.; Su, R.; Tang, J.; Zou, Q. Prediction of human protein subcellular localization using deep learning. J. Parallel Distrib. Comput. 2017, doi:10.1016/j.jpdc.2017.08.009.

42. Davies, M.N.; Secker, A.; Freitas, A.A.; Clark, E.; Timmis, J.; Flower, D.R. Optimizing amino acid groupings for GPCR classification. Bioinformatics 2008, 24, 1980-1986.

43. Tong, J.C.; Tammi, M.T. Prediction of protein allergenicity using local description of amino acid sequence. Front. Biosci. 2007, 13, 6072-6078.

44. Dubchak, I.; Muchnik, I.; Holbrook, S.R.; Kim, S.H. Prediction of protein folding class using global description of amino acid sequence. Proc. Natl. Acad. Sci. USA 1995, 92, 8700-8704.

45. Min, S.; Lee, B.; Yoon, S. Deep learning in bioinformatics. Brief. Bioinform. 2016, 18, 851-869.

46. LeCun, Y.; Bengio, Y.; Hinton, G. Deep learning. Nature 2015, 521, 436-444.

47. Nair, V.; Hinton, G.E. Rectified linear units improve restricted boltzmann machines. In Proceedings of the 27th International Conference on Machine Learning, Haifa, Israel, 21-24 June 2010; pp. 807-814.

48. Cotter, A.; Shamir, O.; Srebro, N.; Sridharan, K. Better mini-batch algorithms via accelerated gradient methods. In Proceedings of the Advances in Neural Information Processing Systems, Granada, Spain, 12-14 December 2011; pp. 1647-1655.

49. Kingma, D.; Ba, J. Adam: A method for stochastic optimization. In Proceedings of the 3rd International Conference for Learning Representations, San Diego, CA, USA, 7-9 May 2015.

50. Hinton, G.; Vinyals, O.; Dean, J. Distilling the knowledge in a neural network. Comput. Sci. 2015, 14, 38-39. 
Article

\title{
UltraPse: A Universal and Extensible Software Platform for Representing Biological Sequences
}

\author{
Pu-Feng Du ${ }^{1}$, Wei Zhao ${ }^{1}$, Yang-Yang Miao ${ }^{1,2}$, Le-Yi Wei ${ }^{1}$ and Likun Wang ${ }^{3, *}$ \\ 1 School of Computer Science and Technology, Tianjin University, Tianjin 300350, China; \\ PufengDu@gmail.com (P.-F.D.); wzhao_cstju@yeah.net (W.Z.); miaoyangyang1998@163.com (Y.-Y.M.); \\ weileyi@tju.edu.cn (L.-Y.W.) \\ 2 School of Chemical Engineering, Tianjin University, Tianjin 300350, China \\ 3 Institute of Systems Biomedicine, Beijing Key Laboratory of Tumor Systems Biology, Department of \\ Pathology, School of Basic Medical Sciences, Peking University Health Science Center, Beijing 100191, China \\ * Correspondence: wanglk@hsc.pku.edu.cn; Tel.: +86-10-8280-5807
}

Received: 10 October 2017; Accepted: 3 November 2017; Published: 14 November 2017

\begin{abstract}
With the avalanche of biological sequences in public databases, one of the most challenging problems in computational biology is to predict their biological functions and cellular attributes. Most of the existing prediction algorithms can only handle fixed-length numerical vectors. Therefore, it is important to be able to represent biological sequences with various lengths using fixed-length numerical vectors. Although several algorithms, as well as software implementations, have been developed to address this problem, these existing programs can only provide a fixed number of representation modes. Every time a new sequence representation mode is developed, a new program will be needed. In this paper, we propose the UltraPse as a universal software platform for this problem. The function of the UltraPse is not only to generate various existing sequence representation modes, but also to simplify all future programming works in developing novel representation modes. The extensibility of UltraPse is particularly enhanced. It allows the users to define their own representation mode, their own physicochemical properties, or even their own types of biological sequences. Moreover, UltraPse is also the fastest software of its kind. The source code package, as well as the executables for both Linux and Windows platforms, can be downloaded from the GitHub repository.
\end{abstract}

Keywords: pseudo-amino acid compositions; pseudo-k nucleotide compositions; extensible software

\section{Introduction}

Over the last two decades, huge numbers of biological sequences have been deposited in public databases. Until today, the number of these sequences is still increasing exponentially. However, the cellular and functional attributes of these sequences, no matter whether they are nucleotide sequences or protein sequences, remain largely unknown. It is a very important task for computational biology to predict the functional and cellular attributes of these sequences.

In the view of machine learning, most of these prediction tasks can be formulated as pattern classification problems. As elaborated in a series of publications [1-8], one of the most challenging parts is to represent a biological sequence with a fixed-length numerical vector, yet still keep a considerable amount of the sequence-order information. This is because almost every existing algorithm for these tasks can only handle fixed-length vectors, but not the sequences.

For protein and peptide sequences, Chou proposed pseudo-amino acid compositions (PseAAC) [9] and amphiphilic pseudo-amino acid compositions (AmPseAAC) [10]. Ever since the concepts of pseudo-factors were introduced, they have rapidly penetrated into almost every area of computational proteomics [11-20]. As elaborated in a review article, the form of classic pseudo-amino acid 
compositions has been generalized to contain various types of information [21], which is known as the general-form pseudo-amino acid compositions. The applications of PseAAC concepts have been summarized in the review papers [22,23].

Recently, the concept of PseAAC has been extended to represent nucleotide sequences [24]. Chen et al. developed pseudo-dinucleotide compositions (PseDNC) to predict DNA recombination hostspots [25]. This formulation was then extended as pseudo-k nucleotide compositions (PseKNC), which have been applied in predicting splicing sites [26], predicting translation initiation sites [27], predicting nucleosome positions [28], predicting promoters [29], predicting DNA methylation sites [30], predicting microRNA precursors [31] and many others [32-41].

In the early days of pseudo-amino acid compositions, every study had to implement PseAAC independently. Although the algorithms in every implementation are identical, different implementations may introduce computational discrepancies due to technical details. For example, different implementations may give results with different precisions. This kind of differences may be amplified by machine-learning based predictors, which may eventually produce different prediction results. For another example, different implementations may have very different computational efficiencies. This means one implementation may only use a second to process a dataset, while another program may require over an hour to achieve the same results on the same dataset with the same parameters.

To solve these problems, a universal implementation of the algorithm should be provided. Many efforts have been made for this purpose [42-52]. The first program focus on the PseAAC formulation is the PseAAC server [43], which was brought online in the year 2008. The PseAAC server can compute Type-I and Type-II PseAAC using six different kinds of physicochemical properties of amino acids. The PseAAC server has a friendly user interface, which is convenient and efficient for small datasets. However, for large datasets and the repeatedly parameter scanning process, the computational efficiency of the PseAAC server is not ideal. The PseAAC-Builder [45], which was released in the year 2012, is dedicated to improving the efficiency. Unlike the PseAAC server, the PseAAC-Builder is a stand-alone program that can be executed locally. It has a simple graphical user interface (GUI) for the users' convenience. It can also be executed in a command line environment. The computational efficiency of PseAAC-Builder is much higher than the PseAAC server, especially in the command line environment. Although the PseAAC-Builder includes over 500 different types of physicochemical properties, it did not provide the ability to compute general form PseAAC. PseAAC-General [46], which is a major upgrade to the PseAAC-Builder, was developed to solve this problem. PseAAC-General provides the ability to compute several commonly used general forms of PseAAC, such as the GO mode, the functional domain mode and the evolutionary mode. The users of PseAAC-General can slightly extend its ability by using Lua scripts.

After Chen et al. proposed the PseKNC representations for nucleotide sequences, similar software and services were needed for DNA and RNA sequences. Chen et al. released the PseKNC [48] and PseKNC-General [49] packages for converting DNA/RNA sequences into its PseKNC or general form of PseKNC representations. Liu et al. developed the repDNA [50], repRNA [51], and Pse-In-One [52] services for more types of descriptors. The Pse-In-One service attempts to be a universal online service that can be applied on both protein and nucleotide sequences.

However, all existing software packages and online services suffer from three problems. (1) Lack of extensibility. Most of the existing software can only be used to produce existing modes of representation. The users cannot extend the software to handle their own novel representation modes. Although PseAAC-General can be extended by using Lua script, it can only be used for protein sequences; (2) Lack of flexibility. Most of the existing software can only handle one type of biological sequences, either nucleotide sequences or protein sequences. Pse-In-One is the only existing service that can handle protein sequence as well as nucleotide sequences. However, no program can handle user-defined sequence types. For example, when studying the protein phosphorylation sites, the modified residues should have different notations of sequences, which are not in the standard 20 letters. The users 
need to define the extra letters to represent the modified residues. As far as we know, no program can handle this kind of sequence; (3) Lack of computational efficiency on large datasets. Most of the existing programs are not designed to handle large datasets. They may need many minutes to process a million sequences. If a user needs to repeatedly scan parameters of a representation, the processing time may be days or even weeks.

In this paper, we proposed the UltraPse program, which is a universal and extensible software platform for all possible sequence representation modes. The UltraPse program unified the processing of nucleotide and protein sequence in one program, as well as the user-defined sequence types. UltraPse supports two forms of extension modules, the BSOs (Binary Shared Objects) and the Lua scripts, which are called the TDFs (Task Definition Files) in UltraPse. The users can develop their own modes by just writing several lines of Lua scripts. UltraPse has very high computational efficiency. It is even faster than the PseAAC-General, which used to be the fastest program of its kind. For the users' convenience, we have integrated many existing modes within the UltraPse. We expect that the UltraPse program can be a useful platform which simplifies all future programming works in developing novel sequence representation modes. All source codes of UltraPse, including some extension modules can be downloaded freely under the term of GNU GPL (GNU General Public License) v3 from the GitHub repository: https://github.com/pufengdu/UltraPse.

\section{Results and Discussion}

\subsection{Computational Efficiency Analysis}

We compared the computational efficiency of UltraPse to that of PseAAC-General and Pse-In-One under the same conditions. As in Figure 1, the UltraPse can process over 120 thousand sequences per second, while PseAAC-General can process about 85 thousand sequences per second. Unfortunately, the Pse-In-One can process only less than one thousand sequences per second. According to these results, the computational efficiency of UltraPse is roughly 1.5 times of the PseAAC-General, and about 185 times of Pse-In-One. Since the algorithms of the three programs are essentially the same, the reason for the efficiency differences resides in the technical details of the implementations.

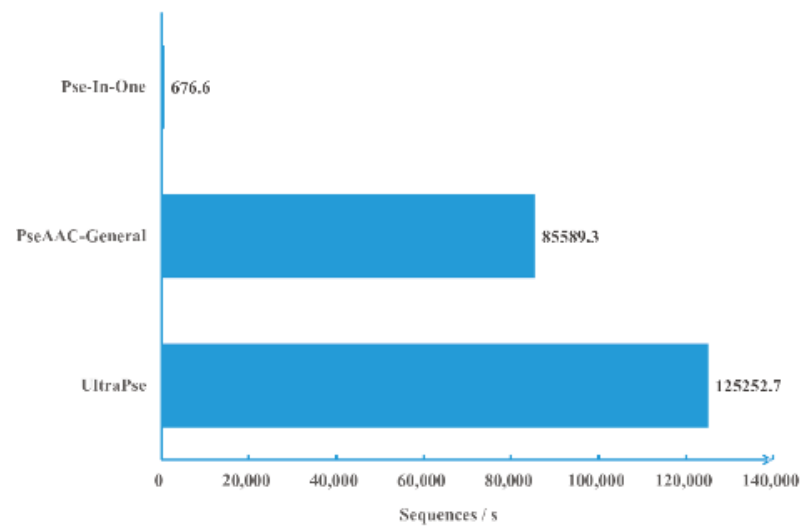

Figure 1. Computational efficiency comparisons. Three programs are compared. The comparison was carried out by letting the three programs compute amino acid compositions on the same dataset on the same machine. Every program was executed with the same parameters for three times. The average execution time was applied in calculating the computational efficiency. The computational efficiency is measured by the average number of sequences that are processed every second. Pse-In-One: A program in literature [52]; PseAAC-General: A program in literature [46]; UltraPse: A program of this work. 


\subsection{Flexibility and Extensibility}

We integrated 35 sequence representation modes within the UltraPse. The representation modes can be organized hierarchically as in Figure 2. The integrated modes can be used to represent protein, as well as DNA and RNA sequences. The modes cover most of the representation modes that can be generated by PseAAC-General, PseKNC-General, and Pse-In-One. Moreover, UltraPse can generate even more modes, for example, the commonly used one-hot encoding mode [53-55]. The sequence representation modes of UltraPse can be extended by using BSOs and TDFs. According to our own works, using UltraPse in developing novel representation modes can save over half of the programing labor.

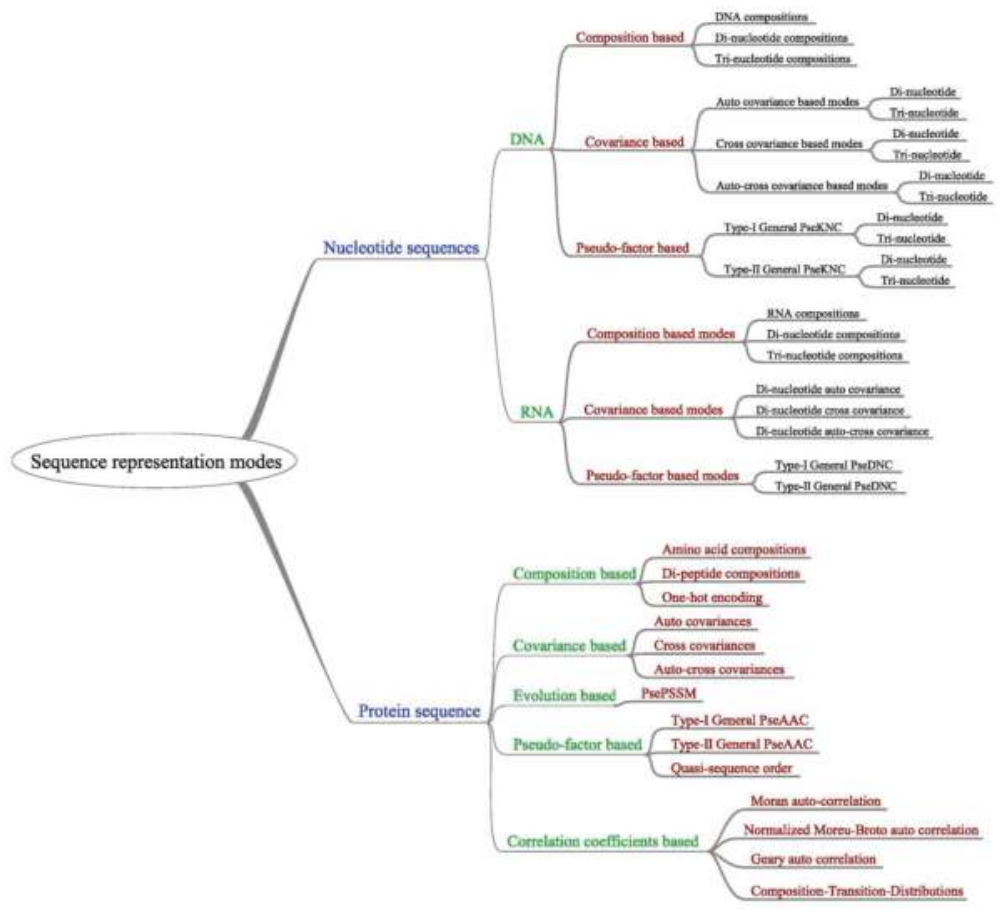

Figure 2. Hierarchical organization of integrated sequence representation modes. UltraPse integrated the sequence representation modes in its distribution package. Most of these modes can also be applied in user-defined sequence types, as long as the users provide proper definitions of the physicochemical properties.

Besides the user-defined representation modes of protein and nucleotide sequences, the users of UltraPse can define their own sequence types using TDFs. They are allowed to choose a set of letters other than the standard ones to represent additional information. For example, a user can use $\mathrm{C}$ for cytidines on a DNA sequence, and $\mathrm{M}$ for methylated cytidines. The choice of the letter $\mathrm{M}$ totally depends on the users. Even more, the users of UltraPse can define their own physicochemical properties with TDFs.

The TDFs of UltraPse is written using Lua language, which is a simple, powerful and extensible programming language which has been applied in bioinformatics software previously [56]. We provide over 20 UltraPse specific functions and interfaces. Users can access and modify UltraPse internal data structures using these functions in TDFs. We compared the flexibility and the extensibility of different software in Table 1. 
Table 1. Software function comparison in terms of flexibility and extensibility.

\begin{tabular}{ccc}
\hline Software Functions & Sequence Types & Extensibility \\
\hline UltraPse & $\begin{array}{c}\text { DNA, RNA, Protein, } \\
\text { User-defined types }\end{array}$ & $\begin{array}{c}\text { Users can define their own sequence } \\
\text { types, representation modes and } \\
\text { physicochemical properties }\end{array}$ \\
\hline PseAAC-General [46] & Protein & $\begin{array}{c}\text { Users can define their own } \\
\text { representation modes }\end{array}$ \\
\hline PseAAC-Builder [45] & DNA, RNA, Protein & No extensibility \\
\hline Pse-In-One [52] & DNA, RNA & $\begin{array}{c}\text { Users can define their own } \\
\text { physicochemical properties }\end{array}$ \\
\hline PseKNC [48] & DNA, RNA & $\begin{array}{l}\text { Users can define their own } \\
\text { physicochemical properties }\end{array}$ \\
\hline PseKNC-General [49] & $\begin{array}{l}\text { Users can define their own } \\
\text { physicochemical properties }\end{array}$ \\
\hline
\end{tabular}

\subsection{Compatibility and Robustness}

UltraPse can recognize FASTA format files that are directly downloaded from one of the following five databases: GenBank, UniProt, EMBL, DDBJ, and RefSeq. The sequence identifiers and comments in these public databases can be automatically recognized. For FASTA file that are not from these public databases, UltraPse can also recognize them as long as the comment line of every sequence is unique in the FASTA file. Besides the FASTA format requirements, there is no additional restriction on input data format. As indicated in Table 2, this is a unique advantage of UltraPse.

According to Chou's five step rule [12,21,57-60], before converting biological sequences into numerical vectors, a high-quality benchmark dataset must be constructed. The construction of a dataset usually includes a step to filter out the sequences containing non-standard letters. For example, $\mathrm{B}, \mathrm{J}$, or $\mathrm{X}$ appear in protein sequences in the UniProt database. However, the sequences containing these letters are hardly suitable for further analysis in many cases. As indicated in Table 2, UltraPse provides a user-controllable data fault tolerant ability. According to users' choice, when one of these sequences is encountered, UltraPse can automatically skip the sequence or abort all further computations. This function is useful in adopting third-party datasets in practical works, because filtering out the sequences usually requires tedious programming work.

Table 2. Software function comparison in terms of data processing ability.

\begin{tabular}{cccc}
\hline Software & Output Formats & Input Formats & Data Fault Tolerant $^{\text {a }}$ \\
\hline UltraPse & SVM $^{\text {b }}$, TSV $^{\mathrm{c}}$, CSV d $^{\text {d }}$ & $\begin{array}{c}\text { Multi-line FASTA (Automatic ID } \\
\text { recognition for UniProt, GenBank, } \\
\text { EMBL, DDBJ and RefSeq) }\end{array}$ & $\begin{array}{c}\text { User-controllable behavior } \\
\text { on data faults }\end{array}$ \\
\hline PseAAC-General [46] & SVM, TSV, CSV & $\begin{array}{c}\text { Single-line FASTA (With } \\
\text { restrictions on comment line) }\end{array}$ & $\begin{array}{c}\text { Automatically ignore and } \\
\text { report data faults }\end{array}$ \\
\hline PseAAC-Builder [45] & SVM, TSV, CSV & $\begin{array}{c}\text { Single-line FASTA (With } \\
\text { restrictions on comment line) }\end{array}$ & $\begin{array}{c}\text { Automatically ignore and } \\
\text { report data faults }\end{array}$ \\
\hline Pse-In-One [52] & SVM, TSV, CSV & Mutlti-line FASTA & $\begin{array}{c}\text { Abort processing on } \\
\text { data faults }\end{array}$ \\
\hline PseKNC [48] & SVM, TSV, CSV & Mutlti-line FASTA & $\begin{array}{c}\text { Abort processing on } \\
\text { data faults }\end{array}$ \\
\hline
\end{tabular}

${ }^{\text {a }}$ Data fault tolerant: The behavior of a software when it encounters some invalid data records. Here, the invalid data records include the sequences with non-standard letter and the sequence without sufficient length; ${ }^{b}$ SVM: data format for libSVM [61]; ${ }^{\mathrm{c}}$ TSV: tab separated vector; ${ }^{\mathrm{d}}$ CSV: comma separated vector; ${ }^{\mathrm{e}}$ Single-line FASTA: the sequence of a record in the file must not spread to multiple lines. Both PseAAC-General and PseAAC-Builder have the same restrictions. 


\subsection{Technical Detail Comparison}

Most state-of-the art software is written in Python, while PseAAC-General and UltraPse are written in $\mathrm{C}++$. This difference eventually made the difference in computational efficiency. Since the computational efficiencies of PseAAC-General and UltraPse are comparable, we can compare several technical details of them.

PseAAC-General is a program that can be extended by using Binary Extension Modules (BEMs). However, it should be noted that, the BEMs of PseAAC-General are completely different to the BSOs in UltraPse. A BEM of PseAAC-General is just a compressed data block. However, how this data block should be used, was still implemented by the PseAAC-General main program. In the UltraPse, a BSO is actually a dynamically loaded library, which contains all the information and instructions for constructing one or more sequence representation modes. Therefore, the BSOs of UltraPse are much more flexible than the BEMs of PseAAC-General.

We have seen that UltraPse has roughly 1.5 times the efficiency of PseAAC-General. This advantage is achieved by an internal representation scheme and a pre-computing mechanism of UltraPse. In PseAAC-General, the sequences are converted to a series of physicochemical properties. The sequence descriptors are then computed according to the corresponding algorithms. However, this intuitive implementation requires repeatedly computing dot-product or Euclidean distance between physicochemical vectors of different amino acids. Since the combination of two different amino acids is limited, we pre-compute the dot-product and Euclidean distance for all possible combinations in UltraPse. The sequences in UltraPse are not converted into a series of physicochemical properties. They are converted into UltraPse internal indices, which can be used to quickly find correct values that have been pre-computed. When computing only the amino acids compositions, the implementations of PseAAC-General and UltraPse are similar. However, UltraPse still benefits from converting all sequences into internal indices first. Because, the amino acids counting procedure becomes simpler, this allows the compiler to do more optimization for speed. This is why UltraPse is faster than PseAAC-General.

\subsection{Future Works in Plan}

Besides the practical application of UltraPse program in research projects, there is still much work to do in terms of software development. The work at first priority is to add an automated unit-testing facility in the source code of UltraPse. Unit-testing is good practice in software engineering to ensure robustness of large scale software. It will be very important for the future versions of UltraPse. The next work in plan is to enable UltraPse support more data formats as input files. As far as we can tell, no existing program in representing biological sequences can handle file formats other than FASTA. We will make the next version of UltraPse handle FASTA, FASTQ, and several other formats of input file.

\subsection{Availability}

The UltraPse software is provided as source codes and binary packages. All the source codes can be downloaded from the GitHub repository (Available online: https://github.com/pufengdu/ UltraPse). The binary distribution packages can also be downloaded from the Release sub-directory in the GitHub repository. Currently, there are binary packages for Windows and Linux platforms. The Windows binary program can be executed directly. The Linux binary package has been tested on a freshly installed Ubuntu Linux Server 16.04.3.

\section{Methods}

\subsection{Efficiency Comparison Protocols}

We performed computational efficiency comparisons on a server with an Intel Xeon X3470 processor and 32 GB memory. To perform a fair comparison, we installed Pse-In-One locally on the server. We also locally compiled and installed PseAAC-General and UltraPse on the same server. 
The testing dataset is the "huge" testing dataset that can be obtained from the official website of PseAAC-General. This dataset contains 516,081 protein sequences. Since the Pse-In-One keeps complaining about non-standard letters and too short sequences in the dataset, we excluded all the sequences that have non-standard letters. The remaining 513,536 protein sequences were fed into three programs independently. All three programs are configured to compute only amino acid compositions. The computational times are measured by the "real" time value of the standard Linux time command. To eliminate random errors, every program was executed consecutively with exactly the same configuration three times. The average computational time was used in calculating computational efficiency.

\subsection{Abstracted Software Design}

We illustrate the internal structure and the data-flows of UltraPse in Figure 3. There are four major parts within UltraPse. They are the FASTA parser, sequence preprocessor, computing engine, and the result writer. The FASTA parser is responsible for loading FASTA format sequences into the memory from a hard drive. It also organizes the sequences according to their identifiers and their sequence types. These sequences are then sent to the sequence preprocessor, where the sequences are converted to UltraPse internal indices according to the sequence type definitions. The computing engine is composed of several mode modules, which are configured according to user requirements. The internal indices go through all mode modules. Eventually, sequence descriptors are generated. The result writer exports these descriptors on the hard drive according to the format requirements.

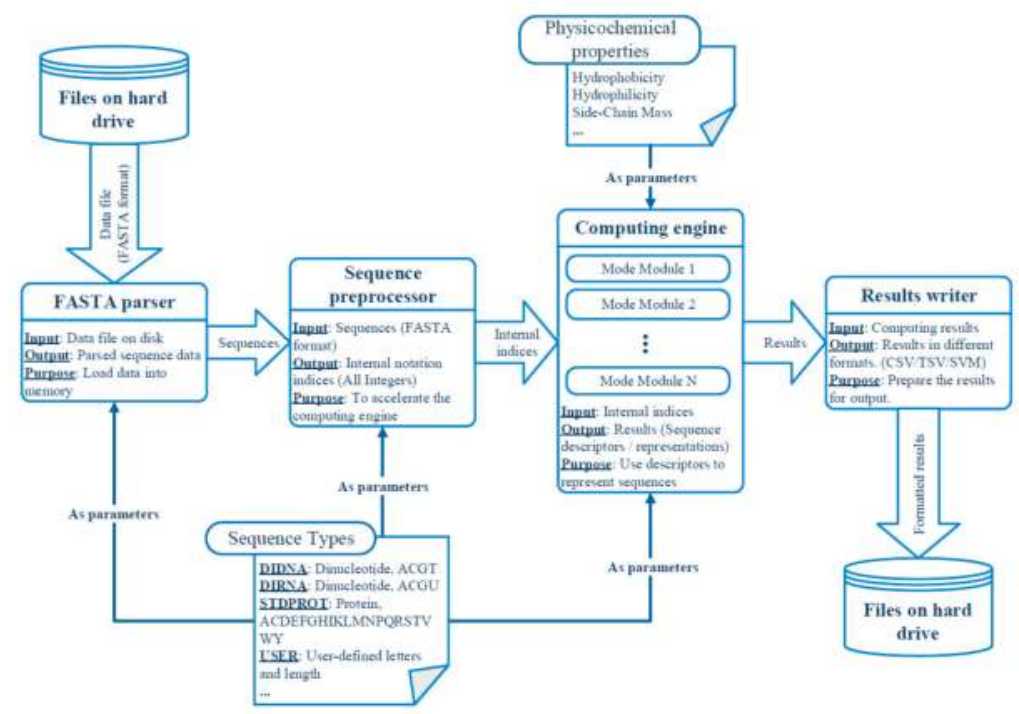

Figure 3. The abstracted software design and data flow chart of UltraPse.

\subsection{Implementation Technology}

The UltraPse main program is written using standard $\mathrm{C}++$ language, following $\mathrm{C}++14$ standard. The destination hardware architecture is $x 86-64$. The dependencies of the UltraPse main program include GNU standard C library and the embedded interpreter of Lua scripting language. The BSOs of UltraPse are also written using $\mathrm{C}++$, following the same rules as the main program.

On the Linux platform, the compiler for producing binary executables is the GNU g++ version 7.2. The users should first install Lua scripting language. The configuration and compilation of UltraPse 
need the library provided by the Lua package. On the Windows platform, the MinGW64 version $\mathrm{g}++$ compiler is applied. Several independent libraries are required to compile the codes. For the convenience of Windows users, we provide a binary executable package for the Windows platform.

The TDFs are provided as platform-independent Lua scripts, which can be viewed, edited, and loaded as their original form. The internal data structures of UltraPse can be accessed by Lua scripts using UltraPse specific functions and interfaces. The details on how to write TDFs can be found in the software manual.

\subsection{A Practical Example}

Figure 4 demonstrate a practical example. The classic pseudo-amino acid composition modes, including type-I and type-II, are implemented using a TDF in UltraPse. The TDF for classic pseudo-amino acid compositions can be found in the "tdfs" subdirectory of UltraPse. The right part of Figure 4 is a part of this TDF. With this TDF, the users only need to specify some parameters on the command line. For example, the "-1 $10-\mathrm{w} 0.05$ " on the command line indicate the value of $\lambda$ and $\omega$ in the PseAAC formulations. Unlike PseAAC-General, where the meanings of all command line options are fixed, the meanings of command line options can be altered by the TDFs in the UltraPse. This is to simplify the development of novel sequence representation modes, where parameters are required to perform correct and efficient computations.

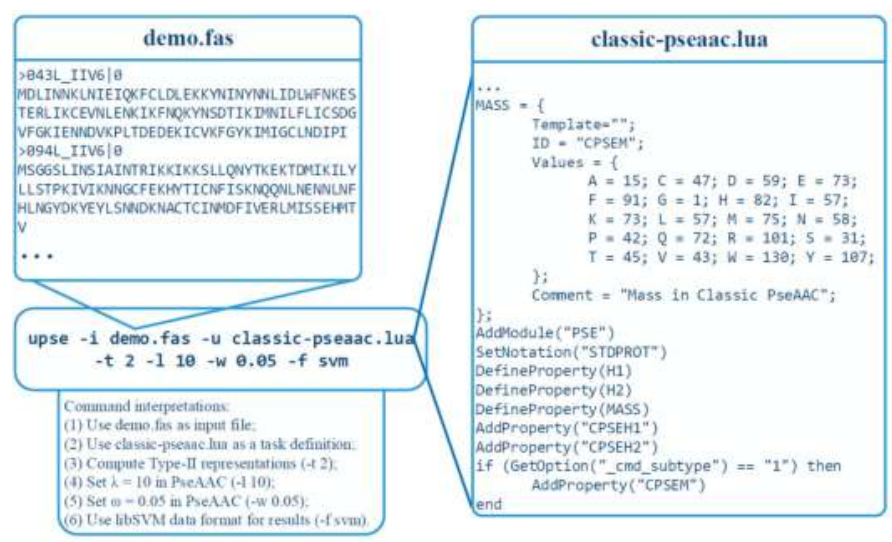

Figure 4. An example on using UltraPse. UltraPse was used to implement classic pseudo-amino acid compositions. A TDF: classic-pseaac.lua, was applied. The FASTA format sequences are stored in the demo.fas file. The command options indicate that the Type 2 PseAAC will be computed with parameters: $\lambda=10$ and $\omega=0.05$. The output format is compatible to libSVM.

\section{Conclusions}

In this paper, we described our new software, the UltraPse (Available online: https://github.com/pufengdu/UltraPse). UltraPse is a universal and extensible software platform for generating biological sequence representations. Since many programs have already been released for various sequence representations, UltraPse has no intention to be a new competitor on the same playground. We expect that UltraPse can work side-by-side with other existing programs, such as PseAAC-General, PseAAC-Builder and Pse-In-One, to accelerate the process of generating sequence representations under various working environments.

Although we have integrated many existing sequence modes within the UltraPse, it should be noted that the major advantage of UltraPse is its flexibility and extensibility. It was designed to be 
a software platform rather than a program with specific functions. It aims at simplifying all future programming works in developing novel sequence representations.

Web servers have already been proved to be a good method in releasing software. However, presenting UltraPse with a web server will severely damage its computational efficiency. Therefore, we do not provide an online web server for UltraPse. We would rather provide it as a local program. The users need to compile and install it on their own servers. The graphical user interfaces (GUI) is useful on platforms like Microsoft Windows. We will develop a GUI for UltraPse on the Windows platform in future.

Acknowledgments: This work is funded by the National Natural Science Foundation of China (NSFC 31401132); National Natural Science Foundation of China (NSFC 61005041); Tianjin Natural Science Foundation (No. 12JCQNJC02300).

Author Contributions: Pu-Feng Du designed the software, wrote most of the codes and the paper in part. Wei Zhao partially wrote the codes and in part tested the software. Yang-Yang Miao partially tested the software and in part wrote the paper. Le-Yi Wei partially wrote the paper. Likun Wang provided technical discussions, and in part wrote the code and the paper.

Conflicts of Interest: The authors declare no conflict of interest.

\section{Abbreviations}

$\begin{array}{ll}\text { AmPseAAC } & \text { Amphiphilic pseudo amino acid composition } \\ \text { BEM } & \text { Binary extension module } \\ \text { BSO } & \text { Binary shared objects } \\ \text { GPL } & \text { General public license } \\ \text { GUI } & \text { Graphical user interfaces } \\ \text { PseAAC } & \text { Pseudo-amino acid composition } \\ \text { PseDNC } & \text { Pseudo-dinucleotide composition } \\ \text { PseKNC } & \text { Pseudo-k nucleotide composition } \\ \text { TDF } & \text { Task definition file }\end{array}$

\section{References}

1. Jiao, Y.-S.; Du, P.-F. Predicting Golgi-resident protein types using pseudo amino acid compositions: Approaches with positional specific physicochemical properties. J. Theor. Biol. 2016, 391, 35-42. [CrossRef] [PubMed]

2. Jiao, Y.-S.; Du, P.-F. Predicting protein submitochondrial locations by incorporating the positional-specific physicochemical properties into Chou's general pseudo-amino acid compositions. J. Theor. Biol. 2017, 416, 81-87. [CrossRef] [PubMed]

3. Nanni, L.; Brahnam, S.; Lumini, A. High performance set of PseAAC and sequence based descriptors for protein classification. J. Theor. Biol. 2010, 266, 1-10. [CrossRef] [PubMed]

4. Nanni, L.; Brahnam, S.; Lumini, A. Prediction of protein structure classes by incorporating different protein descriptors into general Chou's pseudo amino acid composition. J. Theor. Biol. 2014, 360, 109-116. [CrossRef] [PubMed]

5. Li, L.; Yu, S.; Xiao, W.; Li, Y.; Hu, W.; Huang, L.; Zheng, X.; Zhou, S.; Yang, H. Protein submitochondrial localization from integrated sequence representation and SVM-based backward feature extraction. Mol. Biosyst. 2014, 11, 170-177. [CrossRef] [PubMed]

6. Lin, H.; Chen, W.; Yuan, L.-F.; Li, Z.-Q.; Ding, H. Using Over-Represented Tetrapeptides to Predict Protein Submitochondria Locations. Acta Biotheor 2013, 61, 259-268. [CrossRef] [PubMed]

7. Zuo, Y.-C.; Peng, Y.; Liu, L.; Chen, W.; Yang, L.; Fan, G.-L. Predicting peroxidase subcellular location by hybridizing different descriptors of Chou' pseudo amino acid patterns. Anal. Biochem. 2014, 458, 14-19. [CrossRef] [PubMed]

8. Nanni, L.; Lumini, A.; Gupta, D.; Garg, A. Identifying Bacterial Virulent Proteins by Fusing a Set of Classifiers Based on Variants of Chou's Pseudo Amino Acid Composition and on Evolutionary Information. IEEE-ACM Trans. Comput. Biol. Bioinform. 2012, 9, 467-475. [CrossRef] [PubMed] 
9. Chou, K.-C. Prediction of protein cellular attributes using pseudo-amino acid composition. Proteins 2001, 43, 246-255. [CrossRef] [PubMed]

10. Chou, K.-C. Using amphiphilic pseudo amino acid composition to predict enzyme subfamily classes. Bioinformatics 2005, 21, 10-19. [CrossRef] [PubMed]

11. Chou, K.-C. Pseudo Amino Acid Composition and its Applications in Bioinformatics, Proteomics and System Biology. Curr. Proteom. 2009, 6, 262-274. [CrossRef]

12. Qiu, W.-R.; Sun, B.-Q.; Xiao, X.; Xu, Z.-C.; Chou, K.-C. iHyd-PseCp: Identify hydroxyproline and hydroxylysine in proteins by incorporating sequence-coupled effects into general PseAAC. Oncotarget 2016, 7, 44310-44321. [CrossRef] [PubMed]

13. Xu, Y.; Ding, Y.-X.; Ding, J.; Wu, L.-Y.; Deng, N.-Y. Phogly-PseAAC: Prediction of lysine phosphoglycerylation in proteins incorporating with position-specific propensity. J. Theor. Biol. 2015, 379, 10-15. [CrossRef] [PubMed]

14. Jia, J.; Zhang, L.; Liu, Z.; Xiao, X.; Chou, K.-C. pSumo-CD: Predicting sumoylation sites in proteins with covariance discriminant algorithm by incorporating sequence-coupled effects into general PseAAC. Bioinformatics 2016, 32, 3133-3141. [CrossRef] [PubMed]

15. Ahmad, K.; Waris, M.; Hayat, M. Prediction of Protein Submitochondrial Locations by Incorporating Dipeptide Composition into Chou's General Pseudo Amino Acid Composition. J. Membr. Biol. 2016, 249, 293-304. [CrossRef] [PubMed]

16. Feng, P.-M.; Chen, W.; Lin, H.; Chou, K.-C. iHSP-PseRAAAC: Identifying the heat shock protein families using pseudo reduced amino acid alphabet composition. Anal. Biochem. 2013, 442, 118-125. [CrossRef] [PubMed]

17. Lin, W.-Z.; Fang, J.-A.; Xiao, X.; Chou, K.-C. iLoc-Animal: A multi-label learning classifier for predicting subcellular localization of animal proteins. Mol. Biosyst. 2013, 9, 634-644. [CrossRef] [PubMed]

18. Mohabatkar, H.; Mohammad Beigi, M.; Esmaeili, A. Prediction of GABAA receptor proteins using the concept of Chou's pseudo-amino acid composition and support vector machine. J. Theor. Biol. 2011, 281, 18-23. [CrossRef] [PubMed]

19. Jiao, Y.-S.; Du, P.-F. Prediction of Golgi-resident protein types using general form of Chou's pseudo-amino acid compositions: Approaches with minimal redundancy maximal relevance feature selection. J. Theor. Biol. 2016, 402, 38-44. [CrossRef] [PubMed]

20. Du, P.; Wang, L. Predicting human protein subcellular locations by the ensemble of multiple predictors via protein-protein interaction network with edge clustering coefficients. PLoS ONE 2014, 9, e86879. [CrossRef] [PubMed]

21. Chou, K.-C. Some remarks on protein attribute prediction and pseudo amino acid composition. J. Theor. Biol. 2011, 273, 236-247. [CrossRef] [PubMed]

22. Chou, K.-C. Some remarks on predicting multi-label attributes in molecular biosystems. Mol. Biosyst. 2013, 9 , 1092-1100. [CrossRef] [PubMed]

23. Chou, K.-C. Impacts of bioinformatics to medicinal chemistry. Med. Chem. 2015, 11, 218-234. [CrossRef] [PubMed]

24. Chen, W.; Lin, H.; Chou, K.-C. Pseudo nucleotide composition or PseKNC: An effective formulation for analyzing genomic sequences. Mol. Biosyst. 2015, 11, 2620-2634. [CrossRef] [PubMed]

25. Chen, W.; Feng, P.-M.; Lin, H.; Chou, K.-C. iRSpot-PseDNC: Identify recombination spots with pseudo dinucleotide composition. Nucleic. Acids Res. 2013, 41, e68. [CrossRef] [PubMed]

26. Chen, W.; Feng, P.-M.; Lin, H.; Chou, K.-C. iSS-PseDNC: Identifying splicing sites using pseudo dinucleotide composition. Biomed. Res. Int. 2014, 2014, 623149. [CrossRef] [PubMed]

27. Chen, W.; Feng, P.-M.; Deng, E.-Z.; Lin, H.; Chou, K.-C. iTIS-PseTNC: A sequence-based predictor for identifying translation initiation site in human genes using pseudo trinucleotide composition. Anal. Biochem. 2014, 462, 76-83. [CrossRef] [PubMed]

28. Guo, S.-H.; Deng, E.-Z.; Xu, L.-Q.; Ding, H.; Lin, H.; Chen, W.; Chou, K.-C. iNuc-PseKNC: A sequence-based predictor for predicting nucleosome positioning in genomes with pseudo k-tuple nucleotide composition. Bioinformatics 2014, 30, 1522-1529. [CrossRef] [PubMed]

29. Lin, H.; Deng, E.-Z.; Ding, H.; Chen, W.; Chou, K.-C. iPro54-PseKNC: A sequence-based predictor for identifying sigma-54 promoters in prokaryote with pseudo k-tuple nucleotide composition. Nucleic Acids Res. 2014, 42, 12961-12972. [CrossRef] [PubMed] 
30. Chang, C.-C.; Lin, C.-J.; Chen, W.; Feng, P.; Ding, H.; Lin, H.; Chou, K.-C. iRNA-Methyl: Identifying $N^{6}$-methyladenosine sites using pseudo nucleotide composition. Anal. Biochem. 2015, 490, 26-33. [CrossRef]

31. Liu, B.; Fang, L.; Liu, F.; Wang, X.; Chou, K.-C. iMiRNA-PseDPC: MicroRNA precursor identification with a pseudo distance-pair composition approach. J. Biomol. Struct. Dyn. 2016, 34, 223-235. [CrossRef] [PubMed]

32. Chen, W.; Tang, H.; Ye, J.; Lin, H.; Chou, K.-C. iRNA-PseU: Identifying RNA pseudouridine sites. Mol. Ther. Nucleic Acids 2016, 5, e332. [CrossRef] [PubMed]

33. Liu, B.; Long, R.; Chou, K.-C. iDHS-EL: Identifying DNase I hypersensitive sites by fusing three different modes of pseudo nucleotide composition into an ensemble learning framework. Bioinformatics 2016, 32, 2411-2418. [CrossRef] [PubMed]

34. Liu, B.; Yang, F.; Huang, D.-S.; Chou, K.-C. iPromoter-2L: A two-layer predictor for identifying promoters and their types by multi-window-based PseKNC. Bioinformatics 2017. [CrossRef] [PubMed]

35. Iqbal, M.; Hayat, M. "iSS-Hyb-mRMR": Identification of splicing sites using hybrid space of pseudo trinucleotide and pseudo tetranucleotide composition. Comput. Methods Programs Biomed. 2016, 128, 1-11. [CrossRef] [PubMed]

36. Kabir, M.; Iqbal, M.; Ahmad, S.; Hayat, M. iTIS-PseKNC: Identification of Translation Initiation Site in human genes using pseudo k-tuple nucleotides composition. Comput. Biol. Med. 2015, 66, 252-257. [CrossRef] [PubMed]

37. Zhang, M.; Sun, J.-W.; Liu, Z.; Ren, M.-W.; Shen, H.-B.; Yu, D.-J. Improving N(6)-methyladenosine site prediction with heuristic selection of nucleotide physical-chemical properties. Anal. Biochem. 2016, 508, 104-113. [CrossRef] [PubMed]

38. Dong, C.; Yuan, Y.-Z.; Zhang, F.-Z.; Hua, H.-L.; Ye, Y.-N.; Labena, A.A.; Lin, H.; Chen, W.; Guo, F.-B. Combining pseudo dinucleotide composition with the $Z$ curve method to improve the accuracy of predicting DNA elements: A case study in recombination spots. Mol. Biosyst. 2016, 12, 2893-2900. [CrossRef] [PubMed]

39. Liu, B.; Liu, Y.; Huang, D. Recombination Hotspot/Coldspot Identification Combining Three Different Pseudocomponents via an Ensemble Learning Approach. Biomed. Res. Int. 2016, 2016, 8527435. [CrossRef] [PubMed]

40. Qiu, W.-R.; Jiang, S.-Y.; Xu, Z.-C.; Xiao, X.; Chou, K.-C. iRNAm5C-PseDNC: Identifying RNA 5-methylcytosine sites by incorporating physical-chemical properties into pseudo dinucleotide composition. Oncotarget 2017, 8, 41178-41188. [CrossRef] [PubMed]

41. Xu, Z.-C.; Wang, P.; Qiu, W.-R.; Xiao, X. iSS-PC: Identifying Splicing Sites via Physical-Chemical Properties Using Deep Sparse Auto-Encoder. Sci. Rep. 2017, 7, 8222. [CrossRef] [PubMed]

42. Li, Z.R.; Lin, H.H.; Han, L.Y.; Jiang, L.; Chen, X.; Chen, Y.Z. PROFEAT: A web server for computing structural and physicochemical features of proteins and peptides from amino acid sequence. Nucleic Acids Res. 2006, 34, W32-W37. [CrossRef] [PubMed]

43. Shen, H.-B.; Chou, K.-C. PseAAC: A flexible web server for generating various kinds of protein pseudo amino acid composition. Anal. Biochem. 2008, 373, 386-388. [CrossRef] [PubMed]

44. Cao, D.-S.; Xu, Q.-S.; Liang, Y.-Z. Propy: A tool to generate various modes of Chou's PseAAC. Bioinformatics 2013, 29, 960-962. [CrossRef] [PubMed]

45. Du, P.; Wang, X.; Xu, C.; Gao, Y. PseAAC-Builder: A cross-platform stand-alone program for generating various special Chou's pseudo-amino acid compositions. Anal. Biochem. 2012, 425, 117-119. [CrossRef] [PubMed]

46. Du, P.; Gu, S.; Jiao, Y. PseAAC-General: Fast building various modes of general form of Chou's pseudo-amino acid composition for large-scale protein datasets. Int. J. Mol. Sci. 2014, 15, 3495-3506. [CrossRef] [PubMed]

47. Xiao, N.; Cao, D.-S.; Zhu, M.-F.; Xu, Q.-S. protr/ProtrWeb: R package and web server for generating various numerical representation schemes of protein sequences. Bioinformatics 2015, 31, 1857-1859. [CrossRef] [PubMed]

48. Chen, W.; Lei, T.-Y.; Jin, D.-C.; Lin, H.; Chou, K.-C. PseKNC: A flexible web server for generating pseudo K-tuple nucleotide composition. Anal. Biochem. 2014, 456, 53-60. [CrossRef] [PubMed]

49. Chen, W.; Zhang, X.; Brooker, J.; Lin, H.; Zhang, L.; Chou, K.-C. PseKNC-General: A cross-platform package for generating various modes of pseudo nucleotide compositions. Bioinformatics 2015, 31, 119-120. [CrossRef] [PubMed] 
50. Liu, B.; Liu, F.; Fang, L.; Wang, X.; Chou, K.-C. repDNA: A Python package to generate various modes of feature vectors for DNA sequences by incorporating user-defined physicochemical properties and sequence-order effects. Bioinformatics 2015, 31, 1307-1309. [CrossRef] [PubMed]

51. Liu, B.; Liu, F.; Fang, L.; Wang, X.; Chou, K.-C. repRNA: A web server for generating various feature vectors of RNA sequences. Mol. Genet. Genom. 2016, 291, 473-481. [CrossRef] [PubMed]

52. Liu, B.; Liu, F.; Wang, X.; Chen, J.; Fang, L.; Chou, K.-C. Pse-in-One: A web server for generating various modes of pseudo components of DNA, RNA, and protein sequences. Nucleic Acids Res. 2015, 43, W65-W71. [CrossRef] [PubMed]

53. Li, T.; Du, P.; Xu, N. Identifying human kinase-specific protein phosphorylation sites by integrating heterogeneous information from various sources. PLoS ONE 2010, 5, e15411. [CrossRef] [PubMed]

54. Chen, Q.-Y.; Tang, J.; Du, P.-F. Predicting protein lysine phosphoglycerylation sites by hybridizing many sequence based features. Mol. Biosyst. 2017, 13, 874-882. [CrossRef] [PubMed]

55. Lei, G.-C.; Tang, J.; Du, P.-F. Predicting S-sulfenylation Sites Using Physicochemical Properties Differences. Lett. Org. Chem. 2017, 14, 665-672. [CrossRef]

56. Steinbiss, S.; Gremme, G.; Schärfer, C.; Mader, M.; Kurtz, S. AnnotationSketch: A genome annotation drawing library. Bioinformatics 2009, 25, 533-534. [CrossRef] [PubMed]

57. Jia, J.; Liu, Z.; Xiao, X.; Liu, B.; Chou, K.-C. iCar-PseCp: Identify carbonylation sites in proteins by Monte Carlo sampling and incorporating sequence coupled effects into general PseAAC. Oncotarget 2016, 7, 34558-34570. [CrossRef] [PubMed]

58. Qiu, W.-R.; Xiao, X.; Lin, W.-Z.; Chou, K.-C. iMethyl-PseAAC: Identification of protein methylation sites via a pseudo amino acid composition approach. Biomed. Res. Int. 2014, 2014, 947416. [CrossRef] [PubMed]

59. Liu, B.; Xu, J.; Lan, X.; Xu, R.; Zhou, J.; Wang, X.; Chou, K.-C. iDNA-Prot I dis: Identifying DNA-binding proteins by incorporating amino acid distance-pairs and reduced alphabet profile into the general pseudo amino acid composition. PLoS ONE 2014, 9, e106691. [CrossRef] [PubMed]

60. Xu, Y.; Wen, X.; Wen, L.-S.; Wu, L.-Y.; Deng, N.-Y.; Chou, K.-C. iNitro-Tyr: Prediction of nitrotyrosine sites in proteins with general pseudo amino acid composition. PLoS ONE 2014, 9, e105018. [CrossRef] [PubMed]

61. Chang, C.-C.; Lin, C.-J. LIBSVM: A Library for Support Vector Machines. ACM Trans. Intell. Syst. Technol. 2011, 2, 27. [CrossRef] 
Article

\title{
Protein Subcellular Localization with Gaussian Kernel Discriminant Analysis and Its Kernel Parameter Selection
}

\author{
Shunfang Wang ${ }^{1, *,+}$, Bing Nie ${ }^{1,+}$, Kun Yue $^{1, *}, \mathrm{Yu} \mathrm{Fei}^{2, *}$, Wenjia $\mathrm{Li}^{1}$ and Dongshu $\mathrm{Xu}^{1}$ \\ 1 Department of Computer Science and Engineering, School of Information Science and Engineering, \\ Yunnan University, Kunming 650504, China; bingn2017@gmail.com (B.N.); \\ woshiwenzi666@gmail.com (W.L.); qq78316519@gmail.com (D.X.) \\ 2 School of Statistics and Mathematics, Yunnan University of Finance and Economics, Kunming 650221, China \\ * Correspondence: sfwang_66@ynu.edu.cn (S.W.); kyue@ynu.edu.cn (K.Y.); feiyukm@aliyun.com (Y.F.); \\ Tel.: +86-137-6919-6517 (S.W.); +86-871-6593-3093 (K.Y.); +86-139-8765-9718 (Y.F.) \\ + These authors contributed equally to this work.
}

Received: 16 October 2017; Accepted: 5 December 2017; Published: 15 December 2017

\begin{abstract}
Kernel discriminant analysis (KDA) is a dimension reduction and classification algorithm based on nonlinear kernel trick, which can be novelly used to treat high-dimensional and complex biological data before undergoing classification processes such as protein subcellular localization. Kernel parameters make a great impact on the performance of the KDA model. Specifically, for KDA with the popular Gaussian kernel, to select the scale parameter is still a challenging problem. Thus, this paper introduces the KDA method and proposes a new method for Gaussian kernel parameter selection depending on the fact that the differences between reconstruction errors of edge normal samples and those of interior normal samples should be maximized for certain suitable kernel parameters. Experiments with various standard data sets of protein subcellular localization show that the overall accuracy of protein classification prediction with KDA is much higher than that without KDA. Meanwhile, the kernel parameter of KDA has a great impact on the efficiency, and the proposed method can produce an optimum parameter, which makes the new algorithm not only perform as effectively as the traditional ones, but also reduce the computational time and thus improve efficiency.
\end{abstract}

Keywords: protein subcellular localization; kernel parameter selection; kernel discriminant analysis (KDA); Gaussian kernel function; dimension reduction

\section{Introduction}

Some proteins can only play the role in one specific place in the cell while others can play the role in several places in the cell [1]. Generally, a protein can function correctly only when it is localized to a correct subcellular location [2]. Therefore, protein subcellular localization prediction is an important research area of proteomics. It is helpful to predict protein function as well as to understand the interaction and regulation mechanism of proteins [3]. Now, many methods have been used to predict protein subcellular location, such as green fluorescent protein labeling [4], mass spectrometry [5], and so on. However, these traditional experimental methods usually have many technical limitations, resulting in high cost of time and money. Thus, prediction of protein subcellular location based on machine learning has become a focus research in bioinformatics [6-8].

When we use the methods of machine learning to predict protein subcellular location, we must extract features of protein sequences. We can get some vectors after feature extraction, and then we use the classifier to process these vectors. However, these vectors are usually complex due to their high dimensionality and nonlinear property. In order to improve the prediction accuracy of 
protein subcellular location, an appropriate nonlinear method for reducing data dimension should be used before classification. Kernel discriminant analysis (KDA) [9] is a nonlinear reductive dimension algorithm based on kernel trick that has been used in many fields such as facial recognition and fingerprint identification. The KDA method not only reduces data dimensionality but also makes use of the classification information. This paper newly introduces the KDA method to predict protein subcellular location. The algorithm of KDA first maps sample data to a high-dimensional feature space by a kernel function, and then executes linear discriminant analysis (LDA) in the high-dimensional feature space [10], which indicates that kernel parameter selection will significantly affect the algorithm performance.

There are some classical algorithms used to select the parameter of kernel function, such as genetic algorithm, grid searching algorithm, and so on. These methods have high calculation precision but large amounts of calculation. In an effort to reduce computational complexity, recently, Xiao et al. proposed a method based on reconstruction errors of samples and used it to select the parameters of Gaussian kernel principal component analysis (KPCA) for novelty detection [11]. Their methods are applied into the toy data sets and UCI (University of CaliforniaIrvine) benchmark data sets to demonstrate the correctness of the algorithm. However, their innovation in the KPCA method aims at dimensional reduction rather than discriminant analysis, which leads to unsatisfied classification prediction accuracy. Thus, it is necessary to improve the efficiency of the method in [11] especially for some complex data such as biological data.

In this paper, an improved algorithm of selecting parameters of Gaussian kernel in KDA is proposed to analyze complex protein data and predict subcellular location. By maximizing the differences of reconstruction errors between edge normal samples and interior normal samples, the proposed method not only shows the same effect as the traditional grid-searching method, but also reduces the computational time and improves efficiency.

\section{Results and Discussion}

In this section, the proposed method (in Section 3.4) and the grid-searching algorithm (in Section 4.4) are both applied to predict protein subcellular localization. We use two standard data sets as the experimental data. The two used feature expressions are generated from PSSM (position specific scoring matrix) [12], which are the PsePSSM (pseudo-position specific scoring matrix) [12] and the PSSM-S (AAO + PSSM-AAO + PSSM-SAC + PSSM-SD = PSSM-S) [13]. Here AAO means consensus sequence-based occurrence, PSSM-AAO means evolutionary-based occurrence or semi-occurrence of PSSM, PSSM-SD is segmented distribution of PSSM and PSSM-SAC is segmented auto covariance of PSSM. The k-nearest neighbors (KNN) is used as the classifier in which Euclidean distance is adopted for the distance between samples. The flow of experiments is as follows.

- $\quad$ First, for each standard data set, we use the PsePSSM algorithm and the PSSM-S algorithm to extract features, respectively. Then totally we obtain four sample sets, which are GN-1000 (Gram-negative with PsePSSM which contains 1000 features), GN-220 (Gram-negative with PSSM-S which contains 220 features), GP-1000 (Gram-positive with PsePSSM which contains 1000 features) and GP-220 (Gram-positive with PsePSSM which contains 220 features).

- $\quad$ Second, we use the proposed method to select the optimum kernel parameter for the Gaussian KDA model and then use KDA to reduce the dimension of sample sets. The same procedure is also carried out for the traditional grid-searching method to form a comparison with the proposed method.

- Finally, we use the KNN algorithm to classify the reduced dimensional sample sets and use some criterions to evaluate the results and give the comparison results.

Some detailed information in experiments is as follows. For every sample set, we choose the class that contains the most samples to form the training set [8]. Let $S=[0.1,0.2,0.3,0.4,1,2,3,4]$ be a candidate set of the Gaussian kernel parameter, which is proposed at random. When we use the KDA 
algorithm to reduce dimension, the number of retained eigenvectors must be less than or equal to $\mathrm{C}-1$ ( $C$ is the number of classes). Therefore, for sample sets GN-1000 and GN-220, the number of retained eigenvectors, which is denoted as d, can be from 1 to 7 . For the sample sets GP-1000 and GP-220, d can be 1,2 , and 3 . As far as the parameter $u$ is concerned, when it is $5-8 \%$ of the average number of samples, good classification can be achieved [14]. Besides, we demonstrate the robustness of the proposed method with the variation of $u$ in Section 2.2. So here we simply pick a general value for $u$, say 8 . To sum up, in the following experiments, when certain parameters need to be fixed, their default values are as follows. The value of $d$ is 7 for sample sets GN-1000 and GN-220, and 3 for GP-1000 and GP-220; the value of $\mathrm{u}$ is 8 and the $\mathrm{k}$ value in KNN classifier is 20.

\subsection{The Comparison Results of the Overall Accuracy}

\subsubsection{The Accuracy Comparison between the Proposed Method and the Grid-Searching Method}

In this section, first, the proposed method and the grid-searching method are respectively used in the prediction of protein subcellular localization with different $d$ values. The experimental results are presented in Figure 1.
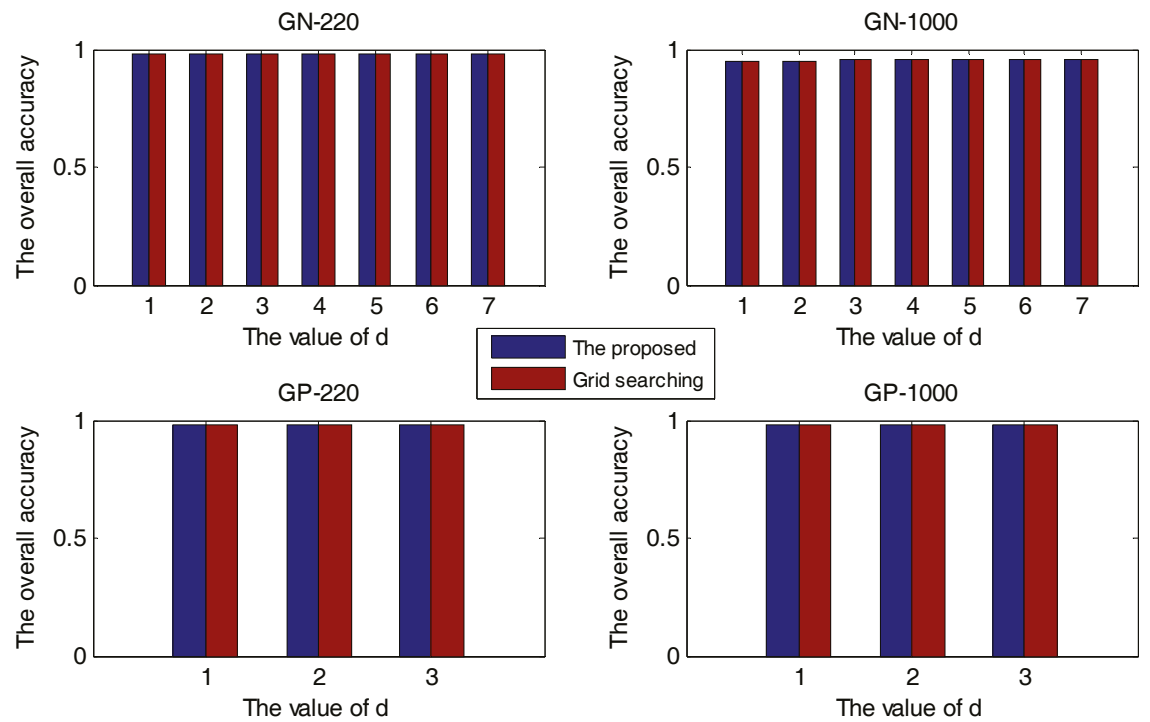

Figure 1. The overall accuracy versus $d$ for four sample sets.

In Figure 1, all four sample sets suggest that when we use the KDA algorithm to reduce dimension, the larger the number of retained eigenvectors, the higher the accuracy. The overall accuracy of the proposed method is always the same as that of the grid-searching method, no matter which value of $\mathrm{d}$. The proposed method is effective for selecting the optimal Gaussian kernel parameter.

Then, in the analyses and experiments, we find that superiority of the proposed method is the low runtime, which is demonstrated in Table 1 and Figure 2. 
Table 1. The overall accuracy and the ratio of runtime for two methods.
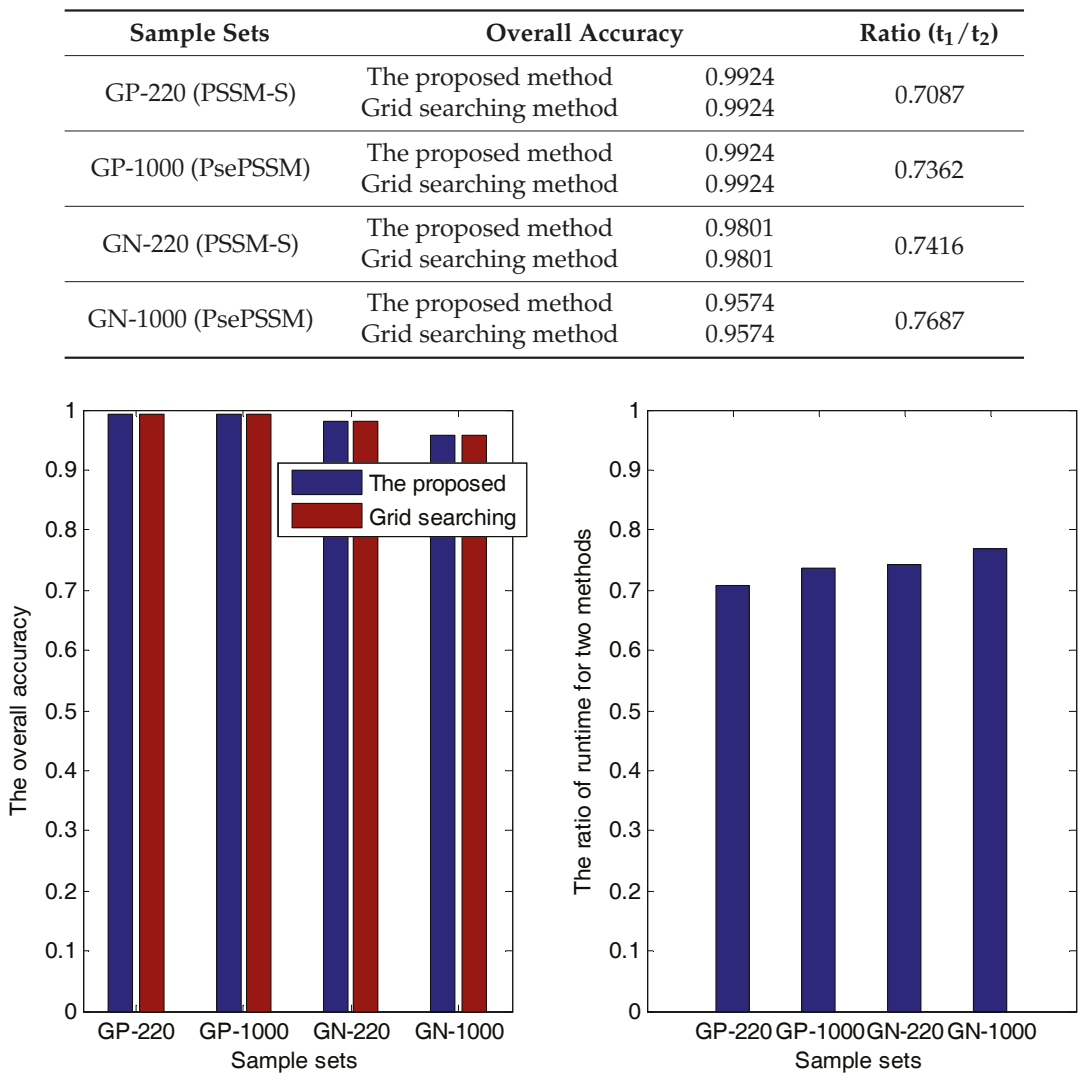

Figure 2. The overall accuracy and the ratio of runtime for two methods.

In Table $1, t_{1}$ and $t_{2}$ are the runtimes of the proposed method and the grid-searching method, respectively. The overall accuracy and the ratio of $t_{1}$ and $t_{2}$ are presented in both Table 1 and Figure 2, from which we can see that for each sample set, the accuracy of the proposed method is always the same as that of the grid-searching method; meanwhile, the runtime of the former is about $70-80 \%$ of that of the latter, indicating that the proposed method has a higher efficiency than the grid-searching method.

\subsubsection{The Comparison between Methods with and without KDA}

In this experiment, we compare the overall accuracies between the cases of using KDA algorithm or not, with $\mathrm{k}$ values of the KNN classifier varying from 1 to 30 . The experimental results are shown in Figure 3.

For each sample set, Figure 3 shows that the accuracy with KDA algorithm to reduce dimension is higher than that of without it. However, the kernel parameter has a great impact on the efficiency of the KDA algorithm, and the proposed method can be used to select the optimum parameter that makes the KDA perform perfect. Therefore, accuracy can be improved by using the proposed method to predict the protein subcellular localization. 

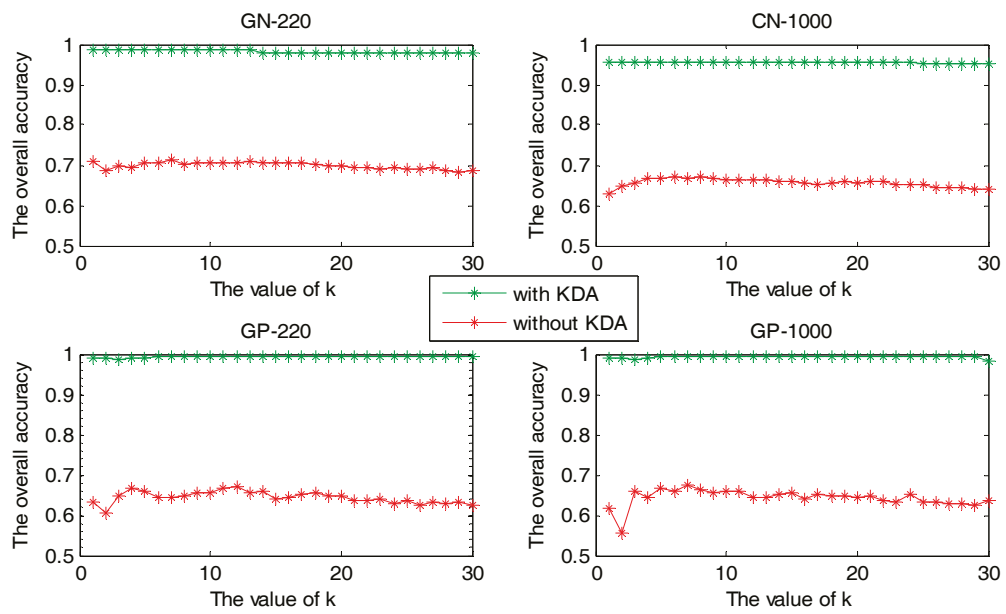

Figure 3. The overall accuracy versus $\mathrm{k}$ value with or without KDA algorithm.

\subsection{The Robustness of the Proposed Method}

In the proposed method, the value of $u$ will have an impact on the radius value of neighborhood so that it can affect the number of the selected internal and edge samples. Figure 4 shows the experimental results when the value of $u$ ranges from 6 to 10, in which the overall accuracies of the proposed method and the grid-searching method are given.

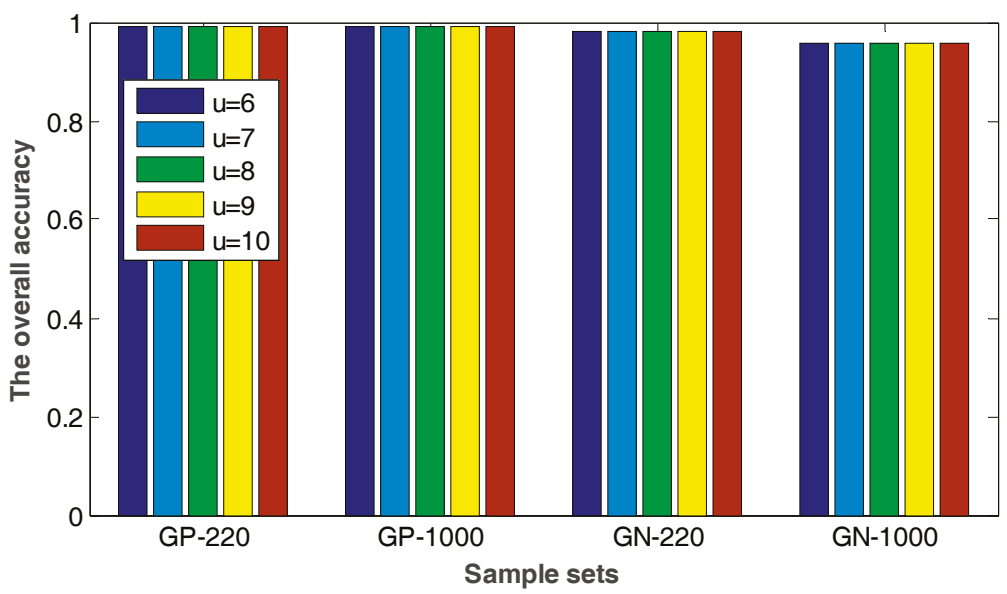

Figure 4. The overall accuracy for four sample sets with different $u$ values.

It is easily seen from Figure 4 that the accuracy keeps invariable with different $u$ values. The number of the selected internal and edge samples has little effect on the performance of the proposed method. Therefore, the method proposed in this paper has a good robustness. 


\subsection{Evaluating the Proposed Method with Some Regular Evaluation Criterions}

In this subsection, we compute the values of some regular evaluation criterions with the proposed method for two standard data sets, which is show in Tables 2 and 3, respectively. In Table 3, "-" means an infinity value, corresponding to the cases when the denominator is 0 in MCC.

Table 2. The values of evaluation criterion with the proposed method for the Gram-positive.

\begin{tabular}{ccccc}
\hline \multirow{2}{*}{ Sample Set } & \multicolumn{4}{c}{ Protein Subcellular Locations } \\
\cline { 2 - 5 } & Cell Membrane & Cell Wall & Cytoplasm & Extracell \\
\hline GP-220 & 1 & Sensitivity \\
GP-1000 & 0.9943 & 0.9444 & 0.9904 & 0.9919 \\
\hline GP-220 & 0.9943 & 0.9444 & 1 & 0.9837 \\
GP-1000 & 0.9971 & 1 & 1 & 09950 \\
GPecificity & 1 & 0.9937 \\
GP-220 & Matthews coefficient correlation (MCC) & 0.9925 \\
GP-1000 & 0.9914 & 0.9709 & 0.9920 \\
GP-220 & 0.9914 & 0.9709 & 0.9921 \\
GP-1000 & \multicolumn{5}{c}{ Overall accuracy (Q) } \\
\hline \multicolumn{5}{c}{0.9924} \\
\hline
\end{tabular}

Table 3. The values of evaluation criterion with the proposed method for the Gram-negative.

\begin{tabular}{|c|c|c|c|c|c|c|c|c|}
\hline \multirow{2}{*}{ Sample Set } & \multicolumn{8}{|c|}{ Protein Subcellular Locations } \\
\hline & (1) & (2) & (3) & (4) & (5) & (6) & (7) & (8) \\
\hline \multicolumn{9}{|c|}{ Sensitivity } \\
\hline GN-220 & 1 & 0.9699 & 1 & 0 & 0.9982 & 0 & 0.9677 & 1 \\
\hline GN-1000 & 1 & 0.9323 & 1 & 0 & 0.9659 & 0 & 0.9516 & 0.9556 \\
\hline \multicolumn{9}{|c|}{ Specificity } \\
\hline GN-220 & 0.9924 & 0.9902 & 1 & 1 & 0.9978 & 1 & 1 & 0.9953 \\
\hline GN-1000 & 0.9608 & 0.9872 & 1 & 1 & 0.9967 & 1 & 1 & 0.9992 \\
\hline \multicolumn{9}{|c|}{ Matthews coefficient correlation (MCC) } \\
\hline GN-220 & 0.9866 & 0.9324 & 1 & - & 0.9956 & - & 0.9823 & 0.9814 \\
\hline GN-1000 & 0.9346 & 0.8957 & 1 & - & 0.9681 & - & 0.9733 & 0.9712 \\
\hline \multicolumn{9}{|c|}{ Overall accuracy $(Q)$} \\
\hline GN-220 & \multicolumn{8}{|c|}{0.9801} \\
\hline GN-1000 & \multicolumn{8}{|c|}{0.9574} \\
\hline
\end{tabular}

(1) Cytoplasm, (2) Extracell, (3) Fimbrium, (4) Flagellum, (5) Inner membrane, (6) Nucleoid, (7) Outer membrane, (8) Periplasm.

Tables 2 and 3 show that the values of the evaluation criterion are close to 1 for the proposed method. Then the selection of the kernel parameter using the proposed method will benefit the protein subcellular localization.

\section{Methods}

\subsection{Protein Subcellular Localization Prediction Based on KDA}

To improve the localization prediction accuracy, it is necessary to reduce dimension of high-dimensional protein data before subcellular classification. The flow of protein subcellular localization prediction is presented in Figure 5. 


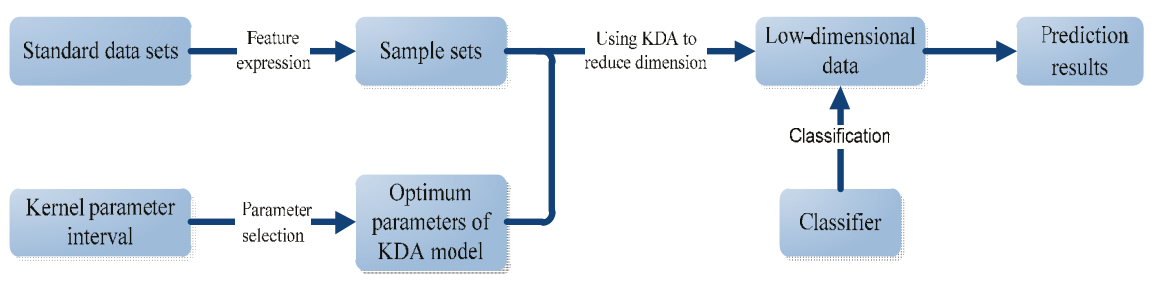

Figure 5. The flow of protein subcellular localization.

As shown in Figure 5, first, for a standard data set, some features of protein sequences such as PSSM-based expressions are extracted to form the sample sets. The specific feature expressions used in this paper are discussed in Section 4.2. Second, the kernel parameter is selected in an interval based on the sample sets to reach its optimal value in KDA model. Third, with this optimal value, we used the KDA to realize the dimension reduction of the sample sets. Lastly, the low dimensional data is treated by certain classifier to realize the classification and the final prediction.

In the whole process of Figure 5, dimension reduction with KDA is very important, in which the kernel selection is a key step and constructs the research focus of this paper. Kernel selection includes the choice of the type of kernel function and the choice of the kernel parameters. In this paper, Gaussian kernel function is adopted for KDA because of its good nature, learning performance, and catholicity. So, the emphasis of this study is to decide the scale parameter of the Gaussian kernel, which plays an important role in the process of dimensionality reduction and has a great influence on prediction results. We put forward a method for selecting the optimum Gaussian kernel parameter with the starting point of reconstruction error idea in [15].

\subsection{Algorithm Principle}

Kernel method constructs a subspace in the feature space by the kernel trick, which makes normal samples locate in or nearby this subspace, while novel samples are far from it. The reconstruction error is the distance of a sample from the feature space to the subspace [11], so the reconstruction errors of normal samples should be different from those of the novel samples. In this paper, we use the Gaussian KDA as the descending algorithms. Since the values of the reconstruction errors are influenced by the Gaussian kernel parameters, the reconstruction errors of normal samples should be differentiated from those of the novel samples by suitable parameters [11].

In the input space, we usually call the samples on the boundary as edge samples, and call those within the boundary as internal samples [16,17]. The edge samples are much closer to novel samples than the internal samples, while the internal samples are much closer to normal states than the edge samples [11]. We usually use the internal samples as the normal samples and use the edge samples as the novel samples, since there are no novel samples in data sets. Therefore, the principle is that the optimal kernel parameter makes the reconstruction errors have a reasonable difference between the internal samples and the edge samples.

\subsection{Kernel Discriminant Analysis (KDA) and Its Reconstruction Error}

KDA is an algorithm by applying kernel trick into linear discriminant analysis (LDA). LDA is an algorithm of linear dimensionality reduction together with classifying discrimination, which aims to find a direction that maximizes the between-class scatter while minimizing the within-class scatter [18]. In order to extend the LDA theory to the nonlinear data, Mika et al. proposed the KDA algorithm, which makes the nonlinear data linearly separable in a much higher dimensional feature space than before [9]. The principle of the KDA algorithm is shown as follows. 
Suppose the $\mathrm{N}$ samples in $\mathrm{X}$ can be divided into $\mathrm{C}$ classes and the ith class contains $\mathrm{N}_{\mathrm{i}}$ samples satisfying $N=\sum_{i=1}^{C} N_{i}$. The between-class scatter matrix $S_{b}^{\phi}$ and the within-class scatter matrix $n_{n}^{\phi}$ of $X$ are defined in the following equations, respectively:

$$
\begin{gathered}
\mathrm{S}_{\mathrm{b}}^{\phi}=\sum_{\mathrm{i}=1}^{\mathrm{C}} \mathrm{N}_{\mathrm{i}}\left(\mathrm{m}_{\mathrm{i}}^{\phi}-\mathrm{m}^{\phi}\right)\left(\mathrm{m}_{\mathrm{i}}^{\phi}-\mathrm{m}^{\phi}\right)^{\mathrm{T}} \\
\mathrm{S}_{\mathrm{W}}^{\phi}=\sum_{\mathrm{i}=1}^{\mathrm{C}} \sum_{\mathrm{j}=1}^{\mathrm{N}_{\mathrm{i}}}\left[\phi\left(\mathrm{x}_{\mathrm{j}}^{\mathrm{i}}\right)-\mathrm{m}_{\mathrm{i}}^{\phi}\right]\left[\phi\left(\mathrm{x}_{\mathrm{j}}^{\mathrm{i}}\right)-\mathrm{m}_{\mathrm{i}}^{\phi}\right]^{\mathrm{T}}
\end{gathered}
$$

where $\mathrm{m}_{\mathrm{i}}^{\phi}=\frac{1}{\mathrm{~N}_{\mathrm{i}}} \sum_{\mathrm{j}=1}^{\mathrm{N}_{\mathrm{i}}} \phi\left(\mathrm{x}_{\mathrm{j}}^{\mathrm{i}}\right)$ is the mean vector of the ith class, and $\mathrm{m}^{\phi}=\frac{1}{\mathrm{~N}} \sum_{\mathrm{i}=1}^{\mathrm{N}} \phi\left(\mathrm{x}_{\mathrm{i}}\right)$ is the total mean of $X$. To find the optimal linear discriminant, we need to maximize $J(W)$ as follows:

$$
\max J(W)=\frac{W^{T} S_{b}^{\phi} W}{W^{T} S_{W}^{\phi} W}
$$

where $\mathrm{W}=\left[\mathrm{w}_{1}, \mathrm{w}_{2}, \cdots, \mathrm{w}_{\mathrm{d}}\right]^{\mathrm{T}}(1 \leq \mathrm{d} \leq \mathrm{C}-1)$ is a projection matrix, and $\mathrm{w}_{\mathrm{k}}(\mathrm{k}=1,2, \cdots, \mathrm{d})$ is a column vector with $\mathrm{N}$ elements. Through certain algebra, it can be deduced that $\mathrm{W}$ is made up of the eigenvectors corresponding to the top d eigenvalues of $S_{\mathrm{w}}^{\phi-1} S_{\mathrm{b}}^{\phi}$. Also, the projection vector $\mathrm{w}_{\mathrm{k}}$ can be represented by a linear combination of the samples in the feature space:

$$
w_{k}=\sum_{j=1}^{N} a_{j} j\left(x_{j}\right)
$$

where $a_{j}^{k}$ is a real coefficient. The projection of the sample $X$ onto $w_{k}$ is given by:

$$
\mathrm{w}_{\mathrm{k}}^{\mathrm{T}} \times \phi(\mathrm{x})=\sum_{\mathrm{i}=1}^{\mathrm{N}} \mathrm{a}_{\mathrm{i}}^{\mathrm{k}} \mathrm{K}\left(\mathrm{x}, \mathrm{x}_{\mathrm{j}}\right)
$$

Let $\mathrm{a}=\left[\mathrm{a}^{1}, \mathrm{a}^{2}, \cdots, \mathrm{a}^{\mathrm{d}}\right]^{\mathrm{T}}$ be the coefficient matrix where $\mathrm{a}^{\mathrm{k}}=\left[\mathrm{a}_{1}^{\mathrm{k}}, \mathrm{a}_{2}^{\mathrm{k}}, \cdots, \mathrm{a}_{\mathrm{N}}^{\mathrm{k}}\right]^{\mathrm{T}}$ is the coefficient vector. Combining Equations (1)-(5), we can obtain the linear discriminant by maximizing the function $\mathrm{J}(\mathrm{a})$ :

$$
\max \mathrm{J}(\mathrm{a})=\frac{\mathrm{a}^{\mathrm{T}} \widetilde{\mathrm{Ma}}}{\mathrm{a}^{\mathrm{T}} \widetilde{\mathrm{La}}}
$$

where $\widetilde{M}=\sum_{i=1}^{C} N_{i}\left(M_{i}-M\right)\left(M_{i}-M\right)^{T}, \widetilde{L}=\sum_{i=1}^{C} K_{i}\left(E-\frac{1}{N_{i}} I\right) K_{i}^{T}$, the kth component of the vector $M_{i}$ is $\left(M_{i}\right)_{k}=\frac{1}{N_{i}} \sum_{j=1}^{N_{i}} K\left(x_{k}, x_{j}^{i}\right)(k=1,2, \cdots, N)$, the $k$ th component of the vector $M$ is $(M)_{k}=$ $\frac{1}{N} \sum_{j=1}^{N} \mathrm{~K}\left(\mathrm{x}_{\mathrm{k}}, \mathrm{x}_{\mathrm{j}}^{\mathrm{i}}\right)(\mathrm{k}=1,2, \cdots, \mathrm{N}), \mathrm{K}_{\mathrm{i}}$ is a $\mathrm{N} \times \mathrm{N}_{\mathrm{i}}$ matrix with $\left(\mathrm{K}_{\mathrm{i}}\right)_{\mathrm{mn}}=\mathrm{K}\left(\mathrm{x}_{\mathrm{m}}, \mathrm{x}_{\mathrm{n}}^{\mathrm{i}}\right)$, E is the $\mathrm{N}_{\mathrm{i}} \times \mathrm{N}_{\mathrm{i}}$ identity matrix, and $\frac{1}{N_{i}} I$ is the $N_{i} \times N_{i}$ matrix that all elements are $\frac{1}{N_{i}}$ [9]. Then, the projection matrix a is made up of the eigenvectors corresponding to the top d eigenvalues of $\widetilde{L}^{-1} \widetilde{M}$.

According to the KDA algorithm principle in (3) or (6), besides the Gaussian kernel parameter s, the number of retained eigenvectors $d$ also affects the algorithm performance. Generally, in this paper, the proposed method is mainly used to screen an optimum $\mathrm{S}$ under a predetermined $\mathrm{d}$ value. 
The Gaussian kernel function is defined as follows:

$$
\mathrm{K}\left(\mathrm{x}_{\mathrm{i}}, \mathrm{x}_{\mathrm{j}}\right)=\exp \left(-\frac{\left\|\mathrm{x}_{\mathrm{i}}-\mathrm{x}_{\mathrm{j}}\right\|^{2}}{\sigma^{2}}\right)
$$

where $\sigma$ is the scale parameter which is generally estimated by s. Note that $\|\phi(x)\|^{2}=K(x, x)=1$.

The kernel-based reconstruction error is defined in the following equation:

$$
\begin{aligned}
\mathrm{RE}(\mathrm{x}) & =\|\phi(\mathrm{x})-\mathrm{Wt}(\mathrm{x})\|^{2}=\|\phi(\mathrm{x})\|^{2}-\|\mathrm{t}(\mathrm{x})\|^{2} \\
& =\mathrm{K}(\mathrm{x}, \mathrm{x})-\|\mathrm{t}(\mathrm{x})\|^{2}
\end{aligned}
$$

where $t(x)$ is the vector obtained by projecting $\phi(x)$ onto a projection matrix a.

\subsection{The Proposed Method for Selecting the Optimum Gaussian Kernel Parameter}

The method of kernel parameter selection relies on the reconstruction errors of the internal samples and the edge samples. Therefore, first we find a method to select the edge samples and the interior samples, then we propose the method for selection of the Gaussian kernel parameter.

\subsubsection{The Method for Selecting Internal and Edge Samples}

Li and Maguire present a border-edge pattern selection method (BEPS) to select the edge samples based on the local geometric information [16]. Xiao et al. [11] modified the BEPS algorithm so that it can select both the edge samples and internal samples. However, their algorithm has the risk of making all samples in the training set become the edge samples. For example, when all samples are distributed on a spherical surface in a three-dimensional space, every sample in the data set will be selected as the edge samples since its neighbors are all located on one side of its tangent plane. In order to solve this problem, this paper innovatively combines the ideas in $[19,20]$ to select the internal and edge samples, respectively, which is not dependent on the local geometric information. The main principle is that the edge sample is usually surrounded by the samples belonging to other classes while the internal sample is usually surrounded by the samples belonging to its same class. Further, the edge samples are usually far from the centroid of this class, while the internal samples are usually close to the centroid. So, a sample will be selected as the edge sample if it is far from the centroid of this class and there are samples around it that belongs to other classes, otherwise it will be selected as the internal sample.

Specifically, suppose the ith class $X_{i}=\left\{x_{1}, x_{2}, \cdots, x_{N_{i}}\right\}$ in the sample set $X$ is picked out as the training set. Denote $c_{i}$ be the centroid of this class:

$$
\mathrm{c}_{\mathrm{i}}=\frac{1}{\mathrm{~N}_{\mathrm{i}}} \sum_{\mathrm{i}=1}^{\mathrm{N}_{\mathrm{i}}} \mathrm{x}_{\mathrm{i}}
$$

We use the median value $\mathrm{m}$ of the distances from all samples in a class to its centroid to measure the distance from a sample to the centroid of this class. A sample is conserved to be far from the centroid of this class if the distance from this sample to the centroid is greater than the median value. Otherwise, the sample is considered to be close to the centroid.

Denote $\operatorname{dist}\left(\mathrm{x}_{\mathrm{i}}, \mathrm{x}_{\mathrm{j}}\right)$ as the distance between any two samples $\mathrm{x}_{\mathrm{i}}$ and $\mathrm{x}_{\mathrm{j}}$, and $\mathrm{N}_{\varepsilon}(\mathrm{x})$ as the $\varepsilon$-neighborhood of $\mathrm{X}$ :

$$
\mathrm{N}_{\varepsilon}(\mathrm{x})=\{\mathrm{y} \mid \operatorname{dist}(\mathrm{x}, \mathrm{y}) \leq \varepsilon, \mathrm{y} \in \mathrm{X}\}
$$

The value of neighborhood $\varepsilon$ is given as follows. Let $u$ be a given number which satisfies $0<\mathrm{u}<\mathrm{N}_{\mathrm{i}}$. Density $\left(\mathrm{X}_{\mathrm{i}}\right)$ is the mean radius of neighborhood of $\mathrm{X}_{\mathrm{i}}$ for the given number $\mathrm{u}$ :

$$
\operatorname{Density}_{\mathrm{u}}\left(\mathrm{X}_{\mathrm{i}}\right)=\frac{1}{\mathrm{~N}_{\mathrm{i}}} \sum_{\mathrm{i}=1}^{\mathrm{N}_{\mathrm{i}}} \operatorname{dist}_{\mathrm{u}}\left(\mathrm{x}_{\mathrm{i}}\right)
$$


where $\operatorname{dist}_{\mathrm{u}}\left(\mathrm{x}_{\mathrm{i}}\right)$ is the distance from $\mathrm{x}_{\mathrm{i}}$ to its $\mathrm{uth}$ nearest neighbor. So, Density $\mathrm{u}_{\mathrm{u}}\left(\mathrm{X}_{\mathrm{i}}\right)$ is used as the value of $\varepsilon$ for the training set $X_{i}$. The flow for the selection of the internal and edge samples is shown in Table 4

Table 4. The Selection of Internal and Edge Samples.

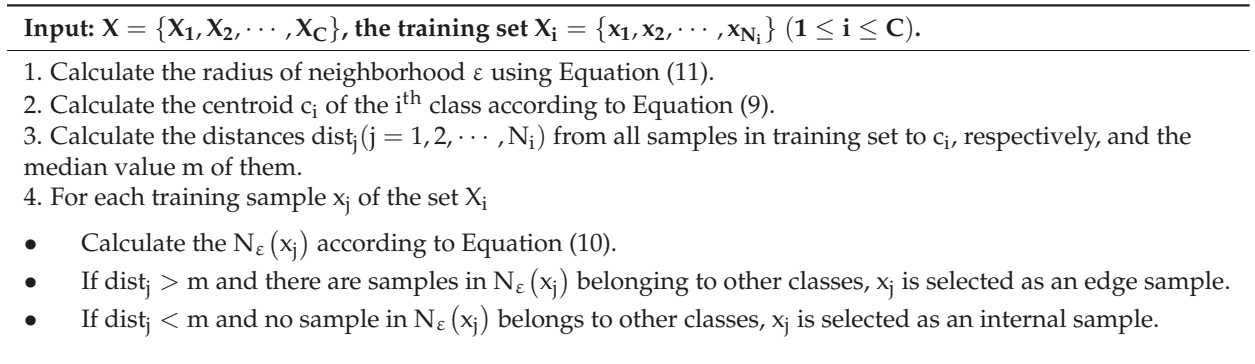

Output: the selected internal sample set $\Omega_{\mathrm{in}}$, the selected edge sample set $\Omega_{\mathrm{ed}}$.

In Table 4, a sample $X$ is considered to be the edge one when the distance from $X$ to the centroid is larger than the median $m$ and there are samples in $N_{\mathcal{E}}(x)$ belonging to other classes in this case. A sample $X$ is considered to be the internal one when the distance from $X$ to the centroid is less than $\mathrm{m}$ and in this case all samples of $\mathrm{N}_{\mathcal{\varepsilon}}(\mathrm{x})$ belong to this class.

\subsubsection{The Proposed Method}

In order to select the optimum kernel parameter, it is necessary to propose a criterion aiming to distinguish reconstruction errors of the edge samples from those of the internal samples. A suitable parameter not only maximizes the difference between reconstruction errors of the internal samples and those of the edge samples, but also minimizes the variance (or standard deviation) of reconstruction errors of the internal samples [11]. According to the rule, an improved objective function is proposed in this paper. The optimal Gaussian kernel parameter $S$ is selected by maximizing this objective function.

$$
\mathrm{s}=\operatorname{argmaxf}(\mathrm{s})=\arg \max _{\mathrm{s}} \frac{\left\|\operatorname{RE}\left(\Omega_{\mathrm{ed}}\right)\right\|_{\infty}-\left\|\operatorname{RE}\left(\Omega_{\mathrm{in}}\right)\right\|_{\infty}}{\operatorname{std}\left\{\operatorname{RE}\left(\Omega_{\text {in }}\right)\right\}}
$$

where $\|\cdot\|_{\infty}$ is the infinite norm which computes the maximum absolute component of a vector and $\operatorname{std}(\cdot)$ is a function of the standard deviation. Note that in the objective function $f(s)$, our key improvement is to use the infinite norm to compute the size of reconstruction error vector since it can lead to a higher accuracy than many other measurements, which has been verified by a series of our experiments. The reason is probably that the maximum component is more reasonable to evaluate the size of a reconstruction error vector than others such as the 1-norm, p-norm $(1<p<+\infty)$ and the minimum component of a reconstruction error vector in [11].

According to (8), when the number of retained eigenvectors is determined, we can select the optimum parameter $\mathrm{s}$ from a candidate set using the proposed method. The optimum parameter ensures that the Gaussian KDA algorithm performs well in dimensionality reduction, which improves the accuracy of protein subcellular location prediction. The proposed method for selecting the Gaussian kernel parameter can be presented in Table 5. 
Table 5. The Method for Selecting the Gaussian KDA Parameter.

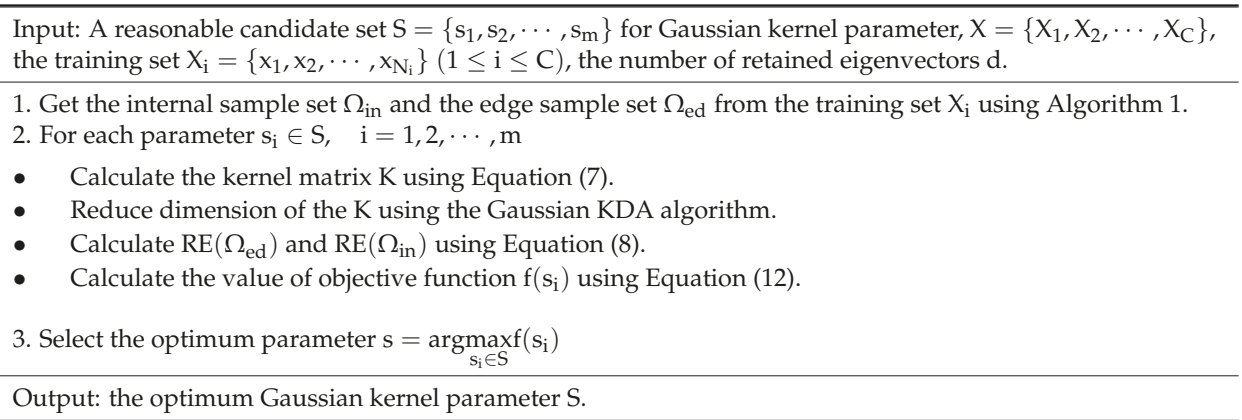

As the end of this section, we want to summarize the position of the proposed method in protein subcellular localization once more. First, two kinds of regularization forms of PSSM are used to extract the features in protein amino acid sequences. Then, the KDA method is performed on the extracted features for dimension reduction and discriminant analysis according to the KDA algorithm principle in Section 3.3 with formulas (1)-(6). During the procedure of KDA, the novelty of our work is to give a new method for selecting the Gaussian kernel parameter, which is summarized in Table 5. Finally, we choose the k-nearest neighbors (KNN) as the classifier to cluster the dimension-reduced data after KDA.

\section{Materials}

In this section, we introduce the other processes in Figure 5 except KDA model and its parameter selection, which are necessary materials for the whole experiment.

\subsection{Standard Data Sets}

In this paper, we use two standard datasets that have been widely used in the literature for Gram-positive and Gram-negative subcellular localizations [13], whose protein sequences all come from the Swiss-Prot database.

For the Gram-positive bacteria, the standard data set we found in the literature $[13,14,21]$ is publicly available on http://www.csbio.sjtu.edu.cn/bioinf/Gpos-multi/Data.htm. There are 523 locative protein sequences in the data set that are distributed in four different subcellular locations. The number of proteins in each location is given in Table 6.

Table 6. The name and the size of each location for the Gram-positive data set.

\begin{tabular}{ccc}
\hline No. & Subcellular Localization & Number of Proteins \\
\hline 1 & cell membrane & 174 \\
2 & cell wall & 18 \\
3 & cytoplasm & 208 \\
4 & extracell & 123 \\
\hline
\end{tabular}

For the Gram-negative bacteria, the standard data set of subcellular localizations is presented in the literature $[13,22]$, which can be downloaded freely from http:/ / www.csbio.sjtu.edu.cn/bioinf/ Gneg-multi/Data.htm. The data set contains 1456 locative protein sequences located in eight different subcellular locations. The number of proteins in each location is shown in Table 7. 
Table 7. The name and the size of each location for the Gram-negative data set.

\begin{tabular}{ccc}
\hline No. & Subcellular Localization & Number of Proteins \\
\hline 1 & cytoplasm & 410 \\
2 & extracell & 133 \\
3 & fimbrium & 32 \\
4 & flagellum & 12 \\
5 & inner membrane & 557 \\
6 & nucleoid & 8 \\
7 & outer membrane & 124 \\
8 & periplasm & 180 \\
\hline
\end{tabular}

\subsection{Feature Expressions and Sample Sets}

In the prediction of protein subcellular localizations with machine learning methods, feature expressions are important information extracted from protein sequences, which have certain proper mathematical algorithms. There are many efficient algorithms used to extract features of protein sequences, in which two of them, PsePSSM [12] and PSSM-S [13], are used in this paper. The two methods rely on the position-specific scoring matrix (PSSM) for benchmarks which is obtained by using the PSI-BLAST algorithm to search the Swiss-Prot database with the parameter E-value of 0.01. The PSSM is defined as follows [12]:

$$
P_{P S S M}=\left[\begin{array}{cccc}
M_{1 \rightarrow 1} & M_{1 \rightarrow 2} & \cdots & M_{1 \rightarrow 20} \\
M_{2 \rightarrow 1} & M_{2 \rightarrow 2} & \cdots & M_{2 \rightarrow 20} \\
\vdots & \vdots & \vdots & \vdots \\
M_{i \rightarrow 1} & M_{i \rightarrow 2} & \cdots & M_{i \rightarrow 20} \\
\vdots & \vdots & \vdots & \vdots \\
M_{L \rightarrow 1} & M_{L \rightarrow 2} & \cdots & M_{L \rightarrow 20}
\end{array}\right]
$$

where $\mathrm{M}_{\mathrm{i} \rightarrow \mathrm{j}}$ represents the score created in the case when the ith amino acid residue of the protein sequence is transformed to the amino acid type $j$ during the evolutionary process [12].

Note that, usually, multiple alignment methods are used to calculate PSSM, whose chief drawback is being time-consuming. The reason why we select PSSM instead of simple multiple alignment in this paper to form the total normalized information content is as follows. First, since our focus is to demonstrate the effectiveness of dimensional reduction algorithm, we need to construct high-dimensional feature expressions such as PsePSSM and PSSM-S, whose dimensions are as high as 1000 and 220, respectively. Second, PSSM has many advantages, such as those described in [23]. As far as the information features are concerned, PSSM has produced the strongest discriminator feature between fold members of protein sequences. Multiple alignment methods are used to calculate PSSM, whose chief drawback is being time-consuming. However, in spite of the time-consuming nature of constructing a PSSM for the new sequence, the extracted feature vectors from PSSM are so informative that are worth the cost of their preparation [23]. Besides, for a new protein sequence, we only need to construct a PSSM for the first time, which could be used repeatedly in the future for producing new normalization forms such as PsePSSM and PSSM-S.

\subsubsection{Pseudo Position-Specific Scoring Matrix (PsePSSM)}

Let $\mathrm{P}$ be a protein sample, whose definition of PsePSSM is given as follows [12]:

$$
\mathrm{P}_{\text {Pse-PSSM }}^{\xi}=\left[\overline{\mathrm{M}}_{1} \overline{\mathrm{M}}_{2} \cdots \overline{\mathrm{M}}_{20} \mathrm{G}_{1}^{\xi} \mathrm{G}_{2}^{\xi} \cdots \mathrm{G}_{20}^{\xi}\right]^{\mathrm{T}}(\xi=0,1,2, \cdots, 49)
$$




$$
\begin{gathered}
\bar{M}_{j}=\frac{1}{L} \sum_{i=1}^{L} M_{i \rightarrow j}(j=1,2, \cdots, 20) \\
G_{j}^{\xi}=\frac{1}{L-\xi} \sum_{i=1}^{L-\xi}\left[M_{i \rightarrow j}-M_{(i+\xi) \rightarrow j}\right]^{2}(j=1,2, \cdots, 20 ; \xi<L)
\end{gathered}
$$

where $\mathrm{L}$ is the length of $\mathrm{P}, \mathrm{G}_{\mathrm{j}}^{\xi}$ is the correlation factor by coupling the $\xi$-most contiguous scores [22]. According to the definition of PsePSSM, a protein sequence can be represented by a 1000-dimensional vector.

\subsubsection{PSSM-S}

Dehzangi et al. [13] put forward a new feature extraction method, PSSM-S, which combines four components: AAO, PSSM-AAO, PSSM-SD, and PSSM-SAC. According to the definition of the PSSM-S, it can be represented a feature vector with $220(20+20+80+100)$ elements.

\subsubsection{Sample Sets}

For the two benchmark data, PsePSSM and PSSM-S are used to extract features, respectively. Finally we get four experimental sample sets GN-1000, GN-220, GP-1000 and GP-220, shown in Table 8.

\begin{tabular}{|c|c|c|c|c|c|}
\hline $\begin{array}{l}\text { Sample } \\
\text { Sets }\end{array}$ & $\begin{array}{l}\text { Benchmarks for } \\
\text { Subcellular Locations }\end{array}$ & $\begin{array}{c}\text { Extraction } \\
\text { Feature Method }\end{array}$ & $\begin{array}{c}\text { The Number of } \\
\text { Classes }\end{array}$ & $\begin{array}{l}\text { The Dimension of } \\
\text { Feature Vector }\end{array}$ & $\begin{array}{c}\text { The Number of } \\
\text { Samples }\end{array}$ \\
\hline GN-1000 & Gram-negative & PsePSSM & 8 & 1000 & 1456 \\
\hline GN-220 & Gram-negative & PSSM-S & 8 & 220 & 1456 \\
\hline GP-220 & Gram-positive & PSSM-S & 4 & 220 & 523 \\
\hline
\end{tabular}

Table 8. Sample sets.

\subsection{Evaluation Criterion}

To evaluate the performance of the proposed method, we use Jackknife cross-validation, which has been widely used to predict protein subcellular localization [13]. The Jackknife test is the most objective and rigorous cross-validation procedure in examining the accuracy of a predictor, which has been used increasingly by investigators to test the power of various predictors [24,25]. In the Jackknife test (also known as leave-one-out cross-validation), every protein is removed one-by-one from the training dataset, and the predictor is trained by the remaining proteins. The isolated protein is then tested by the trained predictor [26]. Let $\mathrm{x}$ be a sample set with $\mathrm{N}$ samples. For each sample, it will be used as the test data, and the remaining $\mathrm{N}-1$ samples will be used to construct the training set [27]. In addition, we use some criterion to assess the experimental results, defined as follows [12]:

$$
\begin{gathered}
\operatorname{MCC}(\mathrm{k})=\frac{\mathrm{TP}_{\mathrm{k}} \times \mathrm{TN}_{\mathrm{k}}-\mathrm{FN}_{\mathrm{k}} \times \mathrm{FP}_{\mathrm{k}}}{\sqrt{\left(\mathrm{TP}_{\mathrm{k}}+\mathrm{FN}_{\mathrm{k}}\right)\left(\mathrm{TP}_{\mathrm{k}}+\mathrm{FP}_{\mathrm{k}}\right)\left(\mathrm{TN}_{\mathrm{k}}+\mathrm{FP}_{\mathrm{k}}\right)\left(\mathrm{TN}_{\mathrm{k}}+\mathrm{FN}_{\mathrm{k}}\right)}} \times 100 \% \\
\operatorname{Sen}(\mathrm{k})=\frac{\mathrm{TP}_{\mathrm{k}}}{\mathrm{TP}_{\mathrm{k}}+\mathrm{FN}_{\mathrm{k}}} \times 100 \% \\
\operatorname{Spe}(\mathrm{k})=\frac{\mathrm{TN}_{\mathrm{k}}}{\mathrm{TP}_{\mathrm{k}}+\mathrm{FP}_{\mathrm{k}}} \times 100 \% \\
\mathrm{Q}=\frac{\sum_{\mathrm{k}=1}^{\mathrm{C}} \mathrm{TP}_{\mathrm{k}}}{\mathrm{N}} \times 100 \%
\end{gathered}
$$

where TP is the number of true positive, TN is the number of true negative, FP is the number of false positive, and FN is the number of false negative [12]. The value of MCC (Matthews coefficient correlation) varies between -1 and 1 , indicating when the classification effect goes from a bad to 
a good one. The values of Specificity (Spe), sensitivity (Sen), and the overall accuracy (Q) all vary between 0 and 1 , and the classification effect is better when their values are closer to 1 , while the classification effect is worse when their values are closer to 0 [13].

\subsection{The Grid Searching Method Used as Contrast}

In this section, we introduce a normal algorithm for searching $\mathrm{S}$, the grid-searching algorithm, which is used as a contrast with the proposed algorithm in Section 3.4.

The grid-searching method is usually used to select the optimum parameter, whose steps are as follows for the candidate parameter set S [28].

- $\quad$ Compute the kernel matrix $k$ for each parameter $s_{i} \in S, i=1,2, \cdots, m$.

- Use the Gaussian KDA to reduce the dimension of K.

- Use the KNN algorithm to classify the reduced dimensional samples.

- Calculate the classification accuracy.

- Repeat the above four steps until all parameters in $\mathrm{S}$ have been traversed. The parameter corresponding to the highest classification accuracy is selected as the optimum parameter.

\section{Conclusions}

Biological data is usually high-dimensional. As a result, it is necessary to reduce dimension to improve the accuracy of the protein subcellular localization prediction. The kernel discriminant analysis (KDA) based on Gaussian kernel function is a suitable algorithm for dimensional reduction in such applications. As is known to all, the selection of a kernel parameter affects the performance of $\mathrm{KDA}$, and thus it is important to choose the proper parameter that makes this algorithm perform well. To handle this problem, we propose a method of the optimum kernel parameter selection, which relies on reconstruction error [15]. Firstly, we use a method to select the edge and internal samples of the training set. Secondly, we compute the reconstruction errors of the selected samples. Finally, we select the optimum kernel parameter that makes the objective function maximum.

The proposed method is applied to the prediction of protein subcellular locations for Gram-negative bacteria and Gram-positive bacteria. Compared with the grid-searching method, the proposed method gives higher efficiency and performance.

Since the performance of the proposed method largely depends on the selection of the internal and edge samples, in the future study, researchers may pay more attention to select more representative internal and edge samples from the biological data set to improve the prediction accuracy of protein subcellular localization. Besides this, it is also meaningful to research how to further improve the proposed method to make it suitable for selecting parameters of other kernels.

Acknowledgments: This research is supported by grants from National Natural Science Foundation of China (No. 11661081, No. 11561071 and No. 61472345) and Natural Science Foundation of Yunnan Province (2017FA032).

Author Contributions: Shunfang Wang and Bing Nie designed the research and the experiments. Wenjia Li, Dongshu $\mathrm{Xu}$, and Bing Nie extracted the feature expressions from the standard data sets, and Bing Nie performed all the other numerical experiments. Shunfang Wang, Bing Nie, Kun Yue, and Yu Fei analyzed the experimental results. Shunfang Wang, Bing Nie, Kun Yue, and Yu Fei wrote this paper. All authors read and approved the final manuscript.

Conflicts of Interest: The authors declare that they have no competing interests.

\section{References}

1. Chou, K.C. Some Remarks on Predicting Multi-Label Attributes in Molecular Biosystems. Mol. Biosyst. 2013, 9, 1092-1100. [CrossRef] [PubMed]

2. Zhang, S.; Huang, B.; Xia, X.F.; Sun, Z.R. Bioinformatics Research in Subcellular Localization of Protein. Prog. Biochem. Biophys. 2007, 34, 573-579. 
3. Zhang, S.B.; Lai, J.H. Machine Learning-based Prediction of Subcellular Localization for Protein. Comput. Sci. 2009, 36, 29-33.

4. Huh, W.K.; Falvo, J.V.; Gerke, L.C.; Carroll, A.S.; Howson, R.W.; Weissman, J.S.; O'Shea, E.K. Global analysis of protein localization in budding yeast. Nature 2003, 425, 686-691. [CrossRef] [PubMed]

5. Dunkley, T.P.J.; Watson, R.; Griffin, J.L.; Dupree, P.; Lilley, K.S. Localization of organelle proteins by isotope tagging (LOPIT). Mol. Cell. Proteom. 2004, 3, 1128-1134. [CrossRef] [PubMed]

6. Hasan, M.A.; Ahmad, S.; Molla, M.K. Protein subcellular localization prediction using multiple kernel learning based support vector machine. Mol. Biosyst. 2017, 13, 785-795. [CrossRef] [PubMed]

7. Teso, S.; Passerini, A. Joint probabilistic-logical refinement of multiple protein feature predictors. BMC Bioinform. 2014, 15, 16. [CrossRef] [PubMed]

8. Wang, S.; Liu, S. Protein Sub-Nuclear Localization Based on Effective Fusion Representations and Dimension Reduction Algorithm LDA. Int. J. Mol. Sci. 2015, 16, 30343-30361. [CrossRef] [PubMed]

9. Baudat, G.; Anouar, F. Generalized Discriminant Analysis Using a Kernel Approach. Neural Comput. 2000, 12, 2385-2404. [CrossRef] [PubMed]

10. Zhang, G.N.; Wang, J.B.; Li, Y.; Miao, Z.; Zhang, Y.F.; Li, H. Person re-identification based on feature fusion and kernel local Fisher discriminant analysis. J. Comput. Appl. 2016, 36, 2597-2600.

11. Xiao, Y.C.; Wang, H.G.; Xu, W.L.; Miao, Z.; Zhang, Y.; Hang, L.I. Model selection of Gaussian kernel PCA for novelty detection. Chemometr. Intell. Lab. 2014, 136, 164-172. [CrossRef]

12. Chou, K.C.; Shen, H.B. MemType-2L: A Web server for predicting membrane proteins and their types by incorporating evolution information through Pse-PSSM. Biochem. Biophys. Res. Commun. 2007, 360, 339-345. [CrossRef] [PubMed]

13. Dehzangi, A.; Heffernan, R.; Sharma, A.; Lyons, J.; Paliwal, K.; Sattar, A. Gram-positive and Gram-negative protein subcellular localization by incorporating evolutionary-based descriptors into Chou's general PseAAC. J. Theor. Biol. 2015, 364, 284-294. [CrossRef] [PubMed]

14. Shen, H.B.; Chou, K.C. Gpos-PLoc: An ensemble classifier for predicting subcellular localization of Gram-positive bacterial proteins. Protein Eng. Des. Sel. 2007, 20, 39-46. [CrossRef] [PubMed]

15. Hoffmann, H. Kernel PCA for novelty detection. Pattern Recogn. 2007, 40, 863-874. [CrossRef]

16. Li, Y.; Maguire, L. Selecting Critical Patterns Based on Local Geometrical and Statistical Information. IEEE Trans. Pattern Anal. 2010, 33, 1189-1201.

17. Wilson, D.R.; Martinez, T.R. Reduction Techniques for Instance-Based Learning Algorithms. Mach. Learn. 2000, 38, 257-286. [CrossRef]

18. Saeidi, R.; Astudillo, R.; Kolossa, D. Uncertain LDA: Including observation uncertainties in discriminative transforms. IEEE Trans. Pattern Anal. 2016, 38, 1479-1488. [CrossRef] [PubMed]

19. Jain, A.K. Data clustering: 50 years beyond K-means. Pattern Recogn. Lett. 2010, 31, 651-666. [CrossRef]

20. Li, R.L.; Hu, Y.F. A Density-Based Method for Reducing the Amount of Training Data in kNN Text Classification. J. Comput. Res. Dev. 2004, 41, 539-545.

21. Chou, K.C.; Shen, H.B. Cell-PLoc 2.0: An improved package of web-servers for predicting subcellular localization of proteins in various organisms. Nat. Sci. 2010, 2, 1090-1103. [CrossRef]

22. Chou, K.C.; Shen, H.B. Large-Scale Predictions of Gram-Negative Bacterial Protein Subcellular Locations. J. Proteome Res. 2007, 5, 3420-3428. [CrossRef] [PubMed]

23. Kavousi, K.; Moshiri, B.; Sadeghi, M.; Araabi, B.N.; Moosavi-Movahedi, A.A. A protein fold classifier formed by fusing different modes of pseudo amino acid composition via PSSM. Comput. Biol. Chem. 2011, 35, 1-9. [CrossRef] [PubMed]

24. Shen, H.B.; Chou, K.C. Nuc-PLoc: A new web-server for predicting protein subnuclear localization by fusing PseAA composition and PsePSSM. Protein Eng. Des. Sel. 2007, 20, 561-567. [CrossRef] [PubMed]

25. Wang, T.; Yang, J. Using the nonlinear dimensionality reduction method for the prediction of subcellular localization of Gram-negative bacterial proteins. Mol. Divers. 2009, 13, 475.

26. Wei, L.Y.; Tang, J.J.; Zou, Q. Local-DPP: An improved DNA-binding protein prediction method by exploring local evolutionary information. Inform. Sci. 2017, 384, 135-144. [CrossRef] 
27. Shen, H.B.; Chou, K.C. Gneg-mPLoc: A top-down strategy to enhance the quality of predicting subcellular localization of Gram-negative bacterial proteins. J. Theor. Biol. 2010, 264, 326-333. [CrossRef] [PubMed]

28. Bing, L.I.; Yao, Q.Z.; Luo, Z.M.; Tian, Y. Gird-pattern method for model selection of support vector machines. Comput. Eng. Appl. 2008, 44, 136-138.

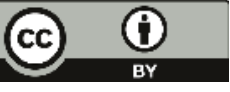

(C) 2017 by the authors. Licensee MDPI, Basel, Switzerland. This article is an open access article distributed under the terms and conditions of the Creative Commons Attribution (CC BY) license (http:/ / creativecommons.org/licenses/by/4.0/). 
Article

\title{
Assessing the Performances of Protein Function Prediction Algorithms from the Perspectives of Identification Accuracy and False Discovery Rate
}

\author{
Chun Yan Yu ${ }^{1,2}$, Xiao Xu Li ${ }^{1,2}$, Hong Yang ${ }^{1,2}$, Ying Hong $\mathrm{Li}^{1,2}$, Wei Wei Xue ${ }^{1}$, Yu Zong Chen ${ }^{3}$, \\ Lin Tao ${ }^{4}$ and Feng $\mathrm{Zhu}{ }^{1,2, *}$ \\ 1 Innovative Drug Research and Bioinformatics Group, School of Pharmaceutical Sciences and Collaborative \\ Innovation Center for Brain Science, Chongqing University, Chongqing 401331, China; \\ yucy@cqu.edu.cn (C.Y.Y.); lixiaoxu@cqu.edu.cn (X.X.L.); yangh0921@cqu.edu.cn (H.Y.); \\ liyh@cqu.edu.cn (Y.H.L.); xueww@cqu.edu.cn (W.W.X.) \\ 2 Innovative Drug Research and Bioinformatics Group, College of Pharmaceutical Sciences, \\ Zhejiang University, Hangzhou 310058, China \\ 3 Bioinformatics and Drug Design Group, Department of Pharmacy, and Center for Computational Science \\ and Engineering, National University of Singapore, Singapore 117543, Singapore; 20121802134@cqu.edu.cn \\ 4 School of Medicine, Hangzhou Normal University, Hangzhou 310012, China; linntaoo@hotmail.com \\ * Correspondence: zhufeng.ns@gmail.com or zhufeng@cqu.edu.cn
}

Received: 22 October 2017; Accepted: 4 January 2018; Published: 8 January 2018

\begin{abstract}
The function of a protein is of great interest in the cutting-edge research of biological mechanisms, disease development and drug/target discovery. Besides experimental explorations, a variety of computational methods have been designed to predict protein function. Among these in silico methods, the prediction of BLAST is based on protein sequence similarity, while that of machine learning is also based on the sequence, but without the consideration of their similarity. This unique characteristic of machine learning makes it a good complement to BLAST and many other approaches in predicting the function of remotely relevant proteins and the homologous proteins of distinct function. However, the identification accuracies of these in silico methods and their false discovery rate have not yet been assessed so far, which greatly limits the usage of these algorithms. Herein, a comprehensive comparison of the performances among four popular prediction algorithms (BLAST, SVM, PNN and KNN) was conducted. In particular, the performance of these methods was systematically assessed by four standard statistical indexes based on the independent test datasets of 93 functional protein families defined by UniProtKB keywords. Moreover, the false discovery rates of these algorithms were evaluated by scanning the genomes of four representative model organisms (Homo sapiens, Arabidopsis thaliana, Saccharomyces cerevisiae and Mycobacterium tuberculosis). As a result, the substantially higher sensitivity of SVM and BLAST was observed compared with that of PNN and KNN. However, the machine learning algorithms (PNN, KNN and SVM) were found capable of substantially reducing the false discovery rate ( $\mathrm{SVM}<\mathrm{PNN}<\mathrm{KNN}$ ). In sum, this study comprehensively assessed the performance of four popular algorithms applied to protein function prediction, which could facilitate the selection of the most appropriate method in the related biomedical research.
\end{abstract}

Keywords: false discovery rate; machine learning; protein function prediction; support vector machine; BLAST

\section{Introduction}

The function of a protein is of great interest in the current research of biological mechanisms [1], disease development [2] and drug/target discovery [3-7], and a variety of databases is available 
for providing functional annotations from the perspectives of the sequence [8], protein-protein interaction [9,10], the biological network [11-15] and many specific functional classes [16-22]. However, a substantial gap is still observed between the total number of protein sequences discovered and that of proteins characterized with known function [23]. To cope with this gap, thousands of high-throughput genome projects are under study [24], and over 13 million sequences have been discovered, but only $1 \%$ of these validated by experimental annotation [25]. Apart from those experimental approaches, many in silico methods have been designed and extensively used to discover protein functions [26]. These include clustering of sequences [27], gene fusion [28], sequence similarity [29,30], evolution study [31], structural comparison [32], protein-protein interaction [33,34], functional classification via the sequence-derived [35-38] and domain [39-43] feature, omics profiling [44-47] and integrated methods, which collectively consider multiple methods and data to promote the performance of function prediction [48-51].

Among these in silico methods [52], the basic local alignment search tool (BLAST) [53] revealing protein functions based on excess sequence similarity [54] demonstrated great capacity and attracted substantial interest from the researchers of this field $[55,56]$. Apart from BLAST, machine learning algorithms have been frequently applied in recent years for functional prediction [57-62], and a variety of online software tools based on machine learning was developed as predictors without considering the similarity in sequence or structure $[36,63]$. This unique characteristic makes machine learning a good complement to other in silico approaches in predicting the function of remotely relevant protein and the homologous proteins of distinct functions $[64,65]$.

So far, three machine learning algorithms, including K-nearest neighbor (KNN), probabilistic neural network (PNN) and support vector machine (SVM), have been extensively explored to classify proteins into certain functional families by analyzing the sequence-based physicochemical property $[64,65]$ and to assess protein functional classes collectively [63]. These algorithms are recognized as powerful alternative methods for predicting the function of both proteins [66-70] and other molecules [71]. However, over one third of the protein sequences in UniProt [26] are still labeled as "putative", "uncharacterized", "unknown function" or "hypothetical", and the difficulty in discovering the function of the remaining proteins is reported to come mainly from the false discovery rate of in silico algorithms [55,56,72]. Moreover, the identification accuracies of those approaches still need to be further improved $[55,56,73]$. Thus, it is urgently needed to assess the identification accuracies and false discovery rates among those different in silico approaches.

In this study, the performances of four popular functional prediction algorithms (BLAST, SVM, KNN and PNN) were comprehensively evaluated from two perspectives. In particular, the identification accuracies (measured by four standard statistical indexes) of various algorithms were systematically evaluated based on the independent test data of 93 functional families. Secondly, the false discovery rates of these algorithms were compared by scanning the genomes of four representative model species (Homo sapiens, Arabidopsis thaliana, Saccharomyces cerevisiae and Mycobacterium tuberculosis). In sum, these findings provided detailed information on the performances of those algorithms that are popular for protein function prediction, which may facilitate the choice of the appropriate algorithm(s) in the related biomedical research.

\section{Results and Discussion}

\subsection{Assessment of the Identification Accuracies Measured by Four Popular Metrics}

The statistical differences in sensitivity (SE) (Figure 1A), specificity (SP) (Figure 1B), accuracy (ACC) (Figure 1C) and Matthews correlation coefficient (MCC) (Figure 1D) among four popular functional prediction algorithms are illustrated. As illustrated in Figure 1A, the SE of BLAST measured by the independent test dataset of 93 families was roughly equivalent to that of SVM, but statistically higher than that of both PNN and KNN. In particular, the SE of 93 functional families was 50.00 99.99\% for SVM, 43.93 99.99\% for BLAST, 65.52 99.99\% for PNN and 51.09 99.99\% for KNN, and the 
SE median values of BLAST, SVM, PNN and KNN equaled 90.59\%, 90.52\%, 84.38\% and 76.54\%, respectively. As shown in Figure 1B, the majority of the SPS of all algorithms surpassed 98.00\%; SPS of 93 functional families were 95.90 99.99\% for SVM, 97.56 99.99\% for BLAST, 98.87 99.99\% for PNN and $97.77 \sim 99.43 \%$ for KNN; and the SP median value of BLAST, SVM, PNN and KNN was 98.90\%, $99.72 \%, 99.67 \%$ and $99.44 \%$, respectively. These results revealed a relatively low level of false discovery rates for all popular functional prediction algorithms.
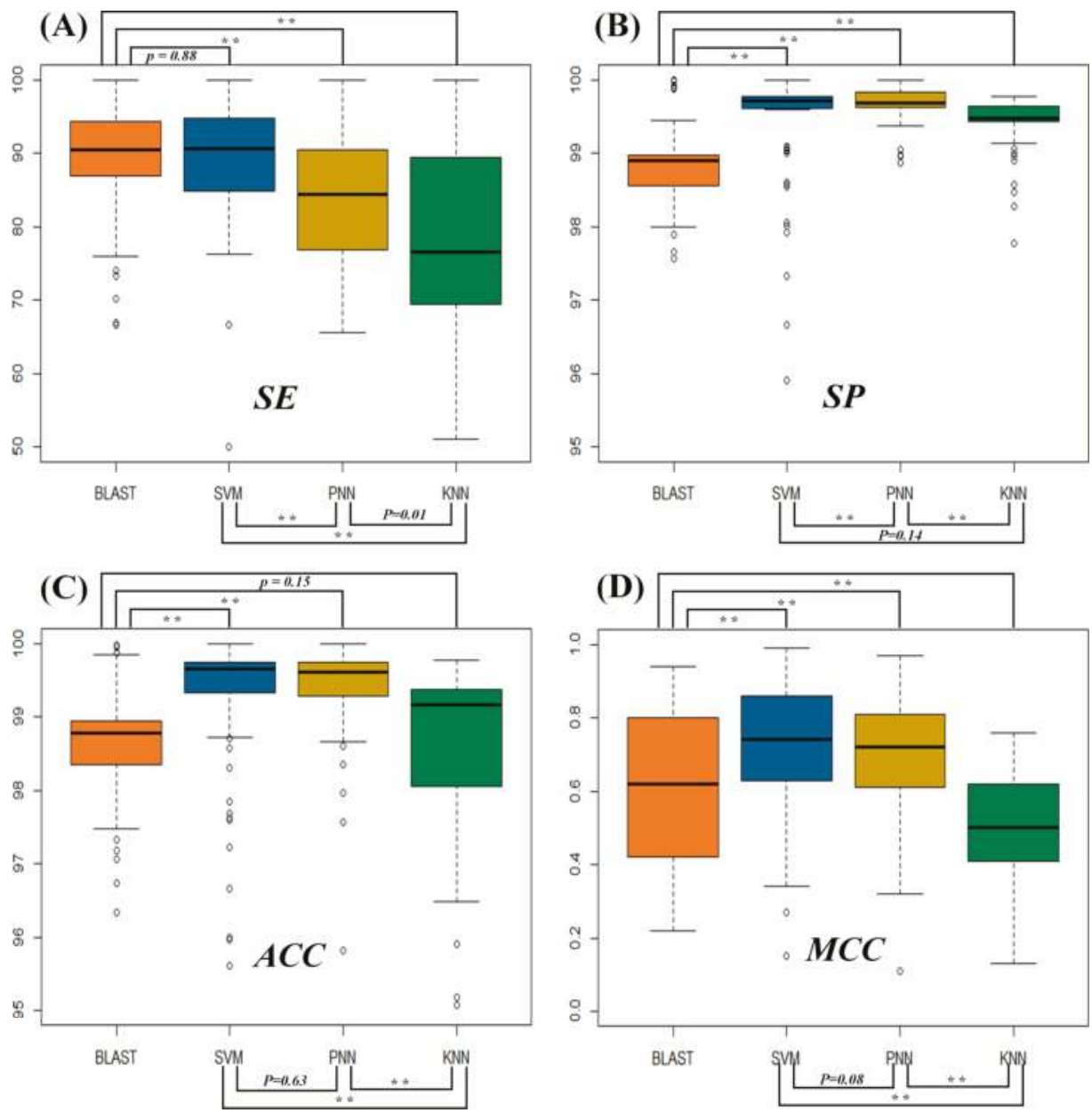

Figure 1. Statistical differences in the performance of four protein function prediction algorithms (BLAST, SVM, PNN and KNN) assessed by four metrics: (A) sensitivity (SE); (B) specificity (SP); (C) accuracy $(A C C)$; and (D) Matthews correlation coefficient $(M C C)$. Significant and moderately significant differences were shown by a $p$-value of $<0.01\left({ }^{* *}\right)$, respectively.

Due to the dominant number of negative samples in the independent test datasets, the statistical difference in ACC was very similar to that of SP (Figure 1C). The majority of the ACCs of all algorithms surpassed $97 \%$. The ACCs of 93 functional families were between 95.61\% and $99.99 \%$ for SVM, between $66.68 \%$ and $99.98 \%$ for BLAST, between $95.81 \%$ and $99.99 \%$ for PNN and between $81.39 \%$ and $99.77 \%$ for KNN. Moreover, median values of ACCs of BLAST, SVM, PNN and KNN equaled 98.78\%, 99.66\%, 
$99.61 \%$ and $99.16 \%$, respectively. MCC was frequently applied to reflect the stability of the protein function predictor and was considered as one of the most comprehensive parameters because of its full consideration of TP, TN, FP and FN. As shown in Figure 1D, the MCC of both SVM and PNN was better than that of BLAST and KNN. The majority of MCCs were over 0.6 and 0.4 for SVM-PNN and BLAST-KNN, respectively. In particular, MCCS of 93 functional families were between 0.15 and 0.99 for SVM, between 0.22 and 0.94 for BLAST, between 0.11 and 0.97 for PNN and between 0.13 and 0.76 for KNN. The median values of MCCs for BLAST, SVM, PNN and KNN equaled 0.62, 0.74, 0.72 and 0.50 , respectively. In sum, there were consistently low levels of the false discovery rate among all algorithms as assessed by the metric $S P$. However, when the positive discovery rates (SEs) and the stability of prediction (MCC) were considered, SVM, PNN and BLAST stood out as more powerful algorithms for protein function prediction.

\subsection{Evaluating the Statistical Differences in SE and MCC among Four Metrics}

For the machine learning algorithms (SVM, PNN and KNN), there was a significant statistical difference in their SEs and MCCs. As shown in Figure 1A, the statistical difference in SEs between SVM and PNN equaled $3.5 \times 10^{-6}$, while that between SVM and KNN was $1.0 \times 10^{-11}$. Moreover, there was a significant statistical difference between PNN and KNN ( $p$-value $=0.01)$. In particular, the number of families with $S E s$ of $>90 \%, \leq 90 \%$ and $>80 \%$ and $\leq 80 \%$ for SVM equaled 49,33 and 11 , respectively; the number of families with $S E s$ of $>90 \%, \leq 90 \%$ and $>80 \%$ and $\leq 80 \%$ for PNN equaled 17, 25 and 20, respectively; and the number of functional families with SEs of $>90 \%, \leq 90 \%$ and $>80 \%$ and $\leq 80 \%$ for KNN equaled 19,13 and 45 , respectively. Similar to the $S E$, the statistical difference in MCC between SVM and PNN was 0.08 , and that between SVM and KNN was $2.2 \times 10^{-16}$. Moreover, there was a clear statistical difference between PNN and KNN ( $p$-value $\left.=2.2 \times 10^{-16}\right)$. In particular, the number of families with MCCs of $>0.85, \leq 0.85$ and $>0.7$ and $\leq 0.7$ for SVM was 26,26 and 41 , respectively; the number of functional families with MCCs of $>0.85, \leq 0.85$ and $>0.7$ and $\leq 0.7$ for PNN equaled 6, 29 and 27, respectively; and there were no protein families with MCCs over 0.7 for KNN. In summary, there were clear ascending trends in both $S E$ and MCC as shown in Figure 1A,D (from KNN to PNN to SVM).

Similar to SVM, BLAST also demonstrated great performances in both SE and MCC. The statistical differences (measured by $p$-value) in the SE and MCC between BLAST and SVM were 0.88 and $2.0 \times 10^{-7}$, respectively. As demonstrated in Table 1 and Table S1, the SE of BLAST surpassed that of SVM in 51 families, but was worse than that of SVM in 40 families. Moreover, the SEs' median values ( $90.52 \%$ for BLAST and $90.59 \%$ for SVM) and mean values ( $88.92 \%$ for BLAST and $89.08 \%$ for SVM) indicated that the $S E$ of SVM was slightly better than that of BLAST and significantly better than that of PNN and KNN. Meanwhile, MCC of SVM was higher than that of BLAST in 68 families, but was lower than that of BLAST in 20 families. The MCCs' median values ( 0.62 for BLAST, 0.74 for SVM) and mean values ( 0.61 for BLAST, 0.73 for SVM) indicated a slight improvement in prediction stabilities by SVM.

The amphibian defense peptide family (KW-0878; KW, keyword) was the family with the highest SE $(99.99 \%)$ for SVM, BLAST and KNN, which was known to be a rich source of antimicrobial peptides with a broad spectrum of antimicrobial activities against pathogenic microorganisms [74-76]. The superior $S E$ of this family may come from its nature as a conserved element of the defense system of various species [77]. 


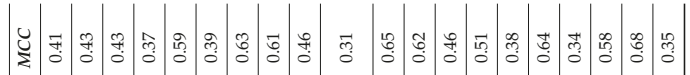

రิ

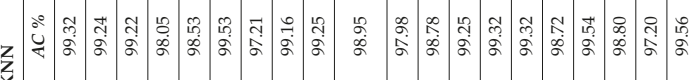

ㅇำ영

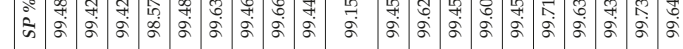

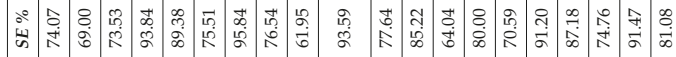

(ิ)

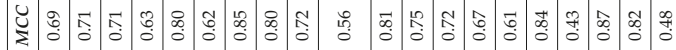

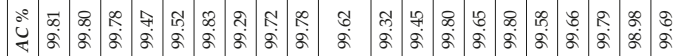

z

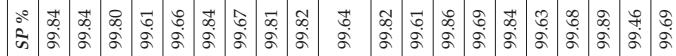

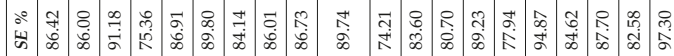

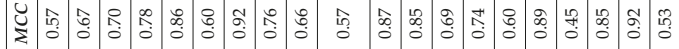

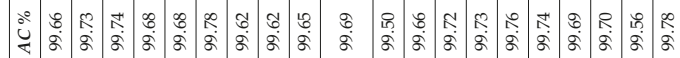

$\sum_{\infty}^{\infty}$

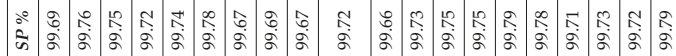

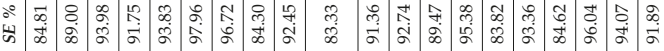

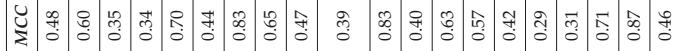

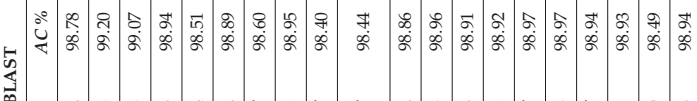

.

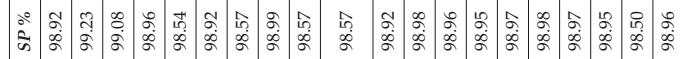

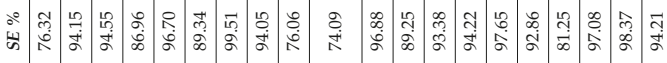

:

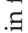

跑

రై

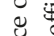

跣

हี

过

है

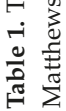

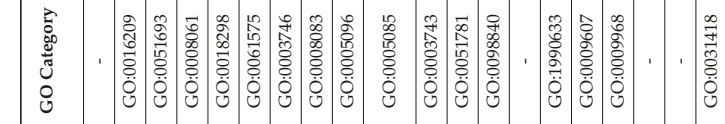

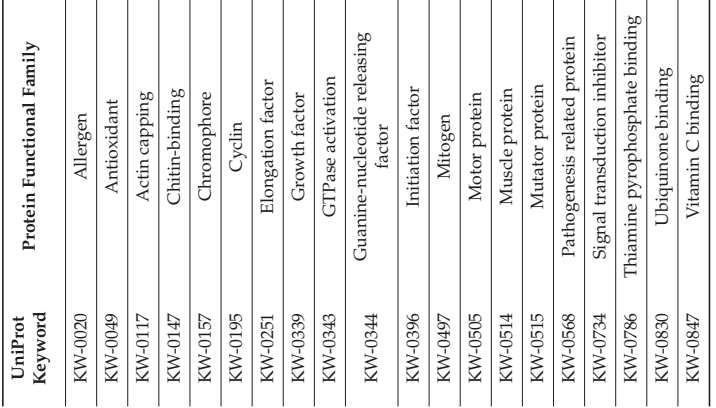




\subsection{In-Depth Assessment of the False Discovery Rate by Genome Scanning}

Genome scanning has been frequently used to evaluate the false discovery rate of function prediction tools $[78,79]$. To have a comprehensive understanding of methods' false discovery rate, the genomes of four model organisms representing four kingdoms (Homo sapiens from Animalia, Arabidopsis thaliana from Plantae, saccharomyces cerevisiae from Fungi and Mycobacterium tuberculosis from Bacteria) were collected. As demonstrated in Table 2 and Table S2, the genome scanning revealed that the number of proteins in any of those 93 studied families predicted by SVM, PNN and KNN did not exceed $10 \%$ of the total number of proteins in the whole genome, and this was the same situation for the majority ( $82 \%$ ) of the 93 studied families by BLAST. The higher number of proteins predicted for a certain functional family may indicate a higher false discovery rate $[78,79]$. For the human genome, the number of proteins identified by SVM was equivalent to or was slightly higher than that of both PNN and KNN, but was significantly lower than that of BLAST (Figure 2a). In addition, the proteins identified by PNN were lower than that of KNN in 11 families and higher in 20 families.
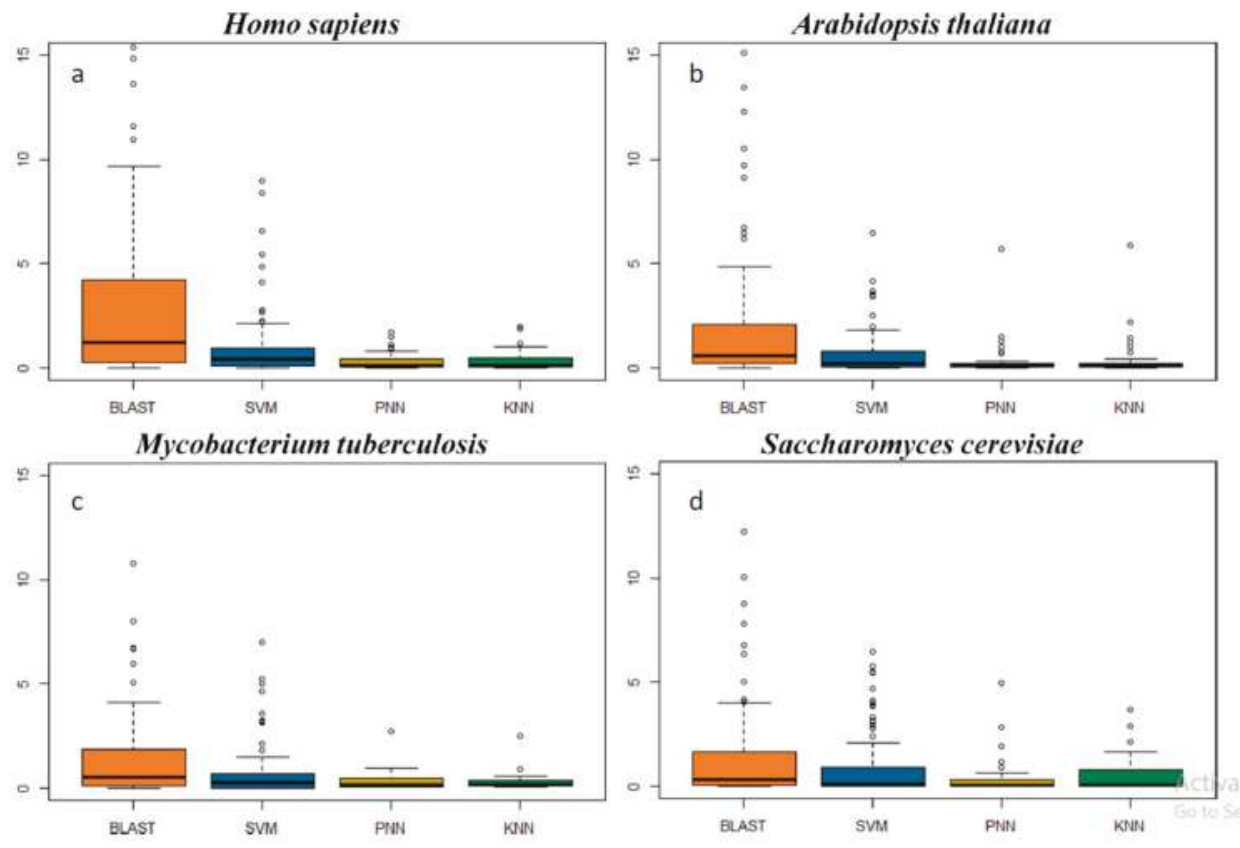

Figure 2. The false discovery rates reflected by the percentage of proteins identified from the genomes of (a) Homo sapiens, (b) Arabidopsis thaliana, (c) Saccharomyces cerevisiae and (d) Mycobacterium tuberculosis. 
Int. J. Mol. Sci. 2018, 19, 183

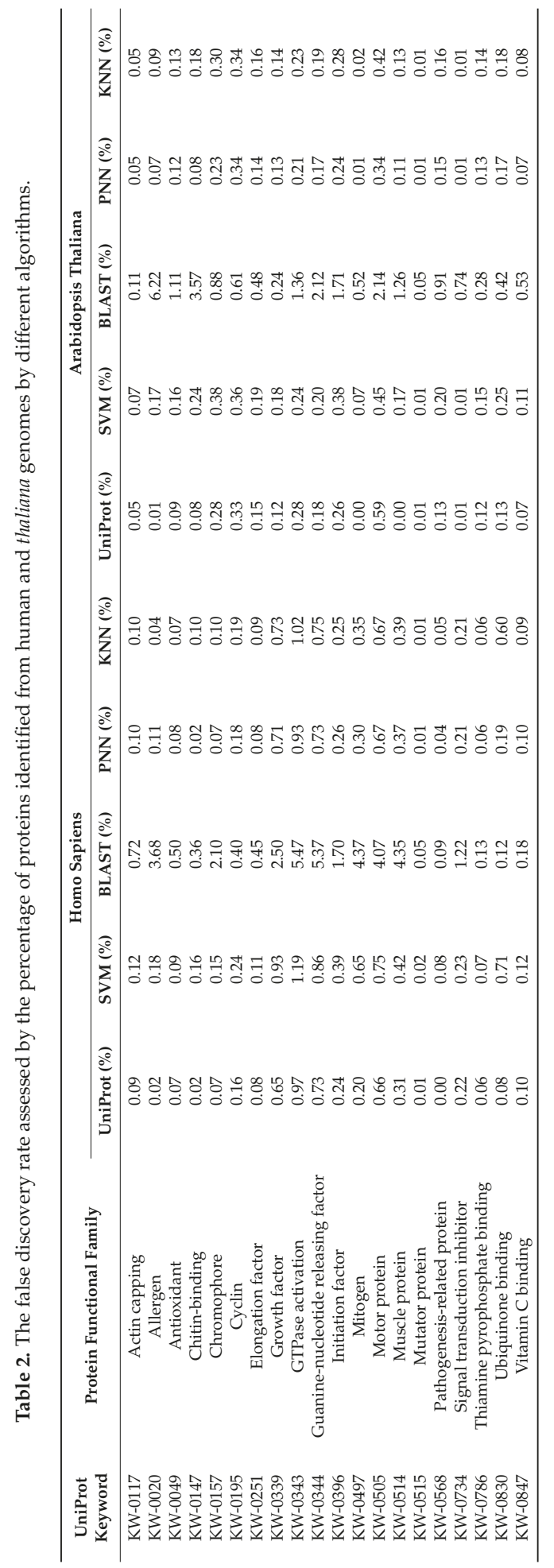


Moreover, 15 protein families only existing in plants, microbes or viruses (Table S3, not existing in the human genome) were collected for assessing the false discovery rate of each algorithm. For example, the covalent protein-RNA linkage family (KW-0191) contained proteins attaching covalently to the RNA molecules in virus [80], and the storage protein (KW-0758) included the proteins as a source of nutrients for the development or growth of the organism in plants. For these families (Table S3), SVM did not identify any proteins from the human genome, while $0.06 \%$ and $0.25 \%$ of the proteins in the human genome were falsely assigned by BLAST to the family of covalent protein-RNA linkage protein and storage protein, respectively. As illustrated in Figure 3, several other families (such as plant defense, virulence) also demonstrated a significantly higher false discovery rate by BLAST than that of SVM.

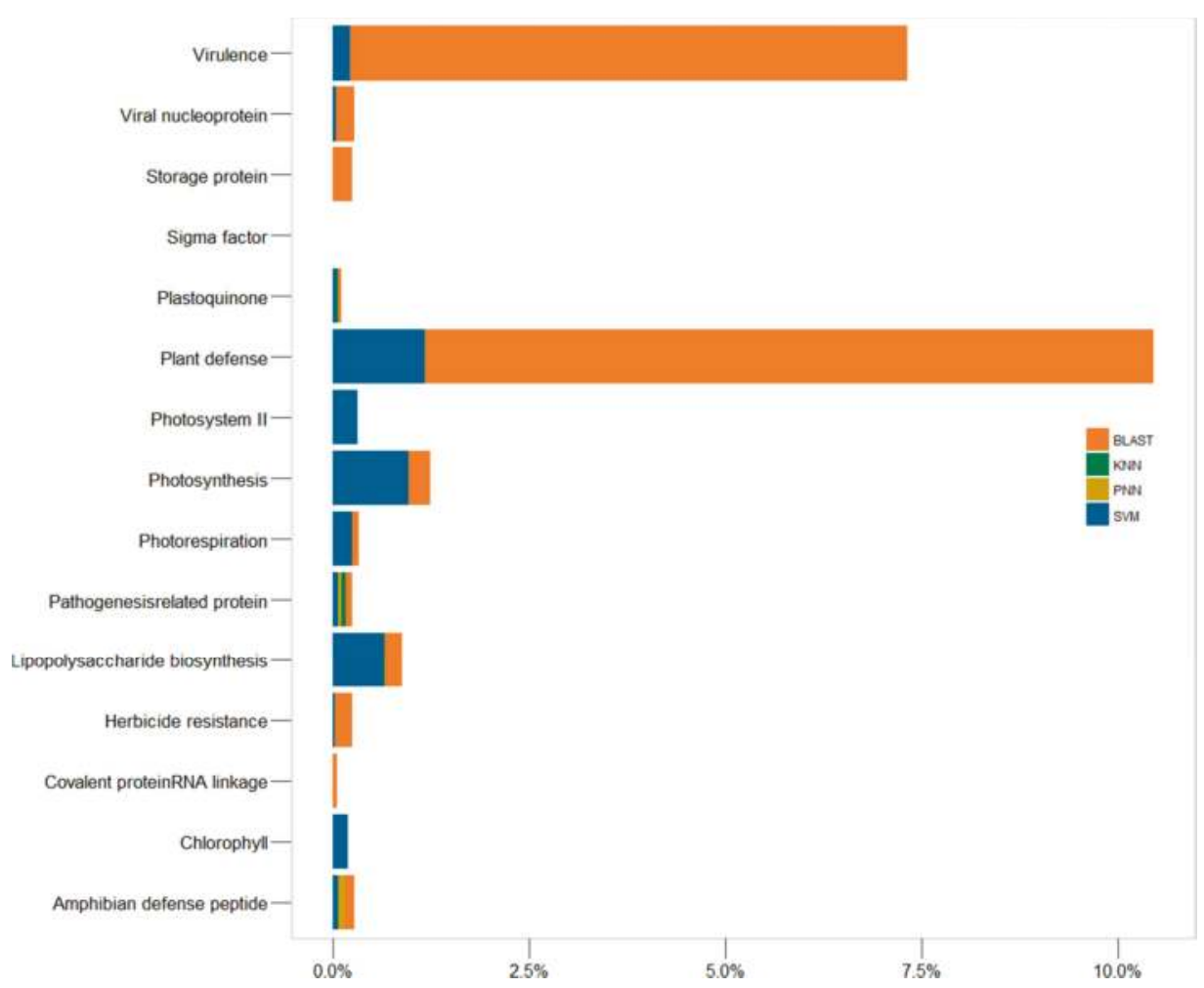

Figure 3. The false discovery rates reflected by the percentage of proteins of 15 protein families only existing in plants, microbes or viruses, but not existing in the human genome identified from the genomes of Homo sapiens.

For the other three genomes, their situation was similar to the human genome. Take the Arabidopsis thaliana genome as an example: proteins identified by SVM were equivalent to or slightly higher than those by PNN and KNN in all protein families, but lower than that of BLAST in 77 families, and the number of protein discovered by PNN was lower than that of KNN in 26 families. In summary, the level of false discovery rate (Figure $2 \mathrm{~b}-\mathrm{d}$ ) could be ordered as BLAST $>$ SVM $>$ PNN and KNN. These results revealed that BLAST was more prone to generate a false discovery rate than the other three machine learning methods (SVM $>\mathrm{PNN} \approx \mathrm{KNN})$.

As reported [81-85], an open web-server is recognized as useful for constructing effective methods and tools. A variety of web-servers have increasing impacts on medical sciences [86], driving medicinal 
chemistry to an unprecedented revolution [87], and efforts will be further made to develop web-based services for the performance assessment discussed in this study.

\section{Materials and Methods}

To construct a valid statistical model for a biology problem based on protein sequences [88-97], a rule of five steps is needed [98]. Firstly, a valid construction of datasets for both training and testing the model is required. Secondly, an effective conversion of the sequence to the digital feature vector is asked to represent their targeted properties. Thirdly, a powerful statistical method should be designed for the functional prediction. Fourthly, the accuracies of the constructed statistic model should be validated correctly. Fifthly, a web-server based on the constructed model may be further developed for public access. The corresponding methods and steps adopted in this study are provided and described below.

\subsection{Collecting the Protein Sequences of Different Functional Families}

Table 1 provides a full list of 93 protein families collected from UniProt [43], and the performances of the popular protein function prediction methods (BLAST, KNN, PNN, SVM) were measured via independent test datasets (the way to generate an independent dataset is shown in the following Section 2.2). These 93 included 12 families of binding molecules (e.g., sodium-, potassium-, SH3- and RNA-binding), 15 ligand families (e.g., plastoquinone ligand, vitamin C ligand and ubiquinone ligand), 58 families defined by Gene Ontology (40 molecular functions and 18 biological processes) and 8 broad families defined by UniProt [43]. All families were contained in the keyword categories of UniProt, and the majority $(82.7 \%$ ) of these 93 families were able to be mapped to GO terms (Table 1). Protein entries that have not been manually annotated and reviewed by UniProtKB curators in a keyword category were not considered for analysis in this study. As a result, 107 49,517 protein-entries from 93 families were collected.

\subsection{Construction of the Training and Testing Datasets}

The independent test dataset was frequently constructed to evaluate the performances of protein function predictors in recent years [99-104]. To construct a valid set of data for building the predictor of each family, the datasets of the training, testing and independent test were generated by a strictly defined process after the data collection described in Section 2.1. Firstly, all proteins of different sequences in a specific family are assigned randomly with a number, which is within the range of the total number of proteins in that family. Secondly, these sequences in each protein function family were sequentially selected based on the number assigned and then iteratively added to the training, testing and independent test datasets. Samples in these datasets are all known as the positive samples. Thirdly, the Pfam families [16] of the proteins of a certain functional family were retrieved from the Pfam database [16] for generating negative samples. The Pfam family with protein(s) of this functional family was defined as the "positive" one, and the remaining families were grouped into the "negative" ones. Finally, 3 representatives were randomly picked out of the negative families and sequentially added to the training, testing and independent test datasets, and samples in these datasets are thus known as the negative samples. It is necessary to emphasize that there was no overlap among the datasets of the training, testing and independent test $[60,61]$.

To assess the false discovery rate among algorithms, the genomes of four model organisms representing four kingdoms (Homo sapiens from Animalia, Arabidopsis thaliana from Plantae, Saccharomyces cerevisiae from Fungi and Mycobacterium tuberculosis from Bacteria) were collected from UniProt. The protein entries without any manual annotation and review by the UniProtKB curators were not taken into consideration. In total, 20,183, 15,169, 6721 and 2166 protein sequences in FASTA format were collected for human, Arabidopsis thaliana, Saccharomyces cerevisiae and Mycobacterium tuberculosis, respectively. 


\subsection{Feature Vectors Used for Representing the Protein Sequence}

The conversion of the protein sequence into the digital feature vector was conducted based on properties of each residue within that protein. These properties include: (1) charge; (2) polarizability; (3) polarity; (4) surface tension; (5) amino acid (AA) composition; (6) van der Waals volume via normalizing; (7) hydrophobicity; (8) solvent accessibility; and (9) protein secondary structure [36,105-107]. Then, 3 features were applied to describe each property [36]. These features contained: (a) composition (No. of AAs of a particular property over the total No. of AAs; (b) transition (the percentage of AAs with a certain property was followed by AAs with a different property); and (c) distribution (the sequence lengths within which the first, one fourth, half, three-quarters and all of the AAs of specific property were localized). The detailed procedure for generating the feature vector from the sequence was described in previous publications $[36,65]$. These features have already been successfully applied to facilitate the prediction of enzyme functional [108] and structural classes [107].

\subsection{Functional Prediction of Protein Constructed by Machine Learning}

To construct the prediction model, the parameters of machine learning methods were optimized using the testing dataset for each training process. Once suitable parameters were discovered, a new training set was constructed by combining the original training and testing datasets, and the corresponding parameters were directly accepted for training a new model. To assess the performance of the constructed models and detect possible over-fitting, the independent test set was further applied. It is necessary to emphasize that all duplicates in the protein sequence were removed during datasets' construction.

\subsection{Construction of Protein Functional Prediction Model Based on Sequence Similarity}

Sequence similarity was assessed by the NCBI Protein-Protein BLAST (Version 2.6.0+) [53,54]. Firstly, the combined training and testing dataset was adopted to form the BLAST database, and the sequences in the independent test dataset were used as queries. The BLAST E-value and percentage sequence identity were usually applied to represent the level of similarity between sequences [109]. The functional variation between proteins was reported to be rare when their sequence identity was more than $40 \%[110,111]$. Thus, an E-value of 0.001 and a sequence identity of $40 \%$ were adopted as the cutoffs in this study to assess the functional conservation of BLAST hits.

\subsection{Assessing the Identification Accuracies of the Studied Methods}

The performance of protein function prediction algorithms was systematically assessed by four popular metrics, sensitivity $(S E)$, specificity $(S P)$, accuracy $(A C C)$ and Matthews correlation coefficient $(M C C)$, based on the independent test datasets generated from the 93 studied families (Supplementary Materials Table S1). All 4 metrics were widely used in assessing the performance of protein function predictors [112-117]. In particular, $S E$ is defined by the percentage of true positive samples correctly identified as "positive" [118,119] (shown in Equation (1)):

$$
S E=\frac{\mathrm{TP}}{\mathrm{TP}+\mathrm{FN}}
$$

$S P$ indicates the proportion of true negative samples that were correctly predicted as "negative" [118,119] (in Equation (2)):

$$
S P=\frac{\mathrm{TN}}{\mathrm{TN}+\mathrm{FP}}
$$

$A C C$ refers to the number of true samples (positive plus negative) divided by the number of all samples studied (shown in Equation (3)):

$$
A C C=\frac{\mathrm{TP}+\mathrm{TN}}{\mathrm{TP}+\mathrm{FN}+\mathrm{TN}+\mathrm{FP}}
$$


The MCC was an important metric reflecting the stability of a protein function predictor, which described the correlation between a predictive value and an actual value [118,119]. It has been considered as one of the most comprehensive parameters in any category of predictors due to its full consideration of all four results. In particular, the MCC could be calculated by Equation 4:

$$
M C C=\frac{(\mathrm{TP} * \mathrm{TN}-\mathrm{FP} * \mathrm{FN})}{\sqrt{(\mathrm{TP}+\mathrm{FN}) *(\mathrm{TP}+\mathrm{FP}) *(\mathrm{TN}+\mathrm{FP}) *(\mathrm{TN}+\mathrm{FN})}}
$$

In particular, those four results were TP (No. of true positive samples), TN (No. of true negative samples), FP (No. of false positive samples) and FN (No. of false negative samples) [118,119]. It is very important to emphasize that these four metrics are applicable to the single-class situations (each protein is grouped into just one family). For the multi-class situations frequently observed in complicated biological networks [81-84] and biomedical researches $[84,89,117]$, different metrics should be defined [120].

\subsection{The Rates of False Discovery of the In Silico Methods Studied Here}

As reported, genome scanning was a comprehensive method to evaluate the capacity of protein functional prediction tools in identifying and classifying protein families [78,79]. In this paper, an evaluation of the false discovery rate of the studied protein function predictors was performed by scanning the genomes of 4 model organisms representing 4 kingdoms (Homo sapiens from Animalia, Arabidopsis thaliana from Plantae, Saccharomyces cerevisiae from Fungi and Mycobacterium tuberculosis from Bacteria). The false discovery rates were assessed by reconstructing the prediction models of those in silico algorithms. In particular, the sequences of proteins in a certain functional family were all put into the reference database for BLAST scanning and were also used to reconstruct the machine learning models using the optimized parameters obtained in Section 3.4. In reality, the total amount of proteins not belonging to a certain family should be much larger than that of proteins in that family. Therefore, a tiny reduction in the value of $S P$ may lead to a significant discovery of false positive hits, which reminded us to use $S P$ as an effective indicator when evaluating the model's false discovery rates.

\section{Conclusions}

This study discovered substantially higher sensitivity $(S P)$ and stability $(M C C)$ of BLAST and SVM than that of PNN and KNN. However, the machine learning algorithms (PNN, KNN and SVM) were found capable of significantly reducing the false discovery rate (with PNN and KNN performed the best). In conclusion, this study comprehensively assessed the performances of popular algorithms applied to protein function prediction, which could facilitate the selection of the appropriate method in the related biomedical research.

Supplementary Materials: The Supplementary Materials are available online at www.mdpi.com/1422-0067/19/ $1 / 183 /$ s1.

Acknowledgments: This work was funded by the Precision Medicine Project of the National Key Research and Development Plan of China (2016YFC0902200); the Innovation Project on Industrial Generic Key Technologies of Chongqing (cstc2015zdcy-ztzx120003); the Fundamental Research Funds for Central Universities (10611CDJXZ238826, CDJZR14468801, CDJKXB14011); and the National Natural Science Foundation of China (21505009).

Author Contributions: Feng Zhu and Lin Tao conceived of and designed the experiments. Chun Yan Yu and Xiao $\mathrm{Xu}$ Li carried out most of the experiments in this paper. Chun Yan Yu, Ying Hong Li, Hong Yang, Wei Wei Xue and $\mathrm{Yu}$ Zong Chen performed the bioinformatics analysis. Feng Zhu wrote the paper. All authors read and approved the final manuscript.

Conflicts of Interest: The authors declare no conflict of interest. 


\section{References}

1. Baldi, P.; Brunak, S.; Chauvin, Y.; Andersen, C.A.; Nielsen, H. Assessing the accuracy of prediction algorithms for classification: An overview. Bioinformatics 2000, 16, 412-424. [CrossRef] [PubMed]

2. Jackson, S.P.; Bartek, J. The DNA-damage response in human biology and disease. Nature 2009, 461, 1071-1078. [CrossRef] [PubMed]

3. Weinberg, S.E.; Chandel, N.S. Targeting mitochondria metabolism for cancer therapy. Nat. Chem. Biol. 2015, 11, 9-15. [CrossRef] [PubMed]

4. Grant, M.A. Integrating computational protein function prediction into drug discovery initiatives. Drug Dev. Res. 2011, 72, 4-16. [CrossRef] [PubMed]

5. Li, B.; Tang, J.; Yang, Q.; Li, S.; Cui, X.; Li, Y.; Chen, Y.; Xue, W.; Li, X.; Zhu, F. Noreva: Normalization and evaluation of MS-based metabolomics data. Nucleic Acids Res. 2017, 45, 162-170. [CrossRef] [PubMed]

6. Li, B.; Tang, J.; Yang, Q.; Cui, X.; Li, S.; Chen, S.; Cao, Q.; Xue, W.; Chen, N.; Zhu, F. Performance evaluation and online realization of data-driven normalization methods used in $\mathrm{l} / \mathrm{ms}$ based untargeted metabolomics analysis. Sci. Rep. 2016, 6, 38881. [CrossRef] [PubMed]

7. Xu, J.; Wang, P.; Yang, H.; Zhou, J.; Li, Y.; Li, X.; Xue, W.; Yu, C.; Tian, Y.; Zhu, F. Comparison of FDA approved kinase targets to clinical trial ones: Insights from their system profiles and drug-target interaction networks. BioMed Res. Int. 2016, 2016, 2509385. [CrossRef] [PubMed]

8. Huerta-Cepas, J.; Szklarczyk, D.; Forslund, K.; Cook, H.; Heller, D.; Walter, M.C.; Rattei, T.; Mende, D.R.; Sunagawa, S.; Kuhn, M.; et al. Eggnog 4.5: A hierarchical orthology framework with improved functional annotations for eukaryotic, prokaryotic and viral sequences. Nucleic Acids Res. 2016, 44, 286-293. [CrossRef] [PubMed]

9. Szklarczyk, D.; Jensen, L.J. Protein-protein interaction databases. Methods Mol. Biol. 2015, 1278, 39-56. [PubMed]

10. Jeanquartier, F.; Jean-Quartier, C.; Holzinger, A. Integrated web visualizations for protein-protein interaction databases. BMC Bioinform. 2015, 16, 195. [CrossRef] [PubMed]

11. Szklarczyk, D.; Santos, A.; von Mering, C.; Jensen, L.J.; Bork, P.; Kuhn, M. Stitch 5: Augmenting protein-chemical interaction networks with tissue and affinity data. Nucleic Acids Res. 2016, 44, 380-384. [CrossRef] [PubMed]

12. Franceschini, A.; Szklarczyk, D.; Frankild, S.; Kuhn, M.; Simonovic, M.; Roth, A.; Lin, J.; Minguez, P.; Bork, P.; von Mering, C.; et al. String v9.1: Protein-protein interaction networks, with increased coverage and integration. Nucleic Acids Res. 2013, 41, 808-815. [CrossRef] [PubMed]

13. Szklarczyk, D.; Franceschini, A.; Wyder, S.; Forslund, K.; Heller, D.; Huerta-Cepas, J.; Simonovic, M.; Roth, A.; Santos, A.; Tsafou, K.P.; et al. String v10: Protein-protein interaction networks, integrated over the tree of life. Nucleic Acids Res. 2015, 43, 447-452. [CrossRef] [PubMed]

14. Szklarczyk, D.; Franceschini, A.; Kuhn, M.; Simonovic, M.; Roth, A.; Minguez, P.; Doerks, T.; Stark, M.; Muller, J.; Bork, P.; et al. The string database in 2011: Functional interaction networks of proteins, globally integrated and scored. Nucleic Acids Res. 2011, 39, 561-568. [CrossRef] [PubMed]

15. Szklarczyk, D.; Morris, J.H.; Cook, H.; Kuhn, M.; Wyder, S.; Simonovic, M.; Santos, A.; Doncheva, N.T.; Roth, A.; Bork, P.; et al. The string database in 2017: Quality-controlled protein-protein association networks, made broadly accessible. Nucleic Acids Res. 2017, 45, 362-368. [CrossRef] [PubMed]

16. Finn, R.D.; Coggill, P.; Eberhardt, R.Y.; Eddy, S.R.; Mistry, J.; Mitchell, A.L.; Potter, S.C.; Punta, M.; Qureshi, M.; Sangrador-Vegas, A.; et al. The pfam protein families database: Towards a more sustainable future. Nucleic Acids Res. 2016, 44, 279-285. [CrossRef] [PubMed]

17. Li, Y.H.; Yu, C.Y.; Li, X.X.; Zhang, P.; Tang, J.; Yang, Q.; Fu, T.; Zhang, X.; Cui, X.; Tu, G.; et al. Therapeutic target database update 2018: Enriched resource for facilitating bench-to-clinic research of targeted therapeutics. Nucleic Acids Res. 2017. [CrossRef]

18. Yang, H.; Qin, C.; Li, Y.H.; Tao, L.; Zhou, J.; Yu, C.Y.; Xu, F.; Chen, Z.; Zhu, F.; Chen, Y.Z. Therapeutic target database update 2016: Enriched resource for bench to clinical drug target and targeted pathway information. Nucleic Acids Res. 2016, 44, 1069-1074. [CrossRef] [PubMed]

19. Zhu, F.; Shi, Z.; Qin, C.; Tao, L.; Liu, X.; Xu, F.; Zhang, L.; Song, Y.; Liu, X.; Zhang, J.; et al. Therapeutic target database update 2012: A resource for facilitating target-oriented drug discovery. Nucleic Acids Res. 2012, 40, 1128-1136. [CrossRef] [PubMed] 
20. Zhu, F.; Han, B.; Kumar, P.; Liu, X.; Ma, X.; Wei, X.; Huang, L.; Guo, Y.; Han, L.; Zheng, C.; et al. Update of ttd: Therapeutic target database. Nucleic Acids Res. 2010, 38, 787-791. [CrossRef] [PubMed]

21. Li, Y.H.; Wang, P.P.; Li, X.X.; Yu, C.Y.; Yang, H.; Zhou, J.; Xue, W.W.; Tan, J.; Zhu, F. The human kinome targeted by FDA approved multi-target drugs and combination products: A comparative study from the drug-target interaction network perspective. PLoS ONE 2016, 11, e0165737. [CrossRef] [PubMed]

22. Zhu, F.; Ma, X.H.; Qin, C.; Tao, L.; Liu, X.; Shi, Z.; Zhang, C.L.; Tan, C.Y.; Chen, Y.Z.; Jiang, Y.Y. Drug discovery prospect from untapped species: Indications from approved natural product drugs. PLoS ONE 2012, 7, e39782. [CrossRef] [PubMed]

23. Erdin, S.; Lisewski, A.M.; Lichtarge, O. Protein function prediction: Towards integration of similarity metrics. Curr. Opin. Struct. Biol. 2011, 21, 180-188. [CrossRef] [PubMed]

24. Sayers, E.W.; Barrett, T.; Benson, D.A.; Bolton, E.; Bryant, S.H.; Canese, K.; Chetvernin, V.; Church, D.M.; Dicuccio, M.; Federhen, S.; et al. Database resources of the national center for biotechnology information. Nucleic Acids Res. 2012, 40, 13-25. [CrossRef] [PubMed]

25. Barrell, D.; Dimmer, E.; Huntley, R.P.; Binns, D.; O'Donovan, C.; Apweiler, R. The goa database in 2009—An integrated gene ontology annotation resource. Nucleic Acids Res. 2009, 37, 396-403. [CrossRef] [PubMed]

26. The UniProt Consortium. Activities at the universal protein resource (UniProt). Nucleic Acids Res. 2014, 42, 191-198.

27. Bork, P.; Koonin, E.V. Predicting functions from protein sequences-where are the bottlenecks? Nat. Genet. 1998, 18, 313-318. [CrossRef] [PubMed]

28. Chitale, M.; Hawkins, T.; Park, C.; Kihara, D. ESG: Extended similarity group method for automated protein function prediction. Bioinformatics 2009, 25, 1739-1745. [CrossRef] [PubMed]

29. Enright, A.J.; Van Dongen, S.; Ouzounis, C.A. An efficient algorithm for large-scale detection of protein families. Nucleic Acids Res. 2002, 30, 1575-1584. [CrossRef] [PubMed]

30. Sahraeian, S.M.; Luo, K.R.; Brenner, S.E. Sifter search: A web server for accurate phylogeny-based protein function prediction. Nucleic Acids Res. 2015, 43, 141-147. [CrossRef] [PubMed]

31. Teichmann, S.A.; Murzin, A.G.; Chothia, C. Determination of protein function, evolution and interactions by structural genomics. Curr. Opin. Struct. Biol. 2001, 11, 354-363. [CrossRef]

32. Enright, A.J.; Iliopoulos, I.; Kyrpides, N.C.; Ouzounis, C.A. Protein interaction maps for complete genomes based on gene fusion events. Nature 1999, 402, 86-90. [CrossRef] [PubMed]

33. Aravind, L. Guilt by association: Contextual information in genome analysis. Genome Res. 2000, 10, $1074-1077$. [CrossRef] [PubMed]

34. Kotlyar, M.; Pastrello, C.; Pivetta, F.; Lo Sardo, A.; Cumbaa, C.; Li, H.; Naranian, T.; Niu, Y.; Ding, Z.; Vafaee, F; et al. In silico prediction of physical protein interactions and characterization of interactome orphans. Nat. Methods 2015, 12, 79-84. [CrossRef] [PubMed]

35. Jensen, L.J.; Gupta, R.; Staerfeldt, H.H.; Brunak, S. Prediction of human protein function according to gene ontology categories. Bioinformatics 2003, 19, 635-642. [CrossRef] [PubMed]

36. Cai, C.Z.; Han, L.Y.; Ji, Z.L.; Chen, X.; Chen, Y.Z. SVM-prot: Web-based support vector machine software for functional classification of a protein from its primary sequence. Nucleic Acids Res. 2003, 31, 3692-3697. [CrossRef] [PubMed]

37. Lobley, A.E.; Nugent, T.; Orengo, C.A.; Jones, D.T. Ffpred: An integrated feature-based function prediction server for vertebrate proteomes. Nucleic Acids Res. 2008, 36, 297-302. [CrossRef] [PubMed]

38. Zhu, F.; Qin, C.; Tao, L.; Liu, X.; Shi, Z.; Ma, X.; Jia, J.; Tan, Y.; Cui, C.; Lin, J.; et al. Clustered patterns of species origins of nature-derived drugs and clues for future bioprospecting. Proc. Natl. Acad. Sci. USA 2011, 108, 12943-12948. [CrossRef] [PubMed]

39. Das, S.; Sillitoe, I.; Lee, D.; Lees, J.G.; Dawson, N.L.; Ward, J.; Orengo, C.A. Cath funfhmmer web server: Protein functional annotations using functional family assignments. Nucleic Acids Res. 2015, 43, 148-153. [CrossRef] [PubMed]

40. Wang, P.; Zhang, X.; Fu, T.; Li, S.; Li, B.; Xue, W.; Yao, X.; Chen, Y.; Zhu, F. Differentiating physicochemical properties between addictive and nonaddictive adhd drugs revealed by molecular dynamics simulation studies. ACS Chem. Neurosci. 2017, 8, 1416-1428. [CrossRef] [PubMed]

41. Xue, W.; Wang, P.; Li, B.; Li, Y.; Xu, X.; Yang, F.; Yao, X.; Chen, Y.Z.; Xu, F.; Zhu, F. Identification of the inhibitory mechanism of fda approved selective serotonin reuptake inhibitors: An insight from molecular dynamics simulation study. Phys. Chem. Chem. Phys. 2016, 18, 3260-3271. [CrossRef] [PubMed] 
42. Zheng, G.; Xue, W.; Wang, P.; Yang, F.; Li, B.; Li, X.; Li, Y.; Yao, X.; Zhu, F. Exploring the inhibitory mechanism of approved selective norepinephrine reuptake inhibitors and reboxetine enantiomers by molecular dynamics study. Sci. Rep. 2016, 6, 26883. [CrossRef] [PubMed]

43. Wang, P.; Yang, F.; Yang, H.; Xu, X.; Liu, D.; Xue, W.; Zhu, F. Identification of dual active agents targeting 5-ht1a and sert by combinatorial virtual screening methods. Biomed. Mater. Eng. 2015, 26 (Suppl. 1), 2233-2239. [CrossRef] [PubMed]

44. Li, D.; Ju, Y.; Zou, Q. Protein folds prediction with hierarchical structured SVM. Curr. Proteom. 2016, 13, 79-85. [CrossRef]

45. Wei, L.; Tang, J.; Zou, Q. Skipcpp-pred: An improved and promising sequence-based predictor for predicting cell-penetrating peptides. BMC Genom. 2017, 18 (Suppl. 7), 742. [CrossRef]

46. Wan, S.; Duan, Y.; Zou, Q. Hpslpred: An ensemble multi-label classifier for human protein subcellular location prediction with imbalanced source. Proteomics 2017, 17. [CrossRef] [PubMed]

47. Wei, L.; Xing, P.; Su, R.; Shi, G.; Ma, Z.S.; Zou, Q. Cppred-rf: A sequence-based predictor for identifying cell-penetrating peptides and their uptake efficiency. J. Proteome Res. 2017, 16, 2044-2053. [CrossRef] [PubMed]

48. Friedberg, I.; Harder, T.; Godzik, A. JAFA: A protein function annotation meta-server. Nucleic Acids Res. 2006, 34, 379-381. [CrossRef] [PubMed]

49. Wass, M.N.; Barton, G.; Sternberg, M.J. Combfunc: Predicting protein function using heterogeneous data sources. Nucleic Acids Res. 2012, 40, 466-470. [CrossRef] [PubMed]

50. Jones, P.; Binns, D.; Chang, H.Y.; Fraser, M.; Li, W.; McAnulla, C.; McWilliam, H.; Maslen, J.; Mitchell, A.; Nuka, G.; et al. Interproscan 5: Genome-scale protein function classification. Bioinformatics 2014, 30, 1236-1240. [CrossRef] [PubMed]

51. Piovesan, D.; Giollo, M.; Leonardi, E.; Ferrari, C.; Tosatto, S.C. Inga: Protein function prediction combining interaction networks, domain assignments and sequence similarity. Nucleic Acids Res. 2015, 43, 134-140. [CrossRef] [PubMed]

52. Bandyopadhyay, S.; Ray, S.; Mukhopadhyay, A.; Maulik, U. A review of in silico approaches for analysis and prediction of hiv-1-human protein-protein interactions. Brief. Bioinform. 2015, 16, 830-851. [CrossRef] [PubMed]

53. Boratyn, G.M.; Camacho, C.; Cooper, P.S.; Coulouris, G.; Fong, A.; Ma, N.; Madden, T.L.; Matten, W.T.; McGinnis, S.D.; Merezhuk, Y.; et al. Blast: A more efficient report with usability improvements. Nucleic Acids Res. 2013, 41, 29-33. [CrossRef] [PubMed]

54. Pearson, W.R. Blast and fasta similarity searching for multiple sequence alignment. Methods Mol. Biol. 2014, 1079, 75-101. [PubMed]

55. Radivojac, P.; Clark, W.T.; Oron, T.R.; Schnoes, A.M.; Wittkop, T.; Sokolov, A.; Graim, K.; Funk, C.; Verspoor, K.; Ben-Hur, A.; et al. A large-scale evaluation of computational protein function prediction. Nat. Methods 2013, 10, 221-227. [CrossRef] [PubMed]

56. Jiang, Y.; Oron, T.R.; Clark, W.T.; Bankapur, A.R.; D’Andrea, D.; Lepore, R.; Funk, C.S.; Kahanda, I.; Verspoor, K.M.; Ben-Hur, A.; et al. An expanded evaluation of protein function prediction methods shows an improvement in accuracy. Genome Biol. 2016, 17, 184. [CrossRef] [PubMed]

57. Liang, Y.; Zhang, S. Predict protein structural class by incorporating two different modes of evolutionary information into chou's general pseudo amino acid composition. J. Mol. Graph. Model. 2017, 78, 110-117. [CrossRef] [PubMed]

58. Pradhan, D.; Padhy, S.; Sahoo, B. Enzyme classification using multiclass support vector machine and feature subset selection. Comput. Biol. Chem. 2017, 70, 211-219. [CrossRef] [PubMed]

59. Meher, P.K.; Sahu, T.K.; Banchariya, A.; Rao, A.R. Dirprot: A computational approach for discriminating insecticide resistant proteins from non-resistant proteins. BMC Bioinform. 2017, 18, 190. [CrossRef] [PubMed]

60. Zhu, F.; Han, L.; Zheng, C.; Xie, B.; Tammi, M.T.; Yang, S.; Wei, Y.; Chen, Y. What are next generation innovative therapeutic targets? Clues from genetic, structural, physicochemical, and systems profiles of successful targets. J. Pharmacol. Exp. Ther. 2009, 330, 304-315. [CrossRef] [PubMed]

61. Zhu, F.; Han, L.Y.; Chen, X.; Lin, H.H.; Ong, S.; Xie, B.; Zhang, H.L.; Chen, Y.Z. Homology-free prediction of functional class of proteins and peptides by support vector machines. Curr. Protein Pept. Sci. 2008, 9, 70-95. [PubMed] 
62. Zhu, F.; Zheng, C.J.; Han, L.Y.; Xie, B.; Jia, J.; Liu, X.; Tammi, M.T.; Yang, S.Y.; Wei, Y.Q.; Chen, Y.Z. Trends in the exploration of anticancer targets and strategies in enhancing the efficacy of drug targeting. Curr. Mol. Pharmacol. 2008, 1, 213-232. [CrossRef] [PubMed]

63. Li, Y.H.; Xu, J.Y.; Tao, L.; Li, X.F.; Li, S.; Zeng, X.; Chen, S.Y.; Zhang, P.; Qin, C.; Zhang, C.; et al. SVM-prot 2016: A web-server for machine learning prediction of protein functional families from sequence irrespective of similarity. PLoS ONE 2016, 11, e0155290. [CrossRef] [PubMed]

64. Cai, C.Z.; Han, L.Y.; Ji, Z.L.; Chen, Y.Z. Enzyme family classification by support vector machines. Proteins 2004, 55, 66-76. [CrossRef] [PubMed]

65. Han, L.Y.; Cai, C.Z.; Ji, Z.L.; Cao, Z.W.; Cui, J.; Chen, Y.Z. Predicting functional family of novel enzymes irrespective of sequence similarity: A statistical learning approach. Nucleic Acids Res. 2004, 32, 6437-6444. [CrossRef] [PubMed]

66. Shen, H.B.; Yang, J.; Chou, K.C. Fuzzy KNN for predicting membrane protein types from pseudo-amino acid composition. J. Theor. Biol. 2006, 240, 9-13. [CrossRef] [PubMed]

67. Nath, N.; Mitchell, J.B. Is EC class predictable from reaction mechanism? BMC Bioinform. 2012, 13, 60. [CrossRef] [PubMed]

68. Naveed, M.; Khan, A. Gpcr-mpredictor: Multi-level prediction of g protein-coupled receptors using genetic ensemble. Amino Acids 2012, 42, 1809-1823. [CrossRef] [PubMed]

69. Hayat, M.; Khan, A. Predicting membrane protein types by fusing composite protein sequence features into pseudo amino acid composition. J. Theor. Biol. 2011, 271, 10-17. [CrossRef] [PubMed]

70. Khan, Z.U.; Hayat, M.; Khan, M.A. Discrimination of acidic and alkaline enzyme using chou's pseudo amino acid composition in conjunction with probabilistic neural network model. J. Theor. Biol. 2015, 365, 197-203. [CrossRef] [PubMed]

71. Li, H.; Yap, C.W.; Ung, C.Y.; Xue, Y.; Li, Z.R.; Han, L.Y.; Lin, H.H.; Chen, Y.Z. Machine learning approaches for predicting compounds that interact with therapeutic and ADMET related proteins. J. Pharm. Sci. 2007, 96, 2838-2860. [CrossRef] [PubMed]

72. Fujimoto, M.S.; Suvorov, A.; Jensen, N.O.; Clement, M.J.; Bybee, S.M. Detecting false positive sequence homology: A machine learning approach. BMC Bioinform. 2016, 17, 101. [CrossRef] [PubMed]

73. Pearson, W.R. Protein function prediction: Problems and pitfalls. Curr. Protoc. Bioinform. 2015, 51, 1-18.

74. Boman, H.G. Peptide antibiotics and their role in innate immunity. Annu. Rev. Immunol. 1995, 13, 61-92. [CrossRef] [PubMed]

75. Hancock, R.E.; Diamond, G. The role of cationic antimicrobial peptides in innate host defences. Trends Microbiol. 2000, 8, 402-410. [CrossRef]

76. Radek, K.; Gallo, R. Antimicrobial peptides: Natural effectors of the innate immune system. Semin. Immunopathol. 2007, 29, 27-43. [CrossRef] [PubMed]

77. Iwamuro, S.; Kobayashi, T. An efficient protocol for DNA amplification of multiple amphibian skin antimicrobial peptide cDNAs. Methods Mol. Biol. 2010, 615, 159-176. [PubMed]

78. Brown, J.B.; Akutsu, T. Identification of novel DNA repair proteins via primary sequence, secondary structure, and homology. BMC Bioinform. 2009, 10, 25. [CrossRef] [PubMed]

79. Crappe, J.; Van Criekinge, W.; Trooskens, G.; Hayakawa, E.; Luyten, W.; Baggerman, G.; Menschaert, G. Combining in silico prediction and ribosome profiling in a genome-wide search for novel putatively coding sorfs. BMC Genom. 2013, 14, 648. [CrossRef] [PubMed]

80. Virgen-Slane, R.; Rozovics, J.M.; Fitzgerald, K.D.; Ngo, T.; Chou, W.; van der Heden van Noort, G.J.; Filippov, D.V.; Gershon, P.D.; Semler, B.L. An RNA virus hijacks an incognito function of a DNA repair enzyme. Proc. Natl. Acad. Sci. USA 2012, 109, 14634-14639. [CrossRef] [PubMed]

81. Cheng, X.; Xiao, X.; Chou, K.C. pLoc-mPlant: Predict subcellular localization of multi-location plant proteins by incorporating the optimal go information into general PseAAC. Mol. Biosyst. 2017, 13, 1722-1727. [CrossRef] [PubMed]

82. Cheng, X.; Xiao, X.; Chou, K.C. pLoc-mEuk: Predict subcellular localization of multi-label eukaryotic proteins by extracting the key go information into general PseAAC. Genomics 2018, 110, 50-58. [CrossRef] [PubMed]

83. Cheng, X.; Xiao, X.; Chou, K.C. pLoc-mVirus: Predict subcellular localization of multi-location virus proteins via incorporating the optimal go information into general PseAAC. Gene 2017, 628, 315-321. [CrossRef] [PubMed] 
84. Cheng, X.; Zhao, S.G.; Lin, W.Z.; Xiao, X.; Chou, K.C. Ploc-manimal: Predict subcellular localization of animal proteins with both single and multiple sites. Bioinformatics 2017, 33, 3524-3531. [CrossRef] [PubMed]

85. Qiu, W.R.; Sun, B.Q.; Xiao, X.; Xu, Z.C.; Jia, J.H.; Chou, K.C. iKCR-PseENs: Identify lysine crotonylation sites in histone proteins with pseudo components and ensemble classifier. Genomics 2017. [CrossRef] [PubMed]

86. Chou, K.C. Impacts of bioinformatics to medicinal chemistry. Med. Chem. 2015, 11, 218-234. [CrossRef] [PubMed]

87. Chou, K.C. An unprecedented revolution in medicinal chemistry driven by the progress of biological science. Curr. Top. Med. Chem. 2017, 17, 2337-2358. [CrossRef] [PubMed]

88. Chen, W.; Feng, P.; Yang, H.; Ding, H.; Lin, H.; Chou, K.C. iRNA-AI: Identifying the adenosine to inosine editing sites in RNA sequences. Oncotarget 2017, 8, 4208-4217. [CrossRef] [PubMed]

89. Cheng, X.; Zhao, S.G.; Xiao, X.; Chou, K.C. iATC-mISF: A multi-label classifier for predicting the classes of anatomical therapeutic chemicals. Bioinformatics 2017, 33, 341-346. [CrossRef] [PubMed]

90. Feng, P.; Ding, H.; Yang, H.; Chen, W.; Lin, H.; Chou, K.C. iRNA-PseCOLL: Identifying the occurrence sites of different rna modifications by incorporating collective effects of nucleotides into PseKNC. Mol. Ther. Nucleic Acids 2017, 7, 155-163. [CrossRef] [PubMed]

91. Liu, B.; Wang, S.; Long, R.; Chou, K.C. iRSpot-EL: Identify recombination spots with an ensemble learning approach. Bioinformatics 2017, 33, 35-41. [CrossRef] [PubMed]

92. Liu, B.; Yang, F.; Chou, K.C. 2l-pirna: A two-layer ensemble classifier for identifying piwi-interacting RNAS and their function. Mol. Ther. Nucleic Acids 2017, 7, 267-277. [CrossRef] [PubMed]

93. Liu, L.M.; Xu, Y.; Chou, K.C. iPGK-PseAAC: Identify lysine phosphoglycerylation sites in proteins by incorporating four different tiers of amino acid pairwise coupling information into the general PseAAC. Med. Chem. 2017, 13, 552-559. [CrossRef] [PubMed]

94. Qiu, W.R.; Jiang, S.Y.; Xu, Z.C.; Xiao, X.; Chou, K.C. iRNAm5C-PseDNC: Identifying RNA 5-methylcytosine sites by incorporating physical-chemical properties into pseudo dinucleotide composition. Oncotarget 2017, 8, 41178-41188. [CrossRef] [PubMed]

95. Qiu, W.R.; Sun, B.Q.; Xiao, X.; Xu, D.; Chou, K.C. iPhos-PseEVO: Identifying human phosphorylated proteins by incorporating evolutionary information into general PseAAC via grey system theory. Mol. Inform. 2017, 36. [CrossRef] [PubMed]

96. Su, Q.; Lu, W.; Du, D.; Chen, F.; Niu, B.; Chou, K.C. Prediction of the aquatic toxicity of aromatic compounds to tetrahymena pyriformis through support vector regression. Oncotarget 2017, 8, 49359-49369. [CrossRef] [PubMed]

97. Xu, Y.; Wang, Z.; Li, C.; Chou, K.C. iPreny-PseAAC: Identify c-terminal cysteine prenylation sites in proteins by incorporating two tiers of sequence couplings into PseAAC. Med. Chem. 2017, 13, 544-551. [CrossRef] [PubMed]

98. Chou, K.C. Some remarks on protein attribute prediction and pseudo amino acid composition. J. Theor. Biol. 2011, 273, 236-247. [CrossRef] [PubMed]

99. Chowdhury, S.Y.; Shatabda, S.; Dehzangi, A. iDNAProt-ES: Identification of DNA-binding proteins using evolutionary and structural features. Sci. Rep. 2017, 7, 14938. [CrossRef] [PubMed]

100. Filos, D.; Chouvarda, I.; Tachmatzidis, D.; Vassilikos, V.; Maglaveras, N. Beat-to-beat p-wave morphology as a predictor of paroxysmal atrial fibrillation. Comput. Methods Progr. Biomed. 2017, 151, 111-121. [CrossRef] [PubMed]

101. Rahimi, M.; Bakhtiarizadeh, M.R.; Mohammadi-Sangcheshmeh, A. Oogenesis_pred: A sequence-based method for predicting oogenesis proteins by six different modes of chou's pseudo amino acid composition. J. Theor. Biol. 2017, 414, 128-136. [CrossRef] [PubMed]

102. Sun, M.A.; Zhang, Q.; Wang, Y.; Ge, W.; Guo, D. Prediction of redox-sensitive cysteines using sequential distance and other sequence-based features. BMC Bioinform. 2016, 17, 316. [CrossRef] [PubMed]

103. Wang, Y.; Li, X.; Tao, B. Improving classification of mature microrna by solving class imbalance problem. Sci. Rep. 2016, 6, 25941. [CrossRef] [PubMed]

104. Meher, P.K.; Sahu, T.K.; Rao, A.R. Prediction of donor splice sites using random forest with a new sequence encoding approach. BioData Min. 2016, 9, 4. [CrossRef] [PubMed]

105. Bock, J.R.; Gough, D.A. Predicting protein-Protein interactions from primary structure. Bioinformatics 2001, 17, 455-460. [CrossRef] [PubMed] 
106. Karchin, R.; Karplus, K.; Haussler, D. Classifying g-protein coupled receptors with support vector machines. Bioinformatics 2002, 18, 147-159. [CrossRef] [PubMed]

107. Dobson, P.D.; Doig, A.J. Distinguishing enzyme structures from non-enzymes without alignments. J. Mol. Biol. 2003, 330, 771-783. [CrossRef]

108. Des Jardins, M.; Karp, P.D.; Krummenacker, M.; Lee, T.J.; Ouzounis, C.A. Prediction of enzyme classification from protein sequence without the use of sequence similarity. Proc. Int. Conf. Intell. Syst. Mol. Biol. 1997, 5, 92-99. [PubMed]

109. Du, R.; Mercante, D.; Fang, Z. An artificial functional family filter in homolog searching in next-generation sequencing metagenomics. PLoS ONE 2013, 8, e58669. [CrossRef] [PubMed]

110. Tian, W.; Skolnick, J. How well is enzyme function conserved as a function of pairwise sequence identity? J. Mol. Biol. 2003, 333, 863-882. [CrossRef] [PubMed]

111. Wommack, K.E.; Bhavsar, J.; Ravel, J. Metagenomics: Read length matters. Appl. Environ. Microbiol. 2008, 74, 1453-1463. [CrossRef] [PubMed]

112. Ju, Z.; He, J.J. Prediction of lysine propionylation sites using biased svm and incorporating four different sequence features into chou's pseaac. J. Mol. Graph. Model. 2017, 76, 356-363. [CrossRef] [PubMed]

113. Jia, J.; Liu, Z.; Xiao, X.; Liu, B.; Chou, K.C. iPPI-Esml: An ensemble classifier for identifying the interactions of proteins by incorporating their physicochemical properties and wavelet transforms into PseAAC. J. Theor. Biol. 2015, 377, 47-56. [CrossRef] [PubMed]

114. Jia, J.; Liu, Z.; Xiao, X.; Liu, B.; Chou, K.C. iCAR-PseCp: Identify carbonylation sites in proteins by monte carlo sampling and incorporating sequence coupled effects into general PseAAC. Oncotarget 2016, 7, 34558-34570. [CrossRef] [PubMed]

115. Liu, B.; Long, R.; Chou, K.C. iDHS-EL: Identifying DNASE I hypersensitive sites by fusing three different modes of pseudo nucleotide composition into an ensemble learning framework. Bioinformatics 2016, 32, 2411-2418. [CrossRef] [PubMed]

116. Liu, Z.; Xiao, X.; Yu, D.J.; Jia, J.; Qiu, W.R.; Chou, K.C. pRNAm-PC: Predicting n(6)-methyladenosine sites in rna sequences via physical-chemical properties. Anal. Biochem. 2016, 497, 60-67. [CrossRef] [PubMed]

117. Qiu, W.R.; Sun, B.Q.; Xiao, X.; Xu, Z.C.; Chou, K.C. iPTM-mLys: Identifying multiple lysine ptm sites and their different types. Bioinformatics 2016, 32, 3116-3123. [CrossRef] [PubMed]

118. Xu, Y.; Shao, X.J.; Wu, L.Y.; Deng, N.Y.; Chou, K.C. iSNO-AAPair: Incorporating amino acid pairwise coupling into pseaac for predicting cysteine s-nitrosylation sites in proteins. Peer] 2013, 1, e171. [CrossRef] [PubMed]

119. Chen, W.; Feng, P.M.; Lin, H.; Chou, K.C. iRSpot-PseDNC: Identify recombination spots with pseudo dinucleotide composition. Nucleic Acids Res. 2013, 41, e68. [CrossRef] [PubMed]

120. Chou, K.C. Some remarks on predicting multi-label attributes in molecular biosystems. Mol. Biosyst. 2013, 9, 1092-1100. [CrossRef] [PubMed]

(C) 2018 by the authors. Licensee MDPI, Basel, Switzerland. This article is an open access article distributed under the terms and conditions of the Creative Commons Attribution (CC BY) license (http:/ / creativecommons.org/licenses/by/4.0/). 



\section{MDPI}

St. Alban-Anlage 66

4052 Basel

Switzerland

Tel. +41616837734

Fax +41 613028918

www.mdpi.com

International Journal of Molecular Sciences Editorial Office

E-mail: ijms@mdpi.com www.mdpi.com/journal/ijms

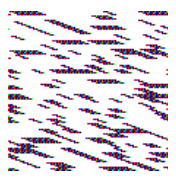



MDPI

St. Alban-Anlage 66

4052 Basel

Switzerland

Tel: +41 616837734

Fax: +41 613028918 